PREMIO TESI DI DOTTORATO

ISSN 2612-8039 (PRINT) | ISSN 2612-8020 (ONLINE)

$-79-$ 
PREMIO TESI DI DOTTORATO

Commissione giudicatrice, anno 2018

Vincenzo Varano, Presidente della Commissione

Tito Arecchi, Area Scientifica

Aldo Bompani, Area delle Scienze Sociali

Mario Caciagli, Area delle Scienze Sociali

Franco Cambi, Area Umanistica

Paolo Felli, Area Tecnologica

Giancarlo Garfagnini, Area Umanistica

Roberto Genesio, Area Tecnologica

Flavio Moroni, Area Biomedica

Adolfo Pazzagli, Area Biomedica

Giuliano Pinto, Area Umanistica

Vincenzo Schettino, Area Scientifica

Luca Uzielli, Area Tecnologica

Graziella Vescovini, Area Umanistica 
Elena Bellini

\section{Ambienti sensoriali "terapeutici" che rendano Abili}

Un progetto integrato di vita per persone con Disturbi dello Spettro Autistico 


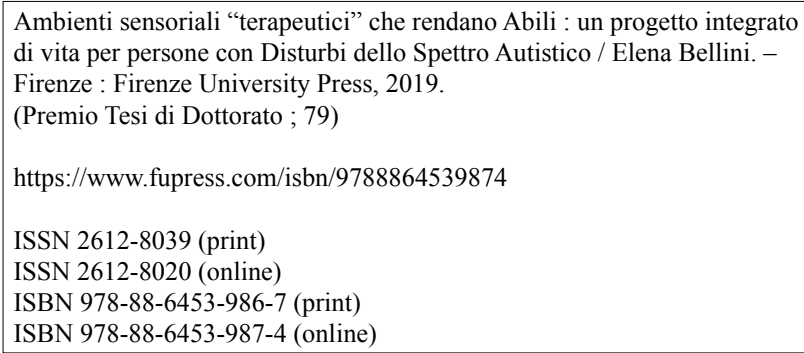

Graphic design: Alberto Pizarro Fernández, Lettera Meccanica SRLs

Peer Review Process

All publications are submitted to an external refereeing process under the responsibility of the FUP Editorial Board and the Scientific Committees of the individual series. The works published in the FUP catalogue are evaluated and approved by the Editorial Board of the publishing house. For a more detailed description of the refereeing process we refer to the official documents published on the website and in the online catalogue (www.fupress.com).

Firenze University Press Editorial Board

M. Garzaniti (Editor-in-Chief), M.E. Alberti, M. Boddi, A. Bucelli, R. Casalbuoni, A. Dolfi, R. Ferrise, M.C. Grisolia, P. Guarnieri, R. Lanfredini, P. Lo Nostro, G. Mari, A. Mariani, P.M. Mariano, S. Marinai, R. Minuti, P. Nanni, A. Orlandi, A. Perulli, G. Pratesi.

The online digital edition is published in Open Access on www.fupress.com.

Content license: the present work is released under Creative Commons Attribution 4.0 International license (CC BY 4.0: http://creativecommons.org/licenses/by/4.0/legalcode). This license allows you to share any part of the work by any means and format, modify it for any purpose, including commercial, as long as appropriate credit is given to the author, any changes made to the work are indicated and a URL link is provided to the license.

Metadata license: all the metadata are released under the Public Domain Dedication license (CC0 1.0 Universal: https://creativecommons.org/publicdomain/zero/1.0/legalcode).

(C) 2019 Author(s)

Published by Firenze University Press

Firenze University Press

Università degli Studi di Firenze

via Cittadella, 7, 50144 Firenze, Italy

www.fupress.com

This book is printed on acid-free paper

Printed in Italy 
A te che mi hai dato tutto, senza chiedere niente 



\section{Sommario}

Premesse

Capitolo 1

I Disturbi dello Spettro Autistico.

Quadro introduttivo e conoscitivo dal punto di vista sanitario e sociale

1.1 Definizione e riferimenti assunti

1.2 L'entità del problema. La scarsità dei dati disponibili

1.3 Le cause dell'autismo: dalla genetica ai fattori ambientali

1.4 I sintomi e i livelli di gravità in base alla diagnosi

1.5 L'approccio Abilitativo. Le recenti Linee Guida italiane

1.6 La normativa sull'autismo

Capitolo 2

Contesti ambientali, spazi per l'autismo e approcci progettuali.

Stato dell'arte

2.1 Design for Autism

2.1.1 Il profilo senso-percettivo. Il rapporto con il contesto ambientale $\quad 43$

2.1.2 Entrare in empatia. Il co-design 48

2.1.3 L'approccio al progetto. Sensory-Sensitive approach/Neuro-Typical approach. Ambiente ecologico/Ambiente strutturato

2.1.4 Descrizione del quadro esigenziale

2.2 Luoghi per l'apprendimento inclusivi e abilitanti.

Una scuola per tutti/una scuola specializzata

2.2.1 Introduzione

2.2.3 Riduzione del disagio sensoriale ed ambientale 
Ambienti sensoriali "terapeutici” che rendano Abili

2.2.4 Salute e benessere

2.2.5 Ambienti sicuri e durevoli 72

2.2.6 Orientamento e comprensione $\quad 73$

2.2.7 Indipendenza ed autonomia. Abilità $\quad 74$

2.2.8 Relazioni sociali. Integrazione e inclusività 75

2.3 Luoghi per l'abitare e l'autonomia.

Residenze protette/adattamento domestico 76

2.3.1 Introduzione. Le Linee Guida esistenti 76

$\begin{array}{ll}\text { 2.3.2 Riduzione del disagio sensoriale ed ambientale } & 79\end{array}$

2.3.3 Salute e benessere $\quad 84$

2.3.4 Ambienti sicuri e durevoli $\quad 85$

2.3.5 Orientamento e comprensione $\quad 88$

$\begin{array}{ll}\text { 2.3.6 Indipendenza ed autonomia. Abilità } & 89\end{array}$

2.3.7 Relazioni sociali. Integrazione e inclusività 92

2.4 Luoghi della cura.

Ospedale accessibile/formazione e assistenza sanitaria 93

2.4.1 Introduzione. Il diritto alla cura 93

2.4.2 L'incidenza dell'ospedalizzazione in casi di autismo.

I numeri, le cause $\quad 94$

2.4.3 Affrontare il percorso di cura.

Criticità e strategie per migliorare l'accessibilità 98

2.4.4 Il Pronto Soccorso.

Alcune risposte per l'area principale di emergenza 101

2.4.5 Nuove frontiere nei contesti dell'emergenza sanitaria. Sensory design $\quad 104$

Capitolo 3

$\begin{array}{ll}\text { Indagine sul campo. Persone 'reali' si raccontano } & 107\end{array}$

$\begin{array}{ll}3.1 \text { Introduzione } & 107\end{array}$

3.2 Testimonianze/Autobiografie e biografie $\quad 108$

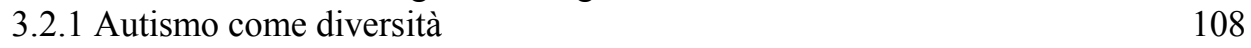

$\begin{array}{ll}3.2 .2 \text { Entrare in empatia } & 111\end{array}$

3.2.3 Ricevere la diagnosi $\quad 113$

$\begin{array}{ll}3.2 .4 \text { Profilo cognitivo } & 114\end{array}$

3.2.5 Profilo senso-percettivo $\quad 115$

$\begin{array}{ll}3.2 .6 \text { Ambiente educativo } & 119\end{array}$

3.2.7 La famiglia 124

3.2.8 Acquisire nuove abilità, diventare adulti e pensare al dopo di noi $\quad 125$

3.3 Questionari e interviste rivolte a genitori di bambini e adulti con

Disturbi dello Spettro Autistico e ad alcune persone con autismo ad alto funzionamento o Asperger 
3.4 Interviste ad adulti con DSA ad alto funzionamento o Asperger 141

$\begin{array}{ll}\text { 3.4.1 A. (maschio, } 25 \text { anni) } & 142\end{array}$

$\begin{array}{ll}\text { 3.4.2 L. (maschio, } 21 \text { anni) } & 143\end{array}$

3.4.3 F. (donna, 55 anni) 143

$\begin{array}{ll}\text { 3.4.4 V. (donna, } 37 \text { anni) } & 145\end{array}$

$\begin{array}{ll}3.4 .5 \mathrm{~F} . \text { (maschio, } 13 \mathrm{anni}) & 146\end{array}$

$\begin{array}{ll}\text { 3.4.6 D. (maschio, } 20 \text { anni) } & 148\end{array}$

3.4.7 C. (maschio, 17 anni) 149

$\begin{array}{ll}3.4 .8 \text { D. (maschio, } 45 \mathrm{nni}) & 149\end{array}$

3.4.9 M. (maschio, 55 anni) 150

3.5 Interviste ai familiari di bambini e ragazzi con DSA 151

3.5.1 Mamma di A. (femmina, 5 anni) 152

3.5.2 Mamma di E. (maschio, 14 anni) 153

3.5.3 Mamma di F. (maschio, 15 anni) 154

3.5.4 Mamma di E. (maschio, 6 anni) 155

3.5.5 Mamma di B. L. e B. M. (maschi, 4 e 11 anni) 156

3.5.6 Mamma di A. (maschio, 10 anni) 157

$\begin{array}{ll}\text { 3.5.7 Mamma di E. (maschio, } 6 \text { anni) } & 158\end{array}$

$\begin{array}{ll}\text { 3.5.8 Mamma di M. (maschio, 5 anni) } & 160\end{array}$

$\begin{array}{ll}\text { 3.5.9 Mamma di E. (maschio, } 18 \text { anni) } & 161\end{array}$

$\begin{array}{ll}\text { 3.5.10 Mamma di D. (maschio, } 7 \text { anni) } & 161\end{array}$

3.5.11 Mamma di M. (maschio, 17 anni) 162

3.6 Colloqui e focus group con caregivers ed esperti sull'autismo 162

Capitolo 4

Casi Studio. Interpretazione e problematiche emergenti 181

4.1 New Struan School - Alloa (UK) - 2005

$\begin{array}{ll}4.1 .1 \text { Il progetto } & 185\end{array}$

4.1.2 Dimensioni e strutturazione degli spazi di apprendimento 186

$\begin{array}{ll}\text { 4.1.3 Aree comuni e spazi esterni } & 187\end{array}$

4.1.4 Comfort luminoso $\quad 187$

$\begin{array}{ll}\text { 4.1.5 Comfort acustico } & 187\end{array}$

4.1.6 Studio del colore $\quad 188$

$\begin{array}{ll}\text { 4.1.7 Ambienti sicuri e durevoli } & 188\end{array}$

4.2 Forbush School for Autism - Hunt Valley (USA) - 2008

$\begin{array}{ll}\text { 4.2.1 Il progetto } & 189\end{array}$

$\begin{array}{lr}\text { 4.2.2 Prossemica e dimensione degli spazi } & 189\end{array}$

$\begin{array}{ll}\text { 4.2.3 Comfort luminoso } & 190\end{array}$

$\begin{array}{ll}\text { 4.2.4 Comfort acustico } & 190\end{array}$

$\begin{array}{ll}\text { 4.2.5 Materiali } & 190\end{array}$ 
4.3 Advance School for Developing Skills of Special Needs Children

- Qattameya (Cairo) - 2008

4.3.1 Inclusione

4.3.2 Distribuzione sensoriale

4.4.3 Orientamento e wayfinding

4.3.4 La strutturazione degli spazi di apprendimento

4.3.5 La strutturazione degli spazi di terapia

193

4.3.6 Spazi di apprendiemnto all'aperto

4.3.7 Strutturazione degli spazi per l'abitare

4.4 Northen School for Autism - Reservoir (Australia) - 2013

4.4.1 Il programma educativo e terapeutico

4.5 Villa delle Rogge - Pordenone - 2012

4.5.2 Distribuzione funzionale

4.6 Sweetwater Spectrum Community - Sonoma (USA) - 2013

4.6.1 Il progetto

4.6.2 Comfort termico e climatico

201

4.6.3 Comfort luminoso

4.6.4 Qualità dell'aria

4.6.5 Materiali e durabilità

4.7 Seniors House - Hinnerup (Danimarca) - 2014

4.7.1 La storia

4.7.2 L'approccio terapeutico

4.7.4 Il verde

4.7.5 Lo studio del colore

4.7.7 Flessibilità degli alloggi 205

$\begin{array}{ll}\text { 4.7.8 Favorire le relazioni } & 205\end{array}$

4.8 Casa Sebastiano - Coredo (Trento) - 2017 


\section{Elena Bellini}

4.8.2 L'architettura (intervista all'arch. Berti)

4.8.3 Materiali, colori, dispositivi e strategie di comfort

4.09 Royal Alexandra Children's Hospital - Brighton (UK) - 2007/2016 208

4.09.1 Il progetto

208

4.09.2 Umanizzazioen degli spazi ospedalieri

209

4.09.3 Abbattere lo stress ospedaliero

4.10 Emma Children's Hospital - Amsterdam (Paesi Bassi) - 2014

4.10.1 Il progetto

4.10.2 Il layout degli spazi

4.10.3 Interazione sensoriale

4.10.4 Orientamento e wayfinding

4.10.5 L'equilibrio sensoriale

4.11 Nemours Children's Hospital - Orlando (USA) - 2014/2016

Capitolo 5

Conclusioni e indicazioni di sintesi

5.1 L'ambiente residenziale

5.1.1 Residenza in famiglia, adattamento domestico

Capitolo 6

Proposta di un ambiente sperimentale:

Lo spazio filtro all'emergenza sanitaria

6.1 La stanza sensoriale di attesa autism-friendly presso il Pronto Soccorso dell'Azienda Ospedaliero-Universitaria di Careggi a Firenze 
6.3 Il progetto della stanza di attesa autism-friendly

6.4 Il sistema di Assistive Technology

6.5 Feedback, limiti e sviluppi futuri del progetto

\section{Capitolo 7}

Proposta di una piattaforma di co-design: Condivisione di dati e conoscenze a supporto della progettazione partecipata

7.1 Introduzione

7.2 Piattaforme web per il design. Cosa già esiste e cosa per l'autismo

7.3 La proposta: DARE (sense)

7.4 Struttura e funzionamento della piattaforma

7.5 Fattibilità e funding.

Conclusioni, limiti della ricerca e sviluppi futuri

Bibliografia 


\section{Premessa}

La prima volta che ho parlato con C. ci siamo conosciuti via Skype. Ero molto nervosa, preoccupata di cosa avrei detto o fatto, di sbagliare qualcosa. Appena ci siamo presentati si leggeva in entrambi un po' di tensione; C. ad esempio aveva dei leggeri movimenti ripetitivi, che pian piano che si è rilassato sono spariti. Avrei voluto dirgli che probabilmente io ero più agitata di lui, che stavo muovendo il piede sotto il tavolo o arricciandomi i capelli intorno alle dita per calmarmi e cercare di sembrare disinvolta, ma non credo sarebbe servito. Cercavo di sorridere e trasmettere attraverso i miei occhi e la mia espressione che ero solo interessata a conoscerlo e sapere di più sul suo modo di vedere le cose. Di solito funziona! Dopo un po' che eravamo al telefono, che avevamo superato la corazza e la freddezza dei primi momenti, che avevamo parlato delle sue esperienze sensoriali, del gatto, della libreria del soggiorno uguale a quella che ho casa, della bellezza di avere un terrazzo dove poter camminare scalzi, del lavoro, degli interessi e tanto altro... mi ha confessato, ormai senza troppo imbarazzo, che era dall'inizio della chiamata che aveva un tessuto liscio sotto il tavolo che stava toccando e passando tra le dita per cercare di regolare i propri sensi e rilassarsi.

Essere adulti è imparare a conoscersi, imparare a capire quelle piccole cose che possono essere di supporto per migliorare la propria vita e superare i propri limiti.

F. mi ha detto durante un'intervista che la cosa che più può essere di sollievo per una persona con autismo é avere la possibilità di "regolare i propri sensi". Non serve molto... uno spazio in penombra e confortevole dove passare qualche minuto dopo le ore di lavoro, per riequilibrarsi dopo tutta una giornata di stress e di tempestamenti sensoriali continui di un "mondo esterno che fa male"; un luogo dove controllare le stimolazioni sensoriali e poter decidere sulla base delle proprie preferenze; un ambiente che non abbia imprevedibilità, ma sia pensato e calibrato sulla base delle proprie esigenze.

La casa è lo spazio dove ognuno di noi si sente maggiormente a proprio agio, è uno dei pochi luoghi che possiamo controllare e, soprattutto, dove possiamo scegliere; e comunque, non è poi così scontato riuscire sempre a trovare una risposta adeguata ai propri bisogni.

Ma cosa succede nel mondo esterno?

I Disturbi dello Spettro Autistico, come definito anche dal DSM V, presentano in generale deficit persistente nella comunicazione e nell'interazione sociale e comportamenti, attività e interessi ristretti e ripetitivi, tra cui rientrano anche iper- o ipo- 
sensibilità sensoriale. La pubblicazione di Olga Bogdashina ${ }^{1}$, riletta dopo due anni che avevo intrapreso questo percorso di ricerca, mi ha molto colpito e aiutato a mettere in ordine tante cose che fino ad allora avevo letto, visto o sentito.

Tutto ciò che conosciamo sul mondo e su noi stessi ha avuto origine attraverso i nostri sensi. Fin da bambini l'apprendimento rispetto al contesto circostante avviene attraverso gli stimoli sensoriali. Gli stimoli sono dall'uomo percepiti attraverso i sensi primari e reinterpretati, fino alla comprensione del contesto e alla costituzione di un concetto a livello cognitivo. L'esperienza dei nostri sensi e la memoria delle esperienze passate costruisce quello che siamo e il nostro modo di vivere e rapportarsi con il mondo esterno. Le sensibilità e le percezioni sensoriali sono pertanto all'origine della comprensione del mondo circostante e dell'interazione con esso e si possono quindi anche ritenere all'origine delle problematiche spesso riscontrate in autismo nella comunicazione e nel rapporto con gli altri e dei cosiddetti comportamenti problema, derivati dalla sovrastimolazione e dall'accumulo di stress e ansia.

Come non pretendiamo che una persona diversamente abile si alzi e cammini, così non possiamo pretendere che una persona con autismo si adatti a noi e ad un mondo progettato perlopiù per neurotipici, solo perché le loro diversità sono poco visibili.

Immaginiamo di stare in una classe e voler ascoltare l'insegnante che spiega, ma non riuscire a distinguere la sua voce rispetto a quelle dei compagni che bisbigliano sullo sfondo. Cade un astuccio pieno di penne ed un frastuono interrompe lo scorrere delle parole... fa male. Le luci iniziano a vibrare e si sente un continuo ronzio che proviene dal soffitto, fastidioso come quello di una mosca nell'orecchio. Ora la maestra dice di scrivere qualcosa sul quaderno, ma aprono una finestra e si sente il rumore del traffico che proviene da fuori, le voci dei passanti. Si sente un odore diverso che fa venire un po' di nausea. Entrano i raggi del sole, al cui interno si vedono tanti piccoli granelli di polvere e seguendo il fascio di luce sul muro si scorgono i riflessi che fanno tanti giochi di colore. Alla parete però ci sono tantissime indicazioni, cartelloni, colori, immagini, con molti dettagli da rielaborare. E all'improvviso di nuovo nel mezzo la maestra... viene fatta una domanda, ma non sentiamo...come rispondere?

Le sovrastimolazione che si genera può limitare la concentrazione e quindi l'apprendimento, ma anche la comunicazione e la relazione con gli altri. Immaginando ciò che può essere provocato in uno spazio così contenuto come un aula, si può facilmente comprendere che non é niente in confronto a quello che possono scaturire gli stimoli di un contesto come un supermercato, un centro commerciale, un ristorante o un luogo pubblico. Il primo aspetto da considerare quindi, cercando di conoscere l'autismo, è cercare di comprendere il sovraccarico di stimoli che provengono dall'ambiente circostante.

Ma anche imparando a conoscere più da vicino l'autismo, siamo sicuri di poter riuscire a capire davvero le persone, che spesso hanno linguaggio verbale assente e

\footnotetext{
${ }^{1}$ Bogdashina, O. (2003) Sensory perceptual issues in Autism and Asperger Syndrome, London: Jessica Kingsley
} 


\section{Elena Bellini}

non possono descriverci quello che provano? E ancora, c'è sempre un "perché" in risposta ad ogni azione o sensazione?

Mi è capitato di intervistare alcuni genitori che hanno ammesso di non saper rispondere ad alcune domande, poiché non erano effettivamente sicuri di quello che il figlio avrebbe provato in quella determinata circostanza o contesto ambientale. Non é detto oltretutto che ci sia sempre una spiegazione logica ai pensieri, alle sensazioni o agli eventi. L. ad esempio mi ha raccontato che non sopporta i rumori, si "protegge" quando è nel traffico mettendo una musica piuttosto alta nelle cuffie che sormonti i rumori dell'esterno, imprevedibili e spaventosi, ma allo stesso tempo una delle sue playlist preferite è un medley composto da suoni di sale gioco e casinò. Com'è possibile provare disagio dai rumori del traffico e non da quelli meccanici e frastornanti delle sale gioco? Mi ha fatto ascoltare le sue playlist, suddivise in base al desiderio di calma e relax piuttosto che di energia, ed è stato utile per entrare in empatia e cercare di carpire qualcosa del suo modo di vedere il mondo, ma non credo di esserci riuscita fino in fondo.

Probabilmente non è possibile comprendere del tutto e, anche riuscendoci, ogni persona è diversa dall'altra, per cui non è possibile pensare di poter avere una risposta pronta ed univoca, adeguata a tutta l'ampiezza dello spettro autistico.

Dovremmo dire quindi che non si può parlare di "progettazione universale" in ambito di autismo?

Quando si parla di "ambienti di vita" spesso ci si concentra sui luoghi dell'abitare e quindi "la casa", ma se riflettiamo sono tantissimi i luoghi dove viviamo tutti i giorni. Parlando con i genitori di alcuni bambini con autismo e con ragazzi e adulti Asperger o ad alto funzionamento, hanno quasi tutti convenuto che la casa è il luogo dove stanno meglio e riescono a ritrovare il loro equilibrio, ma non è sempre così. Mi sono state raccontate situazioni diverse dove al contrario è proprio la residenza a creare disagio poiché non adeguata ai propri bisogni. Una famiglia mi ha contattato tramite un'associazione poiché non sapeva come far fronte ai comportamenti problema del figlio in casa, non riuscendo a mettere più niente nella sua stanza poiché poteva danneggiare qualcosa o soprattutto farsi del male. Non è sempre facile.

Più i ragazzi crescono più le difficoltà aumentano, diventano grandi e forti ed $\mathrm{i}$ genitori sempre più piccoli e deboli. E le famiglie si domandano "che sarà dopo di noi"? Raggiungere autonomia e autodeterminazione é una sfida che va combattuta fin da piccoli, a partire dalla scuola, il centro per la terapia, la casa stessa e la famiglia, al fine di apprendere le abilità quotidiane e sociali e cercare di integrarsi nella comunità. Prima si inizia a comprendere, prima si può lavorare per migliorare la qualità di vita delle persone e delle loro famiglie.

D. mi ha parlato a lungo della scuola e del passaggio all'università come un momento di grande difficoltà e stress, del confronto con gli altri, della difficoltà nell'avere rapporti con i coetanei. Molti adulti Asperger o autistici ad alto funzionamento mi hanno invece descritto l'ambiente di lavoro come il luogo di maggiore stress, come probabilmente si riscontra in generale anche per molte altre persone neurotipiche: è un ambiente "naturale" e non controllabile, dove ci sono tanti stimoli contrastanti, tante persone con cui relazionarsi, in situazioni che a volte possono essere ambigue e poco comprensibili. L. mi ha raccontato ad esempio che non riusciva a lavorare nella stanza con i suoi colleghi poiché c'erano delle luci al neon che gli 
provocavano un forte disagio a livello visivo. Aveva scelto di lavorare nella stanza delle fotocopie poiché era buia e riusciva a sentirsi meglio. Quando hanno posto rimedio migliorando la qualità luminosa ed è tornato nell'ambiente con gli altri, ha dovuto far fronte a tanti altri problemi, come ad esempio la confusione o i rumori derivanti dalle altre persone all'interno dell'open space, per cui adesso ha difficoltà a concentrarsi e rimanere attento sul lavoro. V. dice che mentre lavora non può ascoltare neanche la radio o la musica poiché altrimenti si distrae. In molti, in generale, hanno descritto la difficoltà a sopportare stimoli simultanei.

Sono tanti gli aspetti che possono influire sul benessere generato dall'ambiente, ma chi dice che gli interventi sulla scuola, o ad esempio sull'ambiente di lavoro, pensati per l'autismo, non potrebbero migliorare anche la condizione di stress di tutte le altre persone?

Tutti i bambini hanno bisogno di concentrarsi ed avere un'attenzione maggiore sicuramente favorisce l'apprendimento. E' dimostrato come il luogo di lavoro provochi stress, ansia, frustrazione, depressione o altre patologie croniche. Passiamo gran parte delle nostre giornate e della nostra vita nell'ambiente di lavoro, per cui non possiamo pensare che non abbia influenza sulla nostra qualità di vita.

Probabilmente è vero che non è sempre semplice comprendere il diverso punto di vista di una persona con autismo, le sue particolari esigenze sensoriali, la grande sensibilità che presentano e così via, ma sono veramente così lontani? A me non sembra!

Ho apprezzato moltissimo un'iniziativa di una trasmissione televisiva dell'emittente britannica Channel 4 che si chiama "How Autistic Are You?", che ha promosso un breve test online per capire "quanto autismo c'è in te". L'iniziativa è stata creata con lo scopo di "demistificare uno dei più sconosciuti disordini neurologici della Gran Bretagna" ed aumentare la consapevolezza e la conoscenza di questo spettro, considerando che in Gran Bretagna è stato rilevato che 1 persona su 100 presenta qualche disturbo legato allo spettro autistico. Ovviamente ho fatto il test e non è venuto molto diverso da quello che mi aspettavo dopo aver letto, studiato e parlato con molte persone autistiche o vicine all'autismo.

La cosa che però ho trovato più divertente, durante questo percorso di ricerca, è che tutte le persone che ho intervistato o con cui ho avuto modo di parlare ad un certo punto della discussione mi hanno chiesto: "ma perché hai scelto proprio questo tema di ricerca?"; forse questo risultato potrà aiutarmi a spiegarlo meglio! 


\section{Capitolo 1}

\section{Disturbi dello Spettro Autistico \\ Quadro introduttivo e conoscitivo}

I Disturbi dello Spettro Autistico sono definiti come disturbi neurologici con deficit persistenti nella comunicazione e nell'interazione sociale, che presentano comportamenti, interessi e/o attività ristrette e ripetitive e una caratterizzazione comune di sensibilità all'ambiente, attraverso iper- o ipo- reattività agli stimoli sensoriali (APA, 2013 $)^{2}$. L'Autismo è una sindrome comportamentale causata da un disordine dello sviluppo biologicamente determinato, con esordio nei primi tre anni di vita. Anche se si può evidenziare già nei primi 22-24 mesi di vita, conviene aspettare il trentesimo mese prima di effettuare una diagnosi certa (Mazzone, 2015) ${ }^{3}$. Si configura come una disabilità "permanente" che accompagna la persona per tutta la vita, anche se le caratteristiche del deficit sociale assumono un'espressività variabile nel tempo (Levi, 2005). ${ }^{4}$

"L'autismo...non se ne va...Ciò nondimeno, gli individui autistici possono compensare i loro handicap in misura notevole, e spesso lo fanno. (tuttavia) resta un deficit persistente...qualcosa che non può essere corretto o sostituito". (Frith, 1989) ${ }^{5}$

Nell'autismo grave è frequente l'associazione con il ritardo mentale (Q.I. é inferiore a 70; tende a rimanere stabile a prescindere dall'evoluzione dei sintomi autistici) ${ }^{6}$, con l'epilessia (l'affezione che maggiormente si presenta in casi di autismo; se nella popolazione generale ha un'incidenza del $0,5 \%$, in casi di autismo si aggira intorno a $4-47 \%$, con particolare rischio tra i primi 3 anni di vita e la pubertà) ${ }^{7}$ e i disturbi del sonno. L'autismo si trova inoltre talvolta associato a sindromi o malattie rare a base genetica che compromettono la normale funzionalità del Sistema Nervoso Centrale, quali la sclerosi tuberosa, la sindrome di Rett, la sindrome di Down, la

\footnotetext{
${ }^{2}$ American Psychiatric Association (APA) (2013) DSM V, Diagnostic and Statistical Manual of Mental Disorders $\mathrm{V}$

${ }^{3}$ Mazzone, L. (2015) Un autistico in famiglia, Mondadori, Milano

${ }^{4}$ Levi, G. (a cura di) (2005) Linee Guida Per L'autismo Raccomandazioni Tecniche-Operative Per I Servizi Di Neuropsichiatria Dell'eta' Evolutiva, SINPIA - Societa' Italiana Di Neuropsichiatria Dell'infanzia E Dell'adolescenza, Roma

${ }^{5}$ Frith, U. (1989) Autism: Explaining the Enigma, Wiley-Blackwell, Hoboken, New Jersey, Stati Uniti

${ }^{6}$ Bartolomeo, S. Cerquiglini, A. (2010) Il quotidiano dei soggetti autistici: definizione dei programmi terapeutici e di socializzazione e fabbisogno di servizi, in Giofré, F. (a cura di) (2010) Autismo. Protezione sociale e architettura, Alinea, Firenze, pp. 17-27

${ }^{7}$ ibidem
} 
sindrome di Landau-Kleffner, la fenilchetonuria, la sindrome dell'X fragile (ISS, 2012) $)^{8}$.

\subsection{Definizione e riferimenti assunti}

Il termine autismo é stato usato per la prima volta nel 1911 dallo psichiatra Bleuler, per descrivere un sintomo fondamentale della schizofrenia, riferendosi ad un "distacco completo dalla realtà e dal contatto con il mondo esterno, con la predominanza assoluta della vita interiore" (Bleuler, 1911, cit. In Milton, 2012) ${ }^{9}$. Negli anni '40 Kanner e Asperger sono stati i primi studiosi a definire quasi contemporaneamente questa nuova sindrome come autismo infantile precoce e disturbo del contatto affettivo (Kanner, 1943) ${ }^{10}$ e autistichen psychopathen (Asperger, 1944) ${ }^{11}$, pur lavorando in modo assolutamente indipendente in due diversi luoghi del mondo.

Kanner descrive un complesso di sintomi riscontrati in 11 bambini ( 9 maschi e 2 femmine) diversi dai casi che aveva fino ad allora riscontrato (come schizofrenia e ritardo mentale), facendo riferimento in particolare alla tendenza alla solitudine, al rifiuto di contatto sociale, allo sviluppo anormale del linguaggio (ecolalia) e ad una paura ossessiva dei cambiamenti dell'ambiente, con presenza di comportamenti ripetitivi e stereotipati. I pazienti che ha osservato sembravano vivere in un mondo tutto loro, ignorando chi avevano intorno, passando il tempo dedicandosi per ore a piccoli rituali, come ad esempio far girare il coperchio di una pentola sul pavimento. Allo stesso tempo, erano molto spaventati dai cambiamenti e potevano cadere nel panico alla più piccola variazione dell'ambiente circostante, come la posizione di una sedia o di uno dei giochi preferiti. Alcuni non presentavano linguaggio verbali, altri si limitavano a ripetere alcune frasi oppure parlavano di sé con distacco, in terza persona. Sostenendo che la loro condizione differiva in modo "marcato ed inequivocabile" da tutto quanto la letteratura clinica aveva riportato sino a quel momento, Kanner chiamò questa sindrome autismo (dal greco autos, sé), perché quei bambini sembravano più felici in solitudine (Silberman, 2015) ${ }^{12}$.

Un anno dopo Asperger ha definito un disturbo di una determinata popolazione infantile con sintomi in gran parte simili a quelli descritti da Kanner, ma con capacità cognitive nettamente superiori, sia nel linguaggio che nella capacità di apprende-

\footnotetext{
${ }^{8}$ ISS - Istituto Superiore della Salute (2012) Linee di indirizzo per la promozione ed il miglioramento della qualità della qualità e dell'appropriatezza degli interventi assistenziali nei Disturbi pervasivi dello sviluppo (DPS), con particolare riferimento ai disturbi dello spettro autistico, Ministero della Salute, Roma

${ }^{9}$ Milton, D. (2012) So what exactly is autism?, AET Competence framework for the Department for Education, Birmingham

${ }^{10}$ Kanner, L. (1943) Autisic disturbances and affective contact, The nervous Child, vol. 2, n ${ }^{\circ}$ 3, pp. 217 250 - Traduzione Italiana: Disturbi autistici e contatto affettivo, Psicoterapia e scienze umane, $\mathrm{n}^{\circ} 2$ e 3 , 1989

${ }^{11}$ Asperger, H. (1944) Die “ Autistiche psychopathen” im Kindesalter, Archiv fur psychiatrie und Nervenkrankheiten, vol. 117 - trad. in Frith, U. (1991) Autism and Asperger syndrome, New York: Cambridge University press, pp.37-92

${ }^{12}$ Silberman, S. (2015) Neuro Tribes. The legacy of Autism and the Future of Neurodiversity, AVERY, New York (ed. italiana 2016)
} 


\section{Elena Bellini}

re, descrivendoli come "pensatori astratti"; più avanti si ipotizzò, infatti, che la Sindrome di Asperger fosse caratteristica delle persone autistiche con QI relativamente elevato (Ballerini, 2013) ${ }^{13}$. Nello specifico, quattro tra i pazienti di Asperger, bambini tra i 7 e i 10 anni, scelti come "casi prototipi" per la sua tesi di dottorato, sembravano essere stranamente non in contatto con le altre persone, compresi i genitori. Diversamente dai pazienti di Kanner si esprimevano con proprietà del linguaggio e frasi elaborate, e dimostravano precoci capacità nelle materie scientifiche ed in matematica. Asperger li soprannominò affettuosamente i suoi "piccoli professori" e definì autismo la loro condizione, nonostante sia ancora controverso se quella osservata nella sua clinica fosse la stessa sindrome descritta da Kanner (Silberman, 2015) ${ }^{14}$. Si consideri, in ogni caso, che nel 10\% circa dei bambini autistici, si presentano talenti singolari, che di solito emergono in età molto precoce e si sviluppano a velocità sorprendente (Sacks, 1995) ${ }^{15}$. L'approccio alla diagnosi si basava sul metodo di osservazione intensiva sviluppato da Lazar, ovvero studiando il bambino nel corso della vita quotidiana in classe, durante il gioco, al tavolo dove mangiava e mentre riposava, praticamente vivendo insieme ai bambini stessi. Nel corso di un decennio, alla Heilpadagogik Station Asperger, Asperger e il suo staff (Anni Weiss, lo psichiatra Georg Frankl, lo psicologo Josef Feldner e Suor Viktorine Zak) esaminarono più di duecento bambini che manifestavano un insieme analogamente sorprendente di difficoltà sociali, abilità precoci e attrazioni per le regole, leggi e programmi. Sembravano, inoltre, particolarmente disturbati da cambiamenti imprevisti dell'ambiente o eventi non previsti o non corrispondenti alle loro aspettative. Tendevano a comportarsi in modo rigoroso e ad essere caratterizzati da routines molto strette e ripetitive, come se cercassero in qualche modo di dominare il loro caos. Talvolta, in casi più estremi, la routine si trasformava anche in movimenti ripetitivi e stereotipati, come ad esempio il dondolarsi avanti e indietro, battere su pareti e tavoli, ripetere la stessa frase all'infinito, ecc. Erano fasci di paradossi: precoci e infantili, sofisticati e ingenui, maldestri ma formali, scostanti ma solitari, intonati alla musica delle parole ma insensibili al ritmo dell'interazione reciproca (Silberman, 2015) ${ }^{16}$.

La definizione Autistichen Psychopathen (psicopatici autistici) deriva da un termine del XIX secolo, che va ad indicare lo sfumato confine tra salute e malattia. Asperger utilizzò anche il termine Autismus, ad indicare un' "entità naturale", qualcosa che resta "inconfondibile e costante lungo tutto il corso della vita", affermando che esso abbracciava una gamma incredibilmente ampia di persone, dalle più dotate alle più disabili. Sembravano esistere tante varietà di autismo quante persone autistiche (Silberman, 2015) ${ }^{17}$.

\footnotetext{
${ }^{13}$ Ballerini, L. (2013) I criteri diagnostici per l'autismo. 1943-2013: 70 anni di confusione, Contributo al $1^{\circ}$ simposio Prima rappresentanza e psicopatologia. La psicopatologia precoce del 16 novembre 2013

${ }^{14}$ Silberman, S. (2015) Neuro Tribes. The legacy of Autism and the Future of Neurodiversity, AVERY, New York (ed. italiana 2016)

${ }^{15}$ Sacks, O. (1995) Un antropologo su Marte, Adelphi, Milano

${ }^{16}$ Silberman, S. (2015) Neuro Tribes. The legacy of Autism and the Future of Neurodiversity, AVERY, New York (ed. italiana 2016)

${ }^{17}$ ibidem
} 
Ancora oggi non è chiaro se via sia una differenza radicale tra "autismo infantile classico" e la "sindrome di Asperger" o se piuttosto esista un continuum che va dai casi più gravi di autismo infantile, fino agli individui più dotati e altamente funzionali, o addirittura agli individui con "tratti autistici". La principale differenza probabilmente è che le persone con sindrome di Asperger sono in grado di raccontarci la loro esperienza, i loro sentimenti e i loro stati interiori, mentre le persone con autismo classico non sono in grado di farlo. E' vero anche che solitamente si tende a pensare solo ai bambini, quando si parla di autismo, ma che invece si tratta di una condizione pervasiva dello sviluppo, che riguarda anche ragazzi ed adulti, che solo a volte sono in grado di acquisire discrete capacità di linguaggio o abilità sociali, vivendo una vita autonoma in apparenza piena e "normale", definiti anche persone autistiche "altamente funzionali" (Sacks, 1995) ${ }^{18}$.

Ad ogni modo, la tesi di Asperger é stata per decenni una nota a margine della storica impresa di Leo Kanner, considerato un pioniere di quella che era chiamata appunto "sindorme di Kanner". Contribuirono in questo, da un lato Kanner stesso, che favorì l'idea che il lavoro di Asperger non fosse degno di seria considerazione; dall'altro la situazione socio-politica della Germania, che portò i medici e i ricercatori di America ed Europa a non essere particolarmente inclini a leggere testi tradotti dal tedesco (Silberman, 2015) ${ }^{19}$.

Negli anni successivi un errore interpretativo di Leo Kanner identificato nella "freddezza" nei genitori e nei rapporti con i figli, ha delineato involontariamente le basi per la teoria che la causa dell'autismo fosse individuabile in un ipotetico "rapporto inadeguato" con la madre, definita anche come "madre frigorifero", durata purtroppo per decenni stigmatizzando le famiglie di tutto il mondo. L'ampliamento del concetto kanneriano di "genitore tossico" ha portato negli anni fino alla teoria della "fortezza vuota" 20 di Bettelheim: "il neonato, se completamente abbandonato dagli esseri umani prima di essersi sviluppato abbastanza da cavarsela da solo, morirà, $\mathrm{e}$ se le cure fisiche sono sufficienti per la sua sopravvivenza ma é abbandonato emotivamente o spinto al di là della sua capacità di sopportazione, diventerà autistico" (Bettelheim, cit. in Silberman, 2015 $5^{21}$ ).

Nel 1964 Bernard Rimland, genitore di un bambino autistico, pubblicò 'Infantile Autism: the Syndrome and its Implications for a Neural Theory of Behaviour, con prefazione di Kanner, sostenendo, dopo decenni di confusione, che si trattava di una "disabilità percettiva" congenita, piuttosto che di una forma di psicosi causata da un trauma infantile. Con questa pubblicazione, finalmente, Rimland liberò in qualche modo i genitori dall'opprimente fardello della colpa, rendendo obsoleta la logica dell'internamento dei bambini in istituto "per il loro bene" (Silberman, 2015) ${ }^{22}$.

\footnotetext{
${ }^{18}$ Sacks, O. (1995) Un antropologo su Marte, Adelphi, Milano

${ }^{19}$ Silberman, S. (2015) Neuro Tribes. The legacy of Autism and the Future of Neurodiversity, AVERY, New York (ed. italiana 2016)

${ }^{20}$ Bettheleim, B. (1967) The Empty Fortress: Infantile Autism and the Birth of the Self, The Free Press, New York

${ }^{21}$ ibidem

${ }^{22}$ Silberman, S. (2015) Neuro Tribes. The legacy of Autism and the Future of Neurodiversity, AVERY, New York (ed. italiana 2016)
} 
Proprio alla fine degli anni '70 sarà infatti dimostrata l'importanza del carico genetico e numerosi studi testimoniarono come nessuna deprivazione relazionale potesse generare autismo in assenza di specifica vulnerabilità, rendendo insostenibile l'idea dell'autismo come "conseguenza" di una qualche deprivazione psicologicosociale, depressione, o chiusura del bambino (Barale et al., 2009) ${ }^{23}$.

Uno degli sviluppi più importanti nella storia dell'autismo é stato rappresentato dal lavoro di Wing and Gould (1979), che per primi hanno inserito all'interno dello spettro la sindrome di Asperger. Questo lavoro ha inoltre definito l'autismo come una "triad of impairments" in comunicazione sociale, interazione sociale e immaginazione (interessi e attività ripetitive), classificazione utilizzata fino a pochi anni fa, prima che fosse effettuata la revisione del DSM V dell'APA (2013). Questi ricercatori ipotizzarono che la comparsa simultanea di queste tre menomazioni non fosse casuale e che gli individui autistici, inoltre, non presentassero una reale percezione della mente altrui e della propria, altrimenti detta "teoria della mente" (Sacks, $1995)^{24}$.

Negli anni successivi, tra la fine degli anni '70 e gli anni ' 90 , si sono susseguite quindi varie evoluzioni, tra cui il passaggio dal concetto di "psicosi infantile" a quello di "disturbo generalizzato (o pervasivo) dello sviluppo", prendendo atto che l'autismo è tipicamente una condizione che dura tutta la vita; la differenziazione tra autismo e schizofrenia, identificata per esordio, evoluzione, sintomatologia clinica, epidemiologia, fenomeni tipici, fattori di rischio, distribuzione maschio/femmina, associazione con altre patologie (in particolare con il ritardo mentale e con l'epilessia), genetica; lo sviluppo della triade Wing-Gould, introducendo la nozione di "spettro autistico", ovvero la possibilità di avere una diversa combinazione delle alterazioni nell'ambito dei tre domini della triade; introduzione del "disturbo di Asperger" (Barale et al., 2009) 25 .

A partire dalla metà degli anni '80, Leslie, Baron-Cohen, Frith, Perner e altri (partendo da una rielaborazione del costrutto di Premack e Woodruff) ipotizzarono che all'origine dell'autismo vi fosse un deficit specifico della già accennata "teoria della mente", ovvero la capacità di riflettere sulle emozioni, sui desideri e sulle credenze proprie ed altrui e di comprendere il comportamento degli altri in rapporto non solo a quello che ciascuno di noi sente, desidera o conosce, ma in rapporto a quello che ciascuno di noi pensa che l'altro sente, desidera o conosce (Baron-Cohen et al., 2000) ${ }^{26}$. Il modello dell'autismo come deficit specifico di ToM (Theory of Mind) fu in ogni caso molto presto criticata dagli studiosi, poiché non riusciva a trovare spiegazione una parte importante della sintomatologia autistica (come ad esem-

\footnotetext{
${ }^{23}$ Barale, F. et al. (2009) L'autismo a partire dall'età adulta: nuove conoscenze, criticità, implicazioni abilitative, NÓOs, 15(3):257-91

${ }^{24}$ Sacks, O. (1995) Un antropologo su Marte, Adelphi, Milano

${ }^{25}$ Barale, F. et al. (2009) L'autismo a partire dall'età adulta: nuove conoscenze, criticità, implicazioni abilitative, NÓOs, 15(3):257-91

${ }^{26}$ Baron-Cohen, S. et al. (2000) Understanding other mind. Perspectives from developmental neuroscience, Oxford: Oxford University Press
} 
pio tutta quella riguardante stereotipie, ritualismi, atipie della percezione e dell'attenzione, ecc.) (Barale et al., 2009) ${ }^{27}$.

Dalla fine degli anni '80, si assiste in USA e in Europa alla ripresa di un "filone affettivo", che cerca di riprendere e dare base empirica all'intuizione kanneriana dell'aspetto "primario" della debolezza interattiva autistica. La teoria socio-affettiva parte dal presupposto che l'essere umano nasca con una predisposizione innata ad interagire con l'altro (Hobson, 1993) ${ }^{28}$, definita con diversi termini, quali empatia non inferenziale (Hobson, 1989) ${ }^{29}$ o intersoggettività primaria (Trevarthen et al., $2001)^{30}$. Secondo la teoria socio-affettiva, pertanto, esisterebbe nell'autismo un'innata incapacità di interagire emozionalmente con l'altro, che porterebbe all'incapacità di imparare a riconoscere gli stati mentali degli altri, alla compromissione dei processi di simbolizzazione, al deficit del linguaggio, al deficit della cognizione sociale (Levi, 2005) ${ }^{31}$.

Un'altra ipotesi interpretativa sviluppata negli anni '90 è il modello del deficit di funzioni esecutive (EF). Questo ipotizza che nell'autismo siano compromesse alcune funzioni neuropsicologiche generali, chiamate appunto "funzioni esecutive", che sovraintendono alla pianificazione, controllo, monitoraggio, coordinamento ed esecuzione di azioni e di sequenze di azioni finalizzate (Barale et al., 2009) ${ }^{32}$. Molti dei comportamenti autistici sarebbero, secondo questa teoria, espressione di un deficit di abilità come l'impulsività, l'iperselettività, e la perseverazione (Ozonoff, 1997 ${ }^{33}$; Pennington et al., $1996^{34}$ cit. in Levi, $2005^{35}$ ). Molte caratteristiche di base dell'autismo sono fortemente coerenti con questa ipotesi, come ad esempio la rigidità, le stereotipie, l'intolleranza ai cambiamenti, la difficoltà di programmazione o di modifica degli schemi di risposta, la focalizzazione ristretta, ecc. (Barale et al., $2009)^{36}$.

\footnotetext{
${ }^{27}$ Barale, F. et al. (2009) L'autismo a partire dall'età adulta: nuove conoscenze, criticità, implicazioni abilitative, NÓOs, 15(3):257-91

${ }^{28}$ Hobson, R.P. (1993) Autism and the development of mind, Hove, Sussex:Erlbaum

${ }^{29}$ Hobson, R. P. et al. (1989) Naming emotion in faces and voices: Abilities and disabilities in autism and mental retardation, British Journal of Developmental Psychology, vol. 7, n. 3, Blackwell Publishing Ltd, pp. 237-250

${ }^{30}$ Trevarthen, C. Aitken, K.J. (2001) Infant intersubjectivity: research, theory, and clinical applications, Journal of Child Psychology and Psychiatry, 42: 3-48

31 Levi, G. (a cura di) (2005) Linee Guida Per L'autismo Raccomandazioni Tecniche-Operative Per I Servizi Di Neuropsichiatria Dell'eta' Evolutiva, SINPIA - Societa' Italiana Di Neuropsichiatria Dell'infanzia E Dell'adolescenza, Roma

${ }^{32}$ Barale, F. et al. (2009) L'autismo a partire dall'età adulta: nuove conoscenze, criticità, implicazioni abilitative, NÓOs, 15(3):257-91

${ }^{33}$ Ozonoff, S. (1997) Components of executive function deficits in autism and other disorders, in Russel J. (Ed.) (1997) Autism as an executive disorder, Oxford: Oxford University Press, pp. 179-211

34 Pennington, B.F. Ozonoff, S. (1996) Executive functions and developmental psychopathology, Journal of Child Psychology and Psychiatry, 37: 51-87

${ }^{35}$ Levi, G. (a cura di) (2005) Linee Guida Per L'autismo Raccomandazioni Tecniche-Operative Per I Servizi Di Neuropsichiatria Dell'eta' Evolutiva, SINPIA - Societa' Italiana Di Neuropsichiatria Dell'infanzia E Dell'adolescenza, Roma

${ }^{36}$ Barale, F. et al. (2009) L'autismo a partire dall'età adulta: nuove conoscenze, criticità, implicazioni abilitative, NÓOs, 15(3):257-91
} 


\section{Elena Bellini}

L'ultima teoria sviluppata negli anni più recenti è infine il modello della coerenza centrale (CC) (Frith et al., 1994 $4^{37}$; Happé et al., $1996^{38}$ ), ovvero quella capacità di sintetizzare in un tutto coerente le molteplici esperienze che investono i nostri sensi, non riuscendo a cogliere il significato dello stimolo nel suo complesso (Levi, $2005)^{39}$, ma presentando piuttosto una specifica propensione per i dettagli e le forme astratte dal contesto. Il modello della CC è particolarmente interessante poiché spiega non solo alcuni deficit e particolarità cliniche tipiche dell'autismo, ma anche talune peculiarità, abilità e zolle di capacità (le criticità derivate da cambiamenti minimi, anche impercettibili agli altri, o le capacità di individuare talvolta particolari nascosti) (Barale et al., 2009) ${ }^{40}$.

In ogni caso, uno dei maggiori limiti presentato negli anni recenti sui modelli di autismo delle teorie cognitivo-psicologiche é che sono rivolte principalmente alla mente della persona e non guardano al contesto e alle le relazioni/interazioni tra le persone, che possono generare una totale disconnessione nel senso condiviso di "realtà sociale"(Milton, 2012) ${ }^{41}$. Milton ritiene che sia illogico parlare di deficit "sociale" di un individuo, quanto piuttosto di un problema di empatia, una rottura nella comunicazione tra due persone con due processi mentali di elaborazione delle informazioni differenti. In questo senso definisce un "doppio problema di empatia", basato sul fatto che sia la persona autistica che il neurotipico abbiano una grande difficoltà nel comprendersi reciprocamente, poiché non condividono lo stesso quadro di riferimento nei confronti delle interazioni sociali (Milton, 2012) ${ }^{42}$. Questa interessante teoria sarà approfondita nei successivi capitoli, definendo l'importanza dell'empatia coma base del progetto, affinché si riveli efficace nel rispondere alle esigenze specifiche dell'individuo.

\subsection{L'entità del problema. La scarsità dei dati disponibili}

La World Health Organisaation (WHO) stima la presenza di 1 bambino su 150 con DSA nel mondo, mentre studi più recenti del Centers for Disease Control and Prevention (CDC) in USA contano una media di 1 bambino su 68 (Christensen et al., 2016) ${ }^{43}$. Attualmente l'autismo è 3-4 volte più frequente rispetto a 30 anni fa

\footnotetext{
${ }^{37}$ Frith, U. Happé, F. (1994) Autism: beyond 'theory of mind', Cognition, 50, 115-132

${ }^{38}$ Happé, F. Frith, U. (1996), The neuropsychology of autism, Brain 119:1377-1400

${ }^{39}$ Levi, G. (a cura di) (2005) Linee Guida Per L'autismo Raccomandazioni Tecniche-Operative Per I Servizi Di Neuropsichiatria Dell'eta' Evolutiva, SINPIA - Societa' Italiana Di Neuropsichiatria Dell'infanzia E Dell'adolescenza, Roma

${ }^{40}$ Barale, F. et al. (2009) L'autismo a partire dall'età adulta: nuove conoscenze, criticità, implicazioni abilitative, NÓOs, 15(3):257-91

${ }^{41}$ Milton, D. (2012) So what exactly is autism?, AET Competence framework for the Department for Education, Birmingham

${ }^{42}$ ibidem

${ }^{43}$ Christensen, D.L. Baio, J. Van Naarden Braun, K. et al. (2016) Prevalence and characteristics of autism spectrum disorder among children aged 8 years-Autism and Developmental Disabilities Monitoring Network, 11 sites, United States, 2012, MMWR - Morbity and Mortality Weekly Report, Surveillance Summary 2016, 65(No. SS-3): 1 -23, CDC - Centers for Disease Control and Prevention, United States Department of Health and Human Services
} 
(Fombonne, 2003; Yeargin-Allsopp et al., 2003 cit. in Levi, 2005 ${ }^{44}$ ) e si prevede che già nel prossimo decennio diventi un'emergenza sociale ed economica (Coccia, 2010) ${ }^{45}$. Altri paesi oltre agli USA, tra cui Islanda, Svezia, Finlandia, Danimarca, Canada, Australia, confermano un aumento simile e la commissione Europea ha già da tempo affermato che l'autismo sta diventando la disabilità evolutiva con maggiore evidenza (Coccia, 2010) ${ }^{46}$. In ogni caso l'ipotesi di una vera e propria epidemia di autismo viene confutata da molti autori, che associano questo incremento a diverse cause quali: l'allargamento e la maggiore definizione dei criteri diagnostici, che come vedremo ha portato negli anni ad includere anche forme più lievi di autismo; la diffusione di procedure diagnostiche standardizzate, anche precoci; l'abbassamento dell'età di diagnosi (King, Bearman, 2009) ${ }^{47}$; l'aumento della disponibilità di servizi; la formazione specifica dei medici; un'aumentata sensibilità e conoscenza del disturbo, anche da parte degli operatori e della popolazione in generale (Levi, 2005) ${ }^{48}$, l'eterogeneità delle cause, le diverse manifestazioni cliniche e la peculiarità dell'evoluzione del disturbo (Bertelli et al., 2015) ${ }^{49}$. La ricerca attuale indica come il cambiamento dei criteri diagnostici e di sensibilità non spieghi che il $35 \%$ dell'aumento di prevalenza, a fronte dei fattori biologici ed in particolare l'interfaccia gene-ambiente (epigenetica), considerata negli ultimi anni tra le cause di maggior impatto, come riportato ad esempio nello studio di Nevison ${ }^{50}$ ed evidenziato anche nel prossimo paragrafo. A questo proposito si consideri, inoltre, che se si prendessero in considerazione anche i casi molto lievi, il numero di persone con autismo potrebbe arrivare ad essere anche maggiore; gli studi fatti su tutta la popolazione infatti, e non solo su quella che accede ai servizi sanitari, danno stime di 1 caso su 50 (Vagni, 2015) ${ }^{51}$.

In Italia manca ancora una rilevazione dei dati che possa fornire delle statistiche generali (Coccia, 2010) ${ }^{52}$, ma non ci sono motivi per pensare che la situazione sia diversa, poiché l'autismo non sembra presentare prevalenze geografiche e/o etniche, in quanto é stato riscontrato in tutte le popolazioni del mondo, di ogni razza o am-

\footnotetext{
${ }^{44}$ Levi, G. (a cura di) (2005) Linee Guida Per L'autismo Raccomandazioni Tecniche-Operative Per I Servizi Di Neuropsichiatria Dell'età Evolutiva, SINPIA - Società Italiana Di Neuropsichiatria Dell'infanzia E Dell'adolescenza, Roma

${ }^{45}$ Coccia, G. (2010) La dimensione del fenomeno nelle statistiche ufficiali e nelle indagini ad hoc, in Giofré, F. (a cura di) (2010) Autismo. Protezione sociale e architettura, Alinea, Firenze, pp. 29-39

46 ibidem

${ }^{47}$ King, M. Bearman, P. (2009) Diagnostic change and the increased prevalence of autism, International Journal of Empidiemology, Oct;38(5):1224-34

${ }^{48}$ Levi, G. (a cura di) (2005) Linee Guida Per L'autismo Raccomandazioni Tecniche-Operative Per I Servizi Di Neuropsichiatria Dell'eta' Evolutiva, SINPIA - Societa' Italiana Di Neuropsichiatria Dell'infanzia E Dell'adolescenza, Roma

${ }^{49}$ Bertelli, M. et al. (2015) L'impatto della tipologia residenziale sul percorso riabilitativo della persona con Disturbi dello Spettro Autistico, Autismo e Disturbi dello Sviluppo, vol 13, n. 2, pp. 283.291

${ }_{50}$ Nevison, C. D. (2014) A comparison of temporal trends in United States autism prevalence to trends in suspected environmental factors, Environmental Health, 2014 Sep 5, 13:73

51 Vagni, D. (2015) Lo spettro Autistico, risposte semplici. Per una bonifica semantica dagli stereotipi dell'autismo, Asperger ONLUS per la condivisione della conoscenza

${ }_{52}$ Coccia, G. (2010) La dimensione del fenomeno nelle statistiche ufficiali e nelle indagini ad hoc, in Giofré, F. (a cura di) (2010) Autismo. Protezione sociale e architettura, Alinea, Firenze, pp. 29-39
} 


\section{Elena Bellini}

biente sociale (Levi, 2005) ${ }^{53}$. Ad ogni modo, mentre studi internazionali forniscono un deciso quadro evolutivo del disturbo, in Italia la quantificazione dei soggetti autistici non segue un percorso aggiornato e coordinato sul territorio. Questo avviene anche poiché la diagnosi precoce corretta non è ancora uniformemente diffusa, non si considerano nelle statistiche le persone con autismo e, ancora oggi, molte famiglie "negano" il disturbo, non sono in grado di riconoscerlo, o non si rivelano adeguatamente supportate a farlo (Coccia, 2010) $)^{54}$.

La prevalenza di persone con autismo é di sesso maschile, rilevando 1 sola donna su 4 casi di autismo; 1 su 2 se consideriamo solo il basso funzionamento (con quoziente intellettivo $<50)$; 1 su 8 nel caso della Sindrome di Asperger. In realtà è plausibile pensare che la prevalenza nelle donne sia almeno di 2 su 5 , poiché è più difficile riconoscere l'autismo nel sesso femminile, dato che solitamente tendono ad avere una presentazione diversa dallo stereotipo classico dell'autismo: in caso di alto funzionamento presentano un maggiore interesse sociale e sono più facilmente interessate alle persone, all'arte e alla letteratura; le ragazze Asperger sono considerate dei "camaleonti sociali" e presentano un minor numero di comportamenti problematici; in generale sono spesso più consapevoli e tendono a nascondere i sintomi (Vagni, 2015) ${ }^{55}$.

\subsection{Le cause dell'autismo: dalla genetica ai fattori ambientali}

Non risultano ancora chiare le cause dell'autismo, anche se si possono ricondurre ad una combinazione tra fattori genetici ed ambientali, come ad esempio lo stile di vita, la dieta, l'età dei genitori, l'esposizione a sostanze chimiche, tossiche o virali, la presenza di deficit vitaminici o nutrizionali, la presenza di alcune malattie immunitarie nei genitori, forti stress o complicazioni durante la gravidanza o il parto, ecc. Dagli studi sui gemelli si evidenzia infatti una concordanza che va dal 60 al 90 $\%$ che presuppone che comunque altri fattori oltre ai geni contribuiscono all'autismo (eziopatogenesi) (Hertz-Picciotto, 2011) ${ }^{56}$. Nessun fattore ambientale è sufficiente da solo a provocare l'autismo, ma possono essere eventi scatenanti o concorrenti (Vagni, 2015) ${ }^{57}$. Allo stesso modo non si può ricondurre la causa ad un singolo gene, quanto piuttosto al contributo di molte anomalie genetiche (Venerosi, Chiarotti,

53 Levi, G. (a cura di) (2005) Linee Guida Per L'autismo Raccomandazioni Tecniche-Operative Per I Servizi Di Neuropsichiatria Dell'eta' Evolutiva, SINPIA - Societa' Italiana Di Neuropsichiatria Dell'infanzia E Dell'adolescenza, Roma

54 Coccia, G. (2010) La dimensione del fenomeno nelle statistiche ufficiali e nelle indagini ad hoc, in Giofré, F. (a cura di) (2010) Autismo. Protezione sociale e architettura, Alinea, Firenze, pp. 29-39

${ }_{55}$ Vagni, D. (2015) Lo spettro Autistico, risposte semplici. Per una bonifica semantica dagli stereotipi dell'autismo, Asperger ONLUS per la condivisione della conoscenza

${ }^{56}$ Hertz-Picciotto, I. (2011) Le cause ambientali dell'autismo: lo studio Charge, in Venerosi A, Chiarotti F (Ed.) (2011) Autismo: dalla ricerca al governo clinico, Roma: Istituto Superiore di Sanità (Rapporti ISTISAN 11/33)

57 Vagni, D. (2015) Lo spettro Autistico, risposte semplici. Per una bonifica semantica dagli stereotipi dell'autismo, Asperger ONLUS per la condivisione della conoscenza 
$2011)^{58}$, definendosi per questo spettro autistico. Anche attraverso l'analisi del cervello delle persone autistiche, soprattutto attraverso risonanza magnetica, si è rafforzata la convinzione che l'autismo non riguardi una singola area celebrale, ma piuttosto una difficoltà di connessione tra le diverse aree. Sono state considerate tra le cause anche delle ipotesi ormonali, sostenendo una sorta di "estremizzazione" del cervello maschile, tipicamente meno empatico e più logico di quello femminile, riconducibile alla presenza di alti livelli di testosterone già in epoca fetale; questo spiegherebbe anche la netta prevalenza del disturbo sul genere maschile.

In base alle attuali conoscenze, l'autismo è una patologia psichiatrica con un elevato tasso di ereditarietà e con una significativa concordanza nei gemelli monozigoti; il rischio di avere un altro bambino con autismo è 20 volte più elevato rispetto alla popolazione generale se si è già avuto un figlio affetto (LG 21, 2011-2015) ${ }^{59}$. L'ereditarietà aumenta al diminuire della gravità nello Spettro Autistico, ovvero è più semplice ritrovare tratti autistici nei genitori delle persone con Autismo Lieve (Vagni, 2015) ${ }^{60}$.

Ci sono poi diverse altre ipotesi, pur non supportate da un'adeguata evidenza scientifica, che attribuiscono la causa dell'autismo a vaccini, contaminanti ambientali e alimenti (Mazzone, 2015) ${ }^{61}$.

In particolare, negli anni '90 sono stati riscontrati gravi fattori ambientali derivanti dall'inquinamento alla base delle cause dell'autismo. Più nello specifico, il caso della "Plastic City" di Leominster, città industriale del New England a nord-ovest di Boston, aprì il dibattito sull'incidenza di questa "malattia fino ad allora considerata rara" e l'inquinamento prodotto dalle industrie e dai loro rifiuti tossici. In letteratura, si ha un solo studio di Mary Coleman, condotto nel 1974 con l'aiuto di Rimland e di altri genitori del NSAC - National Society fo Autistic Children, che sosteneva che molti genitori di bambini autistici (1:4) erano stati esposti sul luogo di lavoro a sostanze chimiche tossiche. Non vi sono in ogni caso statistiche precise e correlate e la diagnosi di autismo non era ancora abbastanza definita e precisa per creare in questo senso un'evidenza scientifica. A seguito dello scandalo di Leominster, in ogni caso, iniziarono a spuntare "sciami di autismo" in tutto il Paese, come ad esempio a Brick Township nel New Jersey, dove tra il 1969 e il 1979 erano stati gettati in una discarica quasi 250 milioni di litri di rifiuti settici, ma che anche in questo caso non sono stati dimostrati da studi specifici (Silberman, 2015) ${ }^{62}$.

Nello stesso periodo in cui è iniziata ad affermarsi anche la teoria che afferma la responsabilità dei vaccini ed in particolare del vaccino DPT-difterite-pertossetetano, che era causa di crisi epilettiche, svenimenti, febbre, edema, shock, pianto

\footnotetext{
${ }^{58}$ Venerosi A, Chiarotti F (Ed.) (2011) Autismo: dalla ricerca al governo clinico, Roma: Istituto Superiore di Sanità (Rapporti ISTISAN 11/33)

59 ISS - SNLG (2011) Linee Guida 21 - Il trattamento dei disturbi dello spettro autistico nei bambini e negli adolescenti, Ministero della Salute, Roma (aggiornate nel 2015)

${ }^{60}$ Vagni, D. (2015) Lo spettro Autistico, risposte semplici. Per una bonifica semantica dagli stereotipi dell'autismo, Asperger ONLUS per la condivisione della conoscenza

${ }_{61}^{61}$ Mazzone, L. (2015) Un autistico in famiglia, Mondadori, Milano

${ }^{62}$ Silberman, S. (2015) Neuro Tribes. The legacy of Autism and the Future of Neurodiversity, AVERY, New York (ed. italiana 2016)
} 


\section{Elena Bellini}

acuto e prolungato. Il testo di Coulter e Fisher "DPT: a shot in the dark" che riportava questi dati sulle conseguenze del vaccino, appena menzionava l'autismo, mentre é stato attraverso gli scritti di Rimland che si è diffusa questa idea di correlazione. Un'altra responsabilità in questo senso va attribuita al gastroenterologo inglese Andrew Wakefield, che sosteneva un rapporto diretto tra $i$ danni cerebrali e il vaccino MPR - morbillo-parotite-rosolia, un vaccino che ogni anno previene milioni di morti nel mondo (secondo l'OMS nel 2000 i soggetti che hanno sviluppato il morbillo sono 30-40 milioni, con 770.000 morti, soprattutto nell'Africa subsahariana dove le vaccinazioni e i tassi di cura sono più bassi), suscitando non poche critiche dai funzionari della sanità pubblica britannica (Silberman, 2015) ${ }^{63}$. La copertura mediatica ha avuto la sua corresponsabilità nella diffusione della notizia, che ancora oggi aleggia tra alcune famiglie, pensando che i vaccini possano generare la cosiddetta "regressione autistica" (regressione dello sviluppo con perdita delle competenze acquisite e sviluppo di tutti i sintomi tipici dello spettro autistico). Svariati tentativi di ricercatori indipendenti hanno cercato di confermare la connessione tra autismo e il vaccino MPR, ma sono falliti. Nel 2003, alcuni ricercatori che scrivevano per Archives of Pediatrics and Adolescent Medicine eseguirono una meta-analisi sistematica di più di una decina di studi epidemiologici, concludendo infine che "la letteratura corrente non suggerisce un'associazione tra i disturbi dello spettro autistico e il vaccino MPR. Il rischio di autismo per la vaccinazione resta teorico, mentre le conseguenze della mancata vaccinazione sono reali"(Silberman, 2015) ${ }^{64}$.

\subsection{I sintomi e i livelli di gravità in base alla diagnosi}

E' possibile diagnosticare l'autismo solo attraverso l'osservazione del comportamento del bambino, interviste o questionari ai familiari o educatori. La diagnosi viene solitamente formulata facendo riferimento alle due principali classificazioni internazionali dei disturbi mentali: il DSM - Diagnostic and Statistical Manual of Mental Disorders dell'APA - American Psychiatric Association e 1'ICD - International Classification of Diseases (Classificazione Internazionale dei Disturbi e delle Malattie) dell'OMS - Organizzazione Mondiale della Sanità.

L'ICD-10 (WHO, 1994) ${ }^{65}$ definisce l'autismo all'interno della sezione V denominata "Disturbi psichici e comportamentali", riferendosi in particolare al codice F.84-Alterazione globale dello sviluppo psicologico: un gruppo di sindromi caratterizzato da anormalità qualitative delle interazioni sociali e delle modalità di comunicazione, e da un repertorio limitato, stereotipato, ripetitivo di interessi e di attività. Queste anormalità qualitative sono una caratteristica preminente del funzionamento dell'individuo in tutte le situazioni. Se lo si desidera, si può utilizzare un codice aggiuntivo per specificare una condizione somatica o un ritardo mentale associati, ovvero: F84.0 Autismo Infantile, F84.1 Autismo atipico, F84.2 Sindrome di Rett,

\footnotetext{
${ }^{63}$ Silberman, S. (2015) Neuro Tribes. The legacy of Autism and the Future of Neurodiversity, AVERY, New York (ed. italiana 2016)

${ }^{64}$ ibidem

${ }^{65}$ World Health Organization (WHO) (2007) ICD10, International Classification of diseases 10th revision
} 
F84.3 Sindrome disintegrativa dell'infanzia di altro tipo, F84.4 Sindrome iperattiva associata a ritardo mentale e movimenti stereotipati, F84.5 Sindrome di Asperger.

Con F84.0 Autismo Infantile si identifica una sindrome definita da: (a) presenza di una compromissione dello sviluppo che si manifesta prima dei 3 anni, (b) un tipo caratteristico di funzionamento anormale 1:80-F89 Sindromi e disturbi da alterato Sviluppo psicologico nelle aree dell'interazione sociale, della comunicazione e del comportamento, che è limitato, stereotipato e ripetitivo.

In aggiunta a queste specifiche caratteristiche diagnostiche, è frequente che $\mathrm{i}$ bambini autistici mostrino una varietà di altri problemi non specifici, come fobie, disturbi del sonno è dell'alimentazione, carattere collerico e aggressività (autodiretta). Si definisce come Sindrome autistica, Psicosi infantile, Sindrome di Kanner, mentre esclude la psicopatia autistica (F84.5). In particolare riguarda uno sviluppo anormale o compromesso che si manifesta prima dei tre anni in almeno una delle seguenti aree: (1) comprensione o espressione del linguaggio usato nella comunicazione sociale; (2) sviluppo di attaccamenti sociali selettivi o di interazione sociale reciproca; (3) gioco funzionale o simbolico.

Per effettuare la diagnosi devono essere presenti un totale di almeno sei dei sintomi descritti nei punti (1), (2) e (3), di cui almeno due dei sintomi del punto (1) e almeno un sintomo da ciascuno dei punti (2) e (3):

(1) Compromissioni qualitative dell'interazione sociale sono presenti in almeno due delle seguenti aree: (a) incapacità di utilizzare adeguatamente lo sguardo faccia a faccia, l'espressione facciale, la gestualità e la postura per regolare l'interazione sociale; (b) incapacità a sviluppare (in modo appropriato all'età mentale e nonostante ampie opportunità) rapporti con coetanei che implicano una condivisione di interessi, attività ed emozioni; (c) mancanza di reciprocità socio-emozionale come dimostrato dalla mancanza di risposta alle emozioni delle altre persone; o in accordo al contesto sociale; o debole integrazione di comportamenti sociali, emotivi e comunicativi; (d) mancanza di ricerca spontanea di condividere divertimenti o interessi o risultati con altre persone (ad esempio una difficoltà a mostrare o a donare ad altre persone oggetti di interesse personale);

(2) Compromissioni qualitative nella comunicazione sono presenti in almeno una delle seguenti aree: (a) un ritardo o una totale mancanza dello sviluppo del linguaggio verbale che non è accompagnato da un tentativo di compensazione attraverso l'uso di gesti o della mimica come modalità di comunicazione alternativa (spesso preceduto da una mancanza di lallazione comunicativa); (b) una relativa incapacità ad iniziare o a sostenere una conversazione (a qualsiasi livello di abilità linguistica) in cui vi sia una reciprocità alle comunicazioni dell'altra persona; (c) uso di un linguaggio ripetitivo e stereotipato o uso idiosincrasico di parole e di frasi; (d) assenza di gioco inventivo o (nei primi anni di vita) imitativo;

(3) In almeno una delle seguenti aree sono presenti modelli di comportamento, interessi e attività limitati, ripetitivi e stereotipati: (a) una preoccupazione pervasiva per uno o più interessi limitati e stereotipati che sono anomali nel contenuto e nell'obiettivo, o uno o più interessi che sono anomali per l'intensità e la natura circoscritta, ma non per contenuto od obbiettivi; (b) adesione apparentemente compulsiva a pratiche o rituali specifici e disfunzioni; (c) manierismi motori stereotipati e ripetitivi che implicano il battere o il torcere le mani o le dita, o movimenti complessi di tutto 


\section{Elena Bellini}

il corpo; (d) preoccupazioni per parti di oggetti o per elementi non funzionali dei materiali di gioco (quali l'odore, la sensazione che danno al tatto, il rumore o le vibrazioni che producono) (WHO, 1994). ${ }^{66}$

Con F84.1 Autismo atipico si identifica invece una sindrome che si differenzia dall'autismo infantile sia per l'età di insorgenza sia per il mancato riscontro di tutti i tre requisiti diagnostici. Questa sottocategoria deve essere usata quando vi è una compromissione dello sviluppo che è presente solo dopo i tre anni di età, e quanto manca un'anormalità sufficientemente dimostrabile in una o due delle tre aree richieste per la diagnosi di autismo (cioè, interazioni sociali, comunicazione e comportamento, che è limitato, stereotipato e ripetitivo) pur in presenza delle caratteristiche anomalie nell'altra o nelle altre aree. L'autismo atipico si ha più spesso in individui gravemente ritardati e con un grave disturbo evolutivo specifico della comprensione del linguaggio. Include Psicosi infantile atipica e Ritardo mentale con caratteristiche autistiche (WHO, 1994) ${ }^{67}$.

Per F84.2 Sindrome di Rett si intende una condizione, riscontrata finora solo nel sesso femminile, in cui uno sviluppo che all'inizio è apparentemente normale è seguito da una perdita parziale o completa del linguaggio e delle capacità motorie e dell'uso delle mani insieme con un rallentato accrescimento cranico, in genere con esordio tra i 7 e i 24 mesi. Sono caratteristici la perdita dei movimenti intenzionali delle mani, i movimenti stereotipati di torsione delle mani e l'iperventilazione. Lo sviluppo sociale e quello del gioco si arrestano, ma l'interesse sociale tende ad essere mantenuto. All'età di quattro anni iniziano a manifestarsi atassia e aprassia del tronco, seguiti frequentemente da movimenti coreoatetoidi. Consegue quasi invariabilmente un grave deficit mentale (WHO, 1994) ${ }^{68}$.

F84.3 Sindrome disintegrativa dell'infanzia di altro tipo é una condizione definita da un periodo di sviluppo sicuramente normale, seguito da una chiara perdita delle capacità precedentemente acquisite in diverse aree, nel corso di pochi mesi. Tipicamente, si associano una generale perdita dell'interesse per l'ambiente, manierismi motori ripetitivi, compromissione simil-autistica dell'interazione e che la sindrome è dovuta ad un'encefalopatia associata, ma la diagnosi deve essere posta in base alla caratteristiche del comportamento. Include Demenza infantile, Psicosi disintegrativa, Sindrome di Heller, Psicosi simbiotica (WHO, 1994) ${ }^{69}$.

F84.4 Sindrome iperattiva associata a ritardo mentale e movimenti stereotipati è una sindrome mal definita di incerta validità nosologica. La categoria è destinata ad un gruppo di bambini con grave ritardo mentale (QI al di sotto di SO)che mostrano gravi problemi di iperattività e di deficit attentivo, così come comportamenti stereotipati. Tali bambini tendono a non beneficiare di farmaci stimolanti (a differenza di quelli con QI normale) e possono presentare una grave reazione disforica (qualche volta con rallentamento psicomotorio) quando ricevono tali farmaci. Nell'adolescen-

\footnotetext{
${ }^{66}$ World Health Organization (WHO) (2007) ICD10, International Classification of diseases 10th revision

67 ibidem

68 ibidem

69 ibidem
} 
za, l'iperattività tende ad essere sostituita dall'ipoattività (una caratteristica che non è comune nei bambini ipercinetici con intelligenza normale). Questa sindrome si associa anche con vari deficit dello sviluppo, sia specifici sia globali. Non è chiaro fino a che punto i disturbi comportamentali siano una funzione del basso QI o di un danno organico cerebrale (WHO, 1994) $)^{70}$.

F84.5 Sindrome di Asperger è definita infine come una sindrome di incerta validità nosologica, caratterizzata dallo stesso tipo di anomalia qualitativa dell'interazione sociale che è proprio dell'autismo, insieme ad un repertorio limitato, stereotipato e ripetitivo di interessi e di attività. La sindrome differisce dall'autismo in primo luogo perché non vi è nessun ritardo nel linguaggio o nello sviluppo cognitivo. Questa sindrome e spesso associata a marcata goffaggine. C'è una forte tendenza a persistere nell'adolescenza e nella vita adulta. Nelle fasi precoci della vita adulta si verificano occasionalmente episodi psicotici (WHO, 1994) ${ }^{71}$.

Il DSM - Diagnostic and Statistical Manual of Mental Disorders é un manuale statistico e diagnostico dei disturbi mentali, redatto da una commissione di esperti nominata dall'APA - Associazione Americana degli Psichiatri, elencando le definizioni dei disturbi mentali, i sintomi e i criteri diagnostici che incontrano il consenso degli psichiatri e della comunità scientifica internazionale. La prima pubblicazione del DSM-I risale al 1952, dove si parla di autismo come "reazione schizofrenica, tipo infantile", riportando le definizioni dell'OMS pubblicate nel 1948. La nuova versione del DSM-II é stata invece pubblicata nel 1968, andando a definire in modo più specifico l'autismo, anche se seguendo le teorie di Bettlheim affermate in questo periodo, ovvero riferendosi ad un "comportamento autistico, atipico e ritirato" e una "generale disomogeneità" come prova del "mancato sviluppo di un'identità separata da quella della madre". Il primo volume ad avere una certa consistenza e ad acquisire un utilizzo maggiore in ambito medico e diagnostico, é stato il DSM-III, concepito da Robert Spitzer dell'APA e pubblicato nel 1980, proprio al fine di rendere finalmente operativi i criteri del DSM, allineandoli al tempo stesso agli standard della International Classification of Diseases (ICD), usata in tutto il mondo. Nel DSM-III fu quindi inserito l' "autismo infantile" (sindrome di Kanner), differenziandola dalla schizofrenia e qualificandola come nucleo della nuova categoria dei "disturbi pervasivi dello sviluppo". L'autismo fu quindi definito come "pervasiva mancanza di reattività agli altri" e "resistenza al cambiamento", specificando l'età di esordio precedente ai 30 mesi, confermando nuovamente l'idea di Kanner ed escludendo tutti i bambini che sarebbero poi stati diagnosticati con la sindrome di Asperger. Venne definita anche una lista di controllo delle caratteristiche cliniche che avrebbero dovuto essere presenti per la diagnosi, ovvero "grossolani deficit nello sviluppo del linguaggio" e "risposte bizzarre all'ambiente". I bambini che invece avevano sofferto di una perdita di capacità dopo i 30 mesi, venivano associati ad una diagnosi di "Disturbo pervasivo dello sviluppo a esordio infantile", contraddistinto da "mancanza di adeguata relatività sociale", "attaccamento inadeguato", "asocialità", "iper- o

\footnotetext{
${ }^{70}$ World Health Organization (WHO) (2007) ICD10, International Classification of diseases 10th revision

71 ibidem
} 


\section{Elena Bellini}

ipo- sensibilità agli stimoli sensoriali" e "insistenza sul fare le cose tutte le volte alla stessa maniera". Questa nuova versione del DSM riscosse molto successo ed interesse in ambito medico; d'altro canto i dati su cui si basava il DSM-III erano molto limitati, sommari e provvisori, pertanto nel 1987 fu pubblicata una nuova versione (DSM-III-R) che comprendesse una revisione della letteratura ed elaborasse meglio i criteri, grazie al lavoro di tre studiosi: Lorna Wing, Lynn Waterhouse, Bryna Siegel. In questa revisione si sostituisce il termine "infantile" con "disturbo autistico", definendone la condizione pervasiva, dalla nascita alla morte. La lista di controllo fu inoltre sostituita da una serie di opzioni, che non dovevano considerarsi tutte presenti per definire la diagnosi, ma prevalenti in almeno otto delle sedici voci, di cui almeno due nella sezione A (interazione sociale), una nella B (comunicazione) e una nella C (attività ripetitive). Il primo impatto importante fu che la diagnosi non veniva più considerata "rara", ma attraverso questa rosa di criteri si poteva applicare a popolazioni molto più grandi e diversificate; dopo la pubblicazione del DSM-III e DSMIII-R infatti le stime di prevalenza dell'autismo iniziarono a crescere in tutto il mondo. I primi strumenti di diagnosi e valutazione vennero realizzati da Rimland attraverso delle liste di controllo (E-1 e la successiva E-2), interessanti ma caratterizzate da una serie di pecche metodologiche, poiché fondate sui ricordi dei genitori piuttosto che sull'osservazione clinica diretta. Negli anni '80 una prima svolta venne segnata dalla CARS - Child Autism Rating Scale di Eric Shopler e i colleghi del TEACCH, di particolare utilità nel distinguere l'autismo dalle altre forme di sviluppo ritardato, come la disabilità intellettiva. Classificando i comportamenti la CARS anticipò il modello dell'autismo come spettro del DSM-III-R. In seguito un nuovo strumento completo di valutazione dei problemi di comunicazione, interazione sociale e gioco nei bambini tra 5 e 12 anni, nacque con il nome di ADOS - Autism Diagnostic Observation Schedule, basato su quelli che sarebbero stati i nuovi criteri del DSM-IV, ampliando ancora maggiormente lo spettro. Nella nuova versione del DSM-IV è stata inserita finalmente anche la sindrome di Asperger, adeguandosi alla pubblicazione della decima edizione dell'ICD in cui appunto era stata inserita, benché lo studio di questa condizione fosse ancora agli albori. D'altro canto, dopo la pubblicazione del DSM-IV (1994 guidata da Frances Allen), le diagnosi iniziarono a salire molto velocemente a causa di un errore nella pubblicazione, ovvero si prescrisse che i bambini dovessero mostrare difficoltà di interazione sociale "o" comunicazione e comportamento, invece che "e", scegliendo una sola colonna della categoria "A"; questo in gran parte ha dato origine alla cosiddetta "epidemia dell'autismo" (Silberman, 2015) ${ }^{72}$. Nel 2000 questa versione è stata infatti revisionata pubblicando il DSM-IV-R, volume precedente all'ultima versione del DSM-5 del 2013, diretta dallo psicologo David Kupfer, che ha segnato un cambiamento netto rispetto ai precedenti manuali diagnostici.

L'ICD e il DSM sono stati sempre allineati nella definizione e nei criteri di diagnosi fino alla pubblicazione nel 2013 della nuova edizione del DSM-5, dove sono stati introdotti alcuni cambiamenti rispetto al precedente DSM-IV-R, differenzian-

\footnotetext{
${ }^{72}$ Silberman, S. (2015) Neuro Tribes. The legacy of Autism and the Future of Neurodiversity, AVERY, New York (ed. italiana 2016)
} 
dosi quindi in parte anche rispetto alla definizione dell'OMS (ICD-10 - WHO, $1994)^{73}$. E' in ogni caso in fase di pubblicazione un nuovo ICD-11, dove vi saranno nuove integrazioni anche rispetto alla definizione di autismo. Al momento nella versione Beta Draft dell'ICD-11 classifica l'autismo all'interno dei Mental, behavioural or neurodevelopmental disorders nello specifico dei Neurodevelopmental disorders, con una definizione di Disturbi dello spettro Autistico: "Autism spectrum disorder is characterized by persistent deficits in the ability to initiate and to sustain reciprocal social interaction and social communication, and by a range of restricted, repetitive, and inflexible patterns of behaviour and interests. The onset of the disorder occurs during the developmental period, typically in early childhood, but symptoms may not become fully manifest until later, when social demands exceed limited capacities. Deficits are sufficiently severe to cause impairment in personal, family, social, educational, occupational or other important areas of functioning and are usually a pervasive feature of the individual's functioning observable in all settings, although they may vary according to social, educational, or other context. Individuals along the spectrum exhibit a full range of intellectual functioning and language abilities".

Una delle maggiori differenze del DSM-5 sta proprio nella definizione di Disturbi dello Spettro Autistico, che va a raggruppare in un'unica diagnosi tutti i Disturbi Pervasivi dello Sviluppo, che nella classificazione precedente erano distinti in disturbo autistico, disturbo di Asperger, disturbo disintegrativo della fanciullezza, disturbo pervasivo dello sviluppo non altrimenti specificato e sindrome di Rett (che rappresenta l'unica eccezione, poiché é rimasta comunque al di fuori della classificazione di DSA). Un'altra novità riscontrata dal DSM-5 è inoltre legata alla diagnostica, raggruppando i sintomi in due sole categorie - deficit della comunicazione sociale; comportamenti ristretti e ripetitivi ${ }^{74}$ - rispetto alle tre precedenti del DSM-IV-R menomazione della reciprocità sociale; menomazione del linguaggio/comunicazione; repertori ristretti e ripetitivi di interessi/attività ${ }^{75}$ - che erano a loro volta suddivise in quattro sintomi ciascuna e prevedevano per effettuare la diagnosi almeno un totale di sei sintomi presenti, di cui almeno due nella prima categoria e almeno uno nelle altre due. Oggi invece, con il DSM-5, si ottiene una diagnosi di DSA soddisfacendo almeno tre sintomi nella categoria A, almeno due della B e sempre le categorie $\mathrm{C}$ e $\mathrm{D}$ (si riportano a questo proposito alcune spiegazioni molto chiare e sintetiche di Vagni ${ }^{76}$, allegate ai criteri del DSM-5, poiché molto utili nella definizione e nella diffusione della conoscenza):

A. Deficit persistente nella comunicazione e nell'interazione sociale in diversi contesti, non spiegabile attraverso un ritardo generalizzato dello sviluppo:

\footnotetext{
${ }^{73}$ World Health Organization (WHO) (2007) ICD10, International Classification of diseases 10th revision

${ }^{74}$ American Psychiatric Association (APA) (2013) DSM V, Diagnostic and Statistical Manual of Mental Disorders V

${ }^{75}$ American Psychiatric Association (APA) (2002) DSM IV, Diagnostic and Statistical Manual of Mental Disorders IV

${ }^{76}$ Vagni, D. (2015) Lo spettro Autistico, risposte semplici. Per una bonifica semantica dagli stereotipi dell'autismo, Asperger ONLUS per la condivisione della conoscenza.
} 


\section{Elena Bellini}

1. Deficit nella reciprocità socio-emotiva, che va da un approccio sociale anormale e insuccesso nella normale conversazione (botta e risposta), attraverso una ridotta condivisione di interessi, emozioni, percezione mentale e reazione, fino alla totale mancanza di iniziativa nell'interazione sociale.

2. Deficit nei comportamenti comunicativi non verbali usati per l'interazione sociale, da una scarsa integrazione della comunicazione verbale e non verbale, attraverso anormalità nel contatto oculare e nel linguaggio del corpo o deficit nella comprensione e nell'uso della comunicazione non verbale (intonazione, tono e prosodia della voce), fino alla totale mancanza di espressività facciale e gestualità. Le difficoltà che si riscontrano nei bambini sono soprattutto rivolte alla mancanza di attenzione condivisa, assenza di pointing (indicare), di condivisione di oggetti di interesse, o di triangolazione (non seguire lo sguardo dell'altro). Anche nei casi più lievi permane spesso l'impressione di un linguaggio del corpo "strano", "rigido" o "teatrale" con grande difficoltà di integrare le modalità; ad esempio può essere presente il contatto oculare ma non la coordinazione tra sguardo, gesti e parole, possono ridere nelle occasioni sbagliate, o avere difficoltà a mostrare quando qualcosa va male (Vagni, 2015) ${ }^{77}$.

3. Deficit nella creazione e mantenimento di relazioni appropriate al livello di sviluppo (non comprese quelle con i genitori e caregiver); che vanno dalle difficoltà nell'adattare il comportamento ai diversi contesti sociali, attraverso difficoltà nella condivisione del gioco immaginativo e nel fare amicizie, fino all'apparente assenza di interesse per le persone. Le difficoltà possono manifestarsi attraverso l'emarginazione, la preferenza per attività solitarie o la compagnia di persone più piccole o più grandi, la passività, comportamenti inappropriati rispetto al contesto (che possono apparire aggressivi o ritirati), o la difficoltà nel comprendere od usare ironia, sarcasmo o bugie bianche. E' possibile che la persona voglia amicizie, ma potrebbe non capire bene cosa comporta o qual é il senso dell'amicizia stessa (Vagni, $2015)^{78}$.

B. Pattern di comportamenti, interessi o attività ristretti e ripetitivi:

1. Linguaggio, movimenti o uso di oggetti stereotipati o ripetitivi, come semplici stereotipie motorie, ecolalia (ovvero la ripetizione come un eco di parole, immediata o differita), uso ripetitivo di oggetti, o frasi idiosincratiche. Questi sintomi possono presentarsi in modo molto variabile a seconda dell'età, della gravità, dell'intervento svolto e del supporto sociale. La presenza di stereotipie é più frequente nelle persone con disabilità intellettiva, ad ogni modo molti adulti senza disabilità intellettiva nascondono spesso i comportamenti ripetitivi in pubblico, anche se questo genera molta ansia (Vagni, 2015) ${ }^{79}$.

2. Eccessiva fedeltà alla routine, comportamenti verbali o non verbali riutilizzati o eccessiva riluttanza ai cambiamenti: rituali motori (ad esempio camminare in tondo), insistenza nel fare la stessa strada o mangiare lo stesso cibo, domande incessanti

\footnotetext{
77 Vagni, D. (2015) Lo spettro Autistico, risposte semplici. Per una bonifica semantica dagli stereotipi dell'autismo, Asperger ONLUS per la condivisione della conoscenza.

78 ibidem

79 ibidem
} 
o estremo stress a seguito di piccoli cambiamenti. Per molte persone nello Spettro un piccolo cambiamento è più drammatico di un grande cambiamento, poiché mentre nel primo caso bisogna modificare e cambiare un'immagine del mondo già costituita, nel secondo caso va "solo" creata. Sicuramente prevedere aiuta molto nella comprensione del mondo. Molte persone autistiche hanno difficoltà a modificare ciò che hanno appreso in precedenza, pertanto bisogna cercare di evitare che i bambini apprendano, anche incidentalmente, comportamenti o informazioni errate. Tutte le persone rispondono meglio ai rinforzi piuttosto che alle punizioni, poiché il rinforzo aiuta ad apprendere nuovi comportamenti e aumenta la probabilità che un comportamento si ripeta, mentre una punizione riduce la frequenza di quelli indesiderati. Tuttavia i bambini autistici spesso non hanno a disposizione comportamenti alternativi a quello che si vuole ridurre e non sono in grado di generarli spontaneamente, per cui la punizione non avrebbe molto effetto ma tenderebbero a perseverare ancora di più (Vagni, 2015) ${ }^{80}$.

3. Interessi altamente ristretti e fissati, anormali in intensità (difficoltà di spostare l'attenzione se richiamato) o argomenti: forte attaccamento o interesse per oggetti insoliti, interessi eccessivamente persistenti o circostanziati. L'interesse è un mezzo per rilassarsi, un'attività piacevole, una potente motivazione e uno strumento per superare fobie e paure. Si possono riconoscere quattro diversi stadi di interessi: parte di oggetti (luci, lavatrice, ventilatori), legato ad un interesse prevalentemente sensoriale; categoria di oggetti (tipici ed eccentrici), spesso mezzi di trasporto, animali e oggetti elettronici (questo tipo d'interesse speciale può condurre al collezionismo); interessi complessi ed astratti, come periodi storici, geografia, scienze, etc.; interesse verso una persona, letteratura fantasy o la presenza di molteplici interessi. Lo scopo di un terapista non dovrebbe essere quello di eliminare gli interessi, quanto di espanderli e renderli più flessibili e funzionali (Vagni, 2015) ${ }^{81}$.

4. Iper o Ipo-reattività agli stimoli sensoriali o interessi insoliti verso aspetti sensoriali dell'ambiente: apparente indifferenza al caldo/freddo/dolore, risposta avversa a suoni o consistenze specifiche, eccessivo annusare o toccare gli oggetti, attrazione per luci o oggetti roteanti. Uno degli aspetti dell'autismo a lungo non riconosciuto e finalmente reinserito all'interno del nuovo manuale diagnostico é quello legato alle sensibilità sensoriali. Alcuni adulti con sindrome di Asperger ritengono che nella vita di tutti i giorni la loro sensibilità sensoriale abbia un impatto maggiore rispetto alle problematiche che riguardano lo stringere amicizie, gestire le emozioni e trovare un impiego adatto. La sensibilità più comune è quella verso suoni specifici, ma ci possono essere anche sensibilità tattili, sensibilità riguardanti l'intensità della luce, il gusto e la consistenza di alcuni cibi, o verso certi odori. Possono essere estremamente sensibili ad alcune percezioni sensoriali o trovare eventi apparentemente di routine affascinanti, come ad esempio i modelli di luce su un muro o le foglie fruscianti nel vento; al contrario possono non reagire alle sensazioni che altre persone trovano

\footnotetext{
${ }^{80}$ Vagni, D. (2015) Lo spettro Autistico, risposte semplici. Per una bonifica semantica dagli stereotipi dell'autismo, Asperger ONLUS per la condivisione della conoscenza.

${ }^{81}$ ibidem
} 


\section{Elena Bellini}

sgradevoli, come il calore estremo, il freddo o il dolore (Vagni, 2015) ${ }^{82}$. Ogni sensibilità varia rispetto alle altre da persona a persona e da contesto a contesto e può essere difficile da prevedere e comprendere. Un tratto comune é l'ipersensibilità alle sensazioni che provengono dall'esterno, rispetto ad un'iposensbilità delle sensazioni interne, come ad esempio il senso dell'equilibrio, o la capacità di percepire il proprio corpo, "dove inizia e dove finisce". L'esperienza di stress e sovraccarico, sia emotivo che sensoriale, si possono considerare aspetti universali nell'autismo (Milton, 2012) ${ }^{83}$. Molte persone hanno difficoltà con l'equilibrio, la percezione del movimento, dell'orientamento, dello spazio e la costruzione della propria immagine corporea possono risultare insoliti. Più in generale le persone con sindrome di Asperger o autismo percepiscono il mondo in modo molto diverso, più intenso, a volte quasi doloroso, frammentato, o confusionario (come se ci fosse sempre del rumore a disturbare la comunicazione dei sensi). Altri possono riuscire con difficoltà a separare ciò che é in primo piano dallo sfondo (ad esempio a separare la voce di una persona per seguirne la conversazione se ci sono più persone che parlano), o presentare la sinestesia, ovvero il mescolamento dei canali sensoriali (ad esempio percepire i suoni come immagini o le lettere con dei colori). Anche nel caso dei sensi, imparare a conoscerli riduce l'ansia e le difficoltà. Sappiamo che un'accentuata percezione sensoriale è presente in maniera maggiore nell'infanzia e diminuisce gradualmente durante l'adolescenza, ma possono rimanere caratteristiche permanenti per alcuni adulti, specialmente nelle donne (Vagni, 2015) ${ }^{84}$.

C. I sintomi devono essere presenti nella prima infanzia (ma possono non diventare completamente manifesti finché le esigenze sociali non oltrepassano il limite delle capacità).

D. L'insieme dei sintomi deve limitare e compromettere il funzionamento quotidiano.

In definitiva i sintomi classificabili nelle categorie "deficit di comunicazione sociale" e "comportamenti ripetitivi" coincidono parzialmente con quelli elencati nel DSM IV, con due cambiamenti importanti: il "ritardo/menomazione del linguaggio" non è più fra i sintomi necessari alla diagnosi; la "sensibilità insolita agli stimoli sensoriali” é stata finalmente inserita nel DSM V all'interno della categoria dei "comportamenti ripetitivi".

Infine, come ulteriore novità nel 2013 è stata introdotta la necessità di indicare la gravità della sintomatologia del disturbo dello spettro autistico su una scala di tre punti: Livello 3 (Richiede supporto molto sostanziale); Livello 2 (Richiede supporto sostanziale); Livello 1 (Richiede supporto).

Una delle principali conseguenze dell'introduzione del DSM V, dimostrata dagli studi effettuati successivamente alla sua pubblicazione, è la diminuzione della percentuale di persone diagnosticate con DSA (é stata mostrata una diminuzione di cir-

\footnotetext{
82 Vagni, D. (2015) Lo spettro Autistico, risposte semplici. Per una bonifica semantica dagli stereotipi dell'autismo, Asperger ONLUS per la condivisione della conoscenza.

${ }^{83}$ Milton, D. (2012) So what exactly is autism?, AET Competence framework for the Department for Education, Birmingham

${ }^{84}$ Vagni, D. (2015) Lo spettro Autistico, risposte semplici. Per una bonifica semantica dagli stereotipi dell'autismo, Asperger ONLUS per la condivisione della conoscenza.
} 
ca il 20\%), generando numerose perplessità e dibattiti all'interno della comunità scientifica, tra le persone con autismo e le loro famiglie (Nardocci, 2014) ${ }^{85}$. Il criterio che maggiormente esclude le persone dalla diagnosi di Spettro è l' A.2 - deficit nella comunicazione non-verbale e le persone maggiormente escluse sono bambini molto piccoli e adulti con una manifestazione più lieve del disturbo o con un QI elevato (Vagni, 2015) ${ }^{86}$.

\subsection{L'approccio Abilitativo. Le recenti Linee Guida italiane}

L'autismo é un disturbo pervasivo dello sviluppo, che pervade per tutta la vita e non esistono al momento cure che permettano di guarire. D'altro canto esistono una serie di interventi che possono apportare dei miglioramenti significativi nella qualità della vita della persona, soprattutto nell'acquisizione delle abilità e nello sviluppo delle relazioni con gli altri, in particolare se applicate in modo precoce.

La recente Linea guida "Il trattamento dei disturbi dello spettro autistico nei bambini e negli adolescenti" a cura del Sistema Nazionale Linee Guida dell'Istituto Superiore di Sanità (SNLG-ISS, LG 21, 2001) ha riportato in maniera aggiornata e in accordo con la precedente Linea Guida Scozzese (SIGN - Scottish Intercollegiate Guideline Network, 2007) che i trattamenti che hanno dato maggiore prova di efficacia sono quelli precoci intensivi di tipo abilitativo con valenza comportamentale, cognitivo-comportamentale e psicoeducativa (ISS, 2012) ${ }^{87}$. Ricerche attestano come un intervento intensivo in età prescolare e continuato produca un netto miglioramento per quanto riguarda il quoziente intellettivo, il linguaggio ed il livello d'istruzione (Venerosi, Chiarotti, 2011) ${ }^{88}$. Anche se esistono trattamenti farmacologici che possono ridurre alcuni dei sintomi associati, l'intervento comportamentale precoce risulta essere il più efficace nel trattamento dei sintomi dell'autismo (Venerosi, Chiarotti, 2011) ${ }^{89}$. L'età evolutiva è quindi il momento più proficuo per attivare quegli interventi terapeutici rivolti al comportamento, alle competenze sociali, cognitive e di linguaggio, e allo sviluppo di un soddisfacente adattamento emozionale (Levi, $2005)^{90}$. Dato che ogni persona é diversa dall'altra, un intervento efficace deve prevedere piano abilitativo individualizzato, basato sull'accuratezza della diagnosi e del

\footnotetext{
${ }^{85}$ Nardocci, F. (2014) I Disturbi dello Spettro Autistico: evoluzioni per la classificazione, Relazione svolta al convegno Disabilità intellettive e dello sviluppo quadro diagnostico e interventi efficaci, Milano 10 Marzo 2014

${ }^{86}$ Vagni, D. (2015) Lo spettro Autistico, risposte semplici. Per una bonifica semantica dagli stereotipi dell'autismo, Asperger ONLUS per la condivisione della conoscenza.

87 ISS - Istituto Superiore della Salute (2012) Linee di indirizzo per la promozione ed il miglioramento della qualità della qualità e dell'appropriatezza degli interventi assistenziali nei Disturbi pervasivi dello sviluppo (DPS), con particolare riferimento ai disturbi dello spettro autistico, Ministero della Salute, Roma

${ }^{88}$ Venerosi A, Chiarotti F (Ed.) (2011) Autismo: dalla ricerca al governo clinico, Roma: Istituto Superiore di Sanità (Rapporti ISTISAN 11/33)

89 ibidem

90 Levi, G. (a cura di) (2005) Linee Guida Per L'autismo Raccomandazioni Tecniche-Operative Per I Servizi Di Neuropsichiatria Dell'eta' Evolutiva, SINPIA - Societa' Italiana Di Neuropsichiatria Dell'infanzia E Dell'adolescenza, Roma
} 


\section{Elena Bellini}

profilo cognitivo e funzionale del bambino. L'uso dei farmaci nel trattamento dei soggetti con Disturbi dello Spettro Autistico ha un'efficacia circoscritta al trattamento di alcuni sintomi comportamentali specifici, mentre non è stata dimostrata la sua efficacia nel trattamento di lungo periodo dei sintomi principali della sindrome (ISS, 2012) $)^{91}$. Il piano d'intervento dovrebbe integrare tutte le diverse competenze e i contesti abilitativi, quali la famiglia, la scuola e i servizi socio-sanitari. Recenti studi hanno dimostrato il "costo" dell'autismo per la società e le famiglie nell'arco di vita, anche secondo i fattori demografici, socioeconomici e assistenziali ad esso associati. Ganz (2007) ha calcolato in circa 3,2 milioni di dollari il costo di ciascun soggetto con autismo, per la società e per le famiglie, nell'arco di vita. Un costo in cui rientrano la perdita di produttività e l'assistenza necessaria (circa il 59,3\% delle risorse è imputabile alla perdita di produttività e altri costi indiretti). Nella stessa direzione vanno anche i primi risultati di uno studio attualmente in corso presso l'ISS nell'ambito del "Primo Programma Nazionale di Ricerca Strategica in età evolutiva", volto a quantificare e caratterizzare il carico di sofferenza (hurden) e i bisogni delle famiglie con un figlio affetto da un Disturbo dello Spettro Autistico e i fattori demografici, socioeconomici e assistenziali ad esso associati. Un panorama recentissimo sui bisogni delle famiglie con un componente con autismo è offerto anche dai primi risultati della ricerca "Centralità della persona e della famiglia", della Fondazione Serono e Angsa eseguita dal CENSIS, presentata in Parlamento il 22 novembre 2011. Gli interventi in età evolutiva mirati, specialistici ed intensivi, che utilizzano anche gli ambienti di vita normale (scuola e famiglia), e quelli in età adulta, finalizzati al mantenimento delle abilità acquisite, consentirebbero di ridurre in modo facilmente dimostrabile i costi dell'autismo. In particolare, si ritiene cruciale, nel rispetto dei vigenti Livelli Essenziali di Assistenza (LEA), che tutelano il diritto della persona con autismo a fruire di percorsi di integrazione sociosanitaria ed educativa, che $\mathrm{i}$ servizi sanitari specialistici di diagnosi e trattamento, siano resi accessibili e omogeneamente diffusi in tutte le Regioni e siano parte di una rete coordinata d'intervento, garantendo l'approccio multi professionale, interdisciplinare e d'età, indispensabile per poter affrontare la complessità e l'eterogeneità delle sindromi autistiche. E' altrettanto prioritario il consolidamento di una rete educativo-socio-assistenziale che sia in grado di supportare le famiglie e sostenere i processi di inclusione scolastica e sociale delle persone con autismo, qualunque sia la loro età. (ISS, 2012) ${ }^{92}$.

Nel 2011 sono state redatte quindi in Italia delle Linea Guida ${ }^{93}$ (e aggiornate nel 2015) per il trattamento dei Disturbi dello Spettro Autistico nei bambini e negli adolescenti, focalizzate sulla valutazione, la diagnosi e gli interventi clinici. Questo documento si basa sulla metodologia dello Scottish Intercollegiate Guidelines Network (SIGN) e del National Istitute for Health and Clinical Excellence (NICE) inglese e

\footnotetext{
${ }^{91}$ ISS - Istituto Superiore della Salute (2012) Linee di indirizzo per la promozione ed il miglioramento della qualità della qualità e dell'appropriatezza degli interventi assistenziali nei Disturbi pervasivi dello sviluppo (DPS), con particolare riferimento ai disturbi dello spettro autistico, Ministero della Salute, Roma

${ }_{92}$ ibidem

93 ISS - SNLG (2011) Linee Guida 21 - Il trattamento dei disturbi dello spettro autistico nei bambini e negli adolescenti, Ministero della Salute, Roma (aggiornate nel 2015)
} 
rappresenta una sintesi delle migliori conoscenze disponibili nel panorama di offerte terapeutiche rivolte alle persone con DSA e delle raccomandazioni di comportamento, messe a punto mediante un processo di revisione sistematica della letteratura e delle opinioni di esperti, che possono essere utilizzate come strumento per medici e amministratori sanitari per migliorare la qualità dell'assistenza e razionalizzare l'utilizzo delle risorse (LG 21, 2011-2015) ${ }^{94}$.

In particolare definisce tutte quelle terapie, di tipo farmacologico e nonfarmacologico, considerate efficaci e scientificamente validate al fine di offrire uno strumento operativo al professionista e un supporto ai familiari, in un panorama in cui c'è ancora poca conoscenza diffusa. Tra gli interventi terapeutici analizzati, si fa riferimento a: Supporto per le abilità comunicative; Interventi per la comunicazione sociale e l'interazione. Sono stati individuati vari studi che valutano l'efficacia di interventi volti direttamente a supportare la comunicazione sociale e l'interazione: social stories, interventi che utilizzano nuove tecnologie (video per la proiezione di filmati, computer), interventi mediati da pari (peer), training in teoria della mente e interventi basati sull'imitazione (training in imitazione reciproca o interazione imitativa). Nel complesso le prove scientifiche a oggi disponibili a sostegno dell'efficacia di questi interventi molto eterogenei sono non univoche e non definitive e non ci sono prove che definiscano quale tra i vari approcci di intervento sia il più efficace, per cui la scelta deve essere formulata sulla base di una valutazione delle caratteristiche individuali del soggetto. Secondo il parere degli esperti, è consigliabile adattare l'ambiente comunicativo, sociale e fisico di bambini e adolescenti con disturbi dello spettro autistico: le possibilità comprendono fornire suggerimenti visivi, ridurre le richieste di interazioni sociali complesse, seguire una routine, un programma prevedibile e utilizzare dei suggerimenti, minimizzare le stimolazioni sensoriali disturbanti; TEACCH (Treatment and education of autistic and related communication handicapped children): è un programma di educazione speciale rivolto a bambini con disturbi dello spettro autistico, valutato efficace nel migliorare vari outcome, tra cui abilità motorie (fini e grossolane nel breve termine, solo grossolane nel lungo termine), performance cognitive (nel breve e lungo termine), funzionamento sociale (nel breve e lungo termine) e comunicazione (solo nel breve termine, ma esiti non confermati nel lungo termine). Una limitazione alla forza delle prove di efficacia disponibili a favore del programma TEACCH è costituita dall'essere derivate esclusivamente da studi di coorte (uno singolo, gli altri inclusi nella revisione sistematica), pertanto merita di essere approfondito in ulteriori studi; Programmi intensivi comportamentali: il SIGN si basa sui principi della modificazione comportamentale attraverso l'ABA - analisi comportamentale applicata (Applied behaviour intervention), un programma intensivo (circa 20-40 ore settimanali) che si rivolge ad un intervento precoce rivolto a bambini di età prescolare, solitamente mediato dai genitori, con il supporto di professionisti specializzati. Gli studi sostengono l'efficacia dell'ABA nel migliorare le abilità intellettive (QI), il linguaggio e i comportamenti adattativi e le prove a disposizione, anche se non definitive, consentono di consiglia-

\footnotetext{
${ }^{94}$ ISS - SNLG (2011) Linee Guida 21 - Il trattamento dei disturbi dello spettro autistico nei bambini e negli adolescenti, Ministero della Salute, Roma (aggiornate nel 2015)
} 


\section{Elena Bellini}

re l'utilizzo del modello ABA nel trattamento dei bambini con DSA; Interventi per comportamenti specifici, con la finalità sia di ridurre la frequenza e la gravità del comportamento specifico sia di incrementare lo sviluppo di capacità adattative; La terapia cognitivo comportamentale (Cognitive behavior therapy, CBT), che richiede un certo livello di sviluppo cognitivo e verbale, quantificato in una revisione sistematica come un minimo di QI verbale pari a 69, quindi è rivolta al sottogruppo di soggetti con autismo ad alto funzionamento o sindrome di Asperger. Ne è consigliato l'utilizzo per il trattamento della comorbidità con i disturbi d'ansia e migliorare le capacità di gestione della rabbia; Musicoterapia, anche se non ci sono prove scientifiche sufficienti a formulare una raccomandazione sull'utilizzo della musicoterapia nei Disturbi dello Spettro Autistico (LG 21, 2011-2015) ${ }^{95}$.

Nelle Linee Guida, tra le varie disposizioni, si rileva inoltre che: la terapia con ossigeno iperbarico non produce nei bambini con disturbi dello spettro autistico alcun miglioramento clinico a confronto con il placebo, per cui questo trattamento non è raccomandato; l'equitazione assistita è stata valutata, ma per quanto i risultati siano favorevoli all'intervento sperimentale, non risultano affidabili in quanto manca un comparatore attivo e non è possibile isolare l'effetto specifico dell'intervento dal fatto che i partecipanti del gruppo sperimentale sono seguiti da operatori formati; la Tomatis sound therapy (modulazione delle frequenze per renderle simili a quelle del linguaggio parlato) non ha presentato differenze di effetto tra i gruppi, sperimentale e di controllo; il massaggio tradizionale tailandese è valutato in aggiunta alla terapia standard di integrazione sensoriale ( 2 sessioni a settimana, 1 ora per sessione), erogata da un terapista occupazionale in un centro di riabilitazione, con la sola terapia standard di integrazione sensoriale, ma non si rilevano differenze di efficacia significative tra $\mathrm{i}$ gruppi eccetto che per quanto riguarda i problemi della condotta e l'ansia, in quanto entrambi presentano miglioramenti per quanto riguarda l'iperattività, l'inattenzione-passività e i problemi del sonno; il Qigong sensory training (QST), ovvero l'integrazione del sensory training con un massaggio Qigong basato sulla medicina tradizionale cinese orientato a migliorare le difficoltà a livello del sistema sensoriale, della digestione e del sonno, è stato valutato dai genitori, e confermato dagli insegnanti, come miglioramento significativo per quanto riguarda $\mathrm{i}$ comportamenti autistici e le abilità linguistiche/sociali, il danno sensoriale e l'autoregolazione (minore ipersensibilità agli stimoli sensoriali e maggiore capacità di regolarsi emotivamente, cioè di controllare le proprie reazioni di rabbia e pianto, e di autoconsolarsi); tali miglioramenti sono confermati nel follow up di lungo periodo (dopo 5 mesi dalla conclusione dell'intervento), ma non si sa se i genitori che hanno appreso la tecnica hanno continuato a trattare i bambini; i programmi di esercizio fisico riportano miglioramenti sul comportamento, sulla performance scolastica, sul livello di allenamento e sulla capacità di trascorrere più tempo facendo gli esercizi, in ogni caso $i$ dati ottenuti dalla revisione non forniscono elementi di prova

\footnotetext{
${ }^{95}$ ISS - SNLG (2011) Linee Guida 21 - Il trattamento dei disturbi dello spettro autistico nei bambini e negli adolescenti, Ministero della Salute, Roma (aggiornate nel 2015)
} 
sufficientemente forti a favore degli interventi, perché derivati da studi a disegno non sperimentale (cioè privi di un confronto) (LG 21, 2011-2015) ${ }^{96}$.

Le Linee Guida si sono rilevate uno strumento molto utile, anche se è stato riscontrato come ci sia una disuguaglianza nella conoscenza e nell'applicazione da parte delle diverse Regioni Italiane. Si denota inoltre che le Linee Guida 21 sono rivolte a bambini e adolescenti, ma non si ha nessun tipo di indicazione sugli adulti, per cui ancora oggi manca un documento che in qualche modo guidi l'intervento terapeutico e abilitativo. In Italia non è ancora stata svolta un'indagine puntuale sui servizi disponibili, sui percorsi diagnostici e terapeutici e non è dunque disponibile un'informativa chiara. Le diverse realtà regionali si sono mosse autonomamente a mancanza di una garanzia di un uniforme livello prestazionale nel Paese (Giofré, $2010)^{97}$.

\subsection{La normativa sull'autismo}

Un "diritto" in realtà non è tale se non è assistito da forme di garanzia che ne assicurino l'effettività, in primo luogo intesa in termini di garanzia di un intervento positivo del soggetto pubblico, attraverso una prestazione di servizi (adeguati) e di una vera e propria "giustiziabilità" dei diritti (tutela giurisdizionale) (Giani, 2010) ${ }^{98}$.

Il primo documento a livello internazionale che ha segnato un passo decisivo in ambito di tutela e diritti umani delle persone con disabilità è la Convention on the Rights of Persons with Disabilities and Optional Protocol ${ }^{99}$, approvata dall'Assemblea Generale delle Nazioni Unite nel 2006, con lo scopo di "promuovere, proteggere e garantire il pieno ed uguale godimento di tutti i diritti umani e di tutte le libertà fondamentali da parte delle persone con disabilità, e promuovere il rispetto per la loro intrinseca dignità". Per "persone con disabilità" si intende nello specifico"coloro che presentano durature menomazioni fisiche, mentali, intellettive o sensoriali che in interazione con barriere di diversa natura possono ostacolare la loro piena ed effettiva partecipazione nella società su base di uguaglianza con gli altri".

Con la Legge 3 marzo 2009, n. 18 "Ratifica ed esecuzione della Convenzione delle Nazioni Unite sui diritti delle persone con disabilità, con Protocollo opzionale, fatta a New York il 13 dicembre 2006 e istituzione dell'Osservatorio nazionale sulla condizione delle persone con disabilità l'Italia ha ratificato la Convenzione Onu ed il Protocollo Opzionale, prevedendo, attraverso il lavoro dell'Osservatorio, un Programma di azione biennale sulla disabilità che si occupi e implementi tutte le politiche legate alla disabilità, proponendo ogni volta nuove idee e programmi, anche con

\footnotetext{
${ }^{96}$ ISS - SNLG (2011) Linee Guida 21 - Il trattamento dei disturbi dello spettro autistico nei bambini e negli adolescenti, Ministero della Salute, Roma (aggiornate nel 2015)

${ }^{97}$ Giofré, F. (a cura di) (2010) Autismo. Protezione sociale e architettura, Alinea, Firenze

${ }^{9}$ Giani, L. (2010) Norme e loro attuazione a favore delle persone con problemi cognitivocomportamentali, in Giofré, F. (a cura di) (2010) Autismo. Protezione sociale e architettura, Alinea, Firenze, pp. 29-39

${ }^{99}$ United Nations (2006) Convention on the Rights of Persons with Disabilities and Optional Protocol
} 


\section{Elena Bellini}

l'aiuto e la partecipazione di membri delle Associazioni Italiane maggiormente rappresentative.

Nel caso specifico dei Disturbi dello Spettro Autistico è stato finalmente approvato nel 2015 un disegno di legge, che ha segnato un punto fondamentale nella storia, definendo per la prima volta a livello giuridico l'esistenza dell'autismo, considerata fino ad ora un "disabilità invisibile". In particolare questo testo di legge prevede interventi finalizzati a garantire la tutela della salute, il miglioramento delle condizioni di vita e l'inserimento nella vita sociale delle persone con disturbi dello spettro autistico. Identifica inoltre la necessità di avere un percorso adeguato di assistenza e aggiornamento dei vigenti LEA - Livelli Essenziali di Assistenza - con l'inserimento delle prestazioni della diagnosi precoce, della cura e del trattamento individualizzato, tutelando il diritto della persona con autismo a fruire di percorsi di integrazione sociosanitaria ed educativa e dei servizi sanitari specialistici di diagnosi e trattamento, affinché siano resi accessibili e omogeneamente diffusi in tutte le Regioni e siano parte di una rete coordinata d'intervento (Ddl 344/2015) ${ }^{100}$. La legge sull'autismo decreta infine che l'Istituto superiore di sanità aggiorni le Linee guida sul trattamento dei disturbi dello spettro autistico in tutte le età della vita sulla base ell'evoluzione delle conoscenze fisiopatologiche e terapeutiche derivanti dalla letteratura scientifica e dalle buone pratiche nazionali ed internazionali, e si promuova il coordinamento degli interventi e dei servizi di cui al presente comma per assicurare la continuità dei per-corsi diagnostici, terapeutici e assistenziali nel corso della vita della persona (Ddl 344/2015) ${ }^{101}$.

Ad oggi purtroppo le famiglie risultano ancora abbandonate, specialmente guardando alla vita adulta, senza avere indicazioni sulle terapie abilitative o sulla presa incarico assistenziale per il futuro, dalla residenza, al lavoro, all'autonomia di vita. Di autismo non si muore, non si guarisce ma si può stare meglio, quindi è necessario ipotizzare una presa in carico che duri nel tempo, basata sull'integrazione e l'inclusione sociale, attraverso interventi mirati e specializzati con il supporto della psichiatria, la predisposizione di percorsi di vita autonoma e il lavoro (Marino Lupi - Presidente Autismo Toscana, 2014).

Un primo passo in questo senso é stato fatto dalla legge sul dopo di noi, approvata lo scorso anno al fine di favorire il benessere, la piena inclusione sociale e l'autonomia delle persone con disabilità (D.Lgs. 2232/2016) ${ }^{102}$. Questa legge si rivolge soprattutto ad evitare l'istituzionalizzazione e disciplinare misure di assistenza, cura e protezione nel superiore interesse delle persone con disabilità grave, non determinata dal naturale invecchiamento o da patologie connesse alla senilità, prive di sostegno familiare in quanto mancanti di entrambi i genitori o perché gli stessi non sono in grado di fornire l'adeguato sostegno genitoriale, nonché in vista del venir meno del sostegno familiare, attraverso la progressiva presa in carico della persona

\footnotetext{
${ }^{100}$ Ddl. n. 344, approvato il 18 marzo 2015, Disposizioni in materia di diagnosi, cura e abilitazione delle persone con disturbi dello spettro autistico e di assistenza alle famiglie 101 ibidem

102 L. 26 maggio 2016, n. 2232, in materia di Disposizioni in materia di assistenza in favore delle persone con disabilità grave prive del sostegno familiare
} 
interessata già durante l'esistenza in vita dei genitori (D.Lgs. 2232/2016) ${ }^{103}$, anche attraverso l'istituzione di un Fondo e di sgravi e detrazioni fiscali. In particolare il Fondo è destinato ad attivare e potenziare programmi di intervento di deistituzionalizzazione e supporto alla domiciliarità in abitazioni o gruppi-appartamento che riproducano le condizioni abitative e relazionali della casa familiare e che tengano conto anche delle migliori opportunità offerte dalle nuove tecnologie, al fine di impedire l'isolamento delle persone con disabilità grave; interventi per la permanenza temporanea in una soluzione abitativa extrafamiliare per far fronte ad eventuali situazioni di emergenza, nel rispetto della volontà delle persone con disabilità grave, ove possibile, dei loro genitori o di chi ne tutela gli interessi; realizzare interventi innovativi di residenzialità volti alla creazione di soluzioni alloggiative di tipo familiare e di co-housing; sviluppare programmi di accrescimento della consapevolezza, di abilitazione e di sviluppo delle competenze per la gestione della vita quotidiana e per il raggiungimento del maggior livello di autonomia possibile delle persone con disabilità grave (D.Lgs. 2232/2016) $)^{104}$.

Nuove soluzioni di residenzialità rappresentano quindi uno sviluppo importante nella ricerca, che dovrà essere perseguito anche e soprattutto nell'ottica di un adeguamento dei servizi esistenti. Si rilevano di particolare interesse i "progetti autonomia", ovvero percorsi che inducano all'abilitazione e alla vita indipendente, non solo attraverso l'apprendimento delle abilità quotidiane ma anche grazie e attraverso l'ambiente ed il supporto dato dalla progettazione dei servizi residenziali o alle tecniche di adattamento domestico.

103 L. 26 maggio 2016, n. 2232, in materia di Disposizioni in materia di assistenza in favore delle persone con disabilità grave prive del sostegno familiare

104 ibidem 


\section{Capitolo 2}

Contesti Ambientali e Spazi per l'Autismo. Stato dell'Arte

\subsection{Design for Autism}

\subsubsection{Il profilo senso-percettivo. Il rapporto con il contesto ambientale}

Ciò che i professionisti vedono come "autistico" è solitamente - per ragioni naturali - ciò che vedono, non ciò che le persone autistiche sperimentano. Molte persone con Sindrome di Asperger/autismo ad alto funzionamento definiscono i loro problemi di elaborazione sensoriale come più disabilitanti dei deficit di comunicazione/comportamento sociale (Gunilla Gerland) ${ }^{105}$.

Come visto nei precedenti capitoli una delle differenze maggiori introdotta all'interno del DSM-5 ${ }^{106}$ rispetto al DSM IV ${ }^{107}$ è proprio l'inserimento degli aspetti sensoriali come caratteri essenziali e non più associati alla diagnosi di DSA. Con "aspetti sensoriali" ci si riferisce ad una particolare sensibilità e reattività agli stimoli, come ad esempio un'apparente indifferenza al dolore/alla temperatura, risposta avversa a suoni o consistenze specifiche, eccessivo odorare o toccare degli oggetti, affascinazione visiva di luci o movimenti (APA, 2013) ${ }^{108}$. Fin dagli anni '60-'70 la presenza di possibili anormalità senso-percettive é stata riconosciuta come una delle caratteristiche centrali del disturbo (Rimland, 1964) ${ }^{109}$, sono nate teorie come quella della disfunzione sensoriale (Delacato, 1974) ${ }^{110}$, in cui si definisce l'autismo come disturbo sensoriale integrativo in cui il cervello non é capace di conferire un significato alle sensazioni e di organizzarle in percezioni e infine in concetti (Ayres, $1979)^{111}$, ma le esperienze sensoriali insolite non sono mai state considerate come

\footnotetext{
${ }^{105}$ Bogdashina O. (2003) Le percezioni sensoriali nell'autismo e nella sindrome di Asperger, Uovonero: Crema (edizione italiana del 2011)

${ }^{106}$ American Psychiatric Association (APA) (2013) DSM V, Diagnostic and Statistical Manual of Mental Disorders V

107 American Psychiatric Association (APA) (2002) DSM IV, Diagnostic and Statistical Manual of Mental Disorders IV

${ }^{108}$ American Psychiatric Association (APA) (2013) DSM V, Diagnostic and Statistical Manual of Mental Disorders V

109 Rimland, B. (1964) Infantile Autism: The Syndrome and Its Implications for a Neural Theory of Behavior, Englewood Cliffs, NJ: Prentice-Hall

110 Delacato, C. H. (1974) The ultimate stranger: The autistic child, Knopf Doubleday Publishing Group, New York

${ }^{111}$ Ayres, A. J. (1979) Sensory Integration and the Child, Western Psychological Services, Los Angeles
} 
caratteristiche essenziali dell'autismo nelle principali classificazioni diagnostiche. Benché negli ultimi anni siano state oggetto di numerosi studi, non sono state ancora oggetto di indagini sistematiche che mettano in evidenza il loro ruolo nel causare comportamenti autistici (Bogdashina, 2003) ${ }^{112}$.

Le persone con autismo non rispondono come ci aspettiamo, poiché hanno sistemi di percezione e comunicazione differenti (Bogdashina, 2003) ${ }^{113}$.

Quando si guarda ai comportamenti problema nell'autismo (autostimolazione, autolesionismo, aggressività, attacchi di panico, ansia...), non si guarda altro che alla punta dell'iceberg, senza risalire alle cause del problema, attraverso un approccio basato sui sintomi invece che sulla prevenzione. Secondo questi studi le esperienze sensoriali anomale rappresentano una caratteristica di primaria importanza per spiegare i sintomi di base dell'autismo, poiché possono essere causa di livelli elevati di ansia, comportamenti ossessivi o compulsivi (Bogdashina, 2003) ${ }^{114}$. La scienza ha confermato da tempo che l'integrazione sensoriale avviene nel tronco encefalico, e che le disfunzioni dell'integrazione sensoriale causano l'equivalente di un ingorgo stradale nel cervello (Notbohm, 2015) ${ }^{115}$. Non sempre si è in grado di riconoscere immediatamente le manifestazioni del sovraccarico sensoriale, ma comportamenti ricorrenti sono coprirsi le mani con le orecchie, comportamenti di autostimolazione come dondolarsi, sbattere le mani, vagare senza meta o altre stereotipie, fino ad arrivare a scatti d'ira o di aggressività. In autismo un comportamento, per quanto possa sembrare assurdo o immotivato, ha sempre una causa, non viene mai dal nulla (Notbohm, 2015) ${ }^{116}$. L'autismo é un disturbo neurologico, pertanto bisogna ricordare che i bambini non sono in grado di controllare i propri problemi, né cambiare dei comportamenti senza un aiuto specifico. Prendere in considerazione questo diverso modo di vedere il mondo vuol dire iniziare a condividere le esigenze di una popolazione che spesso riesce a comprendere l'ambiente circostante, ma non riesce a sostenerlo poiché sovraccaricato dalle troppe e continue informazioni sensoriali. Una diversa o alterata percezione sensoriale può essere inoltre un ostacolo alla conoscenza.

Tutto ciò che conosciamo sul mondo e su noi stessi ha avuto origine attraverso $\mathrm{i}$ nostri sensi (Bogdashina, 2003) ${ }^{117}$, che riprende la citazione classica nihil est in intellectu quod prius non fuerit in sensu (niente è nell'intelletto, che prima non sia stato nei sensi), usata come assioma nella filosofia scolastica.

Fin da neonato, l'interazione del bambino con l'ambiente è la fonte primaria di conoscenza. Il bambino deve imparare ad attribuire significato alle sensazioni, immagazzinarle e rielaborarle per comprendere il contesto circostante.

\footnotetext{
112 Bogdashina O. (2003) Le percezioni sensoriali nell'autismo e nella sindrome di Asperger, Uovonero: Crema (edizione italiana del 2011)

113 ibidem

114 ibidem

${ }^{115}$ Notbohm, E. (2015) 10 cose che ogni bambino con autismo vorrebbe che tu sapessi, Edizioni Centro Studi Erickson, Trento (ed. italiana di Notbohm, E. (2012) Ten Things Every Child With Autism Wishes You Knew, Future Horizons Inc., Arlington

116 ibidem

117 Bogdashina O. (2003) Le percezioni sensoriali nell'autismo e nella sindrome di Asperger, Uovonero: Crema (edizione italiana del 2011)
} 


\section{Elena Bellini}

Gli stimoli sensoriali vengono sentiti in modo elementare attraverso le sensazioni, affettive (piacere, dolore) o rappresentative (gusto, tatto, olfatto, calore). La percezione é l'interpretazione di queste sensazioni provenienti dal mondo esterno attraverso una rielaborazione di specifiche aree del cervello, a cui sono connesse associazioni cognitive e memoria (concetti), che danno origine alla comprensione dell'ambiente circostante. La comprensione del contesto avviene quindi attraverso l'interazione con l'ambiente e l'elaborazione sensoriale; se i processi percettivi funzionano bene si é in grado di "dare un senso" all'ambiente (Bogdashina, 2003) ${ }^{118}$. Le persone con autismo possono avere difficoltà nell'acquisizione di queste abilità e soprattutto nell'integrazione di diversi sensi, utili ad esempio a funzioni come quella del parlato e delle interazioni sociali. La sensibilità sensoriale in molti casi può diventare quindi davvero invalidante, poiché compromette la possibilità di una relazione adeguata con l'ambiente circostante. Non è facile trovare la spiegazione di tale vulnerabilità sensoriale: sicuramente l'ipersensibilità agli stimoli riveste un ruolo centrale, $\mathrm{ma}$ in alcune persone è cruciale anche l'incapacità di inibire e filtrare le informazioni sensoriali non rilevanti, o la percezione di una vera e propria "frammentazione" delle proprie sensazioni tattili, uditive ed olfattive. Alcuni studi scientifici sostengono che questo problema è legato ad un'alterazione delle connessioni tra aree specifiche del cervello (Mazzone, 2015) ${ }^{119}$.

Ciò che caratterizza quindi l'autismo è in sintesi una diversa percezione del contesto ambientale, ovvero una diversa conoscenza del mondo, difficile da comprendere e codificare poiché ogni persona è diversa dall'altra, con un modello sensopercettivo sempre differente. Una famosa citazione della Dr Lorna Wing dice che "una volta incontrata una persona con autismo, si è incontrata una persona con autismo" - once you've met one person with autism, you've met one person with autism - non è possibile quindi generalizzare, ma considerare ogni singolo per il suo diverso modo di vedere e sentire il mondo.

$E^{\prime}$ possibile però fare una sintesi di quelle che sono le esperienze sensoriali ricorrenti, pur consci del fatto che variano nella presenza e nella combinazione da persona a persona e anche nello stesso individuo nel corso della sua vita. Olga Bogdashina ha strutturato nel suo lavoro una check-list che cerca di definire il profilo sensoriale della persona attraverso le risposte dei genitori di bambini con DSA ad alcune domande che si riferiscono alla peculiarità sensoriali (vista, udito, tatto, gusto, olfatto, propriocezione, sistema vestibolare) non solo riguardo al presente, ma anche al passato, al fine di identificare possibili punti di forza e di debolezza sensoriale utili per la selezione di metodi di insegnamento e trattamento appropriati (Bogdashina, $2003)^{120}$. L'ottica principale non è curare, ma rendere più tollerabile il mondo circostante. E' molto importante inoltre riuscire a cogliere i primi segni di sovraccarico sensoriale, prevenendo possibili crisi di agitazione e interrompendo l'attività fonte di

\footnotetext{
118 Bogdashina O. (2003) Le percezioni sensoriali nell'autismo e nella sindrome di Asperger, Uovonero: Crema (edizione italiana del 2011)

119 Mazzone, L. (2015) Un autistico in famiglia, Mondadori, Milano

120 Bogdashina O. (2003) Le percezioni sensoriali nell'autismo e nella sindrome di Asperger, Uovonero: Crema (edizione italiana del 2011)
} 
stress (Mazzone, 2015) ${ }^{121}$. Si analizzeranno quindi i fattori negativi esterni per poterli eliminare e ridurre il disagio causato dal contesto, mentre si sfrutteranno i fattori positivi interni alla personalità del bambino per sviluppare l'apprendimento. $\mathrm{Ci}$ sono bambini che effettuano elaborazioni monosensoriali, ovvero utilizzano un senso alla volta per percepire l'ambiente circostante; interrompere tale focalizzazione, soprattutto quando è piacevole, può provocare agitazione e difficoltà a recepire le informazioni provenienti da altri canali sensoriali. A volte le anomalie comportamentali, come urla o stereotipie motorie, non sono che strategie compensatorie o difensive, adottate per ottenere una migliore modulazione di sensazioni troppo intense o per gestire l'eccesso di informazioni sensoriali in entrata. Ci sono infine delle sensazioni che possono dare piacere o rilassare; riconoscere le preferenze sensoriali può essere di aiuto anche per entrare in relazione con la persona con autismo (Mazzone, $2015)^{122}$.

Si consideri inoltre come quando si parla di sensorialità, non ci si riferisce solo ai cinque sensi classici - vista, udito, olfatto, tatto e gusto - ma si contano nel nostro corpo almeno 21 sistemi sensoriali. In particolare oltre ai 5 sensi predominanti si attribuiscono comunemente all'essere umano anche l'equilibriocezione (senso dell'equilibrio o senso vestibolare), la propriocezione e la cinestesia (avvertire l'orientamento e il movimento dei propri arti e del corpo nello spazio), la nocicezione (dolore), il senso temporale (percezione del tempo) e la termocezione (differenze di temperatura) (Notbohm, 2015) ${ }^{123}$.

L'osservazione dei comportamenti sta alla base della comprensione delle anomalie sensoriali; le caratteristiche ricorrenti che possono derivare dallo studio del profilo senso-percettivo si possono riassumere in: iper- o ipo- sensorialità; gestalt (difficoltà nel filtrare le informazioni di sfondo rispetto a quelle in primo piano, rigidità di pensiero e mancanza di generalizzazione); fascinazione per alcuni stimoli; incoerenza della percezione; percezione frammentaria; agnosia sensoriale (difficoltà a interpretare i sensi); elaborazione ritardata; vulnerabilità al sovraccarico sensoriale; elaborazione a singolo canale (difficoltà ad integrare o elaborare stimoli multipli); percezione periferica; arresti di sistema (ad esempio come difesa in caso di sensazioni dolorose); compensazione del senso inaffidabile mediante altri sensi; risonanza o sinestesia (percezione superiore dei sensi, talenti); memoria percettiva o pensiero percettivo (usare metodi adeguati allo stile di apprendimento) (Bogdashina, $2003)^{124}$.

Molte ricerche negli anni hanno dimostrato come la qualità dell'ambiente e degli spazi costruiti sia importante per il benessere dell'uomo e come, allo stesso tempo, l'uomo trasformi l'ambiente sulla base delle proprie necessità. In particolare la psicologia ambientale è quella scienza che si occupa di come l'ambiente influenza il

\footnotetext{
121 Mazzone, L. (2015) Un autistico in famiglia, Mondadori, Milano

122 ibidem

123 Notbohm, E. (2015) 10 cose che ogni bambino con autismo vorrebbe che tu sapessi, Edizioni Centro Studi Erickson, Trento (ed. italiana di Notbohm, E. (2012) Ten Things Every Child With Autism Wishes You Knew, Future Horizons Inc., Arlington

124 Bogdashina O. (2003) Le percezioni sensoriali nell'autismo e nella sindrome di Asperger, Uovonero: Crema (edizione italiana del 2011)
} 


\section{Elena Bellini}

nostro comportamento e la nostra mentre e di come viceversa l'uomo, con la sua mente e il suo comportamento, tende a modificare l'ambiente. Il nostro comportamento e il nostro modo di pensare dipendono strettamente dal dove siamo oltre che dal chi siamo. Per troppo tempo la psicologia si è concentrata solamente sugli aspetti interiori, individuali, come la personalità o l'esame della storia personale, senza inserire la mente umana ed il suo comportamento in un particolare contesto ambientale. Anche per i caratteri più propriamente determinati geneticamente, l'ambiente esercita un'influenza determinante nella crescita e nello sviluppo fenotipico e comportamentale, di fatto non si può separare l'influenza ambientale da quella genetica. L'uomo è l'essere che più è in grado di modificare l'ambiente per adattarlo ai propri scopi e bisogni ed il modo in cui viene cambiato costituisce, per lo psicologo, un indizio molto interessante sul funzionamento della mente umana (Costa, 2013) ${ }^{125}$.

Lo spazio fisico ed il suo significato possono essere interpretati con il concetto di affordance di Gibson secondo il quale la percezione della qualità fisica di un oggetto suggerisce ad un essere umano le azioni e le proprietà appropriate per interagirvi (Gibson, 1977, 1979) ${ }^{126}$; l'interazione tra uomo e ambiente costruito non dipende solo dalla forma fisica dell'architettura, ma anche dalle esperienze passate dell'osservatore, da ciò in cui crede, dalle sue preferenze estetiche, etc. (Baumers, Heylighen, 2010) ${ }^{127}$. Secondo un concetto chiave di Gibson - Ask what's not inside your head but what your head's inside of (Mace, 1977) ${ }^{128}$ - la nostra "testa" è nel mondo e riceve gli inputs all'azione e alla comprensione attraverso le affordances e non è il mondo ad essere già nella nostra testa, avendo una conoscenza precostituita di come interagire con il mondo.

Le qualità ed il significato dell'ambiente non sono però sempre compatibili con le abilità delle persone con autismo, presentandosi come un mondo confuso, non confortevole e spaventoso in cui vivere e andando a provocare quei comportamenti che possano talvolta risultare strani o disturbanti (Gaudion et al., 2015) ${ }^{129}$. Il design non è solo forma e funzione, ma anche un risultato di sensazioni e sentimenti che influenzano il nostro stato di benessere. Per migliorare il nostro stato di benessere continuamente controlliamo, modifichiamo e adattiamo noi stessi rispetto all'ambiente, progettando, riproponendo ed esprimendo nuove affordances; immaginiamo però di avere poco o nessun controllo, non essere in grado ad esempio di abbassare

125 Costa, M. (2013) Psicologia ambientale e architettonica. Come l'ambiente e l'architettura influenzano la mente e il comportamento, Franco Angeli, Milano

${ }^{126}$ Gibson, J. J. (1977) The theory of affordances, in R. Shaw and J. Bransford, eds., Perceiving, acting and knowing, Eribaum, Hillsdale, NJ, USA; Gibson, J. J. (1979) The ecological approach to visual perception, Houghton Mifflin, Boston. USA

${ }^{127}$ Baumers, S. Heylighen, A. et al. (2010) Beyond the Designers' View: How People with Autism Experience Space, Proceedings of the Design Research Society Conference 2010 " Design and Complexity", 7-9 July 2010, Montreal

${ }^{128}$ Mace, W. M. (1977) James J. Gibson's strategy for perceiving: Ask not what's inside your head, but what your head's inside of, in R. Shaw and J. Bransford, eds., Perceiving, acting, and knowing: Toward an ecological psychology, Erlbaum, Hillsdale, NJ, USA, 43-67.

129 Gaudion, K., Hall, A., Myerson, J., and Pellicano, L. (2015) Design and wellbeing: Bridging the empathy gap between neurotypical designers and autistic adults, in Mani, M., and Kandachar, P., Design for Sustainable Well-being and Empowerment, IISc Press and TU Delft, 61-77. 
un suono intollerabilmente forte, o spengere una luce troppo forte (Gaudion et al., $2015)^{130}$, quale risposta darà il nostro cervello agli inputs recepiti?

La sfida dei progettisti é anticipare l'uso e le azioni indotte dall'ambiente; per le sue particolarità nell'elaborare le informazioni le persone autistiche non vedono ed interagiscono con l'ambiente in un modo unico, che influenza l'esperienza spaziale e l'interazione con il mondo circostante (Kinnaer et al., 2016) ${ }^{131}$, pertanto risulta necessario sviluppare un altro metodo per entrare in empatia con queste persone (Gaudion et al., 2015) ${ }^{132}$.

\subsubsection{Entrare in empatia. Il co-design}

"We have to challenge these myths and stereotypes about autism and work to advance an understanding that is based on better quality research, but also on the real life experiences of those of us on the autism spectrum rather than a conversation that talks about us, without us. By taking that step together, we will see more progress for self-advocates, providers and families." (Ne'eman, 2011) ${ }^{133}$.

L'empatia è la capacità di comprendere a pieno lo stato d'animo altrui, attraverso un processo di immedesimazione nei sentimenti e nei pensieri dell'altro. Nel caso dell'autismo si può parlare di un doppio livello di empatia (Milton, 2012) ${ }^{134}$ che deriva un lato dalla difficoltà per i neurotipici di comprendere la mente di una persona con autismo, ma dall'altro la difficoltà per le persone con autismo di comprendere il mondo neurotipico, contemplando una difficoltà di comunicazione tra due soggetti che elaborano le informazioni in modo molto diverso.

"Autreat é un ritiro annuale organizzato da e per persone autistiche in un contesto sociale costruito appositamente per eliminare le fonti di sovraccarico sensoriale e ansia, massimizzando le opportunità per chi é nello spettro autistico di rilassarsi e godersi semplicemente il fatto di essere se stessi, creando contatti con gli altri. Le mie conversazioni ad Autreat, in alcuni casi mediate da tastiere o altri dispositivi di facilitazione alla comunicazione, mi insegnarono, sulla realtà dell'essere autistici, più di quanto non avrebbe potuto fare la lettura di cento casi clinici. Mi offrirono anche, per la prima volta nella mia vita, l'opportunità di trovarmi all'interno di questa minoranza neurologica, cosa che fu illuminante riguardo ad alcune delle difficoltà che i soggetti autistici affrontano in una società non costruita per loro, e smontarono in me alcuni perniciosi stereotipi, come l'idea che queste persone siano prive di sen-

\footnotetext{
130 Gaudion, K., Hall, A., Myerson, J., and Pellicano, L. (2015) Design and wellbeing: Bridging the empathy gap between neurotypical designers and autistic adults, in Mani, M., and Kandachar, P., Design for Sustainable Well-being and Empowerment, IISc Press and TU Delft, 61-77.

${ }_{131}$ Kinnaer et al. (2016) Autism-friendly architecture from the outside in and the inside out: an explorative study based on autobiographies of autistic people, Journal of Housing and the Built Environment, 31:179-195, Springer Science+Business Media Dordecht

${ }^{132}$ Gaudion, K., Hall, A., Myerson, J., and Pellicano, L. (2015) Design and wellbeing: Bridging the empathy gap between neurotypical designers and autistic adults, in Mani, M., and Kandachar, P., Design for Sustainable Well-being and Empowerment, IISc Press and TU Delft, 61-77.

${ }_{133}$ Milton, E. M. D. (2012) The ontological status of autism: The 'double empathy problem' Disability and Society, 1-5, 2

${ }^{134}$ ibidem
} 


\section{Elena Bellini}

so dell'umorismo o di immaginazione creativa. Furono sufficienti quattro soli giorni ad 'Autismland' per farmi apparire il mondo convenzionale come una costante aggressione sensoriale" (Silberman, 2015) ${ }^{135}$.

Dr. Katie Gaudion porta un esempio molto efficace di questo concetto attraverso l'esperienza di co-design effettuata al Kingwood Trust. All'inizio del percorso di ricerca sono state visitate alcune abitazioni di adulti con autismo. La prima visita alla casa di Tom è stata fatta dal progettista da solo, senza la presenza di Tom (Gaudion et al., 2015) ${ }^{136}$. La prima reazione del progettista è stata la descrizione di uno scenario di "distruzione" all'interno dell'alloggio, trovando ad esempio il muro con la vernice sbiadita ed il legno eroso, o il divano di pelle danneggiato. La prima domanda che si è posto uscendo dall'abitazione è stata: come posso riuscire a progettare l'ambiente in modo che questo non avvenga? Settimane dopo il designer ha effettuato una seconda visita nell'appartamento cercando di interagire con Tom e provare a vivere la casa dal suo punto di vista specchiando le sue attività preferite. Ha compreso in questo modo che quelli che sembravano segni di 'distruzione' erano in realtà metodi per Tom di raggiungere una stimolazione sensoriale di tipo tattile, uditiva, propriocettiva, che lo rendesse rilassato e più felice; ad esempio Tom sembrava soddisfatto e rilassato stando seduto a tirare la pelle del suo divano, o appoggiando l'orecchio contro un muro, strofinandolo mentre si ascoltava e sentiva le vibrazioni della musica sulla superficie, scolpendo una sorta di cavità dove posizionare l'orecchio. Non potendo chiedere a Tom quali fossero le sensazioni che gli piacevano nel comportarsi in questo modo, per le difficoltà di comunicazione date dall'assenza di linguaggio verbale, il progettista è stato in grado di comprendere solo attraverso la riproduzione delle azioni e delle esperienze di Tom. La questione quindi non era come riuscire a proteggere dall'atteggiamento "distruttivo" di Tom, ma piuttosto come dare a Tom la possibilità di avere la stimolazione sensoriale di cui necessita e di fare le cose che gli piacciono.

L'aspetto interessante della ricerca di Gaudion è che sposta il punto di vista delle esigenze, passando dalla triade di deficit - triad of impairments (Wing \& Gould, $1979)^{137}$ - identificata nei criteri diagnostici fino al DSM IV e nelle generali descrizioni dell'autismo come difficoltà di comunicazione sociale, interazione sociale e immaginazione sociale, ad una triade di Abilità - strengths (Gaudion et al., 2015) ${ }^{138}$, composte da preferenze sensoriali, interessi speciali e differenti capacità di azione.

L'individuo è così al centro del processo progettuale, supportando il designer nella comprensione e quindi nell'adattamento delle affordances di ogni ambiente,

\footnotetext{
${ }^{135}$ Silberman, S. (2015) Neuro Tribes. The legacy of Autism and the Future of Neurodiversity, AVERY, New York (ed. italiana 2016)

${ }^{136}$ Gaudion, K., Hall, A., Myerson, J., and Pellicano, L. (2015) Design and wellbeing: Bridging the empathy gap between neurotypical designers and autistic adults, in Mani, M., and Kandachar, P., Design for Sustainable Well-being and Empowerment, IISc Press and TU Delft, 61-77.

137 Wing, L. and Gould, J. (1979) Severe impairments of social interaction and associated abnormalities in children: Epidemiology and classification, Journal of Autism and Developmental Disorders, 9, 11-29.

${ }^{138}$ Gaudion, K., Hall, A., Myerson, J., and Pellicano, L. (2015) Design and wellbeing: Bridging the empathy gap between neurotypical designers and autistic adults, in Mani, M., and Kandachar, P., Design for Sustainable Well-being and Empowerment, IISc Press and TU Delft, 61-77.
} 
anticipando le risposte positive del comportamento ed il raggiungimento del benessere (Gaudion et al., 2015) ${ }^{139}$. Non è il metodo progettuale a cambiare ma il punto di vista del progettista, creando empatia e comprensione con la persona con autismo, anche se rimane fondamentale il supporto dello staff/caregivers per permettere di interpretare correttamente le azioni della persona, attraverso l'esperienza nell'osservare e nel comunicare con la persona stessa grazie ad una conoscenza approfondita e duratura; gli operatori hanno infatti sempre un ruolo attivo all'interno del percorso progettuale. Il limite di questo tipo di processo è che non è mai possibile generalizzare, poiché ogni persona è diversa dall'altra e diverso è il suo modo di percepire e avere esperienza del contesto, ancora maggiormente nei casi in cui la sensibilità sensoriale è molto spiccata influenzando in modo importante il benessere e la qualità della vita, come per i disturbi dello spettro autistico.

Allo stesso modo Kinnaer et al. fanno un'analisi approfondita della letteratura in ambito del design for autism, valutando attraverso alcune autobiografie i principi enunciati dalle linee guida o dai contributi delle diverse pubblicazioni sulla base delle esperienze personali delle persone. La loro ricerca dimostra che bisogna essere critici rispetto alle Linee Guida, valutando quello che è stato pubblicato e trasportandolo nel personale contesto, come un'indicazione di partenza utile nello stadio iniziale di progetto/briefing o come suggerimento e spunto per le singole persone con autismo, che possono trovare soluzioni affini e interessanti a cui magari non avevano pensato. Allo stesso tempo considerano come le sei autobiografie di questo studio non possano esser rappresentative di tutto il mondo autistico e che quindi non possa rappresentare un contributo scientifico, in quanto si riferisce ad un esiguo numero di casi, ma possono aiutare ad avvicinare le persone alla condizione reale di vita in casi di disturbi dello spettro autistico ed avere una visione più sfumata e flessibile della realtà (Kinnaer et al., 2016) ${ }^{140}$. Questo pone il primo punto di riflessione a proposito della "non universalità" della risposta progettale autism-friedly e della necessità di strutturare e rielaborare le informazioni sulla base del contesto e della persona stessa.

\subsubsection{L'approccio al progetto. Sensory-Sensitive approach/Neuro-Typical ap- proach. Ambiente ecologico/Ambiente strutturato}

Al fine di dare contributo che fosse in qualche modo invece scientifico in questo ambito, Magda Mostafa ha tentato di trovare un metodo evidence based che consideri la capacità di adattamento di un ambiente sulla base delle preferenze sensoriali della persona, ma che allo stesso tempo rappresenti uno strumento universale di progettazione, di supporto ai professionisti. L'Autism ASPECTSS ${ }^{\mathrm{TM}}$ Design Index

\footnotetext{
139 Gaudion, K., Hall, A., Myerson, J., and Pellicano, L. (2015) Design and wellbeing: Bridging the empathy gap between neurotypical designers and autistic adults, in Mani, M., and Kandachar, P., Design for Sustainable Well-being and Empowerment, IISc Press and TU Delft, 61-77.

${ }^{140}$ Kinnaer et al. (2016) Autism-friendly architecture from the outside in and the inside out: an explorative study based on autobiographies of autistic people, Journal of Housing and the Built Environment, 31:179-195, Springer Science+Business Media Dordecht
} 


\section{Elena Bellini}

(Mostafa, 2014) ${ }^{141}$ deriva dalla composizione di una matrice sensoriale - Sensory Design Matrix (Mostafa, 2008) ${ }^{142}$ - che interseca l'organizzazione spaziale e gli attributi architettonici con la complessità delle diverse sensibilità sensoriali, generando Linee Guida personalizzate in base alle esigenze dell'individuo (residenza) o di gruppi di individui con caratteristiche simili (ambiente pubblico, scolastico). Questo strumento progettuale nasce sulla base di una ricerca sperimentale effettuata in ambito scolastico all'Advance Society for Developing Skills of Special Needs Children (Quattameya, Cairo), dove sono stati fisicamente testati e misurati gli effetti degli interventi applicati alla struttura in termini di apprendimento ed in particolare rispetto all'acustica e alla strutturazione degli spazi secondo una sequenza di routines delle attività educative. Questo metodo si fonda sul concetto base che imparare in uno spazio controllato e strutturato, dove gli stimoli sono ridotti al minimo, possa offrire la possibilità di aumentare l'attenzione e la concentrazione e quindi sviluppare competenze ed abilità da riportare nell'ambiente naturale; per questo motivo si dovrà prevedere una diversa gradualità degli spazi per permettere nel tempo di progredire ed avvicinarsi sempre di più al mondo esterno (Mostafa, 2008) ${ }^{143}$. Rispetto a questo concetto si è sviluppato un ampio dibattito che vede da un lato la Sensory Design Theory ${ }^{144}$ (o Sensory-Sensitive approach ${ }^{145}$ ) e dall'altro il Neuro-Typical appro$\operatorname{ach}^{146}$. Cristopher Henry, pormotore del Neuro-Typical approach, fa un'aspra critica dell'approccio sensoriale-sensitivo poiché secondo la sua ricerca poco valido dal punto di vista scientifico, presentando carenze metodologiche, come un campione poco ampio ed un'evidenza legata principalmente a racconti personali o questionari ai caregivers, creando confusione, incoerenza ed evidenza contraddittoria (Henry, $2015)^{147}$. I limiti di questo approccio progettuale sono legati alla difficoltà di generalizzare e categorizzare i sintomi di reazione agli stimoli sulla base della frequenza, della severità e dell'eventuale cambiamento di reazione nel corso della vita, così come ai livelli di discriminazione dello stesso stimolo, oltre alla difficoltà che la persona riesca a riapplicare le abilità imparate in un contesto di tipo diverso (Henry, $2015)^{148}$. Ad esempio, in molte Linee Guida si suggerisce di eliminare la luce e la vista verso l'esterno per favorire la concentrazione e l'apprendimento in ambito scolastico, ma nessuno studio ha realmente dimostrato beneficio su questo, se non attraverso l'influenza positiva su pochi utenti, mentre molte ricerche hanno dimostrato la

\footnotetext{
${ }^{141}$ Mostafa, M. (2014) Architecture For Autism: Autism ASPECTSS ${ }^{\text {TM }}$ in School Design, in ArchnetIJAR, Volume 8 - Issue 1 - March 2014 - (143-158)

${ }_{142}$ Mostafa, M. (2008) An architecture for autism: Concepts of design intervention for the autistic user, in ArchNet-IJAR : International Journal of Architectural Research, vol.2(1), pp. 189-211

${ }^{143}$ ibidem

144 Mostafa, M. (2015) Architecture for Autism: Built Environment Performance in Accordance to the Autism ASPECTSS ${ }^{\mathrm{TM}}$ Design Index, in Design Principles and practices: an International Journal Annual Review, vol. 8, pp.55-71,Common Ground Publishing, Champaign, Illinois, USA

${ }^{145}$ Henry, C. (2015) A critical analysis of sensorysensitive and "neuro-typical" simulated architectural design in schools for autism, Design for All Institute of India, Special Issue, December 2015, Vol. 10, No. 13 , pp. 53-66

146 ibidem

147 ibidem

148 ibidem
} 
positività della vista verso l'esterno e della luce naturale in termini di salute e produttività (Henry, 2015) ${ }^{149}$. In definitiva, mentre nell'approccio sensoriale si tenta di piegare l'ambiente sula base delle anormalità sensoriali legate all'autismo, nell'approccio neuro-tipico si va a simulare l'ambiente reale in ambiente educativo al fine di apprendere direttamente come adattarsi al contesto sensoriale del mondo che ci circonda. Ad esempio, al Developmental Learning Center in Warren (New Jersey) è stata ricreata una strada principale con negozi, una banca, un laboratorio manifatturiero ed un appartamento completo di soggiorno, cucina, lavanderia, camera e area gioco (Marion, 2006) ${ }^{150}$. Magda Mostafa risponde allo stesso tempo alla critica effettuata, presentando la propria ricerca come una delle poche sperimentate sul campo, in grado di riportare i risultati scientifici ed evidence based (Mostafa, 2015) ${ }^{151}$, presentando misurazioni in ambito dell'attenzione, del tempo di risposta e della diminuzione dei comportamenti di autostimolazione del bambino, a seguito degli interventi effettuati sulla struttura scolastica di sperimentazione. Khare e Mullick hanno inoltre dimostrato attraverso una survey a 18 esperti in autismo ed 14 esperti di educazione in generale di 16 scuole negli USA e 6 in India, come gli interventi da applicare all'ambiente scolastico siano stati considerati molto favorevoli non solo per i bambini con DSA, ma anche per tutti gli altri bambini della scuola (Khare, Mullick, 2010)45.

Si può forse cercare una strada intermedia che prenda in considerazione i diversi approcci, sulla base dell'età, del contesto e delle abilità del singolo bambino e delle esigenze specifiche all'interno dell'ampio Spettro Autistico?

Sicuramente i dati rilevati mostrano un'emergenza, poiché l'autismo sta diventando una delle sfide prevalenti nell'ambiente scolastico. Questo dato è evidente se si comparano i dati di prevalenza di 1 bambino su 68 con DSA, rispetto a 1 bambino su 2000 non vedente, 1 su 769 disabile fisico, 1 su 714 non udente e 1 su 970 diagnosticato con sindrome di Down (Mostafa, 2015) ${ }^{152}$.

Un dibattito simile a quello presentato in ambito scolastico è stato sviluppato anche in ambito residenziale della vita adulta, a proposito del principio ecologico. La visione ecologica nasce dalla ricerca di una coerenza e un'integrazione tra l'intervento terapeutico, il contesto di vita e le prospettive esistenziali (Giddan, Ucelli di Nemi, 2003) ${ }^{153}$, secondo la quale le persone debbano apprendere dall'ambiente naturale stesso e attraverso interventi riabilitativi in un contesto "reale", basato sulle attività della "vita vera" e del "fare insieme": non si fa terapia occupazionale, ma lavoro; non si fa pet therapy, ma si accudiscono gli animali in stalla;

\footnotetext{
149 Henry, C. (2015) A critical analysis of sensorysensitive and "neuro-typical" simulated architectural design in schools for autism, Design for All Institute of India, Special Issue, December 2015, Vol. 10, No. 13 , pp. 53-66

${ }^{150}$ Marion, M. (2006) Bringing The World to the Classroom. EP Magazine, April: 32-35

151 Mostafa, M. (2015) Architecture for Autism: Built Environment Performance in Accordance to the Autism ASPECTSS ${ }^{\mathrm{TM}}$ Design Index, in Design Principles and practices: an International Journal Annual Review, vol. 8, pp.55-71,Common Ground Publishing, Champaign, Illinois, USA

152 ibidem

${ }^{153}$ Giddan, J. J. Ucelli Di Nemi, S. (2003) L'alternativa non urbana: le farm communities per adulti con autismo, in Barale F, Ucelli di Nemi S, Carrà G, Segagni G, eds. (2003) Il disturbo autistico in età adulta, 9: 4, pp. 341-350
} 


\section{Elena Bellini}

non si fa musicoterapia, ma si suona insieme, ecc. Il concetto che sembra maggiormente favorevole a questo approccio é quello delle farm communities, che hanno la capacità di insegnare all'adulto autistico non tanto a svolgere una serie di lavori ed occupazioni (accudire gli animali, pulire la casa, lavorare il legno e la creta) ma, tramite queste attività, a comprendere meglio i meccanismi che regolano la vita sociale e la comunicazione interumana (Barale et al., 2009) ${ }^{154}$. L'alternativa "non urbana" è particolarmente interessante per l'autismo poiché rappresenta un modello di inclusione sociale in un contesto semplice, ma ricco di stimoli e attività significative, coerente e pragmatico, con un ritmo ed una prevedibilità naturale. Le osservazioni e le critiche che sono state rivolte a questo approccio di vita sono legate soprattutto al rischio di segregazione ed isolamento rispetto alla comunità, per cui si dovrà porre attenzione affinché si mantenga un'adeguata integrazione con il contesto e con la società. Ci sono vari esempi di farm communities, sia all'estero che in Italia; d'altro canto esistono ad oggi anche alcuni esempi di "vita urbana" dove l'integrazione sociale è sviluppata proprio attraverso il lavoro, come ad esempio la ristorazione, l'ospitalità, il laboratorio artistico, ecc. Alcuni di questi casi studi saranno presentati all'interno dell'analisi dei percorsi residenziali e di autonomia.

L'altro approccio abilitativo in ambito residenziale prevede invece una strutturazione degli spazi, nell'ottica di sviluppare l'apprendimento delle abilità quotidiane e di supportare gli utenti nella vita di tutti i giorni. Le Linee Guida che saranno presentate anche nel capitolo sulla qualità dell'abitare prevedono indicazioni molto precise sulla strutturazione degli spazi, in base alle routines e alle attività giornaliere, anche coadiuvate da nuove tecnologie dell'assisted living (Ahrentzen, Steele, $2009^{155}$; Brand, $2010^{156}$; Mostafa, 2010 ${ }^{157}$, $2015^{158}$; Kinnaer et al., 2014 ${ }^{159}$ ). Se questo può esser visto come una limitazione alle libertà della persona, d'altro canto può rappresentare un modo per rendere la persona più indipendente, meno controllata e quindi più libera. Se da un lato una strutturazione molto rigida può rendere in qualche modo "schiavi delle istruzioni" integrate nell'ambiente attraverso la strutturazione come facilitazioni e guide da seguire nelle attività giornaliere, non riuscendo a trasportare le abilità acquisite in un contesto invece "naturale", d'altro canto può non

\footnotetext{
154 Barale, F. et al. (2009) L'autismo a partire dall'età adulta: nuove conoscenze, criticità, implicazioni abilitative, NÓOs, 15(3):257-91

155 Ahrentzen, S. Steele, K. (2009) Advancing Full Spectrum Housing: Design for Adults with Autism Spectrum Disorders Technical report, Arizona Board of Regents, Phoenix; Resnik , D. D. et al. (2009) Opening Doors: A discussion of residential options for adults living with autism and related disorders, Collaborative report by the Urban Land Institute Arizona, Southwest Autism Research \& Resource Center and Arizona State University

${ }_{156}$ Brand, A. (2010) Living in the community, London: Helen Hamlyn Centre, Royal College of Art

157 Mostafa, M. (2010) Housing Adaptation for Adults with Autistic Spectrum Disorder, Open House International, Urban International Press, 35, 37-48

${ }_{158}$ Mostafa, M. (2015) Architecture for Autism: Built Environment Performance in Accordance to the Autism ASPECTSS ${ }^{\mathrm{TM}}$ Design Index, in Design Principles and practices: an International Journal Annual Review, vol. 8, pp.55-71,Common Ground Publishing, Champaign, Illinois, USA

159 Kinnaer, M. Baumers, S. Heylighen, A. (2014) How do People with Autism (Like to) Live?, in Langdon, P. M . Lazar, J. Heylighen, A. Dong, H. (a cura di ) (2014) Inclusive Designing. Joining Usability, Acessibility and Inclusion, Springer Cham Heidelberg New York Dordrecht London, part IV, pp. $175-185$
} 
essere così facile (a volte impossibile) apprendere dall'ambiente "naturale" stesso. I progetti autonomia sono per questo esempi interessanti di modelli in cui si cerca di far apprendere nel modo giusto, per avere gli strumenti per vivere nel contesto esterno, accompagnando la persona nella vita di adulto, dando una risposta per il suo futuro e per il raggiungimento di una maggiore indipendenza di vita in ogni ambiente, che sia la residenza, il lavoro o il tempo libero.

\subsubsection{Descrizione del quadro esigenziale}

Come progettare allora per l'autismo? Se per prima cosa è importante entrare in empatia con le persone con DSA, provando a esperire il mondo con "sensorialità di tipo diverso", al fine di indagare e comprendere fino in fondo i loro bisogni, poi è necessario trovare delle risposte alle loro esigenze in termini di soluzioni di progetto. Si è già evidenziato come sia enormemente complesso, forse impossibile, dare una risposta univoca ed universale al problema, poiché ogni persona è diversa dall'altra ed ha un sistema percettivo e sensoriale differenziato e personale. Allo stesso tempo non è possibile pensare di poter rispondere in modo personalizzato in tutti i contesti, fino ad arrivare a quelli pubblici, dove ovviamente si deve tener conto delle innumerevoli esigenze di tutti i diversi individui. Sulla base del contesto e della situazione si dovrà quindi rispondere in modo diverso e flessibile. Nello specifico si considerano tre ambiti all'interno dei luoghi di vita, tra i più comuni e fondamentali nel percorso di vita di una persona con autismo, ovvero residenza, scuola e ambiente sanitario. In ambito residenziale, soprattutto quando si parla di alloggio personale o domicilio di famiglia, si può sviluppare la massima personalizzazione del contesto ambientale. L'adattamento domestico può tener conto delle esigenze della singola persona con autismo, definite attraverso le strategie di empatia e codesign, pur non dimenticando le esigenze dei familiari o delle persone con cui coabita. In particolare, qualora si guardi ad una struttura residenziale per più persone con autismo, si dovrà tener conto delle diverse necessità e quindi andare a conformare lo spazio in modo diversificato tra gli ambienti pubblici, semi-privati e privati, come la camera. L'ambiente dovrà comunque essere flessibile ed adattabile, avvalendosi delle assistive technologies come supporto alla personalizzazione dell'ambiente. In ambito scolastico o sanitario non si può invece progettare fin dall'inizio un ambiente che risponda a pieno alle esigenze di ogni persona con autismo, ma seguire piuttosto delle indicazioni basate su macro-categorie di esigenze, ottenute attraverso la definizione del profilo senso-percettivo analizzato precedentemente. Nello specifico, si consideri che è stato dimostrato come all'interno dell'ambiente scolastico, molte delle soluzioni progettuali valide per la definizione di un ambiente autism-friendly possono considerarsi favorevoli anche per lo sviluppo dell'attenzione, della concentrazione e dell'apprendimento di tutti gli alunni. Inoltre è possibile strutturare l'ambiente in modo da adattarlo al singolo bambino con autismo nel momento in cui si stila il Piano Educativo Individualizzato. D'altro canto, in ambito sanitario, è possibile ancora una volta modificare e adattare l'ambiente neutro attraverso le assistive technolgoies, consentendo anche in un ambiente il più possibile "universale" di ritrovare una personalizzazione ed un adeguamento alle esigenze del singolo. 


\section{Elena Bellini}

Si è scelto quindi di delineare delle macro-categorie di esigenza utili a organizzare e ordinare lo stato dell'arte in ambito della progettazione autism-friendly. Queste categorie saranno anche la base della classificazione di esperienze, progetti e prodotti all'interno della piattaforma web, poiché rilevate come le condizioni di benessere di maggiore importanza ai fine della qualità di vita delle persone con autismo.

Il primo aspetto da tenere in considerazione in un ambiente che sia autismfriendly é sicuramente la riduzione del disagio sensoriale ed ambientale, ovvero evitare il sovraccarico sensoriale che rende il mondo circostante intollerabile e talvolta incomprensibile alle persone con autismo. Un ambiente autism-friendly non è solo un ambiente neutro e privo di stimoli, ma ancor meglio un ambiente dove le persone possono essere in grado di "regolare i propri sensi". In particolare, parlando di sovraccarico sensoriale ci si riferisce alle sensibilità sensoriali più comuni e invalidanti, ovvero a quella uditiva, visiva e olfattiva (come si potrà valutare anche dai risultati delle interviste e dei questionari effettuati). Ci si occuperà quindi di compartimentazione e/o strutturazione degli spazi, distribuzione "sensoriale" degli ambienti, prossemica, qualità acustica (isolamento da e verso l'esterno e tra gli spazi interni, ma soprattutto il riverbero), qualità della luce e qualità dell'aria.

La definizione di salute dell'OMS del 1946 riporta che la salute è uno "stato di completo benessere fisico, psichico e sociale e non semplice assenza di malattia o di infermità". Pertanto migliorare la qualità di vita non è solo la riduzione del disagio ma promuovere una generale condizione di salute. In questo caso non si parla solo di igiene e salubrità, ma anche alle condizioni di comfort ambientale (come ad esempio sensibilità alla temperatura, all'irraggiamento solare, ecc.) e di tutto ciò che può rendere un ambiente "terapeutico" e favorire il benessere della persona (come ad esempio i contesti naturali o gli ambienti multisensoriali).

Promuovere la salute di un persona attraverso l'ambiente vuol dire prima di tutto progettare un ambiente sicuro. Nel caso dei disturbi dello spettro autistico il concetto di sicurezza acquisisce ancora maggiore rilevanza, sia perché le alterazioni percettive, propriocettive e di orientamento spaziale portano le persone con DSA ad essere più facilmente esposte a situazioni di rischio (Mostafa, 2015) ${ }^{160}$, sia perché una frequente sensibilità sotto-sviluppata alla temperatura o al dolore porta rischi maggiori di infortunio (Brand, 2010) ${ }^{161}$. I movimenti, continui, non previsti ed impetuosi, le attività, i comportamenti ripetitivi, le stereotipie e talvolta i comportamenti problema, possono inoltre mettere a dura prova la durabilità delle strutture, dei materiali e dei dispositivi all'interno degli ambienti (Ahrentzen, Steele, 2009 ${ }^{162}$; Beaver, $2011^{163}$; Kinnaer et al., $2016^{164}$ ).

\footnotetext{
${ }^{160}$ Mostafa, M. (2015) Architecture for Autism: Built Environment Performance in Accordance to the Autism ASPECTSS ${ }^{\text {TM }}$ Design Index, in Design Principles and practices: an International Journal Annual Review, vol. 8, pp.55-71, Common Ground Publishing, Champaign, Illinois, USA

${ }^{161}$ Brand, A. (2010) Living in the community, London: Helen Hamlyn Centre, Royal College of Art

162 Ahrentzen, S. Steele, K. (2009) Advancing Full Spectrum Housing: Design for Adults with Autism Spectrum Disorders Technical report, Arizona Board of Regents, Phoenix; Resnik , D. D. et al. (2009) Opening Doors: A discussion of residential options for adults living with autism and related disorders,
} 
Eliminati quelli che Bogdashina chiamava gli effetti negativi esterni ${ }^{165}$, bisogna iniziare a guardare agli aspetti positivi, quindi come rendere le persone "Abili" attraverso lo spazio. Le percezioni sensoriali alterate agiscono prima di tutto sulla comprensione del contesto e possono disorientare all'interno di un ambiente. Per questo motivo lo sviluppo di strategie di orientamento e comprensione sono il primo passo verso l'indipendenza e l'autonomia della persona. In particolare ci si riferisce ad un impianto planimetria che sia semplice e chiaro, ad una distribuzione spaziale che sia efficace e anche al supporto di sistemi di comunicazione e segnaletica di tipo grafico, attraverso immagini, in risposta al pensiero astratto (Grandin, 1995) ${ }^{166}$ tipico delle persone con autismo.

Un ambiente che Abilita è un ambiente che consente, attraverso la progettazione degli spazi, l'apprendimento ed il compimento delle attività quotidiane per quanto possibile in autonomia. L'indipendenza, inoltre, non è solo fare ma anche poter scegliere di fare, come diritto all'autodeterminazione della persona. Ci si rivolgerà in questo senso alla strutturazione degli spazi, sulla base delle routines, per facilitare il compimento delle azioni giornaliere, oltre a sistemi di comunicazione e al progetto di arredi, dispositivi e assistive technologies tali da supportare la persona nel compimento di queste attività.

Abilitare non vuol dire quindi solo riuscire a compiere tutte le attività giornaliere, ma anche stare con gli altri, attraverso l'integrazione nella comunità sociale.

Chi ha detto che le persone con autismo vogliono stare sole?

"Many descriptions of autism and Asperger's describe people like me as "not wanting contact with others" or "preferring to play alone" ... but I'd like to be very clear about my own feelings: I did not ever want to be alone. I played by myself because I was a failure at playing with others. I was alone as a result of my own limitations, and being alone was one of the bitterest disappointments of my young life" (Robison, 2007) ${ }^{167}$.

Lo sviluppo delle relazioni sociali è quindi l'ultimo punto di definizione delle macro-esigenze, rappresentando in qualche modo il "traguardo" di una progettazione Abilitante dove si sorpassa il concetto di accessibilità, verso quello di inclusività. In accordo con l'approccio delle Capability (Biggeri et al., 2010) ${ }^{168}$ si capovolge il punto di vista della disabilità basandosi non su ciò che la persona effettivamente fa, ma sulla gamma di possibilità ed opportunità tra cui può scegliere. Questo set di Capabi-

Collaborative report by the Urban Land Institute Arizona, Southwest Autism Research \& Resource Center and Arizona State University

${ }_{163}$ Beaver, C. (2011) Designing environments for children and adults on the autism spectrum, in Good Autism Practice (GAP), 12(1), pp.7-11

164 Kinnaer et al. (2016) Autism-friendly architecture from the outside in and the inside out: an explorative study based on autobiographies of autistic people, Journal of Housing and the Built Environment, Springer Science+Business Media Dordecht

${ }_{165}$ Bogdashina O. (2003) Le percezioni sensoriali nell'autismo e nella sindrome di Asperger, Uovonero: Crema (edizione italiana del 2011)

${ }^{166}$ Grandin, T. (1995) Thinking in pictures and other reports from my life with autism, Vintage Books, New York

${ }^{167}$ Robison, J. E. (2007) Look Me in the Eye: My Life with Asperger's, New York : Crown Publishers

168 Biggeri, M. (a cura di) (2010) Dalla relazione di cura alla relazione di prossimità. L'approccio della capability alle persone con disabilità, Liguori, Napoli 


\section{Elena Bellini}

lity non dipende solo dalle caratteristiche e delle Abilità personali, ma anche dalle risorse e dalle opportunità sociali. Questo approccio è quindi connesso ad un'idea di giustizia (Sen, 2004 ${ }^{169}$; Nussbaum, $2007^{170}$ ) dove ricercare l'uguaglianza sociale in termini di possibilità e scelte, ovvero benessere e qualità di vita.

Il modello più recente di Qualità di Vita, come approccio orientato alla persona, si basa sulla valutazione della relazione tra l'importanza attribuita ai vari ambiti di vita e la percezione individuale della soddisfazione provata negli stessi, indipendentemente dalle condizioni psico-fisiche di partenza o dal giudizio prognostico (Bertelli et al., $2015^{171}$ ). Rappresenta quindi un modello di riferimento di valutazione basato su quattro dimensioni fondamentali: Importanza, Soddisfazione, Partecipazione decisionale e Opportunità (Brown et al., $1997^{172}$ ), attraverso il quale il progetto riabilitativo globale si configura come sviluppo di abilità specifiche e ottenimento di un livello di soddisfazione generale relativo alla vita (Bertelli et al., 2015 ${ }^{173}$ ).

La stessa Convenzione delle Nazioni Unite sui diritti delle persone con disabilità nell'art. 19 promuove il diritto alla vita nella comunità, ovvero il "diritto di tutte le persone con disabilità a vivere nella società, con la stessa libertà di scelta delle altre persone, e ... la loro piena integrazione e partecipazione nella società, anche assicurando che: (a) le persone con disabilità abbiano la possibilità di scegliere, su base di uguaglianza con gli altri, il proprio luogo di residenza e dove e con chi vivere e non siano obbligate a vivere in una particolare sistemazione; (b) le persone con disabilità abbiano accesso ad una serie di servizi a domicilio o residenziali e ad altri servizi sociali di sostegno, compresa l'assistenza personale necessaria per consentire loro di vivere nella società e di inserirvisi e impedire che siano isolate o vittime di segregazione; (c) i servizi e le strutture sociali destinate a tutta la popolazione siano messe a disposizione, su base di uguaglianza con gli altri, delle persone con disabilità e siano adattate ai loro bisogni. " (Convenzione Nazioni Unite, $2006{ }^{174}$, ratificata in Italia dalla Legge 3 marzo 2009, n. 18)

Riassumendo si identificano quindi le seguenti macro-categorie:

1. Riduzione del disagio sensoriale e ambientale.

2. Salute e benessere.

3. Ambienti sicuri e durevoli.

4. Orientamento e comprensione.

5. Indipendenza e autonomia. Abilità.

6. Relazioni sociali e integrazione. Inclusività.

169 Sen, A. K. (2004) Disability and Justice, keynote speech, second international disability conference, World Bank

170 Nussbaum, M. C. (2007) Le nuove frontiere della giustizia. Disabilità, nazionalità, appartenenza di specie, Il Mulino, Bologna

${ }_{171}$ Bertelli,M. et al. (2015) L'impatto della tipologia residenziale sul percorso riabilitativo della persona con Disturbi dello Spettro Autistico, Autismo e Disturbi dello Sviluppo, vol 13, n. 2, pp. 283.291

172 Brown,I. et al. (1997) Quality of Life Instrument package for adults with development disabilities, Toronto, Centre for Health Promotion, University of Toronto

${ }^{173}$ Bertelli,M. et al. (2015) L'impatto della tipologia residenziale sul percorso riabilitativo della persona con Disturbi dello Spettro Autistico, Autismo e Disturbi dello Sviluppo, vol 13, n. 2, pp. 283.291

${ }^{174}$ United Nations (2006) Convention on the Rights of Persons with Disabilities and Optional Protocol 
Nei successivi capitoli verranno analizzate le categorie di macro-esigenze nel dettaglio facendo riferimento allo stato dell'arte in campo dell'apprendimento e della residenzialità.

\subsection{I luoghi per l'apprendimento inclusivi e abilitanti. Una scuola per tutti/una scuola specializzata}

\subsubsection{Introduzione}

Il tema della scuola e dell'educazione sono centrali in questi anni in Italia in cui si sente la necessità di aggiornare una Normativa Tecnica ferma agli anni '70 (D.M. 18-12-1975) ${ }^{175}$ e di recuperare l'ampio patrimonio edilizio scolastico che ad oggi non risponde più alle esigenze pedagogiche e didattiche, oltre ai requisiti di sicurezza e del risparmio energetico. Nel 2013 il MIUR pubblica delle Linee Guida ${ }^{176}$ aggiornate che definiscono un inquadramento di norme tecniche sugli spazi dell'apprendimento nella nuova scuola - civic center - come centro propulsore della formazione educativa e sociale, ridefinendo l'ambiente educativo integrato e i suoi standard, anche in termini di accessibilità, sicurezza, materiali e tecnologie per il risparmio energetico. Indice inoltre lo scorso anno il Concorso di Idee \#Scuoleinnovative con l'obiettivo di costruire 52 nuovi edifici distribuiti sul territorio nazionale, che dovrebbero anche porre le basi per la stesura della nuova Normativa Tecnica Nazionale.

D'altro canto, sebbene vari siano stati gli studi susseguiti negli anni in merito all'influenza dell'ambiente scolastico sulla qualità dell'apprendimento, sul benessere e comfort del bambino e degli insegnanti, con particolare riguardo all'inclusione e all'integrazione di bambini con bisogni educativi speciali, ancora le Linee Guida non riportano molte indicazioni da questo punto di vista. Restringendo il campo, non esistono ad oggi Linee Guida di riferimento, norme tecniche o standard da seguire nel caso specifico di disturbi o disabilità cognitive ed in particolare ai Disturbi dello Spettro Autistico.

"Non chiediamo ad una persona che non riesce a camminare di fare le scale, ma troviamo mezzi e strumenti per abbattere ciò che costituisce una barriera in modo da ridurre il più possibile la difficoltà. Con l'autismo sembra più faticoso comprenderla difficoltà e fornire degli strumenti compensativi. ...Nei confronti delle persone autistiche spesso non riconosciamo le vere caratteristiche della loro condizione e chiediamo che siano loro ad adattarsi a noi. Ovvero, che imparino a essere concentrati nonostante la confusione, che tollerino i rumori e le luci, che non abbiano comportamenti che secondo noi sono senza significato." (Crivelli, 2003) ${ }^{177}$

\footnotetext{
175 D.M. Dicembre 1975, in materia di Norme tecniche aggiornate relative all'edilizia scolastica, ivi compresi gli indici minimi di funzionalità didattica, edilizia ed urbanistica da osservarsi nella esecuzione di opere di edilizia scolastica

176 MIUR - Ministero dell'Istruzione, dell’Università e della Ricerca (2013) Linee guida per l'edilizia scolastica

177 Bogdashina, O. (2003) Sensory Perceptual Issues in Autism and Asperger Syndrome. Different Sensory Experiences-Different Perceptual World, Jessica Kingsley Pub, London
} 


\section{Elena Bellini}

Questa condizione non si riscontra purtroppo solo in Italia, ma anche negli altri paesi Europei, dove la nazione sicuramente più aggiornata è il Regno Unito, pubblicando nel 2012 il Building Bulletin 102: Designing for Disabled Children and Children with Special Educational Needs ${ }^{178}$, documento tecnico nazionale che guida l'approccio progettuale per bisogni educativi speciali, sia nell'ambiente scolastico tradizionale che in edifici specialistici, a partire dalle strategie, fino alla definizione degli spazi dell'apprendimento e dei dettagli tecnici, presentando anche casi studi di riferimento. Per la prima volta in questo documento si danno indicazioni specifiche sulla progettazione per l'Autismo, con particolare riferimento alle particolari sensibilità sensoriali e all'influenza del contesto ambientale sull'attenzione, la concentrazione e l'apprendimento.

La maggior parte della letteratura in ambito di educazione ed autismo è infatti stata sviluppata in UK, presentando vari studi sperimentali che considerano l'influenza dell'ambiente sensoriale sul comportamento del bambino e sulla qualità dell'apprendimento, definendo indicazioni e linee guida di riferimento in casi di DSA. Prima di entrare nel merito di queste ricerche mi sembra doveroso fare una riflessione sul fatto che il contesto culturale e sociale di riferimento dell'Italia è leggermente diverso rispetto a quello del Regno Unito. In questa realtà si suddividono $\mathrm{i}$ supporti in base alla gravità della diagnosi, ovvero se il bambino viene considerato a "basso funzionamento" o ad "alto funzionamento". I bambini ad alto funzionamento hanno acceso ai servizi di supporto "inclusivi" - insegnanti di supporto a scuola, assistenza specializzata dopo scuola, supporto dei Centri Famiglia fino ai 16 anni -, mentre i bambini a 'basso funzionamento' hanno comunque diritto alle buone pratiche di educazione, ma vengono inseriti in specifici contesti educativi che permettano di esercitare alcuni metodi di insegnamento come il TEACCH, ovvero asili (3-5 anni), scuola primaria (6-11 anni), scuola secondaria (12-16/19 anni), centri residenziali (4-19 anni), centri di respiro (4-19 anni); all'interno delle normali unità ambientali scolastiche si trovano inoltre spazi minimi ritenuti necessari, ovvero sensory room, quiet room, soft room, OT room (terapia occupazionale), piscine per l'idroterapia, 'simulazioni di sale mediche per preparare all'intervento medico, giardini sensoriali (Assirelli, 2010) ${ }^{179}$. Non è un caso quindi che nelle diverse pubblicazioni analizzate per la definizione dello stato dell'arte si parli quasi esclusivamente di Spazi Speciali per l'Educazione e anche i casi studio si riferiscano a Edifici Scolastici Specialistici, dedicati a bambini con DSA o talvolta con diversi tipi di disabilità, ma mai prevedendo una completa integrazione ed inclusione scolastica; anche quando si parla di integrazione all'interno della scuola infatti, si fa comunque riferimento ad un ambiente di classe 'speciale' all'interno dell'unità ambientale scolastica tradizionale, mentre nel contesto italiano l'integrazione tra i bambini e l'inclusione nel contesto

178 Department for Children, Schools and Families, UK (2012) Building Bulletin 102: Designing for Disabled Children and Children with Special Educational Needs, http://media.education.gov.uk/assets/files/pdf/b/building\%20bulletin\%20102.pdf

${ }^{179}$ Assirelli, M. L. (2010) I servizi sociosanitari e relazionali per persone con disturbi dello spettro dell'autismo in Inghilterra: linee di ricerca, indicazioni di progetto e casi studio, in Giofré, F. (a cura di) (2010) Autismo. Protezione sociale e architettura, Alinea, Firenze, pp. 89-102 
sociale avviene direttamente all'interno della classe stessa, come scelta socio-politica e culturale.

Questa riflessione apre le porte ad un grande dibattito: è maggiormente favorevole un ambiente scolastico che sia strutturato su misura dei bisogni specifici di apprendimento dei bambini con autismo, che permetta lo sviluppo degli interessi specifici e l'acquisizione di abilità che garantiscano una futura integrazione nella società, o piuttosto cercare fin da subito l'inclusione all'interno del mondo scolastico tradizionale, dando la possibilità di integrarsi con i propri coetanei, pur considerando specifici Programmi Educativi Integrati ed un supporto adeguato del bambino dal punto di vista del più complesso concetto di salute?

Non vi sono evidenze scientifiche che dimostrino la maggiore efficacia di un approccio rispetto all'altro, o che dimostrino che l'educazione in classi "speciali" sia necessaria o preferibile, salvo alcuni esempi di adeguamento dello spazio aula, in grado di migliorare le condizioni di apprendimento e di mantenimento dell'attenzione. Chi dice però, che questi stessi interventi di adeguamento non possano essere utili per tutti gli alunni e facilitare o potenziare la capacità di apprendimento o l'abbattimento dello stress di tutti i bambini?

Mcallister e Hadjri affrontano il tema dell'integrazione in un'interessante ricerca del 2013 in cui confrontano le note positive e negative dell'inclusione nell'ambiente scolastico tradizionale rispetto alla costituzione di "scuole dell'eccellenza" separate (Mcallister, Hadjri, 2013) ${ }^{180}$. La possibilità di inserimento nel contesto scolastico tradizionale prevede nella loro ipotesi di essere inclusi nelle classi tradizionali con ore didattiche integrative di supporto da parte dello staff o di avere un ambiente dedicato come "risorsa base specializzata" all'interno della scuola (Mcallister, Hadjri, 2013) ${ }^{181}$. Quest'ultima soluzione in particolare è considerata molto favorevole perché consente al bambino di trovarsi all'interno del contesto scolastico tradizionale, usufruendo come gli altri coetanei di tutti gli altri spazi comuni della scuola al di fuori dell'aula, pur avendo una strutturazione dell'ambiente di apprendimento adeguata e specificatamente progettata per le proprie necessità. Mcallister e Hadjri fanno una bellissima metafora della scuola come micro-city ${ }^{182}$; in questo senso l'inclusione dovrebbe essere vista come un'opportunità di crescita e formazione dal punto di vista culturale e sociale del bambino/cittadino, offrendo un'occasione unica per comprendere e conoscere la diversità e promuovere l'accettazione e la tolleranza, dando risposta ai purtroppo numerosi casi di bullismo ed esclusione sociale nei confronti della disabilità, ma anche più in generale del "diverso". Nella micro-city l'aula rappresenta la casa, un ambiente personale, appositamente progettato, sicuro e protetto; il corridoio è la strada, l'area di transizione, dove c'è rumore, movimento, cambiamento ed imprevedibilità (situazione di maggiore stress e ansia in casi di autismo); la hall, la mensa e la biblioteca sono considerati gli spazi agorà, dove avviene la riunione degli studenti e lo scambio tra di loro; le aree gioco corrispondono alla

\footnotetext{
${ }^{180}$ McAllister, K. and Hadjri, K. (2013) Inclusion and the special educational needs (SEN) resource base in mainstream schools: physical factors to maximise effectiveness, Support for Learning, 28: 57-65

${ }^{181}$ ibidem

182 ibidem
} 


\section{Elena Bellini}

piazza, ovvero all'ambiente pubblico e della comunità. Secondo questa base ogni area avrà diversi livelli di privacy e diversi requisiti progettuali che favoriscano l'accettazione e l'interazione, per il comfort ed il benessere di ogni diverso bambino.

Un interessante studio sperimentale di Khare e Mullick, dove sono stati identificati "diciotto parametri di progettazione ambientale" e poi valutati attraverso una survey a 18 esperti in autismo ed 14 esperti di educazione in generale di 16 scuole negli USA e 6 in India, descrive come gli interventi da applicare all'ambiente scolastico siano stati considerati molto favorevoli non solo per i bambini con DSA, ma anche per tutti gli altri bambini della scuola (Khare, Mullick, 2010) ${ }^{183}$.

Si può allora parlare di progettazione universale anche per l'Autismo? Si può quindi auspicare ad una completa inclusione scolastica?

Il dibattito è sicuramente ancora molto vivo e presenta soluzione diverse e contrastanti ed in parte va considerato sulla base di una valutazione integrata delle esigenze specifiche dell'ampio spettro di differenze che stanno all'interno della definizione di Disturbi dello Spettro Autistico. Trovare una soluzione innovativa di ambiente scolastico inclusivo nell'ottica dell'universal design, che tenga conto anche delle difficoltà senso-percettive, riconoscendo quelle caratteristiche dell'autismo che sono spesso "invisibili" e non rilevate (Bogdashina, 2003) ${ }^{184}$, può essere un nuovo sviluppo nell'ambito della ricerca, accettando la complessa sfida di trovare una risposta probabilmente fondata sulla flessibilità, data del fatto che gli individui autistici sono molto diversi nei loro profili senso-percettivi (Bogdashina, 2003) ${ }^{185}$.

Una ricerca rilevante in ambito scolastico in Italia, in corso di sviluppo e sperimentazione all'interno dell'Università di Udine - Dipartimento di Scienze Umane, si occupa di ricerca e studio delle prospettive pedagogiche e dei modelli didattici e di intervento educativo sui temi della didattica inclusiva. In particolare, propone un modello di ricerca e valutazione dell'inclusione scolastica, non solo dal punto di vista del vantaggio per il bambino autistico o con bisogni didattici speciali, ma per tutti gli alunni, che possono potenziare l'apprendimento attraverso un contesto facilitato (Cottini, Morganti, 2015) ${ }^{186}$.

L'inclusione si sostituisce al concetto di integrazione, definendo il diritto di tutti gli individui, qualunque sia la loro condizione, ad avere accesso all'istruzione all'interno di contesti comuni, non separati, per cui l'allievo con disabilità o con altre difficoltà non è visto come un ospite nella scuola e nella classe, ma come parte integrante della stessa. Ritornando al concetto di "uguaglianza sociale" (Sen, $2004^{187}$; Nussbaum, $2007^{188}$ ) e di "Capability" (Biggeri et al., 2010) ${ }^{189}$, tutti gli in-

\footnotetext{
${ }^{183}$ McAllister, K. and Hadjri, K. (2013) Inclusion and the special educational needs (SEN) resource base in mainstream schools: physical factors to maximise effectiveness, Support for Learning, 28: 57-65

184 Bogdashina, O. (2003) Sensory Perceptual Issues in Autism and Asperger Syndrome. Different Sensory Experiences-Different Perceptual World, Jessica Kingsley Pub, London

${ }^{185}$ ibidem

${ }^{186}$ Cottini, L. Morganti, A. (2015) Evidence-Based Education e pedagogia speciale. Principi e modelli per l'inclusione. Carocci, Roma

187 Sen, A. K. (2004) Disability and Justice, keynote speech, second international disability conference, World Bank

${ }^{188}$ Nussbaum, M. C. (2007) Le nuove frontiere della giustizia. Disabilità, nazionalità, appartenenza di specie, Il Mulino, Bologna
} 
dividui devono poter avere le medesime opportunità all'interno della società. Quindi, il contesto scolastico deve poter essere realmente inclusivo, ricercando il successo formativo per tutti gli alunni e, nello specifico, non deve essere il bambino con autismo (o altri bisogni speciali) ad adattarsi alla scuola, ma l'istituto scolastico ad eliminare le barriere e promuovere una strutturazione dell'ambiente tale da favorire l'apprendimento del bambino.

Secondo questa ricerca si possono definire quattro parole chiave per l'autistico a scuola: programmazione, organizzazione (strutturazione), didattica e compagni. E' importante dire infatti che inclusione non vuol dire non avere momenti o attività didattiche dedicate al singolo, ma al contrario si dovrà prevedere un piano educativo personalizzato con la collaborazione di un'equipe multidisciplinare e degli insegnanti, non solo di quello di sostegno, ma di tutto il corpo docente. Sistemi di didattica speciale per l'autismo sono concentrate anche sull'acquisizione di abilità quotidiane e talvolta anche attraverso il supporto della tecnologia; un'esperienza è stata fatta ad esempio con lo strumento del video modeling, dove i compagni hanno registrato dei video che riproducessero le azioni e le attività quotidiane, facilitando e l'apprendimento attraverso la simulazione. I compagni sono una risorsa fondamentale all'interno della classe; la formazione e la sensibilizzazione del corpo studentesco è utile per migliorare il contesto educativo e quindi il clima della classe, a diminuire la competitività e attivare, quando possibile, anche forme di peer tutoring. La strutturazione dell'ambiente infine, anche secondo TEACCH, è utile per favorire e potenziare l'apprendimento; ad esempio è molto importante poter avere una postazione di lavoro individuale, separata dalle altre e anche schermata, per poter focalizzare in alcuni momenti l'attenzione del bambino sull'attività, oppure strategie di comunicazione facilitata attraverso immagini come schemi visivi, o orologi adattati, pur sempre nell'ottica inclusiva di utilizzare strumenti didattici adeguati "per tutti" (Cottini, $2009^{190}$; Cottini, $2011^{191}$ ).

La strutturazione dell'ambiente fisico potrebbe in questo senso essere sviluppata in modo più approfondito, anche dal punto di vista dei requisiti ambientali e sensoriali del contesto. In questo senso, in ambito dell'ambiente scolastico progettato per l'autismo, sono state fatte diverse ricerche a livello internazionale negli ultimi anni, che vanno a definire linee guida, parametri, criteri e standard di progettazione, basate sull'esperienza personale e/o professionale degli autori (Beaver, 2011 ${ }^{192}$; Humphreys, $2011^{193}$ ), sulla revisione della letteratura, sulla sperimentazione e la predisposizione di questionari e survey a esperti di autismo, insegnanti, educatori (Tu-

\footnotetext{
189 Biggeri, M. (a cura di) (2010) Dalla relazione di cura alla relazione di prossimità. L'approccio della capability alle persone con disabilità, Liguori, Napoli

${ }^{190}$ Cottini, L. (2009) Il Bambino con autismo in classe. Quattro parole chiave per l'integrazione, Giunti, Firenze

191 Cottini, L. (2011) L'autismo a scuola. Parole chiave per l'integrazione, Carocci, Roma

192 Beaver, C. (2011) Designing environments for children and adults on the autism spectrum, in Good Autism Practice (GAP), 12(1), pp.7-11

${ }^{193}$ Humphreys, S. (2011) Architecture et Autisme, in Autisme Europe, vol. 55, pp.9-13, Bruxelles
} 


\section{Elena Bellini}

kvesson, Tufvesson, 2007 ${ }^{194}$; Khare, Mullick, 2008 ${ }^{195}-2009^{196}-2010^{197}$; Mostafa, $2008^{198}$; Henriksen, Kaup, $2010^{199}$ ), familiari (Mostafa, $2008^{200}$ ) o agli stessi utenti, ovvero studenti o adulti con autismo (Vogel, 2008) ${ }^{201}$.

All'interno di queste ricerche si è riscontrato un interesse particolare in due studi che hanno affrontato il problema attraverso una sperimentazione diretta sul campo, prevedendo una fase operativa di ricerca che ha coinvolto insegnanti, educatori e terapeuti.

\subsubsection{Due casi studio}

Il primo caso studio, sviluppato da Mcallister e Maguire, ha prodotto un vero e proprio ASD Classroom Design Kit (Mcallister, Maguire, 2012) ${ }^{202}$ con cui gli insegnanti potessero creare la loro "ideal ASD-friendly classroom", sulla base della loro esperienza, attraverso un modello tridimensionale smontabile e ricomponibile, la cui valutazione dei feedback ha dato vita a delle Linee Guida di riferimento per la costituzione di uno spazio educativo dedicato a bambini con autismo. La costituzione del modello è stata fatta attraverso una prima valutazione della letteratura, andando ad identificare 16 criteri base di progettazione, valutati in una seconda fase attraverso una check-list nelle diverse strutture dove sono state visitate 9 classi autism-friendly, scelte tra le 10 classi riqualificate nel 2005 dal Northern Ireland Southern Education and Library Board (SELB) a seguito dell' Evaluating Provision for Autistic Spectrum Disorders in Schools report, ed intervistati gli insegnanti delle diverse strutture, chiedendo una valutazione in punteggio da 1 a 5 dei 16 ASD Friendly Classroom

194 Tufvesson, C. Tufvesson, J. (2009) The building process as a tool towards an all-inclusive school. A Swedish example focusing on children with defined concentration difficulties such as ADHD, autism and Down's syndrome, Journal of Housing and the Built Environment, 24(1), 47-66

195 Khare, R., Mullick, A. (2008) Educational Spaces for Children with Autism; Design Development Process, CIB W 084 Proceedings, Building Comfortable and Liveable Environment for All, Atlanta, USA, pp. 66-75

196 Khare, R., Mullick, A. (2009) Incorporating the Behavioral Dimension in Designing Inclusive Learning Environment for Autism, in ArchNet-IJAR: International Journal of Architectural Research, 3(3), pp.45-64

197 Khare, R. Mullick, A. (2010) Universally Beneficial Educational Space Design for Children with Autism, presented in 'Designing for Children' with focus on 'Play+Learn' Bombay, India

${ }_{198}$ Mostafa, M. (2008) An architecture for autism: Concepts of design intervention for the autistic user, in ArchNet-IJAR : International Journal of Architectural Research , vol.2(1), pp. 189-211

199 Henriksen, K. Kaup, M-1. (2010) Supportive Learning Environments for Children with Autism Spectrum Disorders, in Undergraduate Research Journal for the Human Sciences, 9

${ }^{200}$ Mostafa, M. (2008) An architecture for autism: Concepts of design intervention for the autistic user, in ArchNet-IJAR : International Journal of Architectural Research , vol.2(1), pp. 189-211

201 Vogel, C. (2008) Classroom design for living and learning with autism, Autism Aspergers Digest, May-June

202 McAllister, K. Maguire, B. (2012) A design model: The autism spectrum disorder classroom design kit, in British Journal of Special Education, 39(4), 201-208; McAllister, K. Maguire, B. (2012) Design considerations for the autism spectrum disorder-friendly Key Stage 1 classroom, in Support for Learning, vol 27, n. 3, pp. 103-112, NASEN 
Criteria $^{203}$ : control \& safety - containment, good observation, safe, non-threatening; Classroom character - sense of calm, order, good proportion, non-distracting, proxemics; Classroom usage - flexible+adaptable, controlable, preditctable, nonistitutional; Physical factors - sensory-motor attuned, good quality acoustics, natural light, reduction in detail.

I fattori legati al controllo e alla sicurezza sono stati quelli valutati come più importanti, sia all'interno della classe (favorire l'assenza di angoli, di elementi su cui potersi arrampicare, o elementi mobili come librerie, etc.), che riguardo alla possibilità di avere uno "spazio di transizione" come il guardaroba all'ingresso per facilitare l'accesso e la preparazione del bambino, connettere i bagni all'aula e mantenere la possibilità di osservazione del bambino, anche quando è nelle "aree di quiete" o quando esce all'aperto, attraverso un ambiente protetto esterno direttamente accessibile dall'ambiente di classe. In merito agli altri tre gruppi di parametri il carattere trasversale principale ricercato è la ricerca di un aumento di attenzione e concentrazione del bambino, attraverso la massima riduzione degli stimoli disturbanti o distraenti. Riferendosi ai caratteri dell'aula definiscono infatti come aspetto maggiormente importante l'ordine, la calma e la tranquillità dell'ambiente, riducendo al massimo le distrazioni provenienti dal contesto (ad esempio schermando con tende o oscuranti le finestre che guardano verso l'esterno) e cercando di avere un ambiente di classe dalle dimensioni contenute, soprattutto in merito all'altezza del soffitto, per un senso di contenimento. Allo stesso modo, per quanto riguarda l'uso dell'aula, risulta fondamentale la possibilità di avere uno spazio adeguato per depositare gli oggetti non in uso e garantire una massima flessibilità dello spazio in base alle attività (ad esempio attraverso arredi con rotelle bloccabili). Infine in merito ai fattori fisici, gli aspetti considerati maggiormente sono lo studio della luce (favorire la luce naturale indiretta, regolare e personalizzare la luce artificiale, attraverso diverse zone di luce o la dimmerazione) e dell'acustica (in particolare rispetto ai rumori provenienti dalle altre zone didattiche, sportive e di gioco della scuola), sempre nell'ottica di ridurre le distrazioni, valutando in caso di sovraccarico degli stimoli la necessità di avere uno "spazio della quiete" all'interno o direttamente connesso alla classe dove "ricaricare le batterie" 204 .

Il secondo studio citato è stato invece condotto dalla ricercatrice Magda Mostafa. Nella prima fase della ricerca insegnanti e caregivers sono stati di supporto per l'identificazione di 5 caratteri architettonici e spaziali considerati influenti sul comportamento di bambini con autismo. La valutazione di questi caratteri - acoustics, visual (colors and patterns), visual (lighting), texture, olfactory and spatial sequencing of functions ${ }^{205}$ - attraverso un questionario aperto alle famiglie del FEAT - Families for Early Autism Treatment e agli insegnanti e caregivers dell'Advance

\footnotetext{
${ }^{203}$ McAllister, K. (2010) The ASD friendly classroom - Design complexity, challenge \& characteristics, Paper presented at Design Research Society International Conference (pp. 1-18). Montreal, Canada 204 ibidem

205 Mostafa, M. (2008) An architecture for autism: Concepts of design intervention for the autistic user, in ArchNet-IJAR : International Journal of Architectural Research , vol.2(1), pp. 189-211
} 


\section{Elena Bellini}

Society for Developing Skills of Special Needs Children in Cairo, ha concentrato la scelta su due fattori principali: l'acustica e la sequenza spaziale.

Nella seconda fase della ricerca quindi sono stati fatti due interventi di implementazione nelle strutture dell'Advance Society for Developing Skills of Special Needs Children, ognuno su uno dei due diversi caratteri architettonici: il primo esperimento calcola la differenza di impatto nell'acustica di una stanza per la logopedia, secondo i parametri dell'isolamento acustico (da 65.5 a 52.5 decibels) e riduzione del riverbero (dal 96\% al 57\%) attraverso rivestimenti di pavimento, muri e controsoffitto; il secondo intervento prevede la risistemazione di un layout spaziale di un'aula secondo un percorso a senso unico riferito alle routines delle attività giornaliere, separando gli spazi anche visivamente in base alle funzioni e prevedendo degli "escape spaces" 206 (un ambiente neutro, dove eventualmente inserire diverse risorse di stimolazione sensoriale) per i momenti di sovraccarico o disequilibrio sensoriale del bambino.

Il progetto si fonda sul concetto che imparare in uno spazio controllato e strutturato, dove gli stimoli sono ridotti al minimo, dia la possibilità di aumentare attenzione e concentrazione e quindi apprendere in modo più efficace e veloce, dando la possibilità di acquisire competenze e abilità da usare poi anche nell'ambiente naturale, diventando pian piano meno dipendenti dalle routines33; anche per questo motivo sono previste infatti delle aree ad isolamento acustico graduale differenziato, in modo da consentire nel tempo di progredire da un ambiente completamente insonorizzato ad un'area sempre più vicina al mondo esterno ed evitare il cosiddetto "effetto serra" ${ }^{207}$. I risultati dell'esperimento sono stati ottenuti attraverso l'osservazione di specifici indicatori di comportamento durante un intero anno scolastico su 4 classi di 6 bambini ciascuna tra i 6 e i 10 anni, prevedendo 4 classi come gruppo di controllo (in totale il $25 \%$ del corpo scolastico). Gli indicatori considerati sono stati il livello di attenzione (secondi in cui il bambino rimane concentrato), il tempo di risposta (secondi in cui risponde) e il temperamento comportamentale (misurato attraverso la ricorrenza dell'autostimolazione); per quanto riguarda le routines sono state inoltre valutate anche le abitudini del bambino attraverso una "mappa del comportamento" ${ }^{208}$, utili per la riorganizzazione degli spazi.

Tutti e tre i parametri risultano migliorati a seguito dell'implementazione spaziale di entrambi i fattori; attraverso le mappe del comportamento sono stati inoltre valutati altri aspetti interessanti: l'utilizzo dell'espace space è diminuito nel tempo, risultando necessario successivamente avere solo la consapevolezza della presenza di quell'ambiente nella classe per avere sicurezza e tranquillità; la compartimentazione della classe ha aumentato l'attenzione, grazie alla riduzione delle distrazioni visive ed in generale contenendo le stimolazioni.

Il risultato di questa ricerca é stata una sintesi di indicazioni e riferimenti come Linee Guida di riferimento, ma anche uno strumento di progettazione chiamato

\footnotetext{
206 Mostafa, M. (2008) An architecture for autism: Concepts of design intervention for the autistic user, in ArchNet-IJAR : International Journal of Architectural Research , vol.2(1), pp. 189-211

207 ibidem

208 ibidem
} 
Ambienti sensoriali "terapeutici" che rendano Abili

"sensory design matrix"209, che interseca l'organizzazione spaziale con la complessità delle diverse sensibilità sensoriali tipiche dell'autismo. Nella matrice sensoriale ogni attributo architettonico - Structure: Closure, Proportion, Scale, Orientation, Focus; Balance: Symmetry, Rhythm, Harmony, Balance; Quality: Color, Lighting, Acoustics, Texture, Ventilation; Dynamic: Sequence, Proximity, Routine ${ }^{210}$ - viene analizzato nella sua capacità di rispondere agli specifici bisogni sensoriali, generando Linee Guida personalizzate in base alle esigenze dell'individuo (residenza ) o di gruppi di individui dalle caratteristiche simili (ambiente pubblico, scolastico).

Guardando alle comuni problematiche sensoriali ambientali, sono stati quindi predisposto un Autism ASPECTSS ${ }^{\text {TM }}$ Index $^{211}$, raggruppando i principi progettuali generati dalla matrice:

- Acoustics, controllare l'ambiente acustico al fine di minimizzare i rumori di fondo, il riverbero e l'eco;

- Spatial sequencing, creando un percorso a senso unico basato sulle routines quotidiane;

- Escape space, un ambiente neutro dove rifugiarsi in caso di sovrastimolazione;

- Compartmentalization, suddividere gli spazi di un ambiente o di un edificio sulla base della funzione, differenziandoli anche visivamente attraverso colori o materiali;

- Transition Zones, zone di transizione al fine di regolarsi nel passaggio tra le aree di diverso livello di stimolo;

- Sensory Zoning, organizzazione degli spazi sulla base della qualità sensoriale, piuttosto che sulla funzione, distanziando le aree da basso stimolo (apprendimento, logopedia...) da quelle ad alto stimolo (palestra, musica...);

- Safety, cercare di eliminare qualsiasi tipo di pericolo il bambino, come ad esempio l'acqua troppo calda, o gli spigoli vivi degli arredi.

Riassumendo le diverse indicazioni e linee guida raccolte attraverso lo studio della revisione della letteratura, si possono infine definire queste categorie di interventi favorevoli per l'apprendimento:

\subsubsection{Riduzione del disagio sensoriale ed ambientale}

Il primo aspetto da valutare sono le proporzioni stesse dell'ambiente, al fine che gli spazi siano adeguatamente ampi per rispondere alle necessità di movimento, propriocezione e prossemica dei bambini con autismo (Humphreys, 2011) ${ }^{212}$. Allo stesso tempo un ambiente troppo grande può essere fonte di disagio sensoriale e disorientamento, o generare spazi morti e non utilizzati (Vogel, 2008) ${ }^{213}$, pertanto é con-

\footnotetext{
${ }^{209}$ Mostafa, M. (2008) An architecture for autism: Concepts of design intervention for the autistic user, in ArchNet-IJAR : International Journal of Architectural Research , vol.2(1), pp. 189-211

${ }^{210}$ Ibidem

${ }^{211}$ Mostafa, M. (2014) “Architecture For Autism: Autism ASPECTSS"m in School Design”, in ArchnetIJAR, Volume 8 - Issue 1 - March 2014 - (143-158)

212 Humphreys, S. (2011) Architecture et Autisme, in Autisme Europe, vol. 55, pp.9-13, Bruxelles

213 Vogel, C. (2008) Classroom design for living and learning with autism, Autism Aspergers Digest, May-June
} 


\section{Elena Bellini}

veniente mantenere un giusto equilibrio e differenziare sulla base della tipologia di spazio a cui ci si riferisce:

- sono sconsigliati i soffitti molto alti (Mcallister, Maguire, 2012) ${ }^{214}$;

- si considera al contrario favorevole uno spazio corridoio ampio che riduca lo stress sociale e sensoriale, anche se allo stesso tempo non dovrà essere troppo lungo al fine di semplificare la comprensione e l'orientamento;

- si favoriscono spazi contenuti nelle zone didattiche per l'attenzione e la concentrazione;

- Mcallister e Maguire suggeriscono diverse altezze del controsoffitto all'interno delle aule, per differenziare diverse zone e dare la possibilità di utilizzare lo spazio in modo diverso simultaneamente (Mcallister, Maguire, 2012) ${ }^{215}$;

- vengono indicati inoltre $100 \mathrm{mq}$ come superficie adeguata di aula e servizi annessi (guardaroba all'ingresso, quiet room, cucina per gli insegnanti, ma escludendo ripostiglio e 2 wc per i bambini con area dedicata al cambio) (Mcallister, Maguire, 2012) $)^{216}$.

Il principale obiettivo per la riduzione del disagio è ridurre al minimo il sovraccarico sensoriale attraverso la minimizzazione degli stimoli provenienti dal contesto ambientale, che può causare stress e ansia.

La stessa distribuzione degli ambienti scolastici consente di controllare la sovrastimolazione, organizzando l'ambiente in base ai livelli di stimolo. Separare le funzioni ad alto stimolo sensoriale rispetto a quelle a basso livello - Sensory Zoning $\left(\right.$ Mostafa, 2014) ${ }^{217}$ - consente di isolare le aree didattiche rispetto a quelle di altre attività più distraenti come la palestra, la musica, le aree gioco, o le zone dove si ha una maggiore affluenza di studenti come la hall o lo stesso corridoio, considerata una delle maggiori aree di stress sensoriale (Mcallister, Hadjri, 2013) ${ }^{218}$.

Prevedere degli spazi di transizione (Mcallister, Hadjri, 2013 ${ }^{219}$; Vogel, $2008^{220}$; Mostafa, 2014 ${ }^{221}$ ) permette inoltre al bambino di regolare i propri sensi nel passaggio da una zona ad alto stimolo ad una a basso stimolo, pubblico e privato, o viceversa, soprattutto nel caso in cui si debba iniziare l'attività didattica, che richiede una preparazione e una concentrazione maggiore. A questo scopo si propongono degli spazi filtro all'ingresso delle aule, al fine di supportare il momento di accesso all'apprendimento, aumentando la calma e la tranquillità non solo del bambino, ma di tut-

\footnotetext{
${ }^{214}$ Vogel, C. (2008) Classroom design for living and learning with autism, Autism Aspergers Digest, May-June

${ }_{215}$ McAllister, K. Maguire, B. (2012) Design considerations for the autism spectrum disorder-friendly Key Stage 1 classroom, in Support for Learning, vol 27, n. 3, pp. 103-112, NASEN

216 ibidem

${ }^{217}$ Mostafa, M. (2014) “Architecture For Autism: Autism ASPECTSS ${ }^{\text {тM }}$ in School Design”, in ArchnetIJAR, Volume 8 - Issue 1 - March 2014 - (143-158)

218 McAllister, K. and Hadjri, K. (2013) Inclusion and the special educational needs (SEN) resource base in mainstream schools: physical factors to maximise effectiveness, Support for Learning, 28: 57-65 219 ibidem

220 Vogel, C. (2008) Classroom design for living and learning with autism, Autism Aspergers Digest, May-June

${ }_{221}$ Mostafa, M. (2014) “Architecture For Autism: Autism ASPECTSSTM in School Design”, in Archnet-IJAR, Volume 8 - Issue 1 - March 2014 - (143-158)
} 
ta la classe; una tipologia di spazio "filtro" può essere rappresentata da un disimpegno adibito a guardaroba, dove fermarsi e lasciare le proprie cose (Mcallister, Maguire, $2012^{222}$ ).

Come visto anche dagli esperimenti precedenti, gli aspetti sensoriali che sono considerati di maggiore influenza sul comportamento di un bambino con autismo sono l'acustica (Khare, Mullick, 2009 ${ }^{223}$; Scott, 2009 ${ }^{224}$; Mcallister, 2010 ${ }^{225}$; Humphreys, $2011^{226}$; Mostafa, 2014 ${ }^{227}$ ) e le distrazioni visive.

Le indicazioni di Mostafa sull'acustica precedentemente riportate hanno dimostrato gli effetti positivi degli interventi di isolamento acustico e riduzione di eco e riverbero, anche grazie alle sperimentazioni sul campo in cui sono stati misurati i livelli di attenzione e concentrazione degli studenti dell'Advance Society for Developing Skills of Special Needs Children: il livello di attenzione è risultato triplicato, il tempo di risposta ridotto del $60 \%$ e i comportamenti di auto-stimolazione limitati

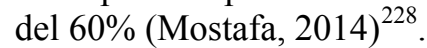

Per quanto riguarda invece le distrazioni visive, si fa riferimento a:

- Ridurre al minimo dettagli, decorazioni, colori e materiali (Vogel, 2008 229 ; Khare, Mullick, 2009 ${ }^{230}$; Humphreys, 2011 ${ }^{231}$; Sanchez, Vazquez, Serrano, $2011^{232}$ ). In particolare si consiglia l'utilizzo di colori tenui, non troppo contrastanti, pur non riducendo l'ambiente ad essere sterile ed istituzionale (Vogel, 2008) ${ }^{233}$; Humphreys propone di utilizzare ove possibile anche un solo materiale o colore sia a pavimento, che a parete o a soffitto Humphreys, $2011^{234}$ ), Mostafa invece propone di mantenere comunque una distinzione tra pavimento ed elementi verticali o soffit-

222 McAllister, K. Maguire, B. (2012) Design considerations for the autism spectrum disorder-friendly Key Stage 1 classroom, in Support for Learning, vol 27, n. 3, pp. 103-112, NASEN

${ }_{223}$ Khare, R., Mullick, A. (2009) Incorporating the Behavioral Dimension in Designing Inclusive Learning Environment for Autism, in ArchNet-IJAR: International Journal of Architectural Research, 3(3), pp.45-64

${ }^{224}$ Scott, I. (2009) Designing learning spaces for children on the autism spectrum, in Good Autism Practice (GAP), 10(1), 36-51

${ }^{225}$ McAllister, K. (2010) The ASD friendly classroom - Design complexity, challenge \& characteristics, Paper presented at Design Research Society International Conference (pp. 1-18). Montreal, Canada

${ }^{226}$ Humphreys, S. (2011) Architecture et Autisme, in Autisme Europe, vol. 55, pp.9-13, Bruxelles

${ }^{227}$ Mostafa, M. (2014) “Architecture For Autism: Autism ASPECTSS ${ }^{\mathrm{TM}}$ in School Design", in ArchnetIJAR, Volume 8 - Issue 1 - March 2014 - (143-158)

228 ibidem

229 Vogel, C. (2008) Classroom design for living and learning with autism, Autism Aspergers Digest, May-June

230 Khare, R., Mullick, A. (2009) Incorporating the Behavioral Dimension in Designing Inclusive Learning Environment for Autism, in ArchNet-IJAR: International Journal of Architectural Research, 3(3), pp.45-64

${ }^{231}$ Humphreys, S. (2011) Architecture et Autisme, in Autisme Europe, vol. 55, pp.9-13, Bruxelles

232 Sanchez, P. A. Vazquez, F. S. Serrano, L. A. (2011) Autism and the built environment, in Willliams, T. (a cura di) (2011) Autism spectrum disorders, Croatia: InTech, pp. 363-380

233 Vogel, C. (2008) Classroom design for living and learning with autism, Autism Aspergers Digest, May-June

${ }^{234}$ Humphreys, S. (2011) Architecture et Autisme, in Autisme Europe, vol. 55, pp.9-13, Bruxelles 


\section{Elena Bellini}

to per favorire l'orientamento e la percezione visiva dell'ambiente di circolazione (Mostafa, 2014) ${ }^{235}$.

- Preferire quando possibile la luce naturale, ma con un'illuminazione di tipo indiretto, diffusa in modo uniforme in tutto l'ambiente (Humphreys, 2011) ${ }^{236}$, al fine di eliminare il fenomeno dell'abbagliamento e i forti contrasti delle ombre, che potrebbero generale distrazioni visive. Questo si può ottenere utilizzando finestre alte a nastro (Humphreys, $2011^{237}$; Scott, 2009 ${ }^{238}$; Mcallister, Maguire, 2012 ${ }^{239}$ ), che consentono inoltre di eliminare anche la visibilità verso l'esterno, oppure attraverso l'utilizzo di tende o oscuranti per filtrare la luce diretta (Mcallister, Maguire, 2012 240 ; Beaver, 2011 ${ }^{241}$ ), o la predisposizione di vetri sabbiati che diffondano la luce in modo più uniforme (Humphreys, 2011) ${ }^{242}$.

- Evitare le luci fluorescenti (Humphreys, 2011243; Beaver, 2011 244; Vogel, $2008^{245}$ ), sia per la qualità luminosa della sorgente, che per lo sfarfallio prodotto dalle luci e per il ronzio prodotto dagli apparecchi, causa di disagio sensoriale. Sono da preferire luci provenienti dall'alto, ad esempio con faretti incassati a soffitto (Scott, $2009)^{246}$, oppure avere la possibilità di utilizzare molteplici tipologie di punti luce e regolarne l'illuminazione, soprattutto nell'intensità attraverso dimmer (Beaver, $2011^{247}$; Sanchez, Vazquez, Serrano, $2011^{248}$ ), per favorire la massima flessibilità e personalizzazione.

- Suddividere l'ambiente attraverso la compartimentazione (Mostafa, 2014) 249 degli spazi, al fine di ridurre al minimo gli stimoli e concentrarsi sull'attività specifica, sia attraverso la separazione fisica degli ambienti che visiva, usando differenti materiali o colori. E' inoltre possibile ottenere lo stesso effetto all'interno della classe

\footnotetext{
${ }^{235}$ Mostafa, M. (2014) “Architecture For Autism: Autism ASPECTSS"M in School Design”, in ArchnetIJAR, Volume 8 - Issue 1 - March 2014 - (143-158)

${ }^{236}$ Humphreys, S. (2011) Architecture et Autisme, in Autisme Europe, vol. 55, pp.9-13, Bruxelles

237 ibidem

238 Scott, I. (2009) Designing learning spaces for children on the autism spectrum, in Good Autism Practice (GAP), 10(1), 36-51

${ }^{239}$ McAllister, K. Maguire, B. (2012) Design considerations for the autism spectrum disorder-friendly Key Stage 1 classroom, in Support for Learning, vol 27, n. 3, pp. 103-112, NASEN

240 ibidem

241 Beaver, C. (2011) Designing environments for children and adults on the autism spectrum, in Good Autism Practice (GAP), 12(1), pp.7-11

${ }^{242}$ Humphreys, S. (2011) Architecture et Autisme, in Autisme Europe, vol. 55, pp.9-13, Bruxelles

243 ibidem

244 Beaver, C. (2011) Designing environments for children and adults on the autism spectrum, in Good Autism Practice (GAP), 12(1), pp.7-11

245 Vogel, C. (2008) Classroom design for living and learning with autism, Autism Aspergers Digest, May-June

246 Scott, I. (2009) Designing learning spaces for children on the autism spectrum, in Good Autism Practice (GAP), 10(1), 36-51

247 Beaver, C. (2011) Designing environments for children and adults on the autism spectrum, in Good Autism Practice (GAP), 12(1), pp.7-11

248 Sanchez, P. A. Vazquez, F. S. Serrano, L. A. (2011) Autism and the built environment, in Willliams, T. (a cura di) (2011) Autism spectrum disorders, Croatia: InTech, pp. 363-380

${ }^{249}$ Mostafa, M. (2014) “Architecture For Autism: Autism ASPECTSS"M in School Design”, in ArchnetIJAR, Volume 8 - Issue 1 - March 2014 - (143-158)
} 
attraverso elementi di arredo mobili, ad esempio "con ruote", pedane o piattaforme mobili, che separino gli spazi in base alle diverse attività, singole o di gruppo (Mcallister, Maguire, 2012 250 ; Vogel, 2008 ${ }^{251}$ ), coinvolgendo anche i bambini e favorendo abilità fisiche, sicurezza e indipendenza (Vogel, 2008 ${ }^{252}$ ). Garantire un certo grado di flessibilità e adattabilità in caso di autismo non vuol dire continuo cambiamento, ma avere la possibilità di trasformare l'ambiente in base alle diverse necessità (Vogel, $2008^{253}$ ). La divisione degli spazi, anche sulla base del programma TEACCH, può essere applicato anche sulla singola postazione studio attraverso elementi di schermo direttamente installati sulla postazione o scaffali laterali, al fine di concentrare la focalizzazione dell'attenzione (Henriksen, Kaup, 2010 ${ }^{254}$; Mcallister, Maguire, $2012^{255}$ ).

- Prevedere spazi ripostiglio adeguati annessi all'aula, che consentano di riporre tutti gli oggetti che non vengono utilizzati durante l'attività didattica specifica (Mcallister, Maguire, 2012) ${ }^{256}$.

Per ricalibrare le situazioni di sovraccarico é infine importante prevedere spazi neutri - "aree di quiete" o escape space (Khare, Mullick, 2009 ${ }^{257}$; Sanchez, Vazquez, Serrano, 2011 ${ }^{258}$; Mcallister, Maguire, 2012 ${ }^{259}$; Mostafa, 2014 ${ }^{260}$ ) - dove il bambino può scegliere di "rifugiarsi" e riequilibrare i propri sensi. Vengono fatti esempi di questi spazi sia in corrispondenza del corridoio o dove $i$ bambini sono sottoposti a stress sociale e sensoriale, che delle stesse aule, dove si consiglia di avere sempre all'interno o in diretta connessione, divisa da pannelli scorrevoli o separazioni vetrate, uno spazio raccolto dove potersi isolare per qualche minuto.

${ }^{250}$ McAllister, K. Maguire, B. (2012) Design considerations for the autism spectrum disorder-friendly Key Stage 1 classroom, in Support for Learning, vol 27, n. 3, pp. 103-112, NASEN

${ }^{251}$ Vogel, C. (2008) Classroom design for living and learning with autism, Autism Aspergers Digest, May-June

${ }^{252}$ ibidem

253 ibidem

254 Henriksen, K. Kaup, M-1. (2010) Supportive Learning Environments for Children with Autism Spectrum Disorders, in Undergraduate Research Journal for the Human Sciences, 9

${ }_{255}$ McAllister, K. Maguire, B. (2012) Design considerations for the autism spectrum disorder-friendly Key Stage 1 classroom, in Support for Learning, vol 27, n. 3, pp. 103-112, NASEN

256 ibidem

257 Khare, R., Mullick, A. (2009) Incorporating the Behavioral Dimension in Designing Inclusive Learning Environment for Autism, in ArchNet-IJAR: International Journal of Architectural Research, 3(3), pp.45-64

${ }^{258}$ Sanchez, P. A. Vazquez, F. S. Serrano, L. A. (2011) Autism and the built environment, in Willliams, T. (a cura di) (2011) Autism spectrum disorders, Croatia: InTech, pp. 363-380

259 McAllister, K. Maguire, B. (2012) Design considerations for the autism spectrum disorder-friendly Key Stage 1 classroom, in Support for Learning, vol 27, n. 3, pp. 103-112, NASEN

260 Mostafa, M. (2014) "Architecture For Autism: Autism ASPECTSSTM in School Design", in Archnet-IJAR, Volume 8 - Issue 1 - March 2014 - (143-158) 


\section{Elena Bellini}

\subsubsection{Salute e benessere}

Sono consigliati materiali naturali, non-tossici, senza emissioni di gas (Scott, $2009^{261}$; Khare, Mullick, $2009^{262}$; Vogel, $2008^{263}$ ) che possano garantire igiene e lavabilità e non accumulare polvere. La ventilazione trasversale é inoltre importante per igiene e salubrità dell'aria, oltre che per una riduzione degli odori, che potrebbero altrimenti infastidire a livello sensoriale (Mostafa, 2008) ${ }^{264}$, ed una regolazione della temperatura; quando non é possibile attraverso le finestre si consigliano sistemi di ventilazione forzata, oppure l'utilizzo di wind catchers e solar assisted wind catchers (Assirelli, 2010) ${ }^{265}$.

Per il benessere 'terapeutico' del bambino può essere inoltre utile prevedere spazi multisensoriali, non solo come luoghi di calma, ma anche come opportunità di sviluppare ed esplorare le diverse esperienze sensoriali, favorendo l'inclusione e l'integrazione tra i bambini (Vogel, 2008 ${ }^{266}$; Khare, Mullick, 2009 ${ }^{267}$; Sanchez, Vazquez, Serrano, $2011^{268}$; Mostafa, 2014 ${ }^{269}$ ). Mostafa propone anche l'utilizzo di un giardino sensoriale, come soluzione di transition zone all'esterno dell'edificio, ma anche come ambito di sperimentazione per il bambino attraverso la grande varietà di spazi e stimoli (percorsi strutturati, giochi d'acqua, diversi materiali e erbe aromatiche..); in questo spazio é inoltre possibile fare "arteterapia", attraverso la libera espressione artistica su una parete-lavagna, ed effettuare attività di orto e giardinaggio, considerate "terapeutiche", sia per il rapporto sviluppato con la natura, che dal punto di vista occupazionale e psicologico, poiché porta a sviluppare motivazione e autostima (Mostafa, 2014 ${ }^{270}$ ).

261 Scott, I. (2009) Designing learning spaces for children on the autism spectrum, in Good Autism Practice (GAP), 10(1), 36-51

262 Khare, R., Mullick, A. (2009) Incorporating the Behavioral Dimension in Designing Inclusive Learning Environment for Autism, in ArchNet-IJAR: International Journal of Architectural Research, 3(3), pp.45-64

263 Vogel, C. (2008) Classroom design for living and learning with autism, Autism Aspergers Digest, May-June

264 Mostafa, M. (2008) An architecture for autism: Concepts of design intervention for the autistic user, in ArchNet-IJAR : International Journal of Architectural Research , vol.2(1), pp. 189-211

265 Assirelli, M. L. (2010) I servizi sociosanitari e relazionali per persone con disturbi dello spettro dell'autismo in Inghilterra: linee di ricerca, indicazioni di progetto e casi studio, in Giofré, F. (a cura di) (2010) Autismo. Protezione sociale e architettura, Alinea, Firenze, pp. 89-102

266 Vogel, C. (2008) Classroom design for living and learning with autism, Autism Aspergers Digest, May-June

267 Khare, R., Mullick, A. (2009) Incorporating the Behavioral Dimension in Designing Inclusive Learning Environment for Autism, in ArchNet-IJAR: International Journal of Architectural Research, 3(3), pp.45-64

268 Sanchez, P. A. Vazquez, F. S. Serrano, L. A. (2011) Autism and the built environment, in Willliams, T. (a cura di) (2011) Autism spectrum disorders, Croatia: InTech, pp. 363-380

${ }^{269}$ Mostafa, M. (2014) “Architecture For Autism: Autism ASPECTSS"M in School Design”, in ArchnetIJAR, Volume 8 - Issue 1 - March 2014 - (143-158)

270 ibidem 
Ambienti sensoriali "terapeutici" che rendano Abili

\subsubsection{Ambienti sicuri e durevoli}

La sicurezza rimane uno degli aspetti costanti e ricorrenti in tutti i diversi studi effettuati (Khare, Mullick, 2009 ${ }^{271}$; Humphreys, 2011 ${ }^{272}$; Mcallister, Maguire, $2012^{273}$; Mostafa, 2014 $4^{274}$ ). L'osservazione ed il controllo sono considerati dagli insegnanti come gli aspetti di maggior importanza e stress per gli educatori, pertanto si ritiene favorevole avere la possibilità di mantenere un controllo visivo continuo, sia durante la didattica che il gioco (Mcallister, Maguire, 2012) ${ }^{275}$. Per questo motivo sono consigliati anche spazi gioco all'aperto protetti, come corti interne direttamente accessibili dall'aula, per permettere momenti di gioco sicuro, anche filtrati rispetto al parco giochi condiviso con le altre classi; in quest'area si potranno organizzare anche attività didattiche e lezioni all'aperto (Humphreys, 20112 ${ }^{276}$; Mcallister, Maguire, $2012^{277}$; Mostafa, 2014 278 ).

Un altro aspetto della sicurezza si riferisce ai materiali, agli arredi e ai dispositivi usati:

- Gli arredi devono essere ben stabili, se "con ruote" con la possibilità di essere fissati;

- non vi devono essere elementi su cui potersi arrampicare;

- evitare spigoli vivi (Humphreys, $2011^{279}$; Mcallister, Maguire, 2012 280 ; Mostafa, $2014^{281}$ );

- limitare la temperatura dell'acqua, poiché qualcuno potrebbe non sentire il calore e bruciarsi (Mostafa, 2014 282 );

- preferire il riscaldamento a pavimento (o a soffitto), rispetto al tradizionale (Humphreys, 2011 283 ), sia perché é possibile avere circuiti a temperatura regolabile,

271 Khare, R., Mullick, A. (2009) Incorporating the Behavioral Dimension in Designing Inclusive Learning Environment for Autism, in ArchNet-IJAR: International Journal of Architectural Research, 3(3), pp.45-64

${ }^{272}$ Humphreys, S. (2011) Architecture et Autisme, in Autisme Europe, vol. 55, pp.9-13, Bruxelles

273 McAllister, K. Maguire, B. (2012) Design considerations for the autism spectrum disorder-friendly

Key Stage 1 classroom, in Support for Learning, vol 27, n. 3, pp. 103-112, NASEN

274 Mostafa, M. (2014) "Architecture For Autism: Autism ASPECTSSTM in School Design", in Archnet-IJAR, Volume 8 - Issue 1 - March 2014 - (143-158)

275 McAllister, K. Maguire, B. (2012) Design considerations for the autism spectrum disorder-friendly Key Stage 1 classroom, in Support for Learning, vol 27, n. 3, pp. 103-112, NASEN

${ }^{276}$ Humphreys, S. (2011) Architecture et Autisme, in Autisme Europe, vol. 55, pp.9-13, Bruxelles

277 McAllister, K. Maguire, B. (2012) Design considerations for the autism spectrum disorder-friendly Key Stage 1 classroom, in Support for Learning, vol 27, n. 3, pp. 103-112, NASEN

278 Mostafa, M. (2014) "Architecture For Autism: Autism ASPECTSS Archnet-IJAR, Volume 8 - Issue 1 - March 2014 - (143-158)

${ }^{279}$ Humphreys, S. (2011) Architecture et Autisme, in Autisme Europe, vol. 55, pp.9-13, Bruxelles

280 McAllister, K. Maguire, B. (2012) Design considerations for the autism spectrum disorder-friendly

Key Stage 1 classroom, in Support for Learning, vol 27, n. 3, pp. 103-112, NASEN

281 Mostafa, M. (2014) "Architecture For Autism: Autism ASPECTSS"м in School Design", in Archnet-IJAR, Volume 8 - Issue 1 - March 2014 - (143-158)

282 ibidem

283 Humphreys, S. (2011) Architecture et Autisme, in Autisme Europe, vol. 55, pp.9-13, Bruxelles 


\section{Elena Bellini}

sia perché consente di proteggere il bambino da eventuali scottature (Assirelli, $2010)^{284}$

- prevedere il vetro infrangibile ad alta sicurezza (Beaver, $2011^{285}$ ).

Considerare infine l'utilizzo di strutture, materiali, arredi e dispositivi che siano durevoli nel tempo, anche se sottoposti a stress e carichi di movimenti ripetitivi e stereotipie a volte presenti in bambini con autismo (Khare, Mullick, 2009 ${ }^{286}$ ), permettendo il gioco libero senza porre troppe restrizioni o continue istruzioni (Sanchez, Vazquez, Serrano, 2011 ${ }^{287}$ ). La scelta di utilizzare materiali "robusti" non deve però dare il senso di un ambiente istituzionale; si consigliano materiali morbidi all'impatto, che prevengano da autolesioni spesso inconsapevoli, facilmente pulibili, manutenibili, o sostituibili (Assirelli, 2010) ${ }^{288}$.

\subsubsection{Orientamento e comprensione}

Definire un disegno chiaro e leggibile degli spazi che dia tranquillità, sia prevedibile (Vogel, 2008) ${ }^{289}$, favorisca l'orientamento (Humphreys, 2011) ${ }^{290}$ e l'utilizzo dell'edificio. L'orientamento può essere guidato anche attraverso istruzioni visive (Vogel, 2008 ${ }^{291}$; Khare, Mullick, 2009 ${ }^{292}$ ) che facilitino la comprensione come immagini, indicazioni scritte, o entrambe; il connubio tra immagini e scritte può essere di supporto anche all'inclusione degli studenti, dando a tutti la stessa possibilità di leggere e comprendere le istruzioni, oltre a favorire l'apprendimento attraverso l'associazione tra parole ed immagini visive (Khare, Mullick, 2009 ${ }^{293}$ ).

Organizzare quindi il layout degli spazi in modo semplice e chiaro dove ogni zona sia identificata per carattere e funzione senza ambiguità d'uso, al fine di migliorare la comprensione. I percorsi devono essere ben evidenziati, conducendo alle diverse aree suddivise per somiglianza attraverso colori, nomi, simboli, separazioni

284 Assirelli, M. L. (2010) I servizi sociosanitari e relazionali per persone con disturbi dello spettro dell'autismo in Inghilterra: linee di ricerca, indicazioni di progetto e casi studio, in Giofré, F. (a cura di) (2010) Autismo. Protezione sociale e architettura, Alinea, Firenze, pp. 89-102

285 Beaver, C. (2011) Designing environments for children and adults on the autism spectrum, in Good Autism Practice (GAP), 12(1), pp.7-11

${ }^{286}$ Khare, R., Mullick, A. (2009) Incorporating the Behavioral Dimension in Designing Inclusive Learning Environment for Autism, in ArchNet-IJAR: International Journal of Architectural Research, 3(3), pp.45-64

287 Sanchez, P. A. Vazquez, F. S. Serrano, L. A. (2011) Autism and the built environment, in Willliams, T. (a cura di) (2011) Autism spectrum disorders, Croatia: InTech, pp. 363-380

288 Assirelli, M. L. (2010) I servizi sociosanitari e relazionali per persone con disturbi dello spettro dell'autismo in Inghilterra: linee di ricerca, indicazioni di progetto e casi studio, in Giofré, F. (a cura di) (2010) Autismo. Protezione sociale e architettura, Alinea, Firenze, pp. 89-102

289 Vogel, C. (2008) Classroom design for living and learning with autism, Autism Aspergers Digest, May-June

290 Humphreys, S. (2011) Architecture et Autisme, in Autisme Europe, vol. 55, pp.9-13, Bruxelles

291 Vogel, C. (2008) Classroom design for living and learning with autism, Autism Aspergers Digest, May-June

${ }^{292}$ Khare, R., Mullick, A. (2009) Incorporating the Behavioral Dimension in Designing Inclusive Learning Environment for Autism, in ArchNet-IJAR: International Journal of Architectural Research, 3(3), pp.45-64

293 ibidem 
visive o elementi distintivi; favorire le informazioni ambientali attraverso i sensi di olfatto, udito, tatto e vista, in modo che anche chi non possiede alcune abilità sensoriali possa essere guidato dalle altre (Vogel, 2008) ${ }^{294}$. Beaver suggerisce l'utilizzo di linee curve per favorire l'orientamento e la circolazione negli spostamenti (Beaver, $2011)^{295}$. La vicinanza della classe all'ingresso e la semplicità del percorso di accesso può infine essere favorevole per minimizzare lo stress e l'ansia di perdersi (Mcallister, Hadjri, 2013) ${ }^{296}$. Una soluzione interessante é inoltre bandire il concetto tradizionale e istituzionale del corridoio, ma piuttosto prevedere uno spazio dove circolare liberamente senza provare un senso di oppressione, ampio e usato come spazio multifunzionale per diverse attività; questo dà un valore aggiunto anche al cliente, giustificando il costo di ogni metro quadro aggiunto al fine di dilatare lo spazio, dando funzioni diverse rispetto al semplice collegamento da A a B (Assirelli, $2010)^{297}$.

\subsubsection{Indipendenza e autonomia. Abilità}

La distribuzione secondo sequenze visive, routines e attività educative (Khare, Mullick, 2009 ${ }^{298}$; Henriksen, Kaup, 2010 ${ }^{299}$; Mostafa, 2014 ${ }^{300}$ ) favorisce e facilita l'apprendimento, dando la possibilità di avere una maggiore indipendenza futura, basata sulle abilità acquisite non solo in campo educativo, ma anche rispetto alle attività di vita quotidiana (Mostafa, 2014 ${ }^{301}$; Khare, Mullick, 2009 ${ }^{302}$ ). Allo stesso tempo una flessibilità e adattabilità dello spazio dà la possibilità di riarrangiare lo spazio o risuddividerlo attraverso arredi e materiali modulari e di sviluppare le abilità fisiche, la sicurezza e l'indipendenza del bambino (Vogel, 2008) ${ }^{303}$.

La possibilità di scelta rappresenta un fattore importante per il senso di appartenenza e autonomia all'interno dell'ambiente scolastico. Ad esempio può essere favo-

\footnotetext{
${ }^{294}$ Vogel, C. (2008) Classroom design for living and learning with autism, Autism Aspergers Digest, May-June

295 Beaver, C. (2011) Designing environments for children and adults on the autism spectrum, in Good Autism Practice (GAP), 12(1), pp.7-11

296 McAllister, K. and Hadjri, K. (2013) Inclusion and the special educational needs (SEN) resource base in mainstream schools: physical factors to maximise effectiveness, Support for Learning, 28: 57-65

${ }^{297}$ Assirelli, M. L. (2010) I servizi sociosanitari e relazionali per persone con disturbi dello spettro dell'autismo in Inghilterra: linee di ricerca, indicazioni di progetto e casi studio, in Giofré, F. (a cura di) (2010) Autismo. Protezione sociale e architettura, Alinea, Firenze, pp. 89-102

298 Khare, R., Mullick, A. (2009) Incorporating the Behavioral Dimension in Designing Inclusive Learning Environment for Autism, in ArchNet-IJAR: International Journal of Architectural Research, 3(3), pp.45-64

${ }^{299}$ Henriksen, K. Kaup, M-1. (2010) Supportive Learning Environments for Children with Autism Spectrum Disorders, in Undergraduate Research Journal for the Human Sciences, 9

${ }^{300}$ Mostafa, M. (2014) “Architecture For Autism: Autism ASPECTSSTM in School Design”, in ArchnetIJAR, Volume 8 - Issue 1 - March 2014 - (143-158)

301 ibidem

302 Khare, R., Mullick, A. (2009) Incorporating the Behavioral Dimension in Designing Inclusive Learning Environment for Autism, in ArchNet-IJAR: International Journal of Architectural Research, 3(3), pp.45-64

${ }^{303}$ Vogel, C. (2008) Classroom design for living and learning with autism, Autism Aspergers Digest, May-June
} 


\section{Elena Bellini}

revole avere diverse possibilità di ingresso nella scuola, attraverso un'entrata principale o una secondaria, più o meno affollata, sulla base del proprio comfort e desiderio di socialità; avere la possibilità di modificare lo spazio all'interno della classe per partecipare ad attività di gruppo o scegliere di fare attività individuali; giocare nello spazio gioco comune, ma avere anche accesso ad una corte o spazio all'aperto 'sicuro' dove scegliere entrare nello spazio ampio di gioco con progressione graduale (Mcallister, Hadjri, 2013) ${ }^{304}$.

Mantenere la possibilità di osservazione continua del bambino, senza essere invasivi, permette oltre alla sicurezza di poter lasciare il bambino libero e quindi favorire la sua indipendenza e autonomia nello spazio (Khare, Mullick, 2009 ${ }^{305}$; Mcallister, Maguire, $2012^{306}$; Mostafa, 2014 ${ }^{307}$ ).

\subsubsection{Relazioni sociali. Integrazione e inclusività}

Un ambiente positivo, non-minaccioso o istituzionale, può favorire e promuove gli incontri, la comunicazione e la relazione con gli altri (Mcallister, 2010 ${ }^{308}$; Vogel, $2008^{309}$ ). Spazi molto grandi possono incutere timore e dispersione, non favorendo la relazione con le persone in un'area caotica e sovraccarica di stimoli; allo stesso modo un ambiente troppo soffocante che costringe alle relazione può creare ansia e stress e quindi bloccare le interazioni interpersonali. Un ambiente flessibile ed equilibrato rappresenta sempre la soluzione migliore, poiché consente di scegliere il grado di interazione sociale. Le zone di transizione o gli spazi di quiete sono in questo caso molto utili poiché possono aiutare a riequilibrarsi e 'accettare' gli altri, favorendo le relazioni piuttosto che isolare, come contrariamente si potrebbe pensare (Vogel, 2008) $)^{310}$. La flessibilità e l'adattabilità dell'ambiente interno alla classe é inoltre utile per favorire i lavori in piccoli gruppi, rispetto a spazi dove focalizzare l'attenzione lavorare individualmente in rapporto uno a uno con l'insegnante (Khare, Mullick, 2009) ${ }^{311}$.

Un suggerimento interessante di Beaver per favorire la socializzazione e l'interazione tra $\mathrm{i}$ bambini è l'utilizzo di spazi di collegamento orizzontale che fungano da

\footnotetext{
${ }^{304}$ McAllister, K. and Hadjri, K. (2013) Inclusion and the special educational needs (SEN) resource base in mainstream schools: physical factors to maximise effectiveness, Support for Learning, 28: 57-65

${ }^{305}$ Khare, R., Mullick, A. (2009) Incorporating the Behavioral Dimension in Designing Inclusive Learning Environment for Autism, in ArchNet-IJAR: International Journal of Architectural Research, 3(3), pp.45-64

${ }^{306}$ McAllister, K. Maguire, B. (2012) Design considerations for the autism spectrum disorder-friendly Key Stage 1 classroom, in Support for Learning, vol 27, n. 3, pp. 103-112, NASEN

${ }^{307}$ Mostafa, M. (2014) “Architecture For Autism: Autism ASPECTSS ${ }^{\mathrm{TM}}$ in School Design”, in ArchnetIJAR, Volume 8 - Issue 1 - March 2014 - (143-158)

${ }^{308}$ McAllister, K. (2010) The ASD friendly classroom - Design complexity, challenge \& characteristics, Paper presented at Design Research Society International Conference (pp. 1-18). Montreal, Canada

${ }^{309}$ Vogel, C. (2008) Classroom design for living and learning with autism, Autism Aspergers Digest, May-June

310 ibidem

311 Khare, R., Mullick, A. (2009) Incorporating the Behavioral Dimension in Designing Inclusive Learning Environment for Autism, in ArchNet-IJAR: International Journal of Architectural Research, 3(3), pp.45-64
} 
corridoi "funzionali", ovvero che vengano utilizzati non solo per gli spostamenti, ma anche come area attrezzata e area gioco (Beaver, 2011) ${ }^{312}$.

Spazi favorevoli di relazione sono infine, oltre alle aree gioco, anche gli ambienti come la palestra dove fare attività di psicomotricità e gli spazi multisensoriali, dove si può favorire a pieno l'integrazione e l'inclusione del bambino (Vogel, 2008) ${ }^{313}$.

\subsection{Luoghi per l'abitare e l'autonomia. Residenze "protette"/adattamento do- mestico}

\subsubsection{Introduzione. Le Linee Guida esistenti}

Guardando alla letteratura scientifica, italiana e perlopiù straniera, vi sono vari studi a proposito dei caratteri e dei requisiti di soluzioni per l'abitare per l'autismo, anche se concentrate soprattutto dal punto di vista delle residenze "protette", piuttosto che in ambito dell'adattamento domestico, dove la letteratura è piuttosto carente. Sono state redatte delle vere e proprie Linee Guida, anche dettagliate, che partono dalla descrizione del contesto, fino ad arrivare ai materiali o alle tecnologie di assistenza all'interno delle abitazioni, tra cui si rilevano come più interessanti le pubblicazioni: Advancing Full Spectrum Housing: Design for Adults with Autism Spectrum Disorders Technical report ${ }^{314}$ e Living in the Community. Housing Design for Adults with Autism ${ }^{315}$.

Per quanto riguarda invece l'adattamento domestico, l'unico esempio rilevato interessante, è lo studio sperimentale di Magda Mostafa sull'applicazione dell'Autism ASPECTSS $^{\text {TM }}$ Design Index all'ambiente residenziale familiare ${ }^{316}$. La prima parte della ricerca ha previsto un'indagine di tipo quali-quantitativo attraverso la diffusione di questionari sul web Families for Early Autism Treatment (FEAT) e l'organizzazione di focus groups con genitori e caregivers. La seconda fase ha previsto invece l'applicazione della metodologia tradotta nell'Autism ASPECTSS TM Design Index su tre casi studio, ovvero tre abitazioni familiari in Cairo di bambini/ragazzi con autismo che abitano con i genitori e uno o più fratelli/sorelle, tra gli 11 e i 17 anni.

Il primo intervento sulle abitazioni è stato quello di analizzare e suddividere le diverse aree sulla base dei livelli sensoriali, con particolare attenzione al bagno e alla cucina, come spazi di maggiore stimolo sensoriale che possono creare un'influenza

\footnotetext{
${ }^{312}$ Beaver, C. (2011) Designing environments for children and adults on the autism spectrum, in Good Autism Practice (GAP), 12(1), pp.7-11

${ }^{313}$ Vogel, C. (2008) Classroom design for living and learning with autism, Autism Aspergers Digest, May-June

314 Ahrentzen, S. Steele, K. (2009) Advancing Full Spectrum Housing: Design for Adults with Autism Spectrum Disorders Technical report, Arizona Board of Regents, Phoenix; Resnik , D. D. et al. (2009) Opening Doors: A discussion of residential options for adults living with autism and related disorders, Collaborative report by the Urban Land Institute Arizona, Southwest Autism Research \& Resource Center and Arizona State University

${ }^{315}$ Brand, A. (2010) Living in the community, London: Helen Hamlyn Centre, Royal College of Art

${ }^{316}$ Mostafa, M. (2014) An Architecture for Autism: Application of the Autism ASPECTSS ${ }^{\mathrm{TM}}$ Design Index to Home Environments, in The International Journal of the Constructed Environment, vol. 20, pp. 25-38
} 


\section{Elena Bellini}

nel comportamento del bambino/ragazzo con autismo. Aspetti particolari che vengono considerati nell'analisi sono inoltre il comfort acustico, sia verso l'esterno dell'abitazione che tra i diversi ambienti della casa; il supporto della conformazione degli spazi e dell'impianto distributivo sulle routines, che possono facilitare il compimento delle azioni quotidiane e l'autonomia del bambino/ragazzo; la sicurezza, rivolta in particolare alle problematiche di propriocezione ed alle difficoltà nel percepire la profondità (progettazione di balconi e finestre). $E^{\prime}$ stato considerato inoltre il layout e la conformazione della camera da letto personale (nei casi studio sempre singola), la posizione del letto, il comfort acustico e visivo (in particolare luminoso), ed eventuali elementi sensoriali in risposta alle ipo- o iper- sensibilità dei bambini/ragazzi con autismo, anche come supporto alle problematiche del sonno.

Lo studio si è dimostrato interessante per l'analisi e le interpretazioni rilevate, ma anche e soprattutto poiché ha constatato che l'oggettività preposta nell'Autism ASPECTSS ${ }^{\text {TM }}$ Design Index si è rivelata comunque soggetta all'interpretazione personale dei familiari e della loro percezione e ha dimostrato la difficoltà nel confrontare punteggi di valutazione non assoluti, ma basati sulla relatività delle caratteristiche personali dei bambini/ragazzi all'interno dell'enorme varietà presente nello spettro autistico e delle differenti sensibilità e percezioni sensoriali. In conclusione, si definisce l'impossibilità ancora una volta di creare degli standard o dei codici normativi specifici per l'autismo, ma si afferma l'utilizzo della matrice sensoriale come strumento efficace di supporto al progetto (Mostafa, 2014) ${ }^{317}$.

Come affrontare quindi la progettazione di una residenza di più persone con autismo, rispondendo alle criticità relative alla standardizzazione? Quale metodologia o approccio al progetto risultano maggiormente efficaci?

Il primo esempio approfondito all'interno della letteratura, Advancing Full Spectrum Housing: Design for Adults with Autism Spectrum Disorders Technical report $^{318}$, è un vero e proprio report, che nasce a seguito di una collaborazione instaurata nel 2004 tra l'Urban Land Institute (ULI) dell'Arizona e il Southwest Autism Research \& Resource Center (SARRC), con supporto nella ricerca da parte dell'Advancing Futures for Adults with Autism (AFAA), per affrontare l'emergenza abitativa a lungo termine di adulti con Disturbi dello Spettro Autistico. E' stato quindi costituito un gruppo di lavoro nel campo immobiliare, finanziario, economico, architettonico e del settore pubblico, che valutasse l'offerta abitativa ed economica del paese nei confronti dei bisogni speciali per l'autismo e definisse infine delle linee guida di indirizzo per i progettisti a supporto del processo decisionale. Le linee guida si basano su una ricerca compiuta sull'analisi di oltre 100 casi studio su residenze

\footnotetext{
${ }^{317}$ Mostafa, M. (2014) An Architecture for Autism: Application of the Autism ASPECTSSTM Design Index to Home Environments, in The International Journal of the Constructed Environment, vol. 20, pp. 25-38

${ }^{318}$ Ahrentzen, S. Steele, K. (2009) Advancing Full Spectrum Housing: Design for Adults with Autism Spectrum Disorders Technical report, Arizona Board of Regents, Phoenix; Resnik , D. D. et al. (2009) Opening Doors: A discussion of residential options for adults living with autism and related disorders, Collaborative report by the Urban Land Institute Arizona, Southwest Autism Research \& Resource Center and Arizona State University
} 
per la disabilità fisica o cognitiva, di cui 17 sono stati selezionati per sopralluogo diretto ed una fase di ricerca più operativa.

La seconda pubblicazione invece, Living in the Community. Housing Design for Adults with Autism ${ }^{319}$, deriva da uno studio affrontato dal Royal College of Art Helen Hamlyn Center a Londra, uno dei maggiori centri di ricerca fondato sul peoplecentred design, da parte del centro per l'autismo Kingswood Trust al fine di "non impedire alle persone con autismo di godere di una vita piena e attiva all'interno della comunità" ${ }^{320}$ ed in particolare comprendere l'influenza del progetto residenziale sul benessere e la salute delle persone con autismo, gli impatti riscontrati e la definizione di good practice come riferimento per architetti, progettisti e decision makers. Le linee guida si basano sul coinvolgimento diretto dell'utente finale (con interviste, alcuni racconti o l'osservazione in ambito di vita quotidiana) attraverso la visita di sette case di persone con autismo, oltre ad interviste ad esperti.

In entrambe le ricerche si identifica come la residenza istituzionale o sanitaria sia ormai obsoleta e non sia in grado di rispondere alle esigenze di vita alle persone con autismo, sia per la qualità dello spazio sovrastimolante, che per l'alto numero di persone presenti all'interno delle strutture, che per la definizione di particolari routines da parte dello staff di riferimento (Brand, 2010) ${ }^{321}$. Si promuovono al contrario delle nuove soluzioni per l'abitare che siano vicine alla tipologia di residenza familiare, sia come dimensione, che come qualità di vita inclusiva ed integrata all'interno della comunità, pur mantenendo il supporto e l'assistenza di operatori specializzati. Brand propone ad esempio, come nuove soluzioni, un'aggregazione di diverse case o di più appartamenti indipendenti in un unico edificio, con al massimo 8 abitanti, consentendo di avere autonomia, una maggiore qualità di vita basata sulle proprie preferenze sensoriali e generali necessità del singolo, ma integrandosi nella comunità $(\text { Brand, 2010 })^{322}$. Influisce molto sull'integrazione e sull'inclusione nella società anche il contesto abitativo. L'ambiente considerato maggiormente favorevole per vivere é un ambiente tranquillo, dove ci sia poca confusione e traffico, dove si possa mantenere un alto grado di tranquillità e privacy, ma che allo stesso tempo consenta l'accessibilità e la raggiungibilità dei servizi primari (servizi medici, gastronomia, farmacia, parchi e spazi aperti); di un centro urbano, anche se piccolo, dove integrarsi socialmente e ove possibile anche a livello lavorativo; della famiglia, se non si abita insieme. E' stato riscontato quindi come si preferisca un'abitazione dalle dimensioni contenute, integrata nel contesto e possibilmente indipendente e distaccata rispetto alle altre case, per migliorare i requisiti di comfort e privacy (Ahrentzen, Steele, $2009^{323}$; Brand, 2010 324 ; Kinnaer et al., $2014^{325}$ ).

\footnotetext{
${ }^{319}$ Brand, A. (2010) Living in the community, London: Helen Hamlyn Centre, Royal College of Art

320 ibidem

321 ibidem

322 ibidem

323 Ahrentzen, S. Steele, K. (2009) Advancing Full Spectrum Housing: Design for Adults with Autism Spectrum Disorders Technical report, Arizona Board of Regents, Phoenix; Resnik , D. D. et al. (2009) Opening Doors: A discussion of residential options for adults living with autism and related disorders, Collaborative report by the Urban Land Institute Arizona, Southwest Autism Research \& Resource Center and Arizona State University
} 


\section{Elena Bellini}

Si presenterà di seguito una sintesi degli aspetti valutati all'interno dello stato dell'arte come di influenza e impatto sulla qualità di vita delle persone con autismo, all'interno dell'ambito delle residenze "protette", alla luce della definizione delle macro-esigenze identificate.

\subsubsection{Riduzione del disagio sensoriale ed ambientale}

Le persone con autismo possono essere spesso inclini all'ansia e allo stress, legato in gran parte alla sensibilità e alla regolazione sensoriale che influiscono in maniera preponderante sulla qualità di vita della persona, come benessere personale e come rapporto con gli altri, causando reazioni insolite o comportamenti problema (Brand, 2010) $)^{326}$. Il progetto deve mirare quindi prima di tutto a ridurre il sovraccarico sensoriale, per abbattere lo stress ed il disagio e consentire alla singola persona di regolare le stimolazioni sulla base delle singole esigenze; questo é possibile attraverso l'orientamento e la distribuzione stessa degli spazi all'interno dell'abitazione. Anche in questo caso si suggerisce una distribuzione sulla base della qualità e dei livelli di stimolazione sensoriale (Sensory Zoning), le aree ad alto stimolo da quelle a basso stimolo ed in particolare le aree comuni, cucina e bagno, che sono le maggiori fonti di distrazione e disturbo sensoriale (Ahrentzen, Steele, 2009 ${ }^{327}$; Brand, 201015; Mostafa, 2010, 2015 $5^{328}$ ). E' considerata favorevole inoltre una suddivisione degli spazi in base all'attività, in modo che ognuno sia dedicato ad una singola funzione specifica, con chiarezza e senza ambiguità (Compartimentalisation; Mostafa, $2010,2015^{329}$ ). Il passaggio tra le diverse aree deve essere filtrato per evitare cambi drastici di ambiente, di funzione e/o stimolazione (Transition Zone - Mostafa, 2010, $2015^{330}$ ). Le separazioni tra queste diverse aree possono essere fisiche, attraverso muri, partizioni, o differenze di livello; oppure visive, attraverso colori, pattern o materiali di finitura. Per questo motivo viene sconsigliato un unico open space, sia per mancanza di chiarezza, che per il sovraccarico sensoriale che potrebbe generare: visivo, acustico ed olfattivo. In questo caso Mostafa consiglia di ritrovare ancora una volta una suddivisione in base alla funzione, anche attraverso colori, pattern, o arre-

\footnotetext{
${ }^{324}$ Brand, A. (2010) Living in the community, London: Helen Hamlyn Centre, Royal College of Art

${ }^{325}$ Kinnaer, M. Baumers, S. Heylighen, A. (2014) How do People with Autism (Like to) Live?, in Langdon, P. M . Lazar, J. Heylighen, A. Dong, H. (a cura di ) (2014) Inclusive Designing. Joining Usability, Acessibility and Inclusion, Springer Cham Heidelberg New York Dordrecht London, part IV, pp. 175-185

326 Brand, A. (2010) Living in the community, London: Helen Hamlyn Centre, Royal College of Art

${ }^{327}$ Ahrentzen, S. Steele, K. (2009) Advancing Full Spectrum Housing: Design for Adults with Autism Spectrum Disorders Technical report, Arizona Board of Regents, Phoenix; Resnik , D. D. et al. (2009) Opening Doors: A discussion of residential options for adults living with autism and related disorders, Collaborative report by the Urban Land Institute Arizona, Southwest Autism Research \& Resource Center and Arizona State University

${ }^{328}$ Mostafa, M. (2010) Housing Adaptation for Adults with Autistic Spectrum Disorder, Open House International, Urban International Press, 35, 37-48; Mostafa, M. (2015) Architecture for Autism: Built Environment Performance in Accordance to the Autism ASPECTSS ${ }^{\mathrm{TM}}$ Design Index, in Design Principles and practices: an International Journal - Annual Review, vol. 8, pp.55-71,Common Ground Publishing, Champaign, Illinois, USA

329 ibidem

${ }^{330}$ ibidem
} 
di, utili per compartimentare spazi più ampi, soprattutto per dedicare zone ad attività che necessitano silenzio e concentrazione (Mostafa, 2010, 2015 ${ }^{331}$ ). Ovviamente ogni persona è diversa dall'altra e le linee guida non possono che ritenersi delle indicazioni di riferimento da adattare ad ogni singolo caso e non da considerare come "regole" universali da seguire. Ad esempio, per quanto riguarda l'open space, Kinnaer et al. riportano una biografia dove invece si considera favorevole per il comfort visivo, poiché consente di tenere continuamente sotto controllo tutto l'ambiente. Allo stesso modo suggerisce infatti porte trasparenti o comunque che diano la possibilità di guardare all'interno dei diversi ambienti (soprattutto quelli comuni) prima di entrare ed eliminare l'inquietudine provocata dall'imprevedibilità di non sapere cosa si può trovare dietro una porta chiusa, o cosa può arrivare dall'esterno quando si è dentro (Kinnaer et al., $2014^{332}$ ).

In ogni caso il principale disagio provocato in casi di autismo proviene sicuramente dal sovraccarico sensoriale, che genera stress ed ansia, ed in particolare dal punto di vista acustico, visivo e talvolta olfattivo, anche se con meno preponderanza.

La stessa geometria degli spazi può andare ad influire in modo importante sull'acustica di un ambiente; ambienti troppo ampi o soffitti molto alti possono generare eco e riverbero, allo stesso modo i corpi scala o i corridoi troppo lunghi (Mostafa, 2010) $)^{333}$. Avere un edificio isolato rispetto agli altri, come valutato precedentemente, é sicuramente un vantaggio dal punto di vista del comfort acustico, così come un contesto tranquillo e senza traffico (Kinnaer et al., 2014 ${ }^{334}$ ). Allo stesso tempo si può prevedere un isolamento a parete, un aumento delle sezioni trasversali, delle intercapedini e dei sistemi di riflessione del suono (Mostafa, 2010 ${ }^{335}$ ) o distaccare le pareti dal pavimento attraverso controsoffitti sospesi (Brand, 2010) ${ }^{336}$. Le aperture e gli infissi rimangono il "punto debole", pertanto si consiglia di usare doppi o tripli vetri, griglie o lamelle per ridurre la permeabilità del suono e tende di cotone spesso o velluto all'interno; in particolare nelle camere e nelle stanze dedicate alla terapia o alla logopedia si suggerisce di limitare al massimo le aperture (Mosta-

${ }^{331}$ Mostafa, M. (2010) Housing Adaptation for Adults with Autistic Spectrum Disorder, Open House International, Urban International Press, 35, 37-48; Mostafa, M. (2015) Architecture for Autism: Built Environment Performance in Accordance to the Autism ASPECTSS ${ }^{\text {TM }}$ Design Index, in Design Principles and practices: an International Journal - Annual Review, vol. 8, pp.55-71,Common Ground Publishing, Champaign, Illinois, USA

${ }^{332}$ Kinnaer, M. Baumers, S. Heylighen, A. (2014) How do People with Autism (Like to) Live?, in Langdon, P. M . Lazar, J. Heylighen, A. Dong, H. (a cura di ) (2014) Inclusive Designing. Joining Usability, Acessibility and Inclusion, Springer Cham Heidelberg New York Dordrecht London, part IV, pp. 175-185

${ }^{333}$ Mostafa, M. (2010) Housing Adaptation for Adults with Autistic Spectrum Disorder, Open House International, Urban International Press, 35, 37-48

${ }^{334}$ Kinnaer, M. Baumers, S. Heylighen, A. (2014) How do People with Autism (Like to) Live?, in Langdon, P. M . Lazar, J. Heylighen, A. Dong, H. (a cura di ) (2014) Inclusive Designing. Joining Usability, Acessibility and Inclusion, Springer Cham Heidelberg New York Dordrecht London, part IV, pp. 175-185

${ }^{35}$ Mostafa, M. (2010) Housing Adaptation for Adults with Autistic Spectrum Disorder, Open House International, Urban International Press, 35, 37-48

${ }^{336}$ Brand, A. (2010) Living in the community, London: Helen Hamlyn Centre, Royal College of Art 


\section{Elena Bellini}

fa, $\left.2010^{337}\right)$. Anche all'interno risulta fondamentale un isolamento delle pareti e dei controsoffitti (Mostafa definisce accettabile un livello acustico compreso tra 125$4000 \mathrm{~Hz}$ ). Particolare attenzione va posta a bagno, cucina e lavanderia, poiché sono le aree di maggiore disturbo sonoro, e a tutti gli apparati ed i dispositivi impiantistici e tecnologici, come il condizionamento dell'aria, che dovranno essere opportunamente isolati (Mostafa, 2010 338 ; Ahrentzen, Steele, 2009 ${ }^{339}$ ). In generale si consigliano materiali fonoassorbenti per rivestire pavimenti, pareti e controsoffitti, per evitare eco e riverbero. Tra i materiali maggiormente usati per il controsoffitto si suggerisce l'utilizzo di pannelli in legno, che oltre ad avere un ottimo assorbimento acustico, aiutano a riscaldare l'ambiente e renderlo più familiare (Whitehurst, $2006)^{340}$. I pavimenti flottanti, oltre ad essere pratici a livello di manutenzione, se isolati, possono rivelarsi utili a garantire maggiore comfort acustico; si suggerisce inoltre di limitare al massimo l'uso della ceramica a pavimento, preferendo pavimenti vinilici fono-assorbenti (ref. Novilon), linoleum, sughero o materiali plastici (rif. Flotex) (Brand, 2010) ${ }^{341}$, materiali naturali come il legno di pino o sisal (Mostafa, $2010)^{342}$. A parete si raccomanda di usare materiali di finitura non riflettenti e fonoassorbenti, tra cui il sughero, materiale interessante per le ottime caratteristiche prestazionali nel ridurre l'eco, ma anche come stimolazione tattile (Mostafa, 2010 ${ }^{343}$ ); Beaver e Whitehurst promuovono invece l'utilizzo di pareti ruvide ed in particolare mattoni facciavista che assorbano maggiormente il suono, in particolare nei corridoi dove c'è maggiore disturbo acustico (Beaver, 2011 ${ }^{344}$; Whitehurst, 2006 ${ }^{345}$ ); viene promosso inoltre l'uso di moquette, tappeti o tessuti, anche a parete, per ridurre il riverbero e migliorare il comfort acustico (Whitehurst, 2006 ${ }^{346}$; Beaver, 2011 347 Ahrentzen, Steele, $2009^{348}$ ).

\footnotetext{
${ }^{337}$ Mostafa, M. (2010) Housing Adaptation for Adults with Autistic Spectrum Disorder, Open House International, Urban International Press, 35, 37-48

338 ibidem

${ }^{339}$ Ahrentzen, S. Steele, K. (2009) Advancing Full Spectrum Housing: Design for Adults with Autism Spectrum Disorders Technical report, Arizona Board of Regents, Phoenix; Resnik , D. D. et al. (2009) Opening Doors: A discussion of residential options for adults living with autism and related disorders, Collaborative report by the Urban Land Institute Arizona, Southwest Autism Research \& Resource Center and Arizona State University

340 Whitehurst, T. (2006) The impact of building design on children with autistic spectrum disorders, in Good Autism Practice, Vol. 7(1), pp31-42; Whitehurst, T. (2006) Evaluation of features specific to an ASD designed living accommodation, in Designing living \& learning environments for children with autism, Sunfield Research Institute

${ }^{341}$ Brand, A. (2010) Living in the community, London: Helen Hamlyn Centre, Royal College of Art

342 Mostafa, M. (2010) Housing Adaptation for Adults with Autistic Spectrum Disorder, Open House International, Urban International Press, 35, 37-48

${ }^{343}$ ibidem

344 Beaver, C. (2011) Designing environments for children and adults on the autism spectrum, in Good Autism Practice (GAP), 12(1), pp.7-11

${ }_{345}$ Whitehurst, T. (2006) The impact of building design on children with autistic spectrum disorders, in Good Autism Practice, Vol. 7(1), pp31-42; Whitehurst, T. (2006) Evaluation of features specific to an ASD designed living accommodation, in Designing living \& learning environments for children with autism, Sunfield Research Institute

346 ibidem
} 
Per quanto riguarda invece il sovraccarico visivo si suggerisce di progettare ambienti molto semplici e puliti, minimizzando i dettagli, su cui alcuni potrebbero focalizzarsi, ed eliminando le decorazioni superflue, i pattern, gli ingombri, gli arredi o gli oggetti di troppo; per questo motivo ampi ripostigli o pareti attrezzate a scomparsa possono rivelarsi utili per riporre tutto ciò che non serve (Ahrentzen, Steele, $2009^{349}$; Kinnaer et al., $2014^{350}$ ). Per lo stesso principio colori e materiali devono essere il più possibile neutri; non usare colori primari o troppo luminosi (Kinnaer et al., 2014 ${ }^{351}$; Ahrentzen, Steele, $2009^{352}$ ), ma piuttosto colori tenui e pastello; Mostafa propone l'utilizzo del bianco caldo o rosa pallido e la ricorrenza di pattern solo in aree controllate come le sensory room o gli escape space, per evitare distrazioni (Mostafa, 2010) ${ }^{353}$; Whitehurst propone invece uno studio condotto dal dr. Di Pauli al Sunfield Research Institute, valutando il rosa e il viola come colori maggiormente positivi da poter usare ampiamente alle pareti, ed il grigio come colore neutro e non riflettente, che favorisce la calma, da utilizzare anche per porte, finestre, infissi e battiscopa (Whitehurst ,2006) ${ }^{354}$.

La luminosità è un altro aspetto molto importante per il comfort sensoriale. La luce naturale è sempre preferibile, ma deve essere comunque controllata e indiretta; Mostafa suggerisce di utilizzare finestre che permettono la vista verso l'esterno solo in ambienti rilassanti, mentre assolutamente considera da evitare le aperture nelle aree dove é richiesta la concentrazione (Mostafa, 2010) ${ }^{355}$; in generale comunque si promuove l'utilizzo di finestre alte a nastro e di tende pesanti (Brand, 2010 ${ }^{356}$; Bea-

347 Beaver, C. (2011) Designing environments for children and adults on the autism spectrum, in Good Autism Practice (GAP), 12(1), pp.7-11

${ }^{348}$ Ahrentzen, S. Steele, K. (2009) Advancing Full Spectrum Housing: Design for Adults with Autism Spectrum Disorders Technical report, Arizona Board of Regents, Phoenix; Resnik , D. D. et al. (2009) Opening Doors: A discussion of residential options for adults living with autism and related disorders, Collaborative report by the Urban Land Institute Arizona, Southwest Autism Research \& Resource Center and Arizona State University

349 ibidem

${ }^{350}$ Kinnaer, M. Baumers, S. Heylighen, A. (2014) How do People with Autism (Like to) Live?, in Langdon, P. M . Lazar, J. Heylighen, A. Dong, H. (a cura di ) (2014) Inclusive Designing. Joining Usability, Acessibility and Inclusion, Springer Cham Heidelberg New York Dordrecht London, part IV, pp. 175-185

ibidem

352 Ahrentzen, S. Steele, K. (2009) Advancing Full Spectrum Housing: Design for Adults with Autism Spectrum Disorders Technical report, Arizona Board of Regents, Phoenix; Resnik , D. D. et al. (2009) Opening Doors: A discussion of residential options for adults living with autism and related disorders, Collaborative report by the Urban Land Institute Arizona, Southwest Autism Research \& Resource Center and Arizona State University

${ }^{353}$ Mostafa, M. (2010) Housing Adaptation for Adults with Autistic Spectrum Disorder, Open House International, Urban International Press, 35, 37-48

354 Whitehurst, T. (2006) The impact of building design on children with autistic spectrum disorders, in Good Autism Practice, Vol. 7(1), pp31-42; Whitehurst, T. (2006) Evaluation of features specific to an ASD designed living accommodation, in Designing living \& learning environments for children with autism, Sunfield Research Institute

${ }^{355}$ Mostafa, M. (2010) Housing Adaptation for Adults with Autistic Spectrum Disorder, Open House International, Urban International Press, 35, 37-48

356 Brand, A. (2010) Living in the community, London: Helen Hamlyn Centre, Royal College of Art 


\section{Elena Bellini}

ver, 2011 ${ }^{357}$; Whitehurst, 2006 ${ }^{358}$; Ahrentzen, Steele, 2009 $9^{359}$ ). Per quanto riguarda invece la luce artificiale si raccomanda un'illuminazione diffusa proveniente dall'alto, come se fosse naturale, con corpi illuminanti eventualmente incassati a soffitto, o con la possibilità di usare diversi punti luce, anche mobili, per personalizzare la qualità luminosa dello spazio (Ahrentzen, Steele, 2009) ${ }^{360}$, anche attraverso l'utilizzo di luci colorate per chi ha particolare sensibilità ai colori (Brand, 2010) ${ }^{361}$. Sono da evitare assolutamente le luci fluorescenti, sia per la qualità luminosa, che per lo sfarfallio ed il ronzio prodotto dal corpo illuminante e si suggerisce l'utilizzo di dimmer per la regolazione dell'illuminazione in base alle proprie esigenze sensoriali (Mostafa, $2010^{362}$; Ahrentzen, Steele, $2009^{363}$ ).

Per riequilibrarsi e autoregolarsi in situazioni di sovraccarico sensoriale sono comunque da prevedere degli escape spaces (Ahrentzen, Steele, 2009 ${ }^{364}$; Mostafa, $2015^{365}$ ), ovvero ambienti neutrali, di dimensione contenuta, fisicamente e visivamente chiusi, dove isolarsi per qualche momento. Allo stesso modo sono utili le sensory rooms (Ahrentzen, Steele, $2009^{366}$; Whitehurst,2006 ${ }^{367}$; Kinnaer et al., 2014 ${ }^{368}$;

${ }^{357}$ Beaver, C. (2011) Designing environments for children and adults on the autism spectrum, in Good Autism Practice (GAP), 12(1), pp.7-11

${ }^{358}$ Whitehurst, T. (2006) The impact of building design on children with autistic spectrum disorders, in Good Autism Practice, Vol. 7(1), pp31-42; Whitehurst, T. (2006) Evaluation of features specific to an ASD designed living accommodation, in Designing living \& learning environments for children with autism, Sunfield Research Institute

${ }^{359}$ Ahrentzen, S. Steele, K. (2009) Advancing Full Spectrum Housing: Design for Adults with Autism Spectrum Disorders Technical report, Arizona Board of Regents, Phoenix; Resnik , D. D. et al. (2009) Opening Doors: A discussion of residential options for adults living with autism and related disorders, Collaborative report by the Urban Land Institute Arizona, Southwest Autism Research \& Resource Center and Arizona State University

${ }^{360}$ ibidem

${ }^{361}$ Brand, A. (2010) Living in the community, London: Helen Hamlyn Centre, Royal College of Art

${ }^{362}$ Mostafa, M. (2010) Housing Adaptation for Adults with Autistic Spectrum Disorder, Open House International, Urban International Press, 35, 37-48

${ }^{363}$ Ahrentzen, S. Steele, K. (2009) Advancing Full Spectrum Housing: Design for Adults with Autism Spectrum Disorders Technical report, Arizona Board of Regents, Phoenix; Resnik, D. D. et al. (2009) Opening Doors: A discussion of residential options for adults living with autism and related disorders, Collaborative report by the Urban Land Institute Arizona, Southwest Autism Research \& Resource Center and Arizona State University

364 ibidem

${ }^{365}$ Mostafa, M. (2010) Housing Adaptation for Adults with Autistic Spectrum Disorder, Open House International, Urban International Press, 35, 37-48

${ }^{366}$ Ahrentzen, S. Steele, K. (2009) Advancing Full Spectrum Housing: Design for Adults with Autism Spectrum Disorders Technical report, Arizona Board of Regents, Phoenix; Resnik, D. D. et al. (2009) Opening Doors: A discussion of residential options for adults living with autism and related disorders, Collaborative report by the Urban Land Institute Arizona, Southwest Autism Research \& Resource Center and Arizona State University

${ }^{367}$ Whitehurst, T. (2006) The impact of building design on children with autistic spectrum disorders, in Good Autism Practice, Vol. 7(1), pp31-42; Whitehurst, T. (2006) Evaluation of features specific to an ASD designed living accommodation, in Designing living \& learning environments for children with autism, Sunfield Research Institute

${ }^{368}$ Kinnaer, M. Baumers, S. Heylighen, A. (2014) How do People with Autism (Like to) Live?, in Langdon, P. M . Lazar, J. Heylighen, A. Dong, H. (a cura di ) (2014) Inclusive Designing. Joining 
Ambienti sensoriali "terapeutici” che rendano Abili

Mostafa, $2010^{369}$ ) per la regolazione dei propri sensi, la calma ed il relax, ma anche la possibilità di ricercare una stimolazione in casi di iposensorialità.

\subsubsection{Salute e benessere}

L'orientamento spaziale é importante a livello distributivo ma anche dal punto di vista ambientale, per garantire il comfort igro-termico degli ambienti e la ventilazione naturale, utile anche a livello olfattivo (Mostafa, 2010) ${ }^{370}$. Le finestre alte favoriscono una ventilazione passiva oltre a migliorare il comfort luminoso e a garantire maggiore sicurezza in alcune situazioni più delicate. Una ventilazione meccanizzata in tutte le stante ed in particolare é inoltre favorevole (Beaver, 2011) ${ }^{371}$ attraverso un sistema di ventilazione controllata ultrasilenzioso ed un sistema di purificazione dell'aria (Ahrentzen, Steele, 2009) ${ }^{372}$. Allo stesso tempo si suggerisce di avere un sistema di controllo personalizzato della ventilazione e del riscaldamento nelle camere (Ahrentzen, Steele, 2009 ${ }^{373}$; Brand, 2010 374 ), poiché ognuno potrebbe avere esigenze molto diverse e non sopportare il calore, o viceversa.

Dal punto di vista delle finiture e degli arredi si suggeriscono materiali naturali, non tossici, lavabili (Beaver, 2011 ${ }^{375}$; Kinnaer et al., 2016 ${ }^{376}$ ), durevoli e resistenti, ipoallergenici, senza emissione di gas o composti organici volatili e senza fragranze (Brand, 2010 377 ; Ahrentzen, Steele, 2009 ${ }^{378}$ ). L'igiene é di fondamentale importanza, anche e soprattutto nelle aree sensoriali dove si interagisce con l'ambiente e si fanno esperienze tattili o di altro tipo. Battiscopa, zoccoli o boiserie alle pareti sono consi-

Usability, Acessibility and Inclusion, Springer Cham Heidelberg New York Dordrecht London, part IV, pp. $175-185$

${ }^{369}$ Mostafa, M. (2010) Housing Adaptation for Adults with Autistic Spectrum Disorder, Open House International, Urban International Press, 35, 37-48

370 ibidem

371 Beaver, C. (2011) Designing environments for children and adults on the autism spectrum, in Good Autism Practice (GAP), 12(1), pp.7-11

${ }^{372}$ Ahrentzen, S. Steele, K. (2009) Advancing Full Spectrum Housing: Design for Adults with Autism Spectrum Disorders Technical report, Arizona Board of Regents, Phoenix; Resnik , D. D. et al. (2009) Opening Doors: A discussion of residential options for adults living with autism and related disorders, Collaborative report by the Urban Land Institute Arizona, Southwest Autism Research \& Resource Center and Arizona State University

373 ibidem

374 Brand, A. (2010) Living in the community, London: Helen Hamlyn Centre, Royal College of Art

375 Beaver, C. (2011) Designing environments for children and adults on the autism spectrum, in Good Autism Practice (GAP), 12(1), pp.7-11

${ }^{376}$ Kinnaer, M. Baumers, S. Heylighen, A. (2014) How do People with Autism (Like to) Live?, in Langdon, P. M . Lazar, J. Heylighen, A. Dong, H. (a cura di ) (2014) Inclusive Designing. Joining Usability, Acessibility and Inclusion, Springer Cham Heidelberg New York Dordrecht London, part IV, pp. $175-185$

377 Brand, A. (2010) Living in the community, London: Helen Hamlyn Centre, Royal College of Art

${ }^{378}$ Ahrentzen, S. Steele, K. (2009) Advancing Full Spectrum Housing: Design for Adults with Autism Spectrum Disorders Technical report, Arizona Board of Regents, Phoenix; Resnik, D. D. et al. (2009) Opening Doors: A discussion of residential options for adults living with autism and related disorders, Collaborative report by the Urban Land Institute Arizona, Southwest Autism Research \& Resource Center and Arizona State University 


\section{Elena Bellini}

gliabili per la durabilità e la pulibilità degli spazi (Ahrentzen, Steele, 2009 ${ }^{379}$ ). Allo stesso tempo si raccomanda di usare materiali che mantengano l'ambiente caldo e familiare, per non riportare a condizioni di residenza sanitaria o di istituzionalizzazione (Whitehurst ,2006 ${ }^{380}$; Brand, $2010^{381}$ ).

Per favorire il benessere della persona si promuovono ambienti naturali, poiché é stato dimostrato che la natura stessa influisca direttamente sul benessere, come una vera e propria 'terapia' (Mostafa, 2010) ${ }^{382}$. I giardini e gli spazi all'aperto sono quindi considerati 'terapeutici' soprattutto se prevedono orti o piante che devono essere curate (Ahrentzen, Steele, 2009) ${ }^{383}$ poiché in questo modo si va sviluppando l'attenzione, l'indipendenza, la soddisfazione e l'autostima della persona. Si possono usare gli spazi all'aperto per diverse attività, anche ricreative, che favoriscano lo scambio sociale o con le famiglie (Ahrentzen, Steele, 2009) ${ }^{384}$, ma anche per aree sensoriali, attraverso l'utilizzo di piante aromatiche, giochi d'acqua, diversi materiali con diverse consistenze e textures, aree relax e diverse zone di stimolazione (Mostafa, $2010)^{385}$.

\subsubsection{Ambienti sicuri e durevoli}

Le alterazioni percettive, propriocettive e di orientamento spaziale portano le persone con autismo ad essere più facilmente esposte a situazioni di rischio (Mostafa, 2015) ${ }^{386}$ e molte possono avere una sensibilità sotto-sviluppata alla temperatura $\mathrm{o}$ al dolore, presentando rischi maggiori di infortunio (Brand, 2010) ${ }^{387}$; bisogna pertanto porre particolare attenzione al sistema edilizio in termini di sicurezza, al fine di poter favorire anche una maggiore indipendenza della persona. Può essere utile

\footnotetext{
${ }^{379}$ Ahrentzen, S. Steele, K. (2009) Advancing Full Spectrum Housing: Design for Adults with Autism Spectrum Disorders Technical report, Arizona Board of Regents, Phoenix; Resnik , D. D. et al. (2009) Opening Doors: A discussion of residential options for adults living with autism and related disorders, Collaborative report by the Urban Land Institute Arizona, Southwest Autism Research \& Resource Center and Arizona State University

380 Whitehurst, T. (2006) The impact of building design on children with autistic spectrum disorders, in Good Autism Practice, Vol. 7(1), pp31-42; Whitehurst, T. (2006) Evaluation of features specific to an ASD designed living accommodation, in Designing living \& learning environments for children with autism, Sunfield Research Institute

381 Brand, A. (2010) Living in the community, London: Helen Hamlyn Centre, Royal College of Art

382 Mostafa, M. (2010) Housing Adaptation for Adults with Autistic Spectrum Disorder, Open House International, Urban International Press, 35, 37-48

${ }^{383}$ Ahrentzen, S. Steele, K. (2009) Advancing Full Spectrum Housing: Design for Adults with Autism Spectrum Disorders Technical report, Arizona Board of Regents, Phoenix; Resnik , D. D. et al. (2009) Opening Doors: A discussion of residential options for adults living with autism and related disorders, Collaborative report by the Urban Land Institute Arizona, Southwest Autism Research \& Resource Center and Arizona State University

384 ibidem

385 Mostafa, M. (2010) Housing Adaptation for Adults with Autistic Spectrum Disorder, Open House International, Urban International Press, 35, 37-48

386 Mostafa, M. (2015) Architecture for Autism: Built Environment Performance in Accordance to the Autism ASPECTSS ${ }^{\text {тм }}$ Design Index, in Design Principles and practices: an International Journal Annual Review, vol. 8, pp.55-71,Common Ground Publishing, Champaign, Illinois, USA 387 Brand, A. (2010) Living in the community, London: Helen Hamlyn Centre, Royal College of Art
} 
strutturare gli spazi al fine di consentire la visibilità all'interno dell'edificio per il controllo e monitoraggio delle persone, senza che l'operatore sia sempre presente e visibile dagli utenti, minando la loro libertà.

Le scale, i balconi e le finestre, sono punti di maggiore rischio, poiché spesso le persone con autismo hanno difficoltà a percepire la profondità spaziale (Kinnaer et al., 2016 ${ }^{388}$; Mostafa, 2010 389 ); sono inoltre da evitare scalini all'ingresso ai fini dell'accessibilità, ma anche le transizioni tra le diverse stanze con scalini o differenza di spessore nei rivestimenti, poiché causa di inciampo (Ahrentzen, Steele, $2009)^{390}$. Per le finestre si suggeriscono vetri doppi o temperati; avvolgibili installati all'interno degli strati di vetro, affinché non vi sia rischio di danneggiarli, movimentati e orientati con controllo remoto; lucchetti all'interno per particolari condizioni di sicurezza (Beaver, 2011) ${ }^{391}$.

I movimenti, continui, non previsti ed impetuosi, le attività, i comportamenti ripetitivi, le stereotipie e talvolta i comportamenti problema, possono mettere a dura prova la durabilità delle strutture, dei materiali e dei dispositivi all'interno dell'abitazione. Si consigliano quindi materiali molto resistenti, o materiali più economici che siano facili da sostituire o riparare (Beaver, 2011 ${ }^{392}$; Kinnaer et al., 2016 ${ }^{393}$ ) Materiali duri e resistenti, adatti per essere usati a pavimento, sono il legno, il bamboo, la ceramica, il linoleum o il marmo (Ahrentzen, Steele, 2009) ${ }^{394}$. Il bagno, la cucina e la lavanderia sono ancora una volta le aree a maggior rischio. Si suggerisce di usare un pavimento con ceramica antiscivolo ed eventualmente, in lavanderia, uno scarico a terra per la raccolta dell'acqua. (Ahrentzen, Steele, 2009) ${ }^{395}$. Per la cucina scegliere un materiale per il piano che sia durevole, resistente al fuoco e al calore e facilmente lavabile; si suggeriscono corian, granito, cemento e quarzo, mentre si sconsigliano le

${ }^{388}$ Kinnaer, M. Baumers, S. Heylighen, A. (2014) How do People with Autism (Like to) Live?, in Langdon, P. M . Lazar, J. Heylighen, A. Dong, H. (a cura di ) (2014) Inclusive Designing. Joining Usability, Acessibility and Inclusion, Springer Cham Heidelberg New York Dordrecht London, part IV, pp. 175-185

${ }_{389}$ Mostafa, M. (2010) Housing Adaptation for Adults with Autistic Spectrum Disorder, Open House International, Urban International Press, 35, 37-48

${ }^{390}$ Ahrentzen, S. Steele, K. (2009) Advancing Full Spectrum Housing: Design for Adults with Autism Spectrum Disorders Technical report, Arizona Board of Regents, Phoenix; Resnik , D. D. et al. (2009) Opening Doors: A discussion of residential options for adults living with autism and related disorders, Collaborative report by the Urban Land Institute Arizona, Southwest Autism Research \& Resource Center and Arizona State University

391 Beaver, C. (2011) Designing environments for children and adults on the autism spectrum, in Good Autism Practice (GAP), 12(1), pp.7-11

392 ibidem

393 Kinnaer, M. Baumers, S. Heylighen, A. (2014) How do People with Autism (Like to) Live?, in Langdon, P. M . Lazar, J. Heylighen, A. Dong, H. (a cura di ) (2014) Inclusive Designing. Joining Usability, Acessibility and Inclusion, Springer Cham Heidelberg New York Dordrecht London, part IV, pp. 175-185

394 Ahrentzen, S. Steele, K. (2009) Advancing Full Spectrum Housing: Design for Adults with Autism Spectrum Disorders Technical report, Arizona Board of Regents, Phoenix; Resnik , D. D. et al. (2009) Opening Doors: A discussion of residential options for adults living with autism and related disorders, Collaborative report by the Urban Land Institute Arizona, Southwest Autism Research \& Resource Center and Arizona State University

395 ibidem 


\section{Elena Bellini}

piastrelle di ceramica, il multistrato ed il laminato (Ahrentzen, Steele, 2009) ${ }^{396}$. Sul piano può essere utile inoltre un cambiamento di materiale per diverse funzioni, ad esempio per l'area taglio e preparazione alimenti (Ahrentzen, Steele, 2009) ${ }^{397}$. Nel bagno e nella cucina un altro problema é rappresentato dalla difficoltà di percepire il caldo e il freddo, o al contrario da una maggiore sensibilità, pertanto si suggerisce l'utilizzo di regolatori della temperatura (Ahrentzen, Steele, 2009) ${ }^{398}$. Un'attenzione particolare per gli impianti é da valutare inoltre in cucina anche dal punto di vista della regolazione automatica della provvisione di energia (Kinnaer et al., 2016) ${ }^{399}$ e dei regolatori per gli elettrodomestici, come i blocchi di sicurezza solitamente usati per i bambini (Mostafa, 2010) ${ }^{400}$.

Nelle scale e nei corridoi se previsti tessuti per il comfort acustico, si raccomanda l'utilizzo di passatoie sicure ben fissate, o in generale di usare riquadri a tappeto o moquette piuttosto che elementi da arrotolare; battiscopa, para spigoli, boiserie o zoccoli a parete sono sempre utili per aumentare la durabilità (Ahrentzen, Steele, $2009)^{401}$.

Il riscaldamento a pannelli radianti é sempre preferibile, a pavimento, o a soffitto ove non possibile (Ahrentzen, Steele, 2009 ${ }^{402}$; Brand, 2010 $0^{403}$; Beaver, $2011^{404}$; Whitehurst, $2006^{405}$ ). All'interno dell'abitazione si consigliano dispositivi tecnologici di controllo, al fine di monitorare la sicurezza: allarme antincendio e rivelatore di fumo, monossido di carbonio, gas, radon o propano, con un segnale vocale piuttosto che sonoro, per non produrre stress improvviso, ma dare indicazioni alle persone; sensori a porte e finestre; chiusura automatica delle porte interne apribili dall'ester-

\footnotetext{
${ }^{396}$ Ahrentzen, S. Steele, K. (2009) Advancing Full Spectrum Housing: Design for Adults with Autism Spectrum Disorders Technical report, Arizona Board of Regents, Phoenix; Resnik , D. D. et al. (2009) Opening Doors: A discussion of residential options for adults living with autism and related disorders, Collaborative report by the Urban Land Institute Arizona, Southwest Autism Research \& Resource Center and Arizona State University

397 ibidem

398 ibidem

399 Kinnaer, M. Baumers, S. Heylighen, A. (2014) How do People with Autism (Like to) Live?, in Langdon, P. M . Lazar, J. Heylighen, A. Dong, H. (a cura di ) (2014) Inclusive Designing. Joining Usability, Acessibility and Inclusion, Springer Cham Heidelberg New York Dordrecht London, part IV, pp. 175-185

${ }^{400}$ Mostafa, M. (2010) Housing Adaptation for Adults with Autistic Spectrum Disorder, Open House International, Urban International Press, 35, 37-48

${ }^{401}$ Ahrentzen, S. Steele, K. (2009) Advancing Full Spectrum Housing: Design for Adults with Autism Spectrum Disorders Technical report, Arizona Board of Regents, Phoenix; Resnik , D. D. et al. (2009) Opening Doors: A discussion of residential options for adults living with autism and related disorders, Collaborative report by the Urban Land Institute Arizona, Southwest Autism Research \& Resource Center and Arizona State University

402 ibidem

403 Brand, A. (2010) Living in the community, London: Helen Hamlyn Centre, Royal College of Art

404 Beaver, C. (2011) Designing environments for children and adults on the autism spectrum, in Good Autism Practice (GAP), 12(1), pp.7-11

405 Whitehurst, T. (2006) The impact of building design on children with autistic spectrum disorders, in Good Autism Practice, Vol. 7(1), pp31-42; Whitehurst, T. (2006) Evaluation of features specific to an ASD designed living accommodation, in Designing living \& learning environments for children with autism, Sunfield Research Institute
} 
no; apertura facilitata dell'ingresso senza chiavi, attraverso un'identificazione a radiofrequenza, biometrica o con tastiere digitali; spegnimento automatico dei dispositivi o degli utensili elettrici; rilevatori di presenza nelle camere o sui letti per sostituire la biancheria eventualmente bagnata; pulsanti di emergenza in ogni stanza; sensori di caduta per persone soggette a convulsioni ed epilessia; integrazione di sistemi di comunicazione facilitata per supportare gli utenti, come immagini, scritte o colori, che gli utenti possono identificare come allarmi (Ahrentzen, Steele, 2009) ${ }^{406}$.

Il giardino o lo spazio esterno é considerato come terapeutico e molto favorevole per e dalle persone con autismo. Ai fini della sicurezza le corti rappresentano una buona soluzione poiché sono facilmente accessibili, a diretto contatto con l'alloggio, chiuse, sicure, private e controllabili. L'accessibilità può essere inoltre incrementata attraverso piante rialzate da terra, che risultano anche maggiormente protette dal calpestio (Ahrentzen, Steele, $2009^{407}$; Beaver, $2011^{408}$; Whitehurst ,2006 ${ }^{409}$ ).

\subsubsection{Orientamento e comprensione}

Una volta eliminate le fonti di possibile disagio all'interno dell'edificio e raggiunta una situazione di comfort si deve consentire, attraverso la progettazione, la comprensione e l'indipendenza da parte della persona, in modo che sia in grado di vivere il più possibile in autonomia. La chiarezza dell'impianto planimetrico è utile in questo senso, al fine di avere una maggiore comprensione degli spazi e di orientamento all'interno dell'edificio, non solo in senso fisico ma anche sociale (Brand, 2010) ${ }^{410}$; si suggeriscono quindi collegamenti semplici e ben coordinati con le zone sensoriali (Mostafa, 2010) ${ }^{411}$, favorendo la leggibilità e la prevedibilità degli spazi e offrendo la possibilità di comprendere cosa ci si aspetta in ogni ambiente, per quale funzione o attività (Brand, 2010) ${ }^{412}$. Le pareti e linee curvilinee possono facilitare $\mathrm{i}$ movimenti nell'edificio, soprattutto in risposta a difficoltà visive, spaziali e propriocettive (Beaver, $2011^{413}$; Whitehurst ,2006 $6^{414}$ ). Linee, colori ed elementi nodali pos-

\footnotetext{
${ }^{406}$ Ahrentzen, S. Steele, K. (2009) Advancing Full Spectrum Housing: Design for Adults with Autism Spectrum Disorders Technical report, Arizona Board of Regents, Phoenix; Resnik , D. D. et al. (2009) Opening Doors: A discussion of residential options for adults living with autism and related disorders, Collaborative report by the Urban Land Institute Arizona, Southwest Autism Research \& Resource Center and Arizona State University

407 ibidem

408 Beaver, C. (2011) Designing environments for children and adults on the autism spectrum, in Good Autism Practice (GAP), 12(1), pp.7-11

409 Whitehurst, T. (2006) The impact of building design on children with autistic spectrum disorders, in Good Autism Practice, Vol. 7(1), pp31-42; Whitehurst, T. (2006) Evaluation of features specific to an ASD designed living accommodation, in Designing living \& learning environments for children with autism, Sunfield Research Institute

${ }^{410}$ Brand, A. (2010) Living in the community, London: Helen Hamlyn Centre, Royal College of Art

${ }^{411}$ Mostafa, M. (2010) Housing Adaptation for Adults with Autistic Spectrum Disorder, Open House International, Urban International Press, 35, 37-48

${ }^{412}$ Brand, A. (2010) Living in the community, London: Helen Hamlyn Centre, Royal College of Art

${ }^{413}$ Beaver, C. (2011) Designing environments for children and adults on the autism spectrum, in Good Autism Practice (GAP), 12(1), pp.7-11
} 


\section{Elena Bellini}

sono essere inoltre utili per l'orientamento (Kinnaer et al., 2016 ${ }^{415}$ ), così come l'utilizzo di indicazioni visive (Mostafa, 2010 416 ). Kinnaer propone infine di identificare e mappare i collegamenti utili nelle zone di vicinato (servizi, lavoro, etc.) poiché migliorano la capacità di orientamento nel contesto urbano e quindi le abilità sociali e la capacità di indipendenza ed integrazione nella comunità (Kinnaer et al., 2016) ${ }^{417}$.

\subsubsection{Indipendenza e autonomia. Abilità}

Uno spazio sicuro e protetto favorisce anche l'indipendenza della persona e l'autostima, derivata dalla possibilità di poter scegliere liberamente e fare le cose da soli, acquisendo l'autodeterminazione e una maggiore autonomia di vita. La sfida è quella di progettare ambienti che sembrino avere opzioni limitate, che non creino ansia e agitazione nella persona con autismo che devi porsi a fare delle scelte, ma allo stesso tempo abbiano la capacità di adattarsi ai diversi bisogni di ogni singolo residente (Brand, 2010) ${ }^{418}$.

Una strategia utile all'aumento di indipendenza prosternale é la predisposizione degli spazi sulla base delle routines quotidiane (Mostafa, 2015 ${ }^{419}$; Brand, 2010 ${ }^{420}$ ), dove ogni ambiente corrisponda ad una funzione specifica e ben delineata (Kinnaer et al., 2016) ${ }^{421}$, anche attraverso un cambio di materiale e colore (Ahrentzen, Steele, $2009)^{422}$. Il layout spaziale deve garantire la prevedibilità, essere facile da comprendere e permettere un accesso visibile a tutte le stanze, agevolato attraverso traspa-

${ }^{414}$ Whitehurst, T. (2006) The impact of building design on children with autistic spectrum disorders, in Good Autism Practice, Vol. 7(1), pp31-42; Whitehurst, T. (2006) Evaluation of features specific to an ASD designed living accommodation, in Designing living \& learning environments for children with autism, Sunfield Research Institute

${ }^{415}$ Kinnaer, M. Baumers, S. Heylighen, A. (2014) How do People with Autism (Like to) Live?, in Langdon, P. M . Lazar, J. Heylighen, A. Dong, H. (a cura di ) (2014) Inclusive Designing. Joining Usability, Acessibility and Inclusion, Springer Cham Heidelberg New York Dordrecht London, part IV, pp. $175-185$

${ }^{416}$ Mostafa, M. (2010) Housing Adaptation for Adults with Autistic Spectrum Disorder, Open House International, Urban International Press, 35, 37-48

${ }^{417}$ Kinnaer, M. Baumers, S. Heylighen, A. (2014) How do People with Autism (Like to) Live?, in Langdon, P. M . Lazar, J. Heylighen, A. Dong, H. (a cura di ) (2014) Inclusive Designing. Joining Usability, Acessibility and Inclusion, Springer Cham Heidelberg New York Dordrecht London, part IV, pp. $175-185$

${ }_{418}$ Brand, A. (2010) Living in the community, London: Helen Hamlyn Centre, Royal College of Art

419 Mostafa, M. (2015) Architecture for Autism: Built Environment Performance in Accordance to the Autism ASPECTSSTM Design Index, in Design Principles and practices: an International Journal Annual Review, vol. 8, pp.55-71,Common Ground Publishing, Champaign, Illinois, USA

${ }^{420}$ Brand, A. (2010) Living in the community, London: Helen Hamlyn Centre, Royal College of Art

${ }^{421}$ Kinnaer, M. Baumers, S. Heylighen, A. (2014) How do People with Autism (Like to) Live?, in Langdon, P. M . Lazar, J. Heylighen, A. Dong, H. (a cura di ) (2014) Inclusive Designing. Joining Usability, Acessibility and Inclusion, Springer Cham Heidelberg New York Dordrecht London, part IV, pp. $175-185$

${ }_{422}$ Ahrentzen, S. Steele, K. (2009) Advancing Full Spectrum Housing: Design for Adults with Autism Spectrum Disorders Technical report, Arizona Board of Regents, Phoenix; Resnik , D. D. et al. (2009) Opening Doors: A discussion of residential options for adults living with autism and related disorders, Collaborative report by the Urban Land Institute Arizona, Southwest Autism Research \& Resource Center and Arizona State University 
renza, aperture, muretti bassi o disimpegni lungo la sequenza spaziale, offrendo la possibilità di guardare dentro uno spazio prima di decidere di entrare (Ahrentzen, Steele, 2009 ${ }^{423}$; Brand, 2010 ${ }^{424}$ ); oppure aree di transizione (Mostafa, 2015 ${ }^{425}$ ) tra le aree dedicate a diverse funzioni o attività. Per facilitare le abilità quotidiane può essere utile organizzare anche il guardaroba e le aree di ripostiglio sulla base delle routines, dividendo vestiti da accessori, oggetti ed attrezzature; allo stesso modo per il bagno (Mostafa, 2010 ${ }^{426}$ ). Prevedere almeno un bagno accessibile, almeno un servizio in adiacenza agli spazi comuni e servizi di dimensione piuttosto ampia, dato che a volte l'uso del bagno é supportato da un operatore (Brand, 2010 ${ }^{427}$; Ahrentzen, Steele, $2009^{428}$ ). Progettare il guardaroba con una struttura che divida gli indumenti per facilitare la preparazione e le attività quotidiane in modo indipendente, ad esempio con scompartimenti per vestiti giornalieri e sistemi di contenitori scorrevoli per una maggiore praticità (Ahrentzen, Steele, 2009) ${ }^{429}$.

Le camere devono invece trasmettere sicurezza e privacy (Whitehurst, 2006) ${ }^{430}$, avere una dimensione contenuta ed un letto tipo alcova, dedicando alla camera la principale funzione del dormire (Mostafa, 2010) ${ }^{431}$. Sono preferibili camere singole con bagno privato, personalizzabili dal punto di vista della luce, con elementi incassati a soffitto o mobili (Ahrentzen, Steele, 2009) ${ }^{432}$, oppure con la scelta del colore a parete (Brand, 2010 ${ }^{433}$; Whitehurst, $2006^{434}$ ). Whitehurst propone inoltre una dispo-

\footnotetext{
${ }^{423}$ Ahrentzen, S. Steele, K. (2009) Advancing Full Spectrum Housing: Design for Adults with Autism Spectrum Disorders Technical report, Arizona Board of Regents, Phoenix; Resnik , D. D. et al. (2009) Opening Doors: A discussion of residential options for adults living with autism and related disorders, Collaborative report by the Urban Land Institute Arizona, Southwest Autism Research \& Resource Center and Arizona State University

${ }_{424}$ Brand, A. (2010) Living in the community, London: Helen Hamlyn Centre, Royal College of Art

${ }^{425}$ Mostafa, M. (2015) Architecture for Autism: Built Environment Performance in Accordance to the Autism ASPECTSS ${ }^{\text {TM }}$ Design Index, in Design Principles and practices: an International Journal Annual Review, vol. 8, pp.55-71,Common Ground Publishing, Champaign, Illinois, USA

${ }^{426}$ Mostafa, M. (2010) Housing Adaptation for Adults with Autistic Spectrum Disorder, Open House International, Urban International Press, 35, 37-48

${ }_{427}$ Brand, A. (2010) Living in the community, London: Helen Hamlyn Centre, Royal College of Art

${ }^{428}$ Ahrentzen, S. Steele, K. (2009) Advancing Full Spectrum Housing: Design for Adults with Autism Spectrum Disorders Technical report, Arizona Board of Regents, Phoenix; Resnik , D. D. et al. (2009) Opening Doors: A discussion of residential options for adults living with autism and related disorders, Collaborative report by the Urban Land Institute Arizona, Southwest Autism Research \& Resource Center and Arizona State University

429 ibidem

430 Whitehurst, T. (2006) The impact of building design on children with autistic spectrum disorders, in Good Autism Practice, Vol. 7(1), pp31-42; Whitehurst, T. (2006) Evaluation of features specific to an ASD designed living accommodation, in Designing living \& learning environments for children with autism, Sunfield Research Institute

${ }^{431}$ Mostafa, M. (2010) Housing Adaptation for Adults with Autistic Spectrum Disorder, Open House International, Urban International Press, 35, 37-48

${ }^{432}$ Ahrentzen, S. Steele, K. (2009) Advancing Full Spectrum Housing: Design for Adults with Autism Spectrum Disorders Technical report, Arizona Board of Regents, Phoenix; Resnik, D. D. et al. (2009) Opening Doors: A discussion of residential options for adults living with autism and related disorders, Collaborative report by the Urban Land Institute Arizona, Southwest Autism Research \& Resource Center and Arizona State University

${ }^{433}$ Brand, A. (2010) Living in the community, London: Helen Hamlyn Centre, Royal College of Art
} 


\section{Elena Bellini}

sizione delle stanze a zig-zag per consentire la vista verso l'esterno senza interferenza con gli altri utenti (come nel caso studio del Sunfield Research Institute); finestre aggiuntive in alto apribili meccanicamente per una maggiore sicurezza; porte arretrate per garantire privacy rispetto allo spazio di circolazione; video e musica all'interno delle stanze (Whitehurst, 2006) ${ }^{435}$.

La cucina deve invece offrire la possibilità di contemporaneità d'uso da parte di più utenti, prevedendo uno spazio piuttosto ampio, con varie postazioni per cucinare ed una grande dispensa/ripostiglio in modo che ognuno possa avere il proprio personale scaffale.

E' preferibile progettare i corridoi secondo un senso di marcia basato sulle routines predefinite, affacciandosi sui diversi spazi comuni e sulla corte giardino, per favorire la luce naturale, la ventilazione e la relazione interpersonale tra gli utenti; l'utilizzo delle maniglie è considerato più semplice rispetto ai pomelli delle porte (Ahrentzen, Steele, 2009) $)^{436}$.

Una facilitazione importante per lo sviluppo delle abilità quotidiane è l'utilizzo di simboli grafici, istruzioni, o schede visive attaccati a parete o sui diversi dispositivi della casa, per facilitare la comprensione e la comunicazione (Whitehurst,2006 ${ }^{437}$; Brand, 2010 ${ }^{438}$ ). L'utilizzo di metodi di comunicazione alternativa può essere utile anche per il rapporto tra utente e operatore, in casi in cui ci sia assenza o limitazione di linguaggio (Brand, 2010) ${ }^{439}$. Colori o scritte possono essere utili per orientarsi ed identificare le funzioni, non solo per quanto riguarda gli ambienti, ma anche per rendere più visibili interruttori o altri dispositivi, giocando sui contrasti (in particolare nella cucina) (Ahrentzen, Steele, 2009) ${ }^{440}$.

${ }^{434}$ Whitehurst, T. (2006) The impact of building design on children with autistic spectrum disorders, in Good Autism Practice, Vol. 7(1), pp31-42; Whitehurst, T. (2006) Evaluation of features specific to an ASD designed living accommodation, in Designing living \& learning environments for children with autism, Sunfield Research Institute

${ }^{435}$ ibidem

${ }^{436}$ Ahrentzen, S. Steele, K. (2009) Advancing Full Spectrum Housing: Design for Adults with Autism Spectrum Disorders Technical report, Arizona Board of Regents, Phoenix; Resnik , D. D. et al. (2009) Opening Doors: A discussion of residential options for adults living with autism and related disorders, Collaborative report by the Urban Land Institute Arizona, Southwest Autism Research \& Resource Center and Arizona State University

${ }^{437}$ Whitehurst, T. (2006) The impact of building design on children with autistic spectrum disorders, in Good Autism Practice, Vol. 7(1), pp31-42; Whitehurst, T. (2006) Evaluation of features specific to an ASD designed living accommodation, in Designing living \& learning environments for children with autism, Sunfield Research Institute

${ }^{438}$ Brand, A. (2010) Living in the community, London: Helen Hamlyn Centre, Royal College of Art

439 ibidem

440 Ahrentzen, S. Steele, K. (2009) Advancing Full Spectrum Housing: Design for Adults with Autism Spectrum Disorders Technical report, Arizona Board of Regents, Phoenix; Resnik, D. D. et al. (2009) Opening Doors: A discussion of residential options for adults living with autism and related disorders, Collaborative report by the Urban Land Institute Arizona, Southwest Autism Research \& Resource Center and Arizona State University 


\subsubsection{Relazioni sociali. Integrazione e inclusività}

Le difficoltà di comunicazione e di interazione sociale tipiche dell'autismo rappresentano le maggiori problematiche nel costituire un rapporto interpersonale ed una completa integrazione nella comunità. Favorire le relazioni all'interno dell'abitazione e con il contesto é quindi utile all'acquisizione di abilità sociali, per evitare la segregazione e l' isolamento della persona.

I collegamenti rappresentano il punto maggiormente favorevole per lo scambio tra le diverse persone, per questo motivo il corridoio diventa un punto importante della casa, non solo pensato come spazio di circolazione, ma come zona di interazione; quest'area può essere quindi attrezzata e avere molteplici funzioni, come aree di sosta o di gioco, nel caso di bambini (Beaver, 2011) ${ }^{441}$. Allo stesso modo si suggeriscono spazi di seduta accanto alle scale o nei collegamenti per favorire la socializzazione nei punti di snodo e quindi incontro tra le persone. Anche l'ingresso é un momento di rapporto con il mondo esterno, che può essere favorito attraverso un portico o una copertura che protegga dalle intemperie e consenta di sostare e favorire la socializzazione e l'interazione con l'esterno (Ahrentzen, Steele, 2009) ${ }^{442}$. All'interno invece, zone legate alla socializzazione circolari sono considerate maggiormente favorevoli alle relazioni e all'interazione (Mostafa, 2010) ${ }^{443}$. Spazi ampi per le zone comuni possono inoltre essere utili per evitare che le persone entrino obbligatoriamente in contatto l'una con l'altra anche quando non sono pronte o non ne hanno voglia (Beaver, 2011) ${ }^{444}$. Pertanto si suggerisce di conformare il layout spaziale in modo che vi siano sempre anche spazi personali, per momenti di privacy o per incontrare la famiglia in privato; ampi spazi comuni per non dare senso di affollamento e che all'interno prevedano "zone di fuga", escape spaces, nicchie, sedute alle finestre, angoli o aree di "isolamento" per garantire una partecipazione anche se più a distanza, che potrebbe essere preferita rispetto al coinvolgimento attivo e rimanere quindi all'interno dell'area comune, scegliendo i gradi di privacy e di interazione sociale (Kinnaer et al., 2014 ${ }^{445}$; Brand, 2010 ${ }^{446}$; Ahrentzen, Steele, 2009 ${ }^{447}$ );

441 Beaver, C. (2011) Designing environments for children and adults on the autism spectrum, in Good Autism Practice (GAP), 12(1), pp.7-11

${ }^{442}$ Ahrentzen, S. Steele, K. (2009) Advancing Full Spectrum Housing: Design for Adults with Autism Spectrum Disorders Technical report, Arizona Board of Regents, Phoenix; Resnik , D. D. et al. (2009) Opening Doors: A discussion of residential options for adults living with autism and related disorders, Collaborative report by the Urban Land Institute Arizona, Southwest Autism Research \& Resource Center and Arizona State University

${ }^{443}$ Mostafa, M. (2010) Housing Adaptation for Adults with Autistic Spectrum Disorder, Open House International, Urban International Press, 35, 37-48

${ }^{444}$ Beaver, C. (2011) Designing environments for children and adults on the autism spectrum, in Good Autism Practice (GAP), 12(1), pp.7-11

${ }^{445}$ Kinnaer, M. Baumers, S. Heylighen, A. (2014) How do People with Autism (Like to) Live?, in Langdon, P. M . Lazar, J. Heylighen, A. Dong, H. (a cura di ) (2014) Inclusive Designing. Joining Usability, Acessibility and Inclusion, Springer Cham Heidelberg New York Dordrecht London, part IV, pp. $175-185$

${ }_{446}$ Brand, A. (2010) Living in the community, London: Helen Hamlyn Centre, Royal College of Art

447 Ahrentzen, S. Steele, K. (2009) Advancing Full Spectrum Housing: Design for Adults with Autism Spectrum Disorders Technical report, Arizona Board of Regents, Phoenix; Resnik , D. D. et al. (2009) 


\section{Elena Bellini}

prevedere diverse aree di living per dare diverse opportunità, pur collegate l'una all'altra in modo da favorire lo scambio (Ahrentzen, Steele, 2009) ${ }^{448}$.

Avere un'area per la posta, messaggi in bacheca, conti da pagare, ecc. può essere infine una strategia per obbligare almeno una volta durante il giorno ad incontrare ed interagire con gli altri (Ahrentzen, Steele, 2009) ${ }^{449}$.

\subsection{Luoghi della cura. Ospedale accessibile/formazione e assistenza sanitaria}

\subsubsection{Introduzione. Il diritto alla cura}

"Le persone con disabilità non hanno bisogni speciali, ..ma hanno gli stessi bisogni di tutte le soggettività empiriche, perché in realtà non sono persone speciali, ma persone umane che soffrono di alcune limitazioni”. (Pessina, 2013) ${ }^{450}$

Così viene introdotto il tema dell'accessibilità e dell'universalità della cura dalla Carta dei diritti delle persone con disabilità in ospedale pubblicata a Roma da Spes contra Spem (2013) come primo documento italiano in questo ambito, scritto sulla base della Convenzione O.N.U. Sui diritti delle Persone con disabilità (approvata il 13 dicembre 2006 e ratificata in Italia con L.18 del 3 marzo 2009 - art 25 "diritto alla cura") e della Carta europea dei diritti del malato (European Charter of Patients' Rights, 2002). Questo documento riporta 14 diritti fondamentali a favore delle persone con disabilità, che si trovano purtroppo spesso a soffrire due volte la malattia, poiché hanno difficoltà nell'esprimere il dolore o i propri bisogni fisiologici e far comprendere agli altri che cosa sentono o provano: Diritto a misure preventive; Diritto all'accesso (Il diritto di una persona con disabilità ad accedere ai servizi sanitari è spesso impedito dalla presenza di barriere architettoniche e/o culturali. In particolare persone con disabilità intellettiva severa o con disturbi dello spettro autistico riscontrano spesso notevoli problemi nell'attesa all'interno dei luoghi di cura, specialmente in una situazione di emergenza come quella del Pronto Soccorso, per cui dovrebbe essere garantito un percorso preferenziale); Diritto alla informazione; Diritto al consenso (diritto ad accedere a tutte le informazioni che possono mettere la persona in grado di partecipare attivamente alle decisioni che riguardano la propria salute, compresa l'eventuale partecipazione alla ricerca scientifica); Diritto alla libera scelta (diritto di esprimere la propria volontà in materia di procedure ed erogazione dei servizi sanitari, salvo non vi sia una comprovata incapacità di intendere e di volere); Diritto alla privacy e alla confidenzialità; Diritto al rispetto del tempo dei pazienti (diritto di usufruire del canale delle emergenze, evitando stress e disagi che possono coinvolgere l'ambiente ospedaliero; diritto al rispetto dei tempi del paziente, sia dell'espressione che della comprensione. Per una persona con Disturbi dello Spettro Autistico, un semplice prelievo del sangue può essere vissuto come una vio-

Opening Doors: A discussion of residential options for adults living with autism and related disorders, Collaborative report by the Urban Land Institute Arizona, Southwest Autism Research \& Resource Center and Arizona State University

${ }^{448}$ ibidem

${ }^{449}$ ibidem

${ }^{450}$ Pessina, A. (2010) Barriere della mente e barriere del corpo. Annotazioni per un' etica della soggettività empirica, in Paradoxa, Etica della condizione umana, Vita e Pensiero, Milano 
lenza e di conseguenza richiedere tempi molto lunghi; è necessario dotarsi di un ambiente idoneo, non avere fretta e spiegare la procedura in collaborazione con i caregivers); Diritto al rispetto di standard di qualità (diritto di accedere a trattamenti e servizi sanitari di alta qualità, senza alcuna differenza tra i pazienti. Soprattutto per le persone con disabilità multipla o disabilità intellettiva medio grave, quando necessitano di procedure diagnostico-terapeutiche ad alto contenuto tecnologico, scarse o costose, vi è la tendenza a non ritenerle idonee non sulla base di un giudizio clinico sulla proporzionalità dell'intervento, ma di un pregiudizio culturale: utilizzarla per una persona con disabilità potrebbe voler dire negarla ad un malato non disabile); Diritto alla sicurezza; Diritto alla innovazione; Diritto a evitare le sofferenze e il dolore non necessari (Il personale sanitario non deve mai ritenere che questi pazienti non siano in grado di provare dolore, poiché spesso non riescono a comunicare in modo esplicito la sofferenza. In casi di disabilità intellettiva severa o Disturbi dello Spettro Autistico la presenza di dolore potrebbe essere esplicitata attraverso comportamenti problema o disturbi emozionali; la collaborazione con i caregivers è fondamentale per la comprensione del paziente. Oltre al dolore fisico può essere inoltre presente un disagio psicologico legato all'ambiente non familiare e alla manipolazione da parte di altre persone. Alcune persone con autismo ad esempio rifuggono spesso il contatto fisico, poiché lo vivono come una vera violenza. Tutti gli interventi sul paziente dovrebbero quindi essere precedute da un colloquio con il paziente o il caregiver. In questi pazienti procedure diagnostiche come la misurazione della pressione arteriosa o la venipuntura, possono risultare particolarmente impegnative e richiedere tempi più lunghi; talvolta è necessario ricorrere anche alla sedazione del paziente. Può rivelarsi utile in persone adulte con questa tipologia di disabilità, l'utilizzo di sistemi di rilevazione del dolore impiegati nei bambini più piccoli.); Diritto a un trattamento personalizzato (Diritto a programmi diagnostici o terapeutici su misura delle personali esigenze. In particolare, in caso di ricovero di persone con disabilità intellettiva o Disturbi dello Spettro Autistico è necessario garantire la presenza continua di un familiare, un caregiver o un operatore specializzato delegato dalla famiglia, che conosca nei dettagli le esigenze del paziente e possa fare da tramite per comunicare con la persona con disabilità, per comprendere le sue emozioni ed esigenze. La degenza può essere particolarmente gravosa per queste persone e quindi accentuare disturbi comportamentali); Diritto al reclamo; Diritto al risarcimento (Spes contra Spem, 2013) ${ }^{451}$.

\subsubsection{L'incidenza dell'ospedalizzazione in casi di autismo. I numeri, le cause}

In Italia non si hanno studi e statistiche di riferimento in merito a dati di accesso ospedaliero e alle cure socio-sanitarie in casi specifici di DSA. Al contrario in ambito europeo ed internazionale sono stati fatti diversi studi che valutano come l'ambito sanitario sia molto frequentato da persone con DSA, con incidenza maggiore rispetto alla popolazione neurotipica; qui di seguito verranno presentati alcuni di questi studi per dare un'idea non solo dei numeri d'incidenza, ma delle motivazioni che portano

${ }^{451}$ Spes contra Spem (2013) Carta Dei Diritti Delle Persone Con Disabilità In Ospedale, Roma 


\section{Elena Bellini}

le persone con autismo in ospedale e delle criticità che si possono riscontrare dal punto di vista medico e psicologico.

Gurney et al. hanno analizzato i dati della National Survey of Children's Health (NSCH) negli Stati Uniti, includendo le informazioni di 483 bambini diagnosticati con autismo, valutando le visite, gli esami o i ricoveri avuti nei precedenti 12 mesi. Dall'indagine è stato riscontrato un bisogno maggiore di bambini con autismo di ricevere cure fisiche, terapie occupazionali o del linguaggio, trattamenti legati a comportamenti problema, emozionali o dello sviluppo e uso di farmaci. Sono state riportate inoltre dai genitori una varietà di condizioni quali depressione, problemi di ansia o del comportamento, ADHD o disturbi dell'attenzione. Non sono state valutate differenze in merito a diabete o asma, ma è stata riscontrata un'incidenza maggiore di allergie di tipo alimentare, respiratorie o della pelle. Per quanto riguarda le visite mediche viene documentato un numero maggiore di visite da parte dei bambini con autismo, in particolare rispetto a quelle preventive e di cure non emergenziali (Gurney et al., 2006) ${ }^{452}$.

Croen et al. hanno analizzato invece il numero ed il costo delle cure di bambini con autismo dai 2 ai 18 anni rispetto agli altri bambini del Kaiser Permanente Medical Care Program della Northern California tra il 2003 e il 2004. E' stata misurata un maggiore incidenza di visite cliniche ( 5.6 vs 2.8$)$, pediatriche $(2.3$ vs 1.6$)$, psichiatriche ( 2.2 vs 0.3$)$ e neurologiche $(5.1 \%$ vs $0.4 \%$;); una maggiore percentuale di accessi ospedalieri, come ricovero ( $3 \%$ vs $1 \%$ ) o ambulatoriali ( $5 \%$ vs $2 \%$ ). Tra i bambini con DSA i disturbi più comuni legati all'ospedalizzazione psichiatrica sono stati i disturbi dell'umore (59\%), disturbi psicotici (20\%), e disturbi del controllo degli impulsi (17\%); per quanto riguarda le ospedalizzazioni non psichiatriche invece sono state più comunemente diagnosticate malattie respiratorie $(17 \%)$, epilessia $(15 \%)$, anomalie congenite (13\%) e disturbi digestivi (13\%). Questa incidenza aumenta ancora di più in età 15-18, relativa soprattutto ai disturbi psichiatrici. La frequenza di visite al Pronto Soccorso é abbastanza simile, ma aumenta drasticamente in età 1518 dove si riscontra il $70 \%$ di accessi al Pronto Soccorso in più rispetto ai ragazzi/e che non presentano disturbi dello spettro autistico. Per quanto riguarda l'uso dei farmaci, risulta maggiore di circa il $40 \%$, con particolare utilizzo di medicinali per i disturbi psichiatrici o gastrointestinali (Croen et al., 2006) ${ }^{453}$.

Lunsky et al. hanno effettuato invece una ricerca nella provincia di Ontario, in Canada, tra il 2010 e il 2012 attraverso un questionario sui servizi sanitari rivolto alle famiglie. Solo nell'infanzia si stima che i casi di ospedalizzazione con DSA sono maggiori di 2,5 volte, oltre ad essere più frequenti gli accessi al Pronto Soccorso. $E^{\prime}$ stato riportato che le problematiche di salute mentale rappresentano il $13 \%$ delle visite tra $\mathrm{i}$ bambini con DSA ed in ogni caso al di sotto dei 21 anni più del $10 \%$ ha effettuato almeno un accesso ospedaliero di tipo psichiatrico. Oltre a presentare casi

${ }^{452}$ Gurney, J. G. et al. (2006) Parental Report of Health Conditions and Health Care Use Among Children With and Without Autism, National Survey of Children's Health, Arch Pediatr Adolesc Med, 160:825-830

${ }^{453}$ Croen, L. A. et al. (2006) A Comparison of Health Care Utilization and Costs of Children With and Without Autism Spectrum Disorders in a Large Group-Model Health Plan, Pediatrics, Volume 118, Number 4, October 2006, pp. e1203-1211 
di comorbidità, le persone con DSA hanno frequenti infortuni rispetto ai coetanei e presentano un maggiore rischio rispetto alle emergenze ospedaliere. E' inoltre piuttosto difficile per questi pazienti comprendere la differenza tra problematiche di tipo medico o psichiatrico, a causa delle difficoltà socio-comunicative. I risultati del questionario riportano che il $18,4 \%$ dei soggetti è stato al Pronto Soccorso almeno una volta; il $69,2 \%$ si è presentato con i familiari, i restanti con il servizio di ambulanza, soprattutto in orario serale $(52,4 \%)$ o pomeridiano $(38, \%)$. Per il $65,4 \%$ si è trattato di visite mediche, perlopiù legate a infortuni $(58,8 \%)$, mentre il $34,6 \%$ legato a crisi di salute mentale, tra cui aggressione $(33,3 \%)$ o trattamenti psicotropi $(33,3 \%)$. Il $23,5 \%$ delle visite ha inoltre presentato casi di disturbi comportamentali come rabbia, agitazione o aggressività. L'interazione con il paziente é avvenuta per l'88,5\% attraverso i familiari o i caregivers e con il paziente direttamente, anche se i genitori hanno riscontrato la necessità di una formazione maggiore del personale, che spesso non ha seguito i loro suggerimenti per interagire e curare il bambino. In generale in un periodo campione di 2 mesi il 13\% dei soggetti ha frequentato il Pronto Soccorso (Lunsky, 2015) ${ }^{454}$.

Iannuzzi et al. hanno analizzato invece nello specifico gli accessi al Pronto Soccorso secondo i dati del Nationwide Emergency Department Sample (NEDS) ${ }^{455}$ del 2010, Healthcare Cost and Utilization Project (HCUP) dell'Agency for Healthcare Research and Quality (AHRQ), il maggiore database disponibile negli Stati Uniti che raccoglie circa 29 milioni di visite PS effettuate in 961 ospedali di 28 stati; rispetto a questo sono stati valutati gli accessi al Pronto Soccorso nelle fasce di età 3$5,6-11,12-15,16-18$ e oltre i 19. Rispetto alle diverse fasce di età è stata riscontrata una grande differenza nelle cause di accesso al PS. I casi di epilessia e convulsioni sono circa il 5,5\% nella fascia 3-5, mentre non sono riportati nella stessa fascia di età ma in casi senza DSA; nella fascia 6-11 sale ancora il numero (circa l'8,4\%) rappresentando il problema più comune, sempre comparato a nessun caso per i pazienti non-DSA, dove si riscontrano soprattutto problemi respiratori $(10,5 \%)$. I disturbi psichiatrici, comportamenti autolesionistici e aggressioni, rappresentano invece la problematica primaria in età $12-15$, contando circa $1 ' 11,5 \%$ delle visite. Nella fascia 16-18 ancora disturbi psichiatrici e epilessia, mentre nel gruppo non-DSA si riscontrano soprattutto distorsioni e stiramenti $(8,8 \%)$. Infine negli adulti con DSA si riscontrano soprattutto casi di epilessia $(8,9 \%)$ e a seguire i disturbi psichiatrici, mentre tra i non-DSA rimangono sempre prevalenti casi di distorsioni e stiramenti $(5 \%)$ (Iannuzzi et al., 2015) ${ }^{456}$. Il fine dello studio è quindi aumentare la consapevolezza che i motivi di accesso al Pronto Soccorso sono differenti in caso di Disturbi dello Spettro Autistico e che le esigenze variano in base alle comorbidità, alle particolari sensibilità e alla difficoltà di relazioni sociali e comunicazione; molti dei comportamenti disadattivi infatti sono espressione di sofferenza fisica e discomfort e che

\footnotetext{
454 Lunsky, Y. et al. (2015) Predictors of emergency service use in adolescents and adults with autism spectrum disorder living with family, Emerg Med Journal, 32:787-792

455 HCUP (2010) Nationwide Emergency Department (NEDS), Healthcare Cost and Utilization Project (HCUP), Agency for Healthcare Research and Quality, Rockville, MD (USA)

${ }^{456}$ Iannuzzi, D. et al. (2015) Brief Report: Emergency Department Utilization by Individuals with Autism, Journal of Autism and Development Disorders 45:1096-1102
} 


\section{Elena Bellini}

quindi alcuni trattamenti possono rivelarsi inopportuni e compromettere la qualità della cura (Iannuzzi et al., 2015) ${ }^{457}$.

Vohra et al. Proseguono il lavoro impostato da Iannuzzi et al., approfondendo il caso degli adulti con DSA, confrontando i dati di sei anni (2006-2011) del Nationwide Emergency Department Sample (NEDS) (Healthcare Cost and Utilization Project - HCUP, 2011) ${ }^{458}$ tra adulti con e senza DSA e analizzando l'utilizzo del Pronto Soccorso rispetto alle visite psichiatriche e non-psichiatriche (per lesioni) (Vohra et al., 2016)10. Riscontrano quindi che il numero delle visite risulta più che duplicato nel corso dei sei anni ed in particolare per quanto riguarda le visite psichiatriche. Le visite psichiatriche $(15 \%)$ e non psichiatriche $(16 \%)$ si rivelano inoltre più frequenti tra gli adulti con DSA e si riscontra tra le visite più comuni cause di: epilessia $(8,8 \%)$, cadute $(6,5 \%)$, schizofrenia $(3,8 \%)$, problemi respiratori $(3,8 \%)$, bipolarismo $(3,2 \%)$ e depressione $(2,5 \%)$ (Vohra et al., 2016) ${ }^{459}$. In questo studio la cooccorrenza specifica dei disturbi psichiatrici nell'adulto con DSA risulta molto alta; questo é' un riscontro frequentemente replicato. In alcuni studi viene riportata una sottostima, che dipende principalmente dall'utilizzo di criteri diagnostici non adattati, soprattutto in casi di compresenza di disabilità intellettiva. In generale, in questa ricerca il problema della vulnerabilità psicologica-psicopatologica complessa della maggior parte delle persone con DSA avrebbe potuto essere trattato di più, anche in relazione agli scopi specifici della ricerca. Rispetto alla prevalenza dei vari disturbi psichiatrici nell'adulto, dati utili si ritrovano nel recente studio di Russel et al. ${ }^{460}$ riferendosi in particolare a disturbi ossessivo compulsivi e dell'ansia.

Lo stesso aumento di accessi al PS riscontrato da Vohra et al. Viene rilevato anche dalla ricerca di Nayfack et al. Dove si analizzano i dati degli accessi pediatrici (1-18 anni) in California nel periodo intercorso tra il 1999 e il 2009, secondo il Unmasked, Confidential Patient Discharge Database dell'Office of Statewide Health Planning and Development (OSHPD). La prima differenza presentata tra la popolazione con o senza autismo é stata rilevata rispetto all'età dei pazienti che nel primo caso sono principalmente tra i 6-10 (31\%) e gli 11-14 anni (32\%), mentre nel secondo vanno dagli 0-5 anni (29\%) e i 15-18 anni (29\%) (Nayfack et al., 2014) ${ }^{461}$. Negli anni si è presentato un aumento costante dei casi di ospedalizzazione, raggiungendo nel 2009 più del triplo degli accessi, soprattutto nella fascia di età 15-18 (aumento del 328\%, dai 155 casi nel 1999 ai 664 nel 2009) e tra i 0-14 anni (aumento del 243\%, dai 230 casi nel 1999 ai 788 nel 2009). I casi di ospedalizzazione di bambini con autismo sono inoltre più frequenti rispetto a quelli senza autismo ( 3 vs $1 \%$ ) ed in

\footnotetext{
457 Iannuzzi, D. et al. (2015) Brief Report: Emergency Department Utilization by Individuals with Autism, Journal of Autism and Development Disorders 45:1096-1102

${ }^{458}$ Healthcare Cost and Utilization Project (HCUP) (2011) HCUP nationwide emergency department sample (NEDS), MD: Agency for Healthcare Research and Quality, Rockville (USA)

${ }_{459}$ Vohra, R. (2016) Emergency Department Use Among Adults with Autism Spectrum Disorders (ASD), Journal of Autism and Development Disorders, 46:1441-1454

${ }^{460}$ Russel, A. J. et al. (2016) The mental health of individuals referred for assessment of autism spectrum disorder in adulthood: A clinical report, Autism, July 2016, 20(5): 623-7

${ }_{461}$ Nayfack, A. M. et al. (2014) Hospitalizations of Children with Autism Increased from 1999 to 2009 , Journal of Autism and Development Disorders, 44:1087-1094
} 
Ambienti sensoriali "terapeutici" che rendano Abili

particolare nell'età dai 15 ai 18 anni, dove si riscontra una frequenza di 3,5 volte superiore (7 vs 2\%) (Nayfack et al., 2014) ${ }^{462}$.

\subsubsection{Affrontare il percorso di cura. Criticità e strategie per migliorare l'acces- sibilità}

La letteratura incentrata sulla qualità degli ambienti sanitari è molto ampia e ha dimostrato l'esigenza di progettare con una visione human-centered, basata sui bisogni specifici delle persone, in un ambito dove le condizioni del contesto ambientale possono avere un impatto non solo sul benessere della persona, come abbattimento dello stress e influenza sull'umore, ma anche sull'evolvere della cura e della guarigione, diminuendo i tempi di ospedalizzazione, soprattutto in utenti con particolare fragilità psico-fisica (Bonaiuto, Bilotta, Fornara, 2004) ${ }^{463}$.

Un progetto di ospedale, o struttura sanitaria, che funzioni, è quello che riesce ad integrare le esigenze dei pazienti con quelle funzionali relative all'intervento medico e all'organizzazione ospedaliera. La difficoltà di una progettazione efficace sta proprio nella complessità di rispondere ai diversi bisogni dei pazienti, ognuno diverso dall'altro, ma anche di risolvere conflitti di esigenze che talvolta si creano tra operatori e pazienti. Anche lo stesso personale sanitario è soggetto a stress associato al contesto ambientale e alle relazioni con i pazienti e le loro famiglie; un aspetto da non sottovalutare, poiché causa cali di produttività e qualità del lavoro e quindi, come conseguenza diretta, cali nella qualità della cura per il paziente stesso.

Tutti gli aspetti considerati nella progettazione di ambienti sanitari che favoriscono la qualità della cura, vanno ad amplificarsi quando ci si rivolte ad un'utenza specifica, maggiormente fragile e vulnerabile, come un'utenza con disabilità, o persone con bisogni particolari. In casi di autismo, in particolare, ci troviamo di fronte ad un'utenza dalla spiccata sensibilità sensoriale, per cui il contesto ambientale ha un'enorme influenza sul benessere, lo stress ed il disagio.

Tra gli aspetti che maggiormente influiscono nella qualità della cura si andranno quindi a considerare quelli che agiscono in particolare sullo stress del paziente, ovvero la capacità di familiarità dell'ambiente, che favorisca la prevedibilità e riduca il senso di ansia dovuto ad ambienti sconosciuti; il comfort visivo, in termini di qualità delle vedute (in particolare se rivolte all'esterno e all'ambiente naturale) e di qualità dell'illuminazione, naturale ed artificiale, che può avere impatto su depressione, agitazione e qualità del sonno; il comfort acustico e le condizioni di privacy, evitando le situazioni di affollamento e regolando le relazioni di prossimità e interazioni tra i pazienti e con il personale; il comfort olfattivo, attraverso la riduzione dei cattivi odori con ventilazione naturale o meccanica; il comfort epidermico, garantendo un adeguato livello di temperatura ed umidità; il senso di comprensione e wayfinding, per pazienti, visitatori e lo stesso personale in termini di efficienza di lavoro; non per

\footnotetext{
462 Nayfack, A. M. et al. (2014) Hospitalizations of Children with Autism Increased from 1999 to 2009 , Journal of Autism and Development Disorders, 44:1087-1094

${ }^{463}$ Bonaiuto, M. Bilotta, E. Fornara, F. (2004) Che cos'è la psicologia architettonica, Carocci editore, Roma
} 


\section{Elena Bellini}

ultima, la capacità di controllo sull'ambiente, al fine di regolare regolare l'intensità, la varietà e la diversità degli stimoli sensoriali presenti nel contesto (Bonaiuto, Bilotta, Fornara, 2004 ${ }^{464}$; Del Nord, 2006 ${ }^{465}$; Ulrich et al., 2004 ${ }^{466}$; Weinberg, Creed, $2000^{467}$ ).

Affrontare il percorso di cura in una struttura ospedaliera per una persona con disabilità, in particolare se cognitiva o con disturbi dello spettro autistico, può rappresentare una vera e propria sfida, in particolare in caso di attesa in un Pronto Soccorso, esami invasivi, o degenza in ospedale. Le particolari sensibilità sensoriali, le comorbidità, l'assunzione di terapie farmacologiche complesse, le difficoltà di comunicazione, l'assenza di autonomia e la conseguente necessità di cure e attenzioni costanti, rendono estremamente fragili questi pazienti e aumentano il rischio comunque connesso a procedure diagnostiche e soprattutto operatorie (Spes contra Spem, 2013) ${ }^{468}$.

Le comorbidità mediche e psicologiche hanno un peso importante nel disagio che può presentarsi per persone con disturbi dello spettro autistico, sono spesso associati: epilessia (stimata tra $11^{\prime} 11 \%$ ed il 39\%, Scarpinato et al. ${ }^{469} ; 29 \%$ secondo Gurney et al.22), disturbi del sonno (stimati tra il 44 e 1'83\%), disturbi gastrointestinali (stimati del $24 \%$, tra cui dolori cronici addominali, diarrea cronica, la più comune, stimata del 12\%23), selettività alimentare, allergie o celiachie, ritardo mentale, disabilità cognitiva e disturbi psichiatrici (26\%) come ansia, ADHD e depressione, oltre a comportamenti problema, aggressività o condizioni di autolesionismo $(32 \%)$, generati dall'ansia e dallo stress e spesso indotti dal contesto ambientale (Scarpinato et al., 2010 ${ }^{470}$; Iannuzzi et al., 2015 ${ }^{471}$; Vohra et al., 2016 ${ }^{472}$; Zwaigenbaum, $2016^{473}$; Nicholas et al., 2016 ${ }^{474}$; Gurney et al., $2006^{475}$ ). Casi di autismo spesso pre-

\footnotetext{
${ }^{464}$ Bonaiuto, M. Bilotta, E. Fornara, F. (2004) Che cos'è la psicologia architettonica, Carocci editore, Roma

${ }^{465}$ Del Nord, R. (2006) Lo stress ambientale nel progetto dell'ospedale pediatrico. Indirizzi tecnici e suggestioni architettoniche, Motta Architettura, Milano

${ }_{466}$ Ulrich, R. Zimring, C. Xiaobo, Q. Anjali, J. Choudhary, R (2004) The role of the physical environment in the hospital of the 21th century: a once-in-a-lifetime opportunity, Report to The Center for Health Design for the Designing the 21st Century Hospital Project, Concord (USA)

${ }^{467}$ Weingerg, A. Creed, F. (2000) Stress and psychiatric disorder in health care professionals and hospital staff, in "The Lacet", 355, February 12, pp.533-537

468 Spes contra Spem (2013) Carta Dei Diritti Delle Persone Con Disabilità In Ospedale, Roma

469 Scarpinato, N. et al. (2010) Caring for the Child With an Autism Spectrum Disorder in the Acute Care Setting, Journal for specialists in Pediatric Nursing, Wiley Periodicals, Inc.

470 ibidem

${ }^{471}$ Iannuzzi, D. et al. (2015) Brief Report: Emergency Department Utilization by Individuals with Autism, Journal of Autism and Development Disorders 45:1096-1102

${ }_{472}$ Vohra, R. (2016) Emergency Department Use Among Adults with Autism Spectrum Disorders (ASD), Journal of Autism and Development Disorders, 46:1441-1454

${ }^{473}$ Zwaigenbaum, L. et al. (2016) Perspectives of Health Care Providers Regarding Emergency Department Care of Children and Youth with Autism Spectrum Disorder, Journal of Autism and Development Disorders, 46:1725-1736

${ }^{474}$ Nicholas, D.B. Zwaigenbaum, L. Muskat, B. et al. (2016) Toward Practice Advancement in Emergency Care for Children With Autism Spectrum Disorder, Pediatrics, 137(S2): S206-S211
} 
sentano inoltre la sindrome di Rett, la sindrome dell'X fragile e la sclerosi tuberosa (Gurney et al., 2006) ${ }^{476}$. Infine si associano ai disturbi dello spettro autistico anche fattori di ipersensibilità uditiva, visiva, tattile o di altro tipo che non rendono il paziente favorevole rispetto alle cure, agli esami e all'ambiente caotico e non familiare del Pronto Soccorso, causando o intensificando i comportamenti disadattivi (Iannuzzi et al., 2015) ${ }^{477}$.

Considerando le difficoltà di un paziente con autismo nell'affrontare un luogo sconosciuto, con diverse persone non familiari con cui dover interagire, sovraccarico uditivo e visivo, avere un ambiente di "privacy" all'interno della propria stanza di degenza, per sé e la propria famiglia, ed essere preparati sulle persone che vi dovranno accedere e gli eventuali interventi da effettuare è sicuramente utile a limitare i livelli di stress e ansia. Una strategia utile può essere anche visitare gli ambienti dove si effettueranno gli interventi, o comprendere le procedure mediche o diagnostiche nel caso di interventi o esami a cui sottoporsi, per cui vi sono vari esempi oggi di video modeling. Uno spazio protetto, isolato acusticamente, dove gli stimoli sensoriali siano ridotti al minimo è molto più confortevole per un bambino con DSA, rispetto all'accedere ad una stanza giochi, dove invece la maggior parte dei bambini riesce a distrarsi e rilassarsi durante la degenza (Scarpinato et al., 2010) ${ }^{478}$. Le persone con autismo possono rilevarsi molto sensibili a luci, rumori e odori delle cliniche mediche; potrebbero inoltre sentirsi molto a disagio nell'essere toccati da altre persone o dagli strumenti sanitari (Gurney et al., 2006) ${ }^{479}$. In generale un ambiente dagli stimoli contenuti, con una particolare attenzione all'acustica e agli stimoli visivi e alla qualità luminosa, riduce lo stress e migliora l'attenzione e la focalizzazione, anche rispetto alle istruzioni degli operatori. La comunicazione è un altro aspetto di criticità, poiché circa il 30\%-50\% delle persone con autismo ha carenza o mancanza di linguaggio funzionale (abilità di trasmettere un messaggio agli altri o comprendere il linguaggio simbolico o rappresentativo) e talvolta anche rispetto al linguaggio non verbale, mancanza di contatto visivo o incapacità di comprensione delle espressioni facciali. Sono stati studiati diversi sistemi di comunicazione aumentativa o alternativa, come le PECS, sia in modalità cartacea che digitale, attraverso dispositivi e programmi digitali (Scarpinato et al., 2010) ${ }^{480}$. In ogni caso il personale infermieristico necessita di una formazione specializzata per favorire l'approccio e le relazioni con il paziente ed ovviamente un continuo supporto da parte di familiari o caregivers che meglio possono essere in grado di interpretare le reazioni ed i segnali tra-

\footnotetext{
${ }^{475}$ Gurney, J. G. et al. (2006) Parental Report of Health Conditions and Health Care Use Among Children With and Without Autism, National Survey of Children's Health, Arch Pediatr Adolesc Med, $160: 825-830$

${ }^{476}$ ibidem

${ }^{477}$ Iannuzzi, D. et al. (2015) Brief Report: Emergency Department Utilization by Individuals with Autism, Journal of Autism and Development Disorders 45:1096-1102

${ }^{478}$ Scarpinato, N. et al. (2010) Caring for the Child With an Autism Spectrum Disorder in the Acute Care Setting, Journal for specialists in Pediatric Nursing, Wiley Periodicals, Inc.

${ }^{479}$ Gurney, J. G. et al. (2006) Parental Report of Health Conditions and Health Care Use Among Children With and Without Autism, National Survey of Children's Health, Arch Pediatr Adolesc Med, 160:825-830

${ }^{480}$ Scarpinato, N. et al. (2010) Caring for the Child With an Autism Spectrum Disorder in the Acute Care Setting, Journal for specialists in Pediatric Nursing, Wiley Periodicals, Inc.
} 


\section{Elena Bellini}

smetti dalle persone con autismo. Questo aspetto è di particolare importanza soprattutto per l'osservazione dei comportamenti per comprendere dolore o sofferenza, che talvolta potrebbe non essere visibile; per questo motivo si consiglia, quando possibile, l'utilizzo di pomate e anestesie locali (Scarpinato et al., 2010) ${ }^{481}$, anche per evitare una frequente fobia verso gli aghi. Un'altra strategia per ridurre l'ansia e migliorare la collaborazione rispetto ai trattamenti può essere il mantenimento delle routines quotidiane, come ad esempio orari dei pasti o riproduzione di determinate attività, e fornire al paziente oggetti o riferimenti familiari (Scarpinato et al., 2010) ${ }^{482}$.

\subsubsection{Il Pronto Soccorso. Alcune risposte per l'area principale di emergenza}

Le barriere comunicative, le difficoltà di relazione, la sensibilità ai cambiamenti delle routines e alle stimolazioni tattili, uditive, visive o olfattive può interferire con gli interventi medici, la diagnosi e i trattamenti; in particolare all'interno delle terapie intensive, dove si devono poter ripetere esami e procedure mediche invasive (Zwaigenbaum et al., 2016) $^{483}$. Il Pronto Soccorso è sicuramente uno degli ambienti maggiormente critici sotto questo punto di vista, per il carico di tensione e pressione e l'intensità del carico sensoriale. Si consideri inoltre come il dipartimento di emergenza rappresenti una situazione critica e di stress anche per lo stesso personale, sia occupazionale che fisico, dovuto all'alto carico lavorativo e intensivo, all'insicurezza rispetto alle proprie capacità, ad un insufficiente controllo operativo, o alla relazione con pazienti e familiari, anche a livello psicologico (Del Nord, 2006) ${ }^{484}$.

Lo studio di Zwaigenbaum et al. si occupa di effettuare delle interviste al personale (medici e infermieri) del Pronto Soccorso per comprendere le difficoltà presentate durante le visite di pazienti con DSA. Le cause riportate di maggiore criticità sono sicuramente le difficoltà comunicative, l'assenza o la carenza di linguaggio verbale e l'aggressività, soprattutto nei bambini più grandi (adolescenti) poiché più difficili da gestire, anche evidentemente per la stazza fisica. Hanno descritto il Pronto Soccorso come un luogo difficile per questi pazienti, poiché pieno di stimoli sensoriali disturbanti, che possono causare sofferenza e stress, di una grande quantità di elementi non familiari simultanei che possono rivelarsi travolgenti, per non parlare delle diverse persone sconosciute che si rivolgono a loro durante le visite e le difficoltà rispetto alla sensibilità tattile, per effettuare esami ed interventi medici. La trasformazione dell'ambiente secondo gli aspetti sensoriali può migliorare molto l'accesso alla cura e il comfort del paziente, ma anche i tempi di attesa e la formazione del personale rappresentano dei requisiti importanti per la qualità della cura (Zwai-

\footnotetext{
481 Scarpinato, N. et al. (2010) Caring for the Child With an Autism Spectrum Disorder in the Acute Care Setting, Journal for specialists in Pediatric Nursing, Wiley Periodicals, Inc.

482 ibidem

483 Zwaigenbaum, L. et al. (2016) Perspectives of Health Care Providers Regarding Emergency Department Care of Children and Youth with Autism Spectrum Disorder, Journal of Autism and Development Disorders, 46:1725-1736

${ }^{484}$ Del Nord, R. (2006) Lo stress ambientale nel progetto dell'ospedale pediatrico. Indirizzi tecnici e suggestioni architettoniche, Motta Architettura, Milano
} 
Ambienti sensoriali "terapeutici" che rendano Abili

genbaum et al., 2016) ${ }^{485}$. Il coinvolgimento dei genitori è in questo senso fondamentale poiché rappresentano gli unici veri esperti e conoscitori dei propri figli, dei loro bisogni, delle loro particolari sensibilità e delle strategie utili a calmare il paziente nei momenti di ansia e stress. L'ambiente stesso deve essere calmante, attraverso il controllo delle stimolazioni sensoriali, minimizzando i fattori di stress per il bambino. Uno spazio privato e tranquillo può essere di aiuto, anche attraverso una stanza personale dedicata ai pazienti con DSA o servizi debitamente progettati per i bambini, che siano accoglienti e contribuiscano a metterli a loro agio. Oltre a mantenere uno stato di calma, anche nelle procedure mediche, può rilevarsi utile usare delle tecniche di distrazione come giochi, TV, videogames, musica o iPads, per occupare il bambino e alleviare l'ansia (Zwaigenbaum et al., 2016) ${ }^{486}$. In particolare il momento di maggiore stress si rivela quello della sala di attesa, dove si ha il maggior grado di imprevedibilità sui tempi e su ciò che accadrà, pertanto uno spazio dedicato al momento dell'attesa è sicuramente da considerarsi favorevole. Nelle sale d'attesa, così come nei corridoi e nelle sale dedicate ai trattamenti, gli aspetti di maggior disagio sono rappresentati dai livelli della luce, che dovrebbe essere regolabile, del rumore e degli odori. Controllare gli aspetti sensoriali permette anche di tollerare maggiormente i tempi di attesa (Zwaigenbaum et al., 2016) ${ }^{487}$.

Nicholas et al. si sono invece occupati di investigare la qualità di cura del Pronto Soccorso attraverso focus groups organizzati tra genitori di bambini con DSA ( $\mathrm{n}=$ 16), operatori del PS $(n=37)$ e direttori sanitari e amministrativi $(n=7)$, reclutati dal PS stesso in due sedi dell'Autism Speaks Autism Treatment Network (ATN): lo Stollery Children's Hospital, Edmonton e Hospital for Sick Children, Toronto. Le osservazioni rilevate sono state ancora una volta rivolte al momento dell'attesa, spesso lungo e stressante per gli utenti, ed il rapporto con il contesto e le persone non familiari con cui interagire. Si considerano favorevoli ambienti calmi e tranquilli, aree di break, elementi di stimolazione sensoriale, strategie di relax, segnalazioni visive e chiarezza per migliorare l'orientamento e la comprensione del contesto. Fondamentale la compartecipazione di familiari e operatori sanitari, anche in caso di persone con DSA adulte, da una parte per aiutare nella comprensione di un linguaggio non verbale, nelle strategie di abbattimento dello stress e nella terapia, dall'altra per la formazione che dovrà avvenire a doppio senso: per gli operatori si prevedono infatti delle sessioni educative su come comportarsi, per facilitare le relazioni con i pazienti; per le famiglie si prevedono invece dei programmi di preparazione all'ospedalizzazione, facendo comprendere in anticipo le procedure ed i trattamenti nei diversi passaggi a coloro che poi dovranno affrontarle (Nicholas et al., 2016) ${ }^{488}$.

Karen Pratt, una Clinical Nurse Specialist all'Evelina Children's Hospital (London, UK) ha messo a punto in collaborazione con la Guy's \& St Thomas' NHS

\footnotetext{
485 Zwaigenbaum, L. et al. (2016) Perspectives of Health Care Providers Regarding Emergency Department Care of Children and Youth with Autism Spectrum Disorder, Journal of Autism and Development Disorders, 46:1725-1736

${ }^{486}$ ibidem

${ }^{487}$ ibidem

${ }^{488}$ Nicholas, D.B. Zwaigenbaum, L. Muskat, B. et al. (2016) Toward Practice Advancement in Emergency Care for Children With Autism Spectrum Disorder, Pediatrics, 137(S2): S206-S211
} 


\section{Elena Bellini}

Foundation Trust e il King's College London, a seguito di interviste informali a operatori sanitari e familiari e focus group, una check-list strutturata come fase di preammissione per pianificare l'accesso ospedaliero ed i servizi terapeutici in caso di disturbi specifici dell'apprendimento e autismo. La checklist si propone quindi di definire un profilo del bambino che rispecchi le sue esigenze: una prima parte riguarda le informazioni sull'età anagrafica e funzionale; la seconda indaga le capacità comunicative del bambino, se presenta o meno linguaggio verbale, o quali metodi alternativi possano rivelarsi efficaci; un'altra parte fondamentale riguarda invece le sensibilità sensoriali del paziente ed i comportamenti problema che possono generare, che cosa può scatenarli e cosa eventualmente può al contrario placarli, anche basandosi sulle precedenti esperienze; definire infine eventuali esigenze specifiche del bambino, anche e soprattutto dal punto di vista terapeutico (es. come effettuare anestesia, o trattamenti medici..), al fine di personalizzare il piano di accesso ospedaliero e renderlo maggiormente efficace per entrambe le parti (Pratt et al., 2011) ${ }^{489}$. La sperimentazione della checklist è stata ben accolta sia dallo staff che dai familiari dimostrandosi molto efficace ed ha già posto in evidenza alcune prime esperienze di criticità, a cui si è cercato di trovare soluzione attraverso il piano terapeutico di accesso: avere la possibilità di arrivare in ospedale in auto e poter sostare durante la cura; avere tempi di attesa ridotti, anche sollevando problemi di richiesta di cibo, che non può essere dato ai bambini prima degli interventi medici, generando comportamenti problema; sopportare il rumore e la confusione, per cui si richiede di poter avere una piccola stanza dedicata con possibilità di attività di distrazione come TV o DVD; particolare sensibilità tattile, che non permette di fare neanche alcune rilevazioni semplici come la misurazione della temperatura e della pressione, soprattutto se da sconosciuti, per cui é richiesta un'anestesia; sensibilità ad alcuni materiali e difficoltà a restare spogliati, per cui lo staff propone di arrivare direttamente vestiti con indumenti $100 \%$ cotone al fine di poter conservare l'abbigliamento personale; difficoltà ad ingerire compresse, per cui lo staff medico dovrà valutare quali medicine sono necessarie, se si possono usare preparazioni liquide o ad esempio mescolarle con la limonata o bevande simili; valutare le strategie per accompagnare il bambino al reparto anestetico (se a piedi o in barella) e con quali metodi distrarlo (TV, DVD...); paura degli aghi, per cui risulta necessario usare un gas anestetico prima di effettuare analisi del sangue o altri esami; avere la possibilità di far risvegliare il bambino nello stesso spazio in cui si trovava prima di addormentarsi e con il genitore presente (Pratt et al., 2011) ${ }^{490}$.

489 Pratt, K. et al. (2011) Ensuring successful admission to hospital for young people with learning difficulties, autism and challenging behaviour: a continuous quality improvement and change management programme, Blackwell Publishing Ltd, Child: care, health and development, 38, 6, 789797

490 ibidem 


\subsubsection{Nuove frontiere nei contesti dell'emergenza sanitaria. Sensory design}

Un'ultima frontiera di innovazione in ambito della progettazione di ambienti sanitari autism-friendly è l'inserimento di ambienti multisensoriali come luoghi di attesa dedicata o ambienti di rilassamento e abbattimento dello stress e dell'ansia.

In generale, è stato dimostrato come l'inserimento di ambienti multisensoriali all'interno o nelle immediate vicinanze di reparti dove i pazienti devono essere sottoposti frequentemente a trattamenti e procedure invasive o comunque sgradevoli può essere di supporto, tranquillizzando il paziente e abbassando i livelli di ansia e di paura. E' stato ad esempio rilevato favorevole per bambini che dovevano frequentemente effettuare prelievi di sangue. Gli spazi di regolazione sensoriale permettono allo spazio di assumere connotati stimolanti e calmanti e si rivelano utili sia direttamente nella stanza dove vengono effettuate procedure cliniche, che in un ambiente dedicato, prima che i pazienti vengano sottoposti a procedure dolorose (come ad esempio le medicazioni per ustioni) o se spaventati poiché devono sottoporsi a interventi chirurgici, al fine di ridurne l'ansia (Del Nord, 2006) ${ }^{491}$.

Le persone con disabilità mentale presentano frequentemente ipo- o ipersensibilità agli stimoli sensoriali, che causa problemi nel regolare autonomamente $\mathrm{i}$ livelli di arousal (Bowman, Jones, 2016) ${ }^{492}$. Gli interventi sensoriali favoriscono il contenimento delle esperienze emozionali, in modo che si riducano i livelli di stress e quindi l'aggressività e i comportamenti adattivi (Sutton, 2013) ${ }^{493}$. Dato che gli ambienti sensoriali riescono a calmare gli utenti e preparare e favorire altri approcci terapeutici, questi interventi vengono spesso usati all'interno di reparti psichiatrici. Molti studi hanno dimostrato come gli ambienti sensoriali siano stati utili in risposta all'isolamento e la segregazione in casi psichiatrici acuti, presentando un abbattimento dello stress dell' $89 \%$ e una riduzione dell'isolamento pari al 54\% (Novak et al., 2012 ${ }^{494}$; Champagne, $2004^{495}$ ). L'intervento sensoriale può essere inoltre utile a distrarre e rifocalizzare l'attenzione e quindi favorire lo stato di calma, agendo sull'abbassamento dei livelli di attivazione/arousal e su una regolazione dell'emozionalità. Gli ambienti sensoriali sono stati utilizzati inoltre in casi di trauma (anche psicologico a seguito di violenza) e riabilitazione, ovvero in situazioni che hanno avuto impatto sulla percezione emozionale della persona, la sensibilità sensoriale, il comportamento e le relazioni e dove l'apporto sensoriale può essere utile nel ricreare

\footnotetext{
491 Del Nord, R. (2006) Lo stress ambientale nel progetto dell'ospedale pediatrico. Indirizzi tecnici e suggestioni architettoniche, Motta Architettura, Milano

492 Bowman, S. Jones, R. (2016) Sensory Interventions for Psychiatric Crisis in Emergency Departments-A New Paradigm, Journal of Psychiatry and Mental Health, 1(1)

${ }_{493}$ Sutton, D. et al. (2013) Optimizing arousal to manage aggression: A pilot study of sensory modulation, International Journal of Mental Health Nursing (2013) 22, 500-511

494 Novak, T. et al. (2012) Pilot study of a sensory room in an acute inpatient psychiatric unit, Australasian Psychiatry Vol 20, Issue 5, pp. 401-406

${ }^{495}$ Champagne, T. (2004) Sensory Approaches in Inpatient Psychiatric Settings. Innovative Alternatives to Seclusion \& Restraint, Journal of Psychological Nursing, VOL. 42, NO. 9
} 


\section{Elena Bellini}

un senso di controllo personale, sicurezza, stabilità e calma (Champagne, 2004 ${ }^{496}$; Sutton, $2013^{497}$; Bowman, Jones, $2016^{498}$ ).

Gli interventi sensoriali si basano su due tipologie di stimolo: quelli esterni derivano dal rapporto degli organi di vista, tatto, olfatto, udito e gusto con il mondo esterno, dando informazioni sul contesto circostante e sulla propria sicurezza; i sensi somatici, come la pressione sulla pelle, la consapevolezza del proprio corpo (propriocezione), dello spazio e dell'equilibrio (vestibolare), comunicano invece un senso di sicurezza interno. I sensi somatici, in particolare, possono favorire la calma. Si definiscono stimolazioni utili a ridurre l'ansia e lo stress: suoni rilassanti o una musica classica, luci colorate (da guardare e regolare), pesci o bolle d'aria che si muovono nell'acqua, oggetti di manipolazione, massaggi attraverso sedie dedicate, o una pressione profonda sul proprio corpo prodotta da elementi come, ad esempio, coperte molto pesanti (Sutton, 2013 ${ }^{499}$; Novak et al., 2012 $2^{500}$; Champagne, $2004^{501}$ ). Individui che hanno subito traumi, che hanno disturbi mentali o comportamenti problematici, sono spesso inconsapevoli dei loro bisogni sensoriali e delle risposte di stress e non riescono a ricalibrare in sensi attraverso quella "dieta sensoriale" che in diversi momenti della giornata ognuno di noi varia, anche in modo non cosciente, al fine di adattarsi all'ambiente e agli stimoli del contesto, per cui ambiente di questo tipo si rivelano particolarmente utili (Champagne, 2004) ${ }^{502}$.

Sutton et al. hanno effettuato un tentativo di intervento di modulazione sensoriale in Nuova Zelanda in tre unità psichiatriche per adulti e una per l'infanzia attraverso una sensory room equipaggiata con diversi elementi di stimolazione sensoriale come una sedia massaggiante, una sedia a dondolo, un pouf, una coperta con tessuto a pelo lungo, coperte molto pesanti, oggetti morbidi, oggetti o palle antistress, player audio portatili con suoni rilassanti, DVD con immagini video, diffusori di aromi, creme per le mani profumate e un sistema illuminotecnico regolabile nei colori. I risultati raccolti hanno dimostrato che gli interventi sensoriali hanno facilitato lo stato di calma, attraverso un ambiente protetto personale e dedicato dove ritirarsi, creare un senso di contenimento, controllare l'ambiente e trovare il proprio comfort, rilassandosi, ma anche favorendo l'orientamento e la consapevolezza del proprio corpo nel tempo e nello spazio. Questi ambienti hanno inoltre favorito le relazioni interpersonali, in particolare con gli operatori sanitari, e supportato l'autoconsapevo-

\footnotetext{
496 Champagne, T. (2004) Sensory Approaches in Inpatient Psychiatric Settings. Innovative Alternatives to Seclusion \& Restraint, Journal of Psychological Nursing, VOL. 42, NO. 9

497 Sutton, D. et al. (2013) Optimizing arousal to manage aggression: A pilot study of sensory modulation, International Journal of Mental Health Nursing (2013) 22, 500-511

498 Bowman, S. Jones, R. (2016) Sensory Interventions for Psychiatric Crisis in Emergency Departments-A New Paradigm, Journal of Psychiatry and Mental Health, 1(1)

499 Sutton, D. et al. (2013) Optimizing arousal to manage aggression: A pilot study of sensory modulation, International Journal of Mental Health Nursing (2013) 22, 500-511

${ }^{500}$ Novak, T. et al. (2012) Pilot study of a sensory room in an acute inpatient psychiatric unit, Australasian Psychiatry Vol 20, Issue 5, pp. 401-406

501 Champagne, T. (2004) Sensory Approaches in Inpatient Psychiatric Settings. Innovative Alternatives to Seclusion \& Restraint, Journal of Psychological Nursing, VOL. 42, NO. 9

502 ibidem
} 
lezza e l'autocontrollo degli utenti (Sutton, 2013) ${ }^{503}$. Si riportano alcuni feedback delle persone che hanno sperimentato l'utilizzo dello spazio sensoriale:

"For me, the most helpful was that opportunity the sensory room gave me to have a quiet, relaxing space on the ward . . . it's a safe space . . . The weighted blanket just gave me a sense of protection and safety".

"I really liked that feeling of being in the [massage] chair, so I got into there and it had that focus of actually centring me . . . I actually found, that sort of made me feel a lot calmer, but . . . you know, alive and not unconnected . . . shutting off senses that were too activated and not helpful".

"I stopped being racy for even just five minutes. I was actually still, you know, and centred and focused. Whereas usually, I'm not like that at all, and when I get unwell, it just magnifies twenty million times, so I'm never resting, you know, and I don't sleep. So, to even be centred for five minutes is a really big deal".

"I wanted the sound of the beach, of the waves, so I had that. And the smell of the hand cream reminded me of sort of sun block . . . and so I was taken back in time to good childhood memories, felt like I was at the beach, tension was relieved and [I was] at peace".

"I found it really good sort of to be in control of the environment. Like, the sound ... . the lighting, it was just being in control of things around you, kind of helps to calm you down" (Sutton, 2013) ${ }^{504}$.

Il Pronto Soccorso rappresenta un'area di emergenza sensoriale, dove le condizioni ambientali sono molto sfavorevoli, in quanto sono spesso presenti molti rumori, anche forti, odori spiacevoli e luci molto intense che possono aumentare il rischio di agitazione e comportamenti aggressivi scaturiti dall'innalzamento dell'arousal fisiologico. Trasformare gli aspetti sensoriali dell'ambiente fisico può rivelarsi uno strumento utile per ridurre l'agitazione e lo stress, soprattutto per quanto riguarda l'accesso delle persone con disabilità mentale. Questo può avvenire trasformando gli spazi di attesa e triage, anche con l'inclusione di sistemi sensoriali come sensory box mobili contenenti elementi auto-calmanti o vere e proprie stanze multisensoriali, senza prescindere, in ogni caso, dalla formazione del personale (Bowman, Jones, $2016)^{505}$.

L'analisi dei casi studio in questo ambito sarà fondamentale per la definizione di un campo ancora molto poco indagato e basato come letteratura più sugli oggetti e gli interventi di stimolazione sensoriale, piuttosto che nella definizione di ambienti sensoriali e di criteri di progettazione sistematica di questi spazi. L'apporto dell'assistive technology e della virtual reality saranno inoltre ambiti di indagine utili nella proposizione di nuove soluzioni legate a nuove frontiere di contesti ambientali, di sicuro interesse per gli sviluppi futuri di ricerca.

\footnotetext{
503 Sutton, D. et al. (2013) Optimizing arousal to manage aggression: A pilot study of sensory modulation, International Journal of Mental Health Nursing (2013) 22, 500-511

504 ibidem

505 Bowman, S. Jones, R. (2016) Sensory Interventions for Psychiatric Crisis in Emergency Departments-A New Paradigm, Journal of Psychiatry and Mental Health, 1(1)
} 


\title{
Capitolo 3
}

\author{
Metodologie d'indagine. Persone "reali" si raccontano
}

\subsection{Introduzione}

Come visto nei precedenti capitoli comprendere l'autismo vuol dire entrare in empatia e provare a fare esperienza del mondo circostante con un sistema sensoriale e percettivo diverso. Anche se probabilmente sarà difficile per una persona neurotipica comprendere fino in fondo il punto di vista di una persona con DSA, come visto anche negli studi del double empathy problem ${ }^{506}$ di Milton, l'unico modo per poter cercare di delineare un quadro esigenziale completo ed adeguato e quindi trovare soluzioni di progetto in risposta alle reali esigenze delle persone con autismo è conoscere e coinvolgere le persone che affrontano queste problematiche ogni giorno e sfruttare le strategie di partecipazione e co-design, al fine di includere la loro "voce". Sono state sperimentate quindi diverse metodologie di indagine per entrare in contatto con il "mondo autistico": una revisione della letteratura autobiografica o biografica; la strutturazione di questionari sulla base delle informazioni rilevate nello studio dello stato dell'arte, anche attraverso il supporto della Fondazione Bambini e Autismo di Pordenone, che ha dato la possibilità di testare lo strumento su alcune famiglie del loro centro, al fine di mettere a punto la chiarezza e la comprensione delle domande, la facilità di risposta in modo autonomo e l'efficacia delle risposte stesse; la diffusione dei questionari sul territorio italiano via web o cartaceo, attraverso i contatti stabiliti con le Associazioni di familiari per l'Autismo e Asperger e ai centri di terapia, per un totale di 61 persone tra i 3 e i 55 anni (di cui 10 donne); la strutturazione di interviste di approfondimento di alcuni aspetti valutati interessanti all'interno del questionario, attraverso interviste a 8 persone con autismo ad alto funzionamento o Asperger tra i 13 e i 55 anni, e 12 familiari di persone a basso funzionamento tra i 4 e i 18 anni, non in grado di comunicare personalmente, presso la Fondazione Bambini e Autismo di Pordenone e ANGSA Novara-Vercelli; colloqui e focus group con operatori, caregivers ed esperti del settore.

"Non esistono due persone con autismo fatte alla stessa maniera: la sua forma o espressione precisa è diversa in ogni singolo caso. L'interazione fra tratti autistici e altre qualità dell'individuo può essere assai complessa (e potenzialmente creativa). Quindi, per una diagnosi clinica può bastare un'occhiata, ma se si opera di compren-

${ }^{506}$ Milton, E. M. D. (2012) The ontological status of autism: The 'double empathy problem' Disability and Society, 1-5, 2 
dere un individuo autistico non basterà nulla di meno che la sua biografia completa" (Oliver Sachs, cit. in Silberman, 2015 ${ }^{507}$ ).

Questa prima fase di analisi ha visto la raccolta di informazioni attraverso autobiografie (Grandin, 2006 $6^{508}$; Gerland, 2003 ${ }^{509}$; De Rosa, 2014 ${ }^{510}$ ), o biografie scritte da familiari, genitori e professionisti (Nicoletti, 2013-2014 ${ }^{511}$; Nobotom, $2005^{512}$; Sachs, 1995 ${ }^{513}$; Peete, 2010 ${ }^{514}$; Arduino, 2014 ${ }^{515}$; Mazzone, 2015 ${ }^{516}$; Silberman, $2015^{517}$ ), che riportano storie e racconti di vita quotidiana, descrizione della vita di tutti i giorni, degli ambienti di vita e delle necessità e i bisogni riscontrati, sia a livello terapeutico, che di abilitazione, che di integrazione nella comunità sociale.

\subsection{Testimonianze/Autobiografie e biografie}

\subsubsection{Autismo come diversità}

Che cos'è l'autismo? Cosa vuol dire essere neurotipici o essere diversi? Qual è la vera normalità? C'è chi pensa che essere autistico sia solo un modo di essere, una parte di ciò che è una persona, una diversità nell'elaborare le informazioni, nel comunicare e relazionarsi con gli altri. Chi invece pensa che l'autismo sia parte intrinseca di se stessi, da cui non vorrebbe mai separarsi poiché ha degli aspetti della propria personalità che altrimenti perderebbe. Chi invece pensa che l'autismo sia una vera e propria prigione, poiché non riesce a comunicare con gli altri. In ogni caso, l'autismo non è una malattia, non si può curare, è un'identità completamente diversa di cui essere orgogliosi, ma soprattutto da conoscere e far conoscere.

"Noi non abbiamo un malattia, e quindi non possiamo essere curati. Semplicemente, noi siamo così." (Notbohm, 2015 - p. 21)

"Se potessi schioccare le dita e non essere autistica, non lo farei. L'autismo è parte di ciò che sono ... Donna Williams afferma: 'L'autismo non è me. L'autismo è

\footnotetext{
${ }^{507}$ Silberman, S. (2015) Neuro Tribes. The legacy of Autism and the Future of Neurodiversity, AVERY, New York (ed. italiana 2016)

${ }^{508}$ Grandin, T. (2006) Thinking in Pictures: And Other Reports from My Life with Autism, Vintage Books,NY.

${ }^{509}$ Gerland, G. (2003) A real person: Life on the outside, London, England: Souvenir Press

${ }^{510}$ De Rosa, F. (2014) Quello che non ho mai detto. Io, il mio autismo e ciò in cui credo, San Paolo Edizioni, Alba (CN)

${ }^{511}$ Nicoletti, G. (2013) Una notte ho sognato che parlavi. Così ho imparato a fare il padre di mio figlio autistico, Mondadori, Milano; Nicoletti, G. (2014) Alla fine qualcosa ci inventeremo. Che ne sarà di mio figlio autistico quando non sarò più al suo fianco, Mondadori, Milano

${ }_{512}$ Notbohm, E. (2005) Ten Things Every Child with Autism Wishes You Knew, Arlington, TX: Future Horizons, Inc.

${ }^{513}$ Sachs, O. (1995) Un antropologo su Marte, Adelphi Edizioni, Milano

${ }^{514}$ Peete, H. R. Peete, R. E. (2010) My Brother Charlie

${ }^{515}$ Arduino, M. (2014) Il bambino che parlava con la luce. Quattro storie di autismo, Einaudi, Torino

${ }^{516}$ Mazzone, L. (2015) Un autistico in famiglia, Mondadori, Milano

${ }^{517}$ Silberman, S. (2015) NeuroTribes. The Legacy of Autism and the Future of Neurodiversity, AVERY, New York

${ }^{518}$ Notbohm, E. (2015) 10 cose che ogni bambino con autismo vorrebbe che tu sapessi, Edizioni Centro Studi Erickson, Trento (ed. italiana di Notbohm, E. (2012) Ten Things Every Child With Autism Wishes You Knew, Future Horizons Inc., Arlington)
} 


\section{Elena Bellini}

solo un problema di elaborazione delle informazioni che governa quello che sono'. Chi ha ragione? Io credo entrambe, perché ci troviamo in punti diversi dello spettro dell'autismo. Io non vorrei perdere la mia capacità di pensare visivamente. Ho trovato il mio posto nel grande continuum." (Grandin, $2001-$ p. 98$)^{519}$

"Il mio autismo è parte di ciò che sono, ma non è tutto ciò che sono. Tu sei una cosa sola? Oppure sei una persona con pensieri, sentimenti, preferenze, idee, talenti e sogni? Sei grasso (sovrappeso), miope (porti gli occhiali) o maldestro (poco coordinato)? Magari sono queste le prime cose che noto quando ti vedo, ma in realtà tu sei più di questo, no?" (Notbohm, $2015-$ p. 24) ${ }^{520}$

"Avveniva lo stesso anche nel caso dei B., la famiglia di autistici che avevo visitato in California; il figlio maggiore, come i genitori, aveva la sindrome di Asperger, mentre quello più piccolo era affetto da autismo classico. La prima vola che arrivai a casa loro l'atmosfera era talmente 'normale' che mi venne il dubbio di essere stato informato male, o di avere sbagliato indirizzo: in loro non c'era alcun segno evidente di 'autismo'. Solo dopo essermi accomodato mi accorsi della pedana elastica, sulla quale tutti i membri della famiglia, di tanto in tanto, amano saltare sbattendo le braccia; della ricca biblioteca di fantascienza; delle strane vignette attaccate alle pareti del bagno; delle istruzioni affisse in cucina (istruzioni per cucinare, apparecchiare la tavola e lavare i piatti), ridicolmente minuziose ed esplicite, a indicare come ogni operazione dovesse essere eseguita in modo preciso e stabilito in precedenza ... la signora B. disse che 'rasentava la normalità' ... 'noi conosciamo le regole e le convenzioni delle persone 'normali', ma non c'è alcun autentico sconfinamento nella normalità' ... 'Impari a scimmiottare il comportamento umano' intervenne il marito ... I B. avevano imparato a erigere una facciata normale, indispensabile se si pensa che avevano una professione, vivevano in un quartiere residenziale, guidavano l'auto, avevano un figlio iscritto a una scuola normale ... riconoscevano il proprio autismo proprio come al college, ciascuno dei due aveva riconosciuto l'autismo dell'altro e ne aveva tratto un tal senso di affinità e di piacere che il matrimonio era stato il coronamento inevitabile ... arrivano a credere che l'autismo vada visto come un modo di essere, una modalità o identità completamente diversa, che deve essere consapevole (e orgogliosa) di sé." (Sacks, 1995 - p. 368-370) (21 $^{521}$

"La letteratura che tratta di autistici è oggi vastissima ma, pur avendone uno in casa, non saprei dire se sono assimilabili a ogni altra forma di disabilità. A volte mi viene da pensare che siano individui nati orfani, indipendentemente dall'esistenza di chi li ha messi al mondo. L'autistico è un perenne estraneo, imprigionato tra gente a lui sconosciuta, inconoscibile, e dalla quale ha pochissime speranze di essere veramente capito. Noi ci affanniamo a portarli a una condizione che giudichiamo salutare, quando in realtà potrebbe essere che loro non abbiano alcuna malattia. Sono so-

\footnotetext{
${ }^{519}$ Grandin, T. (2001) Pensare in immagini e altre testimonianze della mia vita di autistica, Edizioni Centro Studi Erickson, Trento (ed. italiana di Grandin, T. (1995) Thinking in pictures and other reports from my life with autism, Vintage Books, New York)

${ }^{520}$ Notbohm, E. (2015) 10 cose che ogni bambino con autismo vorrebbe che tu sapessi, Edizioni Centro Studi Erickson, Trento (ed. italiana di Notbohm, E. (2012) Ten Things Every Child With Autism Wishes You Knew, Future Horizons Inc., Arlington)

${ }^{521}$ Sacks, O. (1995) Un antropologo su Marte, Adelphi, Milano
} 
lamente disinteressati a condividere i propri pensieri con noi. Ma è davvero importante capirsi? Me lo chiedo osservando mio figlio incartarsi in un 'comportamento problema'. Quando si sbatte su un divano vuol dire che prova disagio, non vede un senso in quello che sta accadendo intorno a lui ed entra in uno stato ansioso. Non pensa che, comunicando il malessere, forse qualcuno potrebbe aiutarlo a risolverlo." (Nicoletti, 2013 - p. 5) (22 $^{5}$

"Non sono convinto che l'autistico sia infelice, questo di lui si pensa solo perché non si pone un grosso problema di quello che faccia il resto del mondo e sorride poco. Io so che mio figlio è felice quando mi accarezza la nuca senza motivi apparenti. Me ne accorgo quando si stende sui cuscinoni e può guardarsi in pace tutti i video di Youtube che crede, senza il fratello che gli si mette accanto per imporgli quelli che piacciono a lui. Tommy è sereno quando sente affetto che non chiede verifica o risposta. Non mi sembra poco. Potessimo tutti sentirsi amati senza dovere necessariamente corrispondere, saremmo visceralmente appagati anche di una carezza." (Nicoletti, 2013 - p. 50) $)^{523}$

"Se il mondo potesse essere infilato sotto un touch screen, per Tommy non ci sarebbero difficoltà. Semplicemente sfiorandolo, potrebbe selezionare le sue priorità, proprio come ore fa, fulmineo, cercando i video della Pimpa o quelli dei Teletubbies, e cito i suoi più fulgidi punti di riferimento assoluti, anche se ha quattordici anni." (Nicoletti, 2013 - p. 54) $)^{524}$

"La normalità è solo la media tra le persone che abbiamo conosciuto. L'idea di normalità che abbiamo in mente non è un concetto ma un nostro limite, soprattutto se esclude qualcuno ... Prendiamo per esempio la mia diversità. Se io e voi ci trovassimo nella stessa stanza, seduti attorno a un tavolo per un incontro, dopo un po' mi vedreste alzarmi, ridere, fare piccole corsette qua e là per la stanza e, con una mano stesa tra la mia bocca e il mio orecchio, raccontare storie tra me e me. E' solo un modo inoffensivo per gestire le mie emozioni, ma vi assicuro che la maggioranza di voi neurotipici entra in ansia per un comportamento ritenuto insolito, se non addirittura sconveniente. Pensate al disagio che provereste e poi chiedetevi se il problema sarebbe reale. Basta constatare che le persone autistiche corrono e parlottano a volte esattamente come $\mathrm{i}$ presbiti hanno bisogno di inforcare i propri occhiali se c'è qualcosa da leggere." (De Rosa, 2016 - p. 33-34) ${ }^{525}$

"All'assemblea della scuola elementare, degli adorabili primini si mettono in fila davanti al microfono per rispondere a turno alla domanda: 'che cosa vuoi diventare nel nuovo millennio?' 'un calciatore!' è la risposta più gettonata ... Bryce aveva va-

\footnotetext{
522 Nicoletti, G. (2013) Una notte ho sognato che parlavi. così ho imparato a fare il padre di mio figlio autistico, Mondadori, Milano

523 ibidem

524 ibidem

525 De Rosa, F. (2014) Quello che non ho mai detto. Io, il mio autismo e ciò in cui credo, Edizioni San Paolo, Cinisello Balsamo (Milano)
} 


\section{Elena Bellini}

lutato la domanda attentamente. 'Penso che vorrei soltanto diventare grande'." (Notbohm, $2015-$ p. 24$)^{526}$

"Ho bisogno che voi mi aiutate a uscire dalla mia prigione: l'autismo ... Vedi, io sono molto solo perché non riuscire a comunicare a voce è un grosso limite. Non riesco proprio a capire come fate voi non autistici a trovare nella vostra testa al volo tutte quelle parole così giuste e a dirle così velocemente ed anche con le espressioni del volto che completano ciò che volete comunicare ... Anch'io però so fare delle cose per voi difficili, come parlare e ascoltare allo stesso tempo o ascoltare e comprendere due persone che parlano contemporaneamente di cose diverse. In sintesi, la mia mente lavora in un modo diverso da quello degli altri e ciò mi mette in difficoltà." (De Rosa, 2016 - p. 94) (27 $^{527}$

"Quando al liceo hanno assegnato alla classe di Andrea il tema 'cosa significa secondo te avere successo nella vita' io subito pensato agli aspetti materiali ... ecco cosa ha scritto, facendomi sentire meschino per i miei pensieri: Il successo nella vita lo troviamo in ognuno di noi. Le persone di successo sanno realizzare i loro sogni e stanno bene con se stessi e con gli altri. Noi siamo unici e in questo troviamo un cammino diverso che ci costruiamo per raggiungere il nostro personale successo. Il nostro compito più difficile è capire cosa vogliamo per noi e non arrenderci agli ostacoli della vita. Fare il nostro sogno è fatica e lavoro che nel tempo ci porta soddisfazione. Oggi il mio grande obiettivo è farmi conoscere chi sono, io ragazzo autistico" (Antonello, 2015 - p.134-135) ${ }^{\mathbf{5 2 8}}$

\subsubsection{Entrare in empatia}

Come comunicare con gli altri e come gli altri ti vedono. Capire una persona con autismo è spesso molto difficile, talvolta forse impossibile; scrivere è spesso un mezzo utile per accedere alle loro emozioni e ai loro pensieri.

"Alcuni ritengono che le persone con autismo non abbiano emozioni. Io ne ho, eccome, ma sono più simili alle emozioni di un bambino che a quelle di un adulto ... Come da bambina, anche ora che sono adulta provo una lieta soddisfazione ... Con la pubertà la paura divenne la mia principale emozione ... Solo la minaccia di essere presa in giro bastava a mettermi paura; avevo paura di attraversare il parcheggio perché temevo che qualcuno mi avrebbe detto qualcosa di brutto ... Gli scritti di Tom McKean e di Therese Joliffe indicano che la paura è un'emozione dominante anche nel loro autismo ... Tony W., un uomo autistico, scrisse nel Journal of Autism Development Disorders che vive in un mondo di fantasie e di timore e che aveva paura di tutto ... Sean Barron racconta che, per i primi cinque o sei anni della sua vita, aveva provato puro terrore. La sua paura si ridusse in parte attraverso l'ambiente fortemente strutturato della sua classe, ma spesso aveva paura e ansia quando si

\footnotetext{
526 Notbohm, E. (2015) 10 cose che ogni bambino con autismo vorrebbe che tu sapessi, Edizioni Centro Studi Erickson, Trento (ed. italiana di Notbohm, E. (2012) Ten Things Every Child With Autism Wishes You Knew, Future Horizons Inc., Arlington)

527 De Rosa, F. (2014) Quello che non ho mai detto. Io, il mio autismo e ciò in cui credo, Edizioni San Paolo, Cinisello Balsamo (Milano)

${ }_{528}$ Antonello, F. Antonello, A. (2015) Sono graditi visi sorridenti, Feltrinelli, Milano
} 
trovava nei corridoi. La paura e l'ansia così intense che provavo una volta sono state quasi eliminate dal farmaco antidepressivo che assumo da tredici anni. La scomparsa, per gran parte, delle paure e degli attacchi di panico ha anche ridotto molte delle mie emozioni. L'emozione più forte che provo oggi è un sentimento di profonda calma e serenità quando lavoro con il bestiame e sento che gli animali, come me, sono rilassati. Questa emozione di grande pace e gioia non svanisce in fretta come le altre mie emozioni. E' come galleggiare sopra le nuvole. Ricevo una sensazione simile ma meno intensa quando uso la stringitrice ... Sono consapevole che mi perdo qualcosa quando gli altri rimangono incantati di fronte a un tramonto magnifico. Cognitivamente so che è bellissimo, ma non provo nulla ...Le mie emozioni sono più semplici di quelle della maggior parte delle persone. Non so cosa siano le emozioni complesse in una relazione umana. Io capisco solo le emozioni semplici, come la paura, la rabbia, la gioia e la tristezza." (Grandin, 2001 - p. 96-98) ) $^{529}$

"Donna Williams descrive le emozioni autistiche in questi termini: 'Io credo che l'autismo sia il risultato del funzionamento inadeguato di qualche tipo di meccanismo che governa le emozioni, tale per cui un corpo e una mente relativamente normali non sono in grado di esprimersi con al profondità di cui potrebbero altrimenti essere capaci." (Grandin, $2001-$ p. 98) ${ }^{530}$

"Kanner rilevò anche che le fissazioni di una persona autistica possono costituire il suo modo di sviluppare una qualche vita sociale e delle amicizie. Oggi, molte persone autistiche sono affascinate dai computer e diventano ottimi programmatori ... Per queste persone Internet è una risorsa straordinaria. Le difficoltà che le persone autistiche hanno con il contatto oculare e le loro movenze goffe on sono visibili quando si comunica attraverso la rete e i messaggi digitati sulla tastiera evitano molti dei problemi sociali che si pongono quando il contatto è faccia a faccia." (Grandin, $2001-$ p. 109) $)^{531}$

"I bambini raccontarono il loro amico come nessun adulto avrebbe potuto fare. $\mathrm{Ne}$ uscì il ritratto di un compagno che 'non sapeva ancora parlare ma poteva dirti tante cose'; 'che se giochi a memory è meglio essere nella stessa squadra perché vince sempre lui'; 'che deve essere sempre tutto in ordine altrimenti si arrabbia, proprio come il mio papà', e che 'non so come fa, ma sa leggere anche se non parla' Vedendolo, capimmo che c'erano tante cose che non conoscevamo di Silvio, e ci rendemmo conto che ciò che noi di solito dicevamo di lui non era quello che i suoi coetanei ritenevano importante." (Arduino, $2014-$ p. 72) (32 $^{532}$

"Ora mi rendo conto che ho sempre parlato con Tommy, non c'è mai stata una volta che lui non riuscisse a farsi capire da me o che io non riuscissi a comunicare con lui. com'è stato possibile con una persona che pronuncia solo monosillabi, parole smozzicate e deformi, al massimo due per volta, mai più di due? La verità è che

\footnotetext{
${ }^{529}$ Grandin, T. (2001) Pensare in immagini e altre testimonianze della mia vita di autistica, Edizioni Centro Studi Erickson, Trento (ed. italiana di Grandin, T. (1995) Thinking in pictures and other reports from my life with autism, Vintage Books, New York)

530 ibidem

531 ibidem

${ }^{532}$ Arduino, M. (2014) Il bambino che parlava con la luce. Quattro storie di autismo, Giulio Einaudi editore, Torino
} 


\section{Elena Bellini}

con poche persone al mondo mi capisco senza fraintendimenti come con lui. Il flusso informativo con l'autistico, una volta assimilato il codice d'interscambio, è inequivocabile. Questo potrebbe contraddire ogni racconto sulle difficoltà di comprendere ed essere compresi da loro. Non sono d'accordo, l'autistico non ha segreti se si parte da un presupposto essenziale: la sua realtà non ha complessità." (Nicoletti, 2013 - p. $72-73)^{533}$

\subsubsection{Ricevere la diagnosi}

Cosa vuol dire ricevere una diagnosi di autismo? Talvolta risulta come un'etichetta, ma talvolta può essere l'accesso a cure e terapie che possono migliorare la qualità della vita, e prima si possono iniziare prima può aumentare il benessere della persona e le possibilità di acquisire abilità e autonomie per un futuro migliore. Una diagnosi di autismo non è semplice da accettare per dei genitori e una grande fonte di stress, soprattutto poiché non si può associare ad un causa oggettiva.

"All'epoca mostravo i sintomi dell'autismo classico: non parlavo, stabilivo scarso contatto oculare, avevo accessi di rabbia, sembravo sorda, non manifestavo interesse per le persone e stavo sempre con lo sguardo fisso nel vuoto. Fui portata da un neurologo e, quando un test dell'udito evidenziò che non ero sorda, mi fu assegnata l'etichetta di 'celebrolesa'. Quarant'anni fa, la maggior parte dei medici non aveva mai sentito parlare di autismo. Pochi anni più tardi, quando questa conoscenza iniziò a diffondersi tra i medici mi fu applicata quell'etichetta. Ricordo bene la frustrazione, all'età di tre anni, di non riuscire a parlare. Questo fu la causa di molti scoppi di rabbia. Capivo quello che gli altri mi dicevano, ma non riuscivo a tirar fuori le parole ... Avevo scoppi di rabbia anche quando mi stancavo o ero disturbata da un rumore eccessivo, come quello delle trombette alle feste di compleanno. Il mio comportamento era come un interruttore automatico che scattava." (Grandin, 2001 - p. 49$50)^{534}$

"Durante la fase di ricerca, confrontammo la qualità della vita di circa sessanta genitori di bambini autistici e di altrettanti genitori di bambini con disabilità intellettiva o paralisi celebrale con quella di un gruppo di bambini a sviluppo tipico. Ebbene, scoprimmo che i genitori di bambini autistici erano i più stressati e in difficoltà, soprattutto quelli che avevano figli con autismo ad alto funzionamento ... Ipotizzammo che il maggiore stress rilevato potesse avere a che fare con l'assoluta mancanza di dati medici certi in grado di spiegare la condizione autistica, e di fornire quindi una causa oggettiva della disabilità ... Bambini belli, senza nessun indizio apparente di malattia ... Accanto a questo peso psicologico, esiste poi un oggettivo carico fisico che i genitori devono affrontare con la crescita del figlio ... La fatica fisica deriva dal dover gestire l'iperattività, contenere i momenti di agitazione, soste-

\footnotetext{
533 Nicoletti, G. (2013) Una notte ho sognato che parlavi. così ho imparato a fare il padre di mio figlio autistico, Mondadori, Milano

534 Grandin, T. (2001) Pensare in immagini e altre testimonianze della mia vita di autistica, Edizioni Centro Studi Erickson, Trento (ed. italiana di Grandin, T. (1995) Thinking in pictures and other reports from my life with autism, Vintage Books, New York)
} 
nere alcuni abbracci troppo energici dei figli maschi adolescenti, o più semplicemente dall'impossibilità di dormire per settimane o mesi." (Mazzone, $2015-$ p.59-60) ${ }^{535}$

"Ma come ho fatto a imparare a scrivere in buon neurotipichese, così che ho preso il diploma e voi mi capite? Il mio autismo è stato diagnosticato molto presto. Ho beneficiato da subito di ottime cure che, lungi dal voler guarire il mio autismo (sarebbe come cercare una cura per trasformare un cavallo in delfino), hanno fatto in modo che la mia forte chiusura non bloccasse le tappe del mio sviluppo mentale in età evolutiva ... Quanti autistici mentalmente perduti avrebbero potuto essere altri Federico se diagnosticati presto, ben supportati nell'età dello sviluppo e molto amati? Forse non molti, ma una buona parte sì. E poi, quanti bambini di due anni, in questo momento sono in bilico su un baratro di un autismo senza ritorno e potrebbero invece salvarsi e scrivere domani un libro? ..Grazie a chi mi ha diagnosticato, a chi mi ha curato, a chi mi ha amato faticando per integrarmi. Mi avete salvato la vita." (De Rosa, $2016-$ p.39) ${ }^{536}$

\subsubsection{Profilo cognitivo}

Un diverso modo di pensare, un diverso modo di apprendere, un diverso modo di vivere.

"Io penso in immagini. Le parole sono come una seconda lingua per me. Io traduco le parole, sia pronunciate che scritte, in filmati a colori, completi di suono, che scorrono come una videocassetta nella mia mente. Quando qualcuno mi parla, traduco immediatamente le sue parole in immagini." (Grandin, $2001-$ p. 23$)^{537}$

"Quando ero bambina, e poi anche da adolescente, credevo che tutti pensassero in immagini. Non avevo idea del fatto che i miei processi di pensiero fossero diversi ... Tutte le soluzioni che ho trovato a problemi di progettazione sono scaturite dalle mie capacità di visualizzare e vedere il mondo in immagini ... Oggi tutti sono entusiasti dei nuovi sistemi di realtà virtuale ... Per me questi sistemi sono rudimentali animazioni ... Non ho bisogno di un sofisticato programma di grafica che produca simulazioni tridimensionali del progetto. Posso fare tutto questo meglio e più rapidamente nella mia testa." (Grandin, 2001 - p. 23-25) (338 $^{53}$

"Le parole più facili da imparare per un bambino autistico sono i sostantivi, perché sono direttamente connessi ad immagini ... I bambini con più basso funzionamento spesso apprendono meglio per associazione, ad esempio con l'aiuto di etichette scritte attaccati ad oggetti nel loro ambiente ... Le parole che definiscono concetti spaziali come 'sopra' e 'sotto' non avevano per me alcun significato finché non ebbi un'immagine visiva per fissarle nella mia memoria ... Visualizzo anche i verbi ... Gli avverbi spesso sollecitano immagini inappropriate - ad esempio, 'bruscamente' mi fa

\footnotetext{
535 Mazzone, L. (2015) Un autistico in famiglia, Mondadori, Milano

${ }^{536}$ De Rosa, F. (2014) Quello che non ho mai detto. Io, il mio autismo e ciò in cui credo, Edizioni San Paolo, Cinisello Balsamo (Milano)

537 Grandin, T. (2001) Pensare in immagini e altre testimonianze della mia vita di autistica, Edizioni Centro Studi Erickson, Trento (ed. italiana di Grandin, T. (1995) Thinking in pictures and other reports from my life with autism, Vintage Books, New York)

538 ibidem
} 


\section{Elena Bellini}

pensare alle bruschette - a meno che non siano accompagnati da un verbo che modifica la mia immagine visiva." (Grandin, 2001- p. 34-35) 339

"Molte persone sono assolutamente disorientate dai simboli autistici, ma per una persona autistica essi possono a volte costituire l'unica realtà tangibile o l'unica coscienza del mondo. "toast", per esempio, può significare "felice" se il bambino si é sentito felice mangiando un toast ... in un'occasione Ted mise gli abiti bagnati nell'armadio, dopo che il dispositivo di asciugatura della lavatrice si era rotto. Era semplicemente passato alla fase successiva della sequenza di lavaggio dei vestiti che aveva appreso meccanicamente. Non aveva senso comune." (Grandin, 2001 -p. $43)^{540}$

\subsubsection{Profilo senso-percettivo}

La grande sensibilità sensoriale delle persone con autismo ha un enorme impatto sul mondo di percepire l'ambiente circostante. Le descrizioni delle sensazioni provate, dal disagio, alla confusione, al dolore fisico, a ciò che al contrario provoca piacere e rilassamento, rappresentano una guida su come "guardare il mondo attorno" e vivere nel contesto circostante.

"Dondolarmi e girare su me stessa erano altri modi per escludere il mondo quando ero sovraccaricata da troppo rumore. Dondolare serviva a calmarmi. Era come prendere una droga che dà assuefazione: più lo facevo e più avevo voglia di farlo." (Grandin, 2001- p. 51)

"Quando la mia logopedista mi prendeva il mento e girava la faccia perché la guardassi, riusciva a tirarmi fuori con forza dal mio mondo privato; per altre persone autistiche, tuttavia, un contatto oculare forzato può indurre la reazione contraria, cioè il sovraccarico celebrale e la completa chiusura. Donna Williams, per esempio, autrice di Nobody nowhere, spiega come lei possa utilizzare soltanto un canale sensoriale alla volta ... Le sue descrizioni della confusione sensoriale forniscono informazioni importanti per comprendere la differenza tra autismo ad alto e basso funzionamento, che io definirei come continuuum di elaborazione sensoriale. A un'estremità del continuum c'è la persona con autismo di Kanner o di Asperger che ha problemi lievi di ipersensibilità sensoriale, mentre all'altra estremità dello spettro c'è la persona con basso funzionamento che percepisce, attraverso i canali sia visivo che uditivo, informazioni confuse e imprecise."(Grandin, 2001 - p. 59) ${ }^{542}$

"Il mondo della persona autistica non verbale è caotico e le crea confusione. E' possibile che un adulto con basso funzionamento che non ha il controllo sfinterico viva in un mondo sensoriale del tutto privo di ordine. E' probabile che questa persona non abbia alcuna consapevolezza dei suoi confini corporei e che gli stimoli visivi, uditivi e tattili siano tutti mescolati insieme. Deve essere qualcosa di simile a osser-

\footnotetext{
539 Grandin, T. (2001) Pensare in immagini e altre testimonianze della mia vita di autistica, Edizioni Centro Studi Erickson, Trento (ed. italiana di Grandin, T. (1995) Thinking in pictures and other reports from my life with autism, Vintage Books, New York)

540 ibidem

541 ibidem

542 ibidem
} 
vare il mondo attraverso un caleidoscopio e contemporaneamente cercare di ascoltare una stazione radio la cui trasmissione è disturbata dalle interferenze. Si aggiunga a questo un guasto nel comando per la regolazione del volume tale per cui il suono passa all'improvviso dal fragore assordante a un brusio impercettibile." (Grandin, $2001-$ p. 66-67) $)^{543}$

"Da sempre, fin da quando posso ricordare, ho odiato essere abbracciata. Volevo provare la sensazione positiva di essere abbracciata, ma era semplicemente troppo opprimente. Era come un'enorme ondata di stimolazioni che sommergeva tutto e io reagivo come un animale selvatico. L'essere toccata innescava una reazione di fuga. Faceva saltare il mio interruttore ... Io ero una di queste persone che cercano la pressione. Quando avevo sei anni i avvolgevo nelle coperte e mi mettevo sotto i cuscini del divano, perché la pressione che esercitavano era rilassante ... Dopo la visita al ranch di mia zia in Arizona, mi venne l'idea di costruire un apparecchio di questo genere, sul modello del passaggio per l'immobilizzazione degli animali che vidi lì per la prima volta ... alcuni giorni più tardi, dopo che ebbi avuto un forte attacco di panico, andai a infilarmi nel passaggio per l'immobilizzazione ... dopo cinque secondi avvertii un'ondata di rilassamento; rimasi nel passaggio per circa mezzora ... dopo, per circa un'ora, mi sentii molto tranquilla e serena. La mia ansia cronica era diminuita. Questa fu la prima volta che mi sentii veramente a mio agio nella mia pelle." (Grandin, 2001 - p. 69) (544 $^{5}$

"Quando ero piccola, per me erano un problema anche i rumori forti; spesso erano dolorosi come il trapano di un dentista che tocca un nervo. Mi facevano veramente male ... I rumori leggeri, ai quali la maggior parte delle persone riesce a non badare, mi distraevano. Quando ero all'università, il rumore dell'asciugacapelli della mia compagna di stanza era per me come quello di un jet in fase di decollo. Alcuni dei suoni più disturbanti per $i$ bambini autistici sono quelli acuti e striduli prodotti dai trapani elettrici, dia frullatori, dalle seghe e dagli aspirapolvere. Le persone con autismo fanno fatica a sopportare l'eco che si crea nelle palestree nei bagni delle scuole. I tipi specifici di suoni che creano disturbo variano da persona a persona. Un suono che a me provocava dolore potrebbe essere piacevole per un altro bambino con autismo ... I bambini con autismo spesso sembrano sordi. Reagiscono ad alcuni suoni ma non ad altri."(Grandin, $2001-$ p. 74) ${ }^{545}$

"Quando due persone parlano contemporaneamente, mi è difficile escludere la voce di una e ascoltare l'altra. I miei orecchi sono come microfoni che raccolgono con la stessa intensità tutti i suoni."(Grandin, 2001 - p. 75) (546 $^{5}$

“Alcune persone hanno gravi problemi di elaborazione visiva e la vista può essere il loro senso meno affidabile. Quando si trovano in luoghi che non conoscono, alcune persone non verbali con autismo si comportano come fossero cieche; altre ancora hanno il problema che, di quando in quando, la vista si eclissa completamen-

\footnotetext{
${ }^{543}$ Grandin, T. (2001) Pensare in immagini e altre testimonianze della mia vita di autistica, Edizioni Centro Studi Erickson, Trento (ed. italiana di Grandin, T. (1995) Thinking in pictures and other reports from my life with autism, Vintage Books, New York)

544 ibidem

545 ibidem

546 ibidem
} 


\section{Elena Bellini}

te e vedono o tutto bianco, come fossero sintonizzate su un canale televisivo vuoto, o tutto nero, come se fosse buio pesto. Varie persone autistiche con vista normale mi hanno detto di avere difficoltà di percezione della profondità e quindi problemi quando si tratta di fare cose come scendere le scale."(Grandin, 2001 - p. 80-81) ${ }^{547}$

"L'illuminazione fluorescente può provocare gravi problemi a molte persone autistiche, perché queste possono vedere uno sfarfallio a sessanta cicli. L'elettricità domestica si accende e si spenge sessanta volte al secondo e alcune persone autistiche riescono a vederlo."(Grandin, $2001-$ p. 82) ${ }^{548}$

"Nelle persone con gravi deficit di elaborazione sensoriale, la vista, l'udito e gli altri sensi si mescolano, soprattutto quando al persona é stanca o turbata."(Grandin, $2001-$ p. 83) $)^{549}$

"Per una persona autistica, la realtà é una massa interagente di eventi, persone, luoghi, suoni e immagini che crea grande confusione. Sembra che nulla abbia confini definiti, ordine o significato. Passo buona parte del mio tempo semplicemente a cercare di capire quale sia la logica che sta dietro alle cose. Routine prestabilite, orari precisi e particolari percorsi e rituali sono tutte cose che mi aiutano a trovare ordine in una vita intollerabilmente caotica.'(Grandin, $2001-$ p. 84) 550

"Immaginate di salire sulle montagne russe più adrenaliniche del mondo (e se non vi piacciono le montagne russe, l'esempio funziona ancora meglio). Disneyland è divertente per andare in vacanza, ma per quanto tempo riuscireste a svolgere le vostre normali occupazioni restando sempre seduti sull'ottovolante? Riuscireste a tenere una riunione, a insegnare a una classe, a cenare piacevolmente in compagnia, a scrivere la relazione e pulire la casa mentre dovete sopportare le vertigini, le urla degli altri occupanti, la spinta fortissima dell'aria, le goccioline inattese e i repentini cambi di direzione, la sensazione di avere i capelli in bocca e i moscerini fra i denti? Potrebbe essere divertente come brivido da provare una volta tanto, ma ammettetelo: dopo una corse di tre minuti avrete voglia di scendere. Per molti bambini con autismo, è una corsa senza cancelletto di uscita, una realtà costante e tutt'altro che divertente."(Notbohm, 2015 - p. 45-46) (51 $^{551}$

"La luce, che tanto lo attraeva, se era troppa lo infastidiva. Anche quando era artificiale, come quella bianca e fredda emessa dai neon che illuminavano il locale dove si svolgevano le sedute di psicomotricità. La terapista, su suggerimento della mamma aveva schermato le lunghe lampade con veli arancioni, e Silvio, dopo un'iniziale titubanza, era infine entrato nella stanza. Questo non fu il solo adattamento

547 Grandin, T. (2001) Pensare in immagini e altre testimonianze della mia vita di autistica, Edizioni Centro Studi Erickson, Trento (ed. italiana di Grandin, T. (1995) Thinking in pictures and other reports from my life with autism, Vintage Books, New York)

548 ibidem

549 ibidem

550 ibidem

551 Notbohm, E. (2015) 10 cose che ogni bambino con autismo vorrebbe che tu sapessi, Edizioni Centro Studi Erickson, Trento (ed. italiana di Notbohm, E. (2012) Ten Things Every Child With Autism Wishes You Knew, Future Horizons Inc., Arlington) 
necessario. Decidemmo di dividere l'ambiente in diverse zona, ciascuna con un significato ben preciso."(Arduino, $2014-$ p. 18) ${ }^{552}$

"Il sono era un altro dei problemi del piccolo. A volte si alzava in piena notte e cominciava ad accendere e spengere le luci della stanza che divideva con i fratelli. I tentativi di farlo riaddormentare, anche portandolo nel lettone, avevano il più delle volte come risultato la rabbia di Silvio, che manifestava il suo disappunto urlando e insisteva a voler schiacciare l'interruttore." (Arduino, 2014 - p. 21-22) ${ }^{553}$

"Nina tornò dalla visita al nido con una notizia importante. Il comune aveva approfittato del periodo di chiusura natalizia del nido per ritinteggiare le pareti del salone di ingresso, che da tempo avevano bisogno di una rinfrescata. erano anche state sostituite le vecchie tende stinte con altre dalle tonalità più vivaci e meno trasparenti. Dal punto di vista percettivo, l'ambiente non era più quello di prima. Visivamente erano cambiate cose essenziali: le pareti avevano un colore diverso e diversa anche la luce, perché le nuove tende non consentivano ai raggi del sole di attraversarle, proiettando le lame oblique di luce su cui si cullavano i granelli di polvere che tanto piacevano a Silvio ... Andrea aveva respinto la mamma che si era fatta la tinta ai capelli e aveva modificato il taglio. Giulio aveva rifiutato di indossare il grembiule nuovo per la scuola: era blu, mentre i precedenti erano sempre stati neri. Piero non voleva più entrare nella macchina del papà perché la targa era stata sostituita ... Insomma, molte reazioni inizialmente incomprensibili si erano poi rivelate conseguenze di cambiamenti sensoriali nell'ambiente di vita del bambino." (Arduino, 2014 - p. $26-27)^{554}$

"Forse fu anche per contrasto con questa atmosfera che rimanemmo così impressionati quando Sara venne ad aprirci e ci trovammo di fronte al buio. Le persiane erano chiuse e a mano a mano che ci inoltravamo dentro la casa, nella penombra, notammo piatti e bicchieri sparsi sul pavimento della cucina, vetri rotti e lampade rovesciate; le tende della sala erano in terra, c'erano riviste e cd sparsi ovunque; un cassetto era aperto e contro il televisore era appoggiato un rettangolo di legno, che più tardi avremmo capito essere l'anta di una credenza divelta della furia di Elia ... Gli domandai se potevamo aprire le persiane, ma lui rispose che voleva il buoi. Gli scrissi su un pezzo di carta che poteva andare in camera mentre noi aiutavamo la mamma a mettere a posto. Lesse ad alta voce e rimase fermo con il foglio in mano; dopo alcuni minuti si alzò e andò nella sua stanza, chiudendosi la porta alle spalle." (Arduino, $2014-$ p. 163) (555 $^{555}$

"Tommy è contento quando si trova in un ambiente che non gli crei problemi e che lo incuriosisca. Il suo tempo di tenuta di una curiosità è brevissimo, anche perché è difficile che sia curioso. Dovrebbe abitare un ambiente in continua mutazione, quasi un caleidoscopio di colori cangianti, una piazza girevole in mezzo a un cerchio di baracconi delle meraviglie. Ogni genitore di ragazzo autistico cova nel suo pro-

\footnotetext{
${ }^{552}$ Arduino, M. (2014) Il bambino che parlava con la luce. Quattro storie di autismo, Giulio Einaudi editore, Torino

553 ibidem

554 ibidem

${ }^{555}$ ibidem
} 


\section{Elena Bellini}

fondo l'idea del paese che non c'è, costruito su misura per suo figlio." (Nicoletti, 2013 - p. 143) $)^{556}$

"Tommy è capace di girare senza fermarsi anche per mezzora: tiene le gambe raccolte sulla seduta come in una posizione yoga e, spingendosi con un braccio dalla scrivania, comincia a girare velocissimo. Sembra che quel movimento gli dia grande relax ... Da solo ha trovato una tecnica che riesce a placare la sua ansia divorante ... Mi viene in mente la macchina degli abbracci che si era costruita Temple Grandin ... Tommy si è ingegnato a immaginare la sua macchina ansiofuga guardando suo padre al lavoro; le prime volte appena mi alzavo, qualunque cosa stesse facendo si sedeva al posto mio e girava un poco; poi questa abitudine è andata in crescendo sino alla folle giravolta, oggi immancabile tappa della sua quotidiana liturgia rituale per essere certo che il mondo non finirà. L'ho mostrato anche al mio amico neuropsichiatra, mentre girava. Lui allora mi ha chiesto di sedermi, girare per qualche secondo e alzarmi in piedi. Naturalmente stavo per cadere, mi girava la testa ed era quasi impossibile reggermi dritto. Il mio amico allora mi ha fatto notare che Tommy poteva girare per mezz'ora, poi si alzava e andava tranquillamente a prendere l'acqua in frigo in un'altra stanza come nulla fosse. Gli autistici hanno proprio un altro cervello: il loro punto d'equilibrio è decentrato rispetto al nostro, in tutti i sensi. Non immaginate come vorrei avere, anche solo per un attimo, il punto di vista "eccentrico" di Tommy e vedere il mondo con il suo colpo d'occhio. Capirei molto più di lui e, forse, capirei molto più pure delle realtà che vedo sempre e comunque con il banalissimo e stantio sguardo dal resto dell'umanità neurotipica." (Nicoletti, 2014 - p. 43$45)^{557}$

"Ho bisogno di non stressare troppo le mie fini capacità percettive, quindi odio gli ambienti rumorosi, con molte luci e mola gente che parla ... Per una passeggiata, quindi, meglio le atmosfere ovattate di un bosco che il caos di un centro commerciale. Datemi poi un input per volta, perché sono molto meno efficace di voi nel decodificare la vostra comunicazione. Posso capirvi ma comunicate piano e con frasi semplici. State tranquilli, poi, perché se siete in ansia per la mia presenza, io che ho l'ansia di non capire bene la situazione penserò che siete in agitazione perché sono inadeguato ... Spiegatemi pacatamente e lentamente dove andiamo, a fare cosa e dove. Per voi sarà ovvio ma per me no. E poi state in silenzio ogni tanto ... Essere diversi è bello e arricchisce." (De Rosa, 2016 - p.35) ${ }^{558}$

\subsubsection{Ambiente educativo}

L'ambiente più importante nella formazione e nello sviluppo di un bambino è l'ambiente educativo. Strutturare un ambiente adeguato, senza stimoli che possano creare un sovraccarico sensoriale, può migliorare l'attenzione e la concentrazione e

\footnotetext{
556 Nicoletti, G. (2013) Una notte ho sognato che parlavi. così ho imparato a fare il padre di mio figlio autistico, Mondadori, Milano

557 Nicoletti, G. (2014) Alla fine qualcosa ci inventeremo. Che ne sarà di mio figlio autistico quando non sarò più al suo fianco, Mondadori, Milano

${ }^{558}$ De Rosa, F. (2014) Quello che non ho mai detto. Io, il mio autismo e ciò in cui credo, Edizioni San Paolo, Cinisello Balsamo (Milano)
} 
quindi favorire l'apprendimento. L'inserimento in ambiente scolastico può essere fonte di molto stress e va preparato per tempo e con attenzione, oltre a valutare un piano educativo personalizzato e la strutturazione dell'ambiente in classe e delle attività da compiere, anche nell'ottica di integrazione con i compagni. Se in parte le difficoltà sensoriali migliorano nel tempo, anche grazie alla strutturazione del contesto, quelle relazionali diventano sempre più complesse, aumentando spesso la diversità rispetto ai coetanei nel crescere e nel confrontarsi.

"Circa la metà dei bambini piccoli con autismo reagiscono bene a programmi leggermente direttivi nei quali sono costantemente incoraggiati a guardare l'insegnante e interagire. Le pareti dell'aula dipinte a colori vivaci servivano per me a rendere divertente l'apprendimento, ma per un bambino con confusione sensoriale potrebbero essere controproducenti perché lo distraggono troppo" (Grandin, 2001 - p. $60)^{559}$

"Più questi bambini sono protetti da stimoli visivi e uditivi che li possono distrarre e più è probabile che il loro sistema nervoso disfunzionale riesca a percepire con accuratezza il linguaggio. Per aiutarli a sentire meglio, è importante che gli insegnanti evitino loro gli stimoli visivi che causerebbero un sovraccarico sensoriale. E' possibile che questi bambini stiano meglio in una stanza silenziosa e poco illuminata, senza luci al neon o decorazioni troppo colorate." (Grandin, 2001 -p. 61) (560 $^{560}$

"Entrando in una di queste scuole durante la ricreazione, ne avevo visto in giardino alcuni che andavano sull'altalena e giocavano a palla. Proprio come se fossero normali, pensai; ma quando mi avvicinai di più vidi che uno di loro si dondolava in modo ossessivo sull'altalena, descrivendo semicerchi terrificanti, spingendosi più in alto possibile; un altro si passava con monotonia una pallina da una mano all'altra; c'era quello che girava su una giostra senza smettere mai; un altro ancora continuava ad allineare i pezzi delle costruzioni senza costruire nulla ma formando file lunghissime. Erano tutti impegnati in attività solitarie e ripetitive ... All'interno, alcuni bambini quando non erano in classe si dondolavano avanti e indietro; altri sbattevano le mani o farfugliavano in modo incomprensibile. A volte mi disse un'insegnante, alcuni di loro avevano improvvisi attacchi di panico o di collera, con esplosioni di urla o agitazioni incontrollabili ... la maggior parte dei bambini sembrava fisicamente normale: era la loro lontananza, la loro inaccessibilità, a essere così misteriosa ... Senza un'istruzione speciale (che per molti era cominciata all'asilo o a casa) questi giovani autistici potevano rimanere profondamente isolati e inabili ... qualcuno di loro avrebbe mai raggiunto una vera autonomia? In altre parole, mi chiedevo se potessero imparare a usare in modo pragmatico i loro automatismi sociali per vivere nel mondo, e inoltre se potessero arrivare ad avere un'autentica vita interiore, seppure profondamente diversa, una vita interiore tipicamente autistica, nota o palesata solo a pochi altri." (Sacks, 1995 - p. 338-339) ${ }^{561}$

559 Grandin, T. (2001) Pensare in immagini e altre testimonianze della mia vita di autistica, Edizioni Centro Studi Erickson, Trento (ed. italiana di Grandin, T. (1995) Thinking in pictures and other reports from my life with autism, Vintage Books, New York)

560 ibidem

561 Sacks, O. (1995) Un antropologo su Marte, Adelphi, Milano 


\section{Elena Bellini}

"L'inserimento scolastico è una delle principali sfide che i genitori di un bambino autistico si trovano ad affrontare ... E' importante sottolineare che non esiste un vero e proprio approccio scolastico all'autismo, e ciò è dovuto alla notevole variabilità dei comportamenti, spesso unici e peculiari. Bisognerebbe, dunque, avvicinarsi a ciascun bambino in modo personalizzato e flessibile, esercitando sempre il pensiero critico." (Mazzone, 2015 - p.42) ${ }^{562}$

"L'ingresso alla scuola elementare rappresenta, comunque un momento traumatico per i bambini autistici e le loro famiglie, perché richiede un impegno diverso, in termini di apprendimento e di organizzazione dell'ambiente classe, che incide sulle modalità di frequenza e sulla percezione che il bambino ha dell'esperienza scolastica. Le richieste sociali aumentano, e non basta, come avveniva alla materna, che il bambino condivida con gli altri soltanto lo spazio fisico. Proprio durante le scuole elementari, infatti, iniziano le preoccupazioni dei genitori riguardo alle potenzialità cognitive e le capacità di apprendimento dei loro figli." (Mazzone, $2015-\mathrm{p} .44)^{563}$

"Alle scuole elementari e medie la ricreazione è un momento cruciale della mattinata, che i bambini autistici tendono a trascorrere totalmente isolati ... Intervenire modulando tali spazi potrebbe essere un obiettivo specifico del sostegno: per esempio, strutturare piccole attività di integrazione coinvolgendo altri membri della classe, inizialmente in piccoli gruppi, potrebbe facilitare il modo di vivere l'intervallo e diminuire l'ansia che tale momento sociale genera sempre nei bambini autistici." (Mazzone, $2015-$ p.46) ${ }^{564}$

"Non è vero che la maggior parte di loro è ritardata. Esistono semplicemente diversi modi di esprimere le potenzialità cognitive, e questo deve essere ben chiaro sia ai genitori sia alla scuola. Affrontare con serietà il percorso scolastico e il problema del sostegno è una delle battaglie più importanti che il sistema dell'istruzione dovrà ingaggiare nei prossimi anni. Infine, è doveroso dedicare un'attenzione specifica ai bambini autistici con un alto funzionamento cognitivo: la mancata comprensione delle loro peculiarità comportamentali e delle loro competenze, in alcuni ambiti didattici anche superiori alla media, può contribuire a generare significativi problemi di autostima o, addirittura, sintomi depressivi." (Mazzone, $2015-$ p.50) ${ }^{565}$

"Attraverso un'analisi retrospettiva capimmo che l'attesa dell'entrata in classe, il suono della campanella e la rumorosa confusione provocata dai compagni innervosivano tremendamente il bambino, che non comprendeva il perché di tanto vociare. Per di più, appena entrati in classe, il compagno di banco invadeva sistematicamente lo spazio di Nico, acuendo un'agitazione che lui non riusciva a smaltire prima di due o tre ore." (Mazzone, $2015-$ p.81) ${ }^{566}$

"Il salone e l'aula erano ambienti che contenevano molti stimoli, com'è giusto che sia in una scuola materna. Per Silvio però, sommerso dalla moltitudine di luci, colori, suoni, parole, odori e da altre sensazioni derivate dalla prossimità con i com-

\footnotetext{
562 Mazzone, L. (2015) Un autistico in famiglia, Mondadori, Milano

563 ibidem

564 ibidem

565 ibidem

566 ibidem
} 
pagni, questi erano perlopiù fonte di confusione e di malessere; se on riusciva a isolarli concentrandosi su attività visive a lui gradite, doveva allontanarsene. Da qualche tempo, inoltre, il bambino aveva accentuato l'abitudine a coprirsi le orecchie con le mani, soprattutto nel salone, e sul suo viso erano comparse smorfie che si accompagnavano alla contrazione dei muscoli e alla chiusura degli occhi. Se non poteva allontanarsi dal bombardamento di stimoli, cercava almeno di proteggersi e limitare i danni." (Arduino, 2014 - p. 37-38) ${ }^{567}$

"Se lo spazio ricco di stimoli del salone amplificava l'autismo di Silvio, quello essenziale, strutturato e poco affollato della stanza evidenziava invece le potenzialità del bambino, favorendone il benessere e la disponibilità al gioco e alla relazione, facendo di lui, cosa molto importante, un compagno con cui gli altri potevano divertirsi e stare bene. La scuola materna cominciò a essere modellata tenendo conto della presenza di Silvio. Ovvio che non si poteva stravolgere una situazione studiata per i bambini con 'sviluppo tipico', tuttavia c'erano aspetti dell'organizzazione dello spazio e attenzione nella proposizione delle attività che potevano e dovevano riflettere il modo di conoscere e di imparare di un soggetto autistico ... All'interno della classe furono delimitati alcuni spazi, ciascuno dei quali aveva una sua specifica funzione. Il banco di Silvio era all'estremità di una delle due file parallele, dal alto opposto rispetto alle finestre. Di fronte a lui sedevano Antonella e Alice, che gli proponevano attività da fare insieme ... Accanto alle finestre era stato invece delimitato, con alcuni scaffali, uno spazio rettangolare, grande a sufficienza per contenere almeno quattro bambini e un adulto, dove erano raccolte varie cose che potevano essere usate da Silvio nei momenti di gioco libero. La vicinanza con le finestre permetteva anche di giocare con la luce. Contro una delle pareti, lontano dalle finestre, erano disposti, a ferro di cavallo, tre banchi. Silvio doveva sedersi in quello centrale: alla sua sinistra venivano collocate le attività che doveva svolgere, a destra un contenitore dove riporre quelle terminate ... Il senso di tale organizzazione dello spazio di lavoro era quello di insegnare al bambino a svolgere alcuni compiti in modo autonomo, senza la presenza dell'insegnante, che era invece indispensabile quando venivano proposti esercizi nuovi o dovevano essere consolidate capacità non ancora acquisite in modo completo; allora Silvio doveva rimanere seduto al proprio banco, con i compagni a fianco e la maestra o l'assistente di fronte ... Lo spazio parlava e diceva a Silvio molto di più di quello che potevano dirgli le parole dell'insegnante. Ogni zona fu contrassegnata da una fotografia che Silvio attaccava su un supporto di velcro quando vi si recava. Per fargli sapere in anticipo cosa gli avrebbe proposto la maestra e dove, fu costruita una sorta di cabina di regia, tecnicamente definita 'agenda', che conteneva le immagini dello spazio di lavoro o di gioco previsto." (Arduino, 2014 - p. 40$43)^{568}$

"Marina aggiunse che l'aula di terza aveva un'esposizione diversa rispetto a quelli dei primi due anni, e che con il cambio dell'ore il sole non la colpiva più alla fine della mattina, quando i bambini stavano per uscire, ma arrivava prima. Silvio tra

\footnotetext{
${ }^{567}$ Arduino, M. (2014) Il bambino che parlava con la luce. Quattro storie di autismo, Giulio Einaudi editore, Torino

${ }^{568}$ ibidem
} 


\section{Elena Bellini}

l'altro, per la posizione del suo banco, era uno dei primi a essere illuminato. Se la spiegazione del disagio di Silvio era quella, spostando il banco o schermando la luce con delle tende il problema si sarebbe dovuto risolvere ... dopo una settimana Marina chiamò per confermare che tutto procedeva bene." (Arduino, $2014-$ p. 65-66) ${ }^{569}$

"L'istituto alberghiero frequentato da Silvio aveva maturato una lunga esperienza con ragazzi che presentavano disabilità, talvolta anche con autistici. Una delle caratteristiche che lo differenziavano dalla maggior parte delle altre scuole superiori, consisteva nel coinvolgimento reale di tutti gli insegnanti nella programmazione delle attività didattiche, mentre altrove lo studente con delle problematiche, soprattutto nei casi più gravi, veniva quasi sempre affidato per intero all'insegnante di sostegno ... Per prima cosa Silvio imparò a usare il pelapatate e a confezionare spiedini di carne e frutta, lavori semplici che avrebbe potuto eseguire anche a casa e che richiedevano un controllo limitato da parte dell'adulto. Era meticoloso fino all'eccesso, non lasciava mai nessun residuo di buccia né alcun segno di imperfezione ... La sua vera passione, però, erano gli spiedini: poteva farne decine e decine senza stancarsi, in modo autonomo. Mentre svolgeva questo compito teneva sul tavolo una guida visiva composta da una sequenza di immagini che gli indicavano quale pezzo di carne o frutta infilare. Silvio seguiva le istruzioni con scrupolo e, completato lo spiedino, lo riponeva in un ampio contenitore. Terminata la materia prima, restava a sedere in attesa che l'insegnante o un compagno gli proponessero qualcos'altro. Il secondo anno vennero aggiunte le attività in sala, che consistevano nel simulare il servizio di una portata. I piatti erano al massimo due, che Silvio prendeva in cucina. La consegna gli veniva data su un vassoio su cui era stato attaccato con il velcro un numero che indicava il tavolo." (Arduino, 2014 - p. 76-77) ${ }^{570}$

"Pensavo a quell'uomo che prende sei euro l'ora per tenere mio figlio per un braccio, mentre la cooperativa che ha il contratto con il Comune ne prende venti. Esattamente quanto costa a me un operatore specializzato che segue con Tommy un programma terapeutico mirato ed efficace, lui fra l'altro si diverte pure. Perché allora devo tollerare una persona senza alcuna formazione su come si tratti un autistico? Quanta gente deve campare grazie al 'problema' di mio figlio? Lo stesso vale per gli operatori che il Comune mi manda a casa come 'sostegno familiare'. Dopo un anno di domande, valutazioni, esami ecc., finalmente otteniamo sei ore a settimana suddivise in due giornate. Devo però scegliere tra una lista di cooperative che mi vengono indicate. Vorrei poter usare gli stessi operatori che pago privatamente, mi sembrerebbe la cosa più razionale... Invece no, devo far lavorare quelli che dicono loro ... se non ha competenze e referenze specifiche su oggetti autistici, mio figlio con lui non l'avrei lasciato solo nemmeno un minuto. almeno questo allora pensai, ma poi dovetti cedere per timore di perdere anche quel servizio e ho cominciato ad abbozzare sul viavai di persone estranee e sempre diverse che mi girano per casa. L'impressione che mi resta è che sia io infine a fornire una giustificazione all'esistenza di una cooperativa, piuttosto che questa fornisca a me un servizio veramente utile. Con mera-

\footnotetext{
${ }^{569}$ Arduino, M. (2014) Il bambino che parlava con la luce. Quattro storie di autismo, Giulio Einaudi editore, Torino

${ }^{570}$ ibidem
} 
viglia ho dovuto scoprire che, anche in questo campo, gli stranieri tamponano un lavoro che probabilmente gli italiani schifano. Peggio per loro, ma che almeno qualcuno si prenda la briga di formare queste persone!" (Nicoletti, 2013 - p. 58-59) ${ }^{571}$

"A scuola capivo e venivo poi interrogato tramite il mio computer portatile, a casa ero aiutato a studiare e cominciai a prendere buoni voti ... Il mio problema non era studiare con profitto, ma il come la scuola era organizzata per tempi e metodi, adatta forse ai miei compagni neurotipici, i quali comunque se ne lamentavano. Come fare a stare fermi e in silenzio per ore ad ascoltare una persona che parla? Difficile per i miei compagni, francamente impossibile per me. E che dire della tempesta emozionale generata quasi ogni ora dal cambiamento generale di tutto il contesto, insegnante, argomento, attività? ... La grande protagonista della mia integrazione, però, è stata la mia classe che é riuscita ad andare oltre i tratti più disorientanti e inquietanti del mio autismo e ha offerto disponibilità e impegno ... Sento tante storie tristi di autistici parcheggiati a scuola per cinque anni se non addirittura oggetto di scherno o di bullismo. chissà quanti di questi avrebbero potuto avere successo se fossero stati aiutati come lo sono stato io." (De Rosa, 2016 - p.69-71) ${ }^{572}$

"Signor giudice, mio figlio, minore e disabile, è stato picchiato nei corridoi della sua scuola, dal preside della sua scuola. Lui e un'intera classe sono rimasti traumatizzati da questo fatto, che in una società civile non si può non considerare di estrema gravità. Ma le istituzioni di questa stessa società civile hanno scelto il silenzio e l'indifferenza, e anche una condanna non varrebbe a compensare i ragazzi di quello che hanno passato, né a rendere meno vergognoso il comportamento di chi avrebbe dovuto prendersi cura di loro e della situazione in cui si trovavano. Ho chiesto la sua opinione in proposito e, sebbene sia affetto da autismo, è riuscito a comunicarmi che desidera lasciar cadere l'accusa. Signor giudice, prendo atto che mio figlio cosiddetto 'disabile' è più abile alla vita e alle relazioni umane di molte altre persone che ricoprono incarichi di prestigio e si responsabilità. Da cittadino, sono preoccupato e offeso. Da padre, sono orgoglioso e fiero. Ritiriamo la denuncia." (Antonello, 2015 p. $160-161)^{573}$

\subsubsection{La famiglia}

Spesso quando si pensa ad una persona autistica e alle sue esigenze, comprese quelle dell'adattamento domestico, spesso si fa l'errore di dimenticare della famiglia, i genitori e soprattutto i fratelli e le sorelle delle persone con autismo. Cosa succede nelle vite dei genitori quando nasce un bambino con autismo? Cosa prova un fratello o una sorella, come vive lo stress e le difficoltà familiari e tra i genitori?

"In un angolo, a osservare, ascoltare ed elaborare tutti i cambiamenti che gli accadono intorno, c'è spesso un altro componente della famiglia: il fratello o la sorella del bambino autistico. Nel caso di autistici a basso funzionamento, si tratta di solito

\footnotetext{
571 Nicoletti, G. (2013) Una notte ho sognato che parlavi. così ho imparato a fare il padre di mio figlio autistico, Mondadori, Milano

572 De Rosa, F. (2014) Quello che non ho mai detto. Io, il mio autismo e ciò in cui credo, Edizioni San Paolo, Cinisello Balsamo (Milano)

573 Antonello, F. Antonello, A. (2015) Sono graditi visi sorridenti, Feltrinelli, Milano
} 


\section{Elena Bellini}

di fratelli o sorelle maggiori ... Nel caso, invece, di un autismo ad alto funzionamento, spesso diagnosticato solo con il passare del tempo, magari quando il bambino è diventato un ragazzo, è possibile trovare nella famiglia anche sorelle o fratelli piccoli ... Il termine siblings nel linguaggio comune è oggi usato, ormai anche in italiano, per riferirsi ai fratelli/sorelle sani di una persona con disabilità ... LA ricerca scientifica, in passato, ha mostrato scarso interesse per i fratelli e le sorelle di bambini con autismo, mentre è ampiamente documentato l'impatto che la nascita di un figlio autistico ha sul benessere familiare, sulle dinamiche di coppia e sulla genesi dello stress genitoriale ... i fratelli e le sorelle di soggetti autistici subiscono le conseguenze non solo delle difficoltà del fratello autistico, ma anche di tutto ciò che vi ruota attorno, come i problemi tra i genitori, l'aumento di stress o l'insorgenza di depressione nelle madri o nei padri. Anche per questo motivo, sarebbe importante che in tutte le famiglie con un figlio autistico si costruisse uno spazio dedicato e pensato per gli altri fratelli." (Mazzone, 2015 - p.91-93) ${ }^{574}$

"Queste rigidità nel comportamento di Silvio erano motivo di forte di disagio per i suoi fratelli, che non volevano più andare insieme a lui in casa d'altri o in locali pubblici ... Luigi e Sandro spiegarono che per loro assistere a certe scenate in presenza di persone non di famiglia era fonte di ansia; il loro comune desiderio, in quelle situazioni, era di non esserci, di poter sparire come per magia. Maria e Gianni dissero che anche loro avevano provato le stesse emozioni ... Mi tornarono alla memoria alcuni casi in cui un genitore, quasi sempre il padre, aveva lasciato la famiglia per iniziare una nuova relazione che spesso si era poi rivelata un semplice pretesto per la fuga ... I sentimenti prevalenti, in loro, erano la vergogna, la rabbia e, soprattutto in Luigi, l'impotenza. In quanto fratello maggiore sentiva infatti la responsabilità di comportarsi da 'terzo genitore', ma soprattutto nelle situazioni anomale non sapeva come gestire le crisi del fratello." (Arduino, 2014 - p. 68-69) ${ }^{575}$

"Andrea, puoi dire qualcosa per tutti qui genitori che hanno un ragazzo con autismo che non riesce a comunicare? Ci sono genitori che non hanno mai avuto un solo pensiero dal loro figlio. 'ciao genitori belli di tutti sono graditi visi sorridenti"' (Antonello, $2015-$ p.198) $)^{576}$

\subsubsection{Acquisire nuove abilità, diventare adulti e pensare al dopo di noi}

Apprendere le abilità quotidiane è una delle cose più importanti per una persona con autismo, poiché può garantirgli una maggiore autonomia ed indipendenza nella vita futura. Cosa succede quando un ragazzo arriva ai 18 anni? Non esiste un'assistenza adeguata, non c'è più la scuola, ma bisogna iniziare ad esplorare nuove realtà come i centri diurni ed i centri per l'autismo. I genitori sognano un futuro migliore per i propri figli ed una soluzione efficace per il dopo di noi, per sapere che in qualche modo vivranno bene anche quando non si potranno più occupare di loro.

\footnotetext{
${ }^{574}$ Mazzone, L. (2015) Un autistico in famiglia, Mondadori, Milano

575 Arduino, M. (2014) Il bambino che parlava con la luce. Quattro storie di autismo, Giulio Einaudi editore, Torino

${ }^{576}$ Antonello, F. Antonello, A. (2015) Sono graditi visi sorridenti, Feltrinelli, Milano
} 
"Quando Maria e Gianni ci spiegarono che era necessario trovare qualcosa di molto motivante per convincere Silvio a sedersi sul water, Annette e io pensammo subito al View Master Super Show Projector: così si chiamava l'aggeggio. Luigi (il fratello di Silvio) si sedette sul water con in mano il proiettore. Davanti a sé aveva la parete bianca, su cui lui stesso aveva disegnato un riquadro che rappresentava uno schermo; quando la mamma lo aveva informato della nostra idea, lui aveva dimostrato molta sagacia obiettando che le immagini potevano essere viste su qualsiasi muro della casa, e che occorreva quindi spiegare al fratellino, in modo chiaro, che invece dovevano essere per forza proiettate su quello di fronte al water ... Come ci aspettavamo, all'inizio il bambino si interessò soltanto a quelle. Allora Luigi iniziò a spengere e riaccendere il proiettore, a intervalli di circa dieci secondo ... Trascorso qualche minuto Maria chiese a Luigi di alzarsi dal water, guidò Silvio a sedersi e gli porse il proiettore aiutandolo ad accenderlo e puntarlo contro al parete. Silvio quasi non se ne accorse, catturato com'era dalla parete magica: e si ritrovò seduto sulla tazza." (Arduino, 2014 - p. 34-35) ) $^{577}$

"Eppure ha ragione lei, e pian piano riesce a dimostrarmelo. Andrea non parla, ma sa leggere. Lisa si è accorta che reagisce non solo alle immagini, ma anche alle parole scritte sui cartelli, come uno che capisce cosa vogliono dire. Miracolo? Eh già, ma non parla. Comincio a capire meglio cos'è l'autismo: una ricetrasmittente che riceve tutto ma trasmette solo rumore bianco. Entra tutto: dettagli, concetti, ogni minimo messaggio. Esce poco o niente. e in questo stesso periodo scopriamo, con l'aiuto di Lisa, la scrittura facilitata. La scrittura facilitata non è prevista dei protocolli ministeriali. E mentirei se dicessi che all'inizio la considero una buona idea. Funziona così: il facilitatore, ovvero la persona addestrata a praticare questa tecnica, prende la mano del ragazzo e la guida sulla tastiera del computer per permettergli di rispondere alle domande che gli vengono poste, inizialmente cose semplici come: "di che colore è la maglietta?" e lui deve rispondere, una lettera alla volta, finché arriva a comporre la parola "gialla" o "blu". E con il tempo può arrivare a scrivere anche pensieri più complessi ... Vedere Andrea concentrato così facile e che è una delle tante cose che la vita gli ha trasformato in un inferno, mi riempie di commozione e di rabbia. " (Antonello, 2015 - p. 132-133)

"Finito il percorso con la neuropsichiatria infantile, al compimento dei 18 anni del figlio, i genitori iniziano a cercare una struttura per adulti a cui appoggiarsi, quantomeno per qualche certificato sanitario. L'esito di queste peregrinazioni, in Italia, è quasi sempre fallimentare. Quanto ai servizi territoriali di psichiatria, neanche a parlarne ... E' più o meno lo stesso per i centri diurni: se una famiglia volesse inserire un figlio autistico adulto in una comunità, lo vedrebbe trattato come uno schizofrenico o un ritardato mentale, e questa sarebbe la peggiore delle sfortune per il paziente. Ecco allora che l'unica, obbligata scelta resta quella di tenerlo in casa. Nei casi più fortunati, quando le energie economiche lo permettono, ingaggiando compagni adulti o rivolgendosi a cooperative, purtroppo non sempre specializzate. In al-

\footnotetext{
577 Arduino, M. (2014) Il bambino che parlava con la luce. Quattro storie di autismo, Giulio Einaudi editore, Torino

${ }_{578}$ Antonello, F. Antonello, A. (2015) Sono graditi visi sorridenti, Feltrinelli, Milano
} 


\section{Elena Bellini}

tri casi, uno dei due genitori deve rinunciare al lavoro per accudire il figlio." (Mazzone, 2015 - p.131) $)^{579}$

"Cosa farà quando noi non potremo preoccuparci di lui?, si chiedevano. Erano domande alle quali noi del Centro non sapevamo rispondere. Per le istituzioni gli adulti con autismo sembravano non esistere. Da anni le associazioni dei genitori denunciavano l'inadeguatezza dei servizi e alcuni fatti di cronaca avevano portato alla luce situazione di grave abbandono delle persone con autismo e delle loro famiglie ... Un paio di anni dopo il termine della scuola superiore, Silvio fu inserito in una struttura aperta di recente in città dove poteva frequentare laboratori e proseguire alcune attività didattiche. Le sue giornate erano sempre piene di cose da fare. Solo il fine settimana continuava a essere un momento vuoto, senza alcun impegno a parte quando l'associazione organizzava gite o serate. Maria e Gianni, insieme a un gruppo di famiglie che avevano il loro stesso problema, stavano progettando una residenza per persone adulte con autismo dove inizialmente i ragazzi avrebbero potuto fermarsi il sabato e la domenica e, in seguito, abitare con il supporto di assistenti ed educatori. L'obiettivo era quello di dare a Silvio e ad altri come lui un posto in cui vivere senza genitori - che in questo modo avrebbero ritrovato un po' di tempo per sé stessi - e senza gravare sui fratelli. Un posto che, a poco a poco, diventasse la loro casa." (Arduino, 2014 - p. 78-79) ${ }^{580}$

"Per lui (Tommy) 'casa' sarà sempre la casa dove è cresciuto, non credo che si abituerebbe a considerarne un'altra. E' come avesse interiorizzato il link 'home' che porta sempre al numero civico dove abita. La prima regola quindi, è che Insettopia sorga vicino a casa .. La città che mi piacerebbe costruire, dentro ogni città, non dovrebbe essere un luogo che potesse essere scambiato con qualcosa che assomigli a un ospedale, una clinica, un manicomio, un carcere. Dovrebbe comunque avere un suo perimetro protetto, che non significa chiuso: io abito nel quartiere delle vecchie caserme umbertine, ormai dismesse, e, ogni volta che passo davanti a quelle mura irte di altane senza più sentinelle, costruisco nella mia mente la nostra città felice .. I nostri figli, è nell'ordine naturale delle cose, ci sopravvivranno, e non sono un carico lieve da amministrare Tanto varrebbe allora costruire sin da oggi la loro città e abituarsi all'idea che ci possa essere in ogni municipio, quartiere, circoscrizione un'Insettopia per chi non si trova a suo perfetto agio tra gli umani. Sarebbe l'esatto opposto della segregazione, almeno nella mia idea. Sarebbe l'equivalente dell'ufficio, del luogo di lavoro, del bar dove si va a prendere l'aperitivo, della palestra, del calcetto, della scuola." (Nicoletti, 2013 - p. 145) ${ }^{581}$

"Immaginate un unico luogo dove si condensino tute le attività gradite e utili ai propri figli ... Immaginate allora un luogo in cui i genitori abbiano la certezza che, come in un campus pensato per le esigenze specifiche dell'autistico, l'attività e il tempo del proprio figlio siano organizzati in maniera costruttiva e confacente alla

\footnotetext{
${ }^{579}$ Mazzone, L. (2015) Un autistico in famiglia, Mondadori, Milano

${ }^{580}$ Arduino, M. (2014) Il bambino che parlava con la luce. Quattro storie di autismo, Giulio Einaudi editore, Torino

581 Nicoletti, G. (2013) Una notte ho sognato che parlavi. così ho imparato a fare il padre di mio figlio autistico, Mondadori, Milano
} 
sua personalità, a sua misura ... Questo luogo però non me lo prefiguro come il giardino di un convento, chiuso verso l'esterno; al contrario, dovrebbe essere un catalizzatore di attività sociali talmente belle, originali e 'strambe' da stimolare anche la curiosità dei neurotipici. La ' nostra città' dovrebbe essere costruita in un'oasi verde $\mathrm{e}$ sospesa dalle forti stimolazioni acustiche e visive del traffico cittadino, delle liturgie ipercomunicative, lontana dall'attrito delle relazioni con un'infinità di altri umani. Allo stesso tempo però, Insettopia non dovrebbe essere emarginata, bensì solo 'sensorialmente alleggerita', quanto basta a non stressare la particolare sensibilità delle persone autistiche. Il che farebbe bene pure a chiunque altro volesse passare per Insettopia, anche solo per attutire il peso quotidiano dell'essere sovraccarico dall'obbligo di socializzazione intensiva e forzata." (Nicoletti, 2014 - p. 55$)^{582}$

"Sono così riusciti a far nascere al posto dei loro pollai una struttura che oggi vale più di quattordici milioni di euro. Per farla non hanno chiesto un soldo a nessuno, hanno tirato su un fabbricato di seimila metri quadrati, con ventidue ettari di terra attorno. Quel luogo è aperto a tutti, non soltanto riservato a chi ci vivrà per un periodo o per sempre. $E^{\prime}$ un centro in cui, per qualche ora al giorno o per soggiorni più lunghi, gli ospiti possono frequentare laboratori e servizi per acquisire abilità e competenze utili nella vita quotidiana o per potenziare le loro capacità relazionali, anche attraverso il lavoro o l'equitazione ricreativa. Per chi viene da fuori ci sono due palazzine destinate a ospitare ragazzi e familiari per dei soggiorni mirati a dare autonomia a chi ha bisogno di essere aiutato ... Alla fine lavoreranno lì cinquanta operatori specializzati, con la supervisione di cinque docenti dell'università di Bologna che si sono prestati per garantire la correttezza scientifica dell'attività ... Al centro della 'città' la coppia Fornino-Valmori ha voluto una struttura residenziale e semiresidenziale per progetti di abilitazione individuali: due edifici con stanze da letto perfettamente a norma secondo i regolamenti vigenti. Altro che un ghetto per disabili: sembra un luogo immaginato per la serenità di chiunque. C'è un ristorante biologico, aperto al pubblico, rifornito con prodotti a chilometro zero, una lavanderia industriale ipermoderna con fornitura, ritiro e lavaggio di pannolini rigenerabili per gli asili nido. Palestre, campi sportivi, aule di abilitazione. e un centro di equitazione ricreativa per disabili che garantisce attività sia al coperto che all'aperto..." (Nicoletti, 2014 - p. $115-116)^{583}$

"Ora mi vien da dire chi se ne frega, sopravvivremo comunque fino al giorno che sarà proprio Tommy a portarmi sulle spalle, come dovette fare Enea con il vecchio Anchise. Io mi attaccherò al suo capoccione bislacco e gli dirò per la miliardesima volta di fermarsi ai semafori e camminare sulle strisce. Mi consolerà pensare che, a quel tempo, gli altri figli efficienti e produttivi avranno già sbattuto i loro genitori a far la muffa in qualche ospizio. Noi ci faremo qualche bella passeggiata ancora assieme. Quando io non ci vedrò quasi più, forse passeremo col rosso." (Mazzone, $2015-$ p. 177$)^{584}$

\footnotetext{
${ }^{582}$ Nicoletti, G. (2013) Una notte ho sognato che parlavi. così ho imparato a fare il padre di mio figlio autistico, Mondadori, Milano

583 ibidem

${ }^{584}$ Mazzone, L. (2015) Un autistico in famiglia, Mondadori, Milano
} 


\section{Elena Bellini}

"E' pazzesco che quando veniamo a vedere questi centri diurni per ragazzi con autismo abbia più ansia io di lui. Che anzi, ci viene volentieri. Non che la Casa Gialla sia un brutto posto. Intendiamoci ... A volte anch'io porto Bogdan al mar o a fare un giro in moto. Forse perché è il primo ragazzo autistico a parte Andrea che ho conosciuto ... O forse perché anche con lui ho toccato con mano che il vero problema di questi ragazzi è la solitudine: trovare amici, non medicine ... Lo so che quando arrivo alla Casa Gialla mi arrabbio. anche s è un bel posto, organizzato e pulito, circondato da campi che Franco fa coltivare ai ragazzi per abituarli a usare le mani e far passare il tempo senza che stiano rinchiusi in una stanza. Vendono anche gli ortaggi che coltivano. Ci sono i laboratori nel seminterrato ... Poi però un volontario non arriva e la situazione precipita. Mica solo qui. Ovunque. Basta che uno non possa, perchè ha un imprevisto, qualcosa da fare, perché questo è volontariato, non il suo lavoro, ed ecco che il meccanismo si inceppa ... Non c'è nessuna soluzione se non quella di chiuderne qualcuno in una stanza. Che è esattamente dove starebbero se stessero a casa, perché un genitore che deve andare a lavorare cosa fa? Li chiude dentro e spera in Dio, che non distruggano la casa o se stessi. Quanto torni trovi quel che trovi ... Quando si fanno del male o sono autolesionisti, diventa completamente impossibile gestirli, devi tenerli d'occhio di continuo, e di conseguenza i genitori - di solito la madre - perdono il lavoro ... Interrompe Franco l'arrivo di un'altra mamma, con un ragazzo grande e grosso che avrà quindici anni e non smette mai di battere $\mathrm{i}$ pugni ovunque, sulla testa, sul uro: chi lo ferma? Lei ha l'aria disperata, borse sotto gli occhi in cui si potrebbe far entrare la spesa di una settimana, una ricrescita di cinque centimetri alla radice di capelli biondi. Grigia, la ricrescita. Grigio-tristezza, grigio-fatica ... 'posso lasciare qui mio figlio un paio d'ore?' nella voce vibra, oltre alla fatica, una vergogna insopprimibile. come se fosse una colpa aver bisogno di due ore ... 'faccia così, lo lasci pure qua anche il pomeriggio, torni a riprenderlo alle sei'. E' come veder spuntare l'alba. Il viso le si riempie di luce e vedo attraverso la fatica che avrà sì e no quarant'anni e che solo ieri era una ragazza, e adesso è disperata, si sente vecchia, si sente sola sotto questo carico enorme. " (Antonello, 2015 p.137-141) ${ }^{585}$

"bravo Andre, ora vai libero, se hai qualcosa da inserire alla fine scrivi quello che vuoi, altrimenti usiamo la frase che hai già scritto. Grazie Andre, numero $1 \ldots$ 'puoi finire con quella frase io voglio dirti mio impegno del continuare a vivere al tuo fianco sicuro che non ci lasceremo in questo mio viaggio la morte non ci sconfigge ma ci lega'. Sì, ma quando non ci sarò più io o la mamma, ti devi arrangiare da solo, Alberto ti può aiutare ma ha la sua vita. Ti spaventa questo... 'no io vi ho dentro tu non avere paura. Vita dura io ho, evidente a tutti, io non lascio, e di aiuto i miei pensieri sono per chi legge e sa andare oltre e crede che non ci sia in ognuno di noi un solo uomo ma diversi talenti, fermiamoci a guardare non con occhi ma con cuore'." (Antonello, 2015 - p.198)

\footnotetext{
${ }_{585}^{585}$ Antonello, F. Antonello, A. (2015) Sono graditi visi sorridenti, Feltrinelli, Milano

586 ibidem
} 


\subsection{Questionari e interviste rivolte a genitori di bambini e adulti con DSA e ad alcune persone con autismo ad alto funzionamento o Asperger}

La definizione di spettro autistico è, come abbiamo, visto molto ampia, per cui vi saranno sempre grandi differenze legate alla percezione e alla sensitività della singola persona, differenziando il profilo di utenza in modo sensibile. Non è possibile quindi delineare dei concetti universali di benessere o disagio, ma può essere utile indagare in questo senso per comprendere se e dove ci sono delle spiccate somiglianze e, soprattutto, quali sono le motivazioni delle differenze. Per questo motivo il questionario è stato concepito per avere un valore quali-quantitativo, prevedendo anche molte domande aperte per lasciare la possibilità, a chi lo volesse, di unire un commento ad un 'si' o un 'no'. Attraverso alcune interviste ai familiari e ad adulti con autismo ad alto funzionamento o Asperger è stato possibile inoltre approfondire queste tematiche e discutere degli stessi argomenti attraverso il loro punto di vista. Non sono "numeri" ma possibilità di affacciarsi ad un mondo completamente diverso dal nostro.

Il questionario quindi è stato strutturato in modo da comprendere prima di tutto quali siano gli ambienti di vita delle persone con DSA, quali criticità si riscontrino negli spazi del costruito e nel contesto ambientale e dove si possa andare ad agire per migliorare la qualità di vita di queste persone. Questo è stato utile per la fase propositiva, al fine di delineare, ove possibile, caratteri simili e famiglie di bisogni e a puntare il dito, al contrario, su quei punti di non convergenza, sulla base delle differenze sensoriali e percettive, che necessitano invece di flessibilità e personalizzazione rispetto al singolo. E' ovvio come nel progetto di residenza ci sia la possibilità ed il dovere, in questo caso, di ascoltare la singola persona e le sue particolari esigenze, ma quando si tratta di uno spazio pubblico? Ogni ambiente avrà un diverso grado di universalità, ma raccogliere informazioni sulle diverse esigenze può essere di supporto a delineare dei suggerimenti per ridurre il disagio e quindi migliorare l'accessibilità di alcuni luoghi, progettare ambienti sicuri, supportare l'autonomia attraverso la comprensione e l'orientamento nei diversi contesti, favorire la relazione con gli altri e l'inclusione.

Ad oggi sono stati realizzati diversi questionari dal punto di vista psicologico ed educativo, che vanno ad indagare i livelli di comunicazione, le abilità e i gradi di autonomia nella vita quotidiana, senza però relazionarsi in modo stretto con gli spazi fisici. Per questo motivo si è tentato, attraverso questo questionario, di indagare nello specifico soprattutto gli aspetti sensoriali e percettivi legati all'ambiente, che influenzano in modo positivo o negativo il rapporto con il contesto ambientale, partendo come riferimento dalla check-list di Olga Bogdashina ${ }^{587}$. La strutturazione delle domande è stata definita a partire dallo studio dello stato dell'arte, al fine di confermare o confutare alcune ipotesi affermate nella letteratura, ma anche indagare quegli aspetti che non sono stati precedentemente approfonditi.

587 Bogdashina O. (2003) Le percezioni sensoriali nell'autismo e nella sindrome di Asperger, Uovonero: Crema (edizione italiana del 2011) 


\section{Elena Bellini}

Il questionario è stato redatto inoltre anche grazie al supporto della Fondazione Bambini e Autismo Onlus di Pordenone, che ha contribuito dal punto di vista delle competenze di tipo psicologico e terapeutico, ma anche facendo da mediatore e facilitatore rispetto alle famiglie. Il questionario è stato infatti, dopo la prima stesura, testato su alcune famiglie della Fondazione, al fine di mettere a punto la chiarezza e la comprensione delle domande, la facilità di risposta in modo autonomo e l'efficacia delle risposte stesse, consentendo così di definire un modello da diffondere su tutto il territorio italiano. Il questionario è stato quindi, a seguito della sperimentazione, inoltrato su tutto il territorio italiano, rivolgendosi alle Associazioni di familiari per l'Autismo e Asperger e ai centri di terapia, e poi distribuito sia online che cartaceo, in base alle preferenze. Alcune delle famiglie che hanno dato disponibilità a rispondere al questionario sono state inoltre intervistate, al fine di commentare, comprendere e approfondire le risposte alle diverse domande, aggiungendo anche racconti sulla vita quotidiana della loro famiglia. Infine sono state intervistate 8 persone con autismo ad alto funzionamento ed Asperger tra i 13 e i 55 anni, presso la Fondazione Bambini e Autismo di Pordenone e ANGSA Novara-Vercelli, attraverso un'intervista strutturata basata sulla raccolta degli stessi dati del questionario, al fine di provare a comprendere un punto di vista diverso e interno al problema sulle stesse tematiche. In totale sono state raccolte le risposte di 61 persone tra i 3 e i 55 anni distribuite sul territorio italiano, anche se in gran parte al centro e al nord Italia, ed in particolare: 13 persone in Piemonte, 3 in Lombardia, 2 in Liguria, 4 in Emilia-Romagna, 16 in Toscana, 3 in Veneto, 13 in Friuli-Venezia-Giulia, 1 in Lazio e 4 in Calabria (Figura 1). Tra queste vi sono 51 uomini e 10 donne (Figura 2), di cui 7 persone tra $i$ 3 e i 5 anni, 10 tra i 6 e gli 11 anni, 9 tra i 12 e i 15 anni, 7 tra i 16 e i 19 anni, 19 tra i 20 e i 30 anni, 6 tra i 30 e i 40 anni, 1 tra i 41 e i 50 anni e 2 persone oltre i 50 anni (Figura 2).

La struttura del questionario prevede una prima parte sull'anagrafica, ovvero età, sesso e luogo di residenza. Dopo di ché si è cercato, attraverso le domande, di andare identificare il profilo dell'utente, definendo l'eventuale presenza di disturbi associati all'autismo (sulla base dei criteri diagnostici del DSM V), le sensibilità sensoriali e quindi, più nel dettaglio, il profilo senso-percettivo della persona.

In particolare i risultati del questionario riportano 39 persone con sensibilità sensoriale spiccata, 43 persone che presentano comportamenti ristretti e ripetitivi o routines, 44 persone con stereotipie, 30 persone che presentano crisi comportamentali, 10 persone con comorbidità (tra cui sindrome di Tourette, disturbo ossessivocompulsivo, trisomia 21, ipovisione, disturbi fisici e scoliosi, problemi gastrici, colite, eosinofila, ipotiroidismo, disfagia, epilessia), 17 persone con disturbi del sonno, 12 persone che hanno difficoltà o selettività nell'alimentazione, 22 persone che assumono regolarmente farmaci, 18 persone con linguaggio completamente assente e 22 persone che si esprimono usando singole parole o frasi (Figura 3).

La sensibilità sensoriale (Figura 4 e 5) maggiormente riscontrata è quella uditiva (43 persone) che, attraverso i commenti lasciati nelle risposte aperte del questionario o durante le interviste, è stata riscontrata come una delle maggiori criticità e causa di stress e disagio, andando ad influire direttamente sull'orientamento, la comprensione, l'attenzione, l'apprendimento, la comunicazione e l'interazione con gli altri. I rumori che causano maggiore disagio sono quelli forti (58 persone) ed improvvisi (53 
persone); il brusio di fondo (37 persone) ed il riverbero; ronzii e fischi impercettibili come il ronzio dei neon o delle prese elettriche, o il ticchettio di un orologio; le frequenze alte e stridule (inizialmente non indagato, comunque 16 risposte di disagio su un totale di 21), in particolare nelle voci di alcune persone, come ad esempio quelle dei bambini (segnalato anche durante le interviste, in particolare le urla ed il pianto). La musica è invece molto apprezzata ( 50 persone), anche se talvolta in modo molto selettivo, e viene spesso ascoltata anche a volume molto alto; in contesti come quello lavorativo d'altro canto può essere fonte di distrazione e quindi rivelarsi negativa. Molti infatti hanno descritto la stimolazione uditiva come una vera e propria causa di distrazione, che non consente di concentrarsi sulle attività o i dialoghi, causando ovviamente difficoltà sia in ambienti educativi che lavorativi, ma anche nelle relazioni sociali; altri addirittura hanno descritto il disagio come dolore fisico. Vivere in un luogo silenzioso è stato segnalato come importante da 43 persone, di cui 20 hanno espresso la necessità di una casa in generale ben isolata acusticamente, sia rispetto ai rumori provenienti dall'esterno (traffico, vicini...) che tra i diversi spazi interni della casa; 16 hanno segnalato di aver bisogno di silenzio in camera da letto per dormire; 6 persone hanno espresso il desiderio di un luogo silenzioso nei momenti in cui ci sono ospiti in casa; 1 persona invece solo rispetto al traffico stradale.

Tra le altre stimolazioni percepite con molta intensità sono state segnalate anche quelle visiva ( 27 persone) ed olfattiva ( 21 persone). Tra le sensibilità visive si è riscontrato il maggiore disagio rispetto alla luce e all'abbagliamento (46 persone), al sovraccarico visivo, espresso anche attraverso il bisogno di "controllo" sullo spazio circostante, oltre che ad una particolare sensibilità legata ai colori. Il tema della luce è molto importante, sia per quanto riguarda quella naturale, poiché in generale sono apprezzati ambienti molto luminosi, che per quella artificiale, preferendo luci calde (20 persone) e regolabili nell'intensità, o ancor meglio diversi punti luce, per differenziare la qualità luminosa degli spazi. La luce del sole, anche se piacevole (18 persone), può rivelarsi fonte di disagio (25 persone), a causa dell'abbagliamento, o di distrazione, attraverso i riflessi e i fasci luminosi che produce negli ambienti interni, per cui è sempre bene che sia regolata attraverso sistemi di oscuramento. Questi stessi riflessi d'altro canto possono essere in alcuni momenti una fonte piacevole di stimolazione sensoriale, così come le luci colorate, che in generale rappresentano una stimolazione favorevole (22 persone). Molti hanno espresso, inoltre, la necessità di passare alcuni momenti in penombra (34 persone) per rilassarsi, o al buio completo, ad esempio per dormire. Per quanto riguarda i colori non è stato molto semplice definire una tendenza specifica: se da un lato c'è chi preferisce ambienti bianchi ed asettici poiché sono poco stimolanti e aiutano la concentrazione, dall'altro lato c'è chi prova disagio in questi ambienti (10 persone); c'è chi ama i colori forti e accesi (17 persone), ma chi al contrario non apprezza i contrasti e si attiva in presenza di colori accesi aumentando l'agitazione e perdendo quindi la concentrazione; i colori tenui e pastello sono in generale apprezzati dalla maggior parte delle persone o quantomeno non provocano nessun tipo di disagio (solo 1 persona). Tutto ciò che riguarda le immagini, le foto, le espressioni artistiche, i video e i multimedia risulta in generale piacevole (41 persone) e può essere un metodo valido di distrazione e rilassamento in momenti di stress, pur sempre con moderazione. Un'altra fonte di sovraccarico visivo e quindi di stress può essere rappresentata infatti dalla presenza 


\section{Elena Bellini}

di troppi oggetti o elementi decorativi, per cui ad esempio è in generale molto apprezzato l'ordine (non è stato indagato fin da subito, ma si è in definitiva riscontrata una prevalenza di 16 risposte positive su un totale di 29), anche inteso come "proprio ordine personale" e "controllo sull'ambiente circostante", a cui le persone intervistate hanno spesso fatto riferimento. Questo aspetto non è tanto legato alla mancanza di flessibilità rispetto agli ambienti, ma ad una necessità di "controllo" che porta ad una rigidità rispetto ai cambiamenti non preavvisati, soprattutto nel caso in cui si tratti delle proprie cose. Questo viene ad esempio esasperato nei bambini, rispetto ai loro giochi, che talvolta non sono in grado di condividere; molte mamme hanno espresso la necessità di avere uno spazio personale dedicato ai giochi per il bambino in modo che possa mantenere il proprio ordine delle cose e scegliere se e come relazionarsi con gli altri, un ambiente protetto, controllato e controllabile, sicuro ed adeguato alle esigenze specifiche del bambino. Spesso la cameretta non è in grado di ottemperare questo compito, poiché viene condivisa con un fratello o una sorella, oppure è troppo piccola, isolata rispetto agli ambienti vissuti della casa, o dedicata solo al sonno; talvolta al contrario alcuni bambini dormono nella stanza dei genitori e usano la camera personale solo come stanza giochi. Per quanto riguarda il "controllo visivo", un ambiente open space può rappresentare un ambiente positivo poiché consente una massima visibilità; d'altro canto molti hanno indicato come sia importante aver la possibilità di riporre gli oggetti, ad esempio con mobili o ripiani a scomparsa, per evitare il sovraccarico visivo; altri ancora invece hanno rivelato utile la differenziazione degli ambienti per funzione, magari anche attraverso colori o materiali, soprattutto per una maggiore chiarezza, orientamento, comprensione e quindi autonomia. In ogni caso la gestione della casa e degli spazi è stata rilevata molto importante e sentita da tutti gli intervistati, riscontrando un interesse particolare nell'essere "registi del cambiamento e delle scelte" all'interno della casa.

Dal punto di vista olfattivo invece si è riscontrato come i profumi e gli aromi siano molto apprezzati (32 persone), ma come allo stesso tempo la maggior parte delle persone siano molto selettive in questo senso e che quindi, talvolta, specialmente in ambienti pubblici in cui non è possibile scegliere l'aroma, sia meglio non avere odore piuttosto che diffondere un aroma che per qualcuno potrebbe rivelarsi spiacevole, soprattutto se forte ed intenso (disagio per 18 persone, segnalato come causa di nausea). Una particolare nota può essere rivolta anche nell'utilizzo di materiali e prodotti naturali che non contengano agenti chimici, come ad esempio la formaldeide, poiché è stata segnalata come causa di forte disagio, o anche svenimento.

Una particolare sensibilità tattile è stata indicata da 16 persone, soprattutto per quanto riguarda $\mathrm{i}$ tessuti, che talvolta può rivelare criticità anche nella scelta degli indumenti o della biancheria e, anche in questo caso, può essere causa di vero e proprio dolore fisico (mal di denti, nausea). Il tatto d'altro canto può essere anche una stimolazione ricercata ( 24 persone), apprezzando le superfici ruvide o lisce, diverse textures, o elementi morbidi ed avvolgenti (29 persone). La studiosa Temple Grandin ha inventato una particolare queeze machine (macchina degli abbracci o stringitrice) che comprime la persona dandole contenimento, una sensazione piacevole e di rilassamento per molte persone con autismo. In questo senso 44 persone hanno indicato interesse e piacere nella ricerca di luoghi raccolti e di isolamento, per i momenti di relax, ancor meglio se al buio o in penombra, per eliminare completamente gli 
stimoli, riequilibrarsi e sentirsi contenuti. Durante le interviste è stato riscontrato come questa necessità sia importante a seguito delle attività giornaliere, come momento di calibrazione e gestione dei propri sensi rispetto al "mondo esterno che fa male", oppure in casi di affluenza maggiore di persone (a casa con ospiti, a scuola, in ambienti non troppo grandi o affollati...). Alcuni hanno raccontato che riescono a rilassarsi riequilibrare i propri sensi in spazi molto piccoli, ad esempio chiudendosi in bagno, o addirittura nell'armadio. In particolare 4 persone hanno indicato che riescono a trovare il modo di isolarsi per qualche momento in ogni luogo della casa, ricreando un proprio angolo in ogni stanza; 38 persone hanno invece indicato la camera come luogo privilegiato per rilassarsi; 16 persone hanno scelto il soggiorno; 1 lo studio e solo 2 persone hanno detto di non averne bisogno. Rispetto agli elementi naturali, il vento può rappresentare una fonte di disagio (20 persone), ma quando si tratta di una brezza o un venticello leggero può rivelarsi anche molto piacevole (20 persone). Questo probabilmente anche perché varie persone hanno espresso disagio per il caldo, soprattutto negli ambienti chiusi, trovando disagio talvolta per il riscaldamento dell'ambiente (riscontrato soprattutto nelle interviste); al contrario altri hanno espresso una sensibilità maggiore al freddo. L'acqua è l'elemento più apprezzato complessivamente (48 persone) e per molti è stato definito come 'elemento essenziale' o 'habitat naturale'. Un'altra passione riscontrata è quella per il verde e la natura (34 persone), che talvolta si rivela anche un hobby, come il giardinaggio.

Da qualcuno è stata inoltre indicata una sensibilità sensoriale propriocettiva (7 persone) e vestibolare ( 5 persone), ma si denota come probabilmente questo quesito non sia stato completamente compreso da tutte le persone che hanno risposto al questionario, poiché durante le interviste si è riscontrato come talvolta non è stata segnalata come sensibilità particolare ma che al contrario la persona presentava vari indicatori di questa presenza, come ad esempio la necessità di spazio "personale" o di ambienti ampi per facilitare i movimenti, la sensibilità rispetto all'altezza e alla gravità, talvolta legata anche ad una difficoltà a salire o scendere le scale, ecc. Questi due ultimi valori pertanto non si considerano del tutto validi ai fini statistici. Per quanto riguarda la dimensione degli ambienti è stato comunque rilevato come gli ambienti grandi siano in generale apprezzati (23 persone) per avere una maggiore libertà di movimento e per un rapporto di prossemica, soprattutto rivolto alla presenta di altre persone; ci sono d'altro canto 15 persone che hanno valutato un ambiente grande come dispersivo e disorientante, o causa di sovraccarico visivo, preferendo invece spazi più piccoli e contenuti. L'altezza è stata rilevata come un altro aspetto importante, soprattutto durante le interviste; se alcuni hanno espresso di apprezzare i soffitti alti in edifici come ad esempio le chiese, al contrario non hanno la stessa percezione in un ambiente casalingo, dove al contrario può talvolta creare disagio. Un altro aspetto riscontrato in più persone è quello di non apprezzare particolarmente gli edifici molto alti, soprattutto se osservati dall'esterno, dal basso, poiché disorientano e disequilibrano. L'altezza e la gravità sono altri due aspetti che possono creare delle criticità, soprattutto rispetto a balconi o terrazze, o alle scale. Anche in questo caso durante le interviste è stato possibile approfondire la tematica, ma non è stata trovata una tendenza specifica; qualcuno ha espresso una preferenza per i parapetti che consentono una visibilità verso l'esterno, per avere una consapevolezza di quello che c'è e non essere costretti ad affacciarsi, che potrebbe rivelarsi anche pericoloso, mentre 


\section{Elena Bellini}

altri hanno indicato una preferenza per una superficie di parapetto opaca per una maggiore sicurezza e per evitare che si possano arrampicare. Le scale talvolta non vengono molto apprezzate, soprattutto per la discesa, poiché creano instabilità, probabilmente legata anche alla difficoltà di percepire la profondità dello spazio, frequente in molte persone con autismo.

Dal punto di vista gustativo è stata segnalata una sensibilità per 13 persone, soprattutto legata alla selettività alimentare, spesso influenzata non tanto dal sapore quanto dalla consistenza, dalla forma, dal colore, dalla mescolanza o dalla temperatura degli alimenti. Un altro aspetto interessante è che talvolta i sapori generalmente considerati sgradevoli non lo sono per alcune persone autistiche, che ad esempio tendono a mangiare saponi o cosmetici, probabilmente collegati all'odore che hanno (riscontrato durante le interviste).

Un dato importante, riscontrato soprattutto durante le interviste, è che le sensibilità sensoriali si amplificano quando associate. Anche se può sembrare una cosa ovvia, questo concetto sta alla base della comprensione dei profili sensoriali, poiché anche sensibilità non così spiccate possono rivelarsi critiche nel momento in cui si hanno molti stimoli contemporaneamente, portando ad un sovraccarico sensoriale e quindi ad una situazione di stress e disagio. D'altro canto l'eliminazione di tutti gli stimoli non sempre è da considerare positiva, ma dipende dal contesto e dalle attività (oltre che ovviamente dalle particolarità della singola persona) per cui si dovrà valutare con attenzione rispetto alla situazione specifica.

Un altro aspetto indagato nel questionario è stato quello degli ambienti di vita, ovvero rilevare quali ambienti vengono maggiormente frequentati e vissuti dalle persone con autismo, quali possano rappresentare delle criticità, sia nel mondo esterno, che nello specifico della casa.

Un primo dato importante è che tra le persone intervistate 53 persone (su 61) vivono nella casa dei propri genitori (Figura 6), mentre solo 5 persone vivono in una residenza assistita (1 persona tra i 16 e i 19 anni, in Calabria; 3 persone tra i 20 e i 30 anni, 2 in Calabria e 1 in Piemonte; 1 persona tra i 31 e i 40 anni, in Calabria); 2 persone vi passano dalle 10 alle 20 ore (entrambi tra i 20 e i 30 anni, 1 in FiuliVenezia-Giulia e 1 in Piemonte), probabilmente in programmi sollievo e/o abilitativi; 1 dalle 20 alle 30 ore (tra i 6 e gli 11 anni, in Toscana) (Figura 7). Tutti i bambini in età tra gli 0 e i 5 anni (in totale 7) vanno alla scuola dell'infanzia; 2 tra le 10 e le 20 ore a settimana e 5 dalle 20 alle 30 ore settimanali (Figura 8). Lo stesso per i bambini tra i 6 e gli 11 anni (10 in totale): 4 bambini vanno alla scuola elementare per $10-20$ ore settimanali, 5 bambini tra le 20 e le 30 ore e solo 1 oltre. Le ore in cui non frequentano la scuola solitamente sono dedicate alle attività terapeutiche integrative. Tra i 12 e i 15 anni si mantiene la frequenza scolastica (9 bambini), di cui 5 bambini tra le 10 e le 20 ore e 4 bambini tra le 20 e le 30 ore settimanali di attività scolastica. Tra i 16 e i 19 anni invece la scuola viene frequentata solo da 3 dei 6 ragazzi, per 20-30 ore. Tra i 20 e i 30 infine vi sono 3 persone che frequentano regolarmente la scuola per $20-30$ ore, 1 persona tra le 10 e le 20 ore e 1 persona tra le 5 e le 10 ore settimanali, rispetto ad un totale di 19 persone. La maggior parte delle persone (33 su 61) fa attività sportive, culturali, o legate al proprio credo religioso (30 tra 1 e 5 ore a settimana, 3 tra 5 e 10 ore) (Figura 9); la frequenza è abbastanza distribuita per età, ma fanno questo tipo di attività soprattutto le persone tra i 6 e i 30 
anni (31 persone su 33). Il centro diurno non è invece generalmente molto frequentato (16 persone su 61) (Figura 10) e soprattutto da persone di età maggiore ai 20 anni. In particolare, 3 persone tra i 20 e i 30 anni, 3 persone tra i 30 e i 40 anni e 1 persona oltre i 50 frequentano il centro diurno quotidianamente (oltre le 30 ore settimanali); 4 persone tra i 20 e i 30 anni e 1 tra gli 0 e i 5 anni lo frequentano tra le 20 e le 30 ore settimanali; 1 persona tra i 12 e i 15 anni lo frequenta per $10-20$ ore settimanali; 2 persone tra i 20 e i 30 anni lo frequentano per 5-10 ore settimanali e 1 persona tra $i$ 17 e i 19 anni lo frequenta per un tempo inferiore alle 5 ore settimanali. Solo 11 persone lavorano regolarmente (Figura 11), di cui 1 oltre i 55 anni lavora a tempo pieno, 3 persone tra i 20 e i 30 anni e 2 tra i 30 e i 40 anni lavorano part-time (20-30 ore settimanali), 1 persona tra i 20 e i 30 anni lavora dalle 10 alle 20 ore e infine alcuni tra i più giovani (2 ragazzi tra i 16 e i 19 anni e 2 tra i 20 e i 30 anni) si stanno avvicinando al lavoro, occupandosene per 1-5 ore settimanali. Infine per quanto riguarda la terapia (Figura 12), 40 persone (su 61) fanno regolarmente attività terapeutiche presso le Associazioni e i Centri per l'Autismo, per lo più in età compresa tra gli $0 \mathrm{e}$ i 19 anni. In particolare, fanno terapia per $1-5$ ore a settimana 28 persone: 3 su 7 tra 0 e 5 anni, 9 su 10 tra i 6 e gli 11 anni, 7 su 9 tra i 12 e i 15 anni, 3 su 6 tra i 16 e i 19 anni, 5 su 19 tra i 20 e i 30 anni, 1 su 5 tra i 30 e i 40 anni.

L'ambiente preferito (Figura 13) dalla maggior parte delle persone è la residenza (37 persone hanno espresso questa preferenza), poiché è il proprio ambiente, familiare, rassicurante, adatto alle proprie personali esigenze, tranquillo, protetto e che consente una maggiore libertà. Alcuni hanno espresso anche una preferenza per la scuola (13 persone), poiché è un'occasione di socializzazione e relazione con gli altri; il centro diurno ( 7 persone) e i luoghi dove fare terapia (11 persone), sempre per la possibilità di interagire con gli altri, anche in questo caso in una situazione maggiormente privilegiata, come qualcuno ha scritto, poiché non si sentono 'diversi' rispetto agli altri; gli ambienti dove fare attività sportiva, culturale o legata al proprio credo religioso ( 7 persone) ed il lavoro (6 persone). Infine qualcuno ha espresso una preferenza rispetto ai luoghi all'aperto, parchi giochi o ambienti naturali, anche se non era tra le domande del questionario, annotandolo in "altro".

Per quanto riguarda invece gli ambienti che possono in qualche modo rivelarsi critici (Figura 13), è stata indicata una prevalenza di difficoltà rispetto ai luoghi sconosciuti (28 persone), poiché non consentono di poter preparare la persona e quindi possono creare ansia e stress. Al secondo posto si trovano i negozi e gli esercizi commerciali (24 persone), o più in generale i luoghi affollati, rumorosi e iperstimolanti, e gli ambienti sanitari (24 persone). In seguito qualcuno ha valutato come critici glia ambienti istituzionali ( 8 persone), la residenza ( 7 persone), la scuola (5 persone), i luoghi all'aperto (4 persone), i luoghi di terapia (3 persone), il centro diurno ( 2 persone) e il lavoro ( 1 persona). Al contrario 12 persone hanno indicato che nessun ambiente può presentare condizioni di criticità.

Talvolta la criticità è rappresentata invece dallo spostamento, soprattutto all'arrivo in un nuovo posto; 23 persone hanno segnalato questa difficoltà, di cui 4 molto elevata e 20 più che altro concentrata all'arrivo, o sull'uso dei mezzi di trasporto; 28 persone invece non hanno espresso particolari criticità negli spostamenti. D'altro canto l'integrazione di diverse attività in un unico edificio è stata considerata positiva per 31 persone rispetto alle 17 che hanno espresso parere negativo. Le motivazio- 
ni per chi considera positiva l'integrazione di più attività in un unico luogo sono perlopiù legate alla comodità, alla difficoltà riscontrata negli spostamenti o nei cambiamenti di ambiente, alla possibilità di avere un ambiente controllato e strutturato, familiare e quindi rassicurante, che favorisca l'integrazione e la socializzazione e l'acquisizione di autonomie.

$\mathrm{Al}$ contrario chi pensa che sia meglio mantenere tutte le attività separate crede che sia meglio avere ogni diverso luogo per una diversa funzione, con le sue caratteristiche e le sue regole, poiché più chiaro e semplice; pensa inoltre che cambiare la routine fa bene, per superare la noia, ma anche per imparare ad adattarsi, favorire l'elasticità mentale e la flessibilità, abituandosi a vivere in tutti i luoghi.

La seconda parte del questionario è stata incentrata sulla casa, per comprendere dove e come vivono le persone con autismo. La maggior parte delle persone (27) vive in un piccolo paese, 15 in periferia, 8 in centro città, 8 in campagna, 2 sul mare e 1 in montagna. La maggior parte delle famiglie è composta da quattro (23 famiglie) o tre persone ( 21 famiglie), mentre 9 nuclei familiari superano le 5 persone e 8 sono nuclei da 2 persone. La maggior parte vive in un appartamento di circa 100-150 mq ( 24 persone) e situato su un solo piano (36 persone); in 7 vivono invece in un appartamento di 50-70 mq, in 12 in un alloggio di 80-95 mq, in 11 in una casa superiore ai $200 \mathrm{mq}$ e in 18 persone in un duplex o triplex.

La stanza preferita (Figura 14) o più usata dalla maggior parte delle persone (39 su 57) è il soggiorno. In base alle note lasciate sui questionari o durante le interviste è stato rilevato che in generale il soggiorno viene preferito poiché è uno spazio grande e complesso, dove fare diverse attività tra cui la vedere TV o ascoltare la musica, rilassarsi sul divano o sui tappeti, e dove, soprattutto, è possibile stare in relazione con i genitori o gli altri componenti della famiglia. Anche la camera personale è molto frequentata (30 persone di cui 16 la considerano la stanza preferita), non solo per dormire e rilassarsi, ma più che altro per fare le proprie attività, passioni e giochi; è stato infatti riscontrato che alcuni bambini/ragazzi dormono con uno dei genitori nella camera matrimoniale. La cucina è anche un ambiente frequentato spesso (27 persone), anche per la passione di qualcuno per la cucina. Il giardino è un altro ambiente abbastanza utilizzato (13 persone sulle 36 che possiedono uno spazio verde privato, di cui 3 lo considerato l'ambiente preferito) per il giardinaggio, ma anche per avere la libertà di muoversi e correre, fare diversi giochi, rilassarsi su un amaca o un dondolo, saltare sul trampolino o andare in piscina. Il bagno viene usato spesso da 9 persone e 3 lo considerano l'ambiente preferito; dalle interviste è emerso come talvolta può essere un ambiente di relax, per la dimensione contenuta e per la stimolazione data dall'acqua, per esempio attraverso la doccia. Molti hanno inoltre espresso il desiderio di possedere un bagno sensoriale o una sorta di "piccola spa" all'interno di questo spazio. La camera dei genitori viene frequentata spesso da 7 persone e 3 la considerano la stanza preferita in casa. Gli altri ambienti sono invece poco frequentati, in particolare camera dei genitori, garage, terrazzo, studio e lavanderia.

Le persona con autismo ha abilità molto variegate in base alla gravità dello spettro. Su un totale di 61 persone 28 sono in grado di stare completamente soli, senza sorveglianza, in tutti gli ambienti della casa, mentre 27 persone possono restare da sole solo in alcuni specifici spazi della casa e 6 persone non possono invece essere 
lasciate assolutamente mai da sole, in nessun ambiente. Tra i 27 sopra citati la maggior parte viene lasciato senza sorveglianza nella camera personale (18 persone) e in soggiorno (16 persone), mentre solo 10 persone vengono lasciate da sole nella camera dei genitori, 7 nella cucina, 6 nel bagno, 3 nel terrazzo, 2 in giardino, 1 nello studio e nessuno in garage e lavanderia. Le motivazioni sono legate soprattutto alla sicurezza, per quanto riguarda la casa nella sua totalità, o all'inadeguatezza dell'ambiente rispetto alle specifiche necessità del figlio/a. Alcuni hanno inoltre descritto la casa come un ambiente critico poiché non abbastanza grande o spaziosa, piena di oggetti "pericolosi" o che non si possono toccare, o non abbastanza silenziosa, soprattutto in rapporto ai vicini e al contesto abitativo. La cucina è considerato uno tra gli ambienti maggiormente critici, sia per i dispositivi elettrici e gli altri rischi insiti nella struttura di questo spazio, ma anche per coloro che ricercano continuamente cibo, per cui qualcuno ha richiesto dispositivi per permettere il blocco del frigo o della dispensa. Il bagno è stato inoltre segnalato come critico per la mancanza di autonomia nella gestione dell'acqua e dei dispositivi presenti. Durante le interviste inoltre sono stati indicati come ambienti critici nella casa anche gli spazi bui, come soffitta e cantina, i corridoi lunghi e il balcone, per motivi di pericolo, ma anche di disagio rispetto all'altezza.

A proposito dell'alloggio è stato poi chiesto se veniva frequentato spesso da ospiti, ma la maggior parte ( 32 persone su 52 ) ha indicato che riceve ospiti solo raramente. Le motivazioni principali sono la difficoltà di avere una regolare vita sociale, di trovare persone sensibili al problema e di andare a rompere le routines della persona con autismo; oltre a questo alcuni hanno specificato che avviene anche poiché non hanno uno spazio adeguato, magari troppo piccolo, o dove la persona con autismo non ha la possibilità ritrovare il suo spazio personale e di isolarsi ad esempio dal rumore delle persone o dai toni di voce, se non tollerati.

In generale 46 persone su 61 hanno affermato che la loro casa risponde ai bisogni di comfort della propria famiglia, ma d'altro canto 33 persone sarebbero disposte a rivedere la sistemazione del proprio alloggio (11 se avessero una disponibilità economica, 3 se coadiuvati da un adeguato supporto tecnico) e solo 13 hanno detto che va bene così com'è, 2 che una riprogettazione non sarebbe utile e 1 che assolutamente non vorrebbe. Nel complesso 36 persone (su 55) hanno detto che la progettazione degli ambienti di vita è sicuramente utile per le persone con autismo, di cui 5 persone per vivere meglio, 4 persone per aumentare l'autonomia, 27 per entrambe le motivazioni; 16 persone invece non hanno saputo rispondere al quesito e solo 3 persone hanno detto che non credevano la progettazione utile.

Un'altra tematica indagata è stato l'utilizzo delle assistive technologies per le acquisizioni di diverse abilità e autonomie. Nello specifico è stato chiesto alle persone se fanno uso di supporti alla comunicazione, programmi di apprendimento e di abilità per le autonomie domestiche, programmi ludici su tablet o supporti digitali, oppure sistemi di domotica e gestione della casa. Nel complesso fanno uso di sistemi di supporto alla comunicazione 35 persone (Figura 15), di cui 14 persone utilizzano regolarmente le PECS, 21 persone preferiscono il tablet e le applicazioni digitali, 14 persone usano le agende $\mathrm{e} i$ programmi visivi per le diverse attività, 11 persone fanno uso delle regole visive e 10 persone delle indicazioni e procedure step by step, soprattutto in bagno e cucina. Delle 26 persone che invece non ne fanno uso, 6 per- 


\section{Elena Bellini}

sone non conosco questi sistemi di comunicazione, 6 persone sono contrarie al loro utilizzo e 10 persone non ne hanno necessità, soprattutto poiché verbali. Per quanto riguarda invece i programmi di apprendimento, vengono regolarmente usati da 17 persone, 12 persone avrebbero piacere a farlo, anche se al momento non lo fanno, 4 persone non li conoscono, 7 non credono che siano utili e 2 sono contrarie al loro utilizzo. Infine solo 6 persone usano i programmi per le autonomie domestiche, 12 persone avrebbero piacere a farlo, anche se al momento non li usano, 8 persone non li conoscono e 10 persone non credono che siano utili. L'utilizzo di programmi digitali ludici da parte di 32 persone mostra comunque che non c'è resistenza nell'utilizzo delle tecnologie; 4 persone non li utilizzano ma avrebbero piacere a farlo, 4 persone non li conoscono, 10 persone non sono interessate, 2 persone pensano che non siano utili e solo 1 persona è contraria al suo utilizzo.

La domotica e la gestione automatizzata della casa non è molto conosciuta (24 persone hanno affermato di non sapere di cosa si tratta), poiché solo una famiglia la utilizza all'interno del proprio alloggio, ma 18 persone avrebbero molto piacere di usarla, se fosse accessibile economicamente. In particolare durante le interviste è stato riscontrato un notevole interesse, anche e soprattutto dalle persone adulte con autismo direttamente intervistate e da alcuni familiari, che lo considerano un notevole supporto per le autonomie quotidiane all'interno della casa. D'altro canto 12 persone hanno espresso che secondo loro non sono invece utili, poiché potrebbero impigrire la persona, oppure rivelarsi una criticità, soprattutto con i bambini, che prenderebbero il programma come un gioco.

In merito alle tecnologie di supporto alla persone sono stati valutati molto positivamente anche gli ambienti multisensoriali terapeutici e di rilassamento (Figura 16), che sono stati utilizzati da 21 persone e piacerebbero a 17 persone, anche se al momento non hanno ancora avuto la possibilità di sperimentarli. La maggior parte ne ha fatto uso in ambienti delle Associazioni o dei Centri per l'Autismo, qualcuno in musei e qualcuno in ambienti sanitari, ma durante le interviste (anche e soprattutto da parte degli utenti adulti con autismo) è stato appurato che molti avrebbero piacere di averli in casa propria, per rilassarsi e "regolare i propri sensi", anche nella forma del bagno sensoriale. D'altro canto ben 18 persone non sanno di cosa si tratta, dimostrando quindi che questi sistemi non sono ancora molto diffusi, mentre solo 3 persone credono che non siano utili.

Un'ultima sezione del questionario è stata infine rivolta agli ambienti sanitari per capire la frequenza di utilizzo da parte delle persone con DSA e le eventuali criticità all'interno di questi spazi, valutati nelle prime domande tra gli ambienti maggiormente problematici. Infatti, 39 persone hanno indicato che considerano andare in ambulatorio o in ospedale un vero e proprio problema (Figura 17). Le reazioni che si riscontrano solitamente (Figura 18) sono agitazione (32 persone), stress (20 persone), fastidio (19 persone), aumento delle stereotipie (12 persone), aggressività (9 persone), lamenti ( 9 persone), sofferenza (6 persone), rabbia (6 persone), ma anche curiosità ( 6 persone $)$, tranquillità ( 4 persone) e eccitazione (4 persone). Nel complesso la maggior parte delle persone (51 in totale) va regolarmente dal medico di famiglia (solo 8 persone non ci vanno mai); 26 persone ci vanno solitamente 1-2 volte all'anno, 12 persone fino a 5 volte, 1 persona fino a 10 e 2 persone anche oltre. Molti si sottopongono anche regolarmente a visite specialistiche, 30 persone rispetto 
alle 18 persone che non ci vanno mai durante l'anno; 20 persone fanno circa 1-2 visite specialistiche all'anno, 7 persone fino a 5 visite e 3 persone un numero di visite superiore alle 10. Il dentista rappresenta una maggiore criticità; 21 persone non ci vanno mai, mentre 30 persone ci vanno regolarmente, di cui 24 persone 1 o 2 volte all'anno, 4 persone fino a 5 volte, due persone anche fino a 10 volte all'anno. Gli esami in ambulatorio invece sono poco frequenti, probabilmente anche per le criticità dovute agli aghi o ad altre procedure mediche; 27 persone non fanno mai esami in ambulatorio, 16 persone fanno regolarmente esami 1 o 2 volte all'anno, 4 persone fino a 5 volte e 1 persona anche fino a 10 volte in un anno. L'ospedale è anche poco frequentato, sia il Pronto Soccorso, che presenta circa 1-2 accessi all'anno per 10 persone, fino a 5 accessi per 1 persona e fino a 10 per 1 altra (12 in totale, contro le 35 che non ci vanno mai); che gli interventi in Day Hospital, rilevate 1-2 all'anno per 3 persone e fino a 5 per 2 persone, contro le 43 che non ci vanno mai; che il ricovero, anche in questo caso mai frequentato da 41 persone e solo 3 persone che vi accedono 1-2 volte all'anno e 2 persone che hanno ricoveri fino a 5 volte all'anno. I reparti maggiormente frequentati sono quello odontoiatrico, neuropsichiatria infantile, pediatria, endocrinologia, fisiatria e ortopedia.

Come ultimo quesito è stato quindi chiesto di valutare quale intervento potesse essere maggiormente efficace per migliorare l'accesso in ambiente sanitario ed in particolare nel Pronto Soccorso (Figura 19), che come si è visto dalla letteratura è stato rilevato l'ambiente maggiormente stressante poiché è un luogo sconosciuto, poco prevedibile, dove i tempi sono veloci, le persone concitate, i rumori molto frequenti e spesso improvvisi, l'attesa è spesso molto lunga e in sale piene di persone, talvolta sofferenti. Gli interventi di miglioramento considerati maggiormente utili vanno a confermare queste ipotesi, ovvero sono state valutati positivamente: la riduzione dei tempi di attesa (51 persone su 55), che possibilmente dovrebbe avvenire in un ambiente dedicato, silenzioso e tranquillo (50 persone), con almeno un familiare o un caregiver sempre presente ( 49 persone) per rassicurare il paziente e fare da mediatore tra lui e lo staff dell'ospedale, facilitando al comunicazione e l'accettazione delle procedure mediche, oltre che la riduzione di stress e comportamenti problema; una stanza dedicata personale (48 persone), sia per gli esami che per la degenza; il personale ospedaliero adeguatamente formato sull'autismo (48 persone), sapendo come relazionarsi e comportarsi con il paziente, per facilitare la comunicazione e l'accettazione delle procedure mediche, oltre che la riduzione di stress e comportamenti problema; ambienti dedicati al rilassamento (43 persone), che possano servire da distrazione per i momenti di attesa e come abbattimento dello stress; oggetti e riferimenti familiari all'interno dell'ambiente (42 persone), al fine di rassicurare il paziente e farlo stare in un ambiente il più possibile simile all'ambiente casa; sistemi di privacy ( 39 persone), per stare più tranquilli, ma anche per non creare disagio ad altri pazienti; la presenza costante della luce del giorno (37 persone), considerata maggiormente positiva rispetto alla luce artificiale, anche perché aiuta a scandire il tempo che passa e i diversi momenti della giornata; la presenza di immagini, video e multimedia (37 persone), come distrazione e abbattimento dello stress, purché siano personalizzabili e regolabili dal singolo, altrimenti potrebbero rivelarsi un'altra fonte di stress; allo stesso modo la musica (33 persone); video che descrivono per step il protocollo delle diverse procedure di intervento medico, come il prelievo o altri e- 


\section{Elena Bellini}

sami diagnostici (35 persone), purché siano usati prima dell'accesso ospedaliero e non durante; una o più poltrone avvolgenti, cuscinoni, materassi o dondoli (33 persone) per il contenimento ed il rilassamento; segnaletica di orientamento attraverso colori e immagini (31 persone) per migliorare l'orientamento ed il wayfinding, ma anche consentire una maggiore integrazione tra i pazienti; ambienti ricreativi e ludici per favorire la socializzazione (30 persone), solo se e quando richiesti dalla persona; una luce calda e soffusa (29 persone), possibilmente regolabile, rispetto soprattutto alle luci fluorescenti e forti tipiche degli ambienti sanitari; colori tenui degli ambienti (28 persone) per ridurre il sovraccarico visivo e l'attivazione, che potrebbe sommarsi all'agitazione già presente nella persona; un sistema di comunicazione attraverso immagini (18 persone) per facilitare la comunicazione tra pazienti, familiari e operatori. Sono invece considerati poco utili spazi formativi (utili per 27 persone), poiché potrebbero per qualcuno essere fonte e accumulo di stress; elementi naturali, piante o pareti verdi (utili per 24 persone); elementi di oscuramento (utili per 26 persone) e penombra nelle stanze (utili per 24 persone); profumi ed aromi (utili per 26 persone), soprattutto poiché potrebbero rivelarsi fonte di stress per qualcuno, mentre sarebbe consigliabile avere un ambiente completamente inodore; superfici ed elementi tattili antistress (utili solo per 16 persone); personale medico senza camice (utile per 23 persone), poiché verrebbe meno la capacità di riconoscere gli operatori, comprendere la loro funzione e quindi avere una maggiore chiarezza.

\subsection{Interviste ad adulti con DSA ad alto funzionamento o Asperger}

Si può aver letto e studiato tutto il possibile sull'autismo, ma se non si è mai incontrato una persona con autismo non si può comprendere di cosa si sta parlando e, probabilmente, anche in questo caso si potrà solo dire di essersi appena affacciati su questo ampio mondo. Il momento più difficile, ma anche il più bello, di questo percorso di ricerca è stato quello delle interviste, poiché pur aver letto tantissimo mi sono accorta di essere totalmente impreparata a parlare con una persona del proprio autismo. Mi ha dato molta soddisfazione sentire F., una tra le ultime intervistate, che diceva "come hai fatto a chiedermi proprio la cosa che stavo pensando?"; non era una domanda strutturata nell'intervista, ma un'associazione banale che siamo abituati a fare in qualsiasi colloquio con un'altra persona nel momento in cui riesci ad entrare in empatia. Lungi da me pensare di essere realmente riuscita a comprendere il "mondo autistico" e che con un'altra persona sarebbe uguale, ma posso dire in quel momento di essere riuscita a comprendere F. Non si tratta che una piccola finestra aperta su un universo, soprattutto se si considera che la maggior parte delle persone con autismo non è in grado di comunicare, ma ci si può affidare solo alla mediazione da parte del genitore, che a sua volta spesso non è in grado di capire e comprendere a pieno il figlio/a. Questo però non vuol dire che non bisogna neanche provarci, poiché a volte ci si può stupire anche del contrario. Quando ho incontrato L. ero insieme alla psicologa che lo segue e ad un'educatrice; non è stato semplice avere altre persone presenti durante l'intervistata poiché crea una sorta di disequilibrio e rende l'incontro più formale e meno spontaneo. Ad ogni modo talvolta comprendo che è inevitabile e utile, poiché gli operatori conoscono a fondo i ragazzi che seguono, sono in grado di comprenderli anche quando non parlano e sanno come relazionarsi ed 
interpretare i loro gesti, oltre che le parole. Ho notato che spesso hanno molto timore delle interviste e hanno ragione, poiché potresti senza volere metterli a disagio o offenderli senza neanche accorgertene. In ogni caso mi avevano detto che L. non era in grado di esprimersi verbalmente, ma solo di dire alcune parole, che sarebbe stato meglio forse leggere insieme le domande e lasciare che L. rispondesse con il supporto di alcuni cartellini con scritto "si", "no", "non ho capito". Ho iniziato l'intervista con gli occhi di L. fissi a guardarmi e non si sono mossi fino a che non abbiamo finito, due grandi bellissimi occhi celesti che luccicavano. Non ha mai usato i facilitatori che avevamo preparato, ma mi ha sempre risposto a voce, a volte anche aggiungendo qualche piccola parola di risposta. Era felice di raccontarmi di sé, io ero felice di ascoltare, l'educatrice e la psicologa piacevolmente sorprese ed emozionate nel vederlo così coinvolto. Credo sia importante ogni tanto anche solo ricordare che non è vero che le persone con disabilità mentale non sono consapevoli e anche se purtroppo spesso non possono comunicarci quello che provano, sentano o pensano, forse bisognerebbe provare a stare vicino ed ascoltare, anche fosse solo un grande silenzio.

Ovviamente non è andata sempre bene, talvolta non sono riuscita ad avvicinarmi a loro con il pensiero, mi sono innervosita, sentita inadeguata e magari lo stesso anche per loro. Però credo che sia stato fondamentale dare un volto umano ad una ricerca che parla di persone.

\subsubsection{A. (maschio, 25 anni)}

A. vive la maggior parte del proprio tempo a casa, anche se è un luogo dove non sta bene poiché è affollata e caotica. Vi abita con 6 persone (i genitori, due fratelli piccoli e due nipoti), benché l'appartamento non sia molto grande, e non ha neanche una camera tutta sua. Ama la musica, soprattutto quella rock e quella classica, suona la batteria, che tiene nella sua camera, partecipando anche ad una band. Ama le macchine ed i motori ed ha anche la patente. E' appassionato di videogames e di tecnologia. Il suo ambiente preferito sarebbe la casa, se solo riuscisse ad avere il proprio spazio, proprio poiché potrebbe farvi tutte le sue attività. Per un periodo ha risolto stando sveglio la notte, quando gli altri componenti della famiglia stavano dormendo, e riposando invece durante il giorno. La scuola non gli piaceva, anche e soprattutto perché era costretto a rispettare degli orari; non era un buon ambiente e non lo considerava utile: "Essere obbligati ad andare in un luogo perché si deve fare, senza che sia utile...e per cosa?" Altri ambienti critici per lui sono gli ambienti sanitari, i negozi ed i centri commerciali, salvo che non sia per andare a comprare i suoi videogames o qualcosa che gli piace. Si sente bene nei luoghi all'aperto, per andare a fare passeggiate, soprattutto in montagna, dove talvolta va per i ritiri con la comunità religiosa dei mormoni a cui appartiene. Le sensibilità maggiori che ha sono uditive e visive. E' disturbato soprattutto dai rumori improvvisi e dalle voci dei bambini, poiché sono stridule e caotiche.. peccato che li abbia anche in casa! A livello visivo invece è soprattutto disturbato dal sole e dall'abbagliamento, ad esempio anche dei fari mentre guida; è invece molto a suo agio al buio. Ha sempre caldo, non gli piace la cucina poiché è una stanza sempre calda e non sopporta il riscaldamento. "Le piante sono fatte per stare in giardino e non nei vasi"; non ama passare il tempo a 


\section{Elena Bellini}

guardare fuori dalla finestra ma piuttosto stare lui direttamente in giardino. Gli piace tantissimo l'acqua, anche il rumore che fa quando scende. Non ha particolari preferenze di colore, gli piacciono i colori vivaci e anche i contrasti, ma per la casa crede che sia meglio un ambiente tutto chiaro. Assolutamente non vorrebbe un pavimento rivestito con parquet, poiché è terrorizzato che possa caderci l'acqua "passandoci lo straccio, o per una perdita dal radiatore", rigonfiare, deteriorarsi, rovinarsi, variare; non gli piacciono i materiali che non sono uniformi. Data la sua sensibilità visiva la luce è piuttosto importante, preferisce un'illuminazione diffusa, soft e possibilmente regolabile nell'intensità. Vorrebbe una casa silenziosa, possibilmente anche isolata.

Parlando invece degli ambienti ospedalieri ha considerato positivo un ambiente di attesa tranquillo e silenzioso, tempi di attesa ridotta, un familiare o caregiver sempre presente, una stanza dedicata, sistemi di privacy, riferimenti familiari, personale formato, segnaletica di orientamento con colori e immagini, profumo e aromi, musica e immagini e video per distrarsi e abbattere lo stress.

\subsubsection{L. (maschio, 21 anni)}

L. vive a casa con i suoi genitori, due volte a settimana va nella residenza di sollievo nella sua città per apprendere a vivere da solo e acquisire abilità quotidiane e autonomie domestiche, va a cavallo, fa terapia una volta a settimana e va due giorni a lavoro presso una struttura gestita dalla fondazione dove fa terapia, dove fanno mosaico, sia sotto forma di prodotti edili (come piastrelle e rivestimenti) che come forma di arte. Il luogo che preferisce in assoluto è quello dove lavora; gli ambienti che invece considera critici sono quelli che non conosce, i luoghi istituzionali e gli ambienti sanitari. Le sensibilità maggiori che ha sono quelle uditive e visive. A livello uditivo gli provocano disagio sopratutto i rumori forti e le voci alte e stridule. A livello visivo invece soffre soprattutto in casi di abbagliamento e preferisce una luce calda e soffusa, anche debole. Non gli piace il buio, ma apprezza la penombra per dormire e rilassarsi. Non ama gli spazi piccoli e raccolti, ma preferisce gli ambienti grandi; se vuole isolarsi per un po' sta nel soggiorno. Il soggiorno è anche il suo spazio preferito in casa, poiché li c'è la tv, i giornali e può stare insieme ai suoi genitori. Gli piacciono i colori accesi, il suo colore preferito è il verde, ma non vorrebbe che le pareti della sua stanza e della sua casa fossero verdi, ma piuttosto tutto bianco. Preferisce gli ambienti silenziosi, non solo per dormire ma anche in generale per vivere bene. Non ha particolare sensibilità a livello di temperatura ma in generale preferisce il freddo. Il vento gli provoca disagio, mentre adora l'acqua.

Dal punto di vista degli ambienti sanitari ha valutato tutti gli interventi positivamente, tranne avere una poltrona o un dondolo per rilassarsi, che ha ritenuto poco utile.

\subsubsection{F. (donna, 55 anni)}

Una donna molto speciale, molto consapevole della sua persona, delle sensazioni che prova e delle sensibilità sensoriali. Dirige due riviste e scrive libri, ha un marito e vive tra due città diverse. E' una persona iperattiva, dorme poco, ha sempre bisogno di far qualcosa. La sua stanza infatti è un 'laboratorio' dove può scrivere e da- 
re libero sfogo alla propria creatività; è appassionata di arti, origami, stoffe, materiali, musica e libri. Tra le altre cose mi ha mostrato un lavoro molto interessante che ha fatto, una sorta di telo/coperta cucito con tanti pezzi di stoffa diversi per toccare e sperimentare diverse textures.. è importante conoscersi! Il suo ambiente preferito è la casa; luoghi critici invece sono quelli sconosciuti, che le creano angoscia, e quelli affollati, come negozi o centri commerciali, poiché creano un dispendio di energia e quindi stanchezza. Ha una spiccata sensibilità all'udito; benché ascolti la musica altissima, anche nelle cuffie, e le piaccia il suono del tamburo, prova disagio per rumori quasi impercettibili, come fischi o ronzii, oltre ovviamente a rumori forti e soprattutto improvvisi. Ha una sensibilità fortissima anche al tatto, sia per i tessuti che per i materiali degli arredi o delle pareti; può anche arrivare a provare mal di denti o male alla nuca in situazioni di disagio sensoriale. Anche dal punto di vista olfattivo è sensibile, soprattutto per le sostanze chimiche come la formaldeide, che può causarle svenimento. Anche il contatto può crearle del disagio, soprattutto in alcune parti del corpo come a metà della schiena, che gli crea nausea. Ha sensibilità anche vestibolare e propriocettiva, ad esempio trovando difficoltà nel movimento, o avendo la tendenza a colpire gli spigoli o gli arredi; ha bisogno di molto spazio, di passaggi liberi, senza ingombri sul percorso. Le piacciono le linee curve, sarebbero adatte secondo lei per questa problematica e per favorire gli spostamenti. Gli ambienti devono essere funzionali, magari anche in sequenza. Le piace l'ordine, anche se è di natura disordinata, ha bisogno di non avere troppe cose o oggetti intorno, anche per un sovraccarico visivo. La cosa migliore sarebbe, secondo lei, avere dei mobili o ripiani a scomparsa nella parete dove poter mettere tutto, ma averlo a portata di mano. Preferisce degli ambienti neutri, anche come colori, poiché alla lunga possono stufare o irritare, anche se piacciono. Avere un ambiente neutro dai colori tenuti permette di poter giocare con i complementi; 'la cosa migliore potrebbe essere avere dei pannelli scorrevoli', da aprire e chiudere in base alla necessità in modo assolutamente flessibile. I colori scuri non le piacciono, soprattutto le creano disagio i pavimenti neri; non le piacciono i contrasti, né le superfici riflettenti. Non le piacciono gli elementi morbidi, sia per dormire che nelle sedute, tranne in alcuni momenti. Per dormire ha bisogno di una superficie dura, radicata a terra; infatti, dorme in un futon in cotone. I materiali degli arredi sono fondamentali, per la tattilità delle superfici, ma anche per le sostanze usate per le vernici e i colori, come detto precedentemente. E' molto sensibile anche ai tessuti, per cui ad esempio non può portare le calze, che non mette neanche in inverno, oppure non usa superfici morbide come il pile. Non sopporta il caldo, soprattutto del riscaldamento, di cui sente l'odore e le crea disagio; le piace invece moltissimo il camino. Gli odori sono infatti un altro aspetto importante, le piacciono i profumi, ma solo quelli naturali come ambra, lavanda, gelsomino; in ogni caso non deve mai essere troppo forte. A livello visivo è molto sensibile alla luce; le piace una casa luminosa, ma deve poter essere in grado di regolare l'intensità della luce e assolutamente non aver neon e luci fluorescenti. Ha la sindrome di Irlen associata (sindrome che impedisce l'elaborazione rapida, facile e corretta delle informazioni visive, presentando un'ipersensibilità verso una o più frequenze dello spettro luminoso), per cui consiglia l'utilizzo di occhiali con lenti colorate, molto utili per migliorare i disturbi di percezione visiva. Le luci colorate le piacciono molto e anche la penombra, che le consente di rilassarsi. Ha raccontato infatti come al rien- 


\section{Elena Bellini}

tro a casa, dopo una giornata di lavoro, sia importantissimo chiudersi in un ambiente buio per dieci-quindici minuti e potersi riequilibrare dopo il tempestamento di stimoli derivanti dal contesto esterno, 'un mondo che fa male'. Gli ambienti piccoli e raccolti le piacciono molto; in ogni stanza ricrea la sua "cuccia", ovvero un angolo dove racchiudersi per rilassarsi e sentirsi a proprio agio. Adora poi le piante ed il verde, che sono la sua passione. Trova disagio con i corridoi lunghi, anche perché non ne capisce il motivo e la funzionalità; allo stesso modo non apprezza open space o stanze polivalenti, ma piuttosto una chiarezza nella distribuzione delle diverse funzioni in diverse stanze. Ha bisogno di molto spazio personale (prossemica), soprattutto in rapporto con gli altri; ad esempio a casa con il marito ognuno ha la propria stanza e i propri spazi; le creano disagio inoltre gli spazi troppo stretti, come ad esempio gli spazi antibagno. Ha dei problemi con l'altezza, sia come vertigini, ad esempio dal balcone (preferisce un parapetto con superficie opaca, oppure se è una ringhiera consiglia di coprirla con fioriere), che con le scale (in particolare a chiocciola) nella discesa, che le crea un senso di instabilità. Non le piacciono inoltre edifici alti come $\mathrm{i}$ grattacieli, preferisce stare a piano terra e possibilmente in una casa con giardino. Apprezza il silenzio, anche per dormire, ed in particolare non sopporta i rumori del traffico. Le piace tutto ciò che è tecnologico, quindi le piacerebbe moltissimo poter avere una gestione automatizzata della casa attraverso sistemi di domotica, anche perché ritiene fondamentale poter controllare e personalizzare tutto l'ambiente. Ha avuto la possibilità di sperimentare degli ambienti sensoriali terapeutici e di rilassamento, anche presso l'associazione per l'autismo della sua città. La considera un'esperienza molto positiva e importantissima per "regolare i propri sensi"; "stare dentro la stanza per pochi minuti può dare beneficio per molto tempo, anche per un mese". Sarebbe importante avere un ambiente di decompressione anche in casa per riequilibrarsi dopo il contatto del mondo esterno e rispondere alla difficoltà data dagli spostamenti e dai cambiamenti di ambiente; la casa deve essere tranquilla e dare il pieno comfort e agio alla persona, poiché rappresenta il proprio spazio personale creato su misura per la propria persona e le proprie esigenze. Il bagno sensoriale potrebbe essere un'ottima soluzione, come una piccola spa in casa, gestita a livello tecnologico.

\subsubsection{V. (donna, 37 anni)}

V. è una mamma di un bambino autistico di 5 anni e mezzo, ha scoperto il suo autismo solo in seguito alla diagnosi del figlio, come spesso succede. E' un'impiegata, lavora part-time, ed è appassionata del ballo latino. Il suo ambiente preferito è la casa poiché c'è tranquillità e può avere sotto controllo l'ambiente in tutti i suoi aspetti. Gli unici luoghi che le creano difficoltà sono quelli molto affollati. Ha una sensibilità soprattutto uditiva, provando disagio per i rumori forti e improvvisi, ma soprattutto per i rumori di fondo come il brusio o le voci in generale, anche se conosciute, poiché le creano distrazione; ad esempio a lavoro non può ascoltare neanche la radio poiché perde la concentrazione. Apprezza i luoghi silenziosi, non solo per dormire ma in generale, e preferisce una casa isolata anche per avere privacy e non esser vista dall'esterno. Anche a livello olfattivo è molto sensibile, soprattutto rispetto agli odori forti, come ad esempio quelli della fattoria, che le creano nausea; d'altro 
canto non sente tutti gli odori, presentando in parte iposensibilità. A livello visivo prova disagio soprattutto verso le luci forti e l'abbagliamento. Le piace una casa molto luminosa, i colori accesi ed i contrasti (ad esempio tra pavimento e pareti). Le piacciono le superfici riflettenti, ma solo a casa, fuori non si specchia mai e prova molto disagio per le superfici riflettenti frammentate. Apprezza il buio, soprattutto per dormire, ma anche la penombra per rilassarsi. Preferisce gli ambienti aperti e spaziosi ed in particolare l'open space dove può tenere tutto sotto controllo; le piace spostare le cose e fare cambiamenti ma tutto deve essere fatto secondo il suo ordine, deve essere lei a decidere e la casa deve essere perfetta in ogni singolo dettaglio. Le piace isolarsi e le è utile per concentrarsi e fare le sue cose, ma lo fa sempre in un angolo del soggiorno o di uno spazio ampio, poiché non le piace stare chiusa in uno spazio più piccolo come ad esempio la camera, ma ha bisogno di uno spazio aperto. Per concentrarsi inoltre è meglio una luce non forte, casomai debole. Il suo ambiente preferito in casa, oltre al soggiorno, è la cucina poiché ama cucinare. Ambienti critici invece possono rivelarsi i corridoi molto lunghi, che le provocano disagio. Le piace il parquet e i pavimenti effetto resina, o se si usano delle piastrelle devono essere il più grandi possibili e senza fughe, poiché le creano disagio. Non sopporta il freddo, né il vento; adora invece l'acqua. Preferisce il riscaldamento radiante a pavimento, che permetterebbe di avere una superficie calda e confortevole a terra, e le piacerebbe avere il camino, anche per la tradizione legata al Natale che le piace moltissimo per la possibilità di mettere tante luci colorate e decorazioni. Soffre di vertigini, non ama l'altezza e non le piace fare le scale. E' una persona iperattiva, deve sempre far qualcosa sennò va in confusione. Ha avuto modo di sperimentare spazi sensoriali terapeutici e di rilassamento presso l'associazione per l'autismo che frequenta, soprattutto per il figlio. Ha apprezzato moltissimo questo ambiente, ha raccontato che le ha dato delle 'sensazioni che fino a quel momento non aveva mai provato, non aveva mai sentito un tale rilassamento, solo stando dentro quello spazio guardando le immagini dello scorrere della cascata'; 'non aveva nessun pensiero', 'anche se stava senza far niente riusciva a sentirsi bene'. Adora le tecnologia, le piacerebbe avere sistemi di domotica e gestione della casa, ad esempio avendo un grande tablet in ogni stanza; pensa che sarebbe molto utile anche per il figlio.

Per quanto riguarda il bambino, pensa inoltre che l'ideale sarebbe averlo sempre sotto controllo e avere sistemi di sicurezza come un lucchetto alla porta per non uscire, inferriate ed una protezione per il balcone (meglio un parapetto non opaco poiché si potrebbe arrampicare per affacciarsi, ma una ringhiera che consenta di guardare giù). La situazione ottima sarebbe poter avere uno spazio grande per il figlio, dove tenere i propri giochi, magari caldo a terra e rivestito con una superficie morbida e gommata per stare a terra e giocare, ancor meglio se collegato con il giardino dove gli piace molto stare. Anche lui adora l'acqua, gli piacciono gli animali e la natura, sporcarsi e toccare le superfici tattili.

\subsubsection{F. (maschio, 13 anni)}

F. vive a casa con i genitori, va a scuola ed è uno scout. Adora la natura e gli animali; il suo ambienti preferito infatti è il bosco. Tra gli ambienti critici ha segnalato la scuola, i luoghi rumorosi come i concerti e gli ambienti dove c'è un profumo 


\section{Elena Bellini}

intenso. Le maggiori sensibilità sono infatti quella olfattiva ed uditiva, benché non senta bene; non vede inoltre bene da lontano. Prova disagio soprattutto per i rumori forti ed il brusio di fondo, mentre gli piacciono i suoni della natura e si rilassa con la musica classica. Preferisce gli ambienti bianchi, ma non prova disagio per i colori forti o i contrasti. Gli piacciono le superfici riflettenti, non ha una particolare sensibilità alla luce (se fredda o calda ad esempio), ma soffre per l'abbagliamento della luce del sole e adora una luce che ha in camera, molto piccola, gialla e caldissima, "perfetta per leggere Dylan Dog”. E' attratto dai riflessi di luce o dai fasci luminosi. Si rilassa in penombra e preferisce il buio, soprattutto per dormire, per cui ha bisogno anche di silenzio. L'ambiente ideale per rilassarsi è buio, grande e fresco, ma anche per studiare e concentrarsi preferisce avere una luce molto bassa e molto silenzio. Il suo ambiente preferito in casa è il bagno, poiché è piccolo e bianco ed è un luogo dove i suoi genitori lo lasciano libero e tranquillo; anche se è piccolo è tutto suo e a volte ci studia anche poiché ci si trova a proprio agio e si può concentrare. La stanza che gli provoca più disagio invece è la cucina, poiché ha una parete gialla che stona con il bianco del resto della casa, e un piatto decorato sul muro che gli dà fastidio, lo vede come un po' inquietante. In generale la casa ha le pareti tutte bianche e il pavimento con piastrelle di colore grigio; gli piace molto così perché è semplice e pulito e il grigio è un bel colore poiché non dà fastidio agli occhi. Gli piacciono sia il freddo che il caldo, ma non troppo perché gli dà fastidio, però considera piacevole avere una superficie riscaldata; infatti gli piacerebbe molto avere un sistema di riscaldamento radiante a pavimento per poter camminare in casa a piedi nudi anche d'inverno. Non usano in casa il condizionatore come sistema di raffrescamento, ma gli piacerebbe, anche se è fondamentale pulirlo bene. Adora l'acqua, gli piace il contatto, ma non la pressione, come ad esempio un abbraccio. Non vorrebbe avere sistemi di domotica e di gestione automatizzata della casa, poiché crede che impigriscano la persona. Ha le idee molte chiare su come dovrebbe essere una casa ideale: 'dovrebbe avere due stanze in più, ambienti di circa 10-20 mq, un computer, una libreria, un posto per studiare e per i videogiochi, una palestra per allenarsi, due bagni, di cui uno dovrebbe essere con la vasca ed uno senza'. Non ha mai avuto esperienza di sistemi sensoriali terapeutici e di rilassamento, ma gli piacerebbe poterli provare.

Dal punto di vista degli ambienti sanitari non ha particolari criticità, ma in generale ha considerato utili tutti gli interventi proposti, tranne: avere riferimenti familiari, avere un sistema di segnaletica e di orientamento per colori e immagini, avere la luce del giorno, avere una luce calda e soffusa, diffondere un profumo o un aroma, poter toccare superfici tattili o antistress, oppure guardare e arricchire l'ambiente con video, foto, immagini o elementi visivi.

Non ha problemi di interazione con gli altri, ha anche la ragazza. E' stato bello infatti continuare a parlare, dopo l'intervista. Avevamo ancora un po' di tempo e l'intervista era stata abbastanza veloce perché aveva risposto in modo molto chiaro e sintetico ai quesiti che gli avevo posto. Dopo essere rimasti qualche minuto in silenzio, dopo aver finito le domande, F. mi ha chiesto 'le è mai capitato di fallire ad un esame?'. F. si riferiva al suo amico, con cui avevo parlato prima di lui, che stava affrontando le difficoltà del primo anno di università. Voleva sapere se è comune avere difficoltà all'inizio e fare i primi esami, fonte di grande stress e ansia per il suo 
compagno. Gli ho detto che si, era normale avere timore le prime volte, o anche sempre; ad esempio gli ho raccontato delle mie difficoltà agli esami orali, dovendosi relazionare con il professore e superare l'ansia del confronto visivo. F. mi ha detto 'ti capisco, succede sempre anche a me'. E' stato in quel momento che abbiamo iniziato a parlare, senza avere domande strutturate, delle cose di tutti i giorni, delle passioni, del tempo libero, degli amici e delle ragazze. Un colloquio informale talvolta può essere più utile a livello empatico di tante altre domande.

\subsubsection{D. (maschio, 20 anni)}

D. vive in casa con i genitori e la sorella, va all'università e frequenta ingegneria informatica; l'informatica è anche il suo hobby. L'ambiente preferito è la casa, la camera soprattutto, poiché è molto introverso e chiuso e trova difficoltà a relazionarsi con gli altri. Gli ambienti che considera maggiormente critici sono i luoghi affollati e rumorosi, tra cui i negozi e gli esercizi commerciali, e gli ambienti sanitari. Pensa che l'ambiente scolastico non sia adeguato alle esigenze delle persone con autismo; ad esempio le aule studio sono poche e non adatte, le pareti sono tutte bianche, sarebbe utile che vi fosse qualcosa pensato per lo stress pre-esame. Lo mettono a disagio gli ambienti tutti bianchi, anche se non ha particolare sensibilità verso i colori. Preferisce la luce fredda e gli piace la luce colorata, quando fatta bene, in particolare di colore arancione. Non tollera la luce del sole e l'abbagliamento e apprezza situazioni di penombra. La situazione ideale sarebbe poter avere una luce regolabile attraverso diversi punti luce, piuttosto che una regolazione attraverso dimmer. Un tipo di luce che gli crea disagio è quella con accensione a sensore; ce né una alla casa dei vicini e non gli permette di guardar fuori poiché é fastidiosa. Gli crea disagio inoltre anche il riflesso della luce; ad esempio i vicini hanno un disco per spaventare gli uccelli, che considera fastidioso e non gli permette di guardare fuori dalla finestra. Prova disagio per rumori forti, improvvisi, brusio di fondo e tonalità sia di bassi che di alti, anche nelle voci delle persone, che non tollera quando sono piatte e ad una sola frequenza; non è particolarmente interessato alla musica. I rumori lo infastidiscono soprattutto poiché perde attenzione e possono influire quindi su comprensione e apprendimento. La casa dovrebbe essere un luogo silenzioso, nel suo alloggio per esempio non tollera il rumore dei vicini, ma non con silenzio assoluto. Non sopporta il caldo mentre gli piace il freddo; crede che sia importante comunque poter regolare la temperatura degli ambienti. Gli piacerebbe avere il camino e uno spazio esterno, come il giardino. Gli piace molto la sensazione di pressione e contenimento, per cui apprezza arredi e poltrone morbidi e avvolgenti. Gli piace il vento, soprattutto sotto forma di brezza, e l'acqua, anche come pioggerellina. a livello olfattivo non apprezza né i profumi, né gli odori forti, solo se corrispondo ad un buon cibo. I movimenti veloci, i cambi di postura e di direzione lo disorientano; prova disagio per altezza e gravità, non ama le scale, la salita e la discesa e ha paura dell'ascensore. Non gli piacciono gli ambienti grandi o molto alti, ogni stanza della casa dovrebbe essere diversa per adeguarsi alle esigenze specifiche dell'ambiente ed in particolare vorrebbe poter arredare la propria camera (non ha una camera propria, ma dorme con la sorella); le porte delle stanze non devono far rumore. "La casa ideale è quella dove si sta bene". 


\section{Elena Bellini}

Per quanto riguarda gli ambienti sanitari, dato che rappresentano una criticità per lui abbastanza importante, ha valutato molto utili tutti gli interventi proposti. Come suggerimenti integrativi ha indicato di usare l'azzurro alle pareti, poiché è un colore rilassante; mettere una musica new age poiché è rilassante; inserire elementi verdi, ma non piante troppo grandi; usare video di preparazione alle procedure mediche, ma spiegate in modo adeguato, anche visivo; migliorare i sistemi di segnaletica ed orientamento.

\subsubsection{C. (maschio, 17 anni)}

C. vive in Francia, fa il liceo scientifico e poi andrà all'università. Va a nuoto, fa palestra, suona il piano e fa teatro. I luoghi che preferisce sono la casa, la scuola e tutti i posti dove ci sono i suoi amici. Gli ambienti che ritiene maggiormente critici invece sono quelli molto affollati, come ad esempio i campus universitari, i negozi e gli esercizi commerciali. E' molto sensibile soprattutto al tatto, all'udito, all'olfatto e al gusto. Gli piace molto la musica e l'ascolta a volume molto alto, ma prova disagio per i rumori forti e improvvisi e per il brusio di fondo. A livello visivo prova disagio invece per gli ambienti completamente bianchi, il sole e l'abbagliamento. Gli piacciono i profumi e sente molto gli odori, che prevalgono sul gusto. Prova disagio anche per i movimenti veloci del corpo e della testa, per le rotazioni su se stesso, il dondolamento, i cambi di direzione e di postura, le situazioni di equilibrio e instabilità e la pressione sul corpo. Le situazioni di disagio e stress, ed in particolare i rumori, influiscono su orientamento, comprensione, concentrazione, apprendimento e interazione con gli altri. Gli piacciono gli elementi morbidi e le superfici tattili, il vento, l'acqua e gli elementi naturali. Gli piacciono gli elementi visivi, video e multimedia, i colori accesi, le superfici riflettenti, i forti contrasti e le luci colorate . Preferisce una luce calda e non forte; apprezza una situazione di penombra per rilassarsi e luoghi di isolamento, contenuti e raccolti. Non ama il freddo, ma preferisce il caldo. Il suo ambiente preferito in casa é la camera personale, il più critico invece la soffitta perché é buia. Non ha preferenze su materiali e colori di pavimenti e pareti, ma considera positiva una differenziazione tra i diversi ambienti. Utilizza sistemi di domotica in casa per la gestione delle luci e pensa che siano piuttosto utili. Non ha mai sperimentato sistemi multisensoriali, ma gli piacerebbe molto farlo. Secondo lui dovrebbero essere spazi o elementi sensoriali anche a casa per rilassarsi, ad esempio una sedia che faccia un massaggio corporeo, della musica classica e in ogni caso spazi ampi. Per quanto riguarda gli ambienti sanitari non ha particolari difficoltà, ma ritiene che tutti gli interventi proposti siano utili, salvo la colorazione tenue degli ambienti e l'utilizzo di elementi naturali, piante o pareti verdi.

\subsubsection{D. (maschio, $45 \mathrm{nni})$}

D. è padre di una ragazza autistica di 24 anni e ha avuto la diagnosi da pochi mesi. Vive a casa con la moglie, la figlia ed il cane, non lavora al momento ed ha sempre avuto un po' di problemi nell'ambito lavorativo, infatti sta compiendo le pratiche per ottenere un'invalidità legata alla diagnosi di autismo. E' un tipo molto attivo e sportivo, ha moltissimi hobby tra cui camminare, giocare a calcio, fare giardi- 
naggio, cucinare, fare decoupage e tutto ciò che riguarda l'informatica. E' inoltre una persona molto religiosa, come tutta la sua famiglia. L'ambiente che preferisce é la casa, mentre considera come maggiormente critici tutti i luoghi affollati, anche all'aperto se vi sono troppe persone, come ad esempio alla stazione. E' molto sensibile a livello uditivo, rispetto ad alcuni tipi di rumori ma soprattutto al sovraccarico dovuto al sentire contemporaneamente diversi suoni, come ad esempio in cucina l'acqua aperta con il rumore della ventola della cappa. Prova disagio con i rumori forti e improvvisi, ma anche continui, come ad esempio il suono dello scrosciare di una cascata; non tollera il rumore di treni e macchine. In questi casi si sente disorientato e perde la concentrazione. E' sensibile a livello tattile, soprattutto con i tessuti. A livello olfattivo non sopporta ad esempio l'odore del forno, gli piacciono invece i profumi, gli odori della campagna e gli aromi del legno. A livello visivo prova disagio per la luce del sole e l'abbagliamento. Preferisce una luce calda, gli piacciono le luci colorate ed una situazione di penombra per rilassarsi. Deve dormire al buio completo o alla luce del giorno, ma non vedere assolutamente i riflessi del sole che entrano, poiché ne è terrorizzato. Anche i fasci di luce, come si vedono ad esempio in discoteca, gli provocano disagio; da giovane ci andava per stare con gli amici ma non si sentiva a proprio agio. Gli piacciono i colori accesi ed i forti contrasti mentre prova disagio negli ambienti completamente bianchi e asettici. A casa ad esempio ha realizzato dei disegni a parete, dato che é anche molto creativo. Gli piace il vento e moltissimo l'acqua. Preferisce il caldo e non ama il freddo. Gli piacciono gli ambienti ampi e anche molto alti, come ad esempio la chiesa, ma apprezza anche i luoghi più raccolti per dei momenti di isolamento. Apprezza molto l'ordine e la simmetria e preferisce gli ambienti luminosi. Il suo spazio preferito in casa è il soggiorno poiché un ambiente composto. Un ambiente critico é invece la cantina, poiché è cupa, e prova disagio con i corridoi lunghi poiché ricordano le corsie degli ospedali ed è uno spazio che non ha funzione. Tende a riempire la casa, è un collezionista di modellini e calamite. E' sensibile anche a livello gustativo e vestibolare e trova disagio soprattutto nei movimenti del collo. Ha avuto esperienza di sistema sensoriali, ad esempio in alcuni centri benessere e gli piacciono moltissimo. Dovrebbero secondo lui esserci anche a casa, come ad esempio un bagno sensoriale, una vasca idromassaggio, la musica, i profumi, ecc. E' un appassionato di tecnologia, di informatica e di tutti gli elettrodomestici; se fosse maggiormente accessibile a livello economico gli piacerebbe molto avere un sistema di domotica per la gestione della casa.

\subsubsection{M. (maschio, 55 anni)}

L'intervista con M. è stata più che altro rivolta alle sensibilità sensoriali, per cui non sono state affrontate alcune tematiche come quella del domicilio o degli ambienti sanitari, ma è stata ad ogni modo utile alla comprensione di molti aspetti percettivi.

M. ha avuto la diagnosi di autismo da pochissimo tempo, a seguito della diagnosi del figlio, come spesso accade. Era un serramentista, ma al momento è disoccupato. E' abituato a riparare tutto, ha molte passioni e collezioni, tra cui la musica. Suona,aveva un gruppo rock e ha cantato nel coro liturgico della parrocchia per sedici anni. L'ambiente che preferisce è la residenza poiché lì si sente a proprio agio. La 


\section{Elena Bellini}

casa in generale deve essere funzionale ed ordinata. Gli ambienti che invece considera maggiormente critici sono i luoghi affollati, anche all'aperto come ad esempio i concerti, e gli ambienti sanitari. Non tollera gli odori medici, né quelli artificiali, come ad esempio la benzina; nel traffico è costretto a chiudere l'auto. I muri alti gli creano un senso di dispersione e disorientamento e si trova a disagio nelle grandi città, dove si sente soffocare. A livello uditivo prova disagio soprattutto sentendo l'eco ed in particolare se sordo. La musica è sempre piacevole, poiché emoziona. Il rumore che considera più fastidioso è l'abbaiare dei cani, ad ogni modo prova disagio ai rumori forti e persistenti come un antifurto, un martello pneumatico, ma anche il ronzio. Non sopporta le frequenze intermedie e prova fastidio nel sentire toni di volte molto alti o piatti e monotoni. Soffre molto il caldo, non gli piace l'umido, né il bagnato. Ha molta sensibilità tattile per cui deve avere sempre vestiti comodi, preferisce le superfici lisce, mentre non tollera quelle ruvide, né la polvere. Trova il sole piacevole, preferisce in generale la luce calda e diffusa, ma per concentrarsi e lavorare pensa sia meglio quella fredda e concentrata; la situazione maggiormente favorevole è avere diversi punti luce, in modo che sia controllabile e non vi siano mai punti bui. La casa deve essere tutta bianca e illuminata, i contrasti vanno usati con moderazione e se si usa il colore deve essere comunque un colore caldo. Non gli piace il buio e preferisce anche dormire avendo il riferimento visivo alla luce del giorno. Ha una fissazione molto forte per i dettagli ed è estremamente preciso. Soffre di vertigini ed ha molta paura dell'altezza. Ha bisogno di muoversi, non sopporta l'idea di essere in qualche modo vincolato, né le situazioni di stress che lo opprimono. Gli piace stare in equilibrio ed essere elastico, è appagante avere la gestione del proprio corpo.

\subsection{Interviste ai familiari di bambini e ragazzi con DSA}

Per comprendere il punto di vista di bambini o ragazzi con autismo a basso funzionamento e non verbali è stato necessario mediare attraverso il colloquio con i genitori. Queste interviste sono state comunque moto utili anche per comprendere il punto di vista dei familiari stessi, che spesso non vengono considerati in ambito progettuale, mentre sono anch'essi componenti del nucleo abitativo, con diverse esigenze e necessità. Questo aspetto non è da sottovalutare in ambito di adattamento domestico e non solo dal punto di vista dei genitori, ma anche da quello dei fratelli e delle sorelle che condividono con loro gli spazi di vita.

"Leo

Mio fratello

Leo è diverso

Eppure gli voglio bene

ancora. Picchia, si tiene per i gomiti, mastica cannucce. Io

affronto tutto questo

perché sono la sua sorella maggiore" (Zelly Rosa, cit. in Silberman, $2015^{588}$ ).

588 Silberman, S. (2015) Neuro Tribes. The legacy of Autism and the Future of Neurodiversity, AVERY, New York (ed. italiana 2016) 


\subsubsection{Mamma di A. (femmina, 5 anni)}

A. vive con i suoi genitori e il fratello, distante di soli 18 mesi, in una casa troppo piccola e poco confortevole. Per questo motivo infatti stanno per cambiare casa e realizzare un nuovo alloggio adatto a tutte le loro esigenze, pensando anche al domani, al 'dopo di noi'. Nella nuova casa infatti ci saranno due piani e già i genitori hanno immaginato che un domani nella taverna a pian terreno potrà esserci un appartamento per A. ed eventualmente per una persona che si prende cura di lei, con il fratello che magari vive sopra. A. è una bambina iperattiva, cammina sulle punte, non parla, presenta comportamenti ristretti e ripetitivi, routines, stereotipie motorie, come lo sfarfallio della mani, e verbali, come riprodurre l'eco della sua voce, disturbi del sonno e forti disturbi alimentari. Ha spiccate sensibilità sensoriali, uditive, tattili, olfattive, gustative e vestibolari. Ha una sensibilità a doppio canale, ovvero per alcune sensazioni prova molto disagio, mentre ne ricerca altre come stimolazione sensoriale che le dà piacere. A livello uditivo le provocano disagio soprattutto i rumori forti e improvvisi e la confusione; se parlano insieme troppe persone si infastidisce e si tappa le orecchie, soprattutto se sono rumori continui e se sono voci o urla stridule di bambini (ad esempio ai compleanni). La musica alta invece la rilassa, in particolare quella classica. Il tatto è una sensazione che le crea molto piacere, tocca sempre gli oggetti e le superfici, in particolare i muri se a finitura ruvida o spatolata. Si agita e si attiva con i colori accesi (come ad esempio il rosso), che le creano eccitazione e solitamente scappa e si va a rifugiare al buio; apprezza infatti molto la penombra o il buio per rilassarsi. Gli ambienti bianchi ed asettici sarebbero per lei molto adatti poiché privi di stimoli. E' molto sensibile alle luci e all'abbagliamento, spesso si vede che strizza gli occhi e con il sole si attiva e si eccita; d'altro canto ricerca una stimolazione visiva attraverso lo sfarfallio delle mani davanti agli occhi. Le piace il vento, "è un vero e proprio animale ad acqua", le piace rilassarsi con elementi morbidi e soffici, come materassi e cuscinoni, o contenersi e comprimersi in coperte e "abbracci alla Temple Grandin" e le stimolazioni sensoriali date dalle luci colorate. Ha avuto modo di provare uno spazio sensoriale presso il Muse di Trento e le è piaciuto moltissimo. Non è attratta invece dagli elementi naturali, non guarda neanche gli animali. Gli ambienti che preferisce in casa sono il soggiorno, dove può stare sul divano e guardare la tv, la camera dei genitori, dove si rilassa completamente e dove dorme, e il bagno, dove solitamente va quando è stressata per farsi una doccia. La camera personale è l'ambiente più critico, poiché è troppo piccola e non ci vuole stare, infatti neanche ci dorme. Il fratello soffre molto di questo e del fatto che non giochi con lui; i momenti più belli sono quelli in cui stanno insieme nel lettone, si cercano e si abbracciano. Nella nuova casa avranno ancora una camera doppia, poiché è bene che stiano insieme, ma molto grande così che siano rispettate le loro esigenze. $\mathrm{Ci}$ sarà inoltre una stanza dedicata ad $\mathrm{A}$. e alle sue attività, come ad esempio fare terapia con la mamma; è importante che abbia un luogo tutto suo. Nella nuova casa applicheranno inoltre a parete delle foto per comprendere l'utilizzo dei diversi spazi. Un altro elemento di criticità per la camera è anche dovuto alla luce, poiché c'è un'unica grande finestra che non si può oscurare; una delle crisi più forti che ha avuto è stata proprio a causa della luce e, per dormire, ha bisogno del buio completo. Suggerisce inoltre di non avere vetrate grandi in casa, usare materiali opa- 


\section{Elena Bellini}

chi in casa e assolutamente non il cristallo. Il silenzio anche è molto importante; meglio una casa isolata con giardino, senza contatto diretto con i vicini, per A. ma anche per i vicini stessi quando durante le notti A. si agita molto. Meglio una casa tutta su un piano, ma con una taverna al piano terreno per ospitare gli amici, così che A. più sempre avere un luogo più isolato e silenzioso sopra, soprattutto per dormire. Prova disagio per altezza e gravità, ha problemi in discesa soprattutto poiché sta sulle punte e quindi è pericoloso, ricerca l'equilibrio, non salta. I disturbi sensoriali sono causa, oltre che di disagio e stress, di impatto sull'orientamento, la comprensione, l'apprendimento, la comunicazione e l'interazione con gli altri.

Per quanto riguarda gli ambienti sanitari considera utili tutti gli interventi proposti, tranne eliminare il camice, migliorare la segnaletica di orientamento per colori o immagini, inserire spazi formativi o ricreativi e di socializzazione. Suggerisce inoltre che per migliorare la loro condizione sarebbe utili che i bambini potessero portare le loro cose e tutto ciò che li possa aiutare ad assecondare le loro abitudini, per non aumentare lo stress; tra questi per esempio il cuscino per dormire, oppure le proprie stoviglie per mangiare o bere, poiché altrimenti potrebbero non farlo.

\subsubsection{Mamma di E. (maschio, 14 anni)}

E. vive in casa con i genitori (sono in 4 persone in totale in casa), va a scuola (16 ore a settimana), fa terapia per 7 ore a settimana, fa atletica ed equitazione, partecipa alle attività dell'oratorio, come passeggiate o attività extra-scolastiche, e vi resta anche per i pasti dal lunedì al venerdì. E' molto sensibile a livello uditivo; da piccolo addirittura percepiva il suono dell'interruttore della luce e si svegliava nella notte, avendo oltretutto disturbi del sonno importanti. Lo disturbano anche i rumori forti e improvvisi e le voci alte. E' molto sensibile anche a livello visivo, in particolare alle luci, e non dorme se non c'è buio completo; preferisce la luce calda e regolabile. E' molto sensibile ai cambiamenti, tutto deve essere sistemato secondo il suo ordine; le porte e gli sportelli ad esempio devono essere sempre chiusi poiché questa è la loro funzionalità. Il suo ambiente preferito è la casa, poiché è un ambiente familiare, dove ha le sue cose, è libero e si può rilassare per rilassarsi; gli serve anche per recuperare il sonno, ad esempio il fine settimana dorme anche nel pomeriggio. I luoghi maggiormente critici invece sono quelli dove c'è affollamento e confusione, ancor peggio se sono stretti e angusti. Ad esempio nella vecchia scuola aveva qualche criticità a entrare poiché l'ingresso era piccolo ed il bancone era vicino all'entrata, per cui non aveva modo di prepararsi prima di entrare; nella nuova scuola invece c'è un ingresso molto ampio e non ha problemi. Preferisce invece gli ambienti grandi, anche i corridoi, lunghi, dove può correre liberamente. Hanno una casa molto grande su tre piani e questo ad esempio gli piace molto poiché può andare su e giù e cambiare ambiente, anche per evitare la noia. Ci può essere tensione invece in luoghi nuovi e sconosciuti, specialmente se non ha il tempo di adattamento alla transizione di ambiente. In casa sta bene in ogni spazio poiché in ogni ambiente ha il suo angolo personale con le sue cose. L'importante è vivere in un ambiente ordinato, pulito e organizzato; anche il contesto abitativo è importante, che sia sereno e sicuro, ad esempio con molte piste ciclabili dove poter andare insieme in sicurezza e tranquillità. 
Gli ambienti sanitari sono molto critici per lui, è un luogo imprevedibile, solitamente c'è molto da attendere, le persone parlano troppo e fanno tante domande. La cosa migliore sarebbe avere una corsia preferenziale di accesso ospedaliero, tempi di attesa brevi e una sala di aspetto tranquilla e silenziosa. Avere poi una stanza dedicata e sistemi di privacy, un familiare o caregiver sempre presente e poter portare le proprie cose. I video dimostrativi delle procedure mediche sono molto utili, ma come preparazione all'accesso ospedaliero, non durante poiché non servirebbero. Sarebbero utili inoltre spazi di rilassamento, formativi e ricreativi di socializzazione, soprattutto ludici.

\subsubsection{Mamma di F. (maschio, 15 anni)}

F. vive in casa con i genitori (sono in 4 persone in totale in casa), va a scuola ( 25 ore a settimana), fa terapia per 5 ore a settimana e deve iniziare musica. Il suo ambiente preferito è la casa poiché è il suo ambiente, pensato ad hoc per lui, che è oltretutto molto legato alle sue routines, e dove non ha disagio a livello uditivo. Gli ambienti maggiormente critici sono quelli affollati, dove c'è traffico, confusione e imprevedibilità. Per lo stesso motivo creano difficoltà anche gli ambienti nuovi, per cui ad esempio vanno sempre allo stesso bar o ristorante. E' particolarmente sensibile a livello uditivo, soprattutto rispetto a rumori forti e improvvisi. Se ci sono troppi rumori o persone che lo guardano si agita, pensa che tutto sia rivolto a lui anche se le persone parlano tra di loro o fanno rumore mentre mangiano, può arrabbiarsi ed avere reazioni anche violente; attraverso le cuffie si calma, le usa a casa anche quando ci sono ospiti ed è stanco. Gli ospiti non vengono spesso infatti, poiché disturbano la routine e invado il suo spazio personale, di cui ha bisogno. Anche a livello visivo è molto sensibile alla luce, soprattutto non tollera la luce fredda e forte, che lo stanca molto; lo disturbano inoltre anche i toni troppo chiari o troppo scuri di colore. $\mathrm{E}^{\prime}$ inoltre sensibile a livello olfattivo, gustativo e vestibolare. In ogni caso riesce a superare il disagio quando fa cose interessanti o che gli piacciono; ad esempio a scuola sopporta anche la luce sbagliata e fastidiosa poiché sta con i suoi compagni. Prova fastidio anche con la luce del sole, infatti preferisce uscire la sera; d'altro canto però gli piace guardare i raggi che entrano dalla finestra e i granelli di polvere che si vedono volare. Gli piace la luce colorata, infatti ha una luce multicolore a fibre ottiche in camera, e la penombra per rilassarsi. Non ha bisogno di buio totale per dormire, ma appena sveglio la mattina non sopporta i contrasti di luci, quindi solitamente va prima in bagno dove le finestre sono più piccole per abituare pian piano gli occhi alla luce. Preferisce gli ambienti ridotti perché più sicuri, anche per momenti di isolamento. Ha avuto esperienza di ambienti sensoriali dal dentista, dove c'era una stanza con musica, luci colorate e acqua. Era molto attratto, anche se forse invece che rilassarlo lo ha eccitato, e l'ha preferite di gran lunga alla stanza dei giochi. Il problema casomai è che non voleva più venir via, voleva spostare le lampade fatte a sfera luminosa ed il dentista era preoccupato che rompesse qualcosa; forse per lui potrebbe essere utile dare l'accesso allo spazio dopo l'intervento come rinforzo o ricompensa. Vivono in una casa colonica molto grande, la sua stanza preferita è il soggiorno, ma sta spesso anche in cucina e nella camera personale, spazio dove dormire e avere privacy. Usa molto spesso anche il bagno, per cui sarebbe meglio 


\section{Elena Bellini}

che fosse in camera, dato che capita di andarci molto frequentemente anche di notte. In questi ambienti può stare anche da solo. La casa deve essere funzionale, meglio se ad un solo piano poiché è pigro a fare le scale. Gli ambienti sanitari sono veramente molto critici per lui, a causa dei rumori dei toni delle persone e degli sguardi insistenti. Non sa esprimere i propri malesseri (si esprime con poche parole o frasi), ha il terrore degli aghi per un trauma subito da piccolo, non tollera i camici bianchi ed è molto sensibile alla bocca e alla parte posteriore della testa per cui non si fa toccare, non si fa neanche tagliare i capelli se non dalla madre. Quando è in tensione reagisce e colpisce e adesso è molto grande e forte quindi può rappresentare davvero un problema. Per gli interventi medici, ma anche per il dentista, è necessario sedarlo, anche se avendo la fobia degli aghi sono costretti a pre-sedarlo con un sedativo liquido per bocca. Per tagliarsi i capelli e farsi la barba ha aiutato un po' l'i-pad in modo da distrarsi. Per superare il trauma dei camici bianchi ha invece seguito un programma e adesso é molto migliorato. D'altro canto il problema del dentista non è ancora stato superato, soprattutto poiché sono gli stessi dentisti che non vogliono prenderlo in cura; ha dovuto cambiare Regione e aderire ad un programma apposito (Progetto Orchidee). Rispetto agli interventi di miglioramento proposti sono stati considerati tutti utili, salvo avere superfici tattili o antistress. Si è suggerito inoltre di dare accesso agli ambienti di relax solo dopo l'intervento medico, come incentivo o ricompensa; rendere gli spazi formativi e ricreativi facoltativi poiché potrebbe esserci un rifiuto; rendere la musica personalizzabile e regolabile personalmente, poiché potrebbe aver bisogno anche di silenzio assoluto; rendere l'ambiente sanitario il più possibile familiare.

\subsubsection{Mamma di E. (maschio, 6 anni)}

E. vive a casa con i genitori, va a scuola (30 ore settimanali), fa terapia per 4 ore alla settimana e va in piscina, che adora poiché ama l'acqua. La piscina è infatti il suo ambiente preferito, mentre trova un po' di criticità a casa, alla fondazione dove fa terapia, negli ambienti sanitari, nei luoghi istituzionali e in tutti luoghi che danno l'idea di "andare dal medico", gli ambienti che non conosce e i luoghi affollati dove c'è confusione, come negozi ed esercizi commerciali. Gli piace stare all'aperto, è iperattivo ed ha continua necessità di stimoli come muoversi, fare le scale, cambiare ambiente; ha un'andatura sulle punte e come stereotipia fa dei piccoli saltelli. Gli piacciono molto le porte scorrevoli e tutto ciò che è automatico. E' molto rigido verso i cambiamenti, le abitudini e le routines, i percorsi e gli ambienti; ad esempio le porte devono stare sempre chiuse e luci accese, legate alla loro funzionalità. Deve fare gli spostamenti in estrema velocità, non è capace di attendere. Ha una sensibilità sensoriale a livello uditivo, visivo, tattile, olfattivo, gustativo, propriocettivo e vestibolare. Prova disagio per i rumori forti e improvvisi, ama la musica, anche se è molto selettivo, e gli piace avere un sottofondo continuo che siano cartoni animati o video di recite registrate. Un ambiente completamente bianco può essere utile per concentrarsi, poiché ha pochi stimoli. Ha bisogno del buio totale per dormire la notte e apprezza la penombra per rilassarsi, anche se gli piace la luce del sole (la va a cercare), i giochi luminosi e le luci colorate; in casa preferisce una luce calda e diffusa. Preferisce gli ambienti grandi per svagarsi, ma quelli più piccoli e contenuti per ri- 
lassarsi. Per calmarsi, quando ha delle crisi, usa l'i-pad, i video e la musica. La casa per il momento risponde ai loro comfort, anche se per il futuro avrebbero bisogno di più spazio, vorrebbero un edificio isolato, con un grande giardino e su più piani, per avere le scale. All'interno della casa sta molto in soggiorno, dove ha i tappeti con i suoi giochi e può guardare la televisione; passa il tempo libero nella camera personale, uno spazio completamente libero dove costruire i suoi giochi, come ad esempio una tenda da cui entrare con un tunnel attraverso il letto, o usare il computer; dorme invece nella camera dei genitori con il padre, poiché non vuole mai stare da solo (neanche il pomeriggio) e anche probabilmente poiché nella cameretta non riescono ad avere buio completo; un luogo ambito è inoltre la taverna poiché c'è la stufa a pellet, che ama guardare, e il basculante di apertura; gli piace moltissimo anche il giardino, dove ha la casa di legno, l'altalena, lo scivolo, un laghetto con un mulino, la piscina (in estate) ed il trampolino elastico. Al contrario l'ambiente più critico è il bagno dove non vuole assolutamente andare, se non per farsi la doccia, poiché adora l'acqua.

Gli ambienti sanitari sono molto critici, per cui ha considerato utili tutti gli interventi proposti. Il suggerimento più importante è che "assomiglino il più possibile alla casa".

\subsubsection{Mamma di B. L. e B. M. (maschi, 4 e 11 anni)}

B. L. e B. M. sono due fratelli con autismo di 4 e 11 anni, vivono in una famiglia numerosa, con i genitori, un altro fratello di 10 anni e una sorella di 6 anni con probabili tratti autistici. B. M. ama studiare, leggere i libri, stare al computer e televisione; va benissimo a scuola, addirittura era in crisi in quarta poiché sapeva più delle maestre. B. L. andrà a scuola il prossimo anno, già scrive e legge e parla 4 lingue (cinese, russo, spagnolo, inglese), che impara guardando i video con il tablet. Lui è iperattivo, gli piace giocare, star fuori, correre e soprattutto la piscina, poiché adora l'acqua; presto inizierà anche a fare nuoto. Anche per i loro interessi, B. M. preferisce la casa, mentre B. L. preferisce i luoghi all'aperto. I luoghi maggiormente critici sono gli ambienti sanitari e dove c'è caos e confusione; nei negozi o al centro commerciali si sono abituati imparando ad attendere, con un rinforzo ad esempio a fine spesa. Vanno ovunque, anche in bar e ristorante, ma scelgono dei posti adatti, che siano ad esempio racchiusi, in modo che siano liberi di muoversi senza scappare, e che non siano pieni di persone; hanno un bar dove vanno di solito ad esempio che é perfetto poiché ha una sala molto grande, sempre mezza vuota e con dei grandi divani dove stendersi. Le sensazioni a cui sono maggiormente sensibili sono quelle uditive e visive. B. L. non tollera i rumori forti e improvvisi e prova un po' di fastidio anche per il brusio; gli piace la musica ma talvolta può creargli disagio; non ama i forti contrasti, ad esempio con un pavimento a scacchiera potrebbe decidere di non camminare su alcuni riquadri di colore; gli creano disagio il sole e l'abbagliamento; gli piace molto il caldo e non tanto il freddo; gli piacciono il vento, l'acqua, gli elementi naturali, le luci colorate, gli elementi morbidi ed i colori accesi, anche a parete; preferisce gli ambienti grandi dove può correre, come ad esempio un grande campo da calcio che hanno vicino casa dove può stare liberamente; è ordinatissimo, apprezza l'open space ma solo per la stanza del soggiorno, mentre è bene che le altre 


\section{Elena Bellini}

funzioni siano tutte separate, in particolare per lo studio, poiché ha bisogno di un ambiente più contenuto e tranquillo. B. M. invece adora la musica; non tollera i rumori forti e improvvisi e le voci stridule, come al voce della sorella; gli piacciono i colori tenui e anche quelli scuri, poiché ha una mente un po' più cupa; preferisce la luce calda e diffusa a quella fredda e apprezza la penombra; gli piace l'acqua, gli elementi morbidi, gli elementi naturali, le luci colorate; gli piacciono sia gli ambienti grandi, che quelli più piccoli e contenuti per isolarsi un po'; è anche lui molto ordinato. B. M. e B. L. stanno in camera con gli altri due fratelli, alternando un figlio più tranquillo con uno più "birichino", ma crede che sarebbe stato meglio il contrario, anche perché sono entrambi ordinatissimi e non sopportano il disordine dei fratelli. Per migliorare l'ordine possono essere utili anche mobili lisci a scomparsa o a incasso, in modo da avere una massima pulizia nella casa. Stanno ristrutturando una nuova casa dove presto si trasferiranno molto più grande, in campagna, con uno spazio esterno enorme. In questo nuovo alloggio ognuno avrà la propria camera personale matrimoniale; vi sarà poi un bagno per i maschi più grande, un bagno per la bambina, uno per i genitori e uno per la zona giorno; la cucina sarà grande e organizzata con una penisola che consenta di separare l'accesso ai fuochi e migliori quindi la sicurezza. Entrambi preferiscono stare il soggiorno e possono stare da soli, senza sorveglianza, nella loro cameretta (a turno ovviamente), dove si racchiudono nei momenti in cui vogliono isolarsi un po'. E' fondamentale avere una stanza ed un bagno personale (come avranno nella futura casa) e un ambiente dove potersi isolare quando c'è bisogno, nei famosi 'cinque minuti', magari con i propri giochi e le proprie cose, tranquillo, silenzioso e isolato. E' importante comunque che la casa sia silenziosa ed avere almeno un angolo (interno o esterno), dove potersi rilassare. Sarebbe bello poter avere in casa anche una palestra e una piscina interna, poiché al cosa più importante è muoversi e fare sport per sfogarsi e stare meglio. Benché la loro casa sia tutta bianca considera utile poter differenziare gli ambienti in base alla diversa funzione. Una nota importante è quella di non usare materiali vinilici o lucidi a pavimento, poiché danno l'impressione che ci sia l'acqua e darebbero problemi a camminarci sopra per paura di affogare. Il legno invece è un materiale molto positivo.

In ambito sanitario ci sono per entrambi numerosi criticità ed è sempre stato una tragedia qualsiasi tipo di visita o intervento medico. Tutti gli interventi proposti sono stati valutati positivamente, salvo la presenza di medici senza camice, poiché al contrario devono essere riconoscibili; luce calda e diffusa, poiché preferisce la luce bianca; la diffusione di profumi e musica; e la manipolazione di elementi o superfici tattili.

\subsubsection{Mamma di A. (maschio, 10 anni)}

A. vive a casa con i genitori e la sorellastra di 25 anni (figlia della madre dal primo matrimonio), va a scuola, fa sport e terapia. L'ambiente preferito è la casa, la camera ed il soggiorno in particolare, ma va volentieri anche a scuola. Ambienti critici sono invece quelli affollati e confusionari, come negozi ed esercizi commerciali, anche perché non tollera l'attesa, e quelli sanitari, ma solo se riguardano lui; quando è andato in ospedale per l'altra sorella che ha partorito non ha avuto problemi, salvo per il pianto del bambino. Le sensazioni che sente con maggiore intensità sono quel- 
la uditiva (non tollera i rumori forti e improvvisi e le voci alte o stridule) e olfattiva (prova disagio per gli odori forti e sgradevoli; in bagno ad esempio spruzza sempre il deodorante appena entra) e prova anche un po' di fastidio per la luce del sole e l'abbagliamento. Inizia adesso a rendersi conto del caldo e del freddo, ma ha problemi a comprendere la mezza stagione, soprattutto per gli indumenti. In casa preferisce il caldo, va da solo ad accendere il riscaldamento, anche perché vuole stare senza indumenti; non fa la stessa cosa con il condizionatore in estate, forse sopporta meglio il caldo del freddo. L'ordine in casa non esiste, ma i suoi giochi devono essere dove e come dice lui, non si possono toccare né spostare. Ci vorrebbe uno spazio personale tutto suo con i giochi, dove poter lasciare tutto com'è ma anche chiudere se viene qualcuno così che i giochi sono al sicuro e non si agita o si arrabbia; in questo modo sarebbe più semplice anche il rapporto con gli altri bambini. Ha problemi ad esempio con il figlio della sorella di 5 anni, poiché non vuole condividere i suoi giochi; in questi casi vanno al piano superiore nella casa dei nonni, poiché è un terreno neutro e così non hanno difficoltà. La camera invece viene usata solo per dormire; A. dorme nella camera matrimoniale con la mamma, mentre il babbo dorme nella doppia con la sorella. L'idea è quella di creare lo spazio per i giochi nella stanza dove adesso dorme la sorella, non appena andrà via di casa, oltre a realizzare un piccolo bagno, che sarebbe per lui molto utile, in corrispondenza del grande corridoio che non ha funzionalità. In generale A. non vuole troppa luce, poiché pensa che $\mathrm{i}$ giochi di rovinino e 'si sciolgano'; per lo stesso motivo non porta i suoi giochi fuori.

Andare in ambienti sanitari è causa di criticità; per fortuna hanno trovato un dentista molto disponibile che lo asseconda; ha due studi, ma preferiscono quello dove c'è una sola stanza poiché in quello con più ambienti vuole andare in giro e perlustrare tutto. Considera positivi gli interventi proposti di miglioramento, salvo i colori tenui degli ambienti, che dovrebbero essere invece a misura di bambino; l'uso di una luce calda e soffusa; l'uso di profumi e aromi, poiché potrebbero anche dare fastidio; elementi naturali e superfici tattili; spazi formativi poiché sarebbero un'ulteriore fonte di stress. Sono molto positivi gli ambienti di relax, anche perché spesso gli capita (anche a scuola durante la ricreazione) di volersi isolare e stare un po' da solo, anche per riequilibrarsi. Immagini video e musica di sottofondo sono positive, ma con la possibilità di essere personalizzate.

\subsubsection{Mamma di E. (maschio, 6 anni)}

E. vive a casa con i genitori e la sorella di 4 anni, il prossimo anno andrà a scuola, fa terapia due volte a settimana, ha un legame molto forte con l'acqua, infatti va in piscina, e fa un corso propedeutico di musica. L'ambiente dove sta maggiormente a suo agio è la casa, anche perché libero e non strutturato, mentre sono luoghi critici gli ambienti istituzionali e i negozi, poiché ha difficoltà ad attendere ed è una richiesta continua. Ha sensibilità spiccata a livello uditivo, olfattivo e gustativo, mentre è iporeattivo a livello visivo e cerca stimolazioni ad esempio muovendo le mani davanti agli occhi, guardando il sole, o attraverso le luci, anche colorate. Gli piace molto la musica, anche se a volte quando è stanco non la sopporta; si infastidisce per i rumori forti ed improvvisi e anche per le voci alte: se si alza la voce urla, evita $i$ gruppi di persone che parlano e si tappa le orecchie. E' attratto dalle superfici riflet- 


\section{Elena Bellini}

tenti, si specchia molto spesso, usando anche i riflessi dei vetri; in soggiorno hanno dovuto toglierlo poiché quando era arrabbiato ci andava contro. Gli piacciono il vento, l'acqua, il sole e gli elementi naturali. Gli piacciono gli estremi, fare il bagno nell'acqua gelata ma bere l'acqua bollente. Per rilassarsi cerca luoghi dove isolarsi e stare in penombra, ad esempio si chiude negli armadi o nelle scatole, o si avvolge nel tappeto. Gli piacciono sia gli ambienti piccoli e raccolti, che quelli grandi ed alti; ad esempio la chiesa gli piace molto, la chiama 'il castello' e chiede di andarci. In generale ricerca le emozioni molto forti, gli piacciono le cose diverse e particolari. In casa la stanza che preferisce è il soggiorno, sta spesso anche in camera, ma per i suoi giochi, non per dormire. La notte infatti dormono tutti insieme nella camera matrimoniale, un letto doppio con mamma e E., uno per il babbo e un lettino per la sorellina; per adesso non riesce a dormire da solo, un domani vorrebbero fare una camera doppia per i bambini. Avrebbe in generale bisogno di una camera tutta sua, per stare da solo e non essere controllato. Adesso lo fa al piano di sopra chiudendosi nell'armadio, ma lo fa al massimo per dieci minuti, poi non ama stare da solo. Se manca per più tempo vuol dire che è andato in bagno a mangiare il sapone! Di solito sta in soggiorno, una stanza molto grande, aperta ma composta da diversi spazi: un angolo tv, un angolo pranzo ed una parte più protetta dove ci sono tutti i suoi giochi, controllata ma più isolata. L'ambiente maggiormente critico è la cucina, poiché tende ad arrampicarsi ovunque per ricercare cibo; servirebbe un sistema di blocco per chiudere frigo e dispensa. In ogni caso va controllato e al momento non può essere lasciato mai da solo, senza sorveglianza. Hanno un sistema di riscaldamento con termocucina a legna ed un condizionatore per il raffrescamento, ma gli dà un po' fastidio a causa del ronzio e lo sveglia durante la notte. La casa è un vecchio fienile di campagna, hanno moltissimo spazio esterno e fanno anche l'orto, anche se non partecipa molto poiché non ama sporcarsi le mani. Per quanto riguarda gli ambienti sanitari ha molte criticità, ha provato con i programmi di training ma non funzionano nel momento in cui cambia contesto. Il dentista è un problema anche perché non riesce a farlo sedere, neanche sul lettino. Ha molta sensibilità alla testa, è un problema anche tagliarsi i capelli, probabilmente infastidito dal ronzio del rasoio elettrico; ora riesce a farlo la mamma con le forbici dandogli un Dvd come rinforzo. La cosa più importante in assoluto, in ambiente ospedaliero, è l'attesa breve e in un ambiente dedicato tranquillo e silenzioso. Una stanza personale sarebbe utile anche per non dare fastidio agli altri, poiché non starebbe mai fermo a letto, tenderebbe a voler andare a giro per l'ospedale. La luce del giorno è utile soprattutto per comprendere la sequenza delle giornate e quando è il momento di dormire. Per quanto riguarda i profumi è meglio che sia inodore. La musica potrebbe essere positiva, ma meglio avvisarlo poiché potrebbe non essere il momento giusto; anche i video e le immagini potrebbero essere positivi. Si immagina una stanza di degenza con un angolo con i giochi, un tavolino, una poltrona, un dondolo o un cuscinone, elementi stimolanti per attrarlo e farlo rimanere nella stanza. Sarebbero sicuramente utili ambienti di relax, magari anche una palestrina, mentre quelli ricreativi o di socializzazione sono funzionali solo se guidati da un adulto, poiché non riesce ad interagire con gli altri se non attraverso attività strutturate. 


\subsubsection{Mamma di M. (maschio, 5 anni)}

M. vive a casa con i genitori (in totale sono in 4 in casa), va volentieri a scuola, ma preferisce la casa per le sue routines ed abitudini. Ha delle criticità solo negli ambienti che non conosce. Presenta iper-sensività agli stimoli uditivi come rumori forti, improvvisi, brusio di fondo, voci alte e stridule, ma ricerca anche stimolazioni, essendo attratto dall'eco, facendo versi e rumori. Ricerca anche stimolazioni di tipo tattile, gli piacciono gli elementi morbidi, quelli da manipolare o anche le diverse superfici tattili. A livello visivo preferisce la luce calda, i colori tenui, non troppo forti né contrastati, senza troppi disegni o decorazioni, poiché altrimenti fa confusione. Ricerca anche in questo caso stimolazioni visive, attraverso lo sfarfallio delle mani. Non tollera molto il caldo, né la luce forte di abbagliamento. Un ambiente completamente bianco potrebbe aiutarlo a concentrarsi. Gli piacciono sapori considerati solitamente spiacevoli, come ad esempio adora mangiare il sapone, non tanto per l'odore ma per la sensazione di bruciore. Preferisce gli ambienti grandi, a quelli più contenuti che ricercava più da piccolo, anche perché gli piace intrattenersi cercando l'eco o correndo. Gli spazi polivalenti potrebbero creargli disagio e disorientamento e potrebbe non accettare il cambiamento, facendogli confusione; meglio che ogni spazio abbia la sua funzione specifica. Il suo ambiente preferito è il soggiorno e la camera personale; non viene mai lasciato da solo, senza sorveglianza, casomai un po' in camera ma non per troppo tempo. Per dormire ha bisogno del buio totale e ha bisogno di un ambiente casa silenzioso. Usa molto anche il giardino, come gioco e sfogo, anche andando in piscina. La casa deve essere ordinata, semplice e pulita. Non devono esserci arredi e sistemi complessi, ogni cosa deve essere sott'occhio. E' rigido rispetto agli spostamenti, ha una sua programmazione mentale di tutte le cose che non deve variare. Un sistema di domotica e gestione automatizzata della casa sarebbe molto utile, soprattutto da adulto.

Per quanto riguarda gli ambienti sanitari si considerano piuttosto critici; quello che spaventa di più è il dentista, non ci è ancora mai andato, ma solo l'idea è un vero incubo. I programmi di training, ad esempio per gli esami del sangue, sono stati molto utili, ma tutto deve corrispondere esattamente al video di preparazione. La prima volta che è stato, prima di effettuare il programma, hanno dovuto reggerlo in tre e pesava solo $16 \mathrm{~kg}$ ! Tutti gli interventi di miglioramento proposti sono stati rilevati utili, tranne la diffusione di profumi (meglio un ambiente inodore), la presenza di medici senza camice, elementi naturali e superfici tattili. La musica deve essere personalizzata e non rappresentare un sottofondo continuo, altrimenti diventa rumore; lo stesso per i video e le immagini, è bene che siano personalizzate, anche scelte direttamente da internet. I colori, se tenui, possono rassicurare ma potrebbero anche confondere; dato che è una situazione di stress é meglio non avere nessuno stimolo. In particolare è utilissima un'attesa ridotta, possibilmente quasi nulla, e in un ambiente adeguato. Un sistema di comunicazione per immagini è molto utile, anche attraverso tablet. 


\subsubsection{Mamma di E. (maschio, 18 anni)}

E. vive in casa con i genitori (sono 5 in totale in casa), frequenta l'IPSIA, istituto professionale meccanico (30 ore settimanali), va in piscina, fa atletica, gli piace andare in bici e fa terapia per 4 ore, due volte settimana. Non ha un ambiente in particolare che preferisce, né critico, salvo il traffico. Quando ha delle tensioni ha bisogno di isolarsi. A scuola ad esempio preferisce il refettorio alla stanza di sostegno, poiché è un ambiente grande, la luce viene dagli shed in alto, le pareti sono azzurre, può sentire l'aroma del caffè proveniente dalle macchinette e può stare da solo, senza gli altri ragazzi. Ha sensibilità di tipo uditivo (rumori forti e improvvisi, voci alte e stridule; se sente rumori che gli danno fastidio si tappa le orecchie), tattile (per i tessuti e gli oggetti, in particolare la ruvidità gli dà rilassamento), olfattiva (attratto dai cosmetici e dai saponi, tende anche a mangiarli), gustativa e propriocettiva. E' rigido nei cambiamenti e sull'ambiente; le luci ad esempio devono stare tutte spente se non servono a qualcosa nello specifico. Dorme in camera con il fratello, che però adesso è all'università dal lunedì al venerdì ed associa la disposizione della camera (letti uniti o separati) alla presenza o meno del fratello; ad esempio è capitato che i letti fossero rimasti uniti ed era disorientato dal fatto che il fratello invece fosse a casa. Tutto deve essere ordinato e sotto controllo. Soffre molto il caldo ed ha bisogno sempre degli occhiali da sole. Ha paura del buio durante gli spostamenti, come nel corridoio, ma va a ricercarlo quando a bisogno di rilassarsi o per dormire. Per rilassarsi ha necessità di un spazio raccolto, lo trova in ogni ambiente diverso, lo sceglie e poi sta sempre lì. Si trova a disagio però negli spazi piccoli se deve condividerli con altre persone; gli piacciono gli ambienti grandi anche perché può giocare con la voce e l'eco (stereotipia verbale).

Andare in ambienti sanitari è un aspetto molto critico, deve essere sedato, ma ha la fobia degli aghi. Il disagio non è tanto legato all'ambiente ma alla motivazione di andarci per la cura, poiché ad esempio in visita ad un nuovo nato non ha avuto nessun problema, ma piuttosto era curioso ed eccitato. Per quanto riguarda gli interventi proposti ritiene che siano tutti utili tranne che il personale non abbia il camice; vi sia segnaletica per immagini o colori di orientamento; venga preparato con video sulle procedure mediche, poiché anche se toglie l'imprevedibilità aumenta l'ansia di ciò che avverrà; la musica o i video, poiché possono distrarre, ma anche eccitare; la penombra; gli elementi naturali; le superfici tattili o antistress, benché forse potrebbero essere utili per le lenzuola, che sono rigide ed hanno un odore sgradevole. La cosa più utile come distrazione sono gli ambienti di rilassamento e quelli ludici, ma ci vorrebbero dei giochi come per i bambini anche per gli adulti. La formazione del personale è inoltre importante, soprattutto per quanto riguarda il tono della voce, che fa il 50\% almeno dello stress percepito.

\subsubsection{Mamma di D. (maschio, 7 anni)}

D. vive a casa con i suoi i genitori, va a scuola (11-20 ore settimanali), fa terapia due volte a settimana e ama andare in bici. Il suo ambiente preferito è la casa, ma gli piace molto stare anche a scuola e in fondazione dove fa terapia. Ambienti critici sono invece per lui i luoghi che non conosce, i negozi e gli esercizi commerciali e 
gli ambienti sanitari. E' sensibile a livello sensoriale soprattutto dal punto di vista uditivo ed in particolare rumori forti e improvvisi, voci alte e stridule; gli piace la musica, ma comunque non a volume alto. Preferisce gli ambienti bianchi e neutri, senza ornamenti, neanche i quadri. E' rigido rispetto ai cambiamenti, tutto deve essere ordinato e sotto controllo e lui deve essere il 'regista del cambiamento'. La sua stanza preferita in casa è la camera personale, mentre non ci sono luoghi che creano disagio o dove non sta da solo, senza sorveglianza. Apprezza i luoghi raccolti e la penombra per rilassarsi, ma non il buio completo, mentre è molto importante che la casa sia silenziosa. Andare in ambienti sanitari è piuttosto problematico per lui. Ha provato in Romania un'esperienza sensoriale in odontoiatria ed é stata molto utile; c'erano in particolare dei video proiettati sul soffitto per distrarre il paziente durante

l'intervento. Sarebbe secondo lei interessante provare degli ambienti sensoriali anche per vedere quali stimolazioni andrebbe a scegliere e quali preferisce. Gli interventi di miglioramento proposti in ambito sanitario sono stati tutti considerati molto utili.

\subsubsection{Mamma di M. (maschio, 17 anni)}

M. vive a casa con i genitori (sono 4 in totale in casa), va a scuola (per 33 ore a settimana), va in piscina, fa terapia per 3 ore e lavora 1 ora a settimana presso una struttura gestita dalla fondazione dove fa terapia, dove fanno mosaico, sia sotto forma di prodotti edili (come piastrelle e rivestimenti) che come forma di arte. Il suo ambiente preferito è la casa, poiché è familiare e rassicurante e ha tutte le sue cose. Non ha un autismo importante quindi non ha particolari criticità in nessun luogo, salvo quelli che non conosce, ma se viene preparato non ci sono problemi. E' molto sensibile invece a livello sensoriale, dal punto di vista uditivo, soprattutto per rumori forti e improvvisi; visivo, in particolare è infastidito dalle luci forti e dal sole; e tattile. Gli piacciono sia gli ambienti grandi che quelli più contenuti e raccolti per i momenti di isolamento. Gli piace molto la grande stanza open space che hanno con soggiorno, cucina e sala da pranzo, dove addirittura va in monopattino. Gli piace molto anche la sua camera dove si isola per rilassarsi e ha anche un bagno personale, benché preferisca quello dei genitori (forse perché è azzurro).

Presenta alcune criticità negli ambienti sanitari, non tanto per l'ambiente, ma per lo stato fisico in cui si trova. Considera utili tutti gli interventi proposti tranne la diffusione di profumi e aromi, mentre sarebbe meglio un ambiente inodore; la musica, che può tranquillizzare, ma non deve essere sempre presente; la presenza di ambienti formativi, poiché rappresenterebbero un ulteriore stress se sta male.

\subsection{Colloqui con caregivers ed esperti sull'autismo}

E' stato utile durante il percorso di ricerca parlare con esperti sull'autismo, per avere un supporto, soprattutto nella prima fase, in ambito medico e psicologico, nella comprensione della sindrome e dei sintomi e come avvicinamento al problema. In particolare dott. Ugo Romualdi (ANFFAS Firenze), dott. Hayek (ospedale Le Scotte Siena), presidente Luciano Pieri (PAMAPI Firenze), dott. Daniele Mugnaini (PAMAPI Firenze), dott. Michele Boschetto (PAMAPI Firenze), dott.ssa Cinzia Raffin (Fondazione Bambini e Autismo Onlus di Pordenone), dott.ssa Marianna Fi- 


\section{Elena Bellini}

lippini (Fondazione Bambini e Autismo Onlus di Pordenone), dott. Bertelli (centro di ricerca CREA), Dott. Antonia Parmeggiani (UNIBO), Dott. Fabiola Cesarini (UNIMORE), Dott. Raffaella Faggioli (Officina Psicoeducativa, Milano), Dott. Riccardo Atzei (Bambini in Movimento, Firenze), dott.ssa Lorenza Fontana (ANGSA Novara-Vercelli Onlus), dott.ssa Stefania Ucelli di Nemi (Cascina di Rossago), dott.ssa Patrizia Galletti (Agrabah Pistoia), Presidente Alvaro Gaggioli (Agrabah Pistoia), dott. Angela Volta (ANGSA Parma), dott. Leonardo Granchi (Casa di Ventignano), Presidente Cinzia Pieraccini (Casa di Ventignano). D'altro canto il punto di vista delle Associazioni, psicologi, educatori e caregivers che ne fanno parte e che vivono e lavorano tutti i giorni con persone con autismo è stato fondamentale per comprendere le scelte quotidiane, dall'adattamento domestico, all'organizzazione del centro e degli spazi per la terapia, ai trattamenti applicati, alle terapie occupazionali, all'avvicinamento al lavoro, fino alle residenze assistite e alle soluzioni per il 'dopo di noi'. E' stato utile inoltre per avere un confronto rispetto alle famiglie o ai ragazzi intervistati, risultando evidente come $\mathrm{i}$ punti di vista tra $\mathrm{i}$ diversi interlocutori fossero molto diversi e tutti utili ed interessanti. In particolare, ho avuto modo di confrontarmi con Anffas Onlus - Associazione Nazionale Famiglie di Persone con Disabilità Intellettiva e/o Relazionale - Firenze, ANGSA La Spezia Onlus - Associazione Nazionale Genitori Soggetti Autistici, ANGSA Liguria, ANGSA Novara Vercelli, ANGSA Parma, Casa di Ventignano - centro diurno per soggetti con autismo, Centro abilitativo per disturbi di spettro autistico PAMAPI - Firenze, Bambini in movimento - Firenze, Fondazione Bambini e Autismo Onlus - Centro Operativo di Pordenone, Fondazione genitori per l'Autismo - Cascina Rossago, Fondazione Marino per l'Autismo Onlus, Associazione SUD - Foggia, AGRABAH - Pistoia, Associazione Davide e Golia - Firenze. Nel capitolo dei casi studio saranno descritte alcune di queste struttura come residenza assistita innovativa. In alcuni casi è stato inoltre possibile parlare con architetti che avevano seguito la progettazione dei centri per l'autismo, tra cui arch. Beretta (Cascina di Rossago) e arch. Giovanni Berti (Casa Sebastiano).

Infine è stato molto utile, per quanto riguarda l'ambiente ospedaliero dialogare con il dott. Scarone dell'Ospedale San Paolo a Milano, a proposito del Progetto DAMA, uno dei pochi programmi italiani dedicati di accessibilità ai servizi sanitari per la disabilità e per l'autismo, e il dott. Hayek e il dott. De Felice del Policlinico Le Scotte a Siena, uno dei più importanti reparti di neuropsichiatria infantile a livello italiano e internazionale, in particolare per quanto riguarda l'autismo e la sindrome di Rett. In entrambe le realtà è stata esplicita l'esigenza di una riqualificazione degli spazi sanitari al fine di adeguarli alle esigenze delle persone con autismo; a Siena inoltre è stata esplicitata l'esigenza di avere un ambiente multisensoriale dedicato ai pazienti e le loro famiglie, o anche un reparto tutto strutturato in modo "sensoriale", al fine di ridurre lo stress ospedaliero, che in questo ambiente raggiunge livelli veramente molto alti. 
Ambienti sensoriali "terapeutici" che rendano Abili

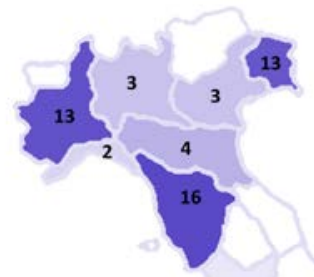

1

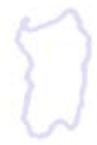

Figura 1. Quadro delle risposte al questionario diffuse sul territorio italiano.

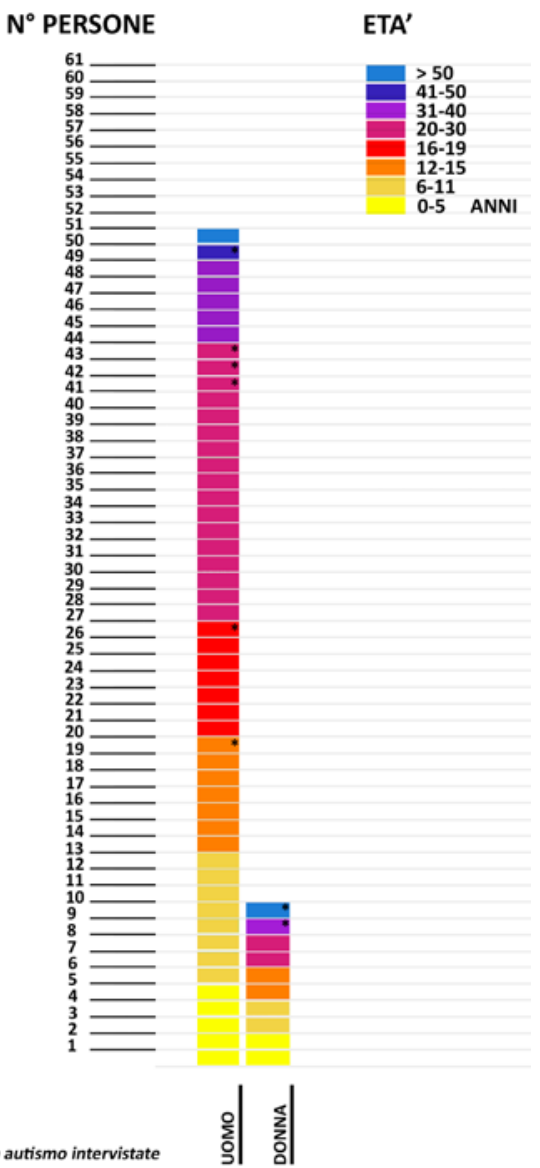

Figura 2. Grafico che rappresenta il sesso e l'età delle persone con autismo, a cui è stato sottoposto il questionario, differenziati rispetto alla loro età. 
Elena Bellini

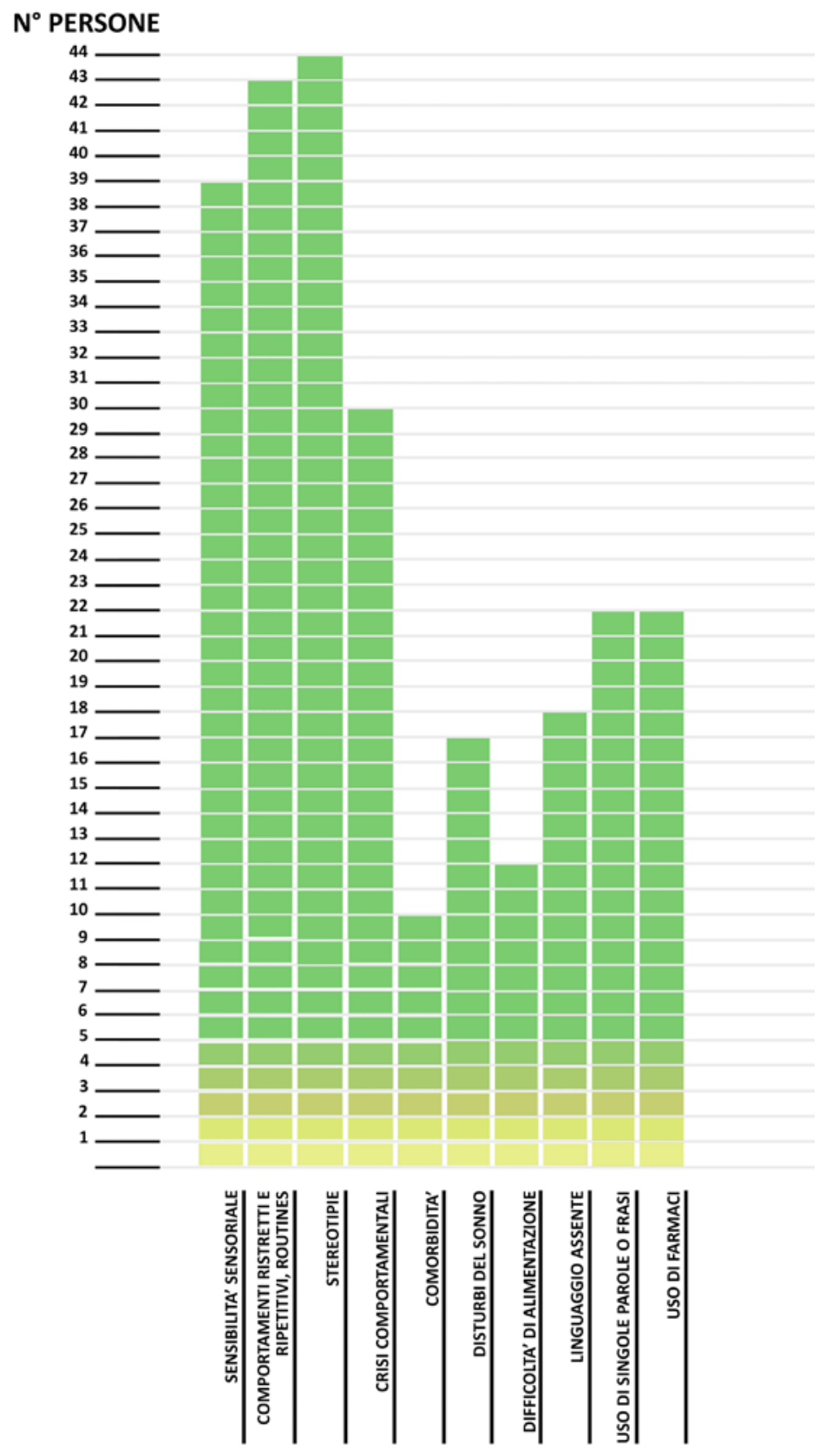

Figura 3. Grafico che rappresenta gli eventuali disturbi presentati dalle persone con autismo, a cui è stato sottoposto il questionario. 
Ambienti sensoriali "terapeutici" che rendano Abili

\section{$N^{\circ}$ PERSONE}
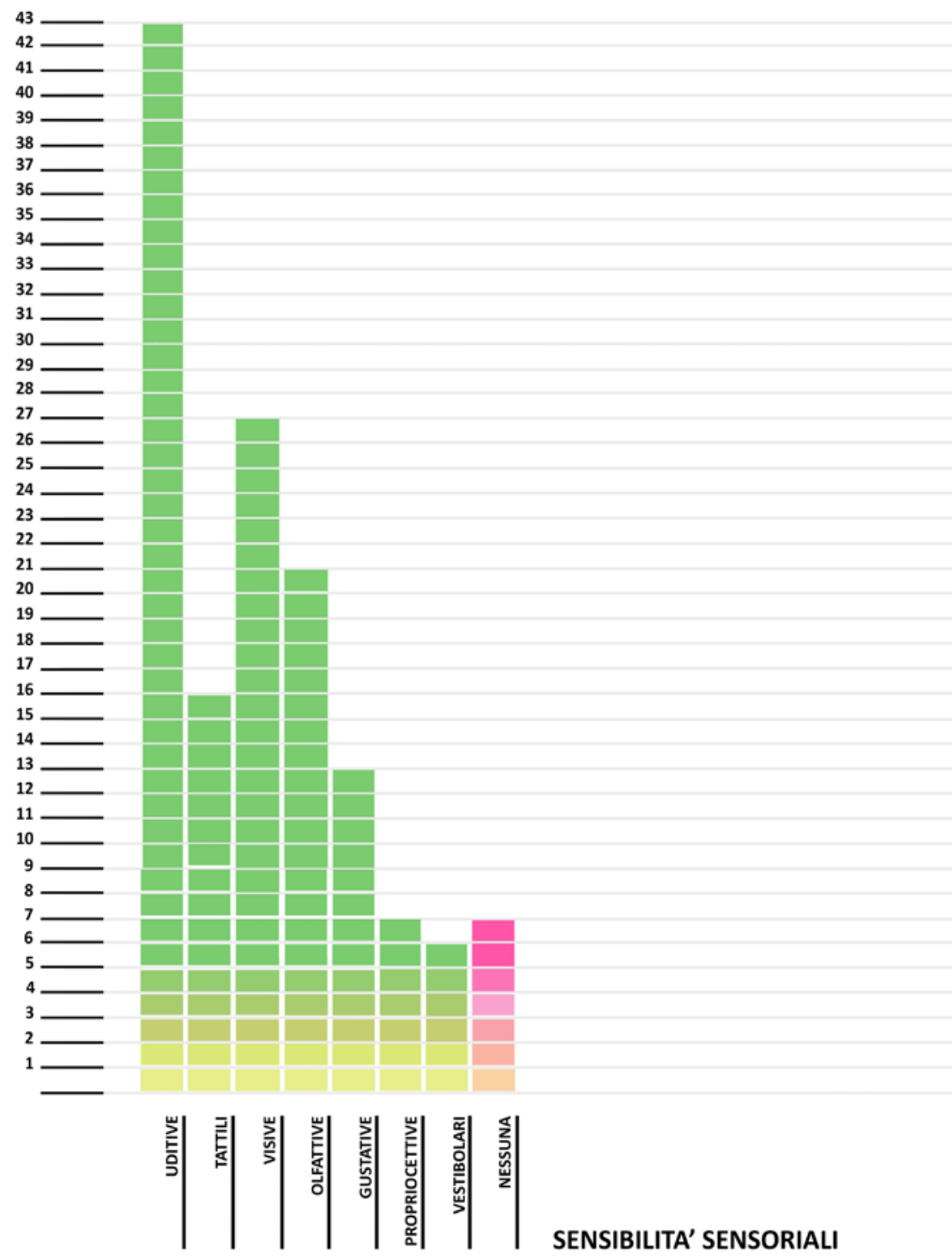

Figura 4. Rappresentazione delle sensibilità sensoriali delle persone con DSA, a cui è stato sottoposto il questionario. 
Elena Bellini

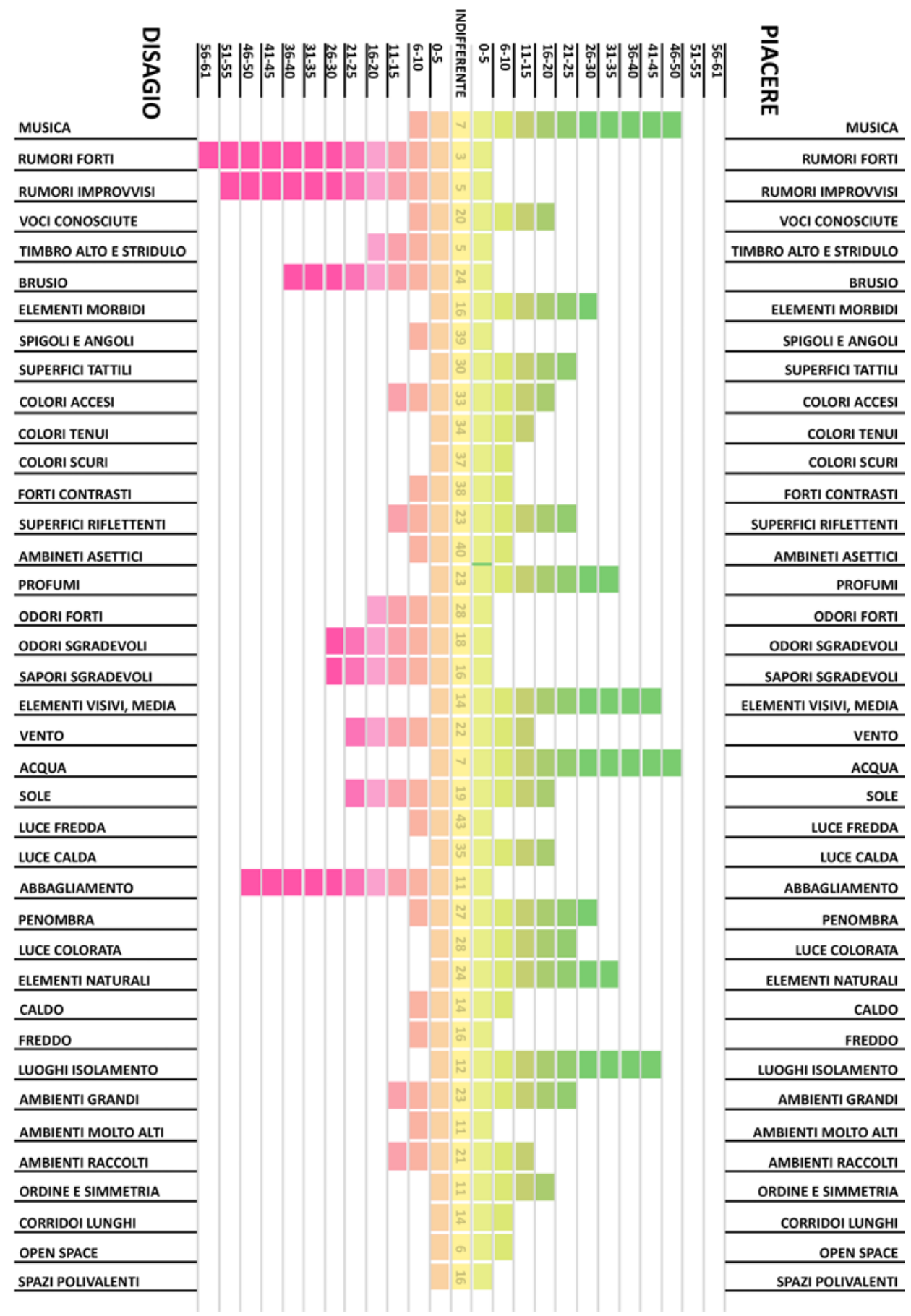

Figura 5. Grafico che rappresenta le sensazioni di piacere o disagio rispetto a differenti stimolazioni sensoriali. 


\section{Quante ore passi di solito a casa in una 'settimana tipo'?}

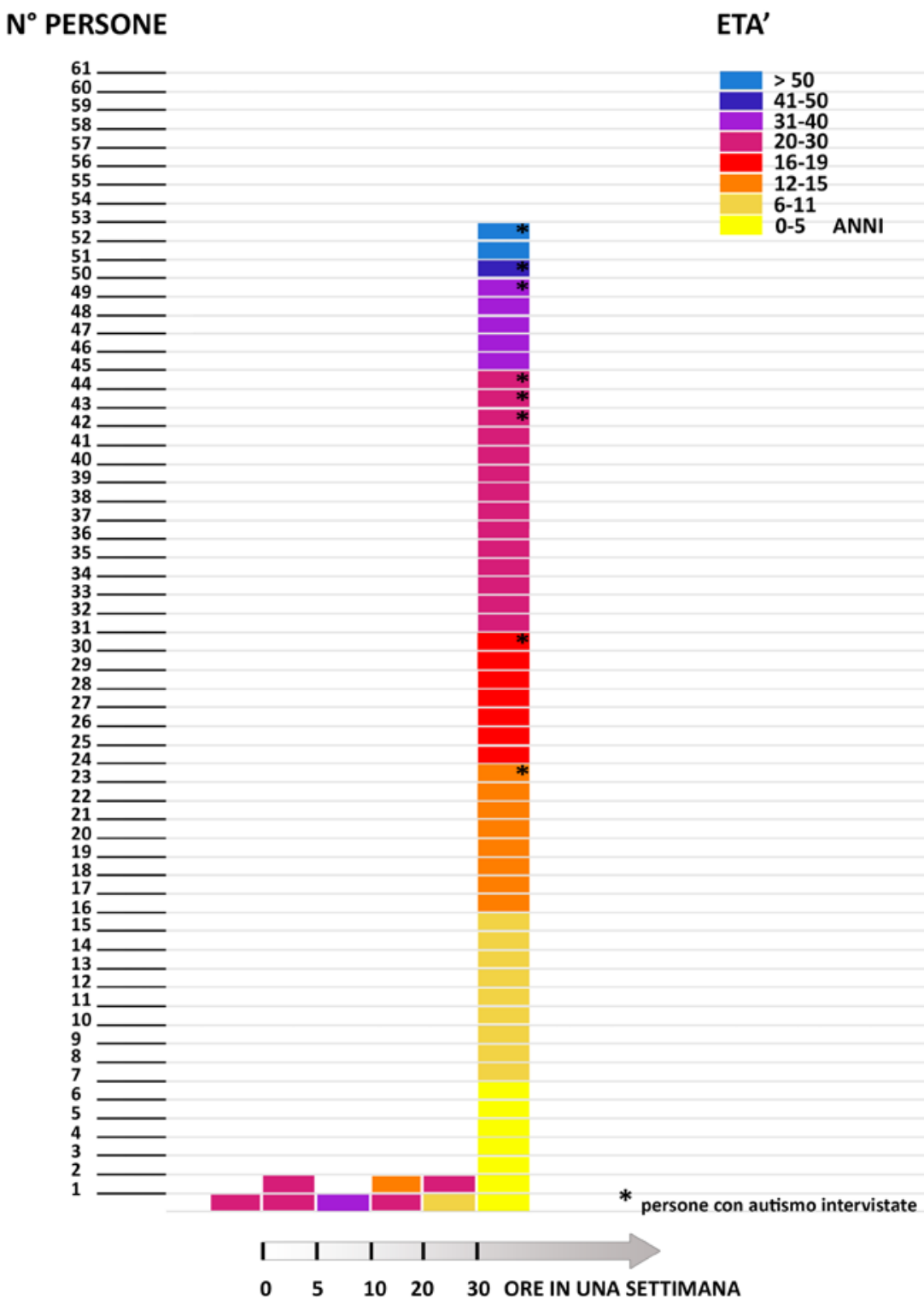

Figura 6. Grafico che rappresenta la quantità di ore in cui le persone con autismo, a cui è stato sottoposto il questionario, sono solite passare nel domicilio familiare in una settimana tipo, anche rispetto alla loro età. 


\section{Quante ore passi di solito in residenza assistita in una 'settimana tipo'?}

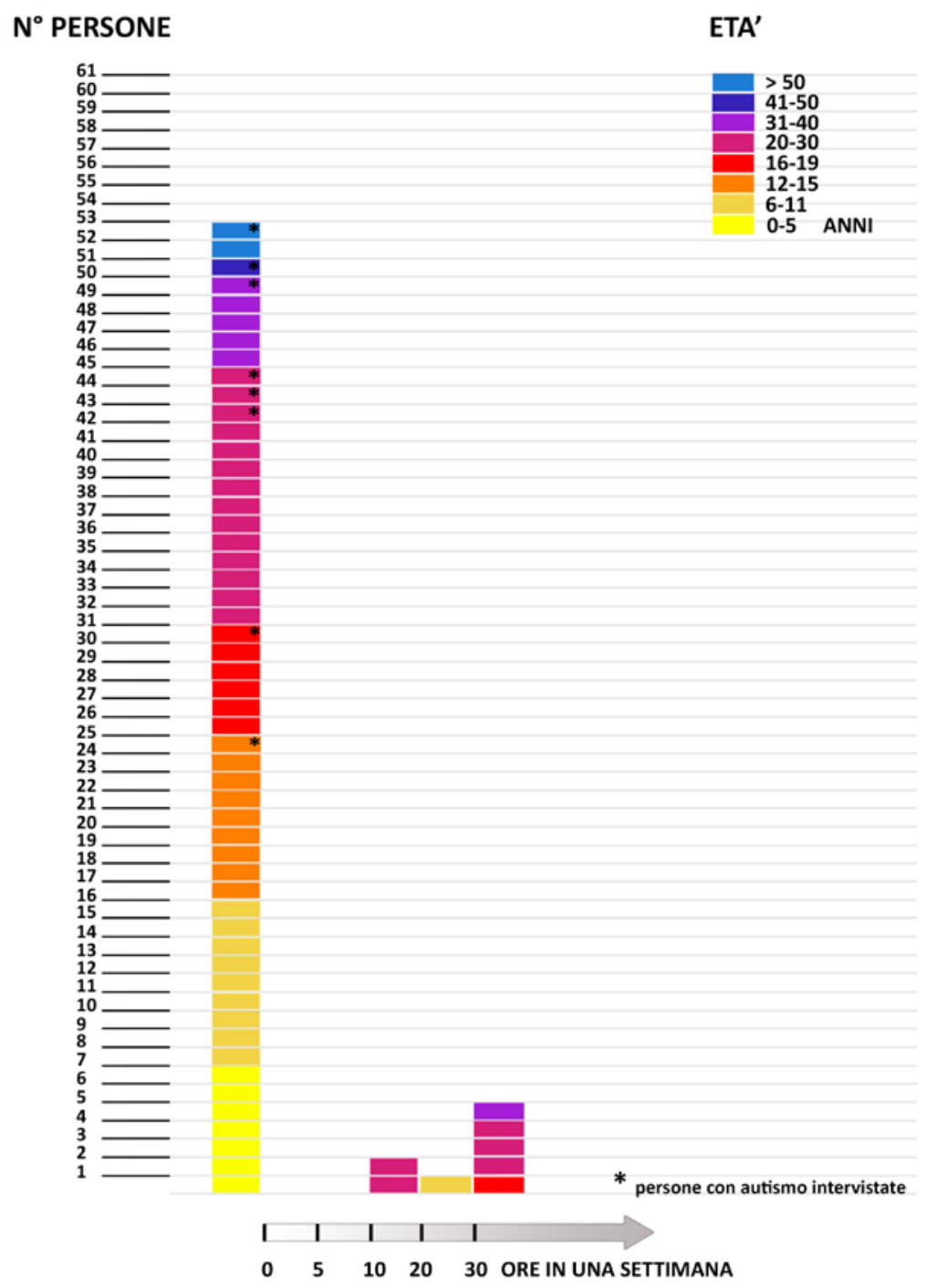

Figura 7. Grafico che rappresenta la quantità di ore in cui le persone con autismo, a cui è stato sottoposto il questionario, sono solite passare in residenza assistita in una settimana tipo, anche rispetto alle loro età. 


\section{Quante ore passi di solito a scuola in una 'settimana tipo'?}

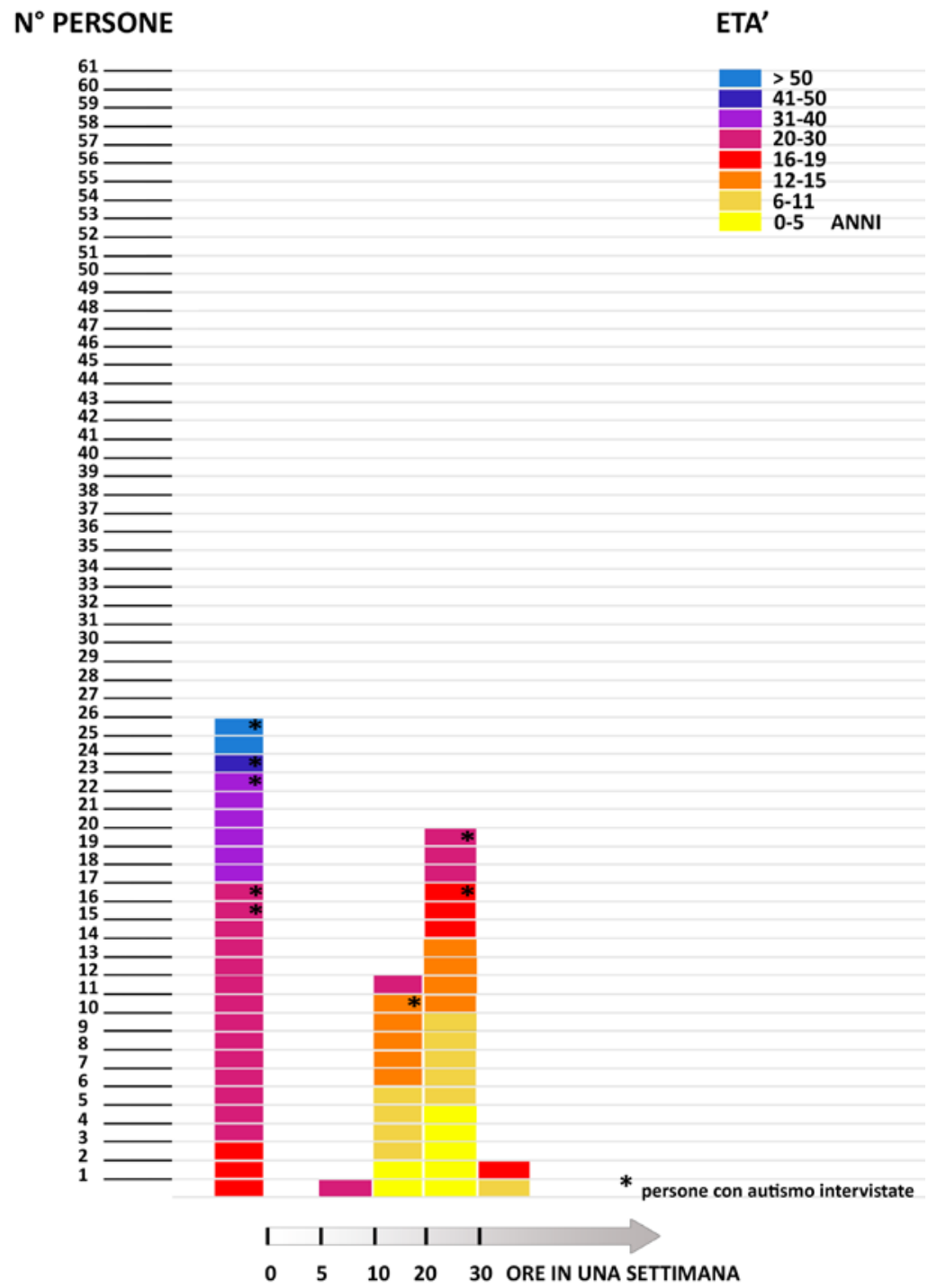

Figura 8. Grafico che rappresenta la quantità di ore in cui le persone con autismo, a cui è stato sottoposto il questionario, sono solite passare a scuola in una settimana tipo, anche rispetto alle loro età. 


\section{Quante ore passi di solito in attività come sport, cultura o religione in una 'settimana tipo'?}

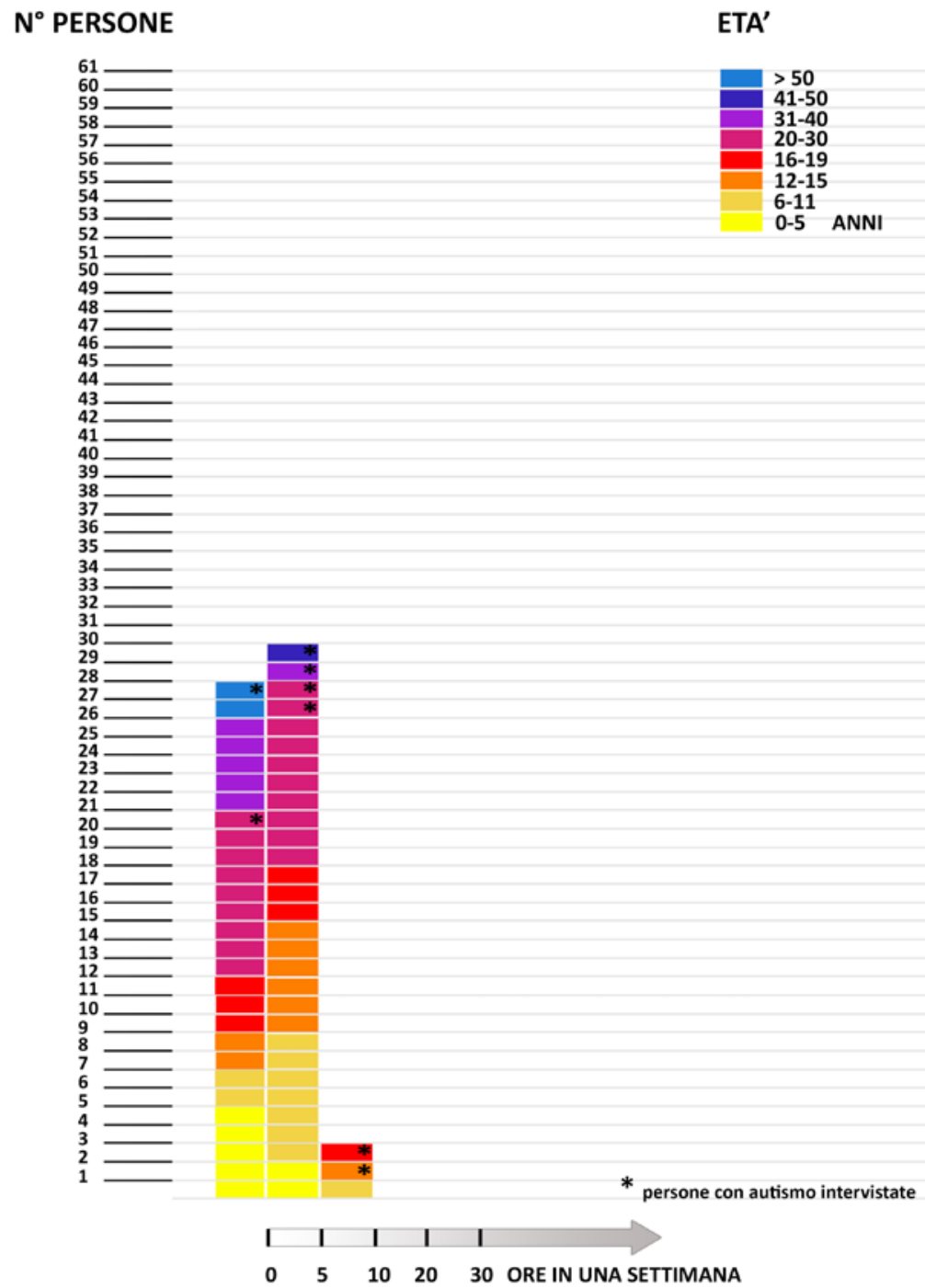

Figura 9. Grafico che rappresenta la quantità di ore in cui le persone con autismo, a cui è stato sottoposto il questionario, sono solite passare facendo sport, attività culturali o religiose, in una settimana tipo, rispetto alle loro età. 
Ambienti sensoriali "terapeutici" che rendano Abili

\section{Quante ore passi di solito in centro diurno in una 'settimana tipo'?}

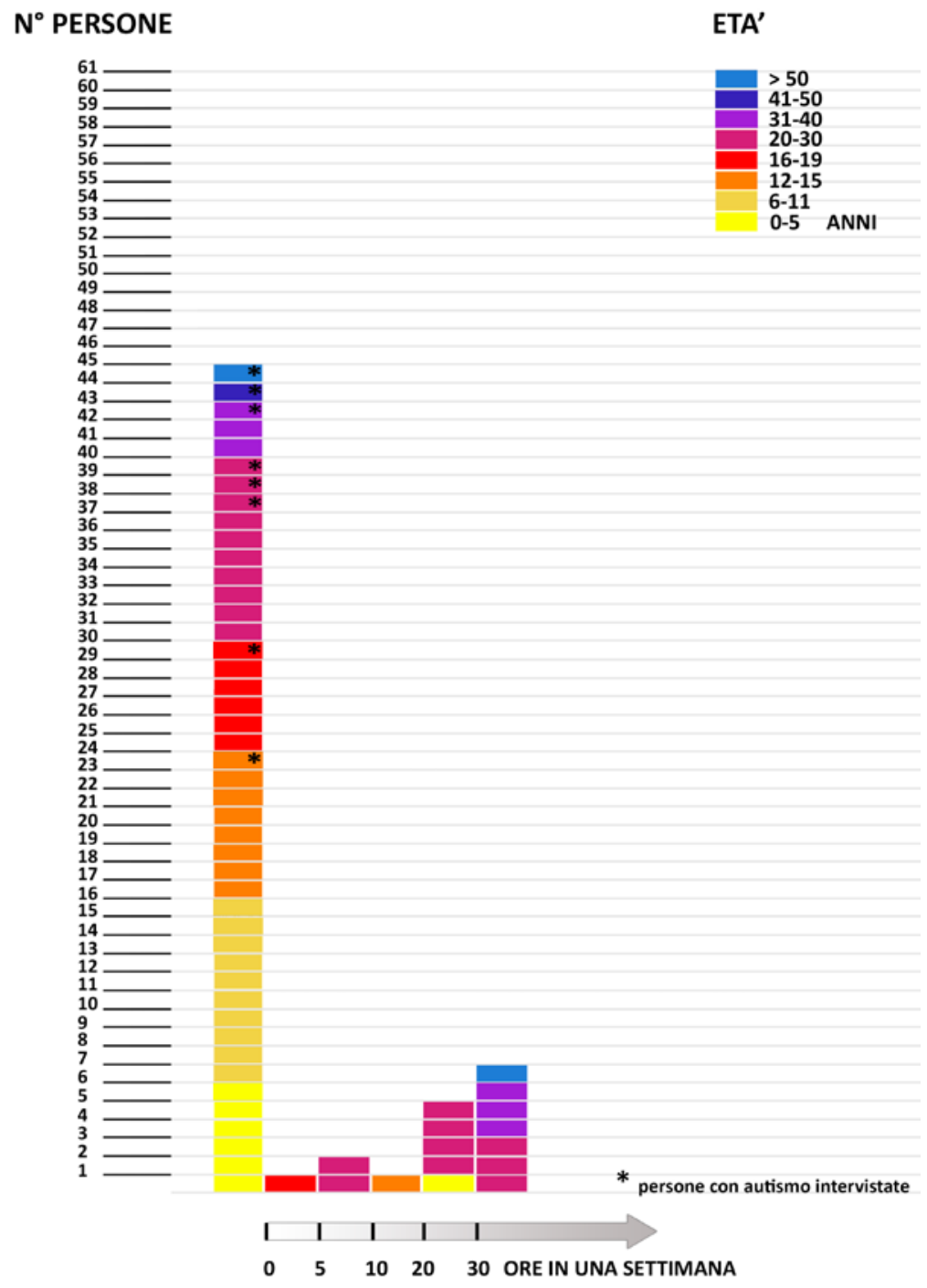

Figura 10. Grafico che rappresenta la quantità di ore in cui le persone con autismo, a cui è stato sottoposto il questionario, sono solite passare in un centro diurno in una settimana tipo, rispetto alle loro età. 
Quante ore passi di solito a lavoro in una 'settimana tipo'?

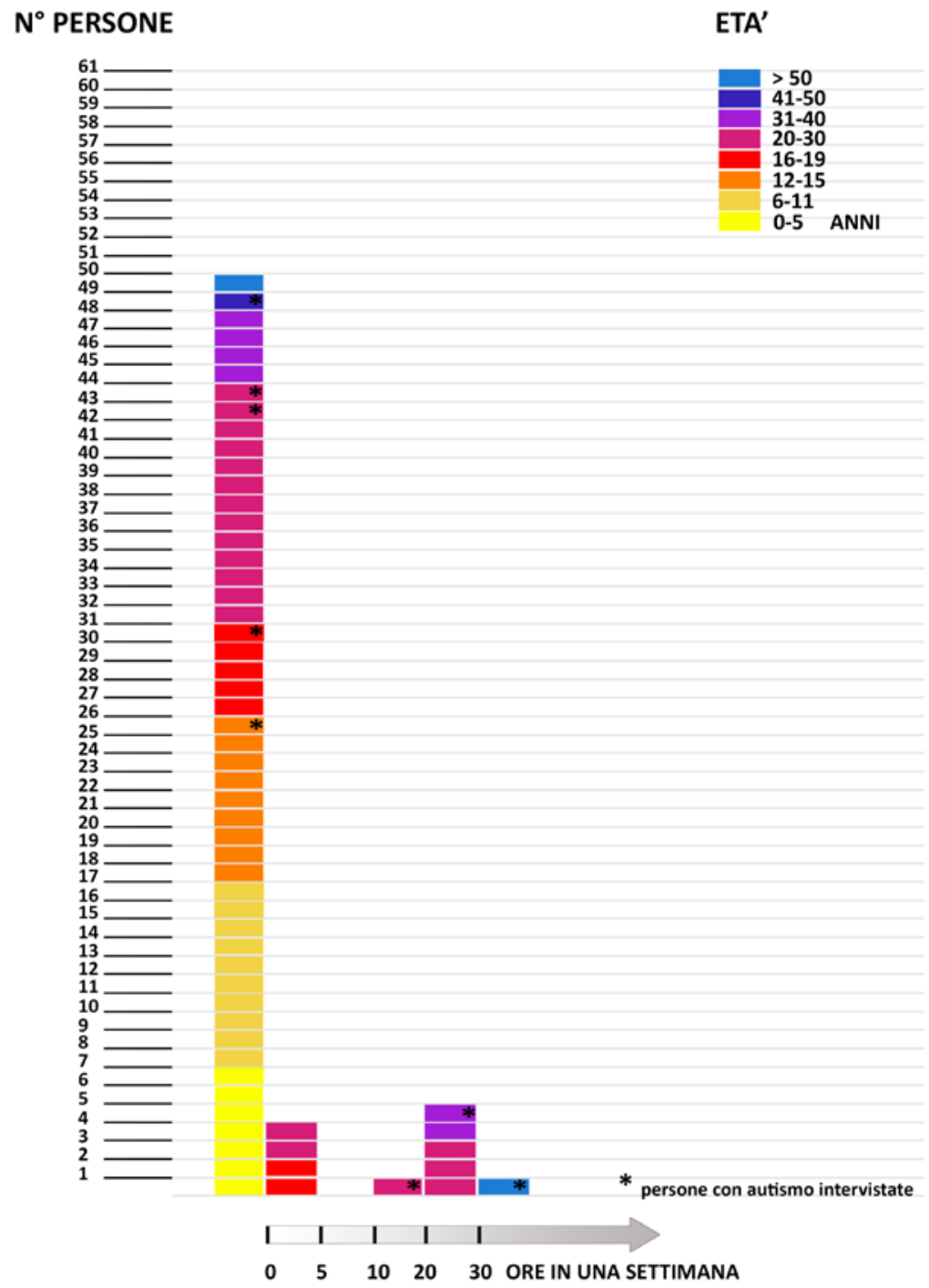

Figura 11. Grafico che rappresenta la quantità di ore in cui le persone con autismo, a cui è stato sottoposto il questionario, sono solite passare a lavoro in una settimana tipo, anche rispetto alle loro età. 
Ambienti sensoriali "terapeutici” che rendano Abili

\section{Quante ore passi di solito fai terapia in una 'settimana tipo'?}

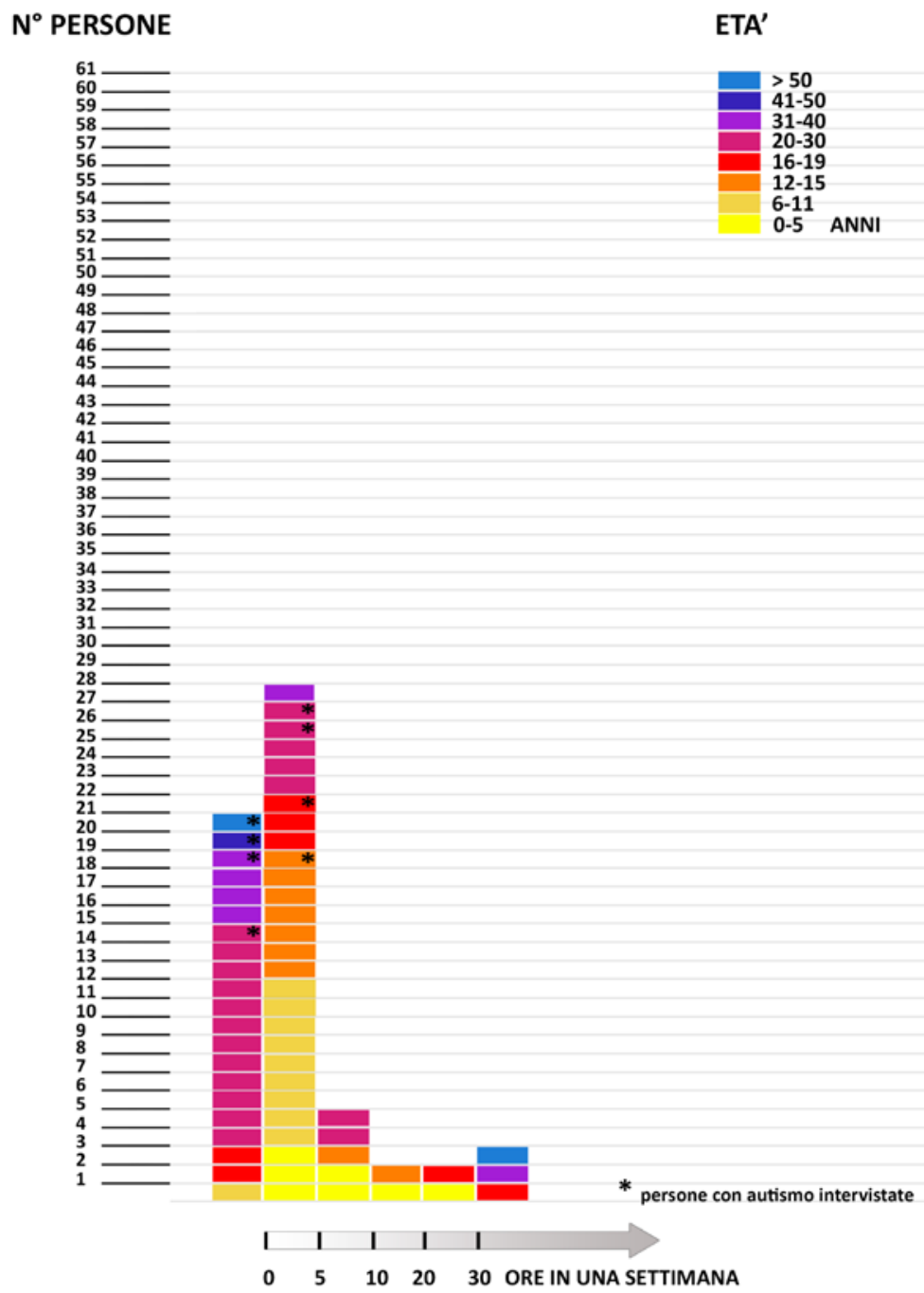

Figura 12. Grafico che rappresenta le ore in cui le persone con autismo, a cui è stato sottoposto il questionario, sono solite fare terapia in una settimana, anche rispetto all'età. 


\section{Elena Bellini}

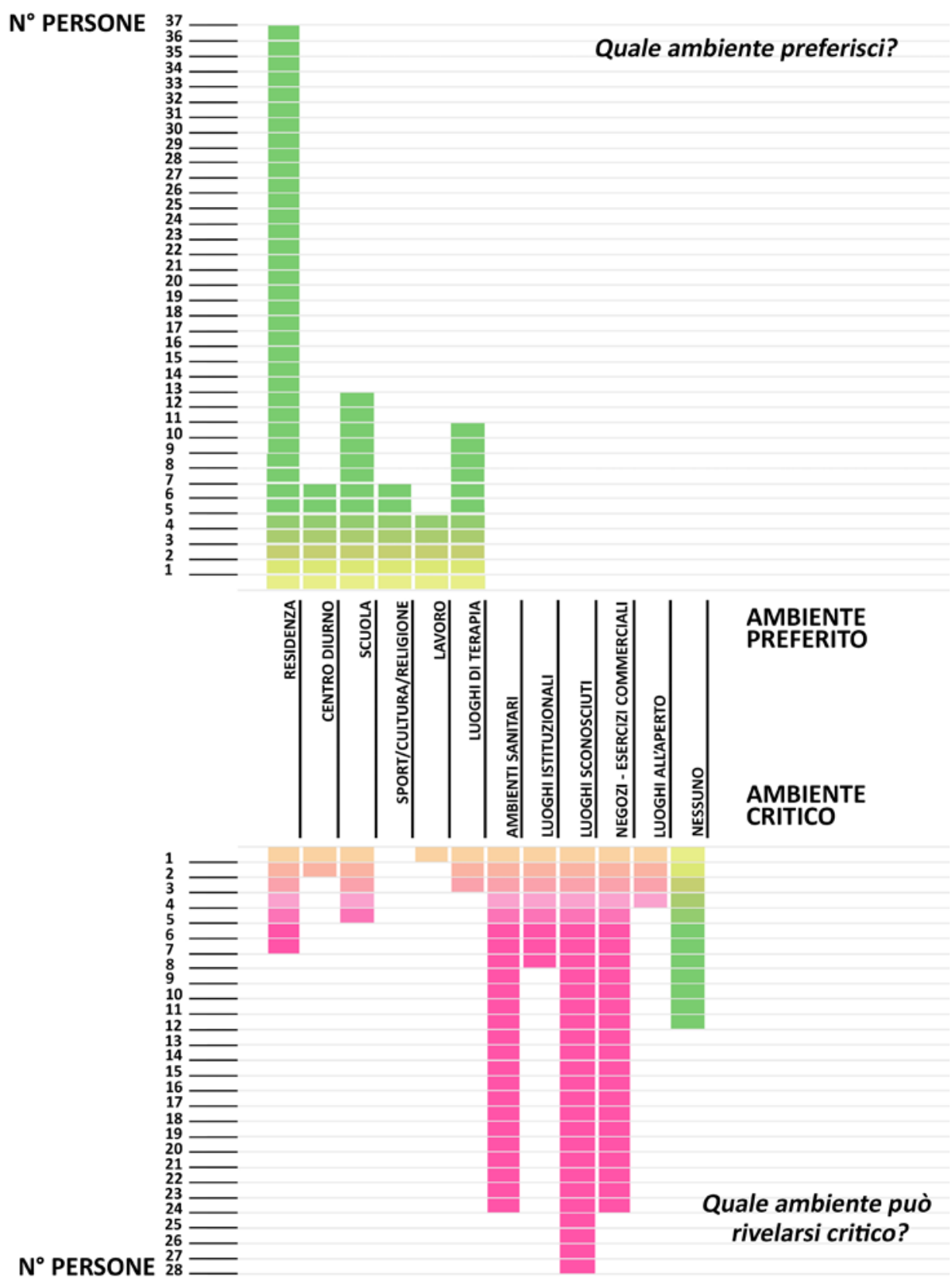

Figura 13. Grafico che rappresenta la quantità gli ambienti preferiti e quelli che possono rivelarsi critici per le persone con autismo, a cui è stato sottoposto il questionario. 
In quali ambienti della casa stai più frequentemente?
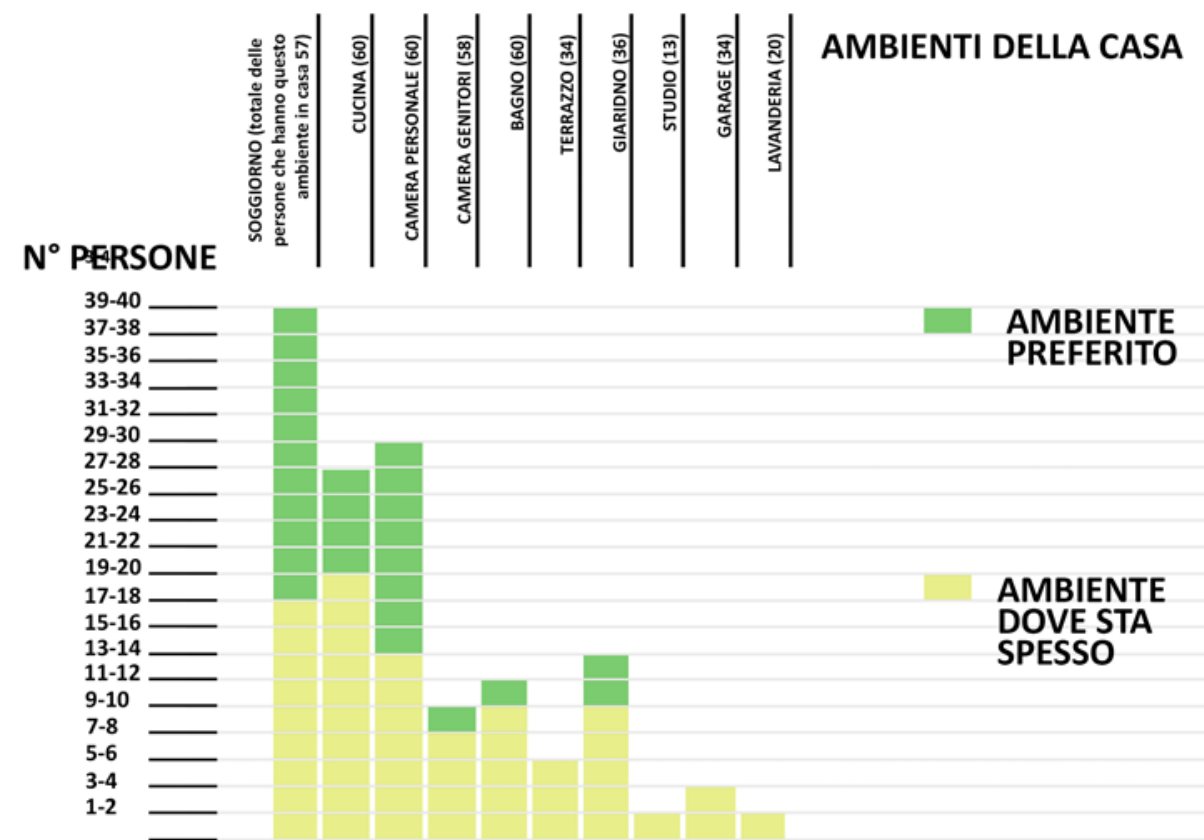

AMBIENTE

PREFERITO

33-34

$$
\text { is } 1111
$$

PREFERITO

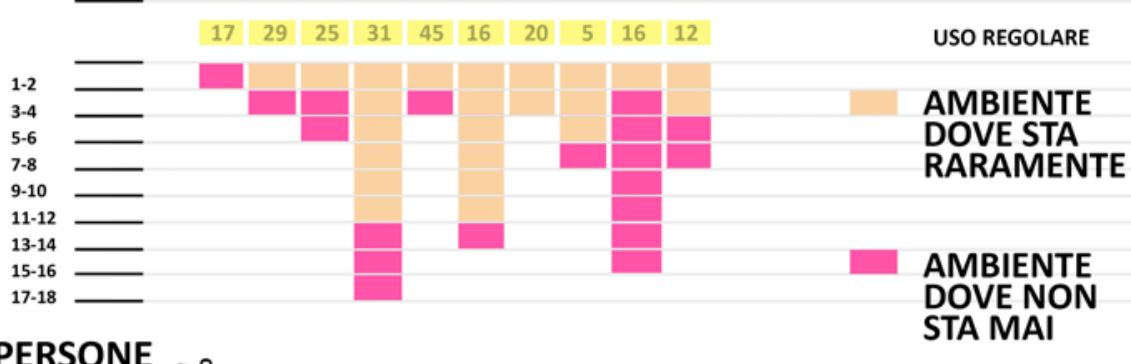

N ${ }^{\circ}$ PERSONE

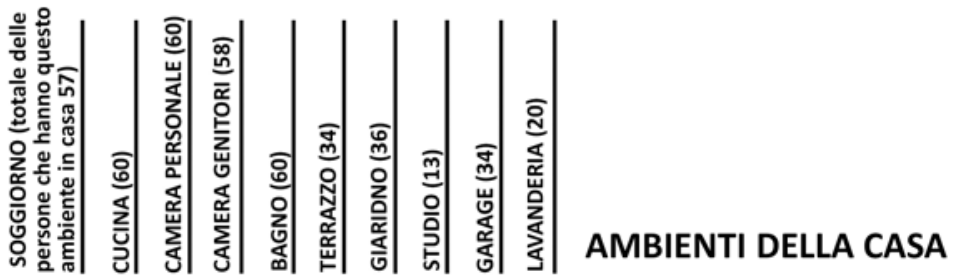

Figura 14. Grafico che rappresenta gli ambienti della casa maggiormente frequentati dalle persone con autismo, a cui è stato sottoposto il questionario. 


\section{Elena Bellini}

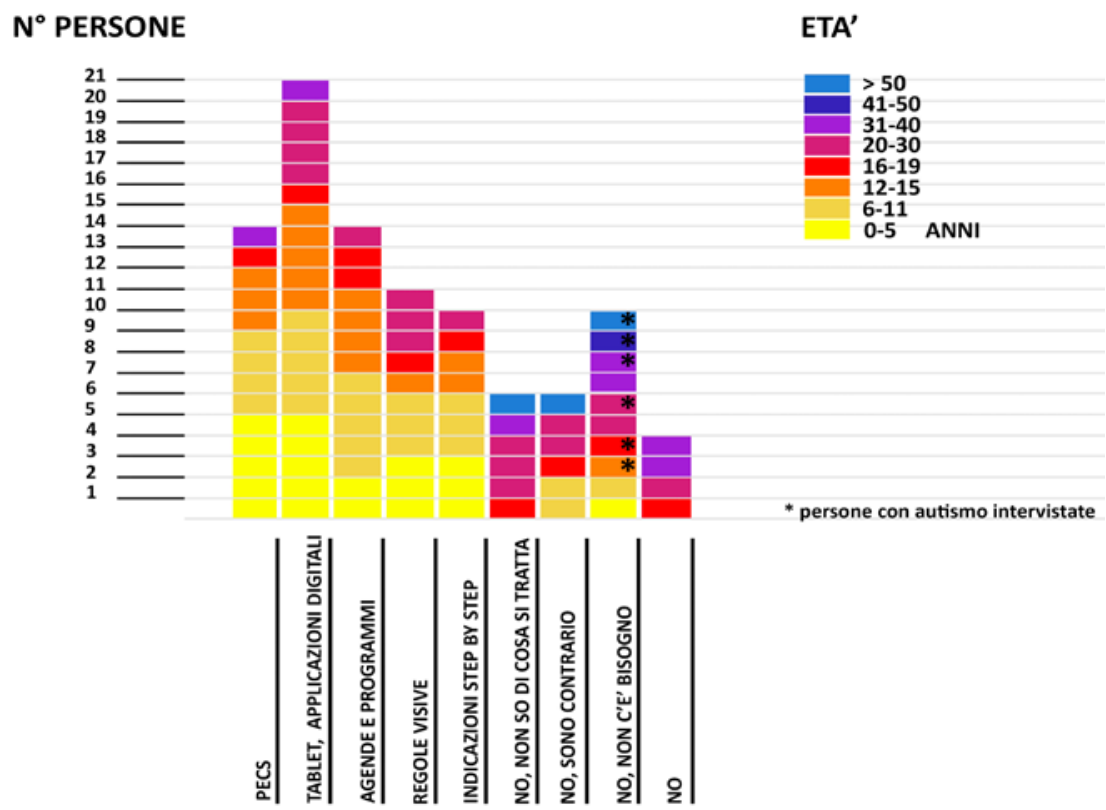

Figura 15. Grafico che rappresenta se le famiglie a cui è stato sottoposto il questionario usano sistemi di supporto alla comunicazione, anche rispetto alle età delle persone con DSA.

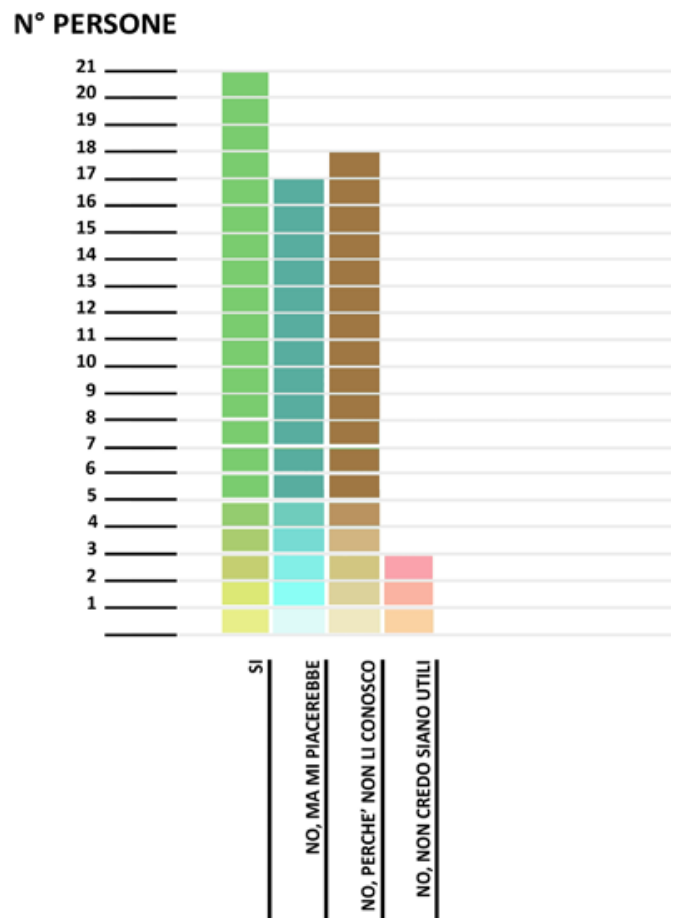

Figura 16. Grafico che rappresenta se le persone con autismo, a cui è stato sottoposto il questionario, hanno esperienza di utilizzo degli ambienti sensoriali terapeutici. 
Ambienti sensoriali "terapeutici" che rendano Abili

\section{Andare in ambienti sanitari può causare delle criticità?}

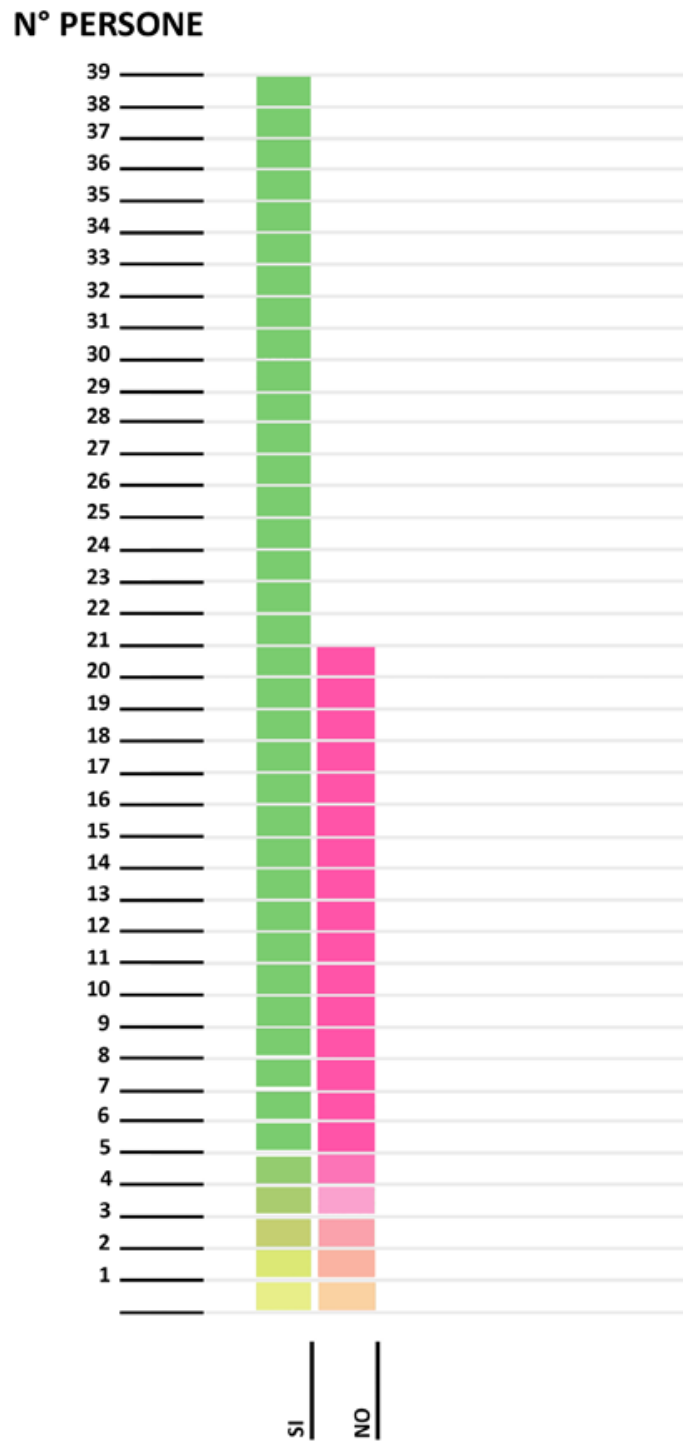

Figura 17. Grafico che rappresenta se gli ambienti sanitari possono rivelarsi critici per le persone con autismo, a cui è stato sottoposto il questionario. 


\section{Quali reazioni ha in caso di accesso ad un ambiente sanitario?}

\section{$\mathbf{N}^{\circ}$ PERSONE}

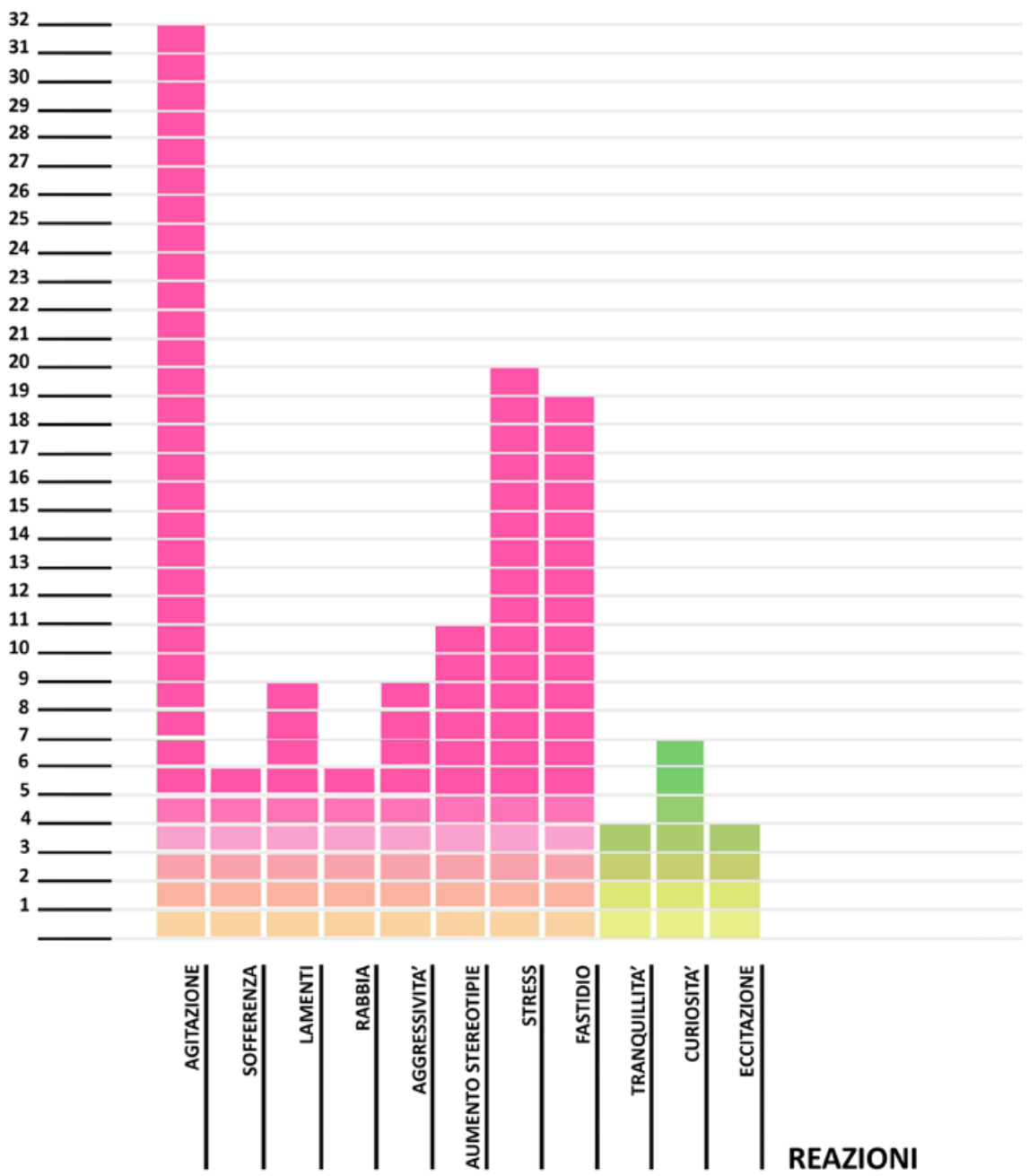

Figura 18. Grafico che rappresenta le reazioni che le persone con autismo, a cui è stato sottoposto il questionario, all'accesso in ambiente sanitario. 
Ambienti sensoriali "terapeutici" che rendano Abili

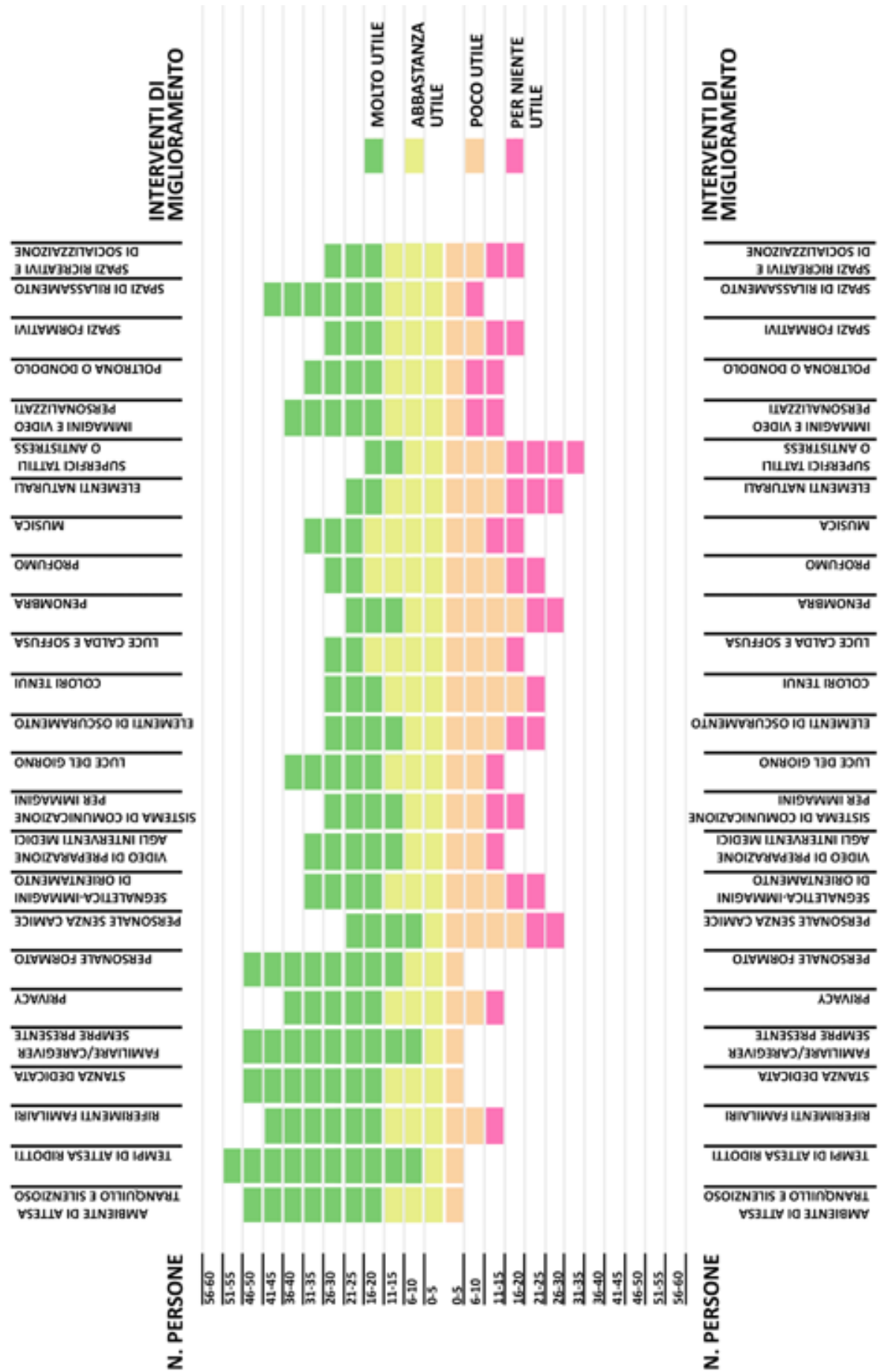

Figura 19. Grafico che rappresenta quali interventi le famiglie a cui è stato sottoposto il questionario, considerano utili al miglioramento dell'accessibilità ospedaliera in casi di autismo. 


\section{Capitolo 4 \\ Casi Studio. Interpretazione e problematiche emergenti}

Come ultima fase di indagine sono stati studiati e visitati alcuni Casi Studio di riferimento importanti per la comprensione e la delimitazione del problema. Sono state visitate strutture residenziali perlopiù in Italia, poiché dallo studio dello stato dell'arte é stato rilevato come la situazione italiana sia piuttosto diversa rispetto a quella estera, dal punto di vista sociale, culturale e normativo. Casi studio internazionali sono stati però studiati al fine di apprendere nuove strategie operative da applicare alla realtà italiana, che ritroveranno interpretazione e applicazione nella fase propositiva. Sono stati scelti i casi più interessanti e all'avanguardia dal punto di vista della progettazione specifica in ambito di Disturbi dello Spettro Autistico, ma anche il più possibile recenti (2002-2017), al fine di guardare alle ultime frontiere di innovazione e ricerca. In particolare, si è cercato di scegliere progetti che fornissero esempi di diversi approcci progettuali (sensoriale/neurotipico, ecologico/strutturato, etc..) e diverse tipologie di soluzioni (residenze temporanee o permanenti, diversa età dell'utenza, ecc.) sulla base delle informazioni ricavate dallo studio dello stato dell'arte, in modo da creare una panoramica completa ed avere modo di identificare e motivare la condivisione di alcune scelte rispetto ad altre.

Le strutture visitate sono perlopiù residenze, che in parte trattano anche gli aspetti educativi e dell'apprendimento o l'inserimento lavorativo, nell'ottica dell'autonomia di vita e del "dopo di noi". In particolare sono state visitate:

- Cascina Rossago (provincia di Pavia), una struttura non molto recente (2002), ma interessante poiché primo esempio in Italia di farm community;

- Villa Le Rogge (Pordenone), come primo esempio in Italia (2012) di residenza temporanea, progettata espressamente per persone con autismo, nell'ottica di un programma di abilitazione per l'autonomia di vita e dell'inserimento lavorativo presso Officina dell'Arte (prodotti unici musivi artigianali, opere di grandi dimensioni e interpretazioni a mosaico di opere d'arte pittoriche, oggettistica decorata a mosaico, packaging e merchandising);

- Casa Sebastiano (provincia di Trento), appena inaugurata (aprile 2017), come struttura terapeutica all'avanguardia, che prevede un'area dedicata alla ricerca e alla formazione degli operatori, stanze per la terapia per utenti di tutte le età, integrando anche attività innovative come l'idroterapia e gli spazi multisensoriali, centro residenziale temporaneo e laboratori per l'autonomia (prevede anche la realizzazione di residenze per il "dopo di noi");

- Aut Aut - La Spezia, ancora in fase di costruzione (inaugurazione prevista nel 2018), residenza, locanda e ristorante, come soluzione indipendente di vita nel "do- 
po di noi", interessante soprattutto per il modello innovativo di integrazione sociale e di inserimento lavorativo (locanda, ristorante/pizzeria, produzione e vendita della pasta, collaborazione con l'adiacente Istituto Alberghiero).

Dal punto di vista dei casi esteri analizzati e approfonditi, sono stati scelti alcuni progetti considerati punti di riferimento in ambito residenziale:

- Sunfield Institute (Londra - UK), anche se non molto recente (2004), selezionato poiché primo esempio europeo di progettazione dedicata esclusivamente all'autismo, con un metodo di ricerca incentrato sulle persone, attraverso la valutazione delle esigenze a partire dall'esperienza della precedente struttura residenziale, sviluppando soluzioni innovative per l'abitare, ed un programma di post-valutazione sull'impatto di gradimento su utenti e operatori, valutato molto positivo;

- Sweetwater Spectrum Community (Sonoma - USA), scelto come esempio recente (2013) internazionale di riferimento in ambito residenziale per l'autismo, per le sue caratteristiche di innovazione, integrazione e qualità della vita, oltre all'interessante percorso di ricerca a monte del progetto, sviluppato nelle Linee Guida "Advancing Full Spectrum Housing: Designing for Adults with Auti sm Spectrum Disorders";

- Seniors House (Hinnerup - Danimarca), selezionato poiché rappresenta un esempio piuttosto recente (2014) ed in particolare la prima residenza specifica per anziani con autismo in Europa, interessante dal punto di vista dell'integrazione e dell'attenzione verso l'anziano, della flessibilità dei moduli abitativi, in particolare studiata in vista delle trasformazioni della vita nel corso degli anni, e della progettazione degli ambienti dedicati specificatamente all'autismo, verso il raggiungimento del comfort e del benessere dei residenti;

- L'eveille du scarabée (Champcevrais, Yonne - France), come esempio piuttosto particolare di residenza moderna (2015), che sperimenta l'utilizzo della tecnologia avanzata, anche per le stimolazioni sensoriali, e tenta di integrare i caratteri dell'architettura più contemporanea in una residenza permanente per adulti con autismo, come soluzione al "dopo di noi".

Per quanto riguarda l'ambiente scolastico la situazione italiana è piuttosto diversa, poiché è stata fatta una scelta politico sociale che garantisce il diritto ad un'istruzione adeguata attraverso una completa integrazione degli alunni con Disturbi dello Spettro Autistico all'interno delle classi tradizionali, supportata da insegnanti di sostegno e sistemi di supporto alla didattica; al contrario in situazioni estere è più comune avere scuole o eventualmente classi "speciali", inserite all'interno di scuole tradizionali. E' stato utile quindi studiare queste diverse realtà per poter capire come andare ad integrarle all'interno dell'ambito scolastico italiano, ovvero quali soluzioni possono rivelarsi utili in modo (per quanto possibile) "universale", migliorando l'attenzione, la concentrazione e la qualità dello studio di tutti gli alunni, oppure dove sia possibile strutturare l'ambiente in modo specifico e personalizzato rispetto al singolo bambino, sviluppando soluzioni di flessibilità. In particolare sono stati analizzati ed approfonditi:

- New Struan School (Alloa - UK), esempio non molto recente (2005), ma punto di riferimento per l'ambiente educativo speciale per bambini con Disturbi dello Spettro Autistico; 


\section{Elena Bellini}

- DLC- Developmental Learning Center (Warren, New Jersey - USA - 2007), esempio molto interessante dal punto di vista dell'affermazione di un diverso metodo educativo - neurotypical approach - che si basa sull'apprendimento attraverso il contesto ambientale "naturale", riproducendo all'interno della scuola una strada tipica di New York, con le strutture pubbliche ed un appartamento residenziale per lo sviluppo delle abilità quotidiane e dell'autonomia di vita;

- Forbush School for Autism (dintorni di Baltimora - USA- 2008), anche in questo caso esempio di riferimento di scuola speciale per Disturbi dello Spettro Autistico, interessante soprattutto per la distribuzione planimetrica e l'organizzazione dello spazio classe e servizi annessi;

- Advance School for Developing Skills of Special Needs Children (Qattameya Cairo - 2008), selezionato come esempio unico di progettazione strutturata per l'apprendimento in casi di autismo e disturbi dello sviluppo, ma soprattutto per il percorso di ricerca che vi sta a monte, per il quale si è sperimentato direttamente con i bambini la sistemazione dell'ambiente al fine di ridurre il disagio sensoriale e migliorare l'attenzione e la concentrazione, con particolare attenzione al comfort acustico, lo zoning sensoriale e la sequenzialità degli spazi;

- CADB - The New York Presbyterian Center for Autism and the Developing Brain (White Plains, New York - USA - 2011), anche se non è una scuola, ma un centro terapeutico e per l'apprendimento, risulta interessante per la particolare conformazione degli spazi;

- Western Autistic School (2011) e Northen School for Autism (2013) in Australia, entrambe progettate da Hede Architects, scuole speciali per bambini e ragazzi con autismo, interessanti per la suddivisione e l'organizzazione degli ambienti educativi.

Per quanto riguarda infine gli ambienti sanitari, non esistono al momento interventi specifici sullo spazio in Italia, salvo il caso della stanza del Pronto Soccorso di Pordenone, che è stato infatti analizzato. Anche all'estero comunque sono pochi gli ambienti conformati specificatamente per l'autismo, salvo alcuni esempi di stanze sensoriali integrate nell'ambiente ospedaliero, anche perché non esistono al momento codici o normative che identifichino standard o parametri di riferimento per l'accessibilità o la qualità degli ambienti di cura specificatamente per le persone con autismo. D'altro canto, sarebbe importante valutare l'adeguamento degli ambienti sanitari al fine di migliorarne l'accessibilità, dato che gli interventi non andrebbero oltretutto in contrasto con gli standard classici della qualità degli ambienti di cura. Pertanto, sono stati identificati esempi esistenti di programmi e protocolli d'intervento in casi di Disturbi dello Spettro Autistico, con particolare attenzione alle situazioni di emergenza sanitaria e Pronto Soccorso, ed è stato invece rilevato utile lo studio di alcuni progetti di ambienti ospedalieri che avessero un'attenzione particolare all'umanizzazione dell'ambiente di cura, alla comunicazione per colori o immagini legata all'orientamento ed il wayfinding e allo sviluppo di sistemi di assistive technology di assistenza e supporto al paziente. Per questo motivo sono stati analizzati quindi alcuni ospedali pediatrici che presentano queste caratteristiche, anche non esclusivamente legate ad una progettazione mirata ai Disturbi dello Spettro Autistico. In particolare quindi sono stati approfonditi, nell'ambito dei programmi e protocolli di intervento: 
- il sistema di assistenza e accesso ospedaliero protetto del Progetto DAMA a Milano, che prevede percorsi di accoglienza medica coordinata a favore dei disabili gravi e gravissimi, in particolare rivolti alla disabilità intellettiva;

- il sistema di accesso ospedaliero protetto in casi di autismo, presso il Pronto Soccorso di Pordenone, che prevede anche uno spazio dedicato e progettato, oltre alla definizione di un'applicazione digitale - Vi.co hospital - che permette di sviluppare percorsi di training e preparazione allintervento medico, al fine di migliorare l'accesso e la qualità della cura del paziente con autismo;

- Nemours Children's Hospital (Orlando - USA), selezionato per il sistema di umanizzazione dell'ambiente ospedaliero con integrazione della sensorialità e del gioco, ma soprattutto per il programma REACH - Respecting Each Awesome Child Here - (2016) di supporto all'accessibilità per l'autismo o altre condizioni comportamentali e dello sviluppo, presso il Pronto Soccorso;

Nell'ambito della qualità ambientale e umanizzazione degli spazi ospedalieri:

- Phoenix Children's Hospital (Phoenix - USA), anche se non molto recente (2008-9), ma primo esempio integrato di umanizzazione degli ambienti ospedalieri, soprattutto per quanto riguarda l'uso del colore e delle luci;

- Royal Alexandra Children's Hospital a Brighton (UK), ospedale pediatrico di riferimento dal punto di vista della qualità ambientale e dell'umanizzazione degli spazi, che lo scorso anno (2016) ha installato uno spazio sensoriale (Sea Life) dedicato al rilassamento dei bambini con autismo, altre disabilità intellettive o bisogni speciali;

- Emma Children's Hospital ad Amsterdam (Paesi Bassi), esempio interessante soprattutto per l'organizzazione degli spazi all'interno del reparto ospedaliero che guarda alla privacy del bambino e al suo rapporto con il contesto esterno, le grafiche di comunicazione ed il sistema efficace di orientamento e wayfinding, l'integrazione di elementi ludici e multisensoriali per l'intrattenimento e l'abbattimento dello stress del paziente;

- Evelina Londron Children's Hospital, ospedale pediatrico nel centro di Londra, uno tra i migliori esempi di umanizzazione dell'ambiente ospedaliero, che presenta inoltre una chiara suddivisione degli spazi e caratterizzazione per colori e immagini rivolte all'orientamento ed al wayfinding, sviluppata ed evoluta nel 2016 attraverso un progetto di psicologia ambientale e lo sviluppo di un'applicazione digitale sperimentata al Pronto Soccorso, per migliorare la comunicazione e l'interazione tra paziente, famiglia e operatore.

Quest'area di sviluppo della ricerca, proprio per la carenza di letteratura e di esempi, anche in ambito internazionale molto recenti e ancora poco sviluppati, sarà inoltre un'area che verrà approfondita come caso sperimentale di progetto e come nuova frontiera dei contesti ambientali.

L'analisi dei Casi Studio presentati avverrà secondo i criteri esigenziali evidenziati a seguito dell'analisi dello Stato dell'Arte, ovvero:

- Riduzione del disagio sensoriale e ambientale.

- Salute e benessere.

- Ambienti sicuri e durevoli.

- Orientamento e comprensione.

- Indipendenza e autonomia. Abilità. 


\section{Elena Bellini}

- Relazioni sociali e integrazione. Inclusività.

A seguito di una descrizione generale, quindi, ogni caso è stato analizzato nello specifico dei diversi criteri esigenziali, andando a definire per punti i lati positivi o critici del progetto, anche mantenendo un'attenzione trasversale per una progettazione legata alla sensibilità sensoriale della persona con autismo. La rilevanza di questi dati, insieme alle parole chiave che descrivono ogni progetto, andranno a comporre la base del materiale che verrà utilizzato all'interno della piattaforma DARE (sense) nella fase applicativa e propositiva del programma di ricerca, nello specifico degli esempi e casi studio di riferimento da proporre e implementare nel tempo. Quest'analisi è stata fondamentale, infine, insieme allo studio dello Stato dell'Arte, nella definizione delle indicazioni di sintesi di progettazione per l'autismo; si riportano a titolo esemplificativo alcune schede.

\subsection{New Struan School}

Sede: Alloa (UK)

Data: 2005

Uso: scuola speciale per bambini con Disturbi dello Spettro Autistico

Utenti: 35 bambini tra i 5 e i 19

Gestione: Scottish Society for Autism

Progettazione: Aitken Turnbull Architecture

Tag: \#scuola \#bambini \#ragazzi \#disagio \#benessere \#sicurezza \#orientamento \#autonomia \#integrazione \#uditiva \#visiva \#olfattiva \#tattile \#propriocettiva

New Struan (Scheda 01) è una scuola indipendente diretta dalla Scottish Society for Autism, che ha anche la funzione di centro di riferimento nazionale per l'autismo, includendo alcune funzioni quali il servizio di consulenza, un centro educativo e di formazione sull'autismo, un centro di diagnosi e di ricerca.

\subsubsection{Il progetto}

Il governo scozzese richiede alle sue scuole di fornire un ambiente sicuro, piacevole e stimolante, adatto a sostenere le attività curricolari, sociali e di svago degli allievi, promuovendo l'inclusione per tutti. Un tema chiave della National Priorities in Education è lo sviluppo di un Framework for Learning dove l'ambiente scolastico potenziato favorisca l'apprendimento e l'insegnamento. Le caratteristiche dell'ambiente che sono in grado di ottimizzare le opportunità di apprendimento si possono riassumere in: spazio che assicura libertà e chiarezza di movimento; adattabilità di luci e riscaldamento; buoni livelli di acustica; arredi e ripostigli adeguati; arredi e colori che stimolino l'apprendimento, ma promuovano anche un'atmosfera calma.

Dall'inizio della progettazione della New Struan, è stato tenuto conto delle difficoltà che possono riscontrare bambini con autismo in ambito scolastico, al fine di creare un ambiente autism-friendly, ovvero: distorsione sensoriale, distorsione percettiva, difficoltà nelle funzioni esecutive (organizzazione personale e pianificazione), difficoltà nella coerenza centrale (fare connessioni, organizzare i dettagli), in- 
flessibilità di pensiero e azione (difficoltà nelle transizioni), difficoltà d'interazione sociale.

L'edificio (Figura 01) ha una forma a "T" dove suddivide un lato più pubblico, quello del "front of house" (la facciata dell'edificio nella sezione orizzontale della "T" da est a ovest), dedicato alla Scottish Society for Autism e agli ambienti di reception, caffetteria e training rooms; e uno invece privato, ovvero la spina della "T", separato da porte di sicurezza, dove sono invece contenuti gli spazi per la diagnosi, l'assistenza e l'educazione dei bambini. La spina va da nord a sud e contiene un atrio ad un solo piano di circolazione con le aule che si affacciano verso est e ovest, dove si trova anche uno spazio gioco all'aperto sicuro. L'atrio, dalla forma di una grande "strada" (Figura 02 - 03), è il cuore sociale dell'edificio ed è importante per sviluppare l'orientamento ed il wayfinding, grazie alla chiara visibilità dell'intera scuola, promuovendo il movimento indipendente tra le diverse aree. E' arricchito dai disegni dei bambini fatti a scuola (Figura 02) per rendere lo spazio personalizzato e familiare, opposto all'idea di istituzionalizzazione.

La street è inondata dalla luce naturale attraverso un atrio vetrato e ben ventilato, che dà subito un immediato senso di benessere. L'ampiezza della street favorisce la prossemica e la propriocezione, dando la possibilità a gruppi di bambini di fruirne contemporaneamente senza scontrarsi o avere la sensazione di caos e confusione. I muri curvi (Fgura 02) conducono il bambino da un'area all'altra, riducendo il numero di angoli acuti, angoli cechi e porte nascoste, diminuendo quindi il livello di ansia e favorendo gli spostamenti.

Lungo la "strada" si accede alle aule e ad alcune zone semi-pubbliche, ovvero il refettorio e la biblioteca, che sono aree aperte per permettere ai bambini di orientarsi in modo indipendente. Si accede alle classi dall'atrio attraverso spazi di soglia - definiti "baie" (Figura 03) - dedicati e personalizzati, che funzionano da filtro, permettendo ai bambini di assimilare il cambiamento di ambiente. I pannelli di vetro su ogni porta amplificano questo aspetto inoltre, dando la possibilità al bambino di guardare dentro la classe, prepararsi e assimilare le informazioni prima di entrare.

\subsubsection{Dimensioni e strutturazione degli spazi di apprendimento}

Le classi sono più piccole rispetto ai soliti standard, accogliendo un massimo di 6 studenti. Le diverse aree all'interno dell'aula sono delineate chiaramente per specifiche attività (ICT, group work, independent work), comprendendo spazi per la formazione in piccoli gruppi, individuale o "one-to-one", connessi visivamente con la classe attraverso pannelli vetrati, che permettono di separarsi, ma non sentirsi isolati rispetto agli altri bambini. In particolare, le aule sono strutturate in modo che ci siano postazioni singole lungo i due lati di muro. Vicino alle finestre invece sono stati collocati dei tavoli circolari per le attività di gruppo. Gli spazi bacheca sono ottimizzati al fine di supportare l'apprendimento attraverso segnali o indicazioni visive. Vi sono scaffali dalla struttura leggera e un ampio spazio ripostiglio per ridurre la confusione ed il sovraccarico visivo. Le pareti finestrate ad altezza bambino lasciano libera la vista dell'aerea gioco esterna, zona completamente sicura e accessibile direttamente dall'aula. Le finestre sono operabili elettronicamente dallo staff per garantire la sicurezza. 


\section{Elena Bellini}

E' prevista inoltre una "quiet room" con muri curvi per offrire un ambiente sensoriale controllato e una zona di calma, in caso di sovraccarico sensoriale.

\subsubsection{Aree comuni e spazi esterni}

Le aree comuni incorporano molti degli obiettivi usati per le classi. Ad esempio la biblioteca è uno spazio aperto a pianta libera dove si comprende immediatamente la distribuzione delle aree dedicate alla lettura rilassata e quelle invece per la ricerca e lo studio. Dalla "street" vi si accede attraverso un ingresso curvo e senza porte, che invita $\mathrm{i}$ bambini e lo staff a entrare e muoversi tra gli scaffali. E' interessante come la qualità dello spazio sia tale da permettere che, benché sia aperto, nessun bambino sia mai scappato da quest'area, così calma e tranquilla.

Una stanza è stata dedicata alla riproduzione di una tipica cucina di famiglia, dove $\mathrm{i}$ bambini possono ap-prendere nuove abilità quotidiane in ambienti simili alla propria casa.

La dining hall e il Café Courtyard creano ulteriori opportunità per i bambini e lo staff di incontrarsi con le altre classi, attraverso relazioni "protette", in queste aree che mantengono le caratteristiche di prevedibilità, favorendo così l'indipendenza.

L'area esterna prevede una pista ciclabile, molti giochi, un parco di palline e un giardino sensoriale, al fine di dare l'opportunità di cooperare in gruppo o indipendentemente, fare esercizio, socializzare o semplicemente rilassarsi. Per i bambini che invece non vogliono giocare ci sono delle sedute nell'area picnic. La recinzione perimetrale è bassa e piacevole esteticamente.

Un ampio parcheggio per lo staff, bus e taxi consente di arrivare in modo sicuro alla scuola.

\subsubsection{Comfort luminoso}

L'illuminazione delle aule è indiretta, provenendo da finestre a nastro dove la luce viene inoltre regolata attraverso brise-soleil che spinge la luce del sole verso il soffitto, realizzato con un'angolatura specifica che ne ottimizza la diffusione in modo riflesso. Un simulatore diurno è utile inoltre per aumentare il livello di illuminazione nei giorni nuvolosi. Porte e finestre hanno infine degli scuri opachi che possono essere manualmente chiusi.

La luce artificiale mima la luce naturale diffondendosi verso il soffitto allo stesso modo ed é inoltre soft e discreta. Sono state evitate luci fluorescenti, che possono disturbare visivamente, e installati corpi illuminanti a parete, che non provocano alcuna oscillazione o vibrazione. Le luci artificiali hanno inoltre un reattore elettrico ad alta frequenza (per evitare lo sfarfallamento) ed un controllo dimmer per la regolazione delle luci.

\subsubsection{Comfort acustico}

Le aule sono isolate con alto standard acustico (150 $\mathrm{mm}$ di parete di muratura in calcestruzzo, con $19 \mathrm{~mm}$ di cartongesso da ogni lato). L'intera area ha la moquette, 
per ridurre il rumore e rendere più soft l'ambiente. Nell'edificio non sono inoltre usati segnali sonori per non turbare gli alunni.

\subsubsection{Studio del colore}

Le finiture, come moquette e colori alle pareti, sono chiaramente indicate per supportare la gerarchia spaziale. Il cambio di colore della moquette facilita la comprensione del cambiamento tra "street" e aula, mentre quello della "baia"/spazio anteriore all'ambiente classe è lo stesso dell'aula per condurre il bambino da un'area all'altra. La moquette è blu scura negli spazi di circolazione, mentre blu chiara negli spazi di sosta come le classi o al biblioteca. Sono stati scelti diversi toni dei colori della "terra", pensati per favorire la calma all'interno dell'edificio, ovvero con colori che hanno un impatto positivo sull'umore.

Le pareti dell'atrio sono neutre, permettendo ai disegni dei bambini attaccati alle pareti di personalizzare lo spazio. L'aula può inoltre cambiare colore, sulla base della volontà dell'insegnante di aggiungere o meno stimoli.

Tutte le porte della scuola sono coordinate come tono di colore, anche se ogni classe ha una porta di colore diverso così che possa essere facilmente riconoscibile dai bambini.

\subsubsection{Ambienti sicuri e durevoli}

Le finiture e gli arredi sono progettati per essere resistenti, con vernici a base d'acqua e sigillanti atossici.

Lo sportello dei dispositivi energetici, elettrici o per l'antincendio è stato rivestito con una superficie riflettente per dare l'illusione di poter vedere tutto il corridoio e creare un senso di spazialità.

Il riscaldamento a pavimento permette di non avere risorsa diretta di calore e di controllare la temperatura in modo separato per ogni area, eliminando i radiatori tradizionali, sgradevoli e visivamente distraenti.

\subsection{Forbush School for Autism}

Sede: dintorni di Baltimora, Hunt Valley, Maryland

Data: 2008

Uso: scuola speciale per bambini con Disturbi dello Spettro Autistico

Utenti: bambini 5-21 (72 studenti +60 operatori)

Progettazione: Cho Benn Holback + Associates, Inc.

Arredi e playground: Lynette Pinhey of Human and Rhode, Inc

Tag: \#scuola \#bambini \#ragazzi \#disagio \#benessere \#sicurezza \#orientamento \#autonomia \#integrazione \#uditiva \#visiva \#propriocettiva

Normative di riferimento: Americans with Disabilities Act (ADA); the International Building Code (IBC) 2006; National Fire Protection Agency (NFPA) 101 Life Safety Code. 


\section{Elena Bellini}

Premi e riconoscimenti: AIA Baltimore; Honorable Mention American Association of School Administrators; AIA Citation Universal Design Case Study; AIA Maryland; Merit Award Inform Magazine Merit Award.

Intervento di recupero e riconversione di un edificio degli anni ' 60 ad uso magazzino in un'area di uffici nella Hunt Valley, nel Maryland, nei dintorni di Baltimora. La struttura (Scheda 02), scuola speciale per bambini con Disturbi dello Spettro Autistico tra i 5 e i 21 anni, fa parte del Sheppard Pratt Health System e prevede classi, uffici, spazi per lo sviluppo educativo e dell'apprendimento e un playground disegnato appositamente per bambini con autismo.

\subsubsection{Il progetto}

Il budget di ristrutturazione non era molto elevato pertanto si sono limitati al massimo gli interventi: è stata realizzata una nuova pensilina colorata, per evidenziare l'ingresso (Figura 04); le ringhiere della scala; le aperture, sulla base della nuova disposizione planimetrica che prevede la distribuzione delle classi ai lati del perimetro dell'edificio per avere un massimo daylighting; segnaletica e colori vivaci della tinteggiatura. I colori scelti fanno parte di una palette diffusa nei corridoi e negli ambienti, al fine di differenziare le diverse funzioni e attività specifiche. Nell'area esterna è stato realizzato inoltre l'accesso all'area attraverso il raggiungimento carrabile, un parcheggio e un appropriato spazio per il gioco. All'interno del playground sono state oltretutto incorporate le rampe per accedere all'ingresso principale del playground.

Il disegno generale degli spazi è semplice e chiaro, per ridurre il disagio sensoriale e migliorare orientamento e comprensione. L'impianto planimetrico (Figura 05 - 06) prevede una main street centrale di distribuzione, illuminata da lucernari a soffitto, che dà accesso a tutti gli spazi della scuola e con della segnaletica visiva organizza lo spazio suddiviso nei tre gruppi di classi, associati per età.

Ogni gruppo di aule contiene, oltre allo spazio educativo, servizi comuni, stanze per la terapia e una "resource room". Lo spazio educativo è strutturato in modo semplice e chiaro in aree di attività differenziate, spazio separato per il lavoro individuale o in piccoli gruppi; ogni spazio educativo prevede inoltre il bagno in adiacenza, accessibile direttamente dall'aula.

All'interno della scuola vi è inoltre una stanza polivalente condivisa, una stanza d'integrazione sensoriale, un appartamento usato per la terapia occupazionale ed il ristorante, distribuiti al centro affinché siano accessibili ai tre gruppi di classi. Anche in questo caso è stato usato un gioco di lucernari circolari a soffitto per integrare l'illuminazione e avere sempre luce del giorno diffusa. Gli uffici amministrativi e la reception sono infine vicini all'ingresso (Figura 07).

\subsubsection{Prossemica e dimensione degli spazi}

Le persone con ASD necessitano di maggiore spazio, per permettere il movimento e l'abbattimento dello stress (prossemica), per cui gli ambienti devono essere abbastanza ampi. La dimensione degli spazi ha avuto quindi un ruolo piuttosto im- 
Ambienti sensoriali "terapeutici" che rendano Abili

portante, come anticipato, per cui è stata aumentata la grandezza delle aule all'aumentare dell'età dei bambini. Il primo gruppo ha uno spazio di circa $46,5 \mathrm{mq}$, il secondo di circa $55,7 \mathrm{mq}$, il terzo di 74,3 mq.

Anche la dimensione dei corridoi è stata ampliata e l'altezza dei soffitti, per dare maggiore libertà di movimento e non sentirsi a disagio con l'affluire degli altri bambini.

\subsubsection{Comfort luminoso}

Un altro aspetto molto studiato è la luce, sia per garantire un'illuminazione naturale costante, diffusa e indiretta per eliminare il fenomeno di abbagliamento, che quella artificiale. Sono stati testati diversi tipi di luce artificiale al fine di scegliere la più confortevole a livello visivo per il bambino, che non producesse lo sfarfallio tipico dei neon. La luce viene inoltre riflessa verso il soffitto in modo da essere diffusa in modo indiretto.

\subsubsection{Comfort acustico}

Per il comfort acustico sono stati invece isolati sia pareti che soffitti; sono stati usati pannelli di rifrazione del suono per eliminare il riverbero, soprattutto negli ambienti più ampi come la palestra e la stanza polivalente, o nel corridoio, dove c'è un maggiore afflusso di bambini; è stato installato un sistema di ventilazione meccanica efficiente ma a basso impatto acustico; sono stati scelti materiali di rivestimento a parete per minimizzare la trasmissione del suono ed il riverbero.

\subsubsection{Materiali}

Sono stati usati, in particolare, pannelli di resina riciclata $3 \mathrm{M}$ con una palette di colori diffusa per modulare gli spazi ed evidenziare le zone dedicate alle diverse attività, riducendo inoltre la rigidità della scatola generale.

Per i pavimenti è stato usato il linoleum Armstrong con un pattern di curve fluttuanti e forme che creino uno spazio accogliente e caldo (Figura 07), migliorino il comfort acustico, nell'abbattere rumore e riverbero e supporti e riducano il rischio di lesioni e infortuni.

Per aumentare la possibilità di personalizzare la propria classe, sono state arricchite le pareti con l'integrazione di pannelli con velcro e si è data inoltre la possibilità di avere pattern flessibili a pavimento.

Se da un lato le linee curve rendono l'ambiente maggiormente accogliente e familiare e possono essere di supporto negli spostamenti, l'utilizzo contemporaneo di molti colori potrebbero essere fonte di sovraccarico. Mentre la differenza di colore a parete aiuta ad evidenziare gli spazi ed è di supporto per la comprensione e l'orientamento, l'uso del colore a pavimento non è corrispondente e si usano spesso tondi e curve di colore in modo contemporaneo e ambiguo. Il pavimento in linoleum, inoltre, ha una finitura lucida e potrebbe contribuire a creare disagio a livello visivo, per la riflessione di luci e colori. 


\section{Elena Bellini}

Tutti i materiali, sia quelli usati per il rivestimento che per l'isolamento, non contengono formaldeide; le vernici ed i materiali hanno un basso livello di emissione di VOC (composti organici volatili); i materiali usati sono antibatterici e antistatici; i prodotti usati sono naturali e rinnovabili.

\subsubsection{La sicurezza}

Per quanto riguarda la sicurezza sono state previste delle pareti composte da pannelli di fibra ad alte prestazioni con contenuti riciclabili (USG), che hanno una notevole resistenza e garantiscono maggiore protezione dagli infortuni, soprattutto in situazioni di comportamenti problema. In questo senso è stata limitata anche l'installazione di elementi sporgenti e sono stati previsti armadi incassati integrati a parete colorati.

\subsection{Advance School for Developing Skills of Special Needs Children}

Sede: Qattameya (Cairo)

Data: 2008

Uso: scuola speciale e residenza per bambini con DSA

Utenti: da 2 a 21 anni; 70 studenti full-time e 50 studenti part-time (massimo).

Gestione: Società Egiziana per lo sviluppo delle Abilità dei bambini con Bisogni

Speciali in Egitto

Progettazione: Magda Mostafa

Tag: \#scuola \#bambini \#ragazzi \#disagio \#benessere \#sicurezza \#orientamento \#autonomia \#integrazione \#uditiva \#visiva \#olfattiva \#tattile \#propriocettiva

Servizio educativo a tempo pieno fornito dalla Società Egiziana per lo sviluppo delle Abilità dei bambini con Bisogni Speciali in Egitto, ed in particolare con autismo (70\% del corpo studentesco), volti all'indipendenza e all'integrazione dei bambini/ragazzi nella società (anche attraverso l'inserimento lavorativo). Il centro (Figura 09) ospita bambini dai 2 ai 21 anni, con un massimo di 70 studenti full-time e circa 50 a part-time e si trova in una periferia di nuovo sviluppo della New Cairo, nel distretto di Qattameya (Cairo, Egitto).

Il progetto (Scheda 03), dell'architetto Magda Mostafa, nasce da un percorso di ricerca e dall'applicazione dei principi di design dell'ASPECTSS ${ }^{\text {TM }}$ index, usato come matrice generatrice delle soluzioni di progetto. L'applicazione di questi principi sull'edificio ha dato vita a delle Linee Guida di spazi autism-friendly.

\subsubsection{Inclusione}

Uno dei problemi fondamentali per i bambini con bisogni speciali è l'inclusione ed il rispetto all'interno della società. Per questo motivo sono stati inseriti all'interno della struttura servizi per la comunità, come spazi commerciali (arts and crafts, centro informatico, forno...), che creano opportunità di interazione e la sensibilizzazione della società sul tema dell'autismo. 


\subsubsection{Distribuzione sensoriale}

Le diverse aree del progetto (Figura 08) sono state organizzate non tanto in modo convenzionale, in base alla funzione, ma piuttosto sulla base della sensorialità delle di verse aree e sul livello di stimolazione (Figura 09). Al fine di ridurre il sovraccarico sensoriale, le aree di deposito, di esposizione, o quelle dedicate al lavoro e aperte al pubblico, sono visivamente e spazialmente separate rispetto alle altre. I servizi, che sono da considerarsi aree ad alto stimolo, come bagni, cucine, stanze del personale o dell'amministrazione, sono inoltre separate dalle aree studio, così come le funzioni come la musica o la psicomotricità. I giardini, la aree di gioco libero o le aree sensoriali faranno da cuscinetto e filtro tra le aree a basso e ad alta stimolazione sensoriale. Al fine di migliorare la qualità degli spazi e la riduzione degli stimoli è stato ottimizzato al massimo, inoltre, l'utilizzo di luce e ventilazione naturale, ed è stata riservata una particolare attenzione nei confronti del comfort acustico.

\subsubsection{Orientamento e wayfinding}

La circolazione all'interno dell'edificio è stata predisposta per essere compiuta in un'unica direzione principale, organizzata sulla base della routines. In questo modo il bambino può sentirsi maggiormente libero e indipendente. Per le indicazioni viene utilizzato il sistema delle PECS come supporto alla comunicazione e all'apprendimento per immagini. La comunicazione attraverso immagini è di supporto alla segnaletica, in modo parallelo alle scritte, al fine di favorire l'apprendimento, attraverso l'associazione di parole e immagini, ma anche la crescita di autostima e inclusione dei bambini con diverse abilità. Lo spazio circolare centrale suddivide le due aree a diversa stimolazione sensoriale e funziona anche da filtro e preparazione tra un'area e l'altra.

Gli spazi di circolazione sono inoltre arricchiti da supporti visivi come colori e pattern, per favorire il wayfinding, anche se non in modo troppo eccessivo, al fine di non causare anche in questo caso una sovrastimolazione visiva.

L'uso di materiali contrastanti tra pavimento, pareti, porte o soffitto, è di supporto inoltre alla definizione e alla differenziazione dei diversi attributi dello spazio, facilitando orientamento e propriocezione.

Nei collegamenti si usa infine al massimo la luce naturale, pur evitando abbagliamento e contrasti delle ombre, che potrebbero sovraccaricare a livello visivo.

\subsubsection{La strutturazione degli spazi di apprendimento}

Il centro educativo e terapeutico comprende 10 aule base, corredate da spazi di osservazione, spazi per i training one to one, stanze per la preparazione degli insegnanti e depositi. Gli spazi educativi sono nell'area della scuola a basso stimolo, per ogni classe sono presenti 7 studenti con almeno 3 insegnanti e assistenti.

L'organizzazione delle aule è stata effettuata per gruppi, in un ambiente a scala ridotta, affinché sia il più possibile familiare. I gruppi sono rivolti verso l'interno dove è possibile ottenere uno spazio open-air interno e contenuto, utilizzato per di- 


\section{Elena Bellini}

verse funzioni, tra cui fare attività didattica all'esterno, rivolta a temi come la natura, il tempo, le abilità motorie etc.

L'aula è divisa in compartimenti, dove ciascuna "stazione" può essere físicamente e visivamente separata dalle altre attraverso partizioni, colori e materiali a pavimento, al fine di focalizzare l'attenzione del bambino sull'attività.

Le risorse didattiche devo essere a disposizione del bambino e degli insegnanti, ma non continuamente visibili, per limitare il sovraccarico visivo, la distrazione e l'attivazione del bambino; per questo motivo sono stati previsti armadi a muro o scaffali con scatole e contenitori dai colori neutri. Queste componenti sono posizionate al centro di due aule al fine di ottimizzare lo spazio.

L'isolamento acustico dell'aula permette di ridurre il rumore proveniente dall'esterno e il riverbero interno.

La luce naturale arriva dall'alto attraverso finestre al di sopra del livello degli occhi e orientate a nord, per eliminare la distrazione visiva, la luce diretta e l'abbagliamento.

All'interno dell'aula è inoltre previsto un “escape space”, ovvero un'area isolata dove potersi rifugiare in un momento di sovrastimolazione, o sopraffazione; questo ambiente é neutro, come se fosse una piccola Snoezelen room, e consente di personalizzare lo spazio in base alle proprie necessità sensoriali. Anche solo la presenza di questo spazio, e non per forza il suo utilizzo regolare, è sufficiente per ridurre i capricci, la rabbia e le esplosioni di violenza, aumentando la produttività della classe. Può essere usata all'inizio o alla fine delle lezioni per calmare il bambino e prepararsi ad essere maggiormente recettivo all'apprendimento.

Accanto all'ambiente classe sono infine previste stanze di osservazione, utili per la partecipazione del genitore con una finestra a specchio in una sola direzione e dispositivi per la registrazione, usato anche per la formazione degli insegnanti e percorsi di training a casa.

\subsubsection{La strutturazione degli spazi di terapia}

L'ambiente educativo è supportato da spazi per la psicomotricità, per la terapia occupazionale, per la fisioterapia, idroterapia con piscina interna; spazi per l'apprendimento di abilità artistiche ed arteterapia come teatro, musicoterapia e del movimento. A parte la logopedia, tutte le attività terapeutiche, come psicomotricità e terapia occupazionale, sono spazi ad alto stimolo.

Ogni area è separata acusticamente e anche in questo caso è previsto l'utilizzo della luce naturale e indiretta, da un'altezza sopra gli occhi per limitare la distrazione visiva e, per quanto riguarda quella artificiale, l'eliminazione di luci fluorescenti (sia per il brusio prodotto che per lo sfarfallio della luce).

Ogni area terapeutica ha bisogno di una diversa qualità dello spazio, sulla base dell'attività svolta. Ad esempio, la psicomotricità necessita di uno spazio lineare che permetta i movimenti nella sua lunghezza; lungo il lato corto invece una parete scorrevole nasconde una zona di 'preparazione' con scaffali e materiale organizzato e riposto, nascosto alla vista dei bambini. L'arteterapia prevede varie attività tra cui pittura, editoria/stampa, scultura e ceramica, collocata nella terrazza esterna. Le attività sono organizzate in stazioni come nelle classi, parzialmente visibili o separate 
spazialmente. Un'area di preparazione professionale è posta al piano terreno, connessa ad un'aerea esterna dove fare falegnameria, carpenteria metallica, o giardinaggio didattico. La piscina coperta interna e l'idroterapia sono poste il più lontano possibile dalle classi e delle zone a basso stimolo; l'area è inoltre racchiusa in un involucro ad alte prestazioni acustiche, anche per evitare il riverbero. L'idroterapia comprende una piscina sensoriale che attiva getti d'acqua rivolti a diverse parti del corpo per offrire stimolazioni tattili. La logopedia è invece prevista nell'area a basso stimolo ed è ben isolata acusticamente, benché per evitare il cosiddetto "effetto serra", siano previste stanze a diverso grado di isolamento, aumentando pian piano le abilità di adattamento al contesto, fino all'immersione nel mondo esterno, ovvero nell'ambiente non controllato.

\subsubsection{Spazi di apprendimento all'aperto}

E' stato dimostrato come l'ambiente esterno sia molto utile in casi di autismo e che favorisca l'apprendimento. Il progetto prevede spazi educativi all'esterno, come spazi patio annessi alle classi, corti suddivise per età, playground per la psicomotricità, spazi per il gioco formale ed informale, un giardino sensoriale e l'orto. Ogni area è completamente accessibile e utilizzabile da tutti i bambini, compreso il parco giochi. Se da un lato la corte esterna è un'occasione di interazione in piccoli gruppi, dall'altro il grande parco giochi può essere usato per un'interazione a più larga scala.

Il giardino sensoriale si trova a l'interno dell'ampio spazio verde e presenta percorsi a diverse textures, giochi d'acqua, una vasca di palline, aree con la sabbia ed un giardino di erbe aromatiche, come cuore centrale dello spazio.

Il giardinaggio didattico è utile anche per la manutenzione di questo spazio, oltre che per l'apprendimento di nuove abilità e i benefici di benessere per la persona.

Lo spazio esterno ha in questo progetto anche valore di spazio di transizione, tra le due aree a diverso livello di stimolazione sensoriale. A livello sensoriale sono particolarmente utili anche i giochi d'acqua, poiché possono mascherare i rumori di fondo.

Sempre all'esterno è prevista inoltre una parete con una tinteggiatura a lavagna, dove è possibile dare libera espressione all'arte. Per chi si vuole invece rilassare sono state previste delle sedute raccolte, a nido, protette dall'irraggiamento solare, anche perché il caldo potrebbe causare delle convulsioni.

\subsubsection{Strutturazione degli spazi per l'abitare}

La struttura residenziale assistita prevede camere per gli studenti singole o doppie (9 alloggi con 9-16 studenti) e bagno di pertinenza, una camera per il supervisore (con bagno), soggiorni comuni e spazi di lavoro (1 per piano, ovvero 3 ), cucina (1 per piano, ovvero 3), uffici e spazio per le famiglie e per gli incontri di gruppo. L'edificio è suddiviso in 3 appartamenti, che assomigliano nello stile e nella morfologia a dei comuni appartamenti egiziani. Gli studenti possono condividere la stanza con un altro studente, con un supervisore, o vivere indipendentemente, in base alle proprie abilità e al livello di indipendenza. 


\section{Elena Bellini}

Gli alloggi vogliono essere una sorta di transizione tra la residenza assistita e quella indipendente, al fine di integrare il bambino/ragazzo nella comunità. Non è pensato per essere un ambiente istituzionale, ma è strutturato al fine di ottimizzare l'apprendimento delle abilità e delle autonomie. Gli alloggi non sono direttamente connessi alla scuola, proprio per non dare un senso di istituzionalizzazione, e al contrario, portare gli studenti a raggiungere a piedi ogni giorno l'ingresso principale della scuola, passando attraverso il traffico e la comunità vicina.

\subsection{Northen School for Autism}

Sede: Reservoir Victoria (Australia)

Data: 2013

Uso: scuola speciale per bambini con Disturbi dello Spettro Autistico

Utenti: 144 bambini (potenzialmente 180) di 5 ai 18 anni anni

Dimensione: $20.400 \mathrm{mq}$ (Sup. lorda)

Gestione: Northern School for Autism (NSA)

Progettazione: Hede Architects

Tag: \#scuola \#bambini \#ragazzi \#disagio \#benessere \#sicurezza \#orientamento

\#autonomia \#integrazione \#visiva \#propriocettiva

Premi e riconoscimenti: Vincitore del 2013 CEFPI Regional Award; Vincitore del 2013 CEFPI Educational Facilities Award for the Australasian Region for New Construction: Entire New School; Selezionato nella longlist $\left(16^{\circ}\right)$ del World Architecture News Awards 2013 - Education Category e nella shortlist del 2013 Victorian Architecture Awards - Sustainability Category.

La Northern School for Autism (Scheda 04) è una scuola speciale del Victorian State Government esclusivamente pensata per i bisogni educativi di studenti con Disturbi dello Spettro Autistico.

\subsubsection{Il programma educativo e terapeutico}

L'impianto scolastico (Figura 10 - 11) è diviso in diversi edifici in base ai livelli scolastici e all' età - Early Years $\sim 5-8$ anni, Middle Years $\sim 9-14$ anni, Later Years $\sim 15-18$ anni - a loro volta suddivisi in classi di circa 6-8 studenti.

Due o tre insegnanti ed assistenti lavorano in cooperazione in ogni classe, con un rapporto insegnante/studente di 1:3, e sono supportati da logopedisti, terapeuti occupazionali, insegnanti specializzati ed un team di insegnanti leader, lavorando in modo transdisciplinare nell'aula, affinché lo studente abbia accesso ad un programma completo ed efficace di terapia.

Il programma educativo prevede di sviluppare abilità comunicative ed interpersonali ed aiutare gli studenti a comprendere ed autoregolare le emozioni ed i comportamenti. I servizi del campus possono riassumersi in: una stanza per la terapia ludica, una stanza per la musica, tre palestre per la terapia occupazionale, una stanza per le arti visive, una cucina per gli studenti, una stanza polivalente, una biblioteca per gli studenti, le 20 aule ed un giardino esterno con playground, aree coperte, aree di sabbia, zone per l'arrampicata e una pista ciclabile. 


\subsubsection{II progetto}

Gli spazi per l'apprendimento sono suddivisi in sub-scuole che hanno accesso indipendente e sono raggruppate attorno agli spazi verdi, favorendo l'accesso individuale all'area gioco da tutti gli spazi educativi. Lo spazio centrale verde si pone come un'area privata e protetta dedicata ai bambini più piccoli; da ogni gruppo di aule si accede comunque ad uno spazio verde dedicato e progettato in base alle diverse età. Questa suddivisione in zone ben distinte aiuta l'orientamento del bambino e favorisce la chiarezza e la comprensione.

Le aule sono distribuite da percorsi di circolazione curvilinei ben definiti, per favorire l'orientamento, che non vanno ad interagire con lo spazio educativo, in modo da ridurre le distrazioni. Ogni aula ha spazi flessibili, consentendo di essere usata sia per lavori in gruppo, che risuddivisa per lavori individuali o di piccoli gruppi; comprende quindi una sala da lavoro, un cortile esterno "privato" e protetto, un angolo cottura ed un ripostiglio ampio. Prevede inoltre un'area di "quiete" di dimensione contenuta, adiacente alla classe, per consentire di isolarsi e calmarsi nei momenti in cui è necessario, o programmi di insegnamento più tranquilli e mirati e $1 \mathrm{o}$ 2 studenti. La distribuzione dello spazio aula, degli arredi e degli spazi esterni consente un continuo controllo da parte dell'insegnante e la possibilità di lasciare il bambino in completa sicurezza, rendendolo maggiormente indipendente.

Le aree rivolte verso sud sono dedicate allo staff e all'amministrazione, consentendo di creare spazi individuali e privati.

La stanza polivalente è pensata anche per svolgere seminari con i genitori, gli insegnanti e la comunità, anche per informarla e sensibilizzarla verso l'autismo.

\subsubsection{Comfort visivo}

Tutta la scuola ha colori con toni soft e naturali, che derivano dai toni della terra, per rispondere alla sensibilità visiva dei bambini. Ogni area si differenzia per colore, dall'esterno, al fine di aumentare la comprensione ed il wayfinding del bambino, oltre che la sua indipendenza. Le aule hanno invece colorazioni neutre all'interno, per ridurre la stimolazione ed aumentare la concentrazione.

\subsubsection{Comfort luminoso}

Alcuni tagli sulla copertura orientati a nord fanno penetrare una luce indiretta all'interno delle aule per un comfort luminoso e la riduzione del sovraccarico sensoriale. Negli spazi educativi vi sono in ogni caso grandi aperture che mantengono un rapporto verso l'esterno e luce naturale continua. La luminosità delle aule attraverso la luce naturale permette inoltre di minimizzare l'uso delle luci artificiali, comunque diffuse dall'alto attraverso elementi incassati a soffitto; sono state evitate luci fluorescenti. 


\section{Elena Bellini}

\subsection{Villa delle Rogge}

Sede: Pordenone

Data: 2012

Uso: residenza temporanea, foresteria, programma respiro, progetto abilità e occupazione lavorativa (Officina dell'Arte)

N. Utenti: 20 settimanali (9 stanze da letto)

Committente: Fondazione Bambini e Autismo Onlus

Tag: \#residenza \#lavoro \#adulti \#disagio \#benessere \#sicurezza \#orientamento \#autonomia \#integrazione \#uditiva \#visiva \#tattile \#propriocettiva

Villa delle Rogge (Scheda 05) nasce come casa/foresteria a carattere temporaneo per persone con autismo, presentandosi come un'assoluta novità nel campo della residenzialità per disabili. La struttura partecipa al progetto "Vivi la città", per l'integrazione nella città degli adulti con autismo che lavorano a Pordenone. E' funzionante 24 ore su 24 per tutta la settimana; permettere a molti adulti con autismo di intraprendere un percorso di autonomia che potrà migliorare la propria qualità di vita e consente alle famiglie di poter avere un periodo di riposo per riuscire a reggere più a lungo l'impegno di cura della persona con autismo (Programma Respiro).

\subsubsection{Il progetto}

La "casa" (Figura 12) sorge su un terreno piantumato che è stato concesso alla Fondazione Bambini e Autismo Onlus in diritto di superficie per 99 anni dal Comune di Pordenone. La Fondazione, nata nel 1998 da una coppia di genitori di un ragazzo autistico, è da anni impegnata nel creare e sperimentare modelli di presa in carico di persone con ASD in grado di dare una risposta articolata ai loro bisogni lungo il corso della vita. Il progetto è un modello innovativo di residenzialità in Italia e in Europa, sia come prototipo di residenza adatta all'accoglimento delle persone con autismo, sia per la flessibilità e la temporaneità della permanenza delle persone nella struttura. Villa delle Rogge è infatti una palestra per l'autonomia dove si imparano alcune regole del vivere sociale che possono poi essere spese nella propria casa e in altri contesti sociali.

La casa ha 9 camere in totale che a turno ospitano oltre 20 persone a settimana. I fruitori del complesso imparano a cucinare i propri pasti, ad utilizzare il bagno in modo autonomo, ad occuparsi delle azioni domestiche più comuni in una casa (fare il letto, stirare, apparecchiare, mettere in ordine, ecc.) e ad utilizzare elettrodomestici e dispositivi che poi ritroveranno nelle loro case. All'interno hanno la possibilità di usufruire di spazi in cui isolarsi dagli stimoli esterni, quando hanno bisogno di autoregolarsi (come ad esempio attraverso la stanza di decompressione) e di vari spazi di relazione a diversi gradi socialità, differenziati in modo da non causare il sovraffollamento degli ambienti e favorire il rispetto della privacy.

\subsubsection{Distribuzione funzionale}

La casa è suddivisa in due piani ed un interrato. 
Al piano terra si trova la zona giorno (Figura 13), filtrata dall'esterno attraverso una confortevole area di reception e attesa subito in adiacenza all'ingresso, composta da soggiorno, sala da pranzo, un'ampia cucina con penisola centrale e doppie postazioni di lavoro per partecipare in più e non darsi fastidio (Figura 14). E' inoltre prevista un'altra piccola cucina, più appartata, utilizzata per i programmi di abilitazione e apprendimento.

Al piano superiore sono invece collocate le camere, nove in tutto, e uno spazio per il personale. Il collegamento avviene attraverso una comoda scala che, attraverso un pianerottolo di arrivo in cristallo, permette di mantenere la visibilità tra i diversi piani, utile sia per i residenti per mantenere la relazione, che per il controllo da parte degli operatori. I colori di ogni stanza sono stati scelti dagli stessi utenti, con arredo abbinato alle colorazioni della parete. L'ambiente è inoltre personalizzato dai rivestimenti sia a pavimento, inserendo dei riquadri in mosaico all'interno del parquet, che nel rivestimento dei bagni, che a parete, attraverso l'affissione di quadri a mosaico realizzati dai ragazzi presso l'Officina dell'Arte. Questo aspetto è fondamentale per i ragazzi poiché li fa "sentire a casa propria" e innesca un sistema di appartenenza e affettività verso la residenza. Durante la visita alla struttura la dott.ssa Raffin mi ha raccontato, a questo proposito, come uno dei ragazzi avesse portato la sorella a Villa delle Rogge per mostrarle la "propria casa" e la propria stanza, orgoglioso e fiero come fosse il suo appartamento personale.

$\mathrm{Al}$ piano interrato infine vi è una palestra, una stanza di decompressione, un ambulatorio e una stanza per la terapia personalizzata o colloquio con lo psicologo.

\subsubsection{Design for all e comfort abitativo}

La casa è curata nei dettagli, come una villa confortevole ed elegante, in cui chiunque si sentirebbe a casa. L'ambiente è completamente accessibile, disegnato veramente "per tutti".

Le colorazioni sono neutre, bianche a parete e parquet naturale a terra, per offrire un ambiente caldo, accogliente e di sapore domestico.

Gli arredi sono moderni e funzionali ed arricchiscono l'alloggio con un po' di colore, rendendolo vissuto e vivace, senza mai esagerare, per non sovraccaricare visivamente la persona.

La casa è luminosa e vi sono ampie vedute verso l'esterno, anche se all'occorrenza è possibile oscurare le vetrate per una regolazione della luce. Le luci artificiali sono calde e soffuse come in un appartamento familiare.

Le porte scorrevoli interne sono state realizzate di larghezza maggiore rispetto a quelle standard, non per necessità, ma per una migliore flessibilità e abitabilità degli spazi. Questo è interessante soprattutto nel soggiorno dove, con questa possibilità, si è in grado di suddividere l'ambiente in aree più piccole dedicate anche all'individuo o a piccoli gruppi, se vogliono fare diverse attività o isolarsi per un po'.

Gli infissi a pian terreno sono ad apertura scorrevole con maniglioni che ne rendono l'uso più semplice. Al piano superiore sono invece bloccati da un sistema meccanico, per una maggiore sicurezza; per lo stesso motivo non sono presenti balconi nelle camere o negli spazi al piano primo.

Il sistema di riscaldamento e rinfrescamento è pensato a pavimento per un maggiore comfort, poiché consente una regolazione manuale e automatica. 


\section{Elena Bellini}

Sono previsti bagni per disabili (previsti dalla normativa, anche se le persone con autismo non ne avrebbero bisogno per il loro tipo di patologia), che sono però al contempo funzionali ed esteticamente curati nei minimi dettagli.

I rivestimenti sono stati scelti dagli stessi ragazzi, utilizzando il mosaico prodotto presso la struttura dell'Officina dell'Arte dove lavorano.

Per il comfort acustico è prevista l'insonorizzazione di pareti e porte, silenziatori per gli impianti, stanze singole o al massimo doppie con bagno privato, importante inoltre per la salvaguardia della privacy e per evitare interferenze tra modi di gestire gli ambienti da parte delle persone con autismo, che amano un ordine personale modificabile con rigidità.

Come sistema di strutturazione degli spazi e comunicazione facilitata per immagini, sono state applicate delle targhette su ogni mobile e contenitore della casa e delle regole visive e istruzioni step by step per guidare l'utente dall'arrivo, per riporre le proprie scarpe nel guardaroba, alle attività da compiere in casa, come in cucina, fino alle più private nelle camere o nei bagni.

\subsubsection{Gli spazi esterni}

Nel giardino sono state realizzate diverse zone per il benessere all'aperto: una vasca Kneipp rivestita dagli stessi utenti con un mosaico di ciottoli, una piccola roggia lungo il camminamento che richiama il toponimo in cui si trova la casa (via Roggiuzzole), entrambi alimentate dall'acqua della pompa di calore, una zona gioco (altalena, scivolo, ecc.) schermata da una siepe per evitare che gli adulti che frequentano la casa possano trovarla inappropriata.

\subsubsection{Officina dell'Arte}

L'Officina dell'Arte è il punto di incontro di mosaicisti, designer, operatori terapeutici e persone con autismo adulte, impegnate nei programmi lavorativi e per la autonomia, dando vita a prodotti unici musivi artigianali, opere di grandi dimensioni e interpretazioni a mosaico di opere d'arte pittoriche. In particolare, all'Officina si realizzano mosaici artistici, oggettistica decorata a mosaico, confezioni di vino etichettate nel laboratorio di packaging e personalizzabili su richiesta, bomboniere per matrimoni, battesimi, anniversari e altre ricorrenze, idee regalo e soluzioni per la regalistica aziendale, merchandising collegato alle mostre d'arte. Il ricavato delle vendite dei prodotti contribuisce a sostenere il Centro e al contempo rappresenta un feedback, in termini di gratificazione e autostima, per le persone con autismo che li hanno creati. Tra i principali lavori a grandi dimensioni sino ad ora eseguiti vi sono due mosaici di 9 mq ciascuno dedicati a Pier Paolo Pasolini e installati nella omonima Scuola Media di Pordenone, che li ha commissionati, e il Mosaico della pace installato nel Parco San Valentino di Pordenone, realizzato su progetto grafico di Francesco Tullio Altan. I lavori artistici realizzati all'Officina vengono invece esposti in mostre ormai annuali, a partire dal 2005.

La struttura, inaugurata nel 2004, nasce da un restauro di un'archeologia industriale, un tempo sede delle storiche Officine Savio, di circa $350 \mathrm{mq}$, e prevede ad oggi un laboratorio professionale di mosaico, un laboratorio di packaging, un labora- 
torio di informatica, uno showroom aperto al pubblico, una palestra con spogliatoi e doccia, e una sala per il tempo libero e il coffee break. Ogni area è caratterizzata e differenziata con un diverso colore (Figura 15 - 16), per facilitare la comprensione e la strutturazione delle attività lavorative. I colori e i rivestimenti a parete son coordinati nel colore, come gli arredi, suddividendo l'ampio spazio in aree aperte e comunicanti, ma indipendenti, per facilitare il lavoro dei ragazzi.

\title{
4.6 Sweetwater Spectrum Community
}

\author{
Sede: Sonoma (USA) \\ Data: 2013 \\ Uso: residenza permanente \\ N. Utenti: 16 residenti adulti \\ Committente: Sweetwater Spectrum, Inc. \\ Progetto: Leddy Maytum Stacy Architects \\ Tag: \#residenza \#lavoro \#adulti \#disagio \#benessere \#sicurezza \#orientamento \\ \#autonomia \#integrazione \#uditiva \#visiva \#olfattiva \#propriocettiva
}

Sweetwater Spectrum (Scheda 06) è un nuovo modello nazionale di supportive housing per una comunità di 16 residenti con autismo, creato per trovare risposta alla crisi dell'housing delle persone con DSA, integrando design for autism, universal design e strategie per la sostenibilità.

\subsubsection{Il progetto}

Sweetwater Spectrum è un'organizzazione no-profit fondata nel 2009 da famiglie, professionisti ed esperti di autismo e leaders della comunità, per provvedere in modo appropriato a soluzioni dell'abitare di alta qualità per aumentare lo sviluppo individuale e l'indipendenza degli adulti con autismo. Il progetto include 4 case, un centro comunitario, una piscina terapeutica e una fattoria urbana. La mission del progetto è permettere ad ogni individuo di raggiungere il massimo potenziale all'interno di una comunità rivolta alle persone con tutte le abilità, promuovendo indipendenza, salute e benessere e creando legami tra territorio, persone e comunità. Sonoma è risultato un contesto favorevole al posizionamento della comunità residenziale per l'autismo poiché è collocata centralmente ad una vasta area di 11.300 mq, vicino ai trasporti pubblici e con una distanza colmabile a piedi o in bici dal centro della città. Si è innescato inoltre uno scambio tra Sweetwater e la comunità locale, ovvero i residenti di Sonoma fanno attività di volontariato a Sweetwater, mentre gli abitanti partecipano nella comunità con lavori part-time, lezioni, attività volontarie e fare parte della banda di zona. La fattoria organica di circa $4.000 \mathrm{mq}$, il frutteto, e la serra supportano le attività rurali e i progetti per i residenti e i volontari della comunità. La produzione dalla fattoria e frutteto è utilizzata quotidianamente dai residenti e anche venduta al mercato locale. La generosa varietà di spazi esterni e la fattoria ha profondamente connesso i residenti di Sweetwater all'ambiente, alla comunità e ai ritmi terapeutici del mondo naturale. Il sito è collocato nel clima temperato e marino della costa interna (entroterra) della Northern California. 


\section{Elena Bellini}

Gli edifici sono distribuiti lungo il perimetro della parte centrale interna del sito, preservando la vista sul paesaggio. Sono stati progettati per garantire flessibilità e adattabilità a lungo e breve termine. La pianificazione del sito è stata inoltre progettata per l'eventuale addizione di altre 4 unità residenziali all'interno dell'area agricola, la conversione della comunità in un co-housing o eventuale residenza per gli anziani se in futuro servirà. La configurazione di ogni casa permette infatti una diversità di opportunità residenziali (donne, uomini, utenti con bisogni simili o abilità simili, coppie che potrebbero formarsi), che possono anche evolvere nel tempo. La configurazione di ogni residenza è simile, inoltre, per promuovere l'efficienza della costruzione e condizioni simili in caso di trasferimento di qualche persona.

Gli edifici hanno forme compatte che riducono al minimo lo spazio servente, ottimizzando gli spazi.

L'ingresso avviene in modo "protetto" attraverso una corte privata; l'edificio presenta poi uno spazio centrale comune come fulcro dell'alloggio e spazi residenziali nelle ali, con due camere private e bagno specchiati in modo simmetrico rispetto al centro.

Gli edifici sono orientati in modo da massimizzare l'apporto solare e creare una varietà di spazi esterni usati per le diverse attività o per il relax. Tutti gli spazi occupati regolarmente hanno viste verso l'esterno e molte con diretta connessione con le zone esterne inclusi i patio, le terrazze comuni e i giardini.

Diversi spazi di relazione sono previsti sia all'esterno che all'interno; la cucina predispone a cucinare, la sala da pranzo favorisce le relazioni, diverse possibilità di seduta danno la possibilità di interagire con la comunità attraverso diversi e preferibili gradi di socialità.

Le finiture principali sono state selezionate per durabilità, ridotta gestione e facilità di manutenzione. Secondo lo stato dell'arte della ricerca sull'autismo, gli spazi sono stati progettati per ridurre le stimolazioni sensoriali (acustica, pattern visivi, odori, ecc.), connettersi con il mondo naturale e creare un ambiente domestico sereno, sicuro, confortevole, leggibile e prevedibile. I materiali e i sistemi dell'edificio promuovono la salute indoor attraverso qualità dell'aria, controllo acustico e comfort termico.

\subsubsection{Comfort termico e climatico}

Il controllo e il comfort climatico sono aspetti critici, proprio a causa della sensibilità sensoriale dei residenti. Oltre alle strategie passive dell'edificio, gli involucri sono ottimizzati con un'alta performance degli infissi e l'isolamento dell'edificio.

Ogni edificio è inoltre progettato per dare un equilibrio di luce naturale con volumi inclinati del tetto, in risposta all'orientamento solare. Beneficiando del clima temperato il $100 \%$ degli edifici sono naturalmente ventilati e raffrescati. Tende da sole, coperture e portici orientati a sud, i sistemi di oscuramento delle facciate est e ovest supportano inoltre il controllo dell'irraggiamento solare ed il comfort termico.

Il sistema di riscaldamento è a pannelli radianti e il sistema di raffrescamento usa un sistema a bassa velocità dato che le ventole a soffitto possono disturbare gli abitanti. 


\subsubsection{Comfort luminoso}

L'orientamento adeguato, la distribuzione delle aperture in facciata e la previsione di lucernari a cono, consente di avere luce naturale al 100\% dell'area residenziale; il 93\% degli spazi usano la luce del giorno come primaria risorsa di luce. In particolare le aree giorno hanno grandi aperture rivolte verso nord, in modo da non avere mai una luce forte e diretta, ma ampia visibilità e relazione con l'esterno; dal lato sud invece aperture alte permettono di migliorare la qualità luminosa dello spazio, riflettendo la luce forte verso il soffitto e diffondendola negli ambienti ampi e chiari, favorendone l'illuminazione naturale; le aree notte invece hanno aperture a est e ovest. L'alta efficienza luminosa è inoltre controllata da interruttori e timer piuttosto che da sensori di occupazione, per non disturbare la particolare sensibilità degli utenti.

\subsubsection{Qualità dell'aria}

Gli edifici sono orientati secondo i venti principali, permettendo la ventilazione naturale di tutti gli spazi residenziali. E' inoltre previsto un sistema di ventilazione a bassa velocità per assicurare la qualità dell'aria interna anche quando le finestre sono chiuse.

\subsubsection{Materiali e durabilità}

I materiali usati stati scelti con particolare attenzione alla salute, all'efficienza delle risorse e ai bisogni specifici degli utenti, incluse vernici, colle e sigillanti senza emissione di composti organici volatili; isolamento in cotone e prodotti del legno senza formaldeide; pavimenti non vinilici e non tossici, ma in linoleum; riscaldamento radiante per ridurre gli allergeni. Muri a secco resistenti all'impatto realizzati con materiali durevoli, porte di tipologia commerciale, rivestimento esterno in cemento, sono inoltre strategie per aumentare la durabilità dei materiali e delle strutture.

\subsubsection{Comfort acustico}

Gli interni presentano un trattamento acustico avanzato per controllare il suono. Le coppie di camere sono inoltre posizionate ai lati opposti della casa per favorire la privacy, anziché in una fila unica come se fosse un dormitorio.

\subsubsection{Sicurezza e assistive technology}

Un controllo domotico digitale dell'edificio monitora tutti i sistemi. I dispositivi ad alta efficienza e i sistemi per la cucina ad induzione sono stati scelti per sicurezza, l'autonomia ed il controllo energetico. L'efficienza luminosa è controllata con timer automatici poiché i sensori distrarrebbero i residenti. 


\section{Elena Bellini}

\subsection{Seniors House}

Sede: Hinnerup (Danimarca)

Data: 2014

Uso: residenza permanente per adulti/anziani con autismo

N. Utenti: 16 residenti

Progetto: Wienberg Architects, Frier Architecture og Møller og Grønborg A/S

Tag: \#residenza \#lavoro \#adulti \#disagio \#benessere \#orientamento

\#autonomia \#integrazione \#uditiva \#visiva \#olfattiva \#propriocettiva

Seniors House (Hinnerup Kollegiet) (Scheda 07), in Danimarca, è una residenza per 16 adulti con autismo, costruita come ambiente di supporto e adattivo dall'inizio dell'età adulta fino all'anzianità. E' uno degli esempi europei più interessanti di residenza dedicata specificatamente all'anzianità in casi di autismo. Ad oggi ospita alcune delle persone con autismo più anziane d'Europa; alcune vi hanno vissuto per 30 anni!

\subsubsection{La storia}

Hinnerup Kollegiet è nata nel 1982, con una comunità di 10 adulti con autismo. I giovani residenti avevano la possibilità di imparare le abilità quotidiane, farsi amici, o partecipare a workshop. Con il passare del tempo e lo sviluppo di nuovi approcci abilitativi, oltre alla consapevolezza che l'autismo è una condizione pervasiva, è sopraggiunta la necessità di avere una residenza permanente. La vecchia residenza non era stata pensata per altri tipi di disabilità, non presentava ad esempio supporti nel bagno per i disabili e non vi era abbastanza spazio nelle camere per accesso alle sedie a rotelle. Per questo motivo nel 2013 è stato deciso di costruire nuovi alloggi, progettati fin dall'inizio in stretta collaborazione con gli Wienberg Architects, per ridurre lo stress, le difficoltà sensoriali e l'ansia tipica di queste persone durante tutta la loro vita. Il coinvolgimento diretto delle persone con autismo e dei loro familiari è stato fondamentale nelle scelte architettoniche e di design, oltre al supporto di Autism Denmark e il Danish National Board of Social Services. Nel 2014 ha quindi aperto Seniors House, il primo ambiente danese progettato unicamente per le persone anziane con ASD.

\subsubsection{L’approccio terapeutico}

Ogni persona è diversa e ha differenti bisogni, per cui ogni decisione va presa nel rispetto del singolo; lo staff lavora insieme al residente, supportandolo e incoraggiandolo, al fine di aumentare comprensione e comunicazione, anche attraverso sistemi di comunicazione visiva e frameworks strutturati. La formazione e lo sviluppo delle abilità si basa sulle capacità di comprendere ed interagire con gli altri, per aumentare l'autonomia in base alle proprie capacità e competenze. Avendo a che fare con persone adulte che stanno transitando verso la pensione, l'approccio del centro è positivo, volto a preparare al cambiamento delle abitudini e delle routines, anche attraverso attività sociali e ricreative. I cambiamenti vengono spiegati ai residen- 
ti attraverso metodi verbali e visivi, anche riguardo a controlli medici e variazioni della routine quotidiana necessarie per il benessere e la salute dell'utente con l'aumentare dell'età.

\subsubsection{Il progetto}

Il progetto della struttura residenziale complessiva é basato sul "Model Program for Sheltered Housing Designed for Elderly People with Autism”, sviluppato nel 2010 dall'organizzazione danese Realdania.

L'idea base dell'architettura è la sicurezza e la tranquillità, trasmessa attraverso il carattere domestico, familiare e non-istituzionale dell'edificio, l'altezza contenuta dei soffitti e le scelte di colore tenui che evitino i gradi contrasti. Ognuno degli appartamenti si differenzia inoltre dagli altri attraverso l'uso del mattone, i colori dei muri o del soffitto interno alle abitazioni individuali.

L'edificio è ad un solo piano per non fare ombra e non impattare rispetto al contesto ed in particolare agli altri due complessi di residenza protetta già esistenti, a cui si connette.

L'impianto planimetrico è semplice e chiaro; presenta blocchi di alloggio separati dove le residenze sono indipendenti e hanno accesso personale ed indipendente, oltre ad uno spazio di pertinenza esterno, ma sono anche connesse attraverso un corridoio interno e spazi comuni di relazione. La conformazione irregolare del corridoio spinge il residente a percorrerlo per vedere cosa ci sarà "dietro l'angolo" e conduce agli ampi spazi centrali da cui si accede ai diversi alloggi, anche per favorire le relazioni tra gli abitanti.

\subsubsection{Il verde}

I tre edifici hanno un percorso verde di accesso alle residenze in un'area con molta vegetazione. Seniors House presenta un trattamento delle facciate esterne con rivestimento verde e, più in generale, è rappresentato dal colore verde come leitmotif dell'edificio, sia all'interno che all'esterno. Questo colore dà un senso di sicurezza e aiuta a creare un sentimento olistico e di tranquillità in un ambiente soft e accogliente. Inoltre sono stati piantati alberi e cespugli fuori dalle molte finestre per consentire una vista continua del verde, ma anche creare un filtro naturale, in modo che i residenti possono sedersi dietro le finestre, gioire della luce del giorno e sentirsi parte della comunità, pur schermati dalla vegetazione.

\subsubsection{Lo studio del colore}

In generale, sia all'esterno che all'interno dell'edificio i colori scelti rappresentano tutte variazioni di tonalità del grigio-verde, trasportando l'atmosfera dell'ambiente naturale esterno all'interno dell'alloggio. La differenza di colore va a marcare la transizione ed il cambiamento di ambiente, ad esempio tra le residenze private e le aree comuni, o tra diverse aree dell'alloggio come cucina e soggiorno. Nel corridoio che connette le unità abitative, le zone di colore sono inoltre state usate per focalizzare la vista e guidare lo staff e i residenti attraverso l'edificio. Mentre 


\section{Elena Bellini}

gli spazi di circolazione sono grigi e neutri, le residenze sono colorate con diversi toni del verde in modo da differenziarle e renderle riconoscibili, ma non impattare con colori troppo accesi e contrastanti.

\subsubsection{Comfort luminoso}

La luce naturale è sempre presente ed abbondante all'interno degli alloggi; le grandi aperture consentono di aprirsi verso l'esterno, anche se schermate per mantenere la privacy e regolare l'intensità della luce.

Le luci artificiali sono invece poste a soffitto, non troppo forti e differenziate rispetto alle diverse zone di luce: gli spazi di relazione centrale, dove il soffitto è più alto, prevedono delle luci a sospensione che creano anche un arredo ed un arricchimento dello spazio; lungo i collegamenti si hanno invece luci a soffitto per avere una luce diffusa, non troppo forte e contrastante; sono previsti inoltre "bagni di luce" nelle nicchie di accesso agli alloggi, attraverso superfici luminose a soffitto, per consentire anche di diffondere la luce verso l'interno e richiamare l'attenzione del residente verso il mondo esterno.

\subsubsection{Flessibilità degli alloggi}

Negli appartamenti è stato incorporato un sistema di divisori e armadi modulari su ruote, che consentono di adattare lo spazio piuttosto che avere soluzioni di distribuzione e arredo permanenti. In questo modo se i residenti sono costretti a letto non sono obbligati a stare in una piccola stanza scura, ma possono ingrandire lo spazio e renderlo più luminoso spostando i divisori e gli armadi.

\subsubsection{Favorire le relazioni}

L'edificio presenta differenze nell'altezza dei soffitti, materiali e colori, oltre a usare un design soft ed organico, per motivare ad uscire dal proprio appartamento e girare per l'edificio. Sia nelle residenze personali che nelle zone comuni, sono inoltre state realizzate delle piccole nicchie con colori e materiali che creano un senso di sicurezza, un soffitto più basso e una piccola panca dove sedersi, dove potersi isolare quando necessario e osservare la vita comune, senza distaccarsene completamente. L'aver posto queste sedute a lato delle porte, in corrispondenza dei collegamenti favorisce inoltre la relazione degli utenti al passaggio lungo i percorsi. Sempre in quest'area, è prevista una finestra da cui affacciarsi dalla cucina e guardare fuori, aumentando le occasioni di relazione, ma anche mantenendo l'accesso alla vita della comunità dall'interno, riducendo l'isolamento e la sensazione di solitudine, comune nelle persone anziane. Le porte sono inoltre caratterizzate dalla possibilità di aprire la porzione superiore dell'anta, come fosse uno sportello, per guardare l'ambiente esterno e le aree comuni prima di accedervi, rendendole prevedibili, ed eventualmente prepararsi alle relazioni sociali. 


\title{
4.8 Casa Sebastiano
}

\author{
Sede: Coredo (Trento) \\ Data: 2017 \\ Uso: residenza temporanea e centro per l'autismo \\ N. Utenti: 9 stanze per i residenti \\ Committente: Fondazione Trentina per l'Autismo \\ Progettazione architettonica ed arredi: arch. Giovanni Berti \\ Progettazione esecutiva e strutturale: ing. Francesco Azzali \\ Tag: \#residenza \#lavoro \#terapia \#adulti \#disagio \#benessere \#sicurezza \\ \#orientamento \#autonomia \#integrazione \#uditiva \#visiva \#tattile \#olfattiva \\ \#propriocettiva
}

Struttura di riferimento per il Trentino (Scheda 08) in merito alla patologia autistica, pensata e costruita per dare risposta a molte famiglie in grave difficoltà. Nata per promuovere e realizzare progetti rivolti al miglioramento della qualità di vita delle persone autistiche di ogni età: bambini, adolescenti e adulti.

\subsubsection{Il progetto}

Il Centro è sorto nel comune di Coredo nell'area "ex-colonia vacanze monopolio", su una superficie di circa 4.000 metri quadrati, raggiungendo una volumetria di 7.000 metri cubi, suddivisi in spazi residenziali (per un totale di 24 persone autistiche in gravi difficoltà familiari e progetti di semi-residenzialità che permettano ai ragazzi autistici di stare periodicamente fuori casa, ambulatoriali e riabilitativi, per il trattamento sociale e sanitario, laboratori ludici e occupazionali, area destinata alla formazione di operatori specializzati, sviluppo di ricerca e innovazione. Le aree per la riabilitazione saranno messe inoltre a disposizione anche per la cura di altre disabilità e problematiche di varia natura, per la riabilitazione motoria, per attività psicoeducative, psicomotorie, ecc. Il Centro, infatti, auspica di aprirsi alla comunità, attraverso integrazione e inclusione, anche lavorativa, attraverso un'azienda agricola biologica, laboratori per il lavoro, attività sportive e ludiche.

\subsubsection{L'architettura (intervista all'arch. Berti)}

Casa Sebastiano nasce dall'idea di diversi blocchi differenziati per colore, aspetto, forma e funzione, reintregrati in un unico edificio che ruota attorno al nucleo centrale (Figura 17 - 18). L'edificio si sviluppa in tre piani, quello inferiore dedicato ai servizi della struttura e terapeutici, il primo livello, dove si trova l'accesso principale, dedicato alle residenze e ai servizi a questa annessi e l'ultimo livello dedicato al centro di riabilitazione e terapia.

In particolare, al piano seminterrato (Figura 19) si trovano cucina, dispensa, sala da pranzo e soggiorno, lavanderia, servizi di deposito e locali tecnici, laboratori occupazionali, idroterapia e stanza multisensoriale. Questi ultimi due aspetti sono stati studiati nel dettaglio poiché ritenuti molto importanti, anche se al momento della visita non ho potuto visitarli, poiché il primo era chiuso ed il secondo ancora in fase di 
realizzazione. I servizi potranno essere usati anche dall'esterno. L'idroterapia è da sempre considerata importante nel lo sviluppo delle persone con autismo, che oltretutto adorano l'acqua. La stanza multisensoriale (Figura 20) si pone come tra le più innovative in Italia, avendo avuto una consulenza da parte di professionisti anglosassoni, e prevede un sistema di proiezione interattiva integrato a parete e a pavimento, che interagisce con l'utente stesso, trasformando l'ambiente in base ai movimenti delle persone, oltre ad un sistema di luci, colori e musica. La sala da pranzo (Figura 19) è un ambiente accogliente e caldo, anche grazie ai materiali e ai colori e alla presenza di luce naturale costante, per favorire il comfort degli utenti e le relazioni. Si è scelto un colore verde chiaro alle pareti, ripreso anche nei sistemi di oscuramento interni, il legno a vista per il soffitto, dove vengono appese anche delle grandi luci lineari che diffondono l'illuminazione dall'alto, ed un sistema di pannelli con isolamento acustico per ridurre il riverbero.

Il piano terra/primo livello (Figura 22) dal lato del giardino, è invece dedicato alla residenza. Le aree dedicate ai residenti sono tutte rivolte verso il giardino e separate attraverso i servizi dall'area più pubblica posizionata verso l'ingresso della struttura. Accanto all'ampio e colorato ingresso infatti si dislocano gli uffici amministrativi, alcuni ambulatori e, progredendo verso la residenza, una stanza polivalente usata come palestra, sala per la musica ed il teatro, cucina e soggiorno dedicata solo agli abitanti.

Le camere (Figura 23 - 24) sono collocate quindi nella parte posteriore. Vi si accede attraverso dei corridoi ampi e curvilinei (Figura 25) per favorire gli spostamenti e la prossemica, colorati con toni caldi e soft. Dove è stato possibile, lungo il percorso, sono state integrate anche aree di sosta con divanetti o sedute per favorire la relazione. Ci sono in tutto nove camere della stessa forma e di dimensione tra i 16 e i 18 $\mathrm{mq}$, associate in tre gruppi, con due camere per gli operatori ed uno spazio di controllo centrale, favorito anche dalle telecamere presenti in tutta la struttura, anche nelle camere stesse. Si affacciano verso il giardino, dove hanno a disposizione anche un balcone a cui possono accedere liberamente poiché protetto dalla struttura in brise-soleil in legno che riveste tutta la facciata, anche per garantire un comfort luminoso ed un oscuramento dall'irraggiamento diretto. Ogni camera ha un ingresso raccolto e contenuto, a lato del bagno privato e prevede poi uno spazio rettangolare arredato in modo familiare, con un letto, una poltrona, un armadio e una scrivania. La dimensione è piuttosto ampia, poiché potrebbe anche essere usata per due persone. I colori della camera e degli arredi sono neutri per non sovraccaricare a livello visivo gli utenti, così come le luci, che sono soft e diffuse. All'interno c'è inoltre la possibilità di avere della musica, integrando un sistema di diffusione sonora a soffitto.

Gli spazi per la terapia e la formazione, al piano superiore (Figura 26), sono al momento state concepite per avere il massimo della flessibilità, al fine di lasciare libertà di utilizzo per le diverse terapie, ancora in corso di avvio. In generale sono accoglienti, con colori neutri e caldi e hanno arredi modulari in legno. Anche in queste stanze c'è un controllo della luce naturale, sempre presente, ed una diffusione della luce artificiale.

All'esterno è previsto un giardino sensoriale (Figura 27), dedicato anche alla cura delle piante, oltre ad un'area coperta con tavolini e un grande spazio verde che circonda la struttura. 


\subsubsection{Materiali, colori, dispositivi e strategie di comfort}

L'uso del legno, parte integrante della struttura dell'edificio, contribuisce a favorire il calore e l'accoglienza dell'ambiente, lasciando le travi a vista e i solai degli ambienti comuni, come il refettorio o la cucina/sala pranzo, ma anche rivestendo le facciate con il brise-soleil. Per ridurre il riverbero, soprattutto nelle aree comuni e di collegamento, sono stati usati inoltre controsoffitti a pannelli acustici, in alcune zone anche in microforato di legno (ingresso). I pavimenti sono invece in linoleum, per necessità di adeguamento alle normative, comfort e facilità di pulizia e manutenzione. Il riscaldamento è radiante a pavimento e c'è un sistema integrato di ventilazione e ricambio dell'aria per il comfort indoor degli utenti.

\subsection{Royal Alexandra Children's Hospital}

Sede: Brighton (UK)

Data: operativo da maggio 2007

Uso: ospedale per bambini

Dimensione: $15,000 \mathrm{~m}^{2}, 100$ posti letto

Committente: Brighton and Sussex University Hospitals NHS Trust

Progettazione: BDP Archietcts

Tag: \#ospedale \#bambini \#disagio \#benessere \#orientamento \#autonomia

\#integrazione \#uditiva \#visiva \#tattile \#olfattiva \#propriocettiva

Premi e riconoscimenti: Civic Trust Award 2009; MLIT (Government of Japan) Award 2009; Prime Minister's Better Public Building Award 2008: Winner; Public Private Finance Award 2008: Operational Project with Best Design; International Academy for Design and Health Award 2007: Healthcare Design Project of the Year

Uno dei soli sette ospedali pediatrici del Regno Unito, "the Alex" (Scheda 09), definito "un ospedale che non sembra un ospedale", si presenta come una sistemazione integrata a misura di bambino di alta qualità terapeutica. E' una struttura che offre servizi terapeutici h24, sia di emergenza, che di cura intensiva, che di servizio ambulatoriale e diagnostico.

\subsubsection{Il progetto}

Iniziato nel 2004 e concluso a maggio del 2007, il nuovo ospedale si presenta come un'arca su nove piani, approdata sulla costa di Brighton . Nella nuova costruzione ha raddoppiato il numero dei letti rispetto al vecchio ospedale, realizzando tre piani aggiuntivi. L'edificio ha un layout semplice che ruota attorno all'atrio vetrato centrale, cuore dell'ospedale sia a livello funzionale, che distributivo, che sociale. Questo spazio, infatti, collega tutti i livelli e garantisce una visione d'insieme dello spazio, sia entrando che muovendosi all'interno dell'ospedale, favorendo orientamento e wayfinding. Questo ambiente centrale, inoltre, garantisce un'ottima illuminazione naturale in tutto l'edificio, grazie anche alla strategia di realizzare i diversi piani con poca profondità. L'hub centrale è anche il cuore sociale della struttura poi- 


\section{Elena Bellini}

ché funge da area di ricezione e zona di attesa degli ambulatori, oltre ad ospitare un caffè ed un negozio.

\subsubsection{Umanizzazione degli spazi ospedalieri}

La struttura family\&children-centered è investita dal colore, dalle grafiche e da sistemi di comunicazione per immagini per favorire l'orientamento e far sentire il bambino in un ambiente familiare, giocoso e poco ospedaliero. Si rileva d'interesse anche l'utilizzo delle immagini che può rappresentare una facilitazione in casi di autismo e allo stesso tempo può essere un'occasione di integrazione e inclusività, permettendo a tutti i bambini di comprendere l'ambiente allo stesso modo, attraverso strumenti comunicativi alternativi. Sono stati usati colori non troppo accesi, materiali caldi e naturali come il legno, linee curve e morbide ed elementi di gioco, che favoriscono l'interazione, il rilassamento e la distrazione del bambino. Il progetto è stato in questo senso facilitato e guidato da una fase di partecipazione e coinvolgimento dei pazienti con una Children's and Young People's Board, attraverso la quale i pazienti hanno dato input sullo sviluppo dell'edificio, arredi, sistemi di wayfinding, grafica e sistemi di comunicazione.

L'ambiente è pensato inoltre per mantenere una vista continua dell'esterno, offrendo panorami sul lungomare di Brighton inquadrati dalle diverse ed irregolari aperture realizzate in facciata, realizzate a misura ed altezza di bambino. Anche le stanze di degenza (singole, da 2 o 4 persone) sono affacciate tutte verso il mare e hanno arredi disegnati specificatamente per i bambini, oltre ad un letto estraibile per i genitori che vogliono rimanere vicino ai propri figli. La presenza costante dei genitori in casi di autismo risulta fondamentale, anche come mediatori rispetto all'interazione con il personale medico e sanitario.

Come altro servizio aggiuntivo sono previsti inoltre, all'ultimo piano dell'edificio, alcuni alloggi per i genitori, completi di servizi come cucina, lavanderia, soggiorni e terrazze.

\subsubsection{Abbattere lo stress ospedaliero}

L'ospedale dà accesso anche ad un'area esterna, dove è stato realizzato un giardino sensoriale coperto da una tettoia, per essere usato durante tutto l'anno. Gli elementi sensoriali, che permettono al bambino di interagire con vista, tatto, udito e olfatto, promuovono lo sviluppo di abilità sociali e offrono uno spazio alternativo dove apprendere, rilassarsi e divertirsi, per minimizzare l'effetto psicologico della visita o della degenza ospedaliera.

All'ultimo piano dell'edificio è inoltre prevista una grandissima area gioco, dove potersi distrarre, divertirsi e socializzare con gli altri bambini, per trovare relax e abbattere lo stress legato alla degenza o all'affrontare un'emergenza sanitaria.

Come altro intervento innovativo nel 2016 è stata inoltre allestita a questo piano una stanza multisensoriale, Sea Life (Figura 28 - 29), per bambini con bisogni complessi. E' un'area terapeutica e di apprendimento, dove potersi rilassare in un ambiente completamente non-sanitario ed evadere temporaneamente, anche quando non si può fisicamente lasciare l'ospedale. Non solo i bambini chiedono di andarci, 
ma anche i più grandi o i teenagers, poiché riescono in questo ambiente a rilassarsi, distrarsi ed abbattere lo stress. E' il primo esempio in tutto il Regno Unito di stanza sensoriale a tema di vita nel fondale marino. E' predisposta con luci e proiettori interattivi, con pesciolini che si muovono sul pavimento e si disperdono quando si toccano. C'è un pannello ad acqua con bolle d'aria e un letto ad acqua dove affondare e rilassarsi al suono dolce dell'acqua e delle bolle. Una medusa gigante luminosa fa effetti di luce e glitter, con fibre ottiche come filamenti con cui giocare e rilassarsi. Si fonda sulla distrazione del bambino rispetto alla cura e alla degenza in ospedale, ma anche come strumento persuasivo, come incentivo a prendere ad esempio le medicine. Gli infermieri inoltre possono giocare in questo spazio con i bambini, anche mentre i genitori si prendono una pausa, migliorando la relazione tra operatore e paziente. Piuttosto che pensare ad un ospedale spaventoso, "the Alex" è diventato in questo modo "il posto con la straordinaria sala dei pesci".

\subsection{Emma Children's Hospital}

Sede: Amsterdam (Paesi Bassi)

Data: ospedale 2014

Uso: ospedale per bambini

Dimensione: $11.000 \mathrm{~m}^{2}$

Progettazione Architettonica: Defesche van den Putte (DVDP)

Interni: OPERA Amsterdam

Tag: \#ospedale \#bambini \#disagio \#benessere \#orientamento

\#integrazione \#uditiva \#visiva \#tattile \#olfattiva \#propriocettiva

Rivoluzione dell'ultimo piano dell'Academic Medical Centre in Amsterdam (Scheda 10); un esempio di ospedale pediatrico children-friendly, la cui chiave è la qualità dello spazio e del rapporto del bambino con esso e con il mondo esterno.

\subsubsection{Il progetto}

Il progetto si fonda sul rispetto e la valorizzazione delle tre principali aree di intimità del bambino/paziente: quella più privata, che deve mantenere protezione e privacy; quella che vede il rapporto del bambino con il contesto circostante immediato all'interno della struttura ospedaliera, che si fonda sullo sviluppo dell'interazione e le relazioni sociali; e quella infine del bambino che si relaziona con il mondo esterno, attraverso un rapporto costante sul contesto circostante. Questa organizzazione degli spazi è molto utile anche in casi di autismo, poiché consente di scegliere quando, se e come relazionarsi con il mondo esterno, avere una strutturazione ben definita dell'ambiente, che favorisce la comprensione e consente di mantenere una percezione complessiva di controllo sul contesto, vicino e lontano, riducendo i livelli di ansia e stress. Per lo stesso scopo l'ambiente è trattato in modo da non dare la sensazione di essere in un ambiente sanitario, ma promuovere la familiarità, per sentirsi in qualche modo a "casa". 


\subsubsection{Il layout degli spazi}

L'impianto planimetrico si fonda su una main street centrale, la "parade", che distribuisce a livello funzionale i diversi dipartimenti dell'ospedale, ma è anche alla base della relazione con il contesto e dell'interazione sociale tra pazienti, familiari e staff. Se i bambini possono usare quest'area per il gioco, i familiari possono trovarvi un momento di distrazione e relax, e lo staff può usarlo come zona di meeting. Il concept del percorso è aprire una strada all'interno di una "città", passando attraverso piazza centrale, zoo, parco, campi sportivi, teatro, ecc. fino a raggiungere la casa del bambino e la sua cameretta. Allo stesso modo, la main street dà accesso a tutte le aree ed i dipartimenti della struttura, oltre alle funzioni speciali come scuola, biblioteca, cinema e ristorante, trasformando l'interno dell'ospedale in una piccola città che conduce alla "casa privata" del bambino, ovvero la camera/stanza di degenza.

L'ampiezza dei collegamenti favorisce inoltre la prossemica e la propriocezione, che potrebbe essere fonte di riduzione dello stress in casi di autismo. Il network delle connessioni prosegue in modo molto semplice e leggibile, per facilitare l'orientamento ed il wayfinding. Tutti i collegamenti sono illuminati da luce naturale e offrono viste sul modo esterno, importante per mantenere un contatto e la percezione del mondo che ci circonda, del passare dei momenti della giornata e del tempo; lungo il percorso sono previste quindi delle aree di sosta, che offrono anche uno sguardo verso il panorama della città e dei polder.

\subsubsection{Interazione sensoriale}

L'interazione del bambino con il contesto avviene attraverso l'ambiente ludico, ovvero giochi con cui intrattenersi, applicazioni digitali, stanze ed installazioni multisensoriali. Usare i canali sensoriali per stimolare le relazioni è una strategia molto efficace anche in casi di autismo, che possono rilevarsi anche utili come strategie di rilassamento, distrazione ed abbattimento dello stress.

\subsubsection{Orientamento e wayfinding}

Il progetto si basa sulla flessibilità, ma ogni reparto è progettato per avere un carattere specifico e soddisfare i bisogni e le esigenze del singolo dipartimento, differenziandosi inoltre per età o per gruppo, come fossero "case separate". Pur avendo un'identità globale, ogni reparto è quindi trattato in modo diverso, attraverso particolari simboli e icone, colori, grafiche e layout spaziali (Figura 30 - 31). Nello specifico è stato realizzato un studio particolareggiato di ogni reparto abbinando in modo integrato un'icona, che si ritrova lungo il percorso (a pavimento, parete o soffitto) (Figura 32 - 33), ad una palette di colori coordinati, un sistema di grafiche ed illustrazioni legate al tema scelto per il reparto e quindi applicate all'ambiente specifico.

Le stanze di degenza sono inoltre evidenziate attraverso un colore che esce a pavimento ed interrompe il percorso, con un numero/grafica di riferimento (Figura 34 $35)$. 
La comunicazione attraverso immagini facilita molto la comprensione di persone con autismo e ne favorisce anche l'inclusività, dando a tutti la stessa possibilità di comprendere attraverso strumenti alternativi.

\subsubsection{L’equilibrio sensoriale}

E' interessante l'equilibrio che si crea tra bassa stimolazione sensoriale, ovvero aree dove il bambino può ritirarsi e godere della privacy e della tranquillità, e zone piene di stimoli, dove attraverso i canali sensoriali il bambino può mettersi in relazione con il mondo esterno. Più in generale, l'ambiente ha una base neutra, di colore bianco, che anche in casi di autismo favorisce lo stato di calma e riduce il sovraccarico sensoriale, su cui vengono applicate puntualmente illustrazioni, colori ed immagini, caratterizzando e differenziando gli ambienti, senza sovraccaricare di stimoli il bambino.

\subsection{Nemours Children's Hospital}

\section{Sede: Orlando (USA)}

Data: 2012

Uso: ospedale per bambini

Dimensione: $16.500 \mathrm{mq}, 140$ letti

Progettazione: Stanley Beaman Sears

Tag: \#ospedale \#bambini \#disagio \#benessere \#orientamento

\#integrazione \#uditiva \#visiva \#tattile \#olfattiva \#propriocettiva

Il nome "Nemours" ha origine dalla parola celtica "nemora": un santuario di alberi abitato dal Dio delle sorgenti curative. Come esplicita il nome stesso dell'ospedale (Scheda 11), il concept del progetto si fonda sulla trasformazione del paesaggio interiore del bambino nel momento in cui sperimenta il viaggio potenzialmente difficile e spaventoso del recupero.

\subsubsection{Il progetto}

L'intervento prevede la concentrazione di pazienti degenti e ambulatoriali in un solo edificio, in ali adiacenti dello stesso piano, condividendo le sale di attesa. L'ospedale (Figura 36) è aperto alle visite h24 quindi prevede anche spazi per i genitori durante la notte, servizi di lavanderia e un desk di reception nella hall di ogni piano. Ampie zone soggiorno e aree gioco danno accesso agli spazi aperti, anch'essi disegnati per il relax, la distrazione e l'abbattimento dello stress: terrazze sul tetto, giochi d'acqua interattivi, un "giardino della scoperta" e un palco per le performances all'aperto. L'apporto positivo dell'ambiente naturale nella cura è stato ampiamente dimostrato, soprattutto se correlato a stimolazioni sensoriali, come nel "giardino della scoperta" e se progettato con linee curve e morbide. Le linee curve sono infatti alla base del progetto dell'esterno; una rampa curvilinea aumenta di un piano il basamento, permettendo al seminterrato, dedicato ai servizi e alle consegne, di es- 


\section{Elena Bellini}

sere illuminato dalla luce del giorno. Il proseguimento di questa linea curva dà quindi vita e forma, attraversando l'edificio, alla sistemazione di piante e giardini.

All'interno del progetto si è prestata particolare al comfort visivo ed in particolare luminoso. In particolare si è progettato l'edificio per ottimizzare il controllo dell'irraggiamento solare che in quest'area è molto intenso, studiando sistemi di oscuramento in facciata che permettano di godere della luce del giorno senza surriscaldare l'ambiente interno. Questo è molto utile in casi di autismo, non solo a livello ambientale, ma poiché permette di regolare il livello di illuminazione indoor, senza che sia mai troppo forte.

L'illuminazione artificiale interattiva dell'edificio è un altro punto di forza del progetto sviluppandosi a soffitto e a parete e variandone il colore attraverso un sistema controllato di illuminazione. Oltre alle luci colorate vengono inoltre riprodotti video di immagini naturali, come cielo e paesaggi. Nei corridoi di connessione viene diffusa una luce bianca calda, che risulta molto favorevole anche in casi di autismo, per cui sono da evitare luci fluorescenti e neon. Nelle stanze di degenza è invece possibile controllare direttamente la luce da parte del paziente attraverso uno schermo interattivo, diffondendosi da una struttura sopra al letto e inondando le pareti di luci dal colore dinamico. Anche questo è fondamentale, poiché permette di personalizzare la colorazione dell'ambiente e l'intensità dell'illuminazione, regolandosi sulla base delle preferenze sensoriali.

L'utilizzo di faretti incassati nell'area di affaccio delle stanze di degenza consente inoltre di avere una caratteristica illuminazione anche dall'esterno dell'edificio, riproducendo in facciata quadri di diverso colore in corrispondenza delle diverse stanze illuminate, come fosse un grande cubo di Rubick, promuovendo anche dall'esterno un impatto giocoso e familiare, che non riconduca ad un ambiente sanitario. Questo sistema di illuminazione, infatti, oltre ad essere un dispositivo di distrazione per i pazienti, è anche un'ottima strategia di umanizzazione dell'ambiente ospedaliero children-centered.

In questo senso sono stati inoltre realizzati in tutto l'ospedale aree gioco e arredi a dimensione bambino, colori vivaci e metodi di interazione, attraverso sedute comode, nicchie a parete dalle dimensioni organiche, a ricreare differenziati spazi a diversi gradi di socializzazione.

Gli spazi sono molto ampi, a favorire propriocezione e prossemica ma allo stesso tempo danno la possibilità di ritrovare spazi raccolti per isolarsi leggermente o riequilibrarsi (Figura 37 - 38), che potrebbe essere molto utile in casi di autismo, riducendo i fattori di stress ambientale e sensoriale, favorendo la partecipazione sociale "protetta" e facilitando quindi le relazioni con gli altri.

Se da un lato tutti questi fattori sono molto positivi, si denota una leggera criticità nello specifico dell'autismo in alcune aree, come l'atrio (Figura 39 - 40), che risultano sovraccariche di stimoli, soprattutto per quanto riguarda l'illuminazione, i tanti stimoli visivi presenti di diversa natura, i colori molto accesi e l'utilizzo di un pavimento lucido che riflette luci e colori e potrebbe essere causa di disagio. Probabilmente sarebbe più efficace ridurre la stimolazione dell'ambiente generale e concentrarla in alcune aree di interazione sensoriale, come l'interessante installazione realizzata a fondo dell'atrio centrale/hall (Figura 41). 
Per quanto riguarda invece la stanza di degenza è interessante mantenere una vista continua verso l'esterno, con un rapporto costante di relazione con la natura e di comprensione del tempo che passa e dei diversi momenti della giornata. L'ambiente anche è neutro e non sovraccarico di stimoli, anche per la scelta dei colori come il bianco ed il blu, colore alla base del rilassamento. Forse potrebbe essere utile una parete attrezzata che vada a nascondere ed integrare tutti gli apparati medicali, per migliorare la familiarità dello spazio e ridurre l'ansia rispetto all'ambiente ospedaliero.

Tutte le soluzioni di progetto sono in ogni caso state condivise con tutti gli stakeholders, inclusi i professionisti, l'amministrazione ed una commissione di genitori e bambini, pazienti dell'ospedale.

\subsubsection{Il programma REACH - Respecting Each Awesome Child Here}

Nemours ha da un anno circa sviluppato un programma di accessibilità per l'autismo o altre condizioni comportamentali e dello sviluppo presso il Pronto Soccorso, incentrato soprattutto sull'eliminazione degli stimoli non necessari, al fine di ridurre il disagio e migliorare l'accoglienza ed il trattamento sanitario.

Molti studi hanno infatti dimostrato che il Pronto Soccorso può causare agitazione e stress a bambini con autismo, dato che si presenta come un ambiente sconosciuto, dove sono presenti contemporaneamente molte persone e tanti suoni, come bambini che gridano e piangono. Anche se non è chiaro, come dati, quanto spesso sia frequentato il Pronto Soccorso, solitamente si registra al Nemours almeno uno o più accessi di bambini con autismo durante il giorno.

Il programma REACH si occupa di identificare i bambini con autismo direttamente all'accesso del Pronto Soccorso e occuparsi dei loro specifici bisogni fino alla fine del percorso ospedaliero. E' molto importante infatti agire subito all'accesso del bambino, quando non è ancora sovrastimolato o agitato, poiché l'ansia del bambino agisce sui genitori e lo stress di entrambi impatta sullo staff e quindi sulla qualità dell'intervento medico. Questa particolarità è una prima differenza del programma REACH rispetto agli altri programmi di assistenza sanitaria.

Il primo passo da compiere poi è fornire un ambiente di attesa adeguato, tranquillo ed alternativo rispetto all'area di attesa comune; in quest'area i bambini possono usare giochi, i-pads, cuffie o elementi sensoriali, per distrarsi ed abbattere lo stress. Un segnale colorato REACH viene appeso inoltre fuori dalla stanza del bambino, anche in caso di esami o diagnostica, affinché lo staff sia consapevole che all'interno vi è un bambino con autismo o altri disordini dello sviluppo e che deve quindi avere un comportamento adeguato. All'interno dei reparti vi sono due stazioni delle infermiere che hanno giochi sensoriali, specifici per bambini con autismo, a disposizione del paziente. In base ai suoi bisogni è inoltre possibile portare nella stanza un riproduttore di musica o un proiettore che illumini i muri con colori o stelle. Durante il tempo passato in ospedale è possibile alleviare i disturbi sensoriali attraverso luci regolabili (con dimmer), la riduzione della presenza di monitor e del numero di persone all'interno della stanza. Oltre al personale debitamente formato, è inoltre importante coinvolgere direttamente il genitore nella scelta dei trattamenti e 


\section{Elena Bellini}

della cura sulla base delle sue specifiche esigenze e sulla sistemazione dell'ambiente affinché abbia il massimo comfort.

Attualmente Nemours sta conducendo studi per valutare l'efficacia del programma all'interno del Pronto Soccorso ed eventualmente espanderlo agli altri reparti.

\subsection{Evelina Londron Children's Hospital}

Sede: London (UK)

Data: ospedale 2005, 'art in site' 2016

Uso: ospedale per bambini

Dimensione: $16.500 \mathrm{mq}, 140$ letti

Committente: Guy's \& St Thomas' NHS Foundation Trust

Progettazione: Hopkins Architects + Buro Happold; sistema di arte e comunicazione digitale per il Pronto Soccorso - ART IN SITE

Tag: \#ospedale \#bambini \#disagio \#benessere \#orientamento \#autonomia \#integrazione \#uditiva \#visiva \#tattile \#olfattiva \#propriocettiva

Premi e riconoscimenti: AIA/UK: Commendation; Civic Trust Award; RIBA National Award; Stirling Prize: People's Choice; British Construction Industry Awards: Judges' Special Award; Building Better Healthcare Awards: Best NHS Hospital Design; FX International: Design Award, Best Public Interior Space; RIBA Regional Award; LABC Built-in Quality Awards: Best Structural Innovation

Evelina Londron Children's Hospital (Scheda 12) è il secondo dei servizi sanitari per bambini più grandi a Londra, dedicato alla cura e all'assistenza del bambino, da prima della nascita fino alla giovane età adulta, e delle loro famiglie. E' parte del Guy's e St. Thomas' Foundation Trust, situato nel pieno centro della città.

\subsubsection{Il progetto}

Realizzato tra il 2002 e il 2004 a seguito di un concorso del RIBA, vinto dallo studio di architetti Hopkins Architects e dallo studio di ingegneria Buro Happold.

La struttura ha un layout semplice e flessibile, composto dall'intersezione di due blocchi molto lunghi con uno spazio centrale a tutto volume, cuore dell'ambiente ospedaliero, dedicato all'interazione sociale.

Un aspetto interessante del progetto è infatti che le partizioni dell'edificio e i servizi sono flessibili, permettendo nel futuro di adattarsi ad eventuali cambiamenti ed evoluzioni del sistema sanitario. I tre livelli più bassi ospitano le funzioni comuni, ovvero il reparto di Terapia Intensiva, tre teatri operatori, il centro di diagnostica, gli ambulatori e il servizio di Day Hospital. Al di sopra tre piani di reparti di degenza pediatrica si affacciano sull'enorme giardino d'inverno centrale, illuminato dall'ampio tetto curvo. Questo spazio, chiamato "la spiaggia" rappresenta il cuore dell'edificio, ospitando un caffè, una sala d'attesa, spazi gioco, spazi formativi e aree per le esibizioni e le mostre. Si accede ai diversi reparti superiori attraverso la colonna rossa di ascensori, dove le camere di degenza si affacciano invece verso il lato opposto, in modo da garantire la privacy. 


\subsubsection{L'umanizzazione degli spazi ospedalieri}

Evelina Children's Hospital è stato completamente studiato a misura di bambino. E' sicuramente all'avanguardia per quanto riguarda l'umanizzazione degli spazi ospedalieri, rendendo fin dall'inizio l'ambiente allegro, colorato e solare. Il giardino d'inverno, prima di tutto, si pone come ambiente non sanitario e presenta ambienti di gioco e socializzazione, offrendo un punto di distrazione ed abbattimento dello stress sia per il bambino, che per le famiglie.

L'illuminazione naturale è sempre presente grazie allo spazio centrale vetrato, ma anche alle ampie aperture delle stanze di degenza, mantenendo un contatto con l'esterno, che favorisce anche la comprensione del tempo che passa; le aperture sono inoltre appositamente schermate per un maggiore comfort luminoso, particolarmente utile in casi di autismo.

I colori usati per i diversi reparti e all'interno delle stanze di degenza sono caldi e vivaci, anche se non troppo forti. L'ambiente è di per sé a base neutra e usa il colore, le immagini o le grafiche, per evidenziare alcune aree e favorire l'orientamento ed il wayfinding.

Le stanze di degenza sono singole; gli impianti medicali sono completamente integrati e nascosti in una parete attrezzata dove è possibile anche aprire un letto per uno dei due genitori, anch'esso integrato nella struttura.

Vi sono sistemi di orientamento e wayfinding, nella camere, come numeri e colori, ma la vera rivoluzione nella comprensione e navigabilità della struttura sta negli spazi comuni.

\subsubsection{Sistemi di orientamento e wayfinding}

Nel 2016 è stato fatto un ulteriore passo avanti, attraverso l'arricchimento dell'ambiente con un sistema comunicativo basato sull'arte digitale e illustrativa, per guidare il bambino all'interno dell'edificio, rassicurarlo, ridurre l'ansia e quindi i comportamenti problema che si possono verificare in situazioni di stress. L'allestimento è frutto di una ricerca di psicologia ambientale effettuata all'interno dell'ospedale, che ha previsto due settimane di osservazione $24 \mathrm{~h}$ per comprendere il funzionamento della struttura, i bisogni e le criticità dei futuri utenti; workshop e sessioni di lavoro per raccogliere i feedback dello staff, dei pazienti e delle loro famiglie sui principi strategici di progetto, anche attraverso prototipi. Il risultato sono una serie di ambientazioni, scenari e personaggi illustrati, generati in collaborazione con bambini e staff. L'artista manga Kiriko Kubo ha disegnato un'intera 'gang' di personaggi che sono andati a caratterizzare ogni piano dell'edificio, differenziandolo rispetto agli altri, anche per migliorare l'orientamento e il wayfinding (Figura 42 43 - 44). I personaggi sono integrati perfettamente con l'ambiente, sbucando dalle porte, attraversando le pareti, strisciando sui soffitti e comparendo su adesivi distribuiti ai bambini, applicate su un ambiente (come si è visto) di base neutra, che permette di non sovraccaricare il bambino a livello visivo e al contrario evidenziare le grafiche puntuali. Il tema è quello dell'ambiente geografico, rappresentando ad ogni piano l'oceano, l'artico, la foresta, la spiaggia, la savana, la montagna ed il cielo. Le illustrazioni offrono un elemento di distrazione per i bambini, oltre che un sistema di 


\section{Elena Bellini}

comunicazione facilitato. La differenziazione dei personaggi è legata anche alla varietà di età dei pazienti e comunque molto apprezzata anche dai teenagers.

\subsubsection{Assistive technology presso il Pronto Soccorso}

Sempre nel 2016 è stata fatta una sperimentazione presso il Pronto Soccorso di un'applicazione digitale per migliorare la comunicazione tra paziente, operatore e famiglia (Figura 45). Sta riscuotendo molto successo e probabilmente verrà presto ampliata a tutta la struttura. L'applicazione è diretta dalla "gang" di personaggi che si ritrovano all'interno dell'ambiente ospedaliero, che aiutano a demistificare il processo di emergenza sanitaria. Delle animazioni interattive (Figura 46 - 47) spiegano le diverse procedure cliniche, come avvengono i prelievi del sangue, le iniezioni, le misurazioni delle pulsazioni e tutto quanto sarà necessario per la visita e l'intervento medico. Alcune storie raffigurano i motivi più frequenti per cui si va al Pronto Soccorso, facendo vedere quali sono le procedure mediche che verranno affrontate nei singoli casi. Allo stesso tempo è possibile fare un tour virtuale nell'ospedale dalla sala di attesa, o dare una spiegazione grafica delle stanze di degenza o degli ambulatori di diagnostica, con l'indicazione dell'utilità dei diversi dispositivi presenti. Infine sono comprese diverse applicazioni di gioco per far distrarre il bambino, ma anche aiutare a comunicare con lo staff. Ad esempio, una di queste dà possibilità di guardare uno scheletro e imparare i nomi delle ossa, ma può essere usata anche il medico per spiegare visivamente al bambino quale parte del corpo si è rotta e come curarlo. All'interno dell'applicazione ci sono inoltre alcune guide per i più grandi sulla pubertà, la salute mentale e la sessualità ed infine alcune note per i genitori, per spiegare cosa fare una volta lasciato l'ospedale e tornati a casa. Un processo di questo tipo è molto importante in casi di autismo, poiché permette di avere la prevedibilità, visitando al struttura e comprendendo in anticipo le procedure mediche, e ridurre quindi lo stato d'ansia, oltre a favorire la comunicazione attraverso le immagini, come sistema alternativo al linguaggio verbale. Oltre a questo un'applicazione di questo tipo può favorire l'integrazione e l'inclusione, poiché è uno strumento utilizzato da tutti i bambini e non specifico per l'autismo.

\subsubsection{Proiezione sensoriale interattiva}

L'ultimo intervento effettuato è un'installazione digitale proiettata su un grande muro, che permette al bambino di giocare con forme e colori, trasformandoli in base ai movimenti del corpo (Figura 48 - 49). E' sicuramente un gioco molto divertente, ma anche un modo per dare la possibilità al bambino di muoversi e distrarsi e abbattere lo stress ospedaliero. 
Ambienti sensoriali "terapeutici" che rendano Abili

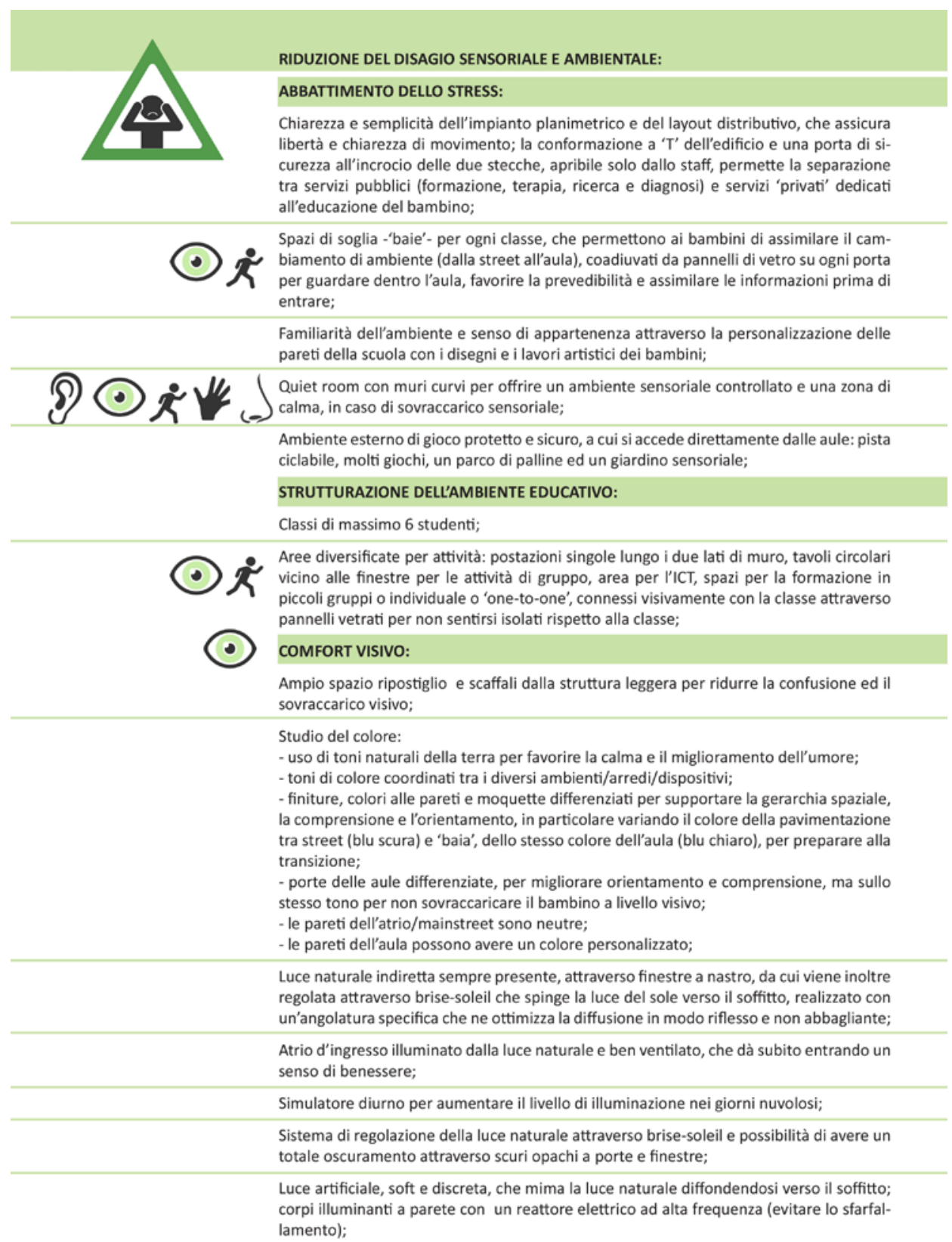

\section{Scheda 01. New Struan School (parte 1)}




\section{Elena Bellini}

Sistema di regolazione della luce artificiale attraverso dimmer;

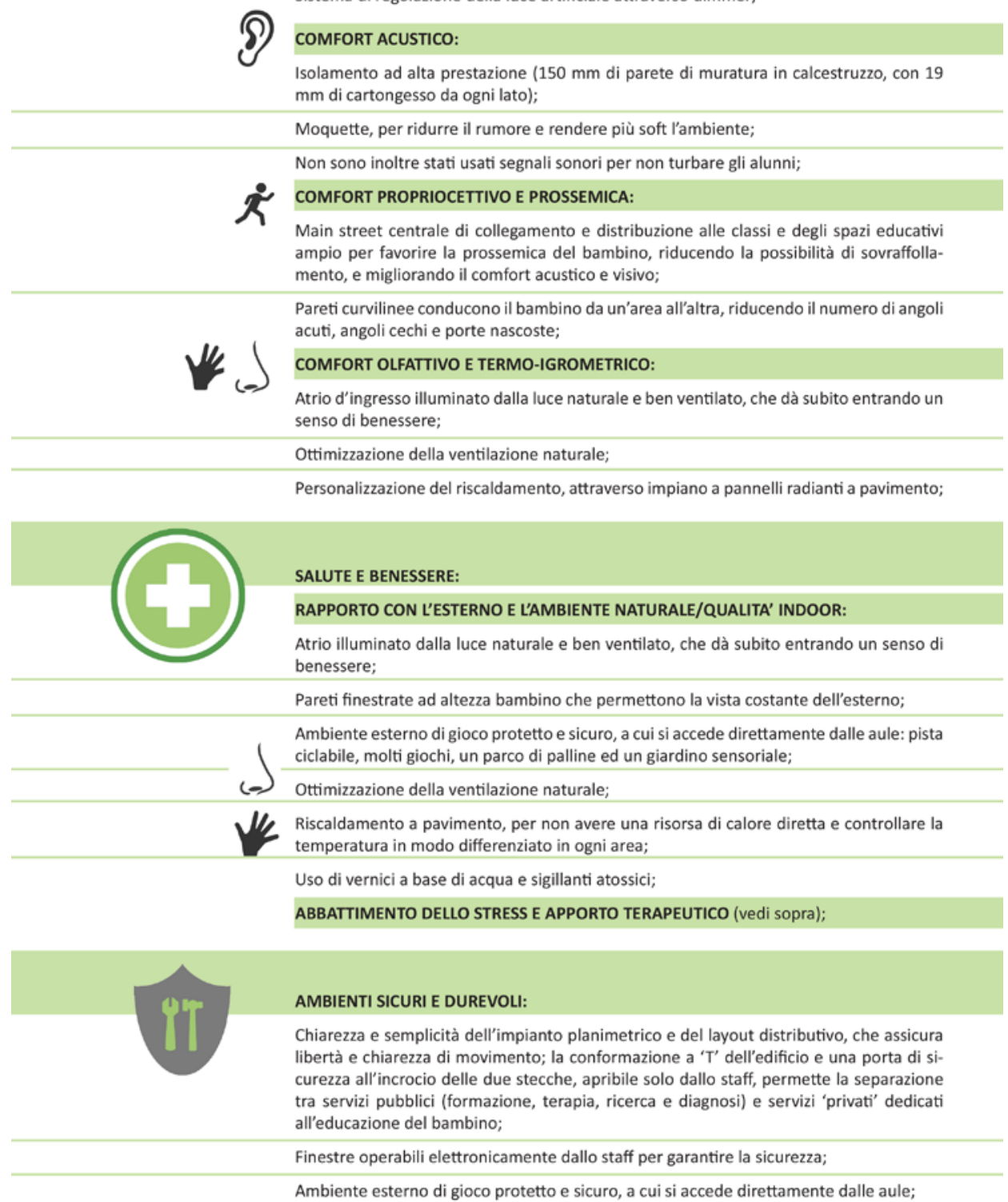

Scheda 01. New Struan School (parte 2) 


\section{Ambienti sensoriali "terapeutici" che rendano Abili}

Recinzione perimetrale, anche se bassa e piacevole a livello estetico, per non impattare la vista per i bambini;

Arredi e finiture progettate per essere resistenti e durevoli;

Quadro dei dispositivi elettrici e antincendio mascherato attraverso un rivestimento della superficie riflettente;

1/. Riscaldamento a pavimento, anche per non avere una risorsa di calore diretta e controllare la temperatura in modo differenziato in ogni area;

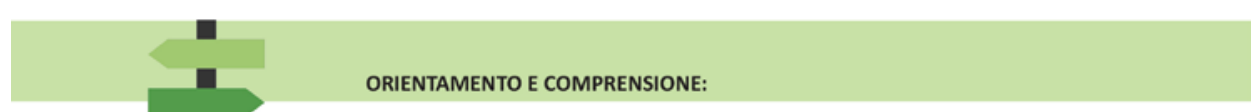

Chiarezza e semplicità dell'impianto planimetrico e del layout distributivo, che assicura libertà e chiarezza di movimento; la conformazione a ' $T$ ' dell'edificio e una porta di sicurezza all'incrocio delle due stecche, apribile solo dallo staff, permette la separazione tra servizi pubblici (formazione, terapia, ricerca e diagnosi) e servizi 'privati' dedicati all'educazione del bambino;

Main street centrale di collegamento e distribuzione alle classi e agli spazi educativi;

Chiara e totale visibilità dell'intera scuola, promuovendo il movimento indipendente tra le diverse aree;

Muri curvi conducono il bambino da un'area all'altra, riducendo il numero di angoli acuti, angoli cechi e porte nascoste, diminuendo quindi il livello di ansia e favorendo gli spostamenti;

Spazi di soglia -'baie'- per ogni classe, che permettono ai bambini di assimilare il cambiamento di ambiente (dalla street all'aula), coadiuvati da pannelli di vetro su ogni porta per guardare dentro l'aula, favorire la prevedibilità e assimilare le informazioni prima di entrare;

Aree comuni aperta a pianta libera di biblioteca e refettorio, a cui si accede direttamente dalla street, per favorire l'indipendenza di circolazione nel bambino e la visibilità totale degli spazi; nella biblioteca si comprende immediatamente la distribuzione delle aree dedicate alla lettura rilassata e quelle invece per la ricerca e lo studio; dalla 'street' vi si accede attraverso un ingresso curvo e senza porte;

\section{Studio del colore:}

- - finiture, colori alle pareti e moquette differenziati per supportare la gerarchia spaziale, la comprensione e l'orientamento, in particolare variando il colore della pavimentazione tra street (blu scura) e 'baia', dello stesso colore dell'aula (blu chiaro), per indicare e preparare alla transizione;

- porte delle aule differenziate, per migliorare orientamento e comprensione, le pareti della classe possono avere un colore personalizzato;

\section{(2) SISTEMA DI COMUNICAZIONE VISIVA ALTERNATIVA:}

Spazi bacheca ottimizzati al fine di supportare l'apprendimento attraverso segnali o indicazioni visive;

\section{AUTONOMIA E INDIPENDENZA. ABILITA':}

\section{STRATEGIE DI PROMOZIONE DEL MOVIMENTO LIBERO DEL BAMBINO:}

Chiarezza e semplicità dell'impianto planimetro e del layout distributivo, che assicura libertà e chiarezza di movimento;

Scheda 01. New Struan School (parte 3) 


\section{Elena Bellini}

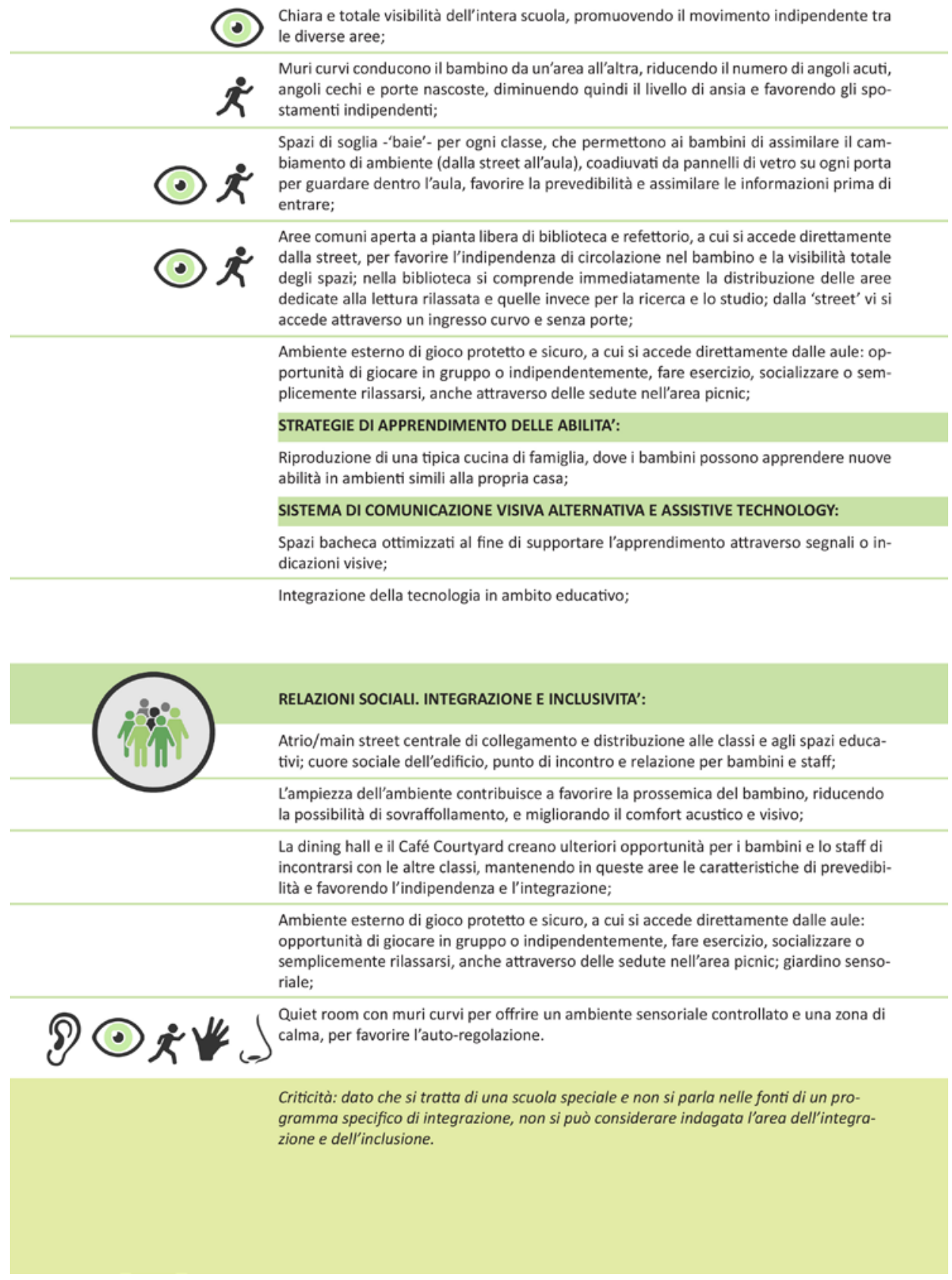

\section{Scheda 01. New Struan School (parte 4)}


Ambienti sensoriali "terapeutici" che rendano Abili

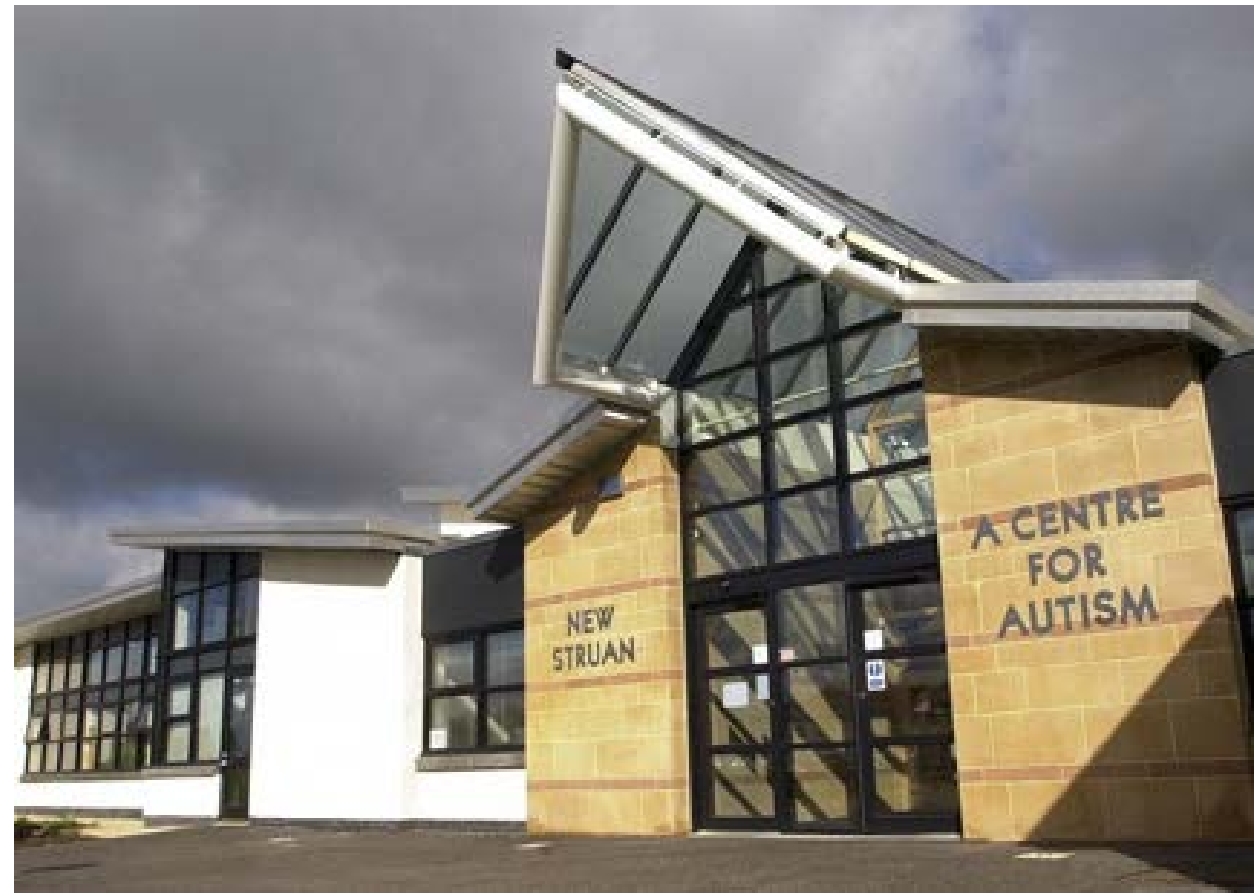

Figura 01. New Struan School (Source: Scottish Autism, photograpgher: Tina Norris)

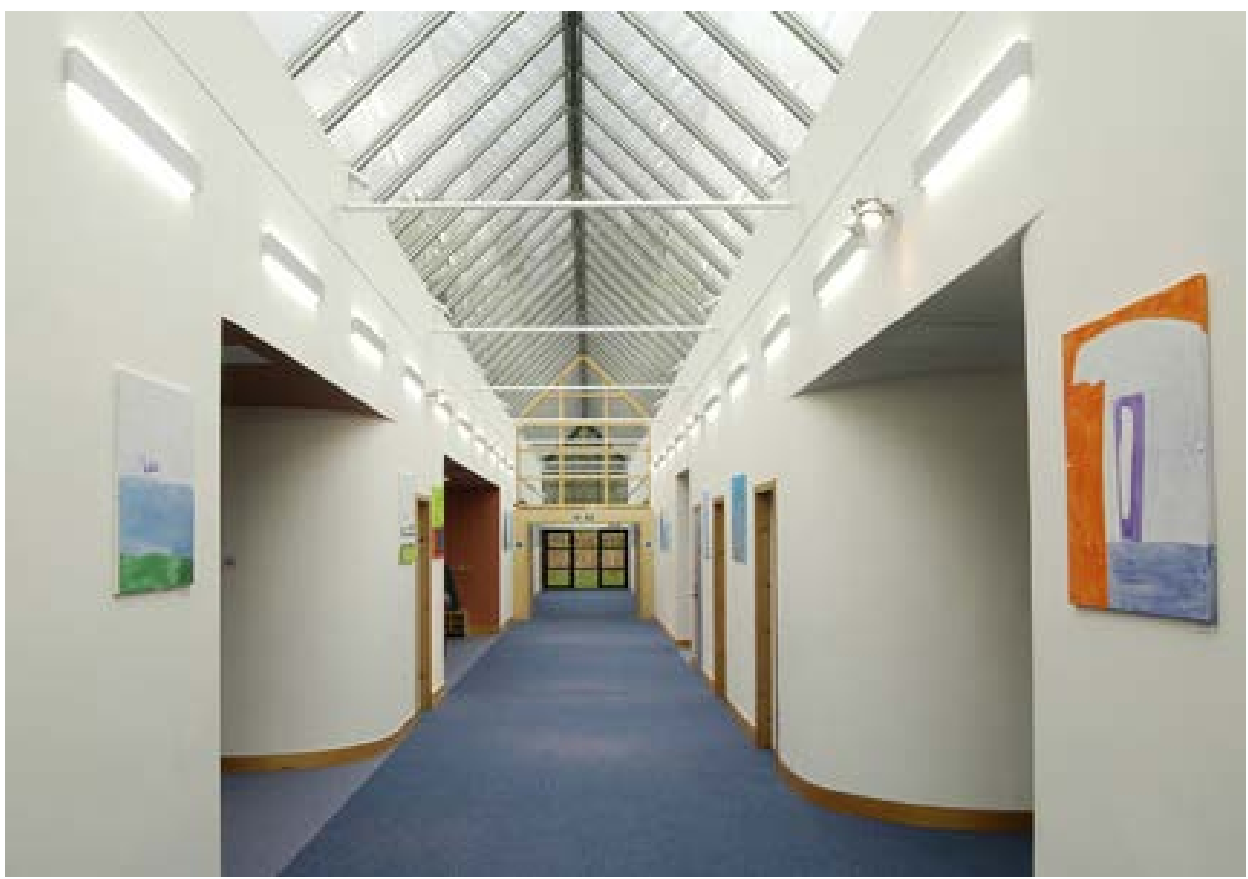

Figura 02. New Struan School (Source: Scottish Autism, photograpgher: Tina Norris) 


\section{Elena Bellini}

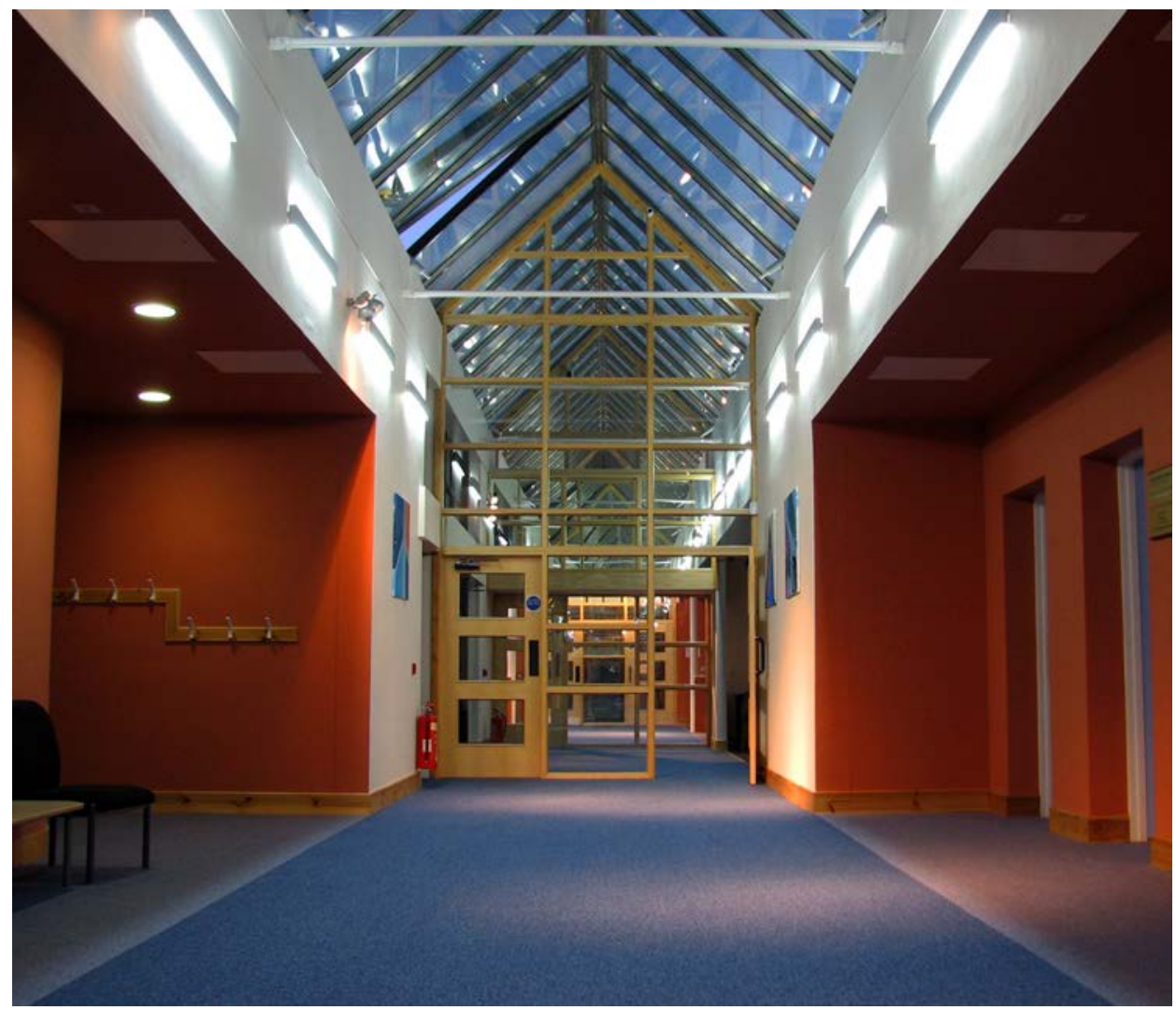

Figura 03. New Struan School (Source: Scottish Autism, photograpgher: Tina Norris) 
Ambienti sensoriali “terapeutici” che rendano Abili

RIDUZIONE DEL DISAGIO SENSORIALE E AMBIENTALE:

\section{ABBATTIMENTO DELLO STRESS:}

Chiarezza e semplicità dell'impianto planimetrico;

Mainstreet centrale di distribuzione che permette una visibilità totale e continua dello

spazio, su cui si affacciano tutti gli spazi educativi;

Divisione netta degli ambienti educativi per gruppi classe in base alle diverse età;

Differenziazione che metta in evidenza gli spazi educativi rispetto alle attività, attraverso

il colore;

(2) Palette di colori diffusi per modulare gli spazi ed evidenziare le attività, vivaci ma non troppo accesi per limitare il sovraccarico visivo;

Rivestimento a pavimento con linee morbide e textures flessibili per personalizzare lo spazio e renderlo caldo e accogliente;

Integrazione di elementi di comunicazione con velcro a parete per personalizzare le clas-

si e favorire la comunicazione visiva attraverso immagini;

Stanza d'integrazione sensoriale;

STRUTTURAZIONE DELLO SPAZIO EDUCATIVO:

Strutturato in modo semplice e chiaro in aree di attività differenziate, spazio separato per il lavoro individuale o in piccoli gruppi;

Condivisione tra le diverse aule di servizi comuni, stanze per la terapia e una 'resource room', per non sovraccaricare il bambino a livello sensoriale;

Ogni ambiente educativo prevede il bagno in adiacenza, con accesso diretto dall'aula;

\section{(-) COMFORT VISIVO:}

Illuminazione naturale indiretta costante e diffusa;

Aule distribuite lungo il perimetro per avere un massimo daylighting e spazi comuni centrali illuminati da un gioco di lucernari circolari;

Luce artificiale testata per un massimo comfort visivo per il bambino, che non produca lo sfarfallio tipico dei neon; luce riflessa sul soffitto per una maggiore diffusione indiretta;

(3) COMFORT uditivo:

Isolamento acustico di pareti e soffitti;

Sistema di ventilazione meccanica efficiente a basso impatto acustico;

Materiali di rivestimento a parete in resina e linoleum a pavimento per minimizzare la trasmissione del suono ed il riverbero;

Uso di pannelli di rifrazione sonora a soffitto per migliorare il comfort acustico, soprattutto negli ambienti maggiormente ampi, come palestra e stanza polivalente, o nei corridoi;

Scheda 02. Forbush School for Autism (parte 1) 


\section{Elena Bellini}

\section{COMFORT PROPRIOCETTIVO E PROSSEMICA:}

Spazi comuni ampi per non sovraccaricare il bambino a livello di stimoli visivi e uditivi, o sovraffollamento;

Ampi corridoi e altezza dei soffitti, per dare maggiore libertà di movimento e non sentirsi a disagio con l'affluire degli altri bambini;

Dimensione delle aule in base all'età: $46,5 \mathrm{mq}, 55,7 \mathrm{mq}, 74,3 \mathrm{mq}$.

Criticità:

- Se da un lato le linee curve rendono l'ambiente maggiormente accogliente e familiare e possono essere di supporto negli spostamenti, l'utilizzo contemporaneo di molti colori potrebbe essere fonte di sovraccarico.

- Mentre la differenza di colore a parete aiuta ad evidenziare gli spazi ed è di supporto per la comprensione e l'orientamento, I'uso del colore a pavimento non è corrispondente e si usano spesso tondi e curve di colore in modo contemporaneo e ambiguo.

- Il pavimento in linoleum, inoltre, ha una finitura lucida e potrebbe contribuire a creare disagio a livello visivo, per la riflessione di luci e colori.

SALUTE E BENESSERE:

Materiali e prodotti naturali e riciclabili; antibatterici e antistatici; non contengono formaldeide; hanno un basso livello di emissione di VOC (composti organici volatili);

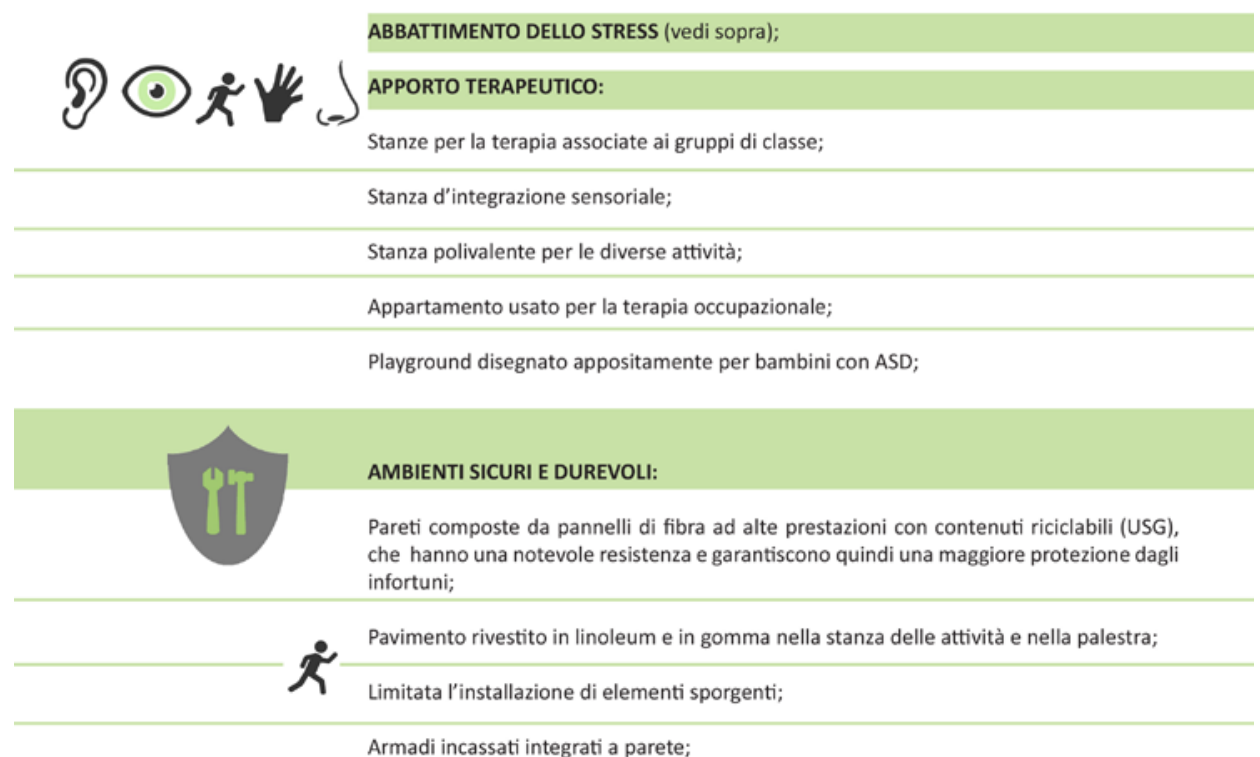

Scheda 02. Forbush School for Autism (parte 2) 
Ambienti sensoriali “terapeutici” che rendano Abili

ORIENTAMENTO E COMPRENSIONE:

Riconoscibilità dell'ingresso attraverso una pensilina colorata;

Chiarezza e semplicità dell’impianto planimetrico;

Mainstreet centrale di distribuzione che permette una visibilità totale e continua dello

spazio, su cui si affacciano tutti gli spazi educativi;

Divisione netta degli ambienti educativi per gruppi classe in base alle diverse età;

Differenziazione che metta in evidenza gli spazi educativi rispetto alle attività, attraverso

il colore;

(-) Palette di colori diffusi per modulare gli spazi ed evidenziare le attività, vivaci ma non troppo accesi per limitare il sovraccarico visivo;

Rivestimento a pavimento con linee morbide e textures flessibili per personalizzare lo
spazio e renderlo caldo e accogliente;

Ogni classe prevede il bagno in adiacenza, accessibile direttamente dall'aula;

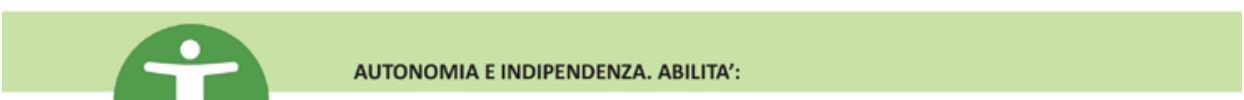

Personalizzazione e strutturazione dello spazio educativo, per ottimizzare le attività della classe sulla base del percorso di apprendimento;

Divisione netta degli ambienti educativi per gruppi classe in base alle diverse età;

Strutturazione semplice e chiara in aree di attività differenziate all'interno dell'aula; spazio separato per il lavoro individuale o in piccoli gruppi; ogni classe prevede inoltre il bagno in adiacenza, accessibile direttamente dall'aula;

\section{STRATEGIE DI APPRENDIMENTO DELLE ABILITA'}

Appartamento per la terapia occupazionale e l'apprendimento delle abilità quotidiane;

Stanze per la terapia associate ai gruppi di classe;

Scheda 02. Forbush School for Autism (parte 3) 


\section{Elena Bellini}

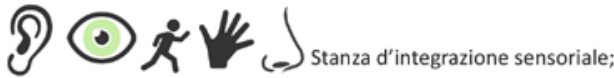

Stanza polivalente per le diverse attività;

\section{SISTEMA DI COMUNICAZIONE VISIVA ALTERNATIVA:} (1) Comunicazione visiva attraverso segnaletica; installazione di immagini e quanto utile alle
attività educative nelle classi attraverso l'integrazione di velcro a parete;

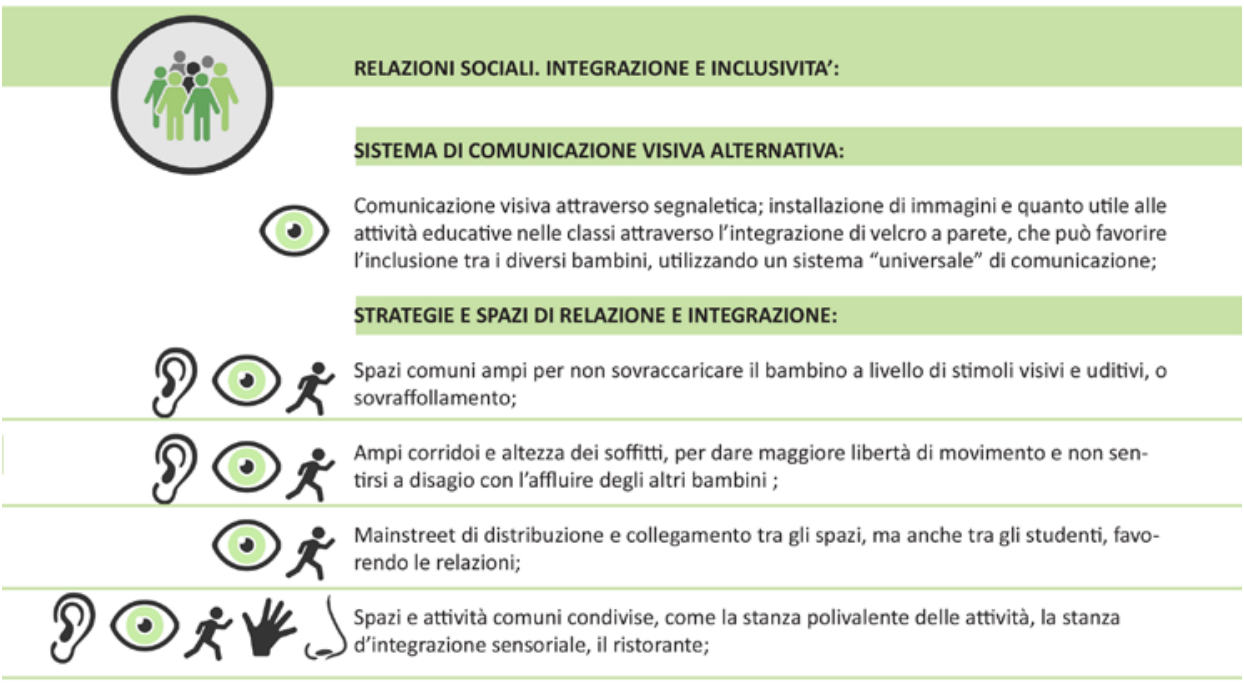

Playground esterno, appositamente disegnato per bambini con DSA.

Scheda 02. Forbush School for Autism (parte 4)

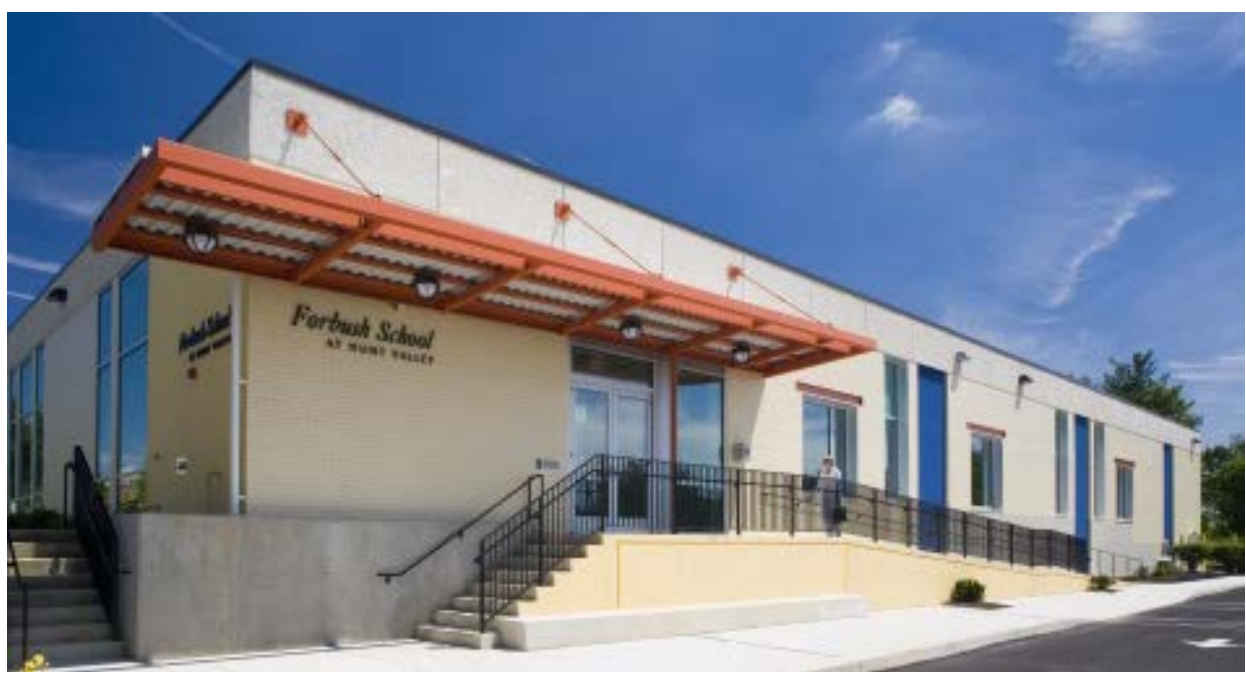

Figura 04. Forbush School for Autism (Source: Universal Design Case Study Collection at: https://www.universaldesigncasestudies.org) 
Ambienti sensoriali "terapeutici" che rendano Abili

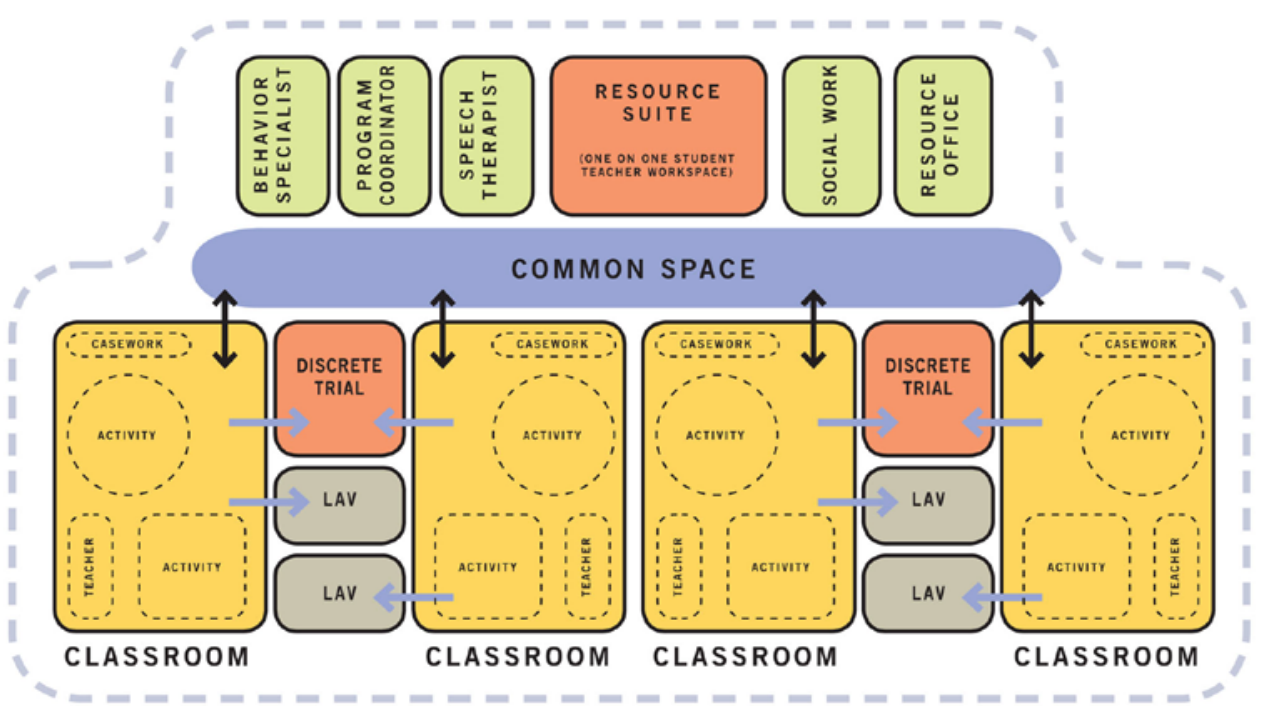

Figura 05. Forbush School for Autism: planimetria (Source: Universal Design Case Study Collection at: https://www.universaldesigncasestudies.org)

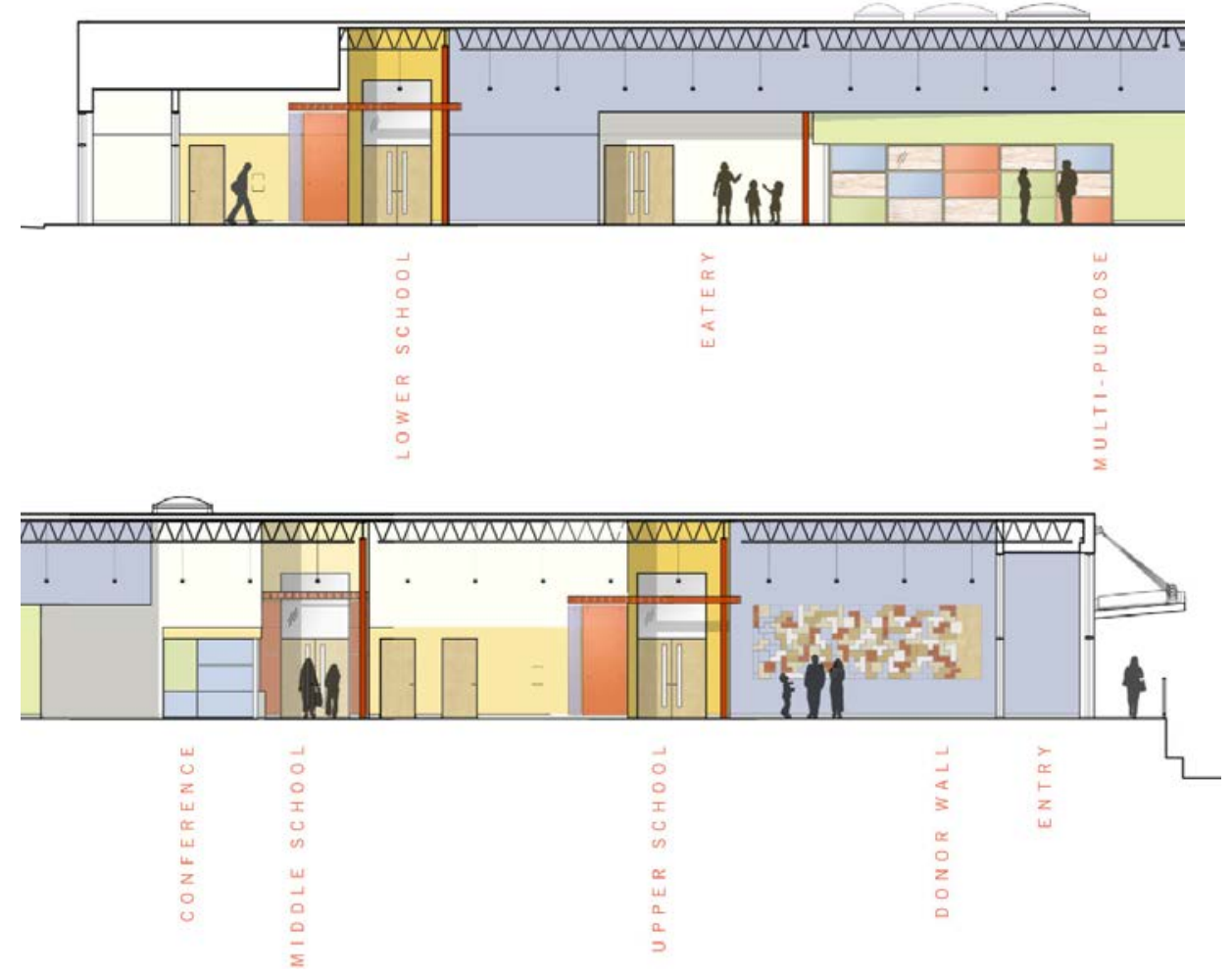

Figura 06. Forbush School for Autism: sezione (Source: Universal Design Case Study Collection at: https://www.universaldesigncasestudies.org) 


\section{Elena Bellini}

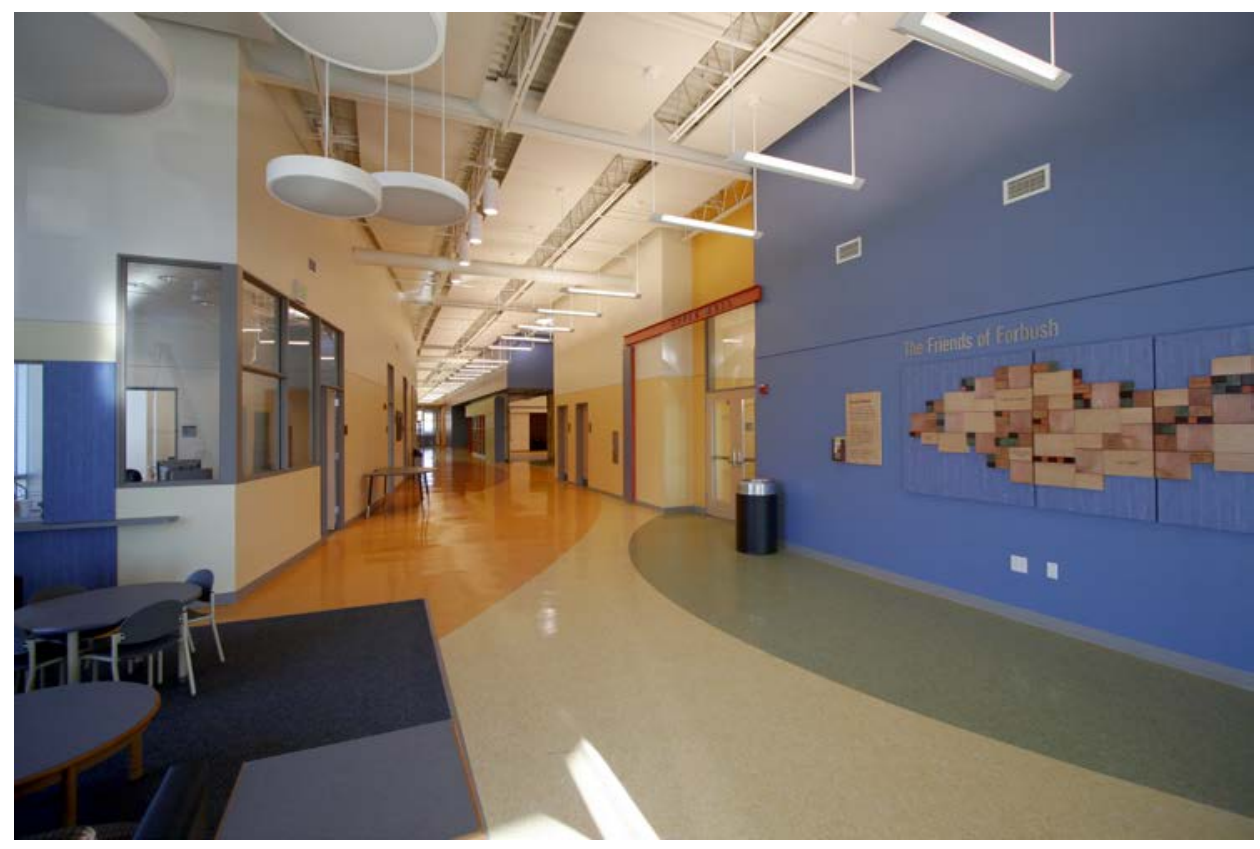

Figura 07. Forbush School for Autism: ingresso della scuola (Source: Universal Design Case Study Collection at: https://www.universaldesigncasestudies.org) 
Ambienti sensoriali “terapeutici” che rendano Abili

RIDUZIONE DEL DISAGIO SENSORIALE E AMBIENTALE:

\section{ABBATTIMENTO DELLO STRESS:}

Sensory zoning: distribuzione delle funzioni in modo da suddividere le attività ad alto stimolo da quelle a basso stimolo e limitare quindi le distrazioni ed il sovraccarico sensoriale;

Spazi di transizione come spazio di circolare centrale, area verde centrale e giardino sensoriale, aree gioco e aree sensoriali, posizionate tra zone ad alto e zone a basso stimolo, per regolare le stimolazioni sensoriali, prepararsi al passaggio tra le diverse aree, migliorare l'indipendenza e l'autonomia e favorire gli spostamenti;

\section{STRUTTURAZIONE DELL'AMBIENTE EDUCATIVO:}

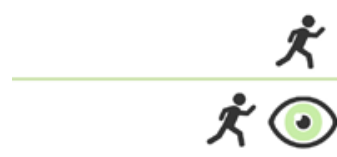

Classi di dimensione contenuta - 7 studenti e insegnanti/assistenti - organizzate per grup$\mathrm{pi}$, rispecchiando la familiarità dell'ambiente;

Compartimenti/stazioni all'interno dell'aula, legate alle diverse attività e distribuite secondo una routine per facilitare attenzione e apprendimento; ogni stazione può essere separata fisicamente o visivamente attraverso diversi materiali e colori;

Armadi a muro o scaffali con scatole dai colori neutri per riporre gli oggetti e i materiali educativi e limitare il sovraccarico visivo;

Escape spaze/Sensory room all'interno delle aule per sostenere la sovrastimolazione e l'autoregolazione, oltre a personalizzare lo spazio;

Spazio open-air interno e contenuto, utilizzato per diverse funzioni, tra cui fare attività didattica all'esterno, rivolta a temi come la natura, il tempo, le abilità motorie etc.

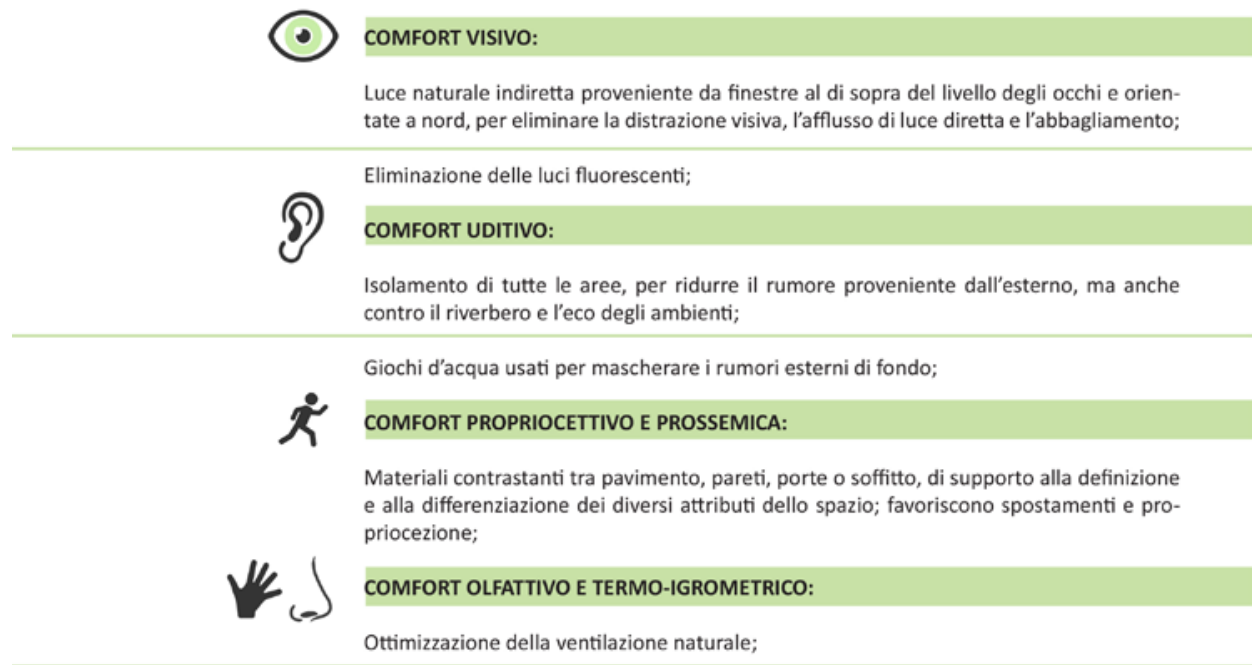

Cortile centrale interno su cui affacciano tutte le aule, per favorire il controllo termo-igrometrico;

Scheda 03. Advance School for Developing Skills of Special Needs Children (parte 1) 


\section{Elena Bellini}

\section{(1) \\ Psicomotricità: spazio adeguato, rettangolare e ampio con una porzione separata da una parete scorrevole usata come ripostiglio e preparazione;

Logopedia: collocata nell’area a basso stimolo, prevede spazi con diversi livelli di isola-
mento acustico per evitare l' 'effetto serra';

Giardino sensoriale e spazio esterno: percorsi sensoriali, giochi d'acqua, vasche di palline, vasche di sabbia, giardino di erbe aromatiche, parete tinteggiata a lavagna per la libera espressione dell'arte, sedute raccolte 'a nido'; la manutenzione ed il giardinaggio sono attività considerate terapeutiche, oltre che utili all'acquisizione di nuove abilità;

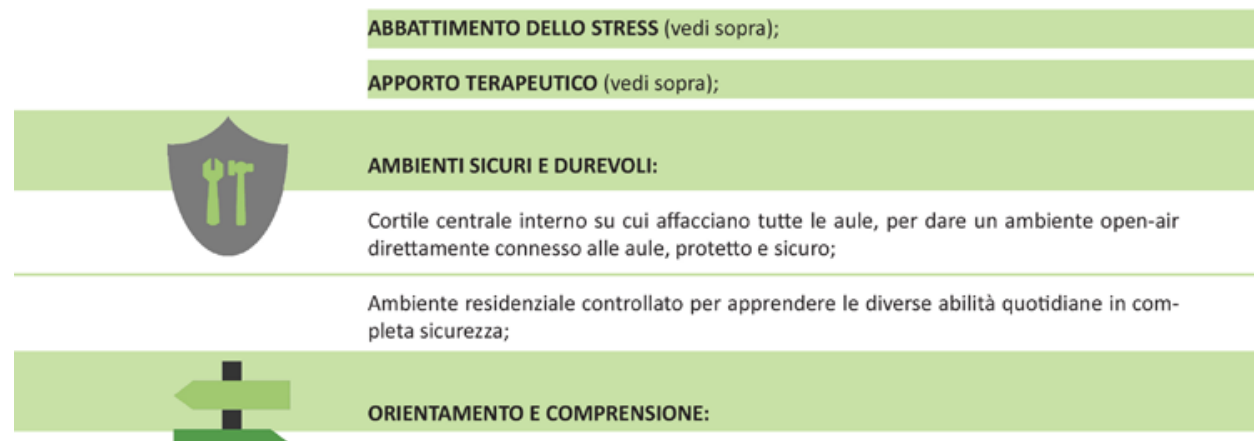

Sensory zoning: distribuzione delle funzioni in modo da suddividere le attività ad alto stimolo da quelle a basso stimolo e limitare quindi le distrazioni ed il sovraccarico sensoriale;

Spazi di transizione, come spazio di circolare centrale, area verde centrale e giardino sensoriale, aree gioco e aree sensoriali, posizionate tra zone ad alto e zone a basso stimolo, per regolare le stimolazioni sensoriali, prepararsi al passaggio tra le diverse aree, migliorare l'indipendenza e l'autonomia e favorire gli spostamenti;

Scheda 03. Advance School for Developing Skills of Special Needs Children (parte 2) 
Ambienti sensoriali "terapeutici” che rendano Abili

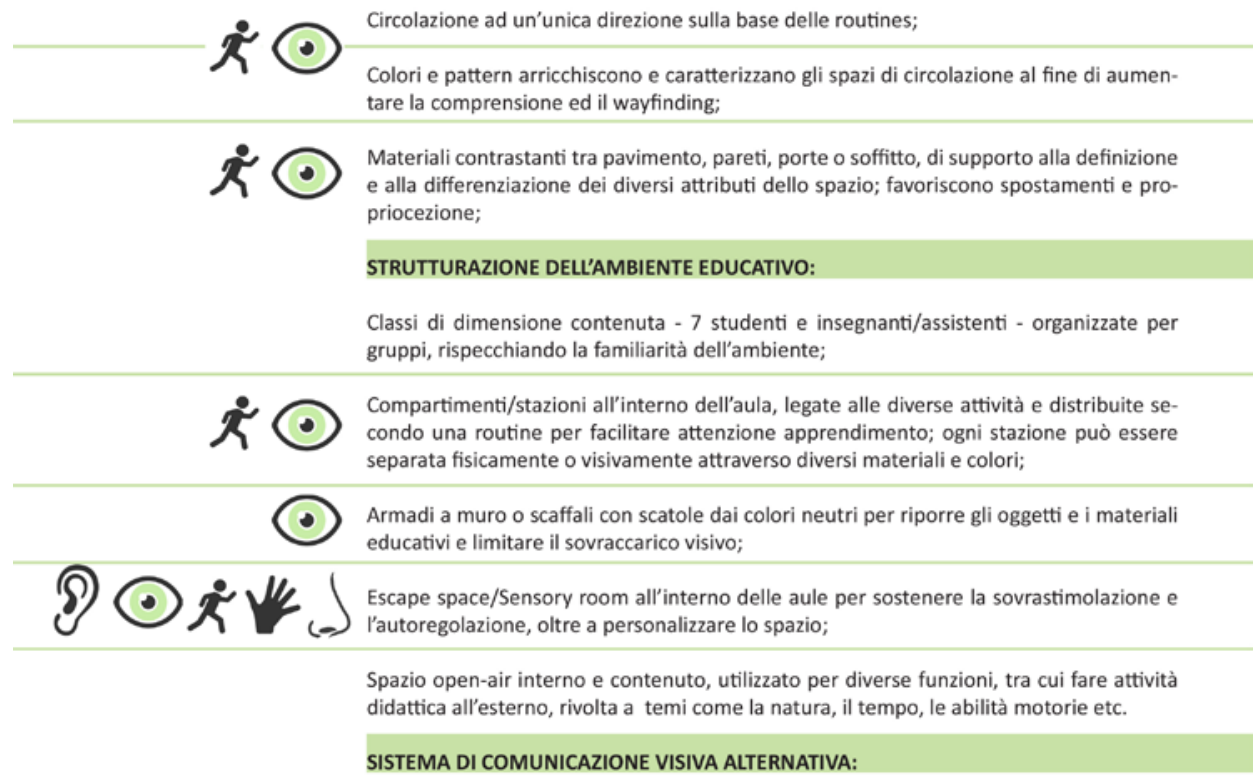

Sistema di comunicazione attraverso PECS, come supporto alla comunicazione; l'associazione di parole e immagini favorisce l'apprendimento, ma anche l'inclusione e l'autostima di bambini con diverse abilità;

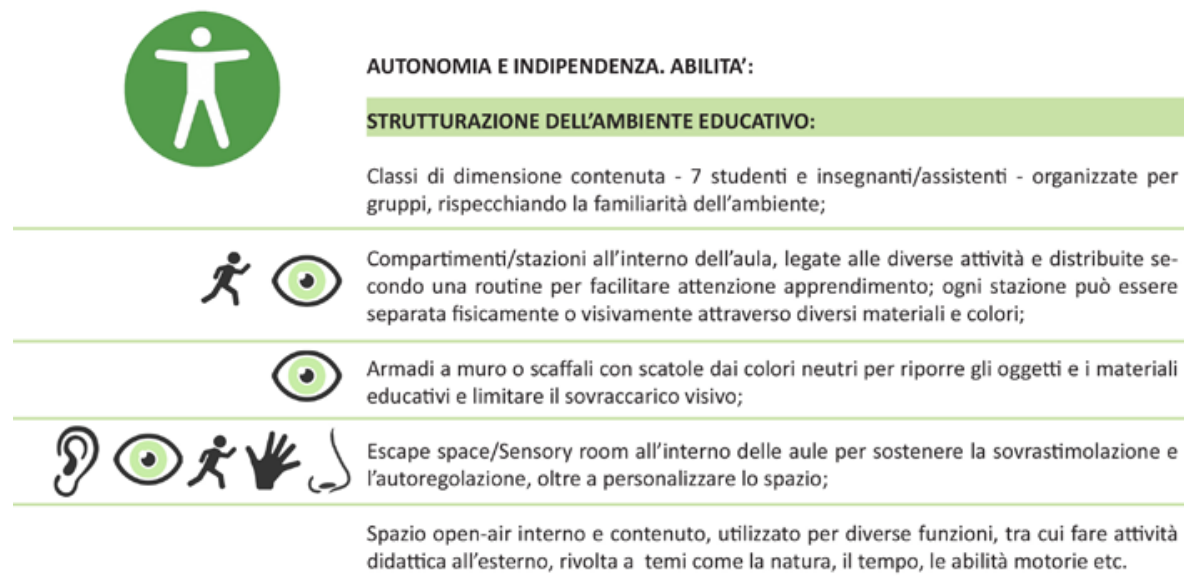

Scheda 03. Advance School for Developing Skills of Special Needs Children (parte 3) 


\section{Elena Bellini}

\section{SISTEMA DI COMUNICAZIONE VISIVA ALTERNATIVA:}

Sistema di comunicazione attraverso PECS, come supporto alla comunicazione; l'associazione di parole e immagini favorisce l'apprendimento, ma anche l'inclusione e l'autostima di bambini con diverse abilità;

\section{STRATEGIE DI APPRENDIMENTO DELLE ABILITA':}

Terapia occupazionale e attitudinale (falegnameria, carpenteria metallica, giardinaggio...) e inserimento lavorativo;

Logopedia: collocata nell'area a basso stimolo, prevede spazi con diversi livelli di isolamento acustico per evitare I' 'effetto serra' ed aumentare l'integrazione nel mondo esterno;

Manutenzione di giardino sensoriale e spazio esterno;

Spazi residenziali di transizione tra residenza assistita e autonoma per l'apprendimento controllato delle abilità quotidiane, nell'ottica di una futura integrazione nella comunità sociale; gli alloggi, non connessi alla scuola, permettono di fare un percorso verso la struttura scolastica ogni giorno in modo indipendente e iniziare ad integrarsi nella comunità.

RELAZIONI SOCIALI. INTEGRAZIONE E INCLUSIVITA':

Diversi livelli di isolamento acustico progettati per evitare l' 'effetto serra' ed aumentare l'integrazione nel mondo esterno;

\section{SISTEMA DI COMUNICAZIONE VISIVA ALTERNATIVA:}

(-) Sistema di comunicazione attraverso PECS, come supporto alla comunicazione; l'associazione di parole e immagini favorisce l'apprendimento, ma anche l'inclusione e l'autostima di bambini con diverse abilità;

\section{STRATEGIE E SPAZI DI RELAZIONE E INTEGRAZIONE:}

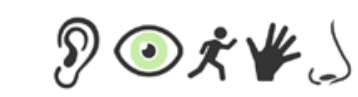

Giardino sensoriale e spazio esterno: percorsi sensoriali, giochi d'acqua, vasche di palline, vasche di sabbia, giardino di erbe aromatiche, parete tinteggiata a lavagna per la libera espressione dell'arte, sedute raccolte 'a nido';

Piscina sensoriale e idroterapia;

Terapia occupazionale e attitudinale (falegnameria, carpenteria metallica, giardinaggio...) e inserimento lavorativo;

Manutenzione di giardino sensoriale e spazio esterno;

Servizi commerciali aperti alla comunità integrati all'interno della struttura, al fine di aprirsi verso l'esterno e aumentare la consapevolezza l'informazione sull'autismo;

Spazi residenziali di transizione tra residenza assistita e autonoma per l'apprendimento controllato delle abilità quotidiane, nell'ottica di una futura integrazione nella comunità sociale; gli alloggi, non connessi alla scuola, permettono di fare un percorso verso la struttura scolastica ogni giorno in modo indipendente e iniziare ad integrarsi nella comunità.

Scheda 03. Advance School for Developing Skills of Special Needs Children (parte 4) 
Ambienti sensoriali "terapeutici" che rendano Abili

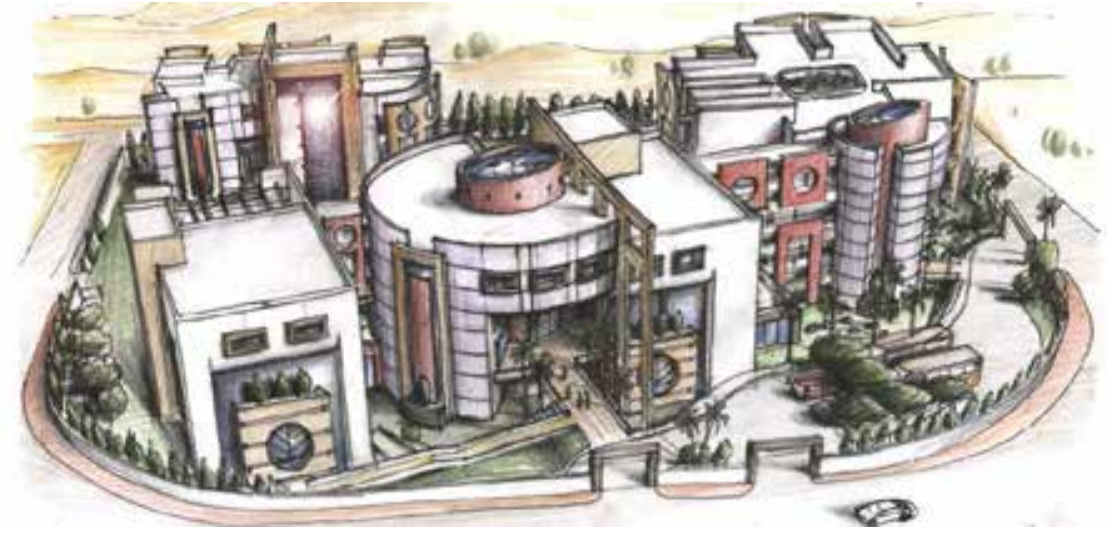

Figura 08. Advance School for Developing Skills of Special Needs Children

(Source: Mostafa, M. (2014) Architecture For Autism: Autism ASPECTSSTM in School Design, in Archnet-IJAR, Volume 8 -Issue 1 - March 2014 - (143-158) - Copyright to Progressive Architects and Magda Mostafa)

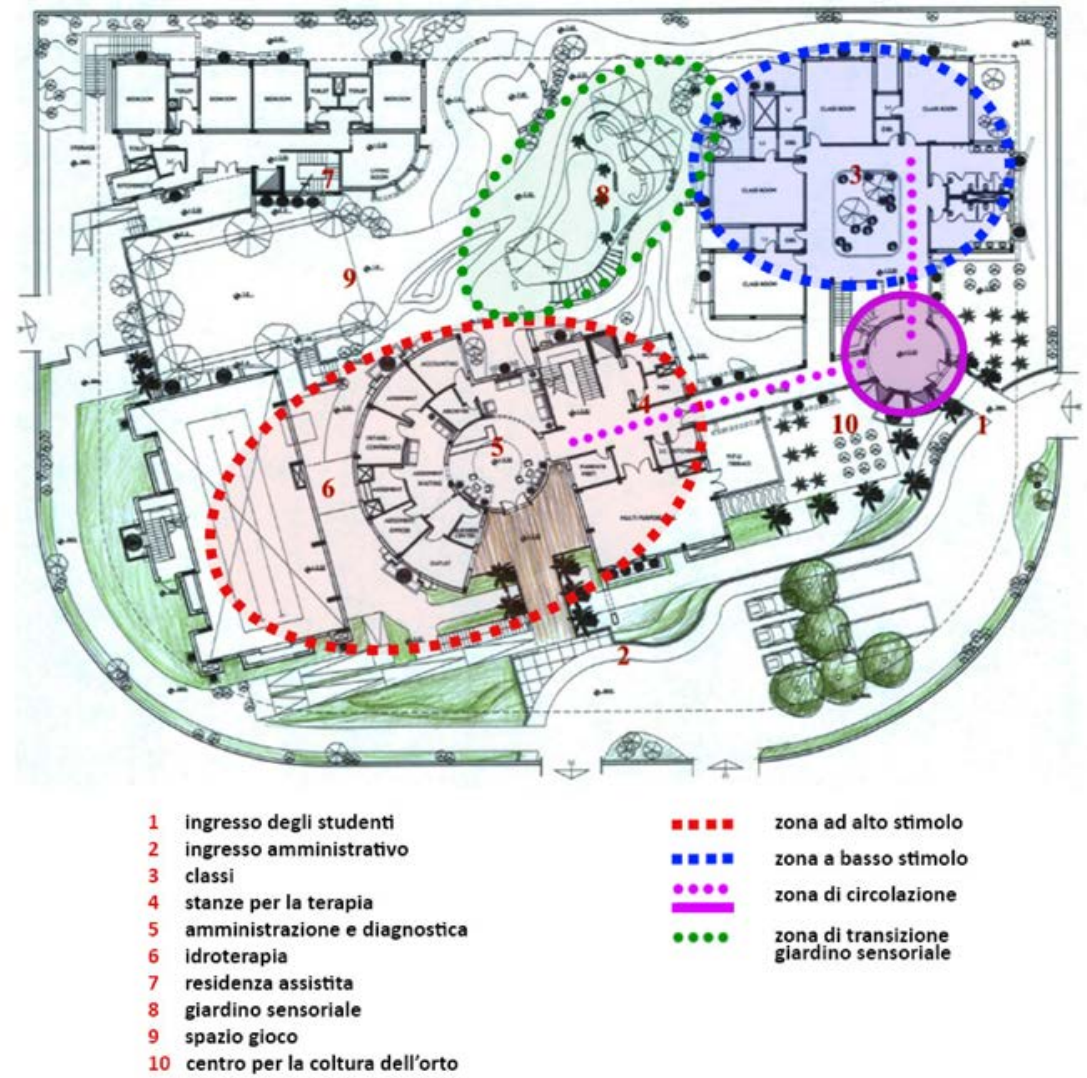

Figura 09. Advance School for Developing Skills of Special Needs Children (Source: Mostafa, M. (2014) Architecture For Autism: Autism ASPECTSS ${ }^{\text {TM }}$ in School Design, in 


\section{Elena Bellini}

Archnet-IJAR, Volume 8 -Issue 1 - March 2014 - (143-158) - Copyright to Progressive Architects and Magda Mostafa)

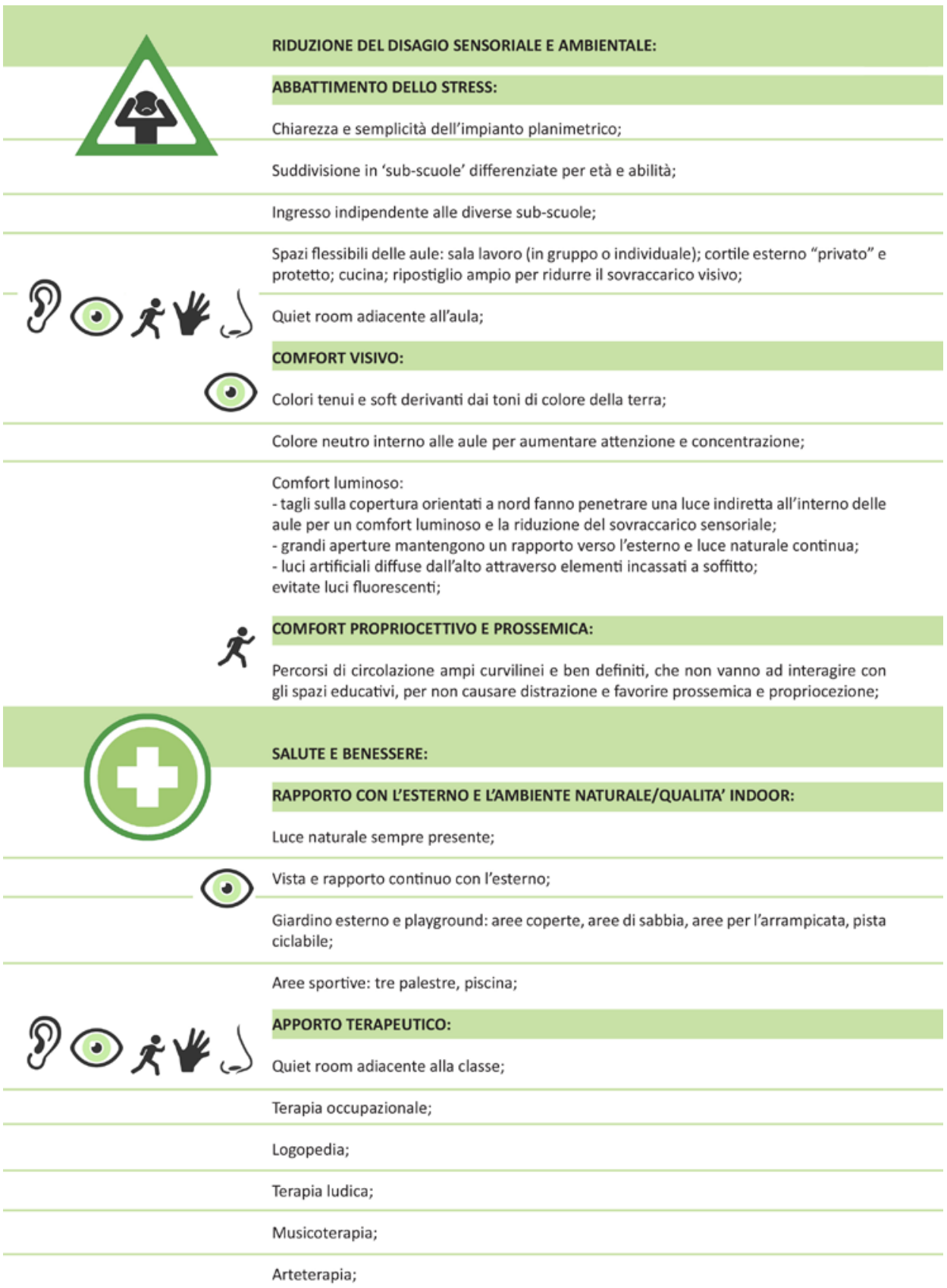

Scheda 04. Northen School for Autism (parte 1) 
Ambienti sensoriali "terapeutici” che rendano Abili

AMBIENTI SICURI E DUREVOLI:
$\begin{aligned} & \text { Distribuzione di spazi, arredi e spazi esterni tale da consentire la sicurezza e l'indipen- } \\ & \text { denza del bambino; }\end{aligned}$
Possibilità di visibilità e controllo continuo da parte dell'operatore/insegnante;

Cortile esterno all'aula dedicato e playground differenziato per ogni età/abilità;

\begin{tabular}{l} 
ORIENTAMENTO E COMPRENSIONE: \\
Chiarezza e semplicità dell'impianto planimetrico; \\
Suddivisione in 'sub-scuole' differenziate per età e abilità; \\
\hline $\begin{array}{l}\text { Ingresso indipendente alle diverse sub-scuole; } \\
\text { classi, per non causare distrazione e favorire prossemica e propriocezione; }\end{array}$ \\
\hline Differenziazione di ogni aree attraverso il colore, per favorire orientamento e wayfin- \\
ding, ma anche indipendenza;
\end{tabular}

Spazi flessibili delle aule: sala lavoro (in gruppo o individuale); cortile esterno "privato" e protetto; cucina; ripostiglio ampio per ridurre il sovraccarico visivo;

Cortile esterno all'aula dedicato e playground differenziato per ogni età/abilità;

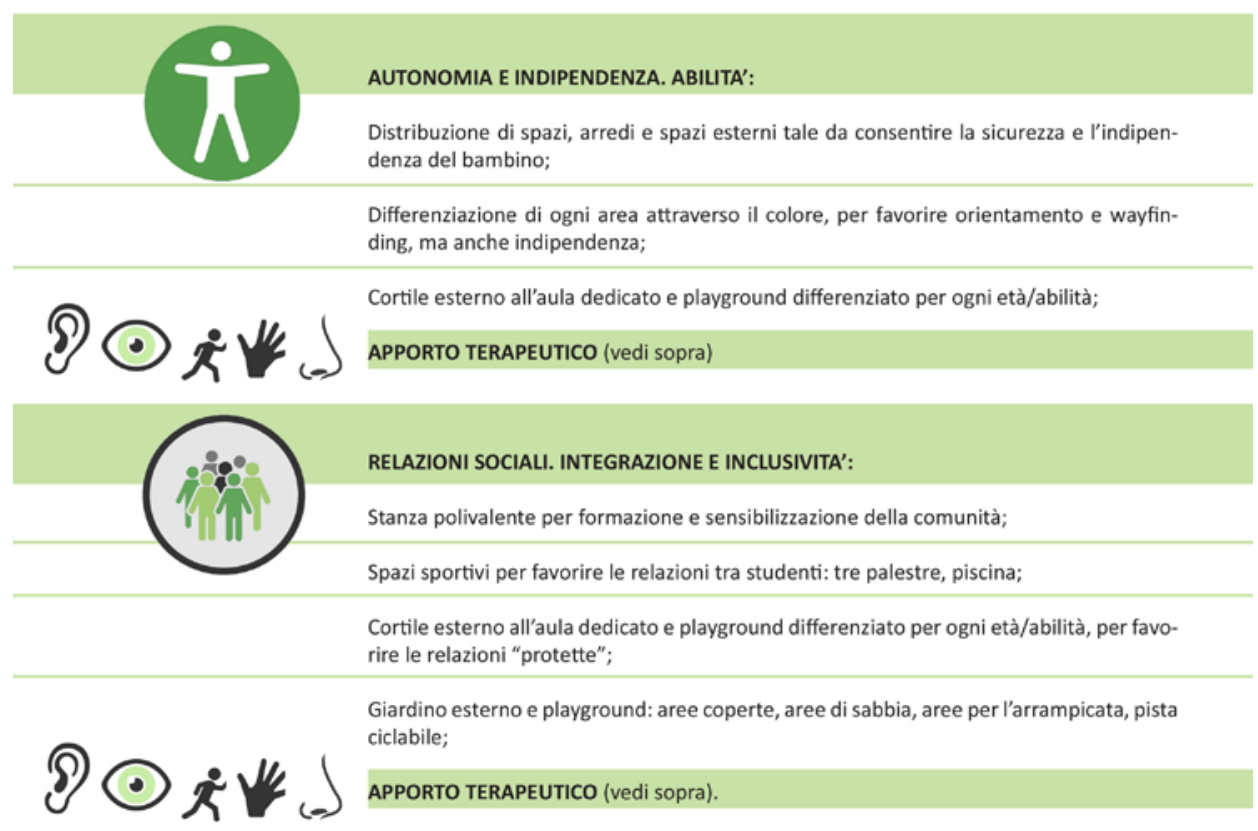

Scheda 04. Northen School for Autism (parte 2) 


\section{Elena Bellini}

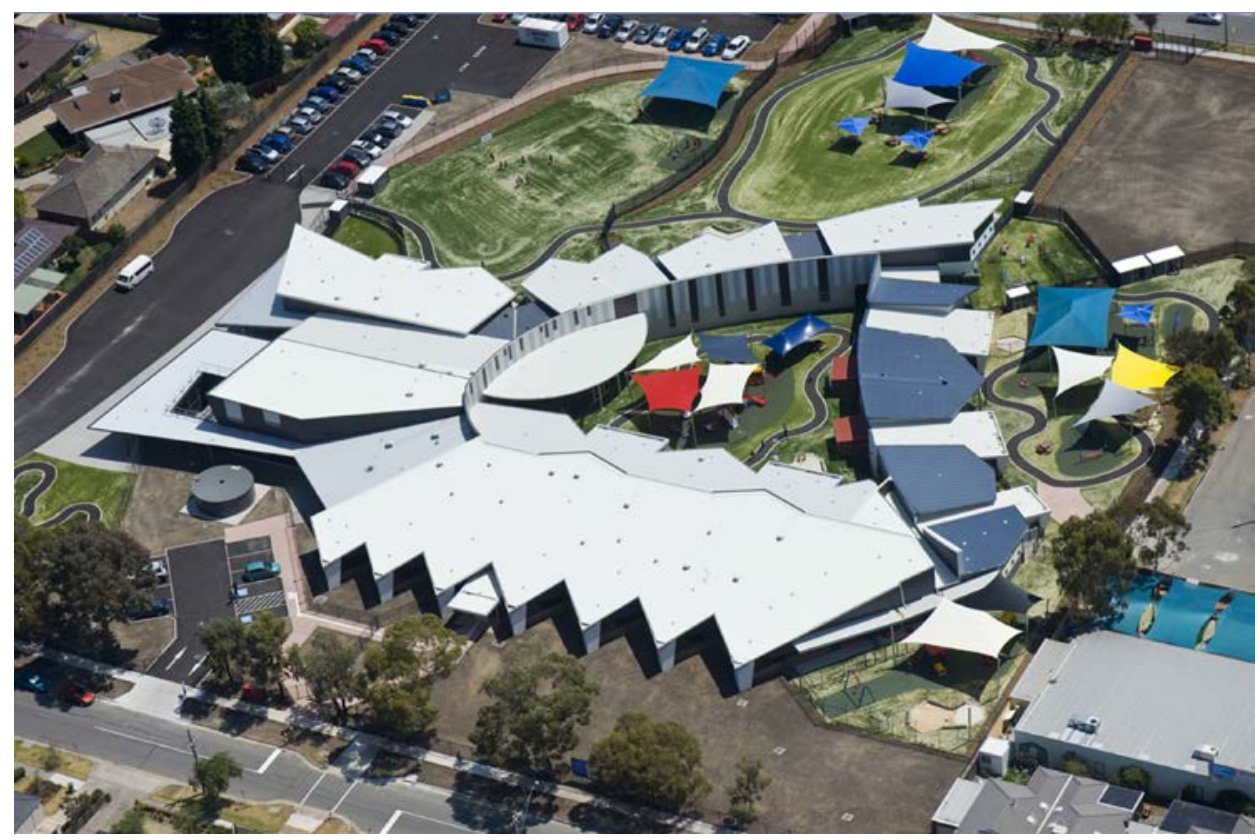

Figura 10. Northen School for Autism

(Source: Mostafa, M. (2015), Architecture for Autism: Built Environment Performance in Accordance to the Autism ASPECTSS ${ }^{\mathrm{TM}}$ Design Index, in Design Principles and Practices: An International Journal - Annual Review, vol 8, pp.55-71 - Copyright to Progressive Architects and Magda Mostafa) 
Ambienti sensoriali “terapeutici” che rendano Abili

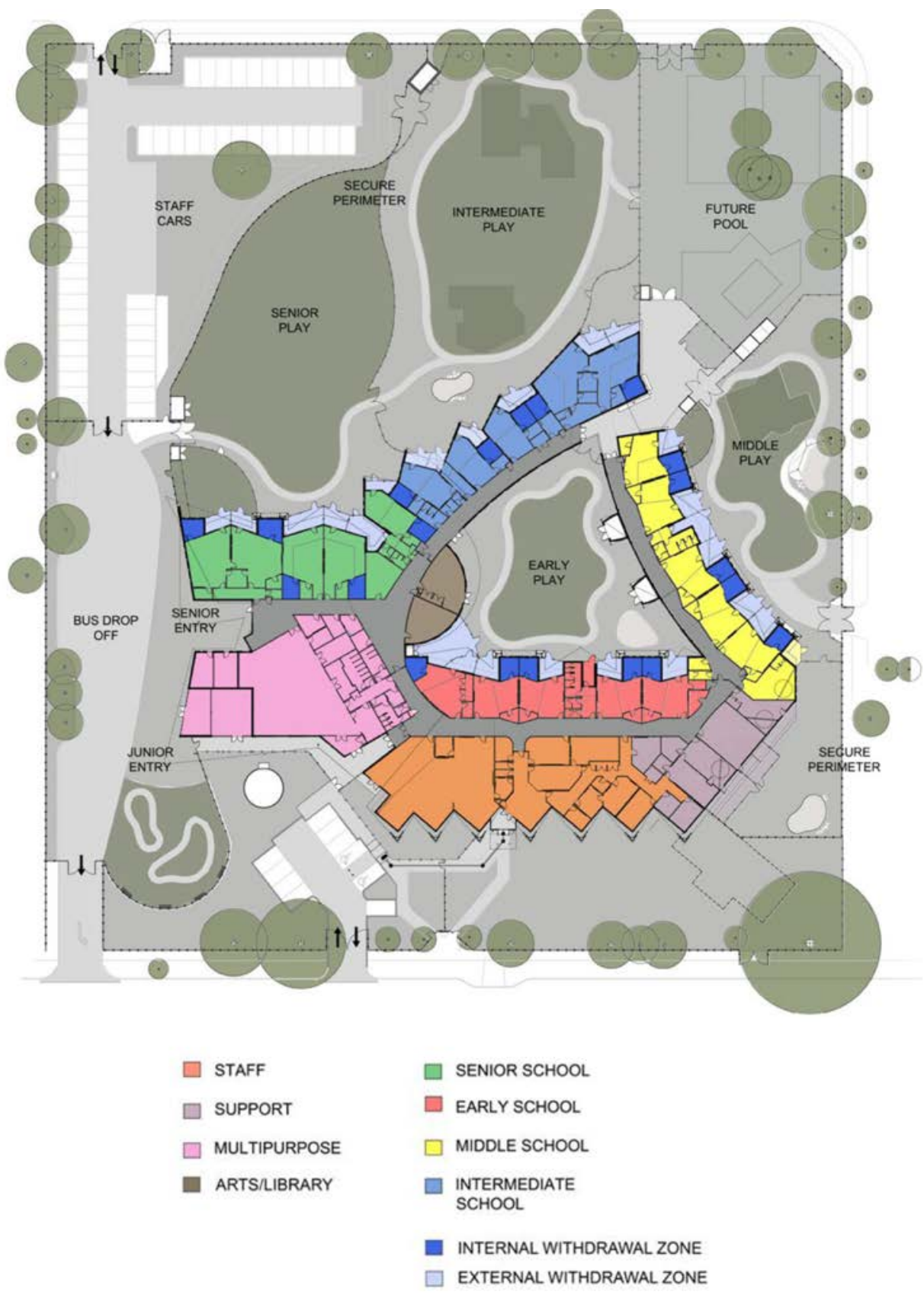

Figura 11. Northen School for Autism: planimetria generale (Source: Mostafa, M. (2015), Architecture for Autism: Built Environment Performance in Accordance to the Autism ASPECTSS ${ }^{\text {TM }}$ Design Index, in Design Principles and Practices: An International Journal Annual Review, vol 8, pp.55-71 - Copyright to Progressive Architects and Magda Mostafa) 


\section{Elena Bellini}

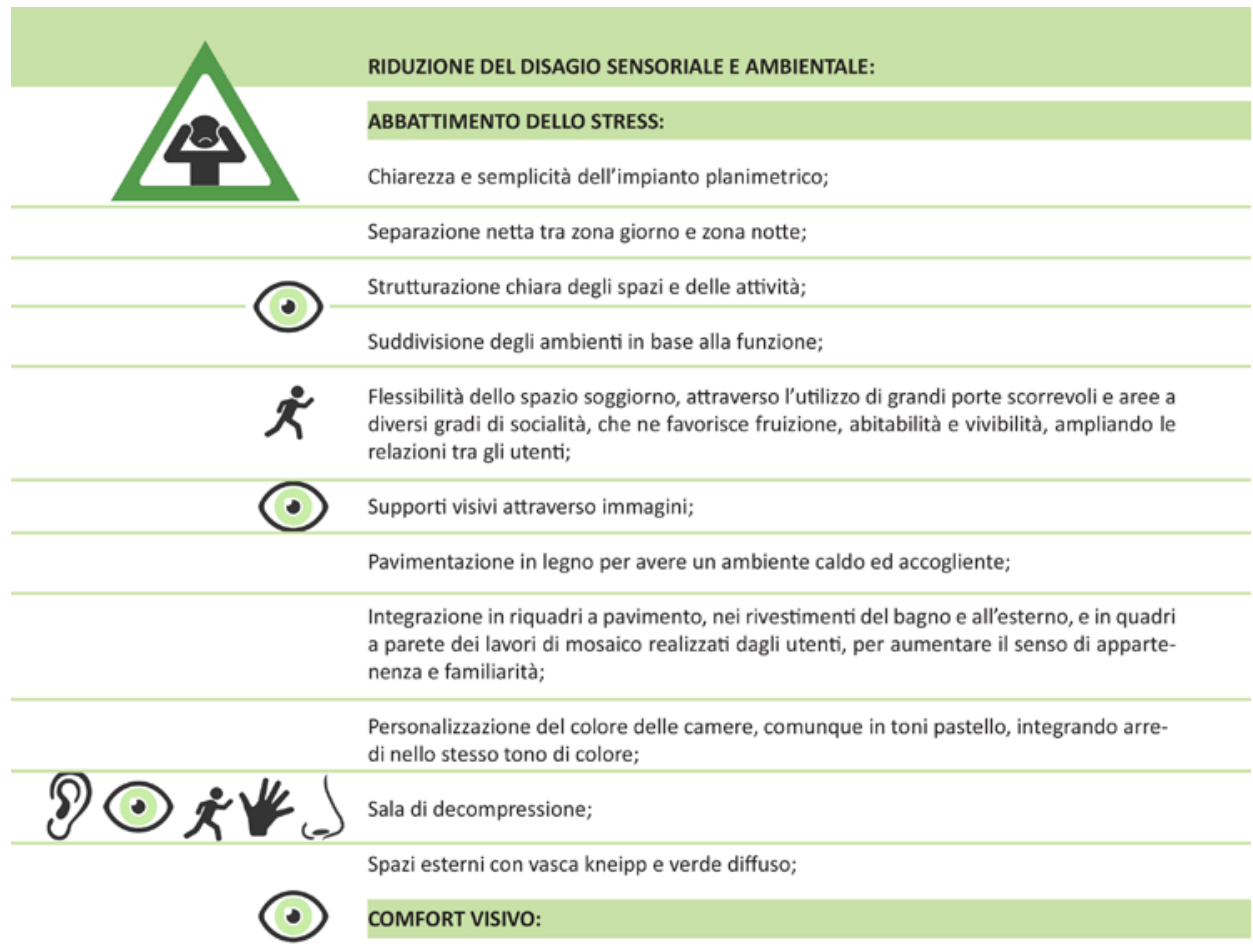

Colori neutri dell'ambiente, per non sovraccaricare a livello visivo;

Luce naturale sempre presente, oltre alla possibilità di oscurare dall'interno;

Luce artificiale calda e diffusa, integrata a soffitto o a sospensione;

COMFORT ACUSTICO:

Isolamento di pareti e porte;

Applicazione di silenziatori a parete;

COMFORT PROPRIOCETTIVO E PROSSEMICA:

Diversi gradi di socialità nel soggiorno, spazio flessibile, suddivisibile in diverse aree attraverso porte scorrevoli;

Ampia cucina con penisola centrale con doppia postazione per permettere il lavoro contemporaneo di più persone senza darsi fastidio;

\section{I// COMFORT TERMO-IgROMETRICO:}

Sistema di riscaldamento e raffrescamento a pavimento che consente una regolazione autonoma della temperatura, differenziata per le diverse aree della casa;

Scheda 05. Villa delle Rogge (parte 1) 
Ambienti sensoriali "terapeutici” che rendano Abili

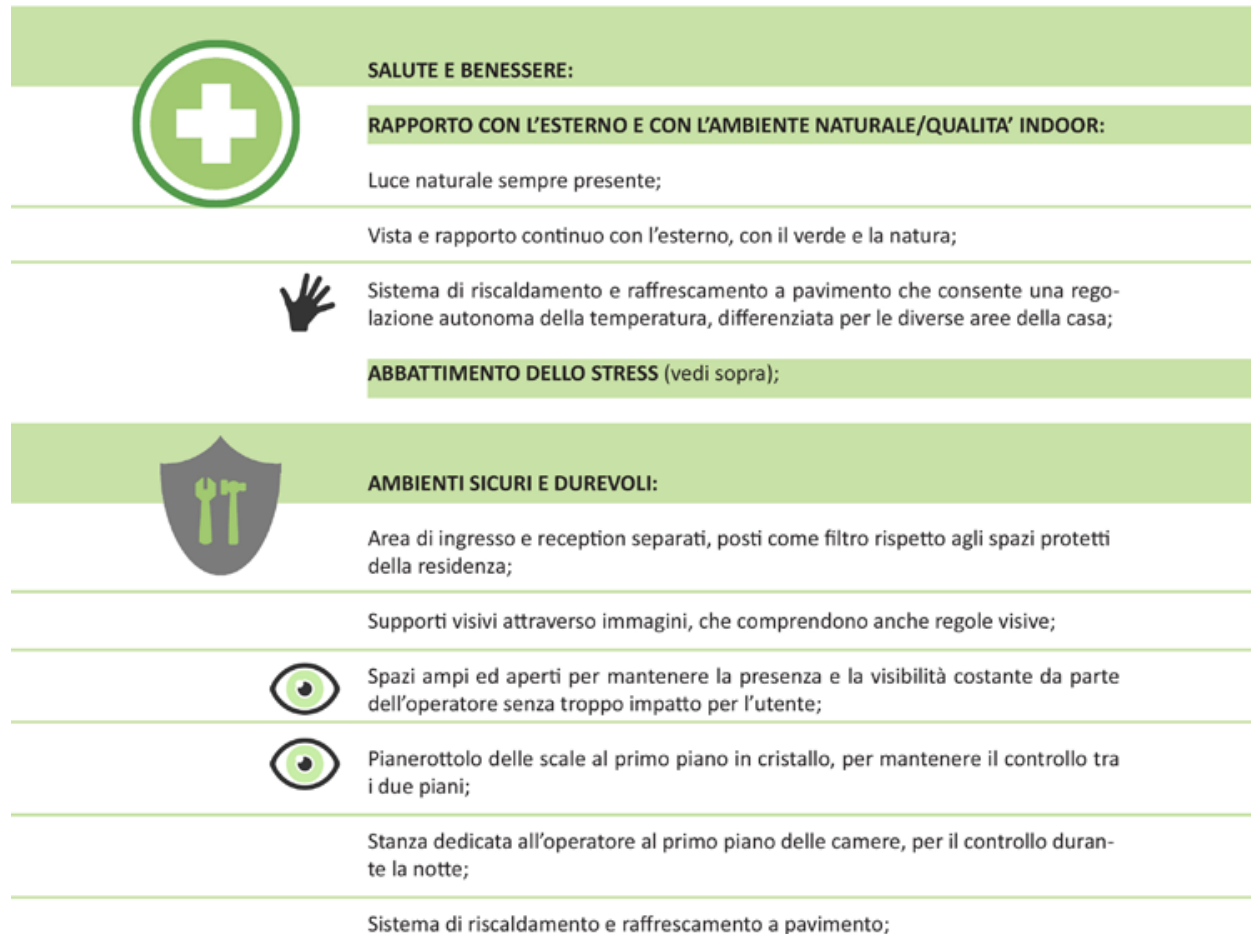

Sistema di riscaldamento e raffrescamento a pavimento;

Blocco delle finestre nelle camere al primo piano.

\begin{tabular}{l} 
ORIENTAMENTO E COMPRENSIONE: \\
Chiarezza e semplicità dell'impianto planimetrico; \\
Separazione netta tra zona giorno e zona notte; \\
\hline Strutturazione chiara degli spazi e delle attività;
\end{tabular}

Flessibilità dello spazio soggiorno, attraverso l'utilizzo di grandi porte scorrevoli e aree a diversi gradi di socialità, che ne favorisce fruizione, abitabilità e vivibilità, ampliando le relazioni tra gli utenti;

Riconoscibilità delle stanze da letto attraverso la scelta personalizzata del colore, comun-
que in toni pastello, integrando gli arredi nello stesso tono di colore;

\section{(-) Pianerottolo in cristallo che consente una relazione tra i piani ed una prevedibilità rispetto al nuovo ambiente;}

\section{SUPPORTI VISIVI:}

- Uso di sistema di comunicazione facilitato attraverso immagini, regole e agende visive, indicazioni step by step (soprattutto in cucina, bagno, guardaroba...) per supportare le attività e le abilità quotidiane;

Strutturazione dello spazio cucina con etichette che descrivono cosa c’è nei diversi ripiani.

Scheda 05. Villa delle Rogge (parte 2) 


\section{Elena Bellini}

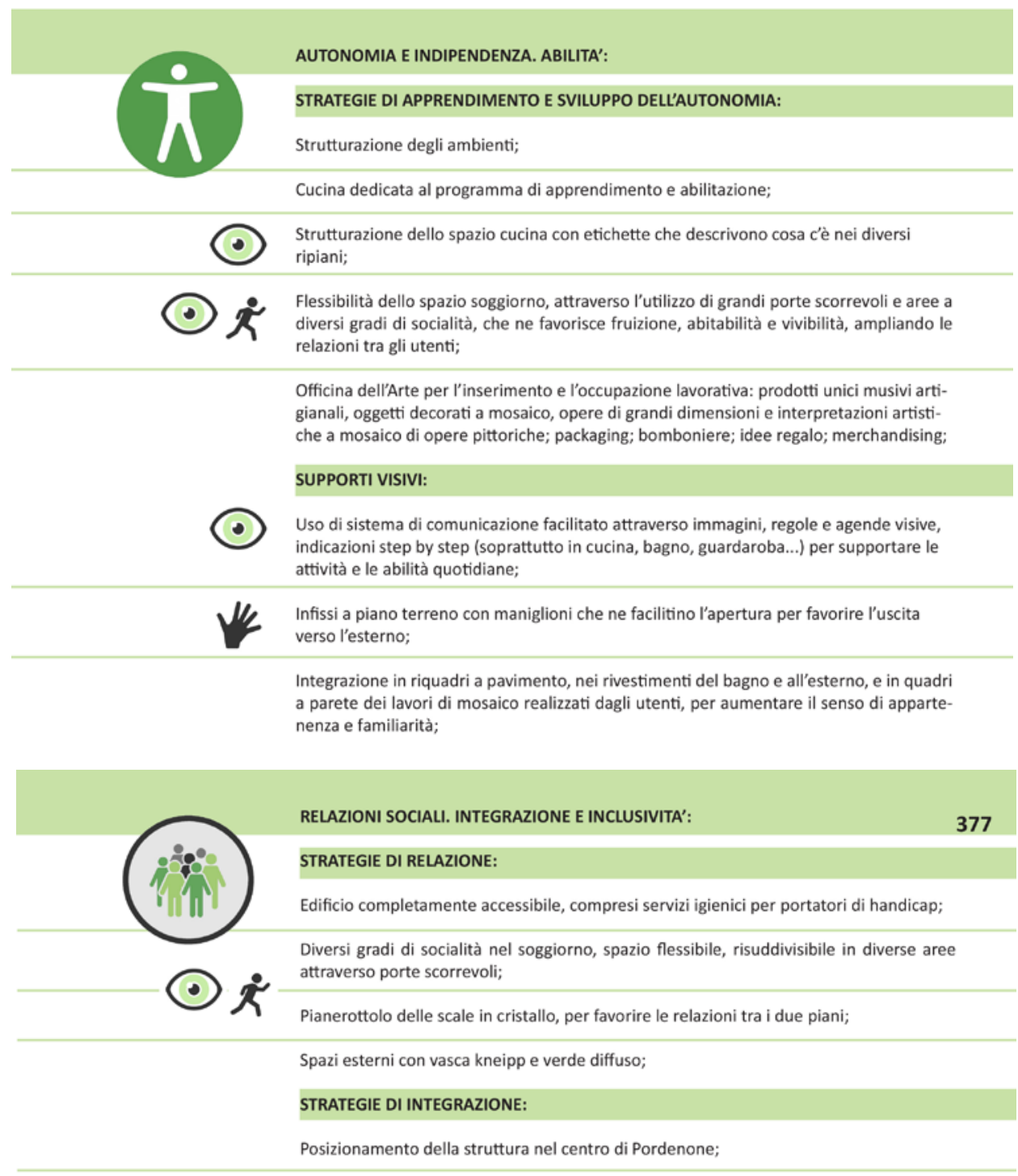

Rapporto diretto con la comunità sociale, anche attraverso programmi di integrazione e abilitazione rispetto alle attività e alle abilità sociali quotidiane;

Officina dell'Arte per l'inserimento e l'occupazione lavorativa: prodotti unici musivi artigianali, oggetti decorati a mosaico, opere di grandi dimensioni e interpretazioni artistiche a mosaico di opere pittoriche; packaging; bomboniere; idee regalo; merchandising.

Scheda 05. Villa delle Rogge (parte 3) 
Ambienti sensoriali "terapeutici" che rendano Abili

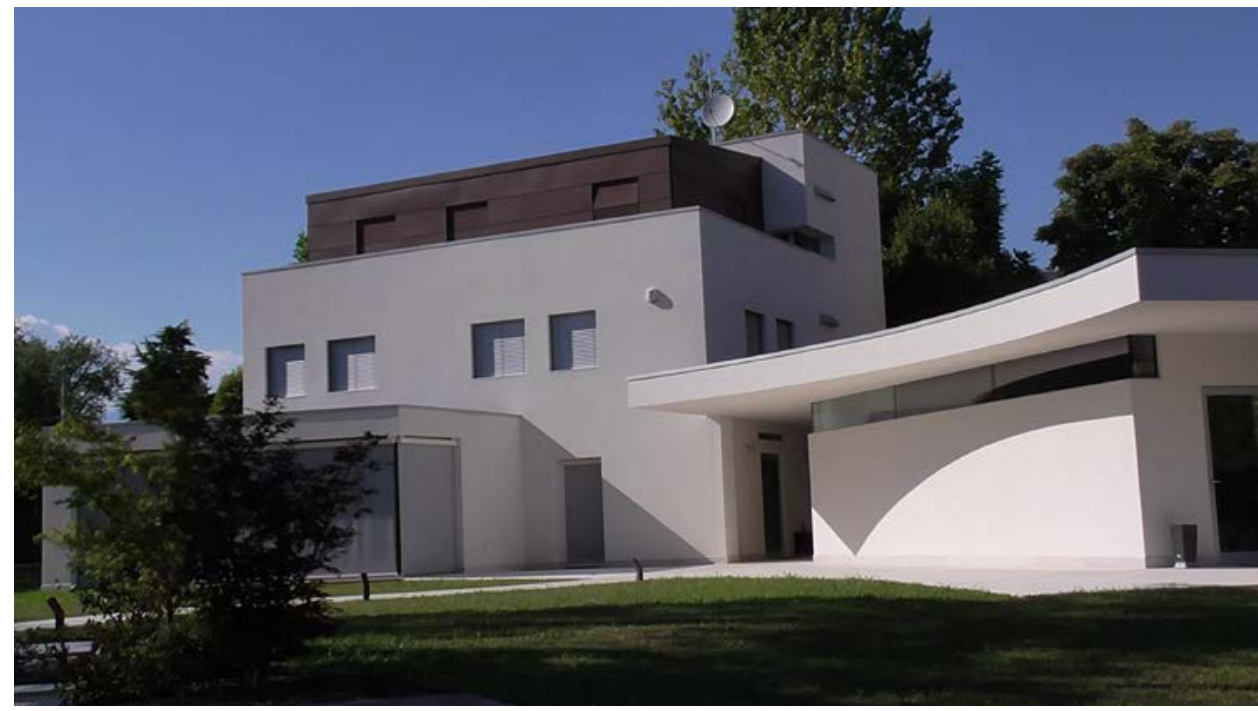

Figura 12. Villa delle Rogge (Source: materiale fornito da Fondazione Bambini e Autismo Onlus, Pordenone)

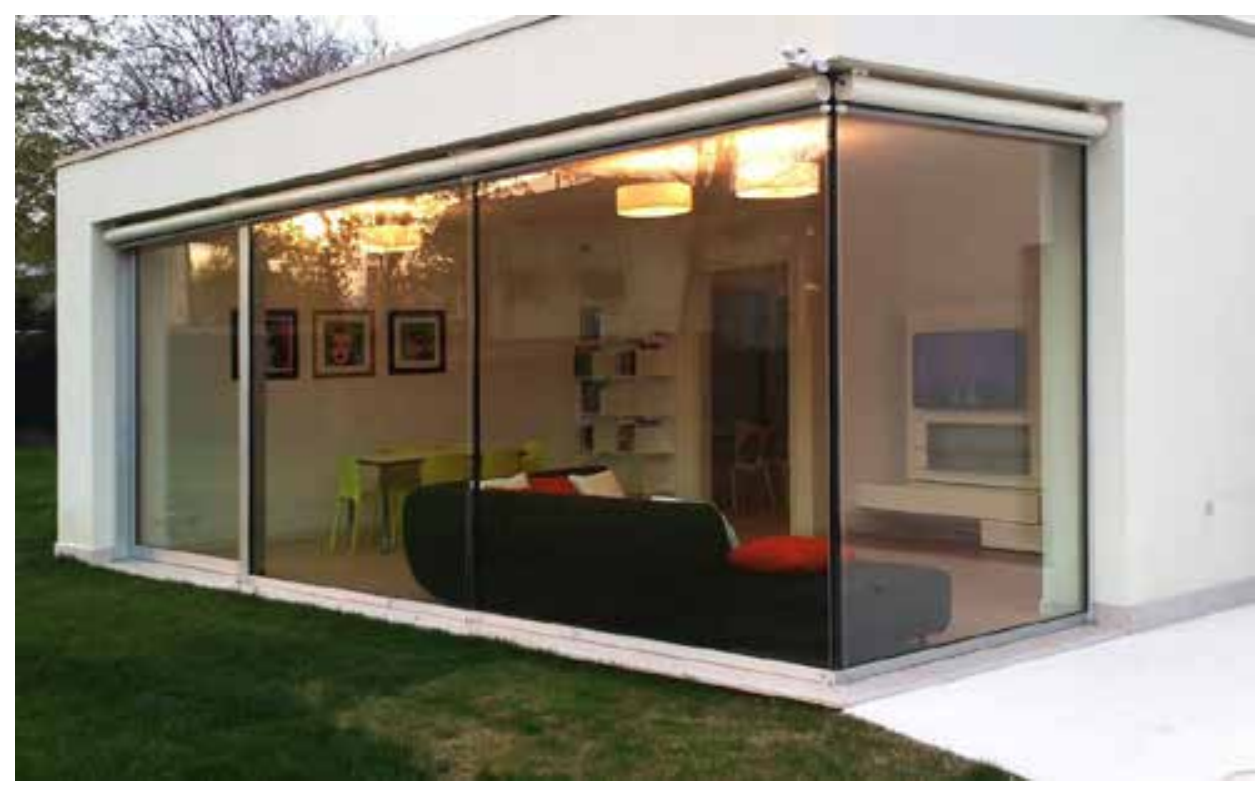

Figura 13. Villa delle Rogge: vista dello spazio soggiorno (Source: materiale fornito da Fondazione Bambini e Autismo Onlus, Pordenone) 


\section{Elena Bellini}

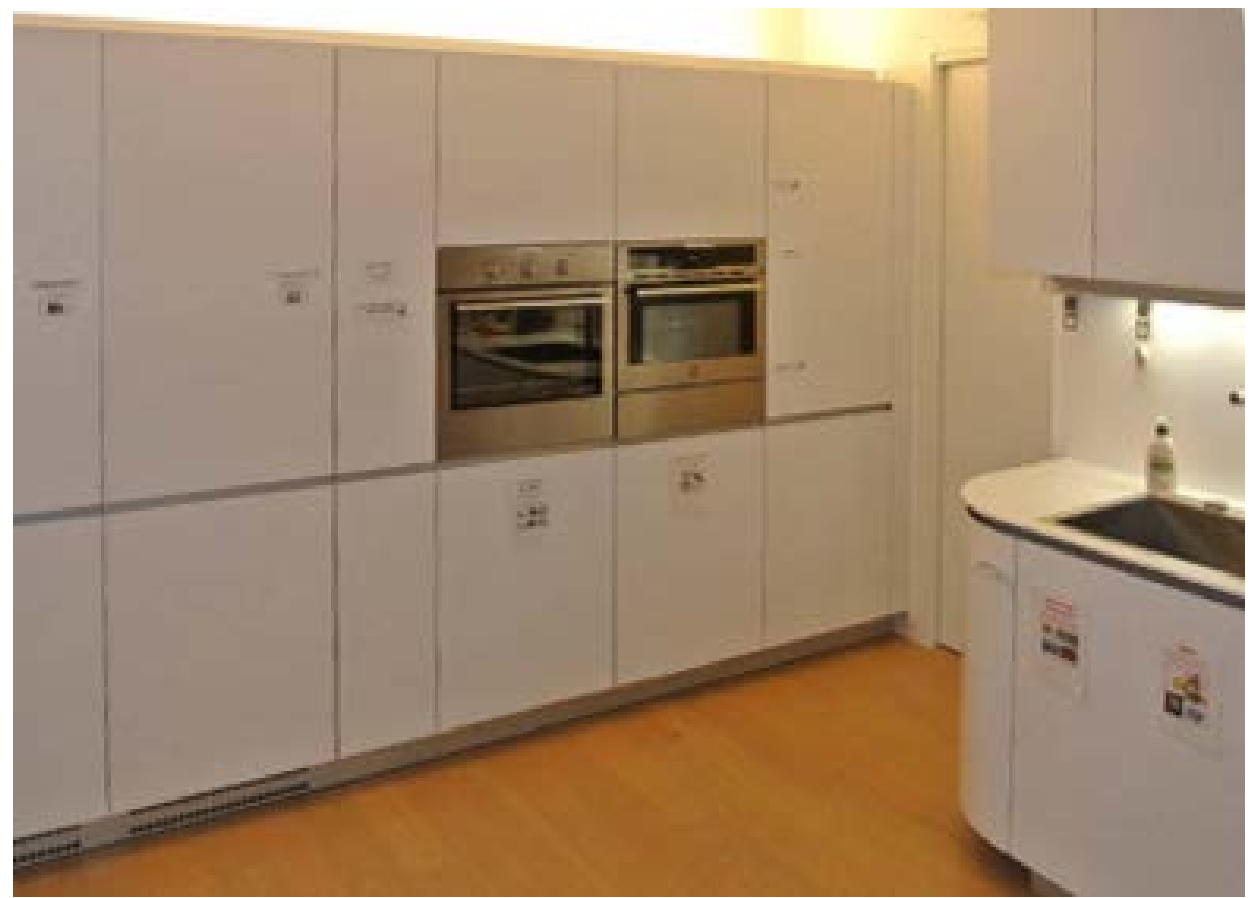

Figura 14. Villa delle Rogge: cucina (Source: materiale fornito da Fondazione Bambini e Autismo Onlus, Pordenone)

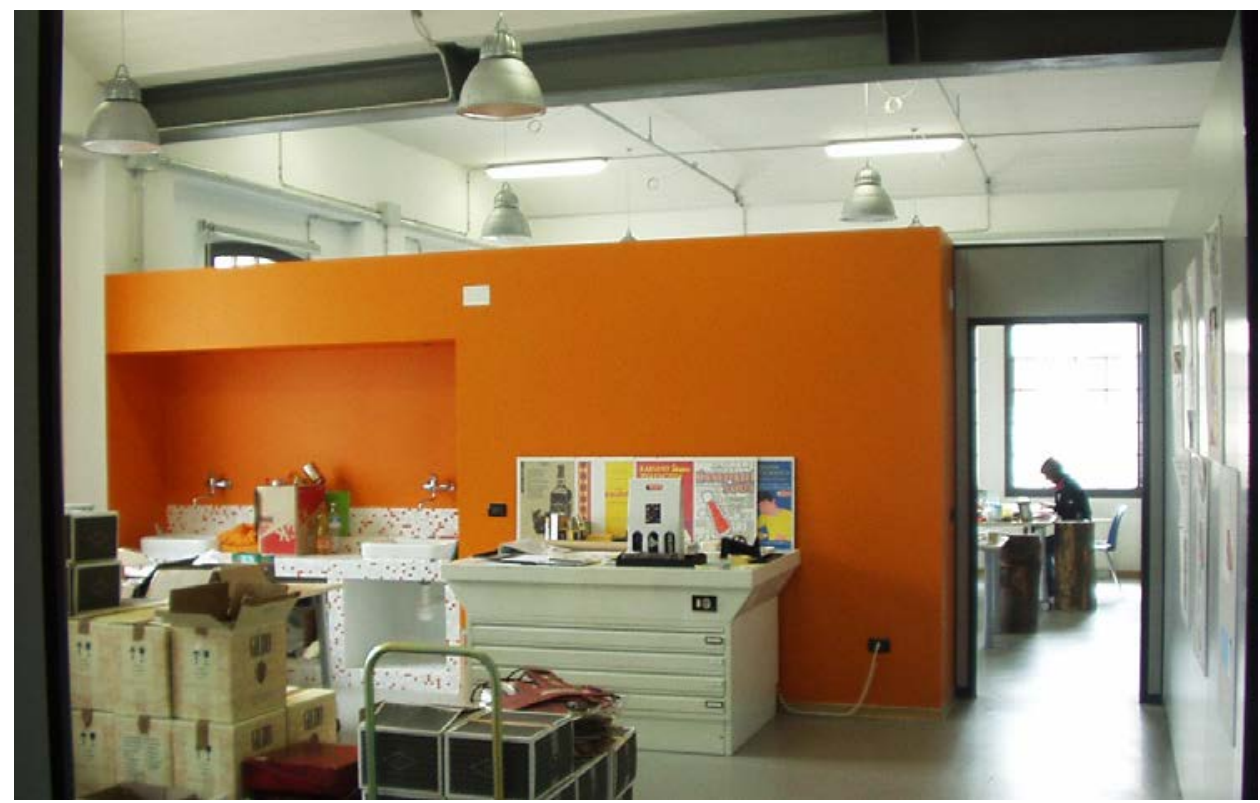

Figura 15. Officina dell'Arte (Source: materiale fornito da Fondazione Bambini e Autismo Onlus, Pordenone) 
Ambienti sensoriali "terapeutici" che rendano Abili

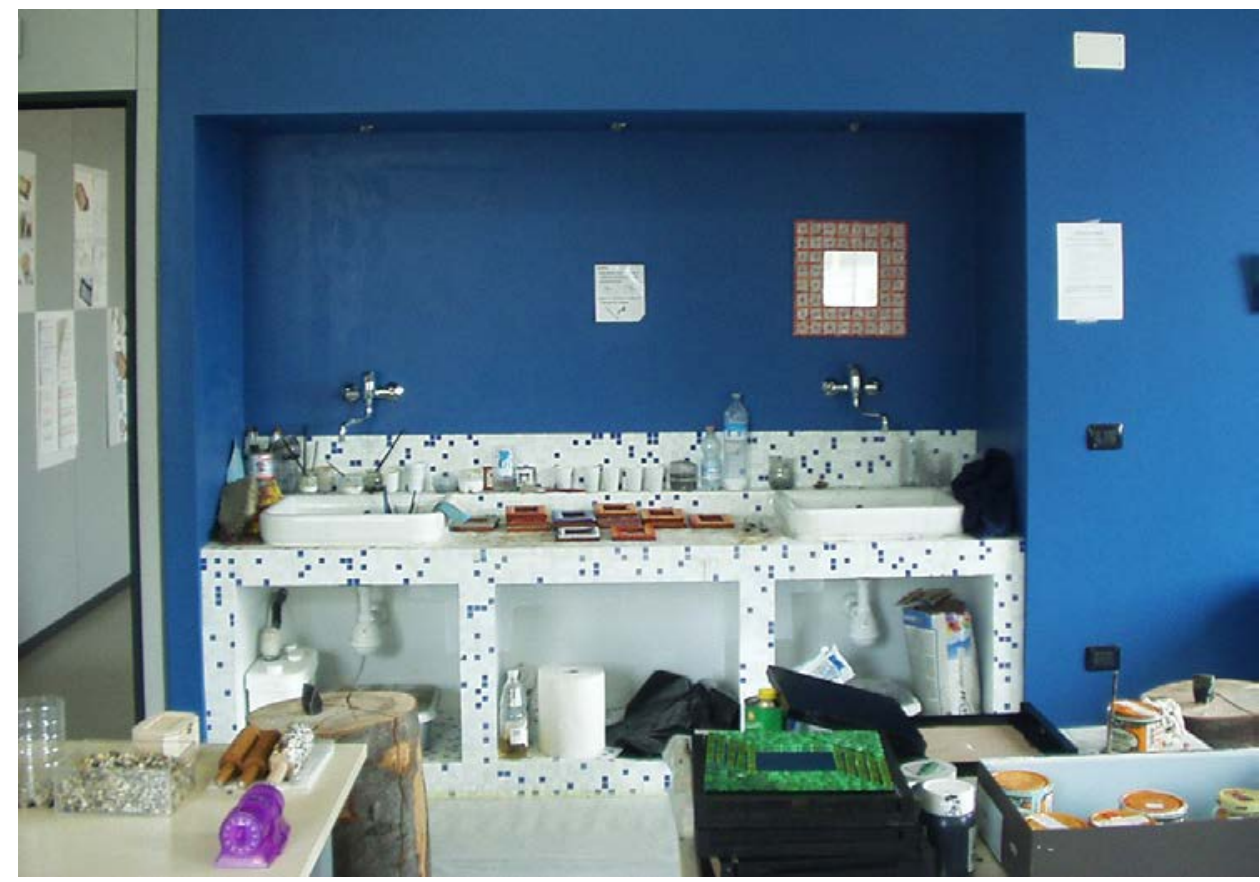

Figura 16. Officina dell'Arte (Source: materiale fornito da Fondazione Bambini e Autismo Onlus, Pordenone)

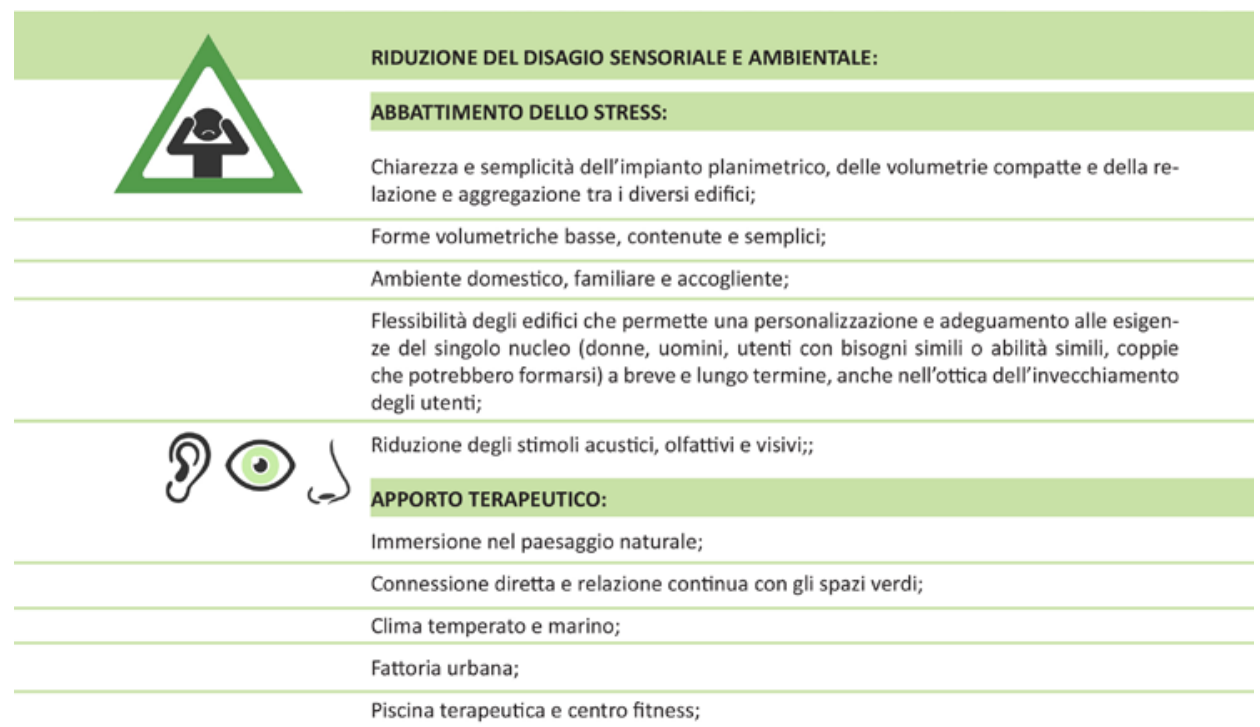

Scheda 06. Sweetwater Spectrum Community (parte 1) 


\section{Elena Bellini}

Giardino delle "Amache" per i momenti di relax all'esterno;

Biblioteca e area relax; arteterapia e altre attività di terapia occupazionale;

COMFORT VISIVO:

Distribuzione planimetrica ottimizzata rispetto all'orientamento solare;

Illuminazione naturale sempre presente: volumi inclinati del tetto, in risposta all'orientamento solare;

Progettazione e controllo della luce:

- grandi finestre in facciata con possibilità di oscuramento,

- livello di finestre alte per aiutare la diffusione indiretta della luce e lucernari a cono dove necessario,

- le aree giorno hanno grandi aperture rivolte verso nord, in modo da non avere mai una luce forte e diretta, ma ampia visibilità e relazione con l'esterno,

- dal lato sud invece aperture alte permettono di migliorare la qualità luminosa dello spazio, riflettendo la luce forte verso il soffitto e diffondendola negli ambienti ampi e chiari,

- le aree notte invece hanno aperture a est e ovest;

Interruttori e timer controllano la luce artificiale, piuttosto che sensori, per non disturbare l'utente;

Colori neutri e caldi dell'ambiente;

Materiali naturali, caldi e familiari, come l'uso del legno;

COMFORT ACUSTICO:

Infissi ad alta prestazione acustica;

Posizionamento opposto delle coppie di camere per mantenere la privacy;

Alta prestazione dell'isolamento acustico indoor;

COMFORT PROPRIOCETTIVO E PROSSEMICA:

Spazi ampi e liberi per favorire movimento e prossemica;

Diversi gradi di socialità sviluppati all'interno e all'esterno dell'edificio;

COMFORT OLFATTIVO E TERMO-IGROMETRICO:

Ventilazione naturale: orientamento secondo i venti principali;

Qualità dell'aria: sistema di ventilazione meccanica di supporto a bassa velocità per non disturbare gli utenti:

Riscaldamento a pannelli radianti a pavimento;

ASSISTIVE TECHNOLOGY:

Controllo domotico di tutto l'edificio, dispositivi ad alta efficienza (sicurezza e risparmio energetico), timer automatici per il controllo della luce;

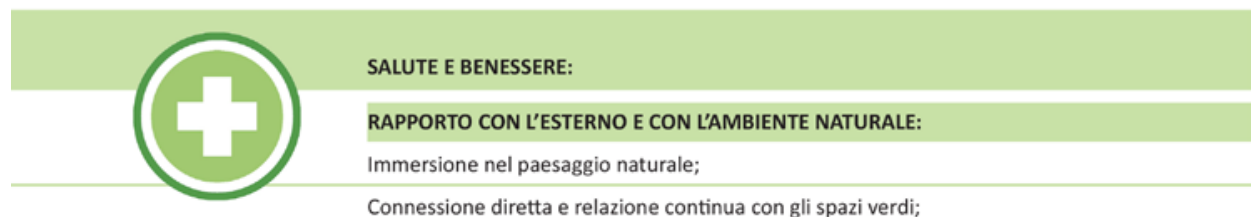

Scheda 06. Sweetwater Spectrum Community (parte 2) 
Ambienti sensoriali "terapeutici” che rendano Abili

Clima temperato e marino;

Fattoria urbana;

COMFORT E QUALITA' INDOOR:

Distribuzione planimetrica ottimizzata rispetto all'orientamento solare;

Illuminazione naturale sempre presente: volumi inclinati del tetto, in risposta all'orien-

tamento solare;

Ventilazione naturale: orientamento secondo i venti principali;

Qualità dell'aria: sistema di ventilazione meccanica di supporto a bassa velocità per non disturbare gli utenti;

Materiali naturali, caldi e familiari, come l'uso del legno;

Vernici, colle e sigillanti senza emissione di VOC:

Isolamento in cotone e derivati del legno senza uso di formaldeide;

Pavimenti non vinilici e non tossici (linoleum);

II/. Riscaldamento radiante per ridurre gli allergeni;

\section{ASSISTIVE TECHNOLOGY:}

Controllo domotico di tutto l'edificio, dispositivi ad alta efficienza (sicurezza e risparmio energetico), timer automatici per il controllo della luce;

ABBATTIMENTO DELLO STRESS E APPORTO TERAPEUTICO (vedi sopra);

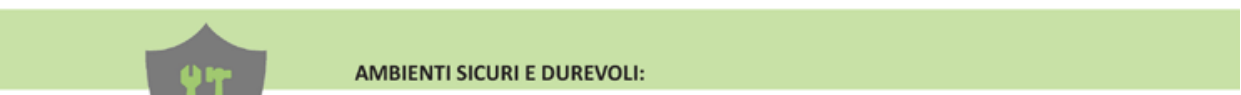

Flessibilità degli edifici che permette una personalizzazione e adeguamento alle esigenze del singolo nucleo (donne, uomini, utenti con bisogni simili o abilità simili, coppie che potrebbero formarsi) a breve e lungo termine, anche nell'ottica dell'invecchiamento degli utenti;

Finiture generali scelte per facilità di manutenzione, ridotta gestione e durabilità:

- Muri a secco resistenti agli impatti;

- Porte di tipologia commerciale;

- Rivestimento esterno in cemento;

\section{ASSISTIVE TECHNOLOGY:}

Controllo domotico di tutto l'edificio, dispositivi ad alta efficienza (sicurezza e risparmio energetico), timer automatici per il controllo della luce;

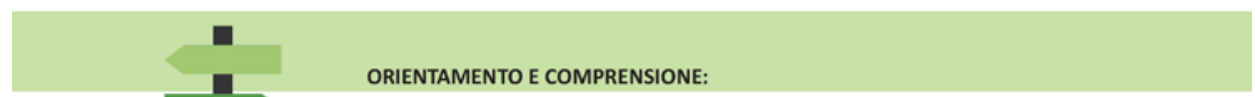

Chiarezza e semplicità dell'impianto planimetrico: corte privata di ingresso; spazio centrale comune come fulcro dell'alloggio; spazi residenziali con due camere private e bagno specchiati in modo simmetrico rispetto al centro; collegamenti distributivi ottimizzati e ridotti al minimo; piano superiore a pianta libera dedicato alle attività e servizi sulla fascia posteriore dell'edificio;

Forme volumetriche basse, contenute e semplici;

Scheda 06. Sweetwater Spectrum Community (parte 3) 


\section{Elena Bellini}

Spazi serventi ridotti al minimo, no presenza di corridoi che possono causare disagio nelle persone con autismo, dovuto anche alla mancanza di funzionalità;

Spazi ampi e liberi per favorire movimento e prossemica;

Diversi gradi di socialità sviluppati all'interno e all'esterno dell'edificio;

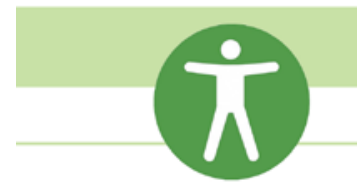

AUTONOMIA E INDIPENDENZA. ABILITA':

Vicinanza con la comunità locale(raggiungibile anche a piedi o in bici);

Partecipazione della comunità (condivisione del progetto e volontariato) e dei residenti alla vita sociale e lavorativa (partecipazione al mercato di zona con i prodotti coltivati nella fattoria e lavori part-time in città);

Fattoria urbana;

Flessibilità degli edifici: possibilità di variare l'impianto e vivere in familgia, in cohousing o adattamento a residenza per anziani, per rispondere alle diverse esigenze ed abilità e adattarsi con l'evolversi del tempo e della situazione;

\section{ASSISTIVE TECHNOLOGY:}

Controllo domotico di tutto l'edificio, dispositivi ad alta efficienza (sicurezza e risparmio energetico), timer automatici per il controllo della luce;

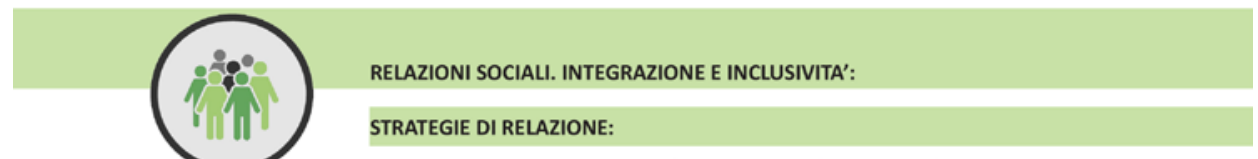

Spazi di relazione progettati sia all'interno che all'esterno delle abitazioni:

- la cucina invoglia a cucinare;

- vi sono diverse possibilità di sedute;

- Diversi spazi e gradi di socialità facilitano le relazioni;

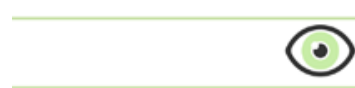

Visibilità continua e connessione con l'ambiente esterno inclusi i patio, le terrazze comuni e i giardini;

Flessibilità degli edifici: possibilità di variare l'impianto e vivere in famiglia, in cohousing o adattamento a residenza per anziani, per rispondere alle diverse esigenze ed abilità e adattarsi con l'evolversi del tempo e della situazione;

\section{STRATEGIE DI INTEGRAZIONE:}

Vicinanza con la comunità locale(raggiungibile anche a piedi o in bici);

Partecipazione della comunità (condivisione del progetto e volontariato) e dei residenti alla vita sociale e lavorativa (partecipazione al mercato di zona con i prodotti coltivati nella fattoria e lavori part-time in città);

Fattoria urbana;

APPORTO TERAPEUTICO (vedi sopra).

Scheda 06. Sweetwater Spectrum Community (parte 4) 
Ambienti sensoriali "terapeutici" che rendano Abili

RIDUZIONE DEL DISAGIO SENSORIALE E AMBIENTALE:

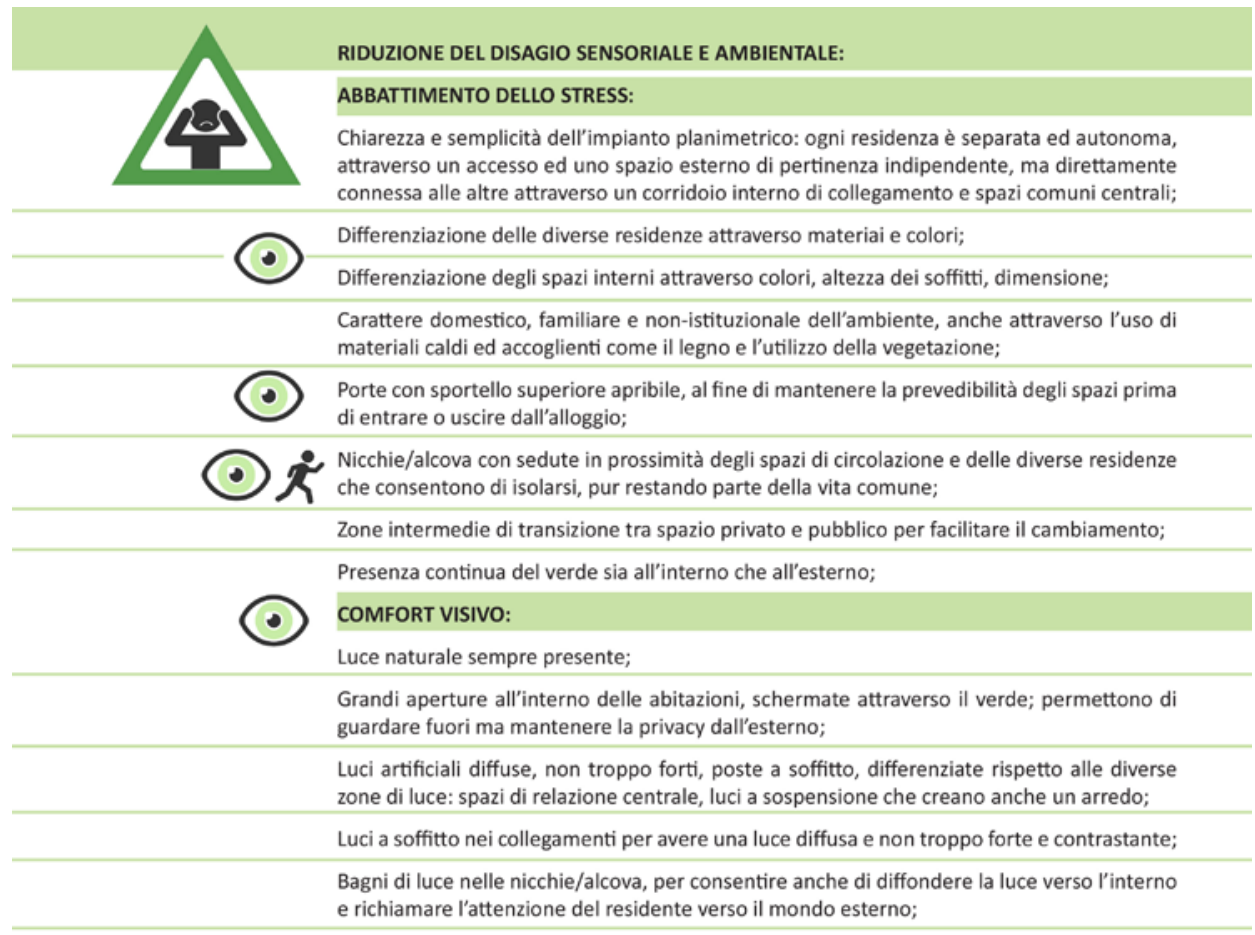

Colori tenui scelti tra i diversi toni del verde-grigio: spazi di circolazione neutri, di colore grigio; evidenziate le diverse residenze con diversi toni del verde;

Scheda 07. Seniors house (parte 1) 


\section{Elena Bellini}

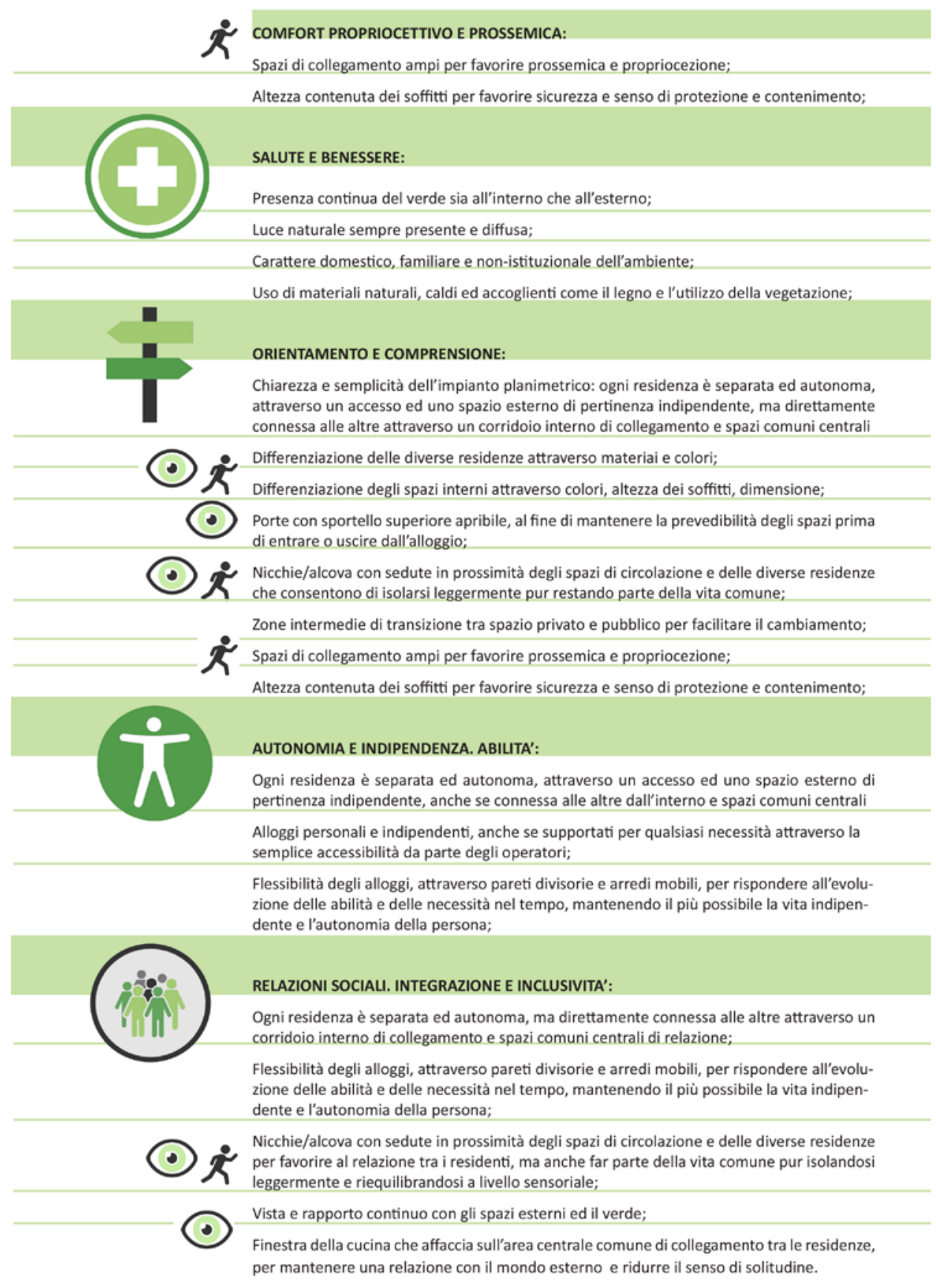

Scheda 07. Seniors house (parte 2) 
Ambienti sensoriali "terapeutici" che rendano Abili

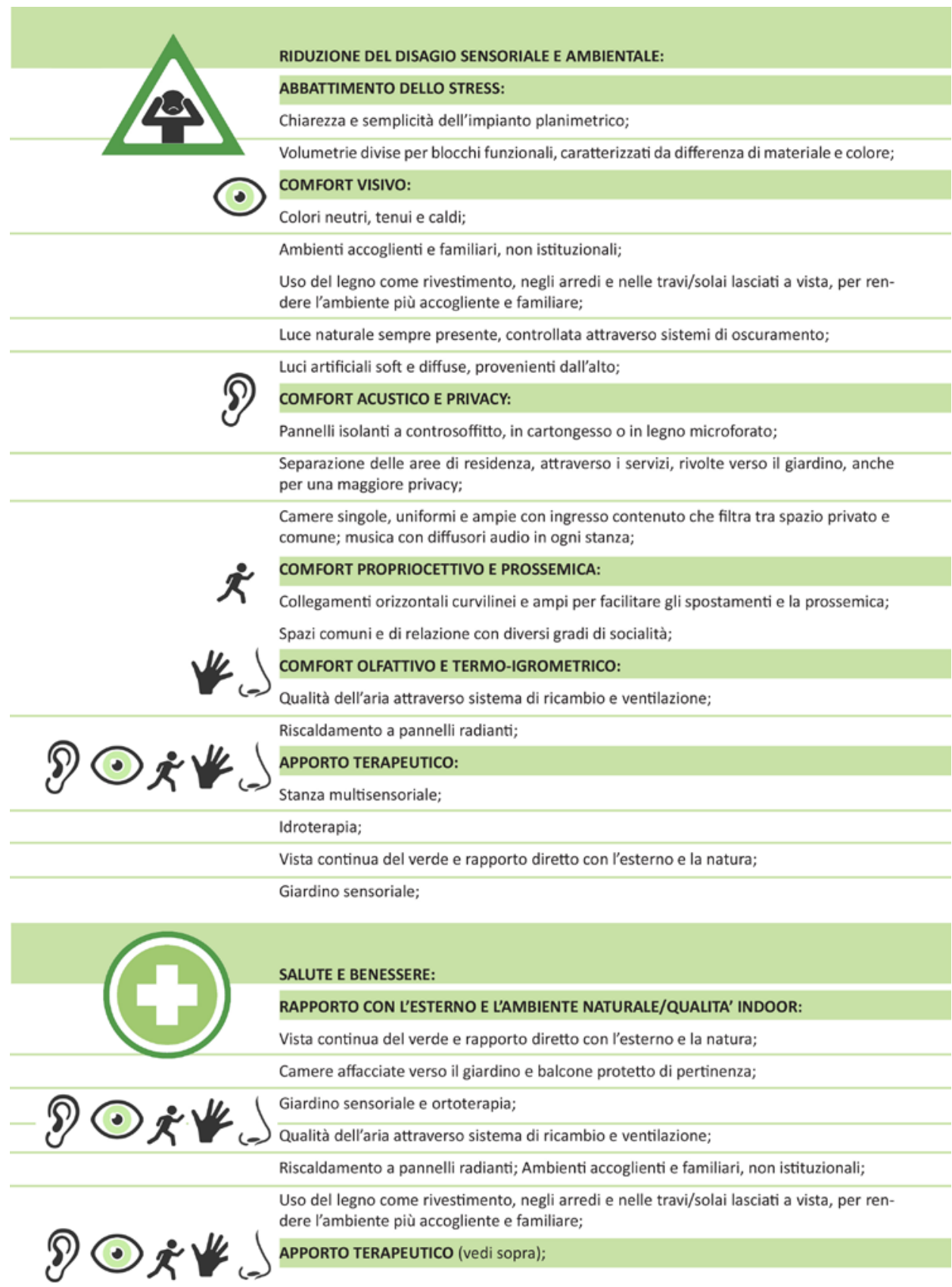

Scheda 08. Casa Sebastiano (parte 1) 


\section{Elena Bellini}

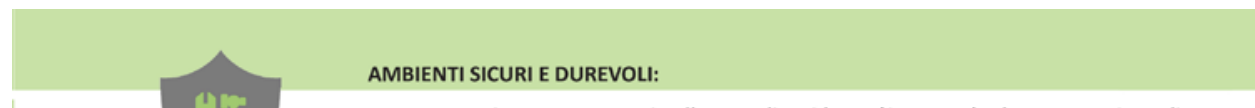

Due operatori sempre presenti nelle aree di residenza ( 9 camere), oltre a postazione di controllo centrale;

Telecamere presenti in tutta la struttura;

Pavimento rivestito in linoleum, per necessità di normative sanitarie, facilità di manutenzione e gestione, pulibilità, durabilità;

Rivestimento delle facciate con brise-soleil in legno per rendere gli affacci e i balcon della struttura protetti;

Riscaldamento a pannelli radianti;

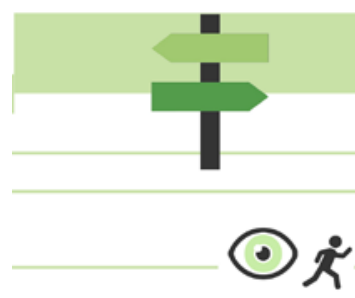

ORIENTAMENTO E COMPRENSIONE:

Chiarezza e semplicità dell'impianto planimetrico;

Volumetrie divise per blocchi funzionali, caratterizzati da differenza di materiale e colore;

Separazione delle aree di residenza, attraverso i servizi, rivolte verso il giardino, anche per una maggiore privacy;

Collegamenti orizzontali curvilinei e ampi per facilitare gli spostamenti e la prossemica;

(Q) Camere singole, uniformi e ampie con ingresso contenuto che filtra tra spazio privato e comune; musica con diffusori audio in ogni stanza;

AUTONOMIA E INDIPENDENZA. ABILITA':
Residenze temporanee e Programma "Autonomia";
Laboratori ludici, di musica e teatro, e occupazionali; Laboratori per il lavoro;
Terapie riabilitative aperte anche all'esterno;
Azienda agricola biologica;

Azienda agricola biologica;

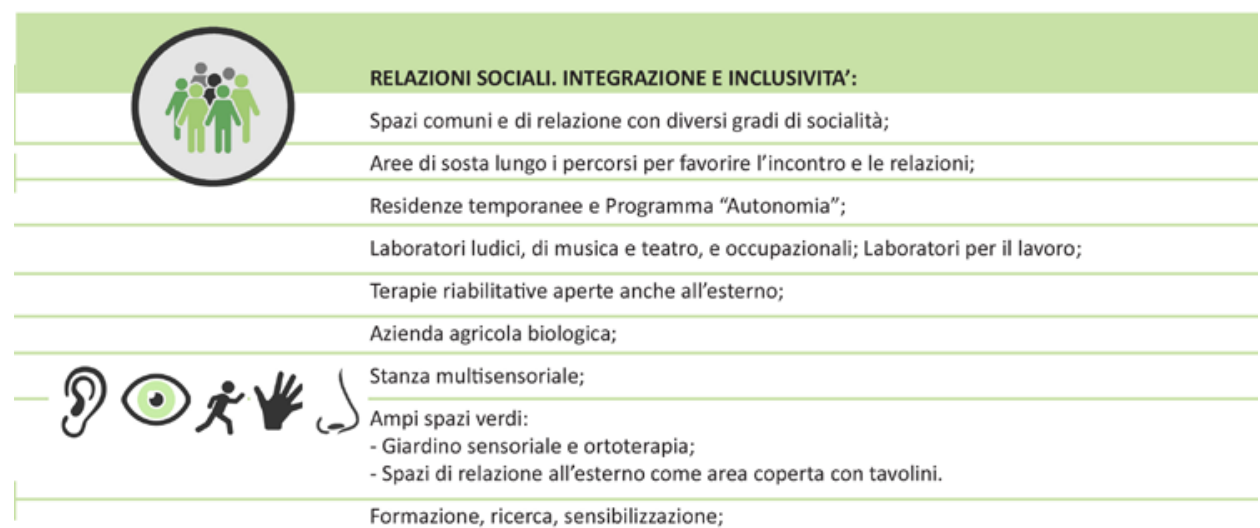

Scheda 08. Casa Sebastiano (parte 2) 
Ambienti sensoriali "terapeutici" che rendano Abili

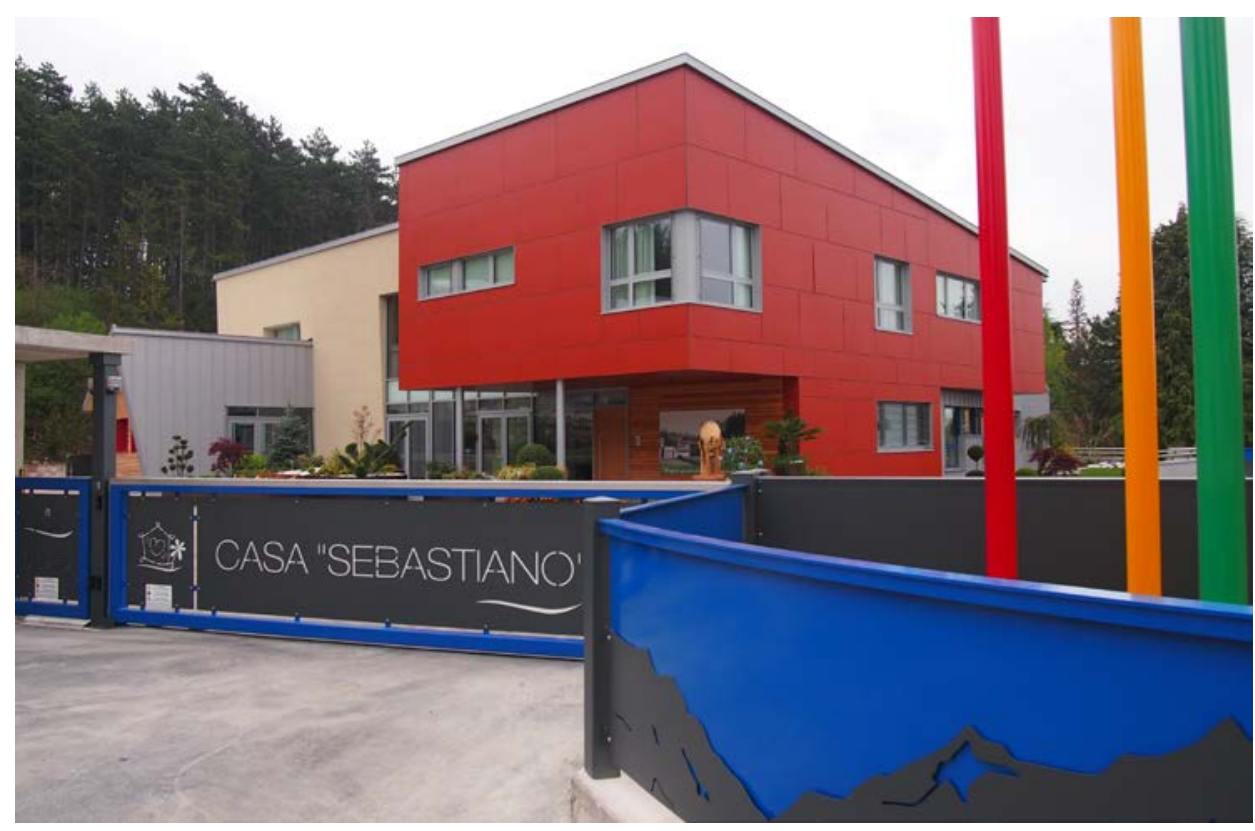

Figura 17. Casa Sebastiano (foto dell'autore)

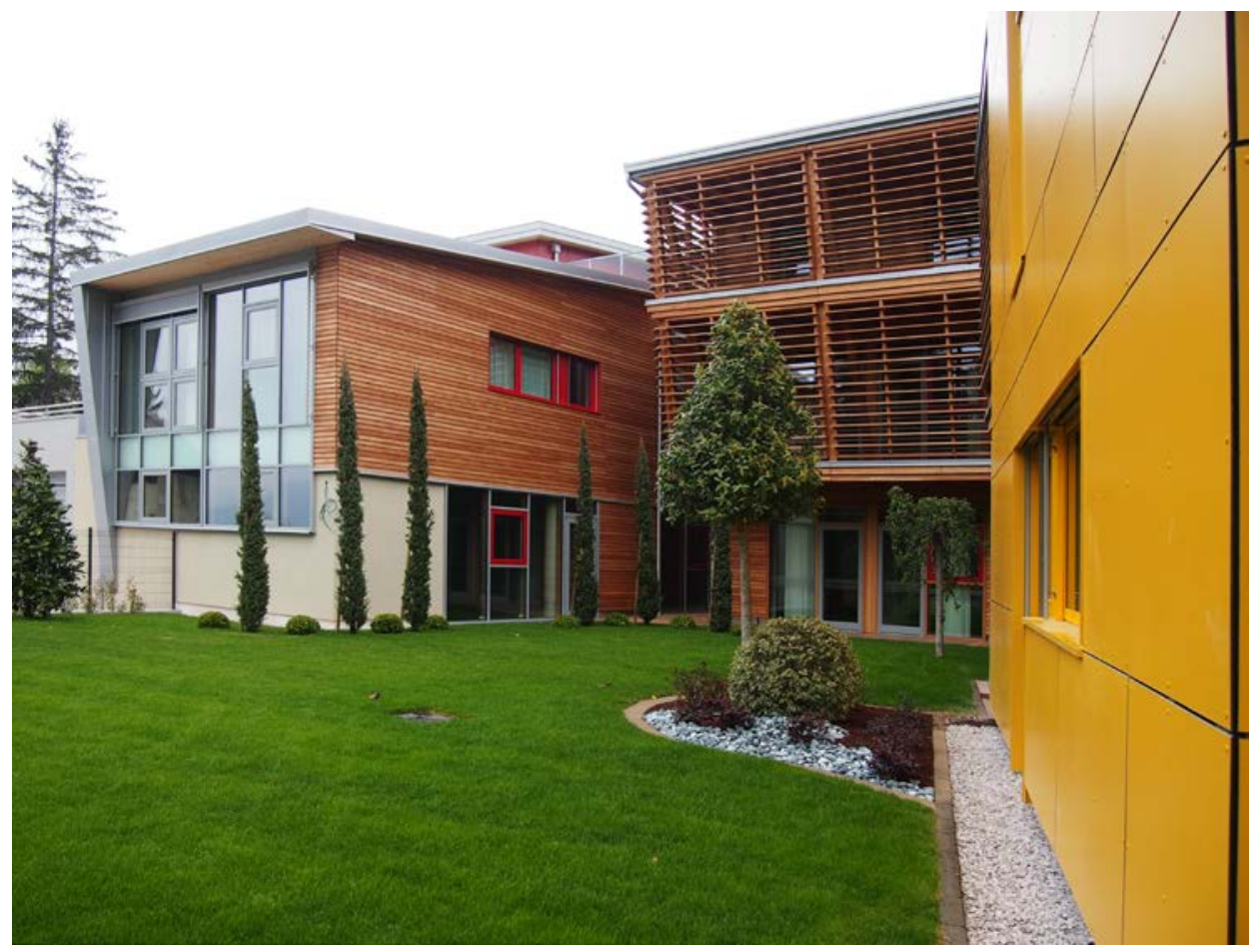

Figura 18. Casa Sebastiano (foto dell'autore) 


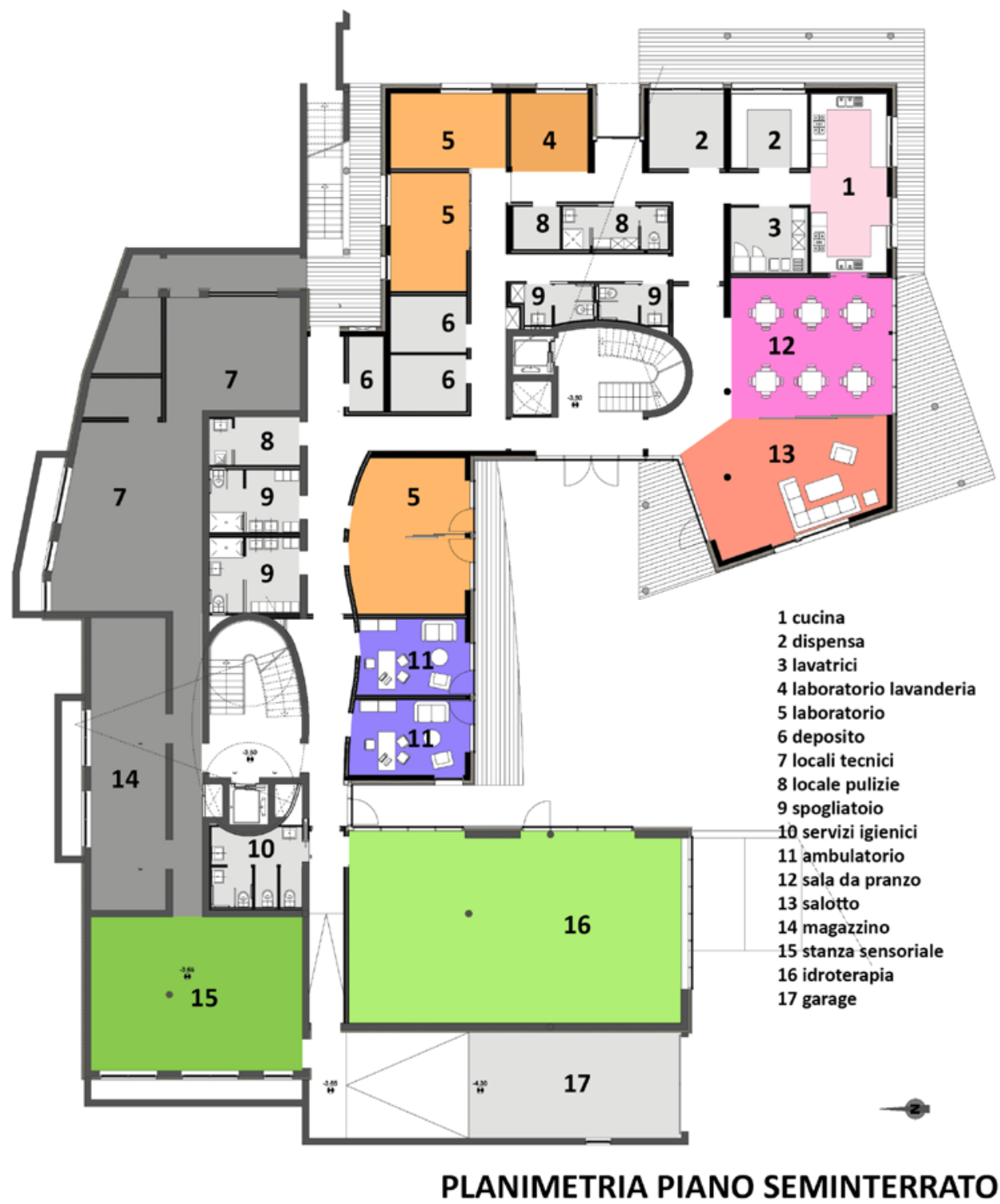

Figura 19. Casa Sebastiano: planimetria piano seminterrato (Source: materiale fornito dall'arch. Giovanni Berti, progettista, e ridisegnato dall'autore) 
Ambienti sensoriali "terapeutici” che rendano Abili

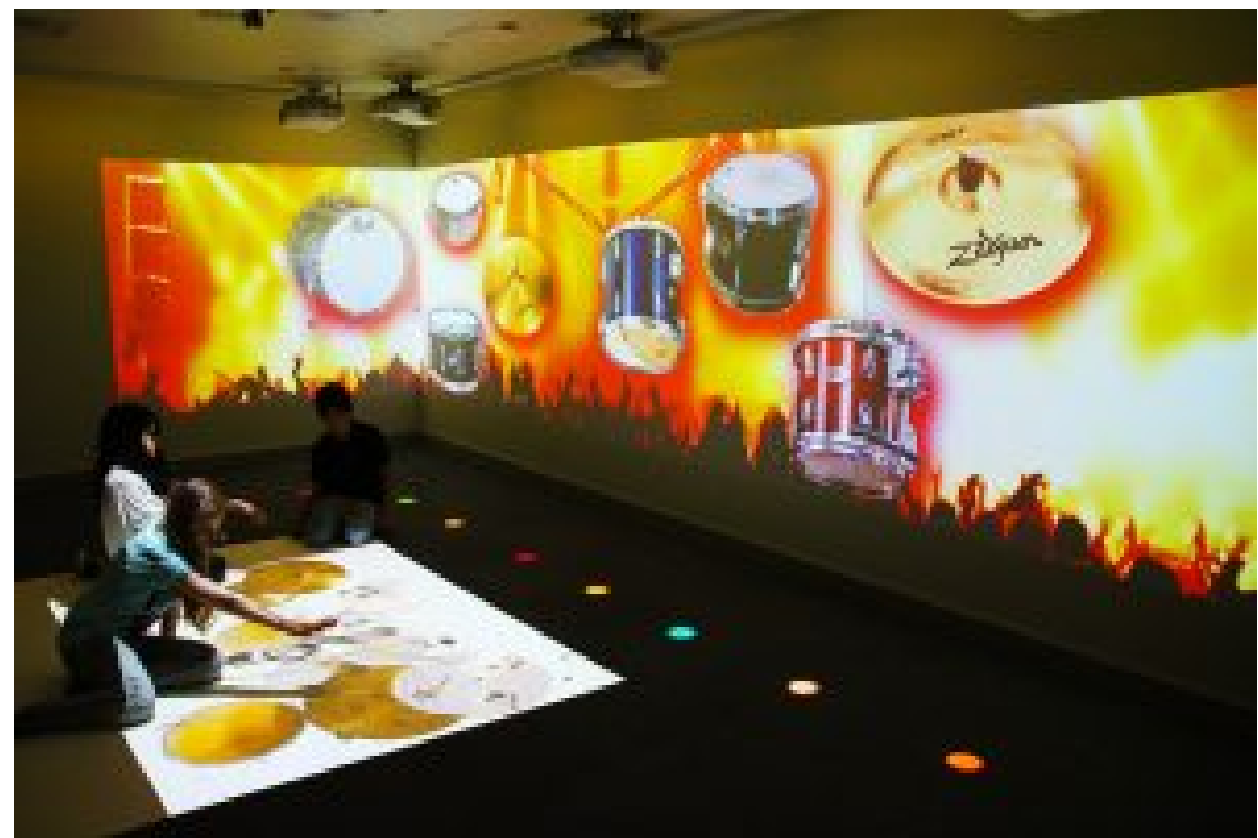

Figura 20. Casa Sebastiano: stanza multisensoriale (Source: Fondazione Trentina per l'Autismo)

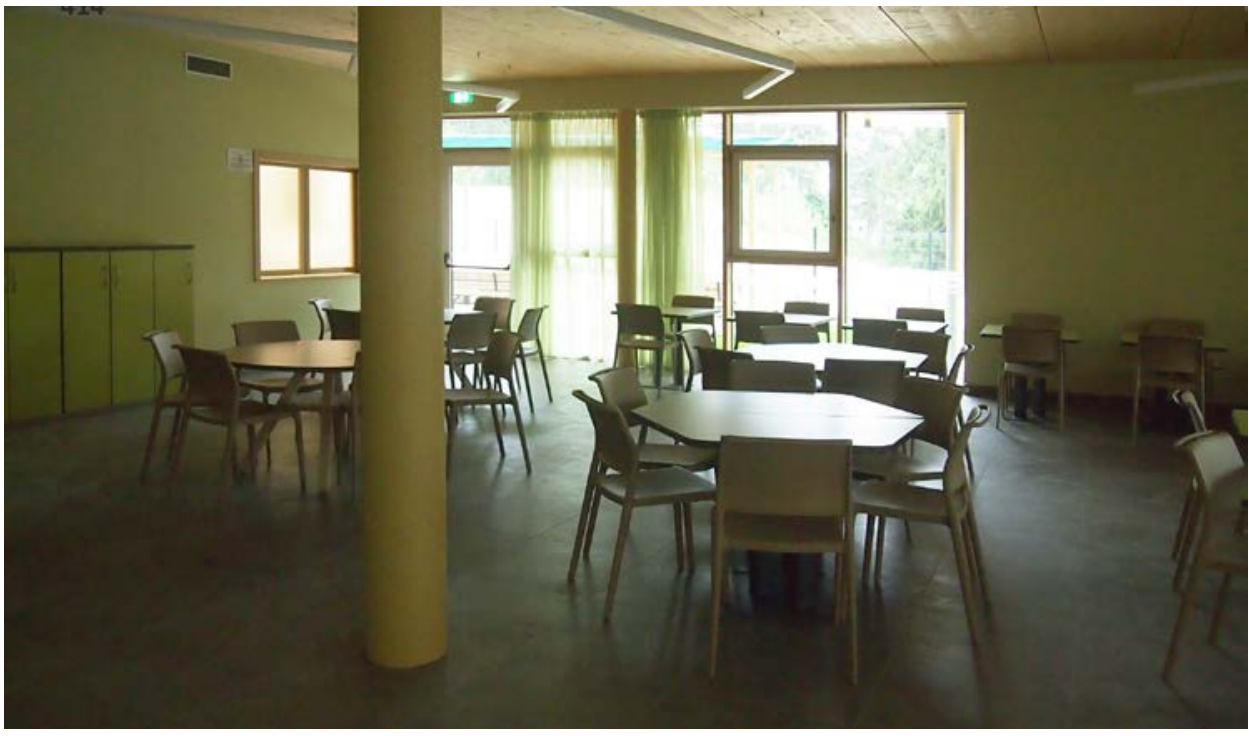

Figura 21. Casa Sebastiano: sala da pranzo comune (foto dell'autore) 


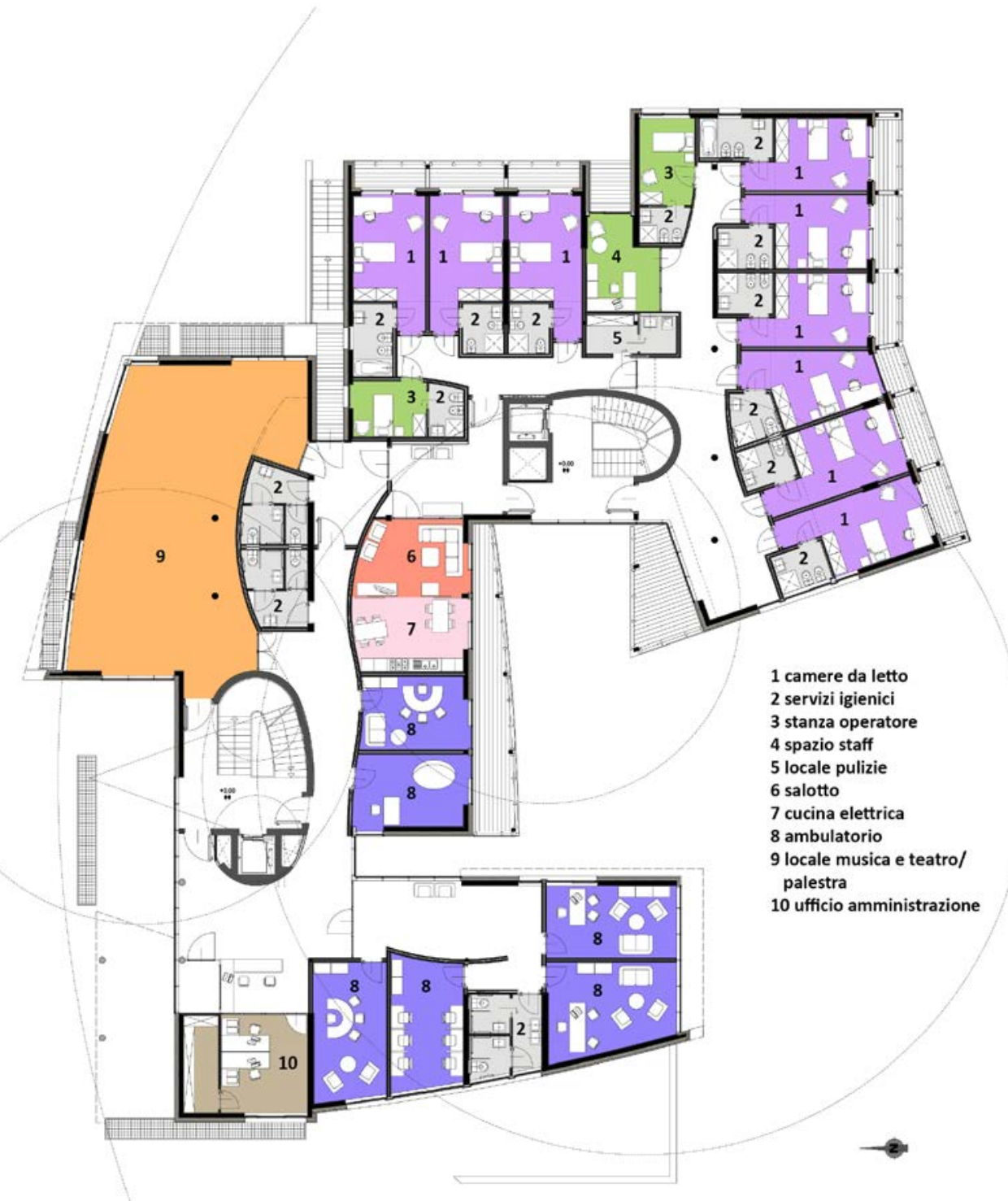

\section{PLANIMETRIA PIANO TERRA}

Figura 22. Casa Sebastiano: planimetria piano terra (Source: materiale fornito dall'arch. Giovanni Berti, progettista, e ridisegnato dall'autore) 
Ambienti sensoriali "terapeutici" che rendano Abili
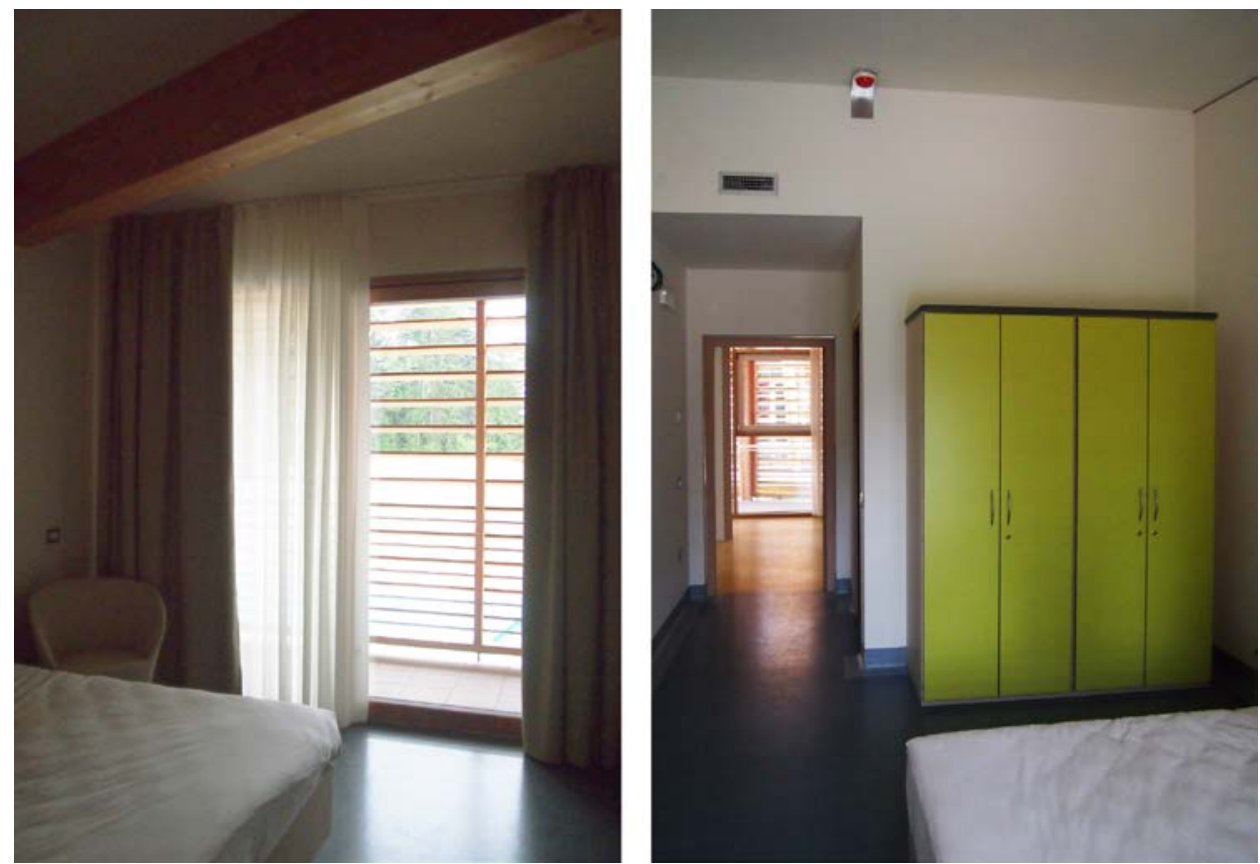

Figura 23 e 24. Casa Sebastiano: camera tipo (foto dall'autore)

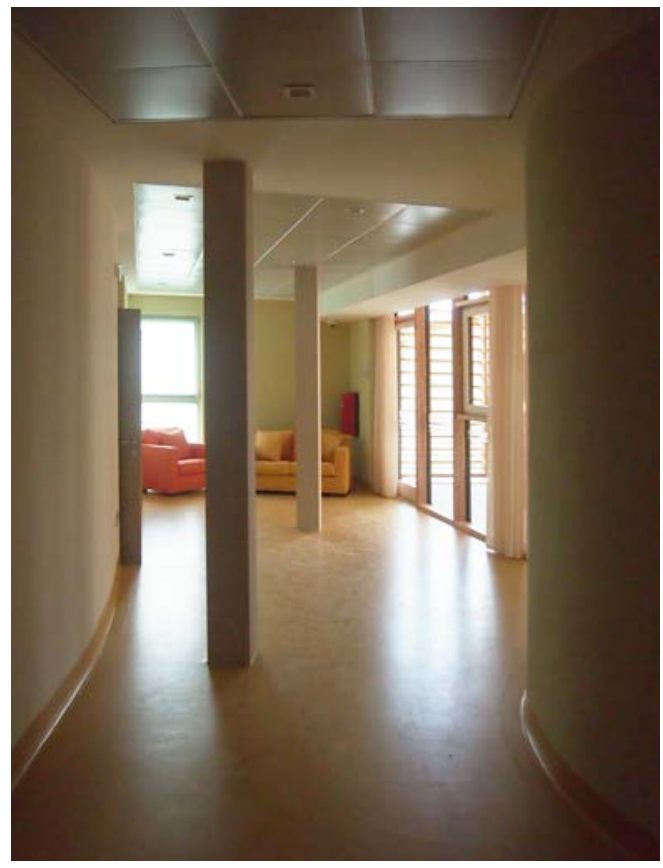

Figura 25. Casa Sebastiano: spazio di distribuzione al piano delle camere (foto dall'autore) 


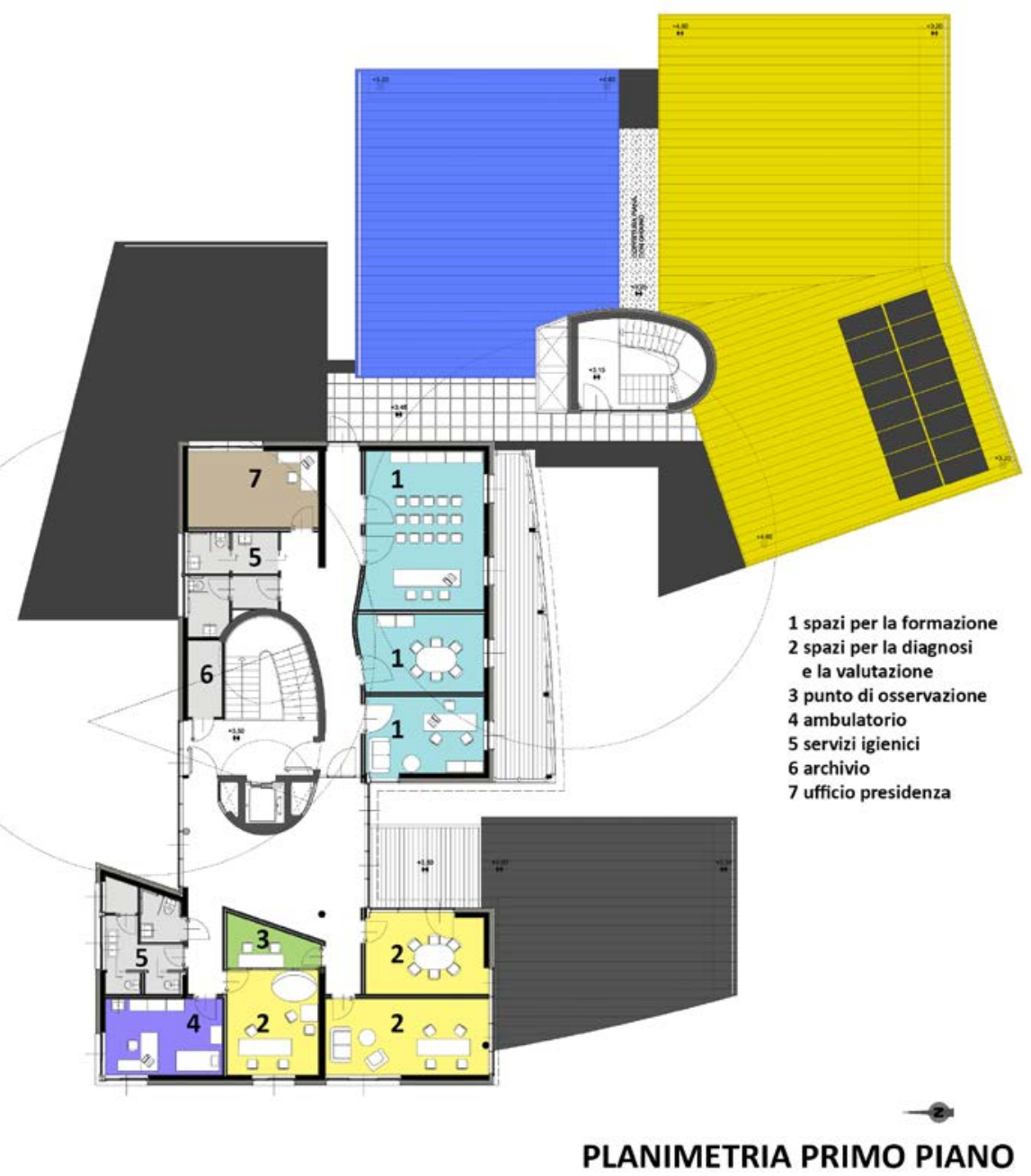

Figura 26. Casa Sebastiano: planimetria piano primo (Source: materiale fornito dall'arch. Giovanni Berti, progettista, e ridisegnato dall'autore) 
Ambienti sensoriali "terapeutici" che rendano Abili

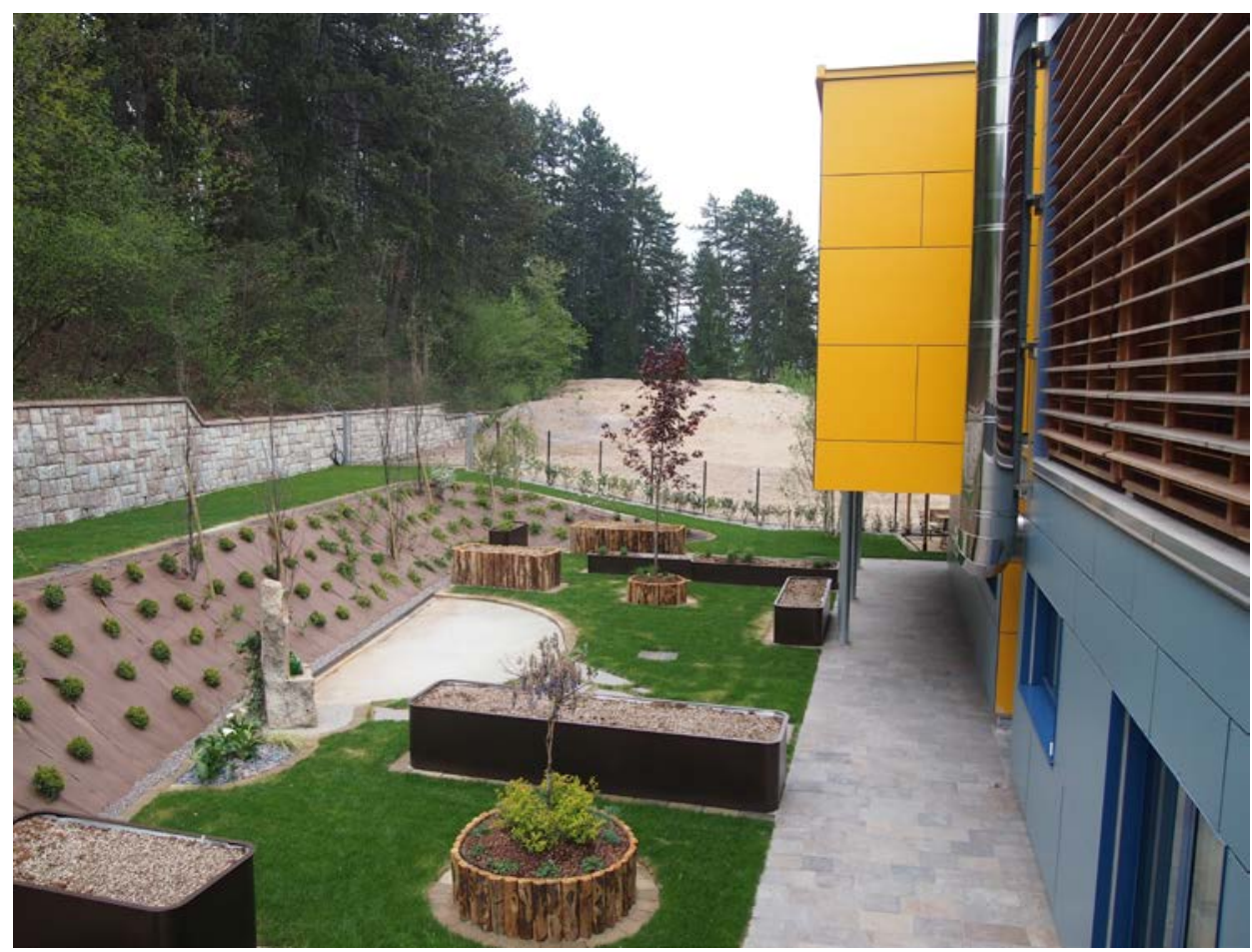

Figura 27. Casa Sebastiano: giardino sensoriale (foto dall'autore) 


\section{Elena Bellini}

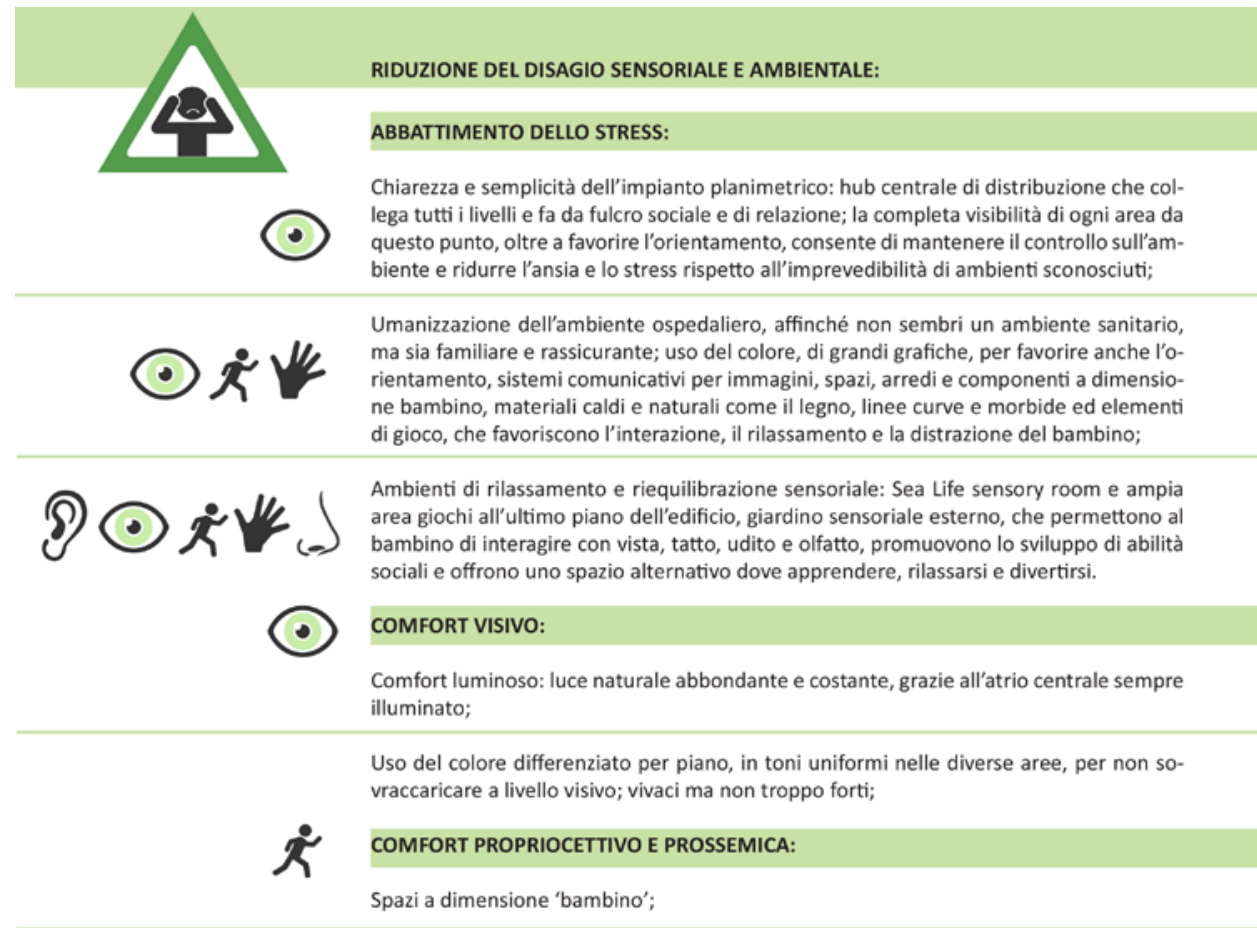

Ampi spazi di collegamento ad andamento curvilineo, per facilitare gli spostamenti e ridurre lo stress nelle aree comuni dove si possono avere situazioni di sovraffollamento.

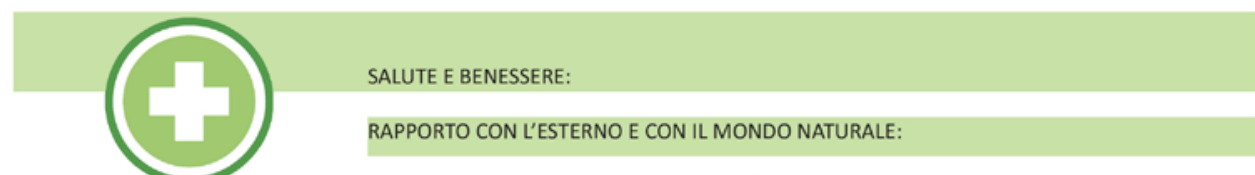

Luce naturale sempre presente grazie all'atrio centrale ad illuminazione abbondante e costante; percezione dei ritmi della giornata e del tempo che passa;

Vista del panorama marino da tutti gli ambienti dell'ospedale, comprese le stanze di de-
genza, caratterizzate da aperture a misura e altezza di bambino, per favorire distrazione e rilassamento;

$$
\text { ABBATTIMENTO DELLO STRESS E APPORTO TERAPEUTICO: }
$$

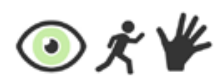

Umanizzazione dell'ambiente ospedaliero, affinché non sembri un ambiente sanitario, ma sia familiare e rassicurante; uso del colore, di grandi grafiche, per favorire anche l'orientamento, sistemi comunicativi per immagini, spazi, arredi e componenti a dimensione bambino, materiali caldi e naturali come il legno, linee curve e morbide ed elementi di gioco, che favoriscono l'interazione, il rilassamento e la distrazione del bambino;

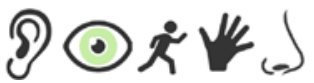

Ambienti di rilassamento e riequilibrazione sensoriale: Sea Life sensory room e ampia area giochi all'ultimo piano dell'edificio, giardino sensoriale esterno;

Scheda 09. Royal Alexandra Children's Hospital (parte 1) 
Ambienti sensoriali "terapeutici” che rendano Abili

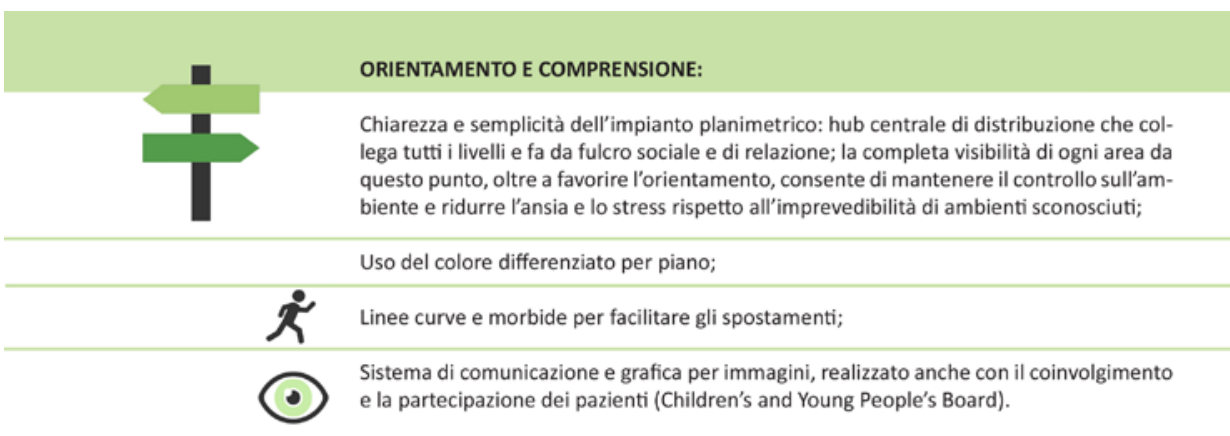

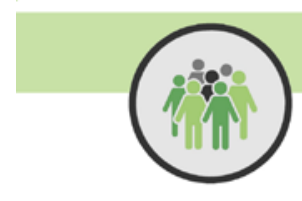

RELAZIONI SOCIALI. INTEGRAZIONE E INCLUSIVITA':

Ospedale family\&chilndren centered a partire dalla conformazione stessa dell'ospedale a forma di arca, l'uso dei colori e della grafica per immagini, la realizzazione di aperture sul panorama marino a misura ed altezza di bambino, arredi e complementi pensati e disegnati appositamente per $\mathrm{i}$ bambini, materiali caldi e naturali come il legno, linee curve e morbide ed elementi di gioco, che favoriscono l'interazione, il rilassamento e la distrazione;

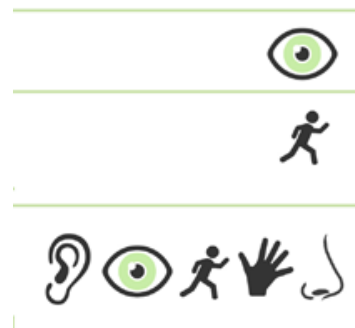

Riferimenti visivi e interazione continua: affaccio diretto di tutti i piani sull'ambiente fulcro centrale, per aumentare la visibilità e l'interazione tra i diversi livelli;

Diversi gradi di socialità degli spazi gioco e di relazione in tutto l'ospedale: piano terra dedicato alla socializzazione e all'interazione con gli altri, ospitando anche la caffetteria, ultimo piano dedicato al gioco e alla distrazione ed il rilassamento del bambino;

Ambienti di rilassamento e riequilibrazione sensoriale: Sea Life sensory room e giardino sensoriale esterno, che permettono al bambino di interagire con vista, tatto, udito e olfatto, promuovono lo sviluppo di abilità sociali e offrono uno spazio alternativo dove apprendere, rilassarsi e divertirsi;

Sistema di comunicazione e grafica per immagini, realizzato anche con il coinvolgimento e la partecipazione dei pazienti (Children's and Young People's Board), promuovendo l'inclusività, permettendo a tutti i bambini di comprendere l'ambiente allo stesso modo, attraverso strumenti comunicativi alternativi.

Scheda 09. Royal Alexandra Children's Hospital (parte 2) 


\section{Elena Bellini}

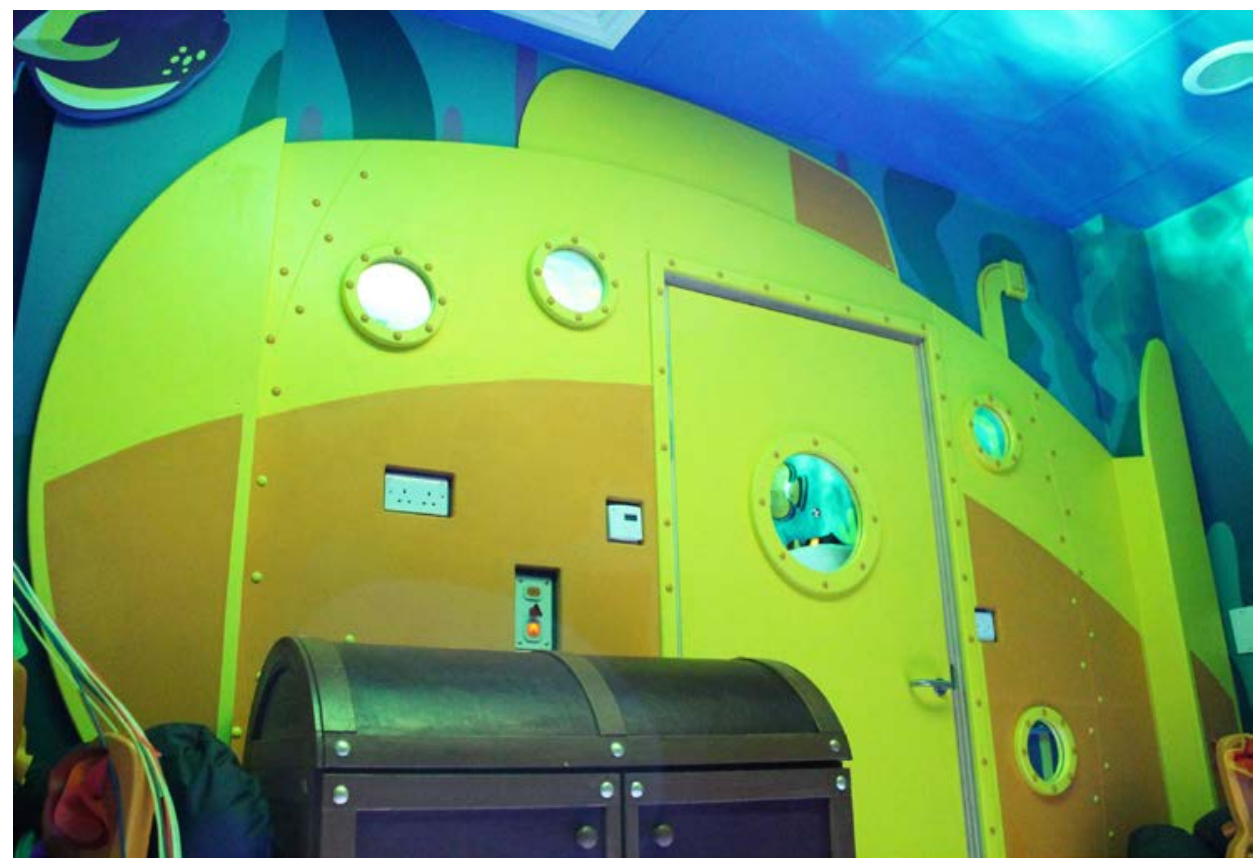

Figura 28. Royal Alexandra Children's Hospital: stanza multisensoriale (Source: Rockinghorse Children's Charity)

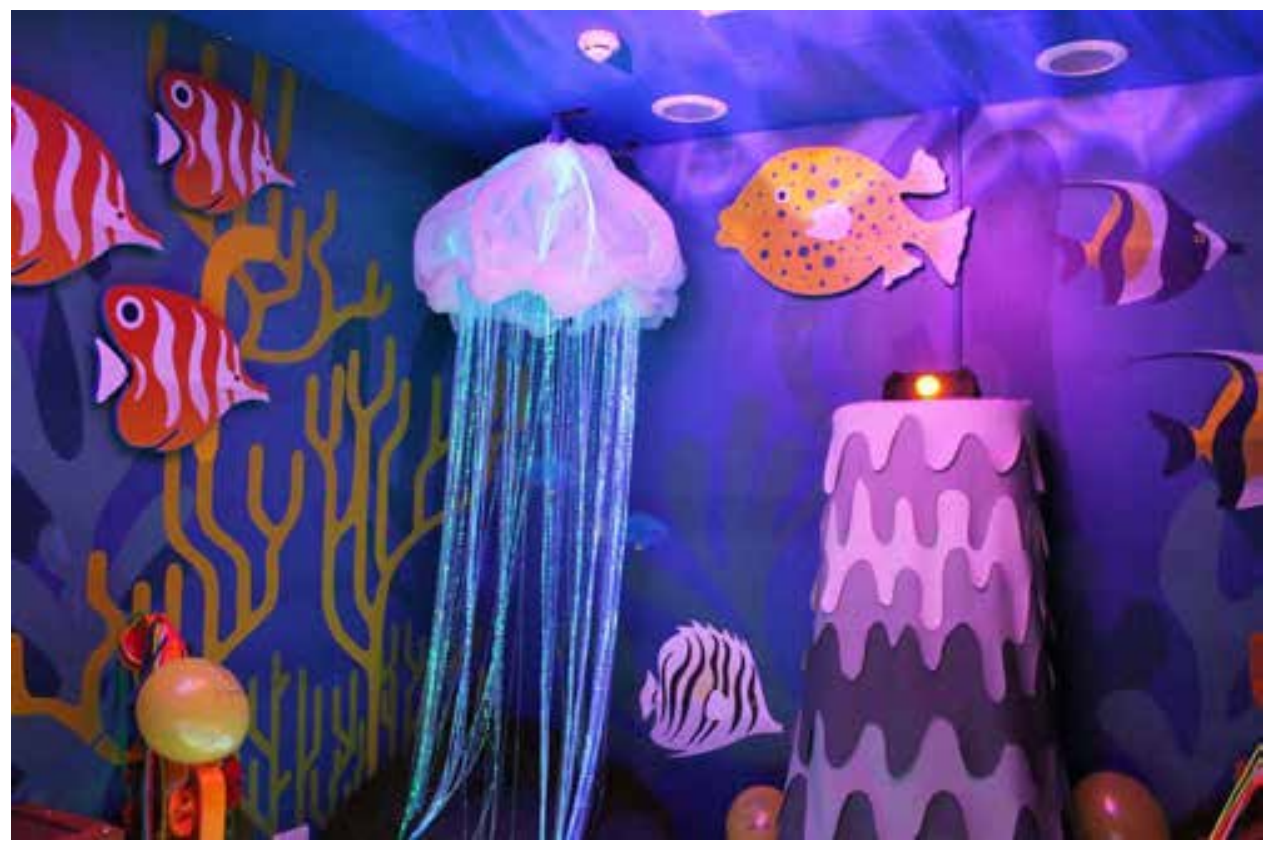

Figura 29. Royal Alexandra Children's Hospital: stanza multisensoriale (Source: Rockinghorse Children's Charity) 


\section{Ambienti sensoriali "terapeutici" che rendano Abili}

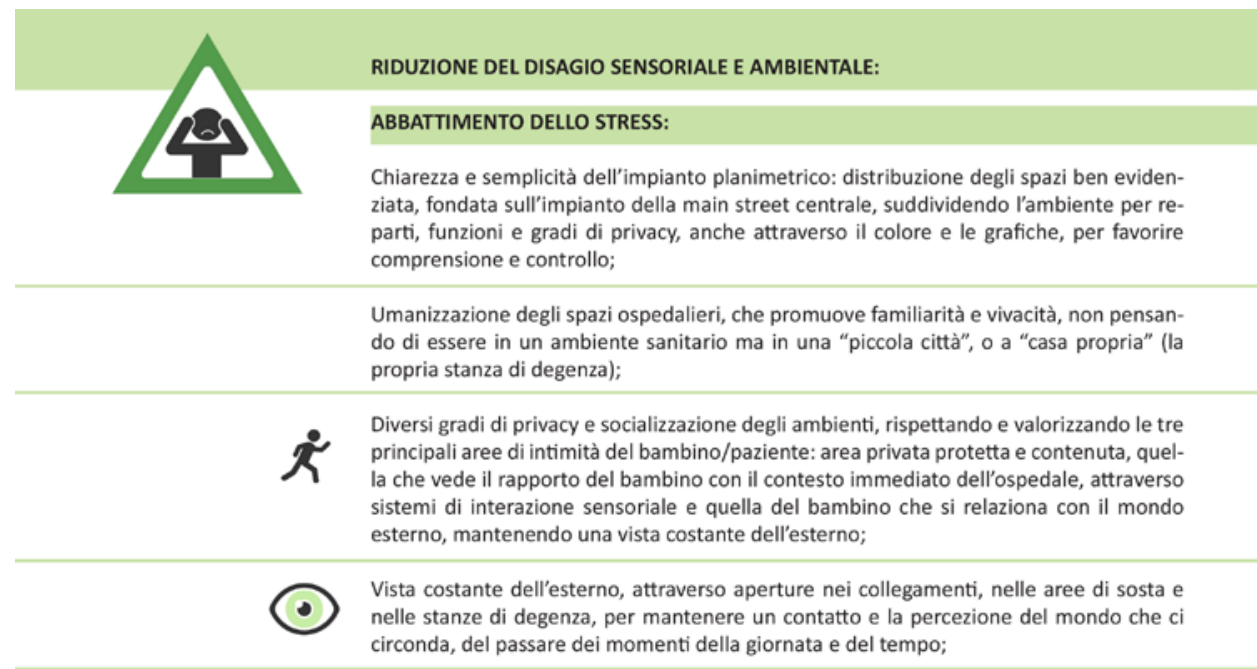

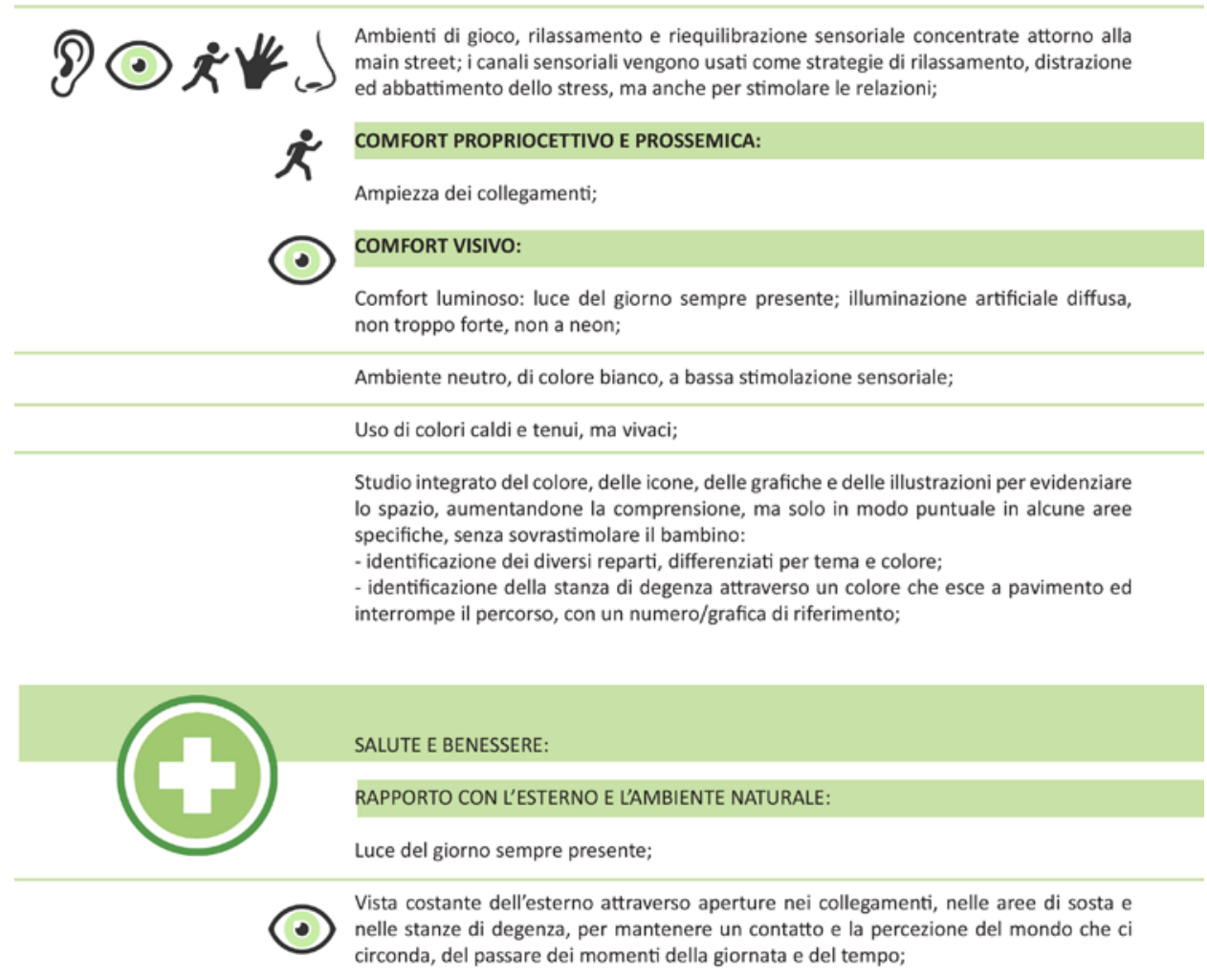

Scheda 10. Emma Children's Hospital (parte 1) 


\section{Elena Bellini}

\section{ABBATTIMENTO DELLO STRESS E APPORTO TERAPEUTICO:}

Umanizzazione degli spazi ospedalieri, che promuove familiarità e vivacità, non pensando di essere in un ambiente sanitario ma in una "piccola città", o a "casa propria" (la propria stanza di degenza);

Diversi gradi di privacy e socializzazione degli ambienti, rispettando e valorizzando le tre principali aree di intimità del bambino/paziente: area privata protetta e contenuta, quella che vede il rapporto del bambino con il contesto immediato dell'ospedale, attraverso sistemi di interazione sensoriale e quella del bambino che si relaziona con il mondo esterno, mantenendo una vista costante dell'esterno;

Ambienti di gioco, rilassamento e riequilibrazione sensoriale concentrate attorno alla main street; i canali sensoriali vengono usati come strategie di rilassamento, distrazione ed abbattimento dello stress, ma anche per stimolare le relazioni;

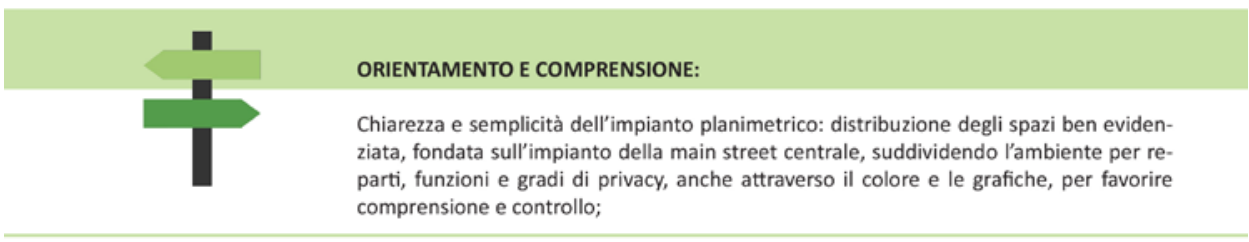

Main street - Parade - di distribuzione funzionale e sviluppo dell'interazione sociale tra bambini, famiglie e staff;

Diversi gradi di privacy e socializzazione degli ambienti, rispettando e valorizzando le tre principali aree di intimità del bambino/paziente: area privata protetta e contenuta, quella che vede il rapporto del bambino con il contesto immediato dell'ospedale, attraverso sistemi di interazione sensoriale e quella del bambino che si relaziona con il mondo esterno, mantenendo una vista costante dell'esterno;

Vista costante dell'esterno, attraverso aperture nei collegamenti, nelle aree di sosta e nelle stanze di degenza, per mantenere un contatto e la percezione del mondo che ci circonda, del passare dei momenti della giornata e del tempo;

Studio integrato del colore, delle icone, delle grafiche e delle illustrazioni per evidenziare lo spazio, aumentandone la comprensione, ma solo in modo puntuale in alcune aree specifiche, senza sovrastimolare il bambino:

- identificazione dei diversi reparti, differenziati per tema e colore;

- identificazione della stanza di degenza attraverso un colore che esce a pavimento ed interrompe il percorso, con un numero/grafica di riferimento;

Segnaletica sotto forma di immagini, riprodotta a terra, in alto appesa a soffitto e sui pannelli informativi all'ingresso di ogni reparto ed in ogni hall;

Colore e grafica numerica che esce dalle stanze di degenza apparendo sul corridoio di collegamento, per essere immediatamente visibile;

\section{Scheda 10. Emma Children's Hospital (parte 2)}


Ambienti sensoriali "terapeutici" che rendano Abili

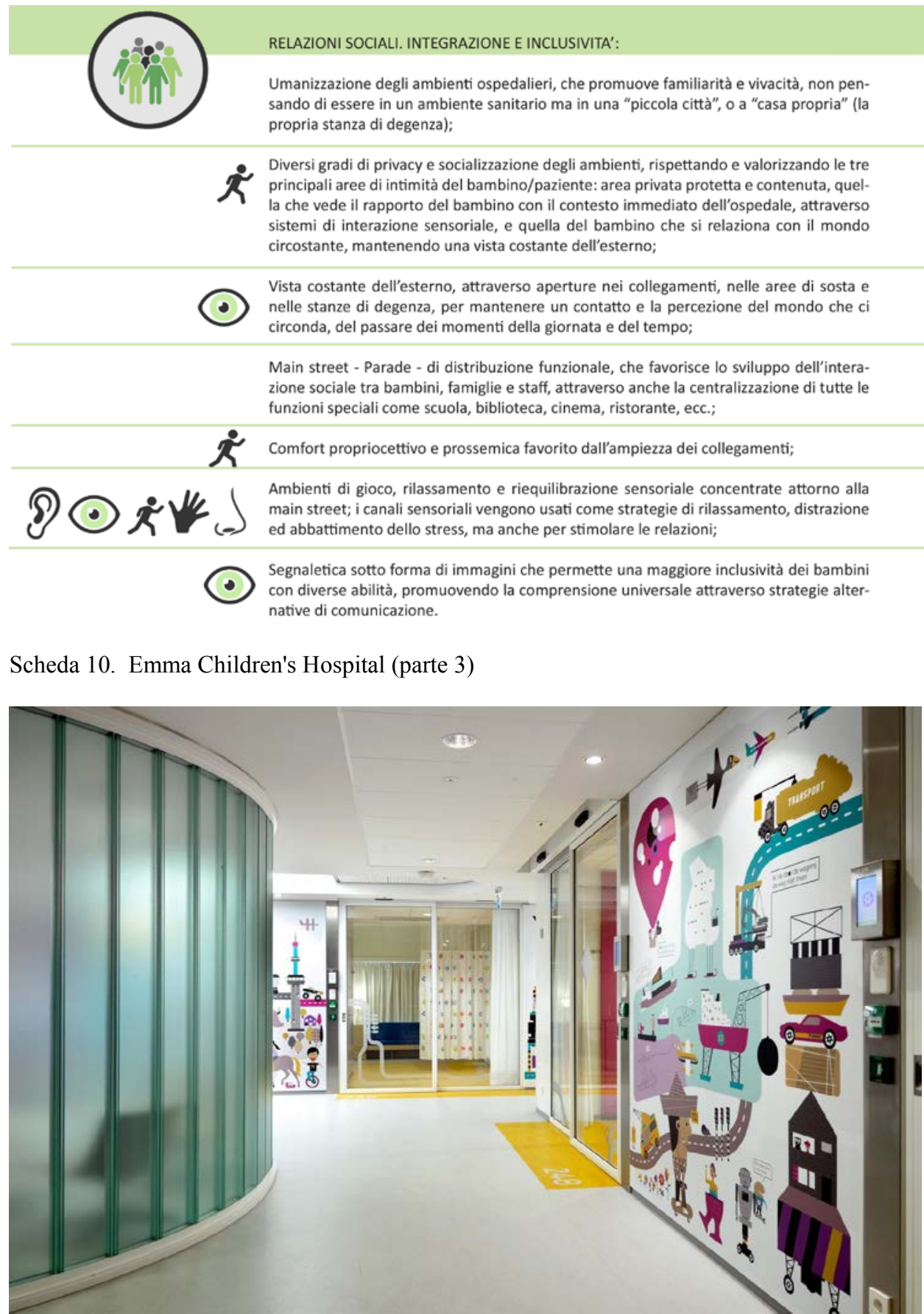

Figura 30. Emma Children's Hospital (Source: Defesche van den Putte, in short: DVDP, an office for urban design and architecture,located in Amsterdam, The Netherlands - photographer: Mike Bink) 


\section{Elena Bellini}

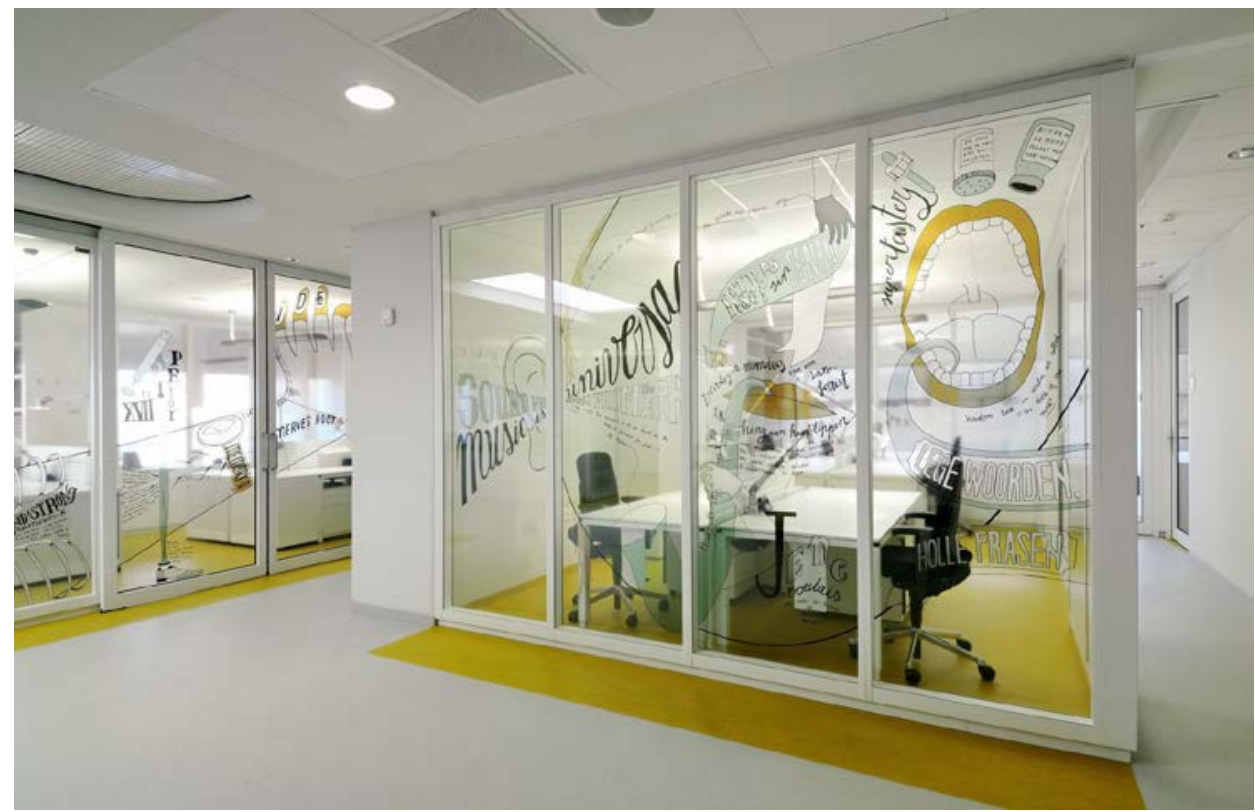

Figura 31. Emma Children's Hospital (Source: Defesche van den Putte, in short: DVDP, an office for urban design and architecture, located in Amsterdam, The Netherlands - photographer: Mike Bink)

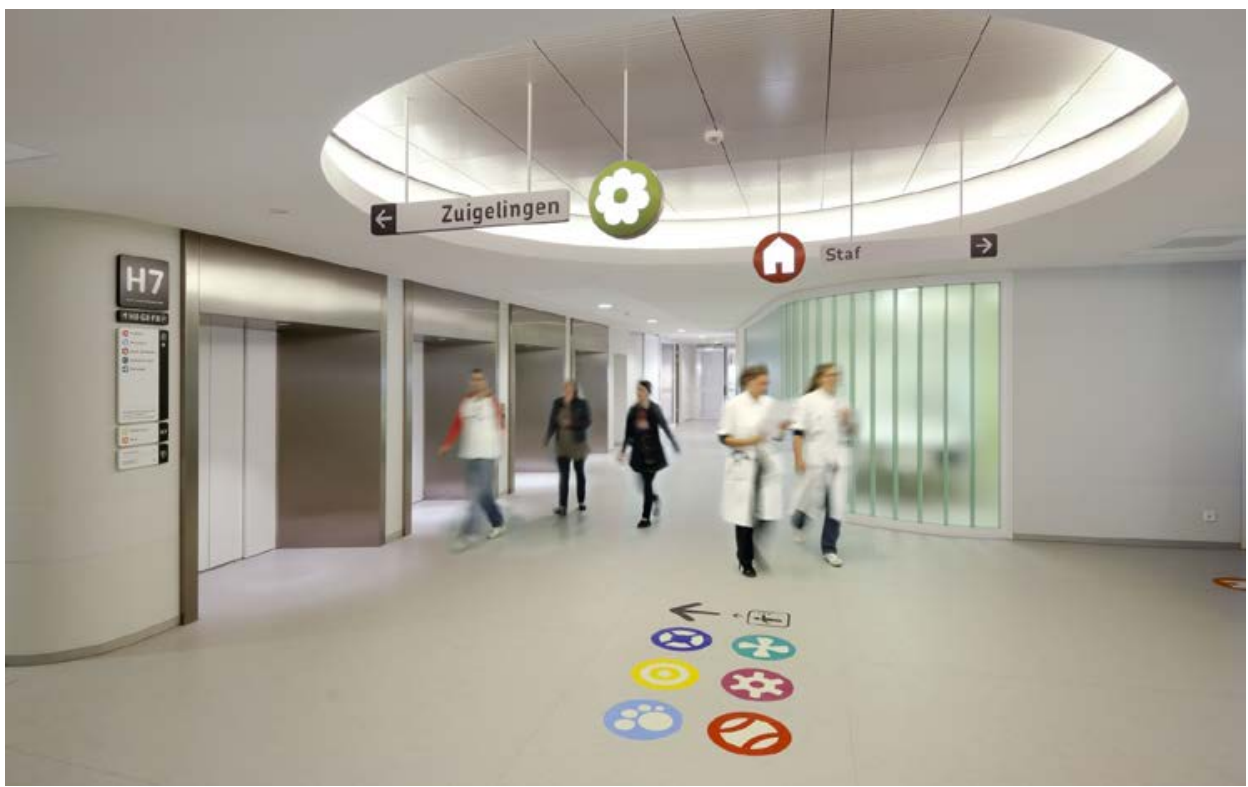

Figura 32. Emma Children's Hospital (Source: Defesche van den Putte, in short: DVDP, an office for urban design and architecture, located in Amsterdam, The Netherlands - photographer: Mike Bink) 
Ambienti sensoriali "terapeutici" che rendano Abili

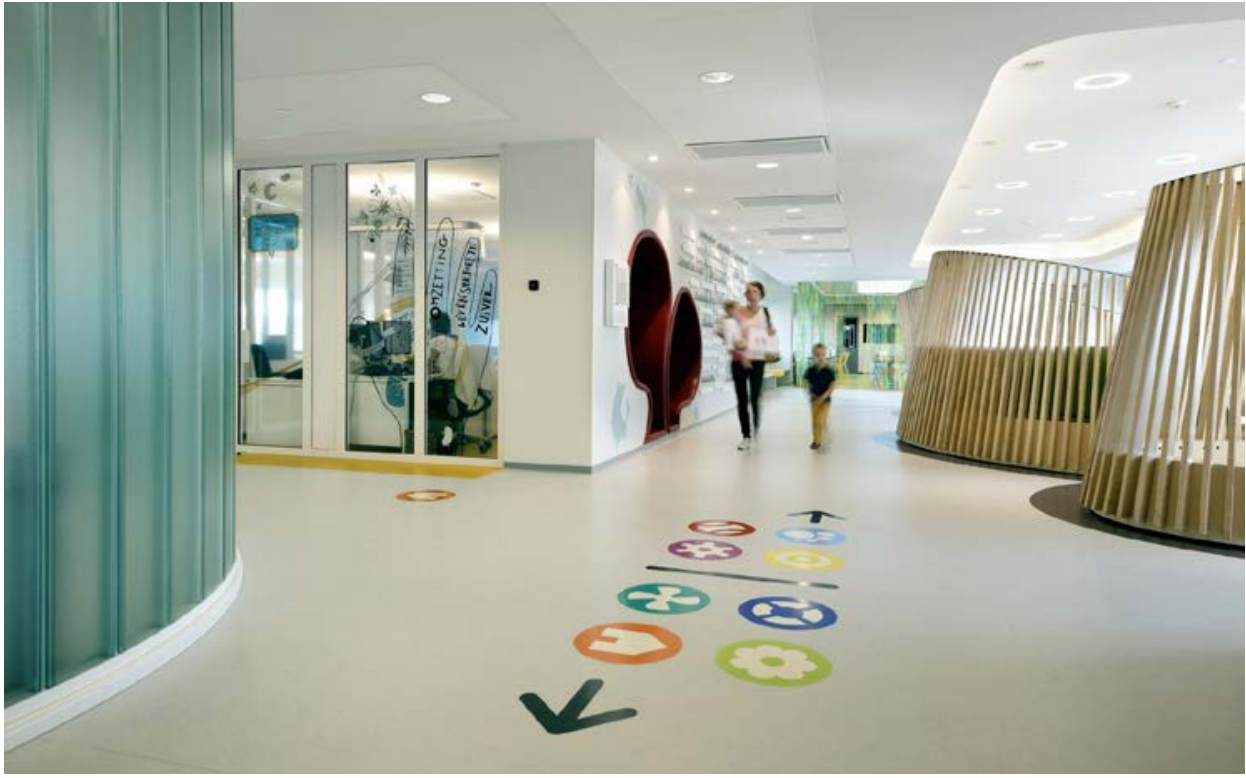

Figura 33. Emma Children's Hospital (Source: Defesche van den Putte, in short: DVDP, an office for urban design and architecture,located in Amsterdam, The Netherlands - photographer: Mike Bink)

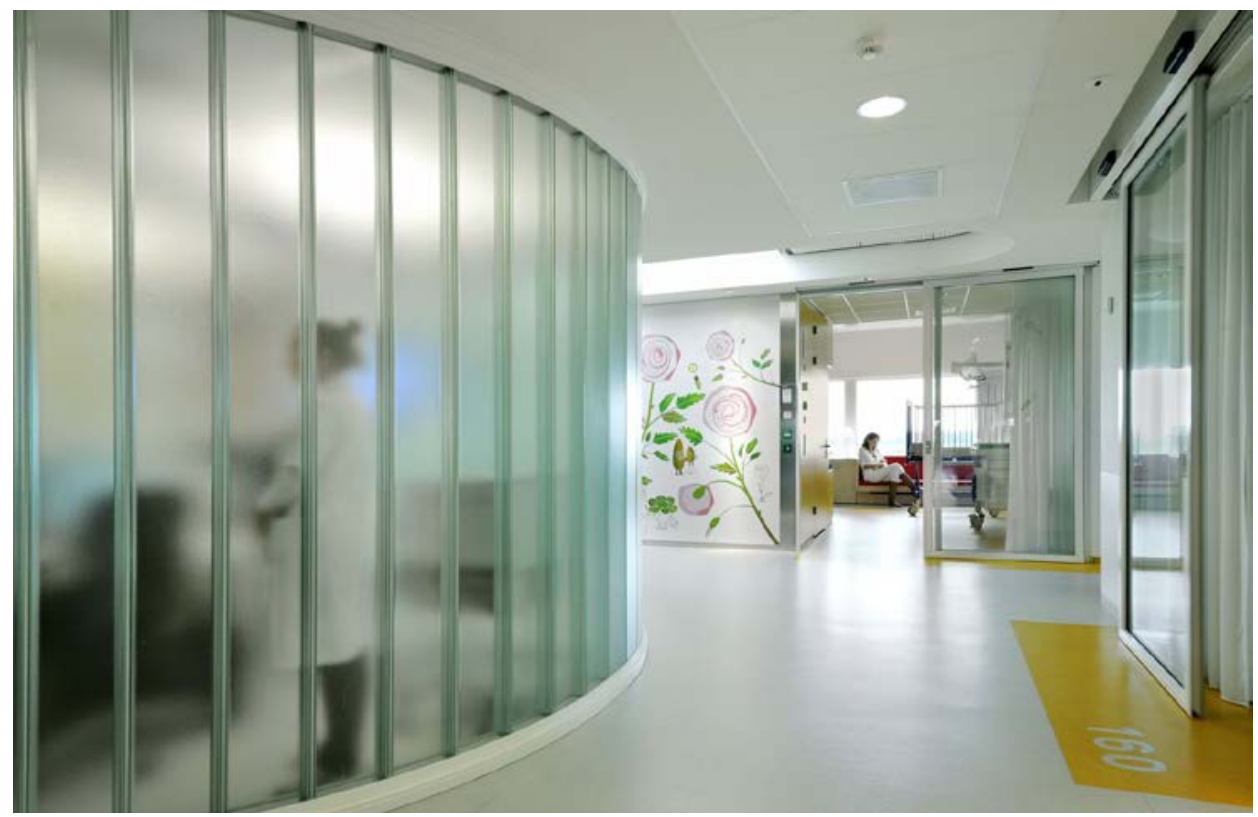

Figura 34. Emma Children's Hospital (Source: Defesche van den Putte, in short: DVDP, an office for urban design and architecture,located in Amsterdam, The Netherlands - photographer: Mike Bink) 


\section{Elena Bellini}

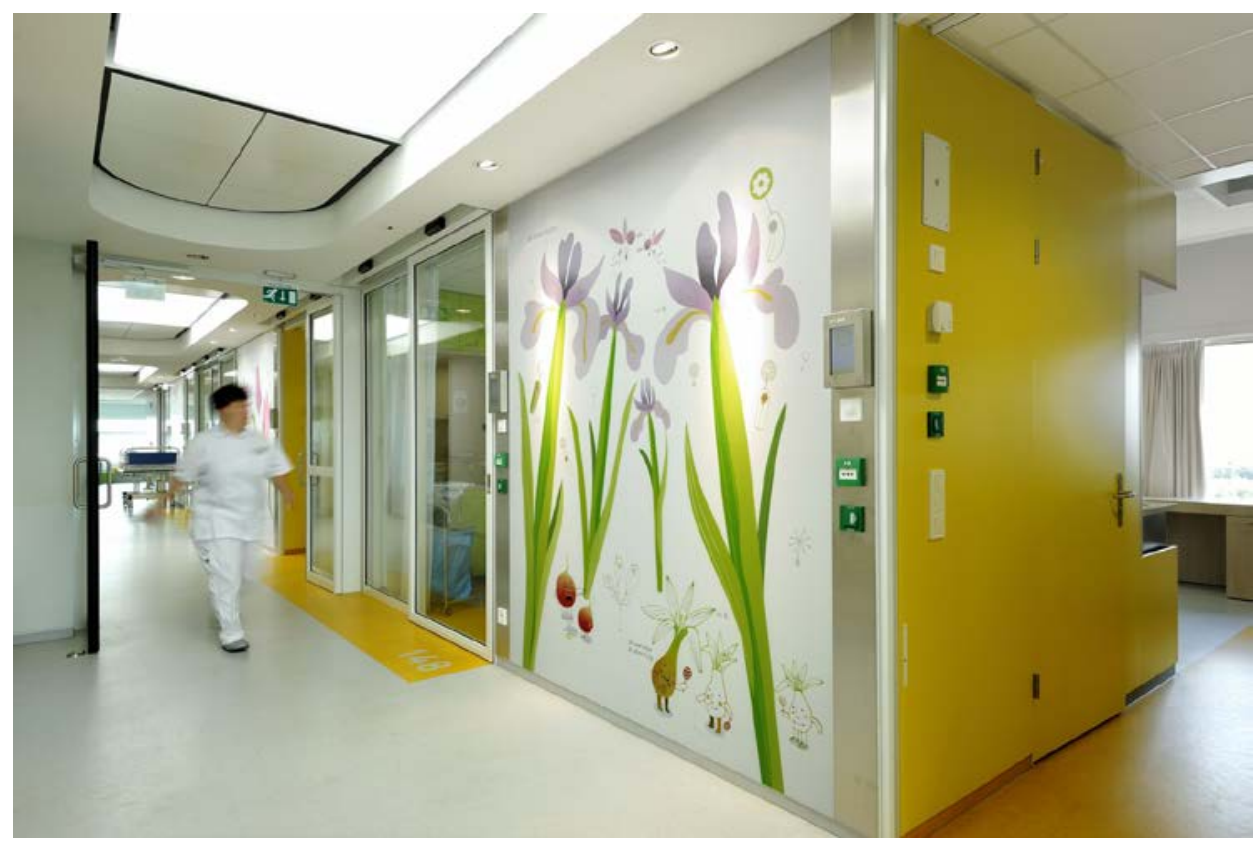

Figura 35. Emma Children's Hospital (Source: Defesche van den Putte, in short: DVDP, an office for urban design and architecture,located in Amsterdam, The Netherlands - photographer: Mike Bink) 
Ambienti sensoriali "terapeutici" che rendano Abili

RIDUZIONE DEL DISAGIO SENSORIALE E AMBIENTALE:

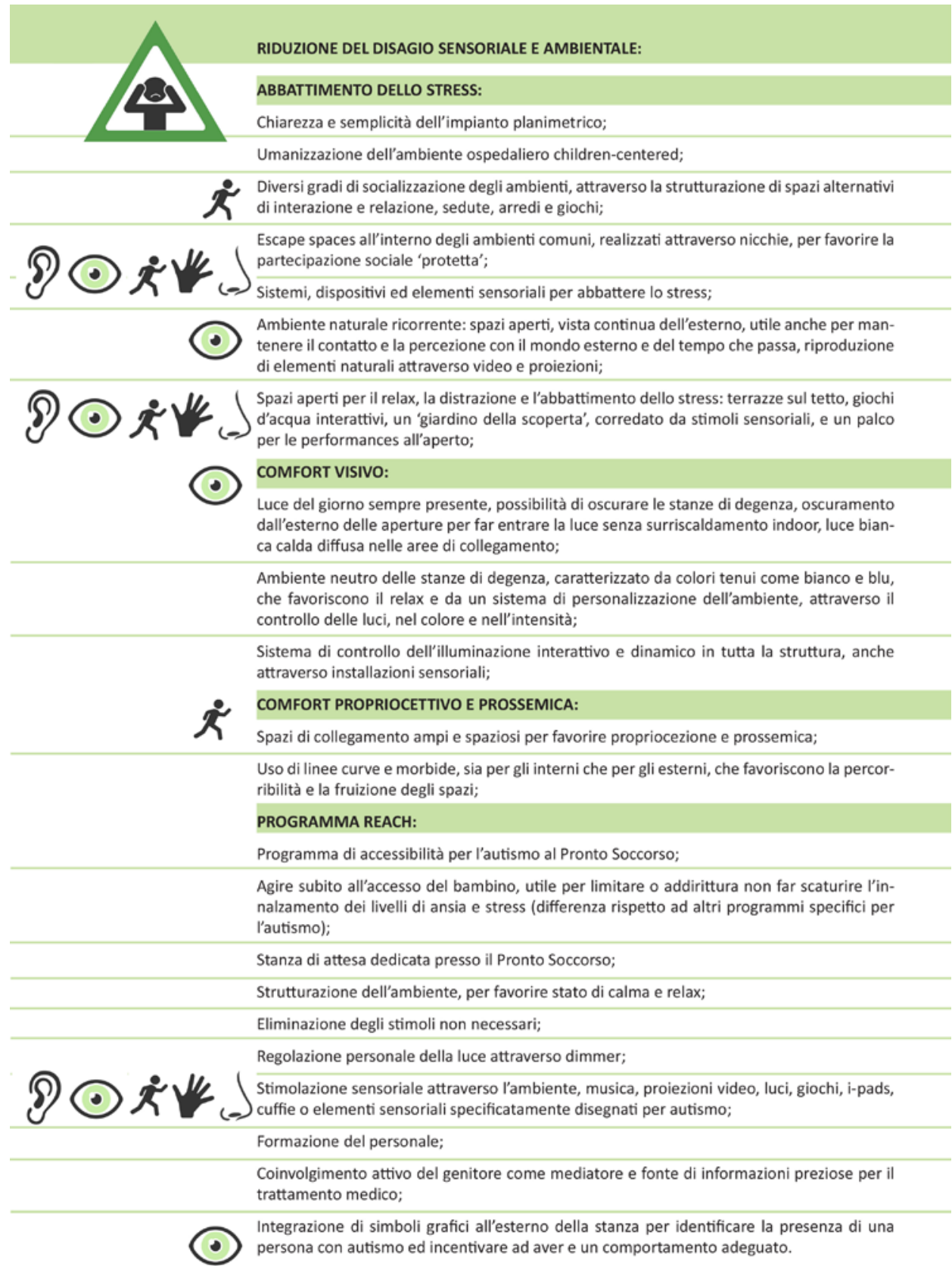

Scheda 11. Nemours Children's Hospital (parte 1) 


\section{Elena Bellini}

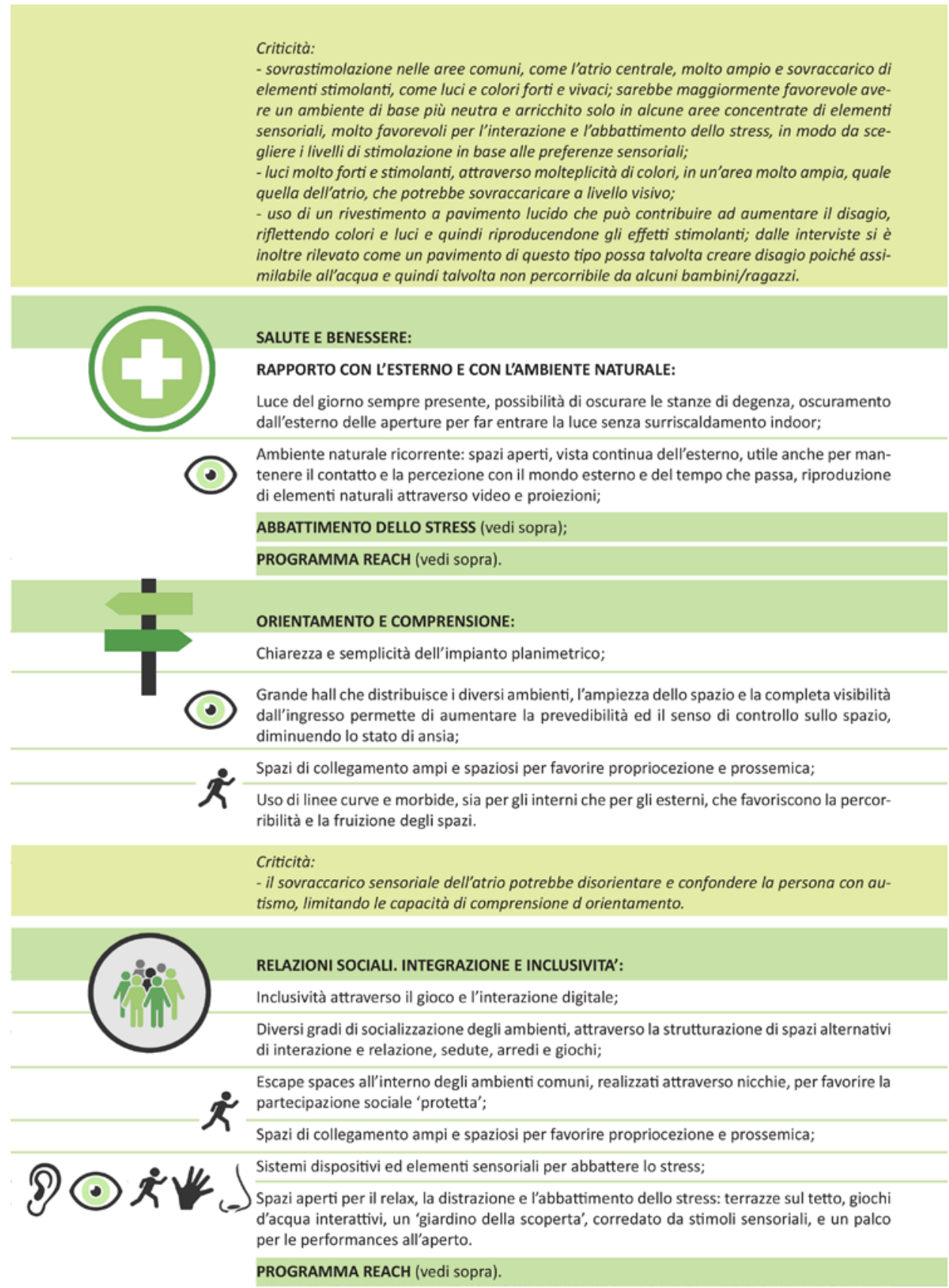

Scheda 11. Nemours Children's Hospital (parte 2) 


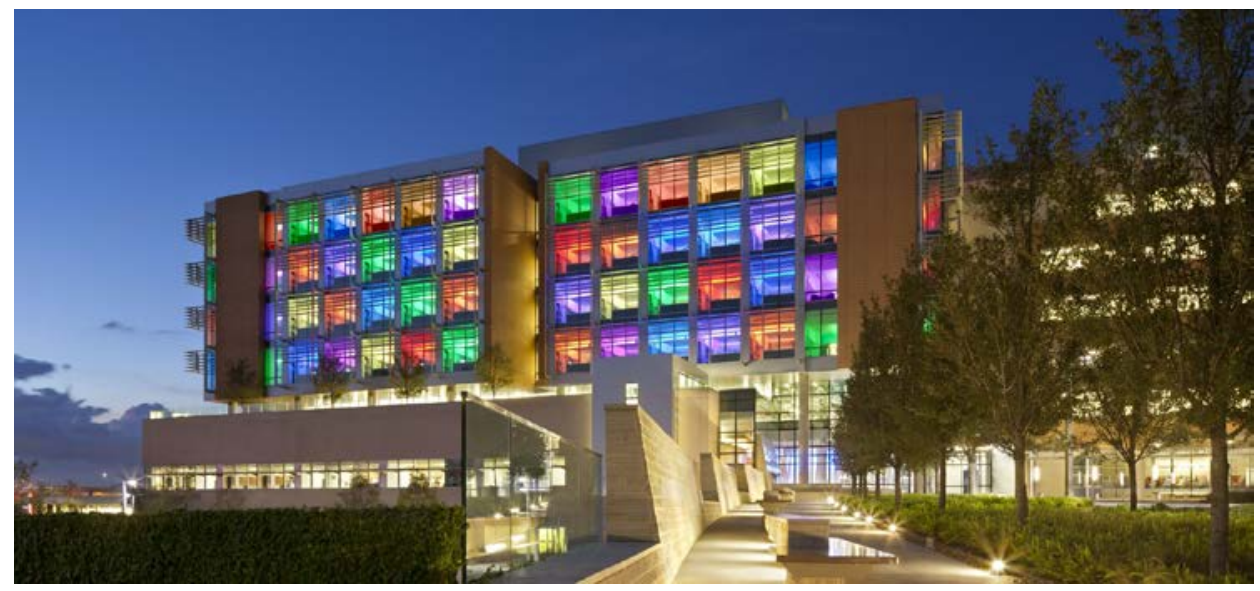

Figura 36. Nemours Children's Hospital (Source: EYP Architecture \& Engineering)

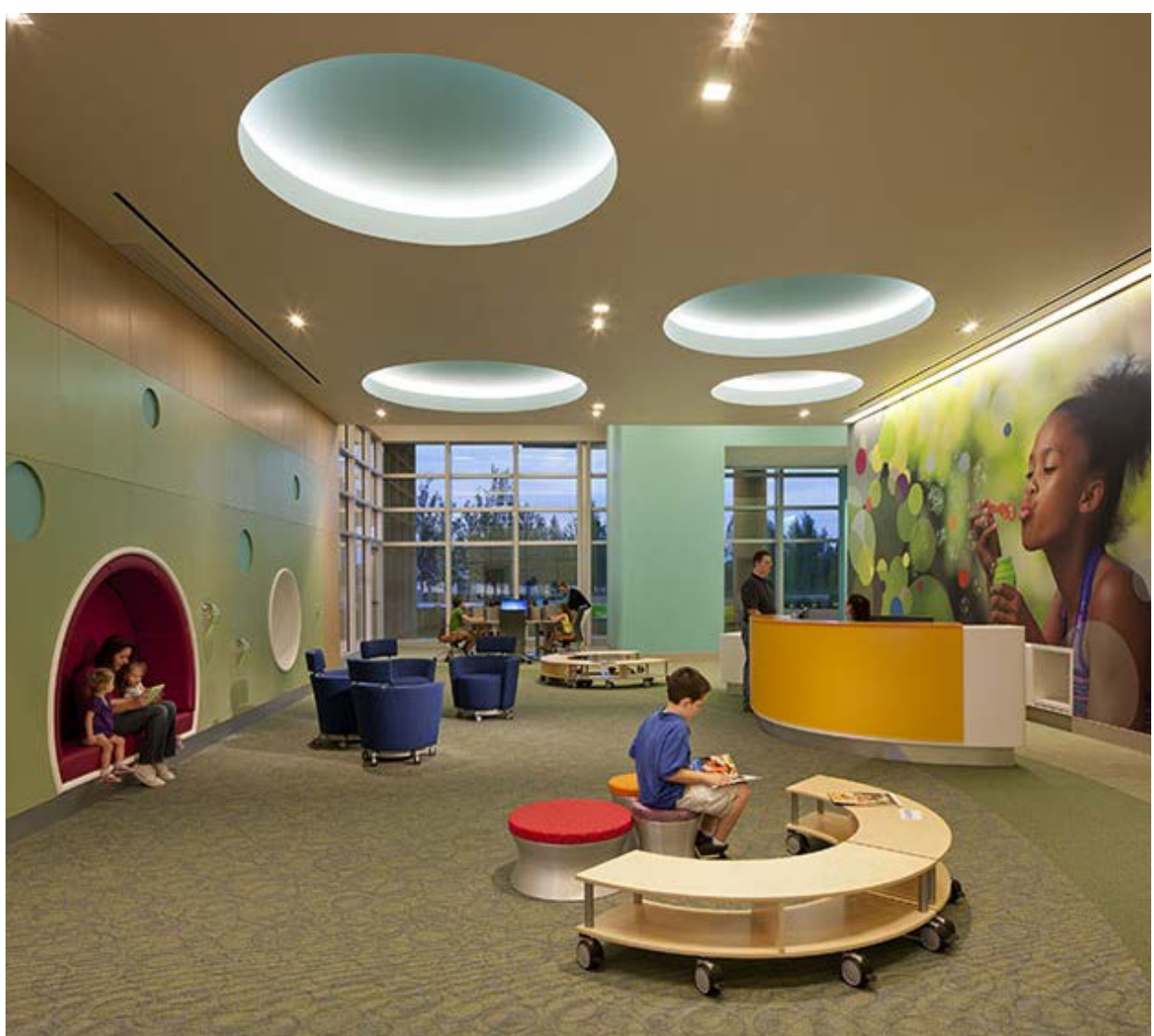

Figura 37. Nemours Children's Hospital: spazio per i bambini (Source: EYP Architecture \& Engineering) 


\section{Elena Bellini}

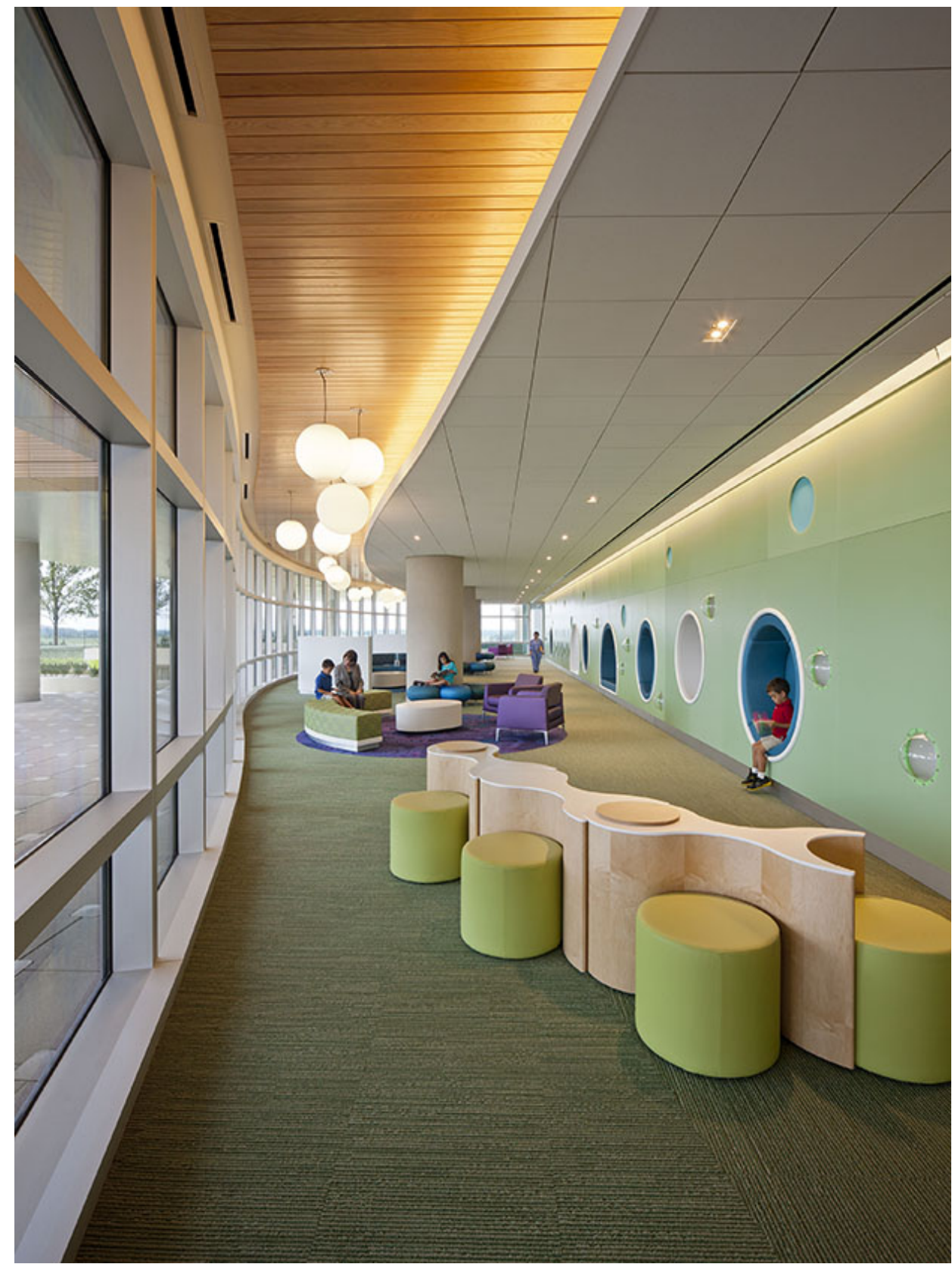

Figura 38. Nemours Children's Hospital: spaizo di attesa per la sala operatoria (Source: EYP Architecture \& Engineering) 
Ambienti sensoriali "terapeutici" che rendano Abili

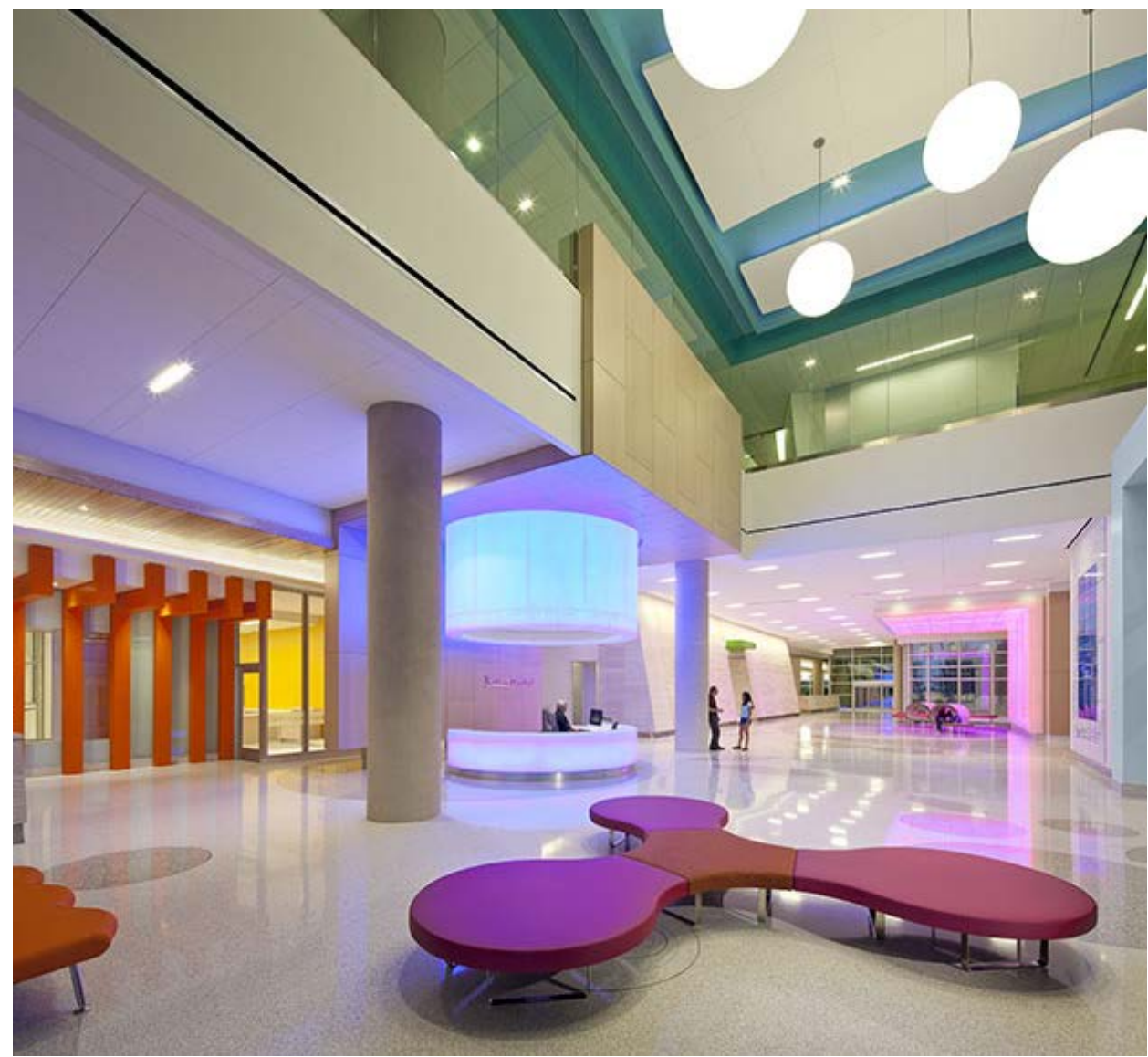

Figura 39. Nemours Children's Hospital: hall di ingresso e reception (Source: EYP Architecture \& Engineering) 


\section{Elena Bellini}

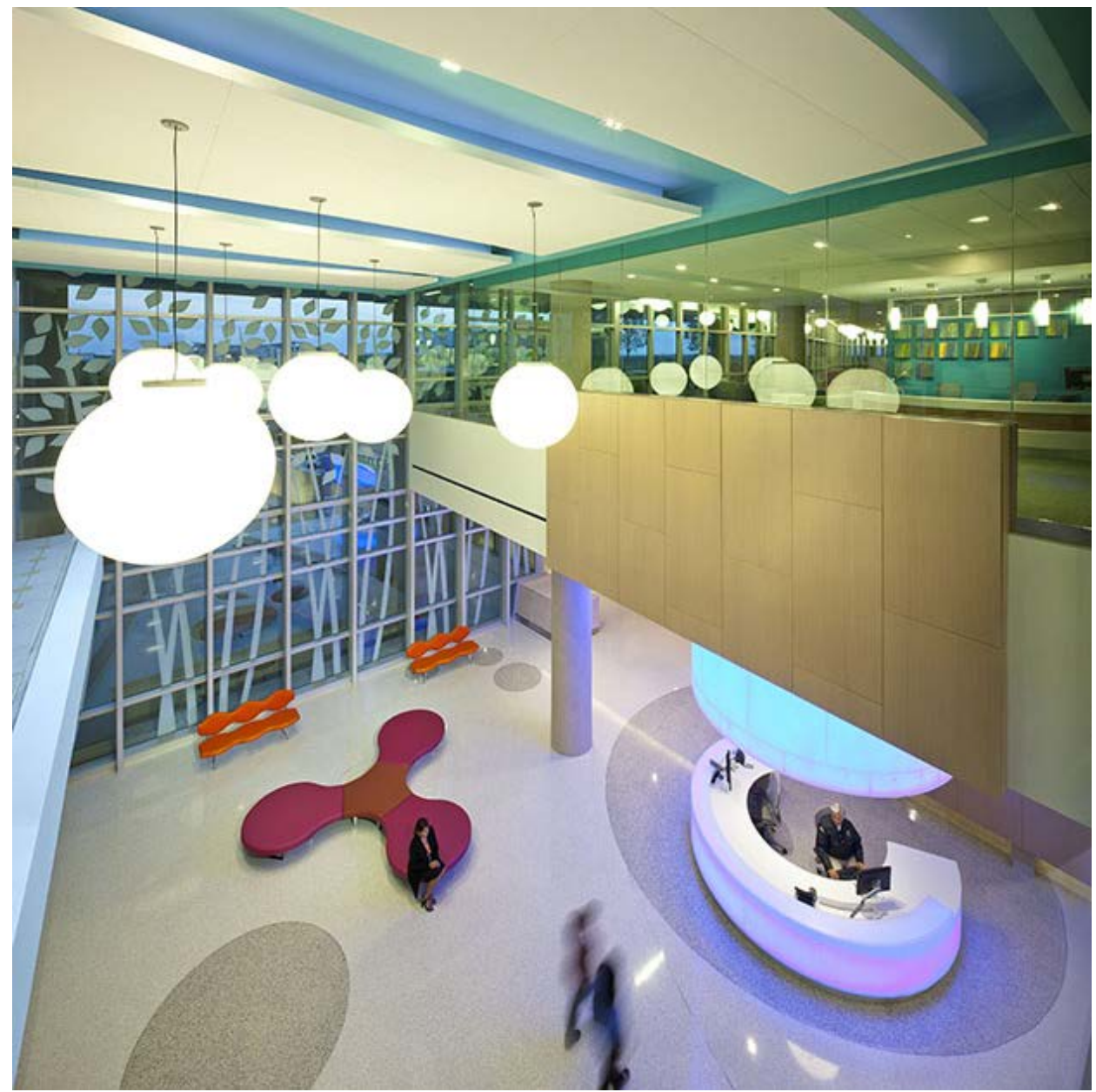

Figura 40. Nemours Children's Hospital: hall di ingresso e reception (Source: EYP Architecture \& Engineering) 


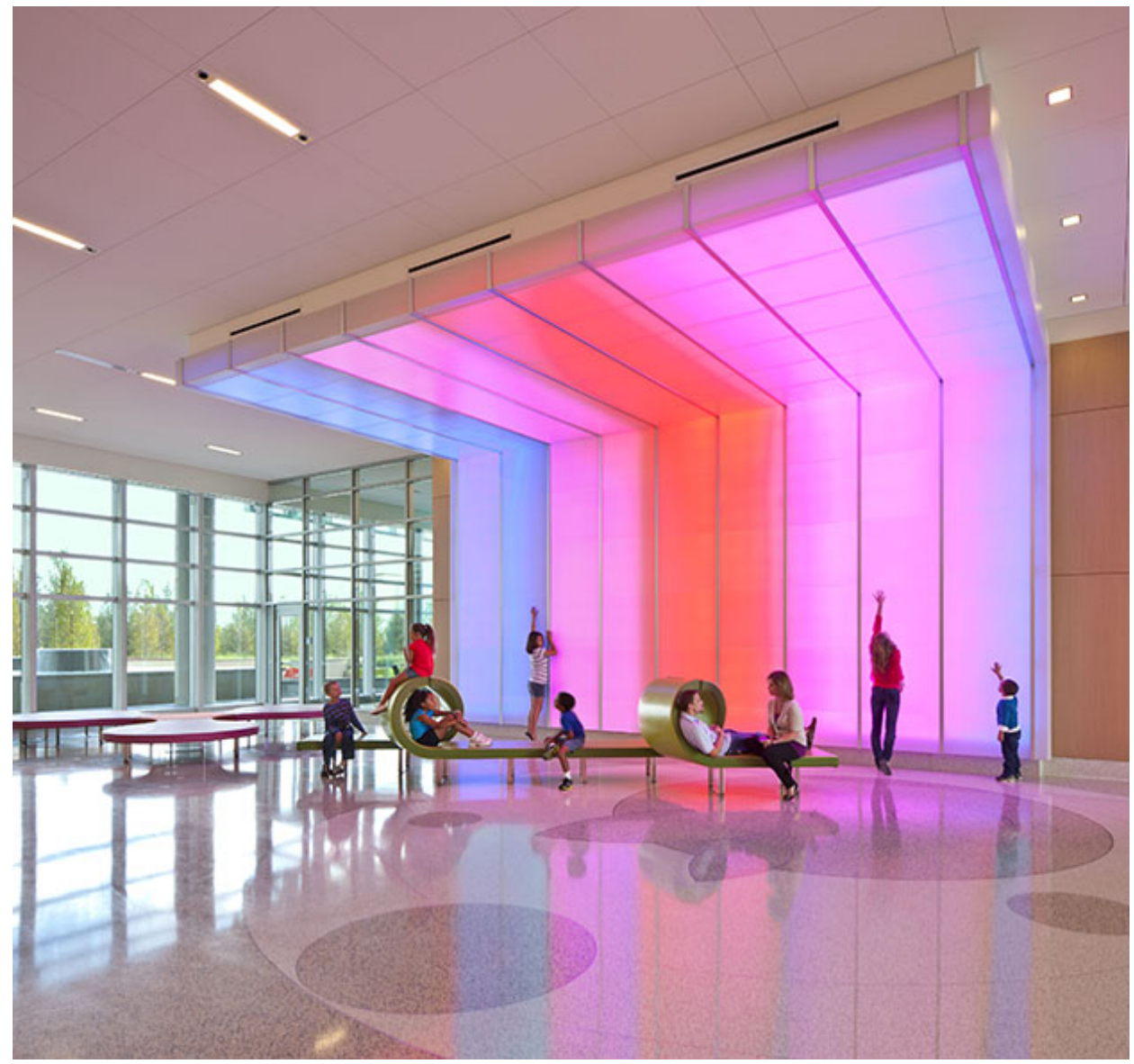

Figura 41. Nemours Children's Hospital: area di aspetto, installazione multisensoriale (Source: EYP Architecture \& Engineering) 


\section{Elena Bellini}

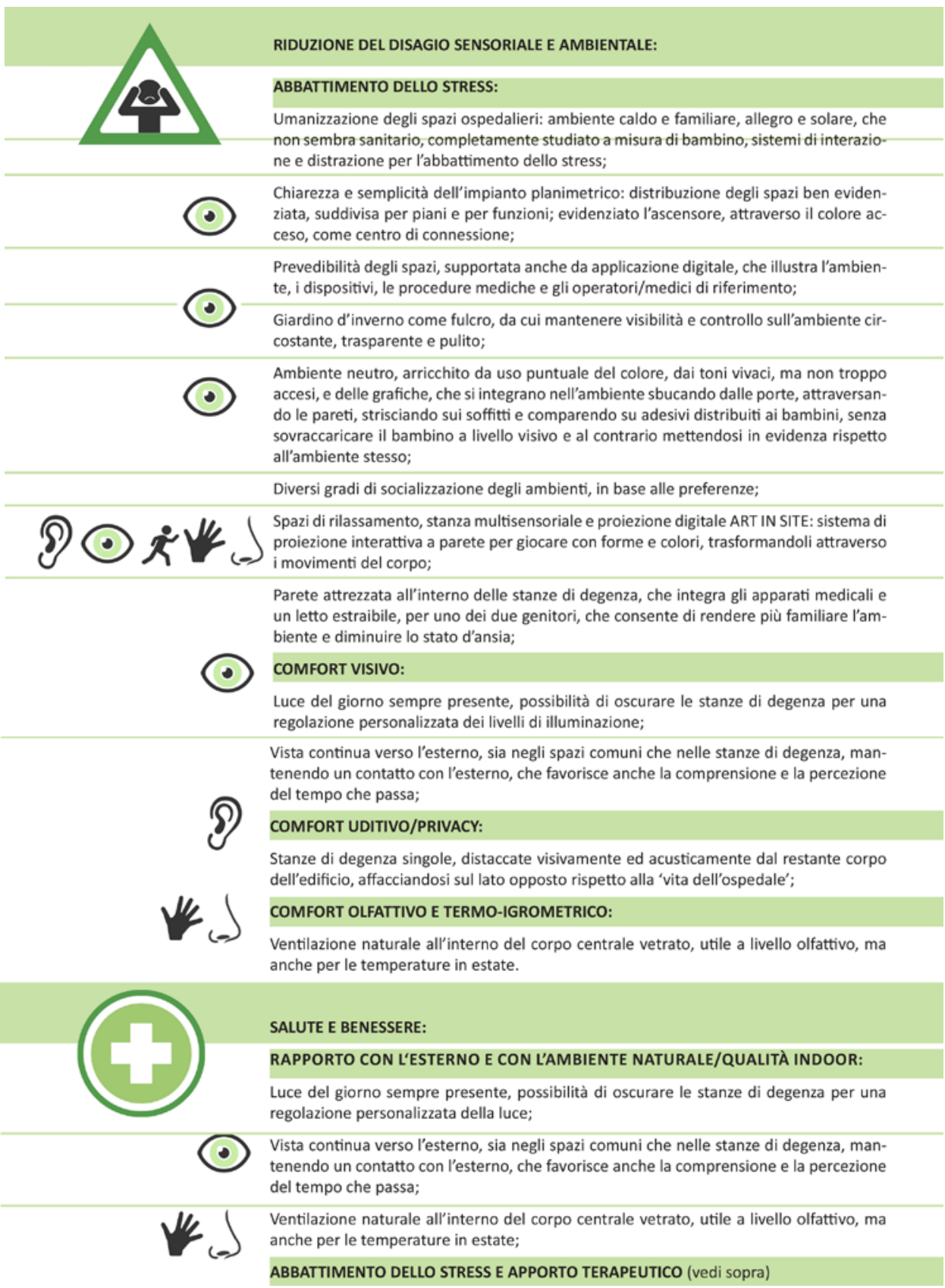

Scheda 12. Evelina Londron Children's Hospital (parte 1) 
Centro di servizi dedicati alle disabilità del neurosviluppo, integrando diverse discipline tra cui quelle sanitarie, scolastiche, dei servizi sociali e terapeutici come la logopedia, la terapia occupazionale, fisioterapisti, dietologi, psicomotricità, ecc.

\section{ASSISTIVE TECHNOLOGY:}

Applicazione digitale per il supporto alla persona, prima, durante e dopo l'intervento medico, anche attraverso raccomandazioni e informazioni sulla salute, fisica e mentale;

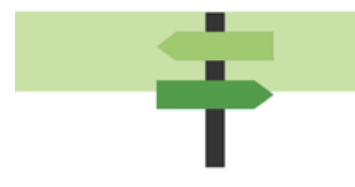

ORIENTAMENTO E COMPRENSIONE:

Chiarezza e semplicità dell'impianto planimetrico: distribuzione degli spazi ben evidenziata, suddivisa per piani e per funzioni; evidenziato l'ascensore, attraverso il colore acceso, come centro di connessione;

Giardino d'inverno come fulcro, da cui mantenere visibilità e controllo sull'ambiente circostante, avendo un affaccio diretto di tutti i piani sull'ambiente centrale, riferimenti visivi e interazione continua;

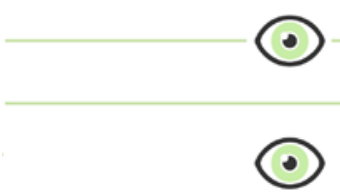

Sistema di arte e illustrazione digitale per favorire l'orientamento ed il wayfinding: colori, ambientazione, scenari e 'gang' di personaggi differenziati per ogni piano;

Ambiente neutro, arricchito da uso puntuale del colore, e delle grafiche, che si integrano nell'ambiente sbucando dalle porte, attraversando le pareti, strisciando sui soffitti e comparendo su adesivi distribuiti ai bambini, senza sovraccaricare il bambino a livello visivo e al contrario mettendosi in evidenza rispetto all'ambiente stesso;

\section{ASSISTIVE TECHNOLOGY:}

Applicazione digitale che illustra l'ambiente (tour virtuale), i dispositivi (descrizione degli spazi e dei dispositivi utili per le procedure mediche), le procedure mediche (storie che raccontano le motivazioni per cui si va solitamente al Pronto Soccorso, gli interventi per curarsi e le classiche procedure di visita o di diagnosi) e gli operatori/medici di riferimento; giochi che distraggono e spiegano anche il funzionamento del corpo umano, aiutando la comunicazione tra medici/operatori e pazienti/famiglie attraverso le immagini.

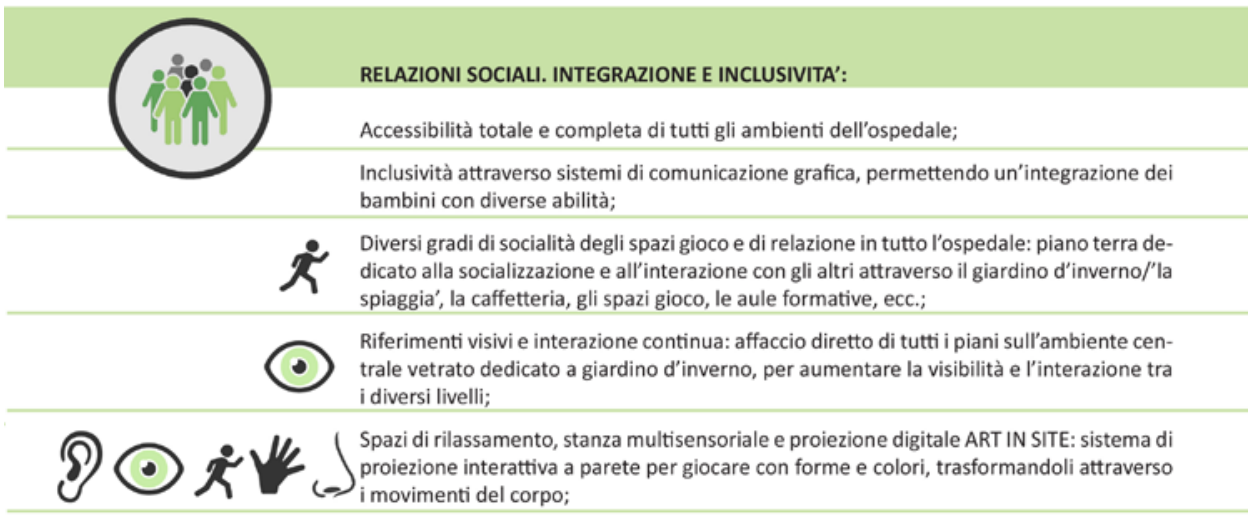
Informazioni e conoscenza all'interno dell'applicazione digitale sulle diverse problematiche e patologie, alle diverse età del bambino, con focus anche sulla salute mentale.

Scheda 12. Evelina Londron Children's Hospital (parte 2) 


\section{Elena Bellini}

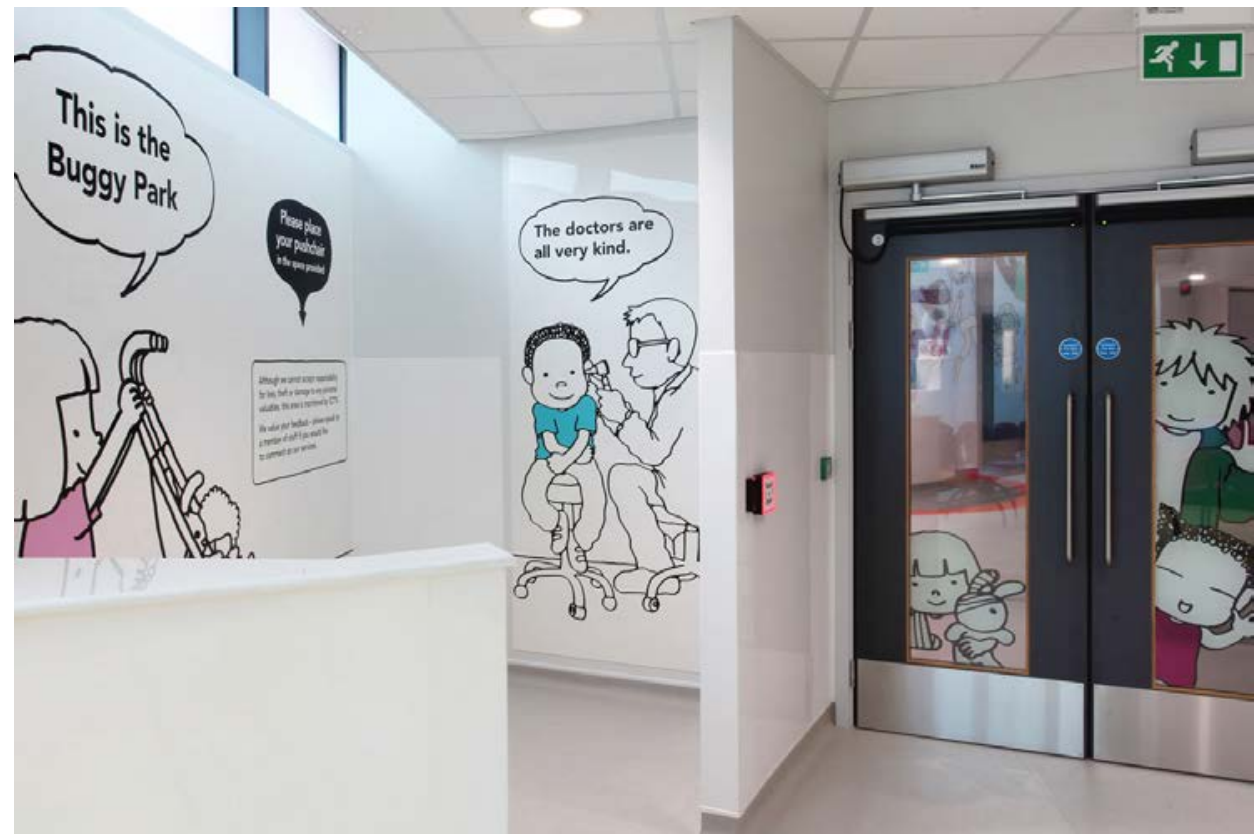

Figura 42. Evelina Londron Children's Hospital: sistema di grafica a parete (Source: Art in Site)

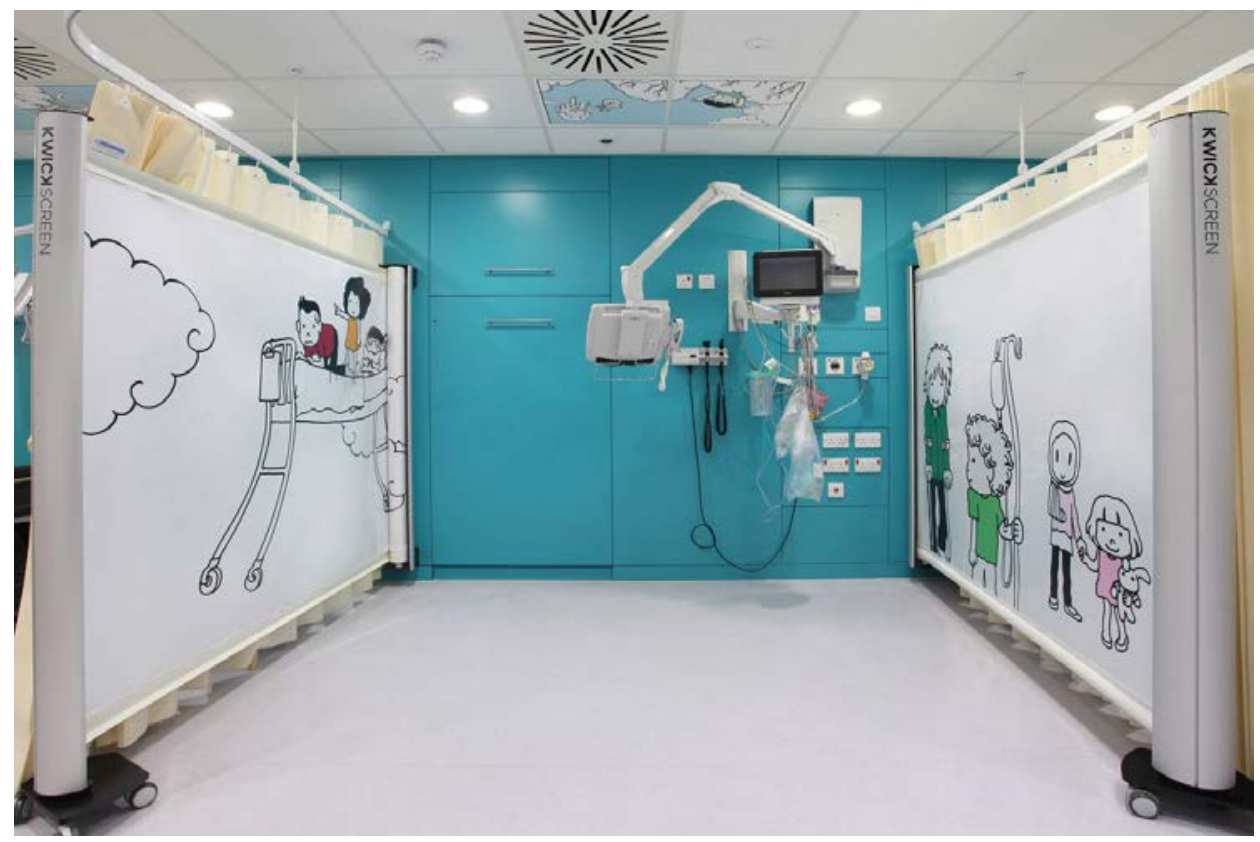

Figura 43. Evelina Londron Children's Hospital: sistema di grafica a parete (Source: Art in Site) 
Ambienti sensoriali "terapeutici” che rendano Abili

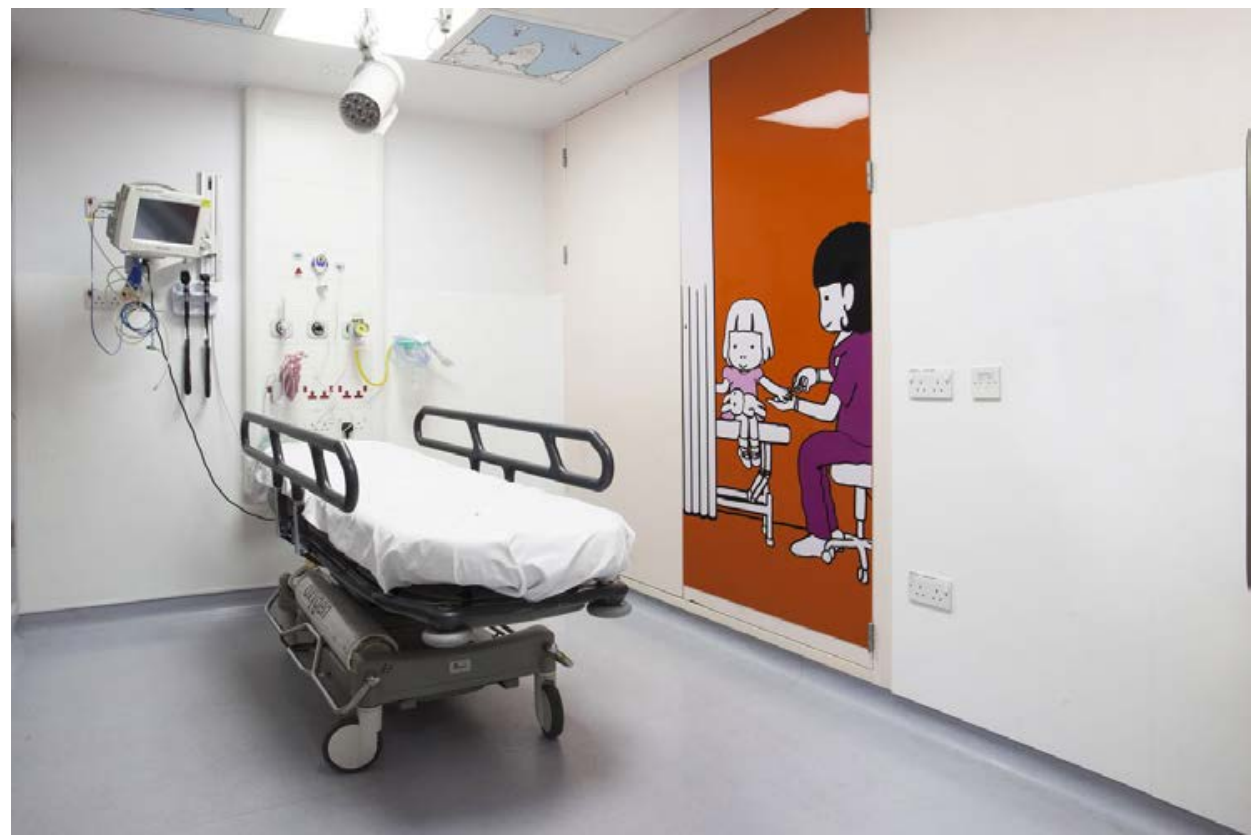

Figura 44. Evelina Londron Children's Hospital: sistema di grafica a parete (Source: Art in Site)

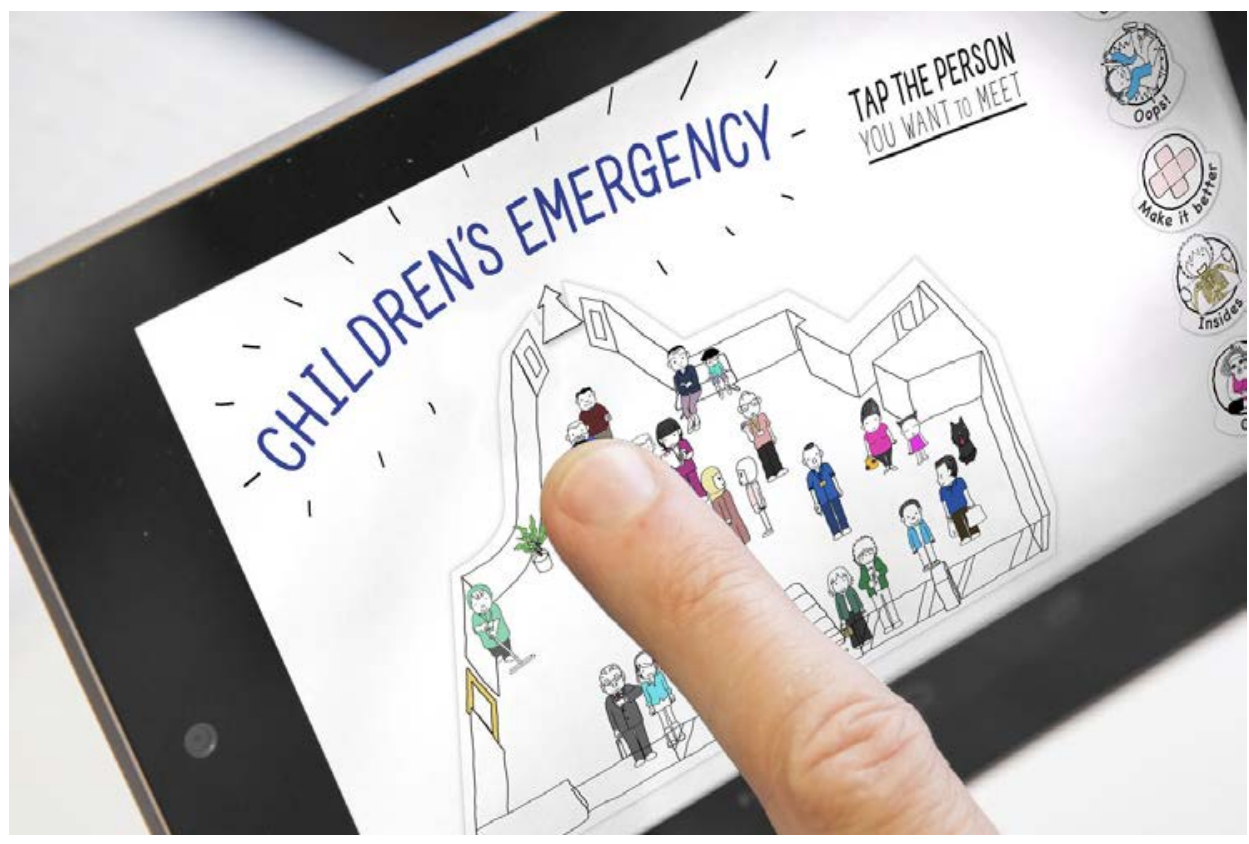

Figura 45. Evelina Londron Children's Hospital: sistema di grafica e applicazione digitale per la comunicazione (Source: Art in Site) 


\section{Elena Bellini}

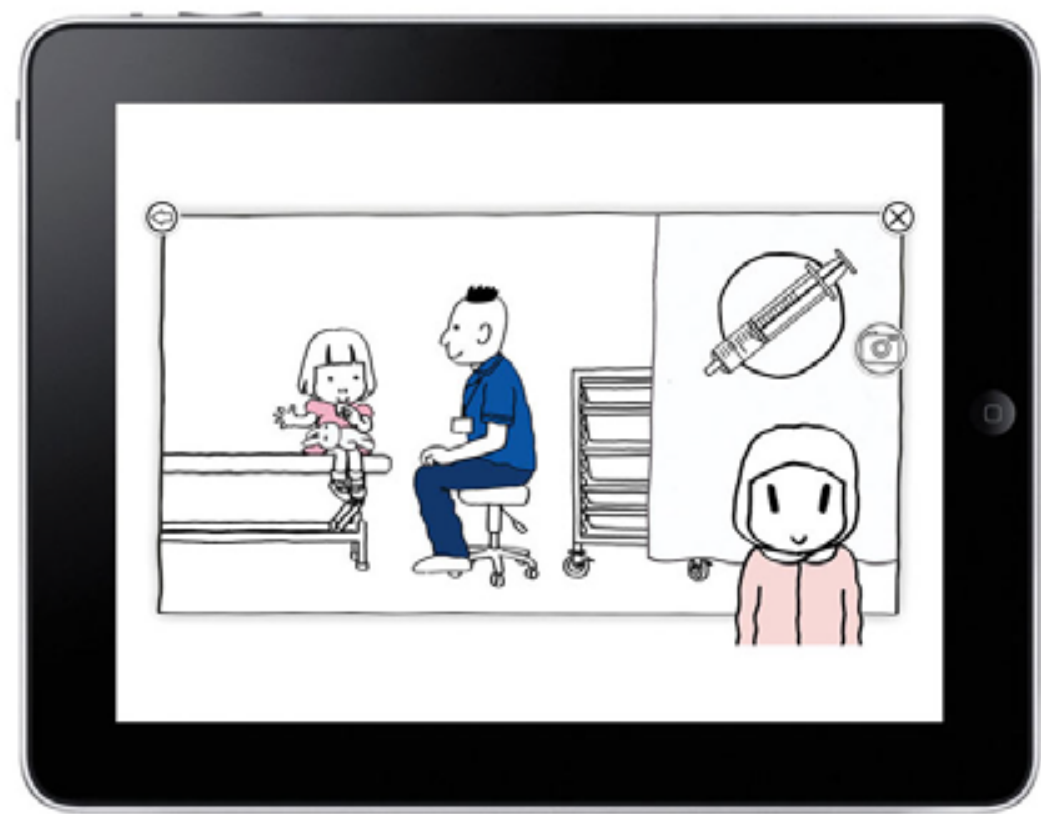

Figura 46. Evelina Londron Children's Hospital: sistema di grafica e applicazione digitale per la comunicazione (Source: Art in Site)

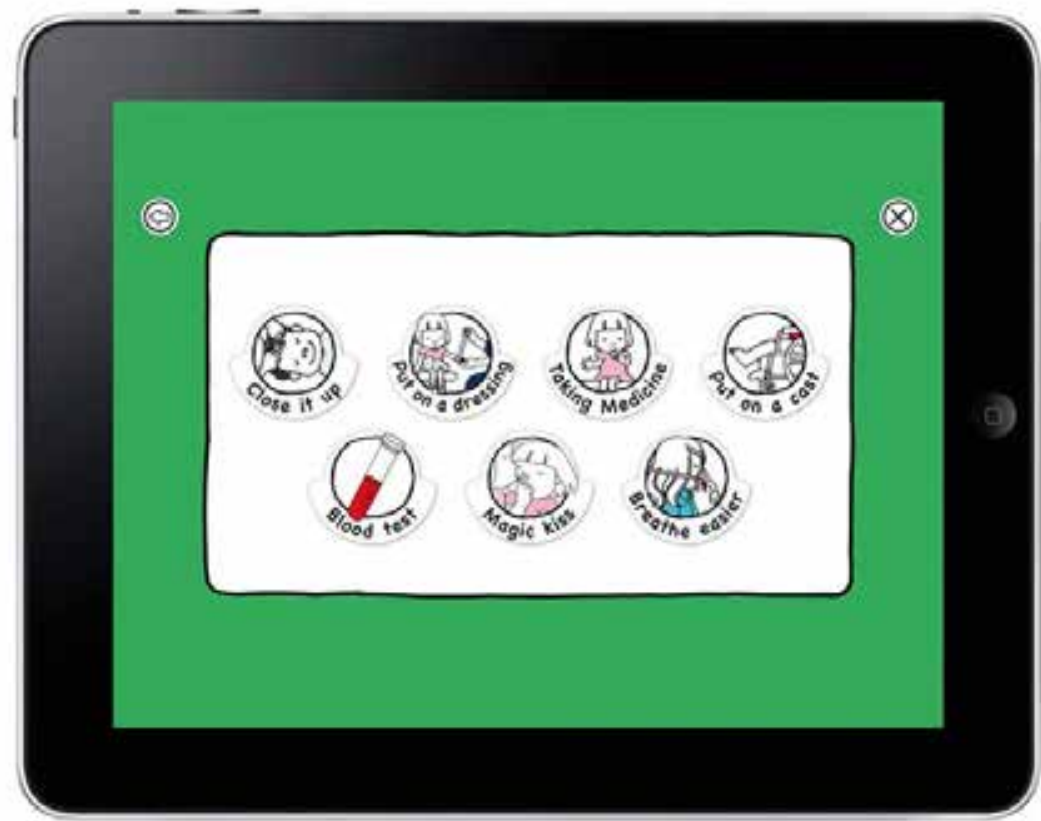

Figura 47. Evelina Londron Children's Hospital: sistema di grafica e applicazione digitale per la comunicazione (Source: Art in Site) 
Ambienti sensoriali "terapeutici” che rendano Abili

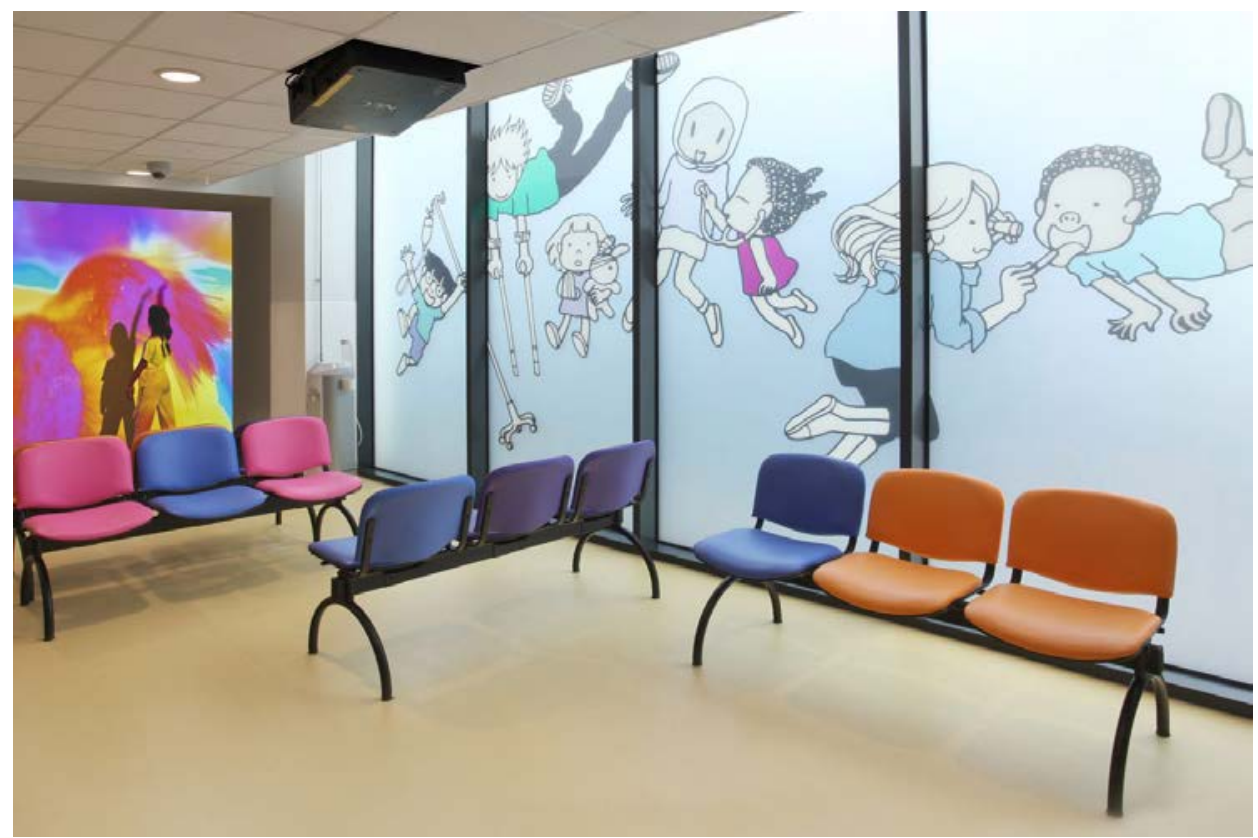

Figura 48. Evelina Londron Children's Hospital: sistema di grafica a parete, parete interattiva multisensoriale (Source: Art in Site)

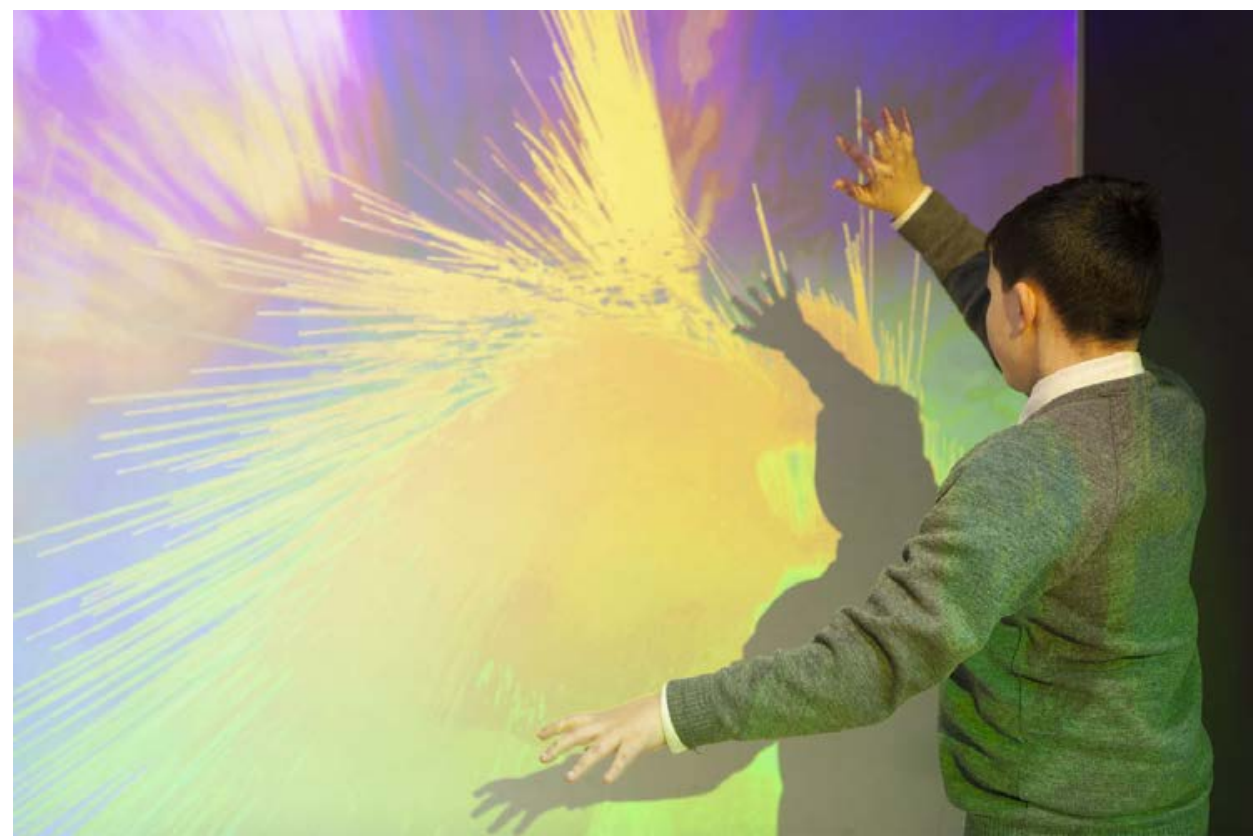

Figura 49. Evelina Londron Children's Hospital: parete interattiva multisensoriale (Source: Art in Site) 


\section{Capitolo 5 \\ Conclusioni e indicazioni di sintesi}

Ognuno dei tre ambiti di studio analizzati per quanto riguarda lo stato dell'arte sulla progettazione autism-friendly (residenziale, educativo, sanitario) e dei casi studio di sperimentazione sul campo, ha dimostrato la grande difficoltà nel definire $\mathrm{e}$ sintetizzare regole generali evidence based e, soprattutto, universali. Non bisogna quindi aver paura di affermare che stilare delle Linee Guida di progettazione per l'autismo potrebbe rivelarsi sterile e che, allo stesso modo, sarebbe complesso arrivare a delle norme in termini di specifiche prestazioni, riscontrando oltretutto una carenza di dati che diano evidenza sull'efficacia dei diversi interventi. Non si possono ridurre le persone con autismo a tipizzazioni poiché si rischierebbe di produrre regole e norme che rispondono solo ad una piccola porzione delle innumerevoli e importanti esigenze della popolazione all'interno dello spettro. Attraverso questa ricerca si mira quindi a trovare supporto in nuovi metodi progettuali che siano collaborativi, dinamici e flessibili, e che consentano di rilevare i bisogni specifici delle singole persone e rispondere in modo adeguato per ottenere un miglioramento reale della qualità di vita. In particolare, si cerca di integrare il progetto degli ambienti in un complessivo progetto di vita, che veda quindi la collaborazione delle competenze di diverse professionalità e multidisciplinarietà per un fine comune: dare dignità $\mathrm{e}$ capacità di autodeterminazione a queste persone, nelle diverse fasi della loro vita.

E' stato ampiamente dimostrato come l'ambiente sia d'impatto nella vita e nel benessere di ogni uomo e come, in casi di autismo, si amplifichi questo concetto per le particolari sensibilità che rendono queste persone ipersensibili alle condizioni del contesto ambientale. Un progetto di vita complessivo è quello che trova risposta ed adeguamento in ogni ambiente di vita vissuto dalla persona. E' vero altresì che ogni ambiente è parte di un diverso contesto e andrà trattato nella progettazione sulla base delle condizioni al contorno, oltre che delle esigenze della persona che utilizzerà quell'ambito specifico. Si ritiene tuttavia che, per ogni tipo di ambiente, si possano definire le priorità tra macro-categorie di esigenze fondamentali e i requisiti con maggiore influenza in base agli obiettivi esigenziali connessi al profilo sensopercettivo del singolo e al programma funzionale dell'intervento: residenza temporanea, residenza in famiglia, residenza di adulto autonomo, scuola inclusiva, scuola "speciale", etc. 


\subsection{L'ambiente residenziale}

Nel caso dell'ambito residenziale, è possibile (e doveroso) personalizzare al massimo le risposte progettuali in funzione della singola persona. Prima di tutto si dovrà in ogni caso valutare la condizione di vita della persona, ovvero se si tratta di residenza permanente o temporanea, se in famiglia o in autonomia, se in età evolutiva, adulta o anziana, se rivolta a programmi di abilitazione, ecc. Anche il target è importante, come età, ma anche come caratteristiche specifiche all'interno del continuum dello spettro autistico: se si tratta di un target vario, oppure rivolto perlopiù a persone ad alto o basso funzionamento. Non si può prescindere quindi dal complessivo progetto di vita della persona, età, genere, abilità, esigenze e obiettivi di benessere e qualità di vita.

Dallo studio condotto dello stato dell'arte, dei casi studio e dalle indagini effettuate sul campo, si possono quindi riassumere alcune indicazioni. Di seguito si svilupperanno quindi questee considerazioni sulla base di tre condizioni specifiche:

- residenza in famiglia, adattamento domestico;

- residenza temporanea "abilitante";

- residenza in autonomia, o cosiddetta "dopo di noi".

\subsubsection{Residenza in famiglia, adattamento domestico}

L'adattamento domestico é considerato come un prerequisito, poiché permette alla persona di continuare a vivere nella propria abitazione attraverso il supporto di una progettazione adeguata degli spazi e di ausili e tecnologie utili all'aumento dell'autonomia della persona stessa.

In particolare, all'interno dei percorsi di adattamento domestico sarà necessario, attraverso la valutazione degli spazi residenziali da parte di un'equipe multidisciplinare, relazionare i comportamenti e le criticità dell'ambiente, ovvero le condizioni di comfort e benessere per poter definire nello specifico degli interventi puntuali e mirati, contemplando le esigenze della persona con autismo, ma anche di tutta la sua famiglia che abita nello stesso alloggio.

Le interviste effettuate ai familiari di persone con autismo, o agli stessi adulti ad alto funzionamento o Asperger, sono un primo passo verso un'indagine mirata al comfort abitativo. Dai risultati delle indagini quali-quantitative si può affermare che gli aspetti maggiormente importanti della casa, da valutare e progettare nello specifico, sono:

- l'organizzazione e la distribuzione degli spazi funzionali della casa, al fine di favorire la routine ed il compimento delle azioni quotidiane; separare gli ambienti critici, distraenti a livello sensoriale (come cucina e bagno), migliorando la sicurezza e la fruibilità autonoma dell'alloggio; relazionare gli spazi in base alla loro funzionalità (bagno adiacente alla camera da letto personale, spazio dedicato indipendente per le proprie attività, complessità e ampiezza dello spazio giorno, ecc.). Nella Figura 01 ad esempio, si riporta uno studio di layout di abitazione privata tratta dall'ana- 


\section{Elena Bellini}

lisi progettuale di Magda Mostafa (Mostafa, 2014) ${ }^{589}$ : sono stati analizzati tre alloggi del Cairo sulla base della stimolazione sensoriale dei diversi spazi, ponendo particolare attenzione a bagno e cucina, come aree di maggiore stimolo e distrazione, e progettazione del layout specifico della camera da letto personalizzato in base alle esigenze sensoriali del bambino. In questo caso, si è scelto di posizionare la camera all'inizio dell'area notte per limitare il percorso da compiere, diminuendo le distrazioni. All'interno della stanza è stato posizionato il letto sul lato più protetto della stanza, sia rispetto ai rumori esterni, che da quelli interni (cucina). E' stata inoltre scelta la stanza più piccola, poiché favorisse il senso di contenimento e attaccato il letto al muro per sfruttare le proprietà tattili della parete e migliorare le criticità relative al sonno. La luce proveniente dalla finestra entra infine dal lato nord, quindi non forte e diretta. Questo rappresenta uno dei pochi esempi in letteratura di adattamento domestico, basato sulla distribuzione degli spazi funzionali sulla base della sensorialità introducendo altri aspetti, che seguiranno nei prossimi punti;

- una differenziazione degli spazi chiara per funzione ed attività. Nel caso studio di Seniors House (Hinnerup, Danmark), ad esempio, gli spazi dell'alloggio sono suddivisi per colore, per facilitare la comprensione dell'ambiente: la cucina in verde, racchiusa in una nicchia; il soggiorno bianco, suddiviso attraverso mobili su ruote, per rendere lo spazio flessibile, é caratterizzato da una differenza di altezza da cui proviene una luce zenitale, maggiormente gradevole poiché indiretta e diffusa in modo più uniforme; la camera in rosa, anch'essa suddivisa attraverso mobili su ruote al fine di rendere l'ambiente adattabile alle diverse necessità, come ad esempio se l'utente è costretto a letto;

- una dimensione dei diversi ambienti adeguata alla sua funzionalità specifica, ma anche alle particolari esigenze della persona, che potrebbe preferire ambienti maggiormente ampi, o al contrario contenuti, come risulta anche dalle interviste. Può essere utile quindi, ad esempio, mantenere una dimensione ampia per il soggiorno, che è uno spazio comune, anche differenziato per zone, mentre uno spazio più contenuto per la camera, dove si ha bisogno di una sensazione di contenimento;

- il mantenimento del controllo da parte della persona con autismo, sia visivo, che di strutturazioni degli spazi rispetto alle attività, poiché, come si è visto, rappresenta un punto importante per il comfort e l'abbattimento dello stress;

- la progettazione del soggiorno, come area maggiormente frequentata oltre alla camera da letto personale, affinché sia uno spazio ampio e luminoso, ordinato ed organizzato in modo da non sovraccaricare visivamente la persona; che offra la possibilità di regolare le stimolazioni sensoriali e, in particolare, luce, audio, temperatura e ventilazione. Lo spazio soggiorno deve essere progettato al fine di favorire la relazione con gli altri componenti della famiglia, ma prevedere anche un'area più raccolta e contenuta (un angolo, una porzione di stanza, una nicchia) dedicata al rilassamento ed eventualmente all'isolamento parziale all'interno dell'ambiente, dove si può

\footnotetext{
${ }^{589}$ Mostafa, M. (2014) Architecture for Autism: Application of the Autism ASPECTSSTM Design Index to Home Environments, in The International Journal of the Constructed Environment, Volume 4, Issue 2, April 2014, pp.25-38.
} 
ritrovare l'organizzazione di oggetti ed elementi ludici e di intrattenimento per la persona con autismo;

- la camera da letto é legata invece al mantenimento della privacy, all'autonomia della persona e alla necessità dimostrata di un ambiente dedicato esclusivo, che potrebbe essere rappresentato dalla stanza da letto, o ancora meglio, da un altro spazio indipendente nella casa. La camera da letto è legata profondamente al supporto rispetto ai disturbi del sonno, molto frequenti in casi di autismo, e deve permettere ad esempio la regolazione dell'illuminazione artificiale, la possibilità di oscurare completamente la stanza dalla luce del giorno, la regolazione di temperatura e ventilazione, ma anche elementi sensoriali che consentano un supporto nelle ore notturne, favorendo la stimolazione controllata e l'abbattimento dello stress. La posizione del letto è preferibile opposta alla porta, per non guardare l'ingresso, che potrebbe provocare ansia e agitazione; è da considerare inoltre la possibilità di affiancare il letto al muro, per offrire un ambiente più raccolto e di contenimento, come un'alcova, che potrebbe favorire anche una stimolazione di tipo tattile; è da valutare infine il posizionamento della stanza e del letto, nello specifico, sulla base degli spazi adiacenti e dell'eventuale provenienza di rumori dall'esterno o dall'interno. Un altro aspetto importante è la vicinanza del bagno, che potrebbe essere favorevole durante la notte, dato che in molti casi è usato frequentemente, ma anche per lo sviluppo delle abilità quotidiane di cura e igiene del corpo; in questo senso ha un'importanza anche il guardaroba e la sua strutturazione, come supporto al compimento delle azioni in modo indipendente. La progettazione della camera in ogni caso é legata alle esigenze a agli interessi specifici della persona e dovrà essere personalizzata in ogni dettaglio, valutando le attività e routine quotidiane;

- la progettazione della camera introduce inoltre un aspetto importante all'interno della famiglia, ovvero il rapporto della persona con autismo con eventuali fratelli o sorelle, o con gli stessi genitori. Si sono riportati vari esempi in cui il bambino con autismo dorme con uno dei due genitori, presenta delle criticità nella relazione con il fratello o la sorella, o non riesce a dormire da solo; una corretta progettazione degli spazi, incentrata sulle esigenze e gli obiettivi della famiglia, può essere parte di un percorso di sviluppo e miglioramento delle relazioni all'interno del nucleo familiare;

- un ambiente critico nella casa è rappresentato dalla cucina (tra gli spazi rilevati come maggiormente problematici, insieme al bagno), soprattutto per motivi di sicurezza e/o disturbo sensoriale, per cui sarà necessaria una progettazione condivisa dell'arredo al fine di garantire sicurezza e comfort, ma anche per sviluppare l'abilitazione e l'autonomia della persona nelle funzioni quotidiane. Le assistive technologies sono in questo senso molto utili, poiché permettono un controllo dei dispositivi e degli impianti e favoriscono l'autonomia della persona, attraverso il supporto e la facilitazione nell'utilizzo dei dispositivi stessi;

- anche il bagno, come anticipato, rappresenta una criticità poiché sovrastimolante, ma allo stesso tempo può esser valutato come una risorsa in ambito dell'adattamento e dell'integrazione degli spazi sensoriali all'interno del domicilio, che potrebbero in questo modo anche risolvere le criticità insite rispetto a questo ambiente. E' preferibile una stanza ampia, che consenta il movimento facile e comodo, anche sulla base delle eventuali difficoltà propriocettive; la doccia deve essere anche ampia e preferibilmente con piatto doccia a pavimento, per evitare scalini che potrebbe- 
ro essere causa di inciampo; è da valutare con la singola persona la presenza o meno dello specchio e la sua grandezza; per quanto riguarda la luce è sempre favorevole un'illuminazione regolabile e da valutare secondo la persona in colore ed intensità; sono da considerare infine tutti gli aspetti legati alla sicurezza, quali ad esempio la scelta dei materiali che siano pratici ed antiscivolo, ma anche l'eventuale integrazione di dispositivi e supporti a livello tecnologico, ad esempio per quanto riguarda la regolazione della temperatura dell'acqua, in base alle abilità della persona;

- all'interno del bagno e della cucina é inoltre da considerare l'integrazione di elementi di strutturazione per immagini, come PECS (Figura 02), sequenze o regole visive, o procedimenti step by step per favorire l'apprendimento delle autonomie domestiche;

- altri punti critici nella casa possono essere rappresentati dai corridoi, poiché talvolta rappresentano una causa di disagio, che dovranno essere ottimizzati nella casa e progettati in modo da essere il più possibile corti e funzionali. Sono esempi di soluzione applicativa interessante, la strutturazione dello spazio corridoio nel Sunfield Institute (Stourbridge West Midlands, UK), dove gli spazi di circolazione hanno funzionalità di ambienti comuni, sia attraverso le sedute, che attraverso spazi di ripostiglio per gli operatori, e la distribuzione planimetrica delle residenze di Sweetwater Spectrum Community \& Farm (Sonoma, USA), da cui si può percepire il limitato utilizzo degli spazi serventi, salvo un breve corridoio di accesso alle camere, completamente aperto sul cortile centrale;

- le scale ed i balconi/logge sono punti critici per la sicurezza, soprattutto da considerare sulla base dei disturbi propriocettivi e vestibolari, alla difficoltà nel percepire la profondità o ai problemi di equilibrio;

- i ripostigli, il guardaroba e le pareti attrezzate rappresentano ambiti di solito poco indagati, ma che invece possono risultare in questo caso molto importanti per la riduzione del sovraccarico sensoriale (soprattutto visivo), il mantenimento del controllo e l'organizzazione dell'ambiente quotidiano, per facilitare anche le routines e lo sviluppo dell'autonomia personale. La strutturazione dello spazio guardaroba (Figura 03), ad esempio, può permettere alle persona di apprendere a vestirsi da solo e rendersi maggiormente indipendente nelle azioni quotidiane;

- un altro aspetto, in questo senso, che sembra marginale ma può rivelarsi importante nello sviluppo delle abilità è la scelta delle tipologie di apertura e chiusura di porte e finestre, o degli stessi arredi e mobili contenitori, da un lato per la sicurezza (finestre ai piani alti, porte di ingresso all'abitazione, ecc.), dall'altro per la capacità di aprire e chiudere in modo indipendente; alcune persone potrebbero infatti avere delle difficoltà di manipolazione, ad esempio ad impugnare pomelli, o difficoltà a percepire la forza nell'apertura di scorrevoli, per cui è preferibile avere un blocco finale, ecc..;

- a livello di comfort, sono da considerarsi maggiormente importanti il comfort acustico (isolamento da e verso l'esterno e tra i diversi spazi della casa, in particolare per quanto riguarda la camera da letto, ed eventualmente negli spazi più ampi o con soffitti alti rispetto al riverbero); visivo (colori, textures, materiali, ma soprattutto illuminazione naturale e artificiale, differenziate e personalizzabili); olfattivo (ventilazione naturale e meccanica efficiente, possibilità di diffondere aromi se graditi e utili nel rilassamento dell'utente); termo-igrometrico (particolarmente importante 
poiché spesso non viene tollerato ad esempio il riscaldamento, come rilevato dalle interviste, per cui conviene valutarlo direttamente rispetto alle esigenze specifiche della singola persona; anche il riscaldamento a pannelli radianti, che solitamente si rivela utile per la particolarità di essere regolabile in modo differenziato in ogni stanza, talvolta potrebbe non essere tollerato);

- i materiali e i colori della casa sono da tenere di conto per il comfort, la sicurezza, la durabilità ed il benessere degli utenti; anche in questo caso non avrebbe senso definire quali siano più adatti, salvo in merito alla durabilità, poiché è necessaria una personalizzazione completa in accordo con le esigenze generali della famiglia. Si é visto, anche dalle interviste, come siano variabili le preferenze in questo ambito, da chi preferisce un ambiente completamente asettico, poiché favorisce calma e concentrazione, a chi al contrario preferisce l'uso di elementi vivaci e di colore; ma quale colore? Non è sempre vero, ad esempio, che nella casa i colori tenui o la mancanza di contrasti sono maggiormente favorevoli, poiché dipende dal contesto e dall'attività, ma soprattutto dalla persona;

- le luci sono un aspetto importante da considerare poiché è stata riscontrata una sensibilità particolare ed è stato rilevato come, per tutti gli intervistati, l'abbagliamento e la luce troppo forte, sia naturale che artificiale, solitamente provochino disagio. La luce naturale è sempre apprezzabile, ma deve avere la possibilità di essere regolabile nell'intensità e talvolta completamente oscurabile, come ad esempio nel caso della camera da letto (Figura 04), per cui è fondamentale in alcuni casi avere buio assoluto. La luce zenitale è spesso molto favorevole poiché indiretta, ma anche le vedute sono molto importanti per mantenere un contatto diretto tra interno ed esterno. Per quanto riguarda invece la luce artificiale, la migliore opzione è dare una varietà di punti luce e la possibilità di regolarli manualmente nell'intensità, ma anche nel colore. Anche rispetto al semplice "bianco" vi sono delle preferenze rispetto alla temperatura della luce; ad esempio, anche se solitamente si tende a dire che una luce diffusa e calda é migliore, talvolta si preferisce una luce fredda per il lavoro o la concentrazione. I sistemi di regolazione della luce, in colore ed intensità, sono ottimali; una luce colorata potrebbe talvolta essere preferibile, come stimolazione sensoriale, anche ad esempio per favorire il sonno;

- sono da considerare sistemi di supporto e assistive technologies integrate nell'abitazione per favorire l'indipendenza, il monitoraggio, la sicurezza, la regolazione degli stimoli sensoriali e l'adattamento in modo flessibile all'interno degli spazi abitativi.

$\mathrm{Nel}$ caso in cui si stia valutando dove scegliere la propria abitazione, sulla base delle esperienze raccolte e dello stato dell'arte, risulta sicuramente favorevole un edificio isolato rispetto al condominio, poiché mantiene livelli di privacy e comfort (ad esempio acustico) rispetto ai vicini, meglio ancora se prevede uno spazio esterno dedicato a giardino. In ogni caso, è da considerarsi importante anche lo sviluppo della relazione di vicinato e la possibilità di avere servizi primari in prossimità dell'abitazione, per mantenere lo sviluppo dell'indipendenza e della abilità quotidiane, nell'ottica di un'integrazione nella società comune. 


\subsubsection{Residenza temporanea "abilitante"}

Il tema della residenza può essere affrontato anche in rapporto a soluzioni di progetti abilità e abitare temporaneo, poiché permettono al giovane ragazzo con autismo di apprendere nuove capacità di vita indipendente e concorrono ad aumentare la qualità di vita della persona, in termini di autodeterminazione. In questo senso soluzioni interessanti, come quelle viste nei casi studio di Pordenone e di La Spezia, sono le possibilità di integrare in modo complessivo il progetto di vita, anche attraverso l'inserimento lavorativo. Tra le cose rilevate come più interessanti, all'interno del processo di indagine quali-quantitativa, si denota come gli ambienti di relazione con gli altri o le aree di lavoro risultino tra gli spazi maggiormente preferiti da parte delle persone con autismo, dimostrando quindi l'esigenza di stare con gli altri ed integrarsi nella società comune.

Residenze temporanee e "residenze abilità" sono ancora nuove come soluzioni di progetto, per cui si necessita di una sperimentazione in merito, che potrebbe rappresentare un futuro sviluppo della ricerca.

Come prima conseguenza, nella progettazione di una residenza per più persone con autismo, diminuisce il grado di personalizzazione, che si limiterà perlopiù agli spazi privati, e si rivelerà ancor più complesso nelle situazioni temporanee, dove gli ambienti vengono usati in successione da diverse persone. Oltre alle indicazioni generali, che poi si ritroveranno parlando di residenze permanenti per persone con autismo, si può dire che nel caso del "progetto abilità" la conformazione degli spazi deve andare a braccetto con la strutturazione del programma abilitativo e terapeutico. Il programma deve essere assolutamente personalizzato sull'individuo, pertanto questi spazi dovranno essere il più possibile flessibili ed adattabili. Allo steso tempo dovranno, come aspetto e conformazione, riportare all'ambiente "casa", familiare e accogliente, anche nei materiali, nei colori e negli arredi, che potrebbero essere personalizzati, ad esempio, nella stanza da letto, al fine di ampliare il senso di appartenenza. La dimensione della 'casa' deve essere appunto contenuta, per riportare all'idea di residenza familiare, pensata per 4-6 persone. L'impianto generale della struttura deve essere semplice e chiaro, diminuendo al massimo lo spazio servente a favore di spazi di vita e relazione ampi e ben proporzionati; sono da evitare, ad esempio, soffitti molto alti o ambienti troppo grandi e vuoti, che possono generare anche eco e riverbero. La struttura può prevedere un solo spazio 'casa', con la presenza di uno o due operatori, o diversi alloggi indipendenti, come nell'esempio di Seniors House, composto da alloggi minimi individuali, tutti connessi dall'interno, che diventa uno spazio di incontro, favorendo le relazioni con gli altri abitanti.

Il contesto preferibile è quello della piccola città o paese, dove ci possa essere la possibilità di avere uno spazio esterno verde, essere isolati rispetto ai vicini, ma mantenere un contatto diretto e continuo con la comunità sociale, integrandosi anche a livello lavorativo. E' importante quindi che tutti i servizi ed il lavoro siano facilmente e velocemente raggiungibili, anche a piedi o in bicicletta, per consentire alla persona di muoversi anche indipendentemente. Il rapporto con la natura è importante e terapeutico, ma in questo caso risulta di particolare importanza il rapporto con il contesto urbano. 
Gli ambienti, i materiali e i colori devono essere il più possibile neutri, poiché devono adattarsi a più persone e quindi essere il più possibile flessibili; in ogni caso non devono ricondurre ad un ambiente asettico, sanitario o istituzionale, quindi devono comunque essere caldi e accoglienti, come ad esempio l'uso del legno o di altri materiali naturali.

La luce naturale deve sempre essere presente, anche se regolabile, e la luce artificiale differenziata per i diversi spazi, flessibile e regolabile in colore ed intensità, come visto anche nell'ambiente di adattamento domestico.

Il comfort acustico deve essere valutato attraverso strategie di isolamento e abbattimento del riverbero, dato che in questo contesto potrebbero esserci ambienti più ampi e con una maggiore concentrazione di persone.

Si deve prevedere una ventilazione naturale e meccanica, anche per un comfort olfattivo, e, per quanto riguarda il sistema di riscaldamento e rinfrescamento, sono preferibili sistemi a pannelli radianti, poiché regolabili in modo separato nelle diverse aree della casa e migliori a livello di comfort.

La conformazione della struttura della residenza varia rispetto alle attività, ma non viene in ogni caso utilizzata per $24 \mathrm{~h}$, poiché è previsto l'inserimento lavorativo all'interno del percorso di abilitazione. Pertanto gli spazi maggiormente importanti sono da considerarsi: la camera da letto, come stanza personale e momento di privacy, relax e regolazione sensoriale; il soggiorno (e/o la sala da pranzo), come luogo comune incentrato nello sviluppo delle relazioni; la cucina, come laboratorio abilitativo; il bagno, come sviluppo di abilità personali, autonomia ed indipendenza.

A questo proposito, un ambito interessante è rappresentato dalla progettazione degli spazi comuni, al fine di favorire le relazioni e non far sì che la sera ognuno si"'rintani" nella propria stanza. Soluzioni intelligenti di progettazione di questi spazi, sono sicuramente la previsione di aree di socialità diversificata, poiché la comunicazione a piccoli gruppi è più semplice e tollerabile; soluzioni di 'partecipazione protetta' sono inoltre auspicabili per avere possibilità di auto-regolazione nei momenti di stress o disturbo sensoriale; un ambiente con differenze di altezza o materiale, diversi tipi di sedute e aggregabilità, arredi flessibili e modulari, é sicuramente utile, insieme ad una programmazione di attività che possano in qualche modo "creare gruppo". Un esempio interessante è il già citato Seniors House (Hinnerup, Denmark) dove gli spazi comuni vengono usati come luogo di relazione, favoriti dall'uso di aree di "partecipazione protetta": nicchie differenziate in colori e materiali, allestite con sedute, luci e piante, collocate come aree di sosta lungo il percorso; l'affaccio diretto dell'alloggio attraverso un'apertura a nastro nella cucina sui collegamenti interni; l'utilizzo di una porta con una doppia apertura, totale ad anta e a metà, con uno sportello superiore apribile, che permette di guardare l'esterno prima di uscire dall'appartamento, dando la possibilità di prepararsi e auto-regolarsi.

Per quanto riguarda la camera, valgono tutte le indicazioni fornite per la camera da letto in ambiente domestico e familiare, considerando però che può essere usata in successione da più persone (anche se singola). Una soluzione efficace potrebbe essere quella di avere spazi adattabili sulla base delle preferenze sensoriali attraverso il supporto della tecnologia, come se fossero piccole sensory room; questo può rappresentare inoltre un dispositivo utile per l'autoregolazione o l'abbattimento dello 


\section{Elena Bellini}

stress, soprattutto la sera, rientrando a casa dopo la giornata di lavoro o di attività (molto importante, come visto dalle interviste) e come supporto per il sonno.

E' auspicabile un bagno per ogni stanza, per mantenere la privacy, soprattutto considerando la convivenza con altre persone adulte, e facilitare l'accesso nelle ore notturne; restano valide tutte le indicazioni presentate per l'ambiente domestico, oltre alla grande importanza in questo caso dei supporti visivi come programma di abilitazione integrato.

Per quanto riguarda la cucina, infine, è bene che sia uno spazio molto ampio e che presenti più postazioni di lavoro, poiché può essere un momento importante di relazione e abilitazione. Anche in questo caso l'integrazione di supporti visivi risulta fondamentale, anche come inclusione rispetto alle diverse abilità; sarebbe interessante riuscire ad integrarla anche a livello digitale, ad esempio attraverso sistemi che aiutano nella preparazione di alcuni alimenti molto semplici, con indicazioni step by step. L'uso delle assistive technologies è inoltre fondamentale come supporto alla sicurezza, attraverso la regolazione e l'automazione di tutti i dispositivi elettrici. In ogni caso, questo programma deve considerarsi temporaneo, quindi è importante anche valutare il passaggio e la transizione verso la vita autonoma.

In generale, si dovrà comunque prevedere un adeguamento della struttura per la sicurezza (apertura di finestre e porte, vetri infrangibili, arredi durevoli, funzionali, senza spigoli vivi, ecc.), in modo che gli utenti possano sempre muoversi autonomamente, senza avere rischi ma senza essere continuamente controllati. In questo senso un sistema a telecamere è preferibile rispetto al controllo continuo e visivo da parte degli operatori, che devono essere parte integrante della comunità, senza creare impatto sugli utenti, ma solo supporto nei momenti di crisi o difficoltà.

\subsubsection{Residenza in autonomia, o cosiddetta "dopo di noi"}

Come ultimo sviluppo dell'ambito residenziale, vi è poi la soluzione abitativa in autonomia, o cosiddetta 'dopo di noi'. Sono da preferire in questo senso soluzioni di vita indipendente integrata, attraverso la conformazione di diversi alloggi indipendenti, come nel caso di Seniors House e Sweetwater. In entrambi è importante l'estrema flessibilità, poiché prevedono un'evoluzione nel tempo sulla base delle proprie abilità, ma anche del semplice invecchiamento. Sono entrambe interessanti inoltre poiché mirano alla vita indipendente in un contesto di relazione comune, che non causi mai l'isolamento della persona. Nel caso invece in cui si preveda una soluzione di co-housing in un'unica struttura abitativa, è da prevedere per poche persone, 4 o 6 al massimo, allontanandosi dal concetto istituzionale tipico delle residenze assistite, ma riportare al contrario ad una soluzione familiare e una dimensione di 'casa'.

La natura ed il rapporto con l'ambiente rurale è stato dimostrato molto positivo in molte situazioni, anche come soluzione di integrazione lavorativa, ma è bene che sia realizzata in un ambiente comunque prossimo alla vita urbana, anche trattandosi di un piccolo paese, che per dimensione ben si associa con situazioni di relazione sociale protetta.

In sintesi, tantissimi sono i temi su cui confrontarsi all'interno del contesto abitativo, tali che necessiterebbero un lavoro a parte concentrato solo su questo; si posso- 
no in ogni caso riassumere alcuni requisiti specifici rilevati all'interno del percorso di ricerca, ovvero:

- il comfort acustico: è preferibile un'abitazione isolata rispetto ad un appartamento in condominio, anche per avere maggiore privacy; è necessario un adeguato isolamento contro i rumori esterni e interni e una riduzione del riverbero, specialmente negli spazi più ampi;

- il comfort luminoso: è preferibile un ambiente con molta luce naturale, ma con la possibilità di poter regolare l'apporto solare e anche di oscurare completamente l'ambiente, quando necessario, come per rilassarsi e "auto-regolarsi" o per dormire (necessario talvolta il buio totale); sono preferibili luci calde e diffuse, regolabili nell'intensità e se possibile anche nella gradazione di colore; è preferibile l'utilizzo di diversi punti luce; sono da eliminare, in ogni caso, l'utilizzo di neon o luci fluorescenti; da indagare rispetto al singolo caso l'utilizzo di luci colorate;

- il comfort visivo: l'ambiente "casa" deve poter essere "sotto controllo" da parte della persona con autismo; l'ordine è in generale preferibile e soprattutto non avere un sovraccarico di informazioni visive, avendo la possibilità di riporre gli oggetti in mobili, spazi o elementi a scomparsa; colori e dettagli sulle finiture sono da usare in modo molto calibrato, avendo un equilibrio tra ambiente caldo ed accogliente e ambiente neutro e distensivo, evitando colori troppo accesi o contrasti troppo forti, che potrebbero creare disagio, o comunque a lungo stancare;

- scale, logge, balconi sono da trattare con attenzione, poiché possono rappresentare delle criticità sulla base del disagio rispetto all'altezza o all'instabilità data, ad esempio, dalla discesa delle scale (in particolare se a chiocciola o poco ampie);

- la ventilazione degli ambienti è importante, anche a livello olfattivo;

- è da valutare inoltre l'utilizzo di diversi sistemi di riscaldamento o raffrescamento, soprattutto poiché molte persone con autismo hanno particolare sensibilità (in particolare verso il caldo) e quindi potrebbero non sopportare o comunque preferire alcuni dispositivi rispetto ad altri, oppure potrebbero provare disagio dovuto al rumore o al ronzio dei dispositivi. I sistemi a pannelli radianti sono solitamente considerati favorevoli in questo senso poiché sono regolabili in modo autonomo in ogni stanza, sono sicuri, non sono visibili e non fanno rumore;

- la distribuzione e la strutturazione degli ambienti è da considerare in base ad un progetto di vita della persona, alle routines e alle attività specifiche giornaliere; se ne consiglia la valutazione multidisciplinare e attraverso un determinato piano terapeutico e "abilitativo"; un altro aspetto da considerare è inoltre la relazione tra i diversi componenti della casa ed eventuali ambienti che possono rivelarsi critici (soprattutto la cucina), valutando le strategie di sicurezza e filtro rispetto a questi spazi; in ogni caso sono comunque preferibili ambienti separati e diversificati per diverse funzioni, eventualmente anche distinti attraverso caratteri, materiali o colori, senza sovraccaricare a livello visivo;

- sono da evitare corridoi stretti e molto lunghi, o ambienti troppo angusti;

- é da valutare il dimensionamento generale degli ambienti rispetto all'utilizzo dei diversi spazi e alla concentrazione di persone. Gli spazi comuni è bene che siano ampi e variegati nella composizione, per consentire diversi gradi di socialità agli utenti e non sovraccaricarli a livello sensoriale, mentre la camera da letto è spesso 


\section{Elena Bellini}

preferibile di dimensione contenuta, per ricreare una sorta di nido o alcova dove sentirsi contenuti;

- anche arredi e complementi saranno da valutare sulla base delle abilità della persona, come ad esempio sistemi di apertura di porte o mobili, sistemi di strutturazione degli spazi ripostiglio o guardaroba, ecc. con particolare attenzione a bagno e cucina;

- l'integrazione di dispositivi di assistive technologies è da considerarsi come una risorsa importante nella gestione dell'ambiente casa, come facilitazione e supporto, ma anche come strategia di personalizzazione dell'ambiente;

- l'utilizzo di sistemi sensoriali terapeutici (Figura 05) risulta di particolare importanza, poiché permette di avere la possibilità di ricalibrarsi o "regolare i propri sensi", anche in momenti di crisi o sovraccarico sensoriale, o nella notte per rispondere ai disturbi del sonno; questo può avvenire attraverso uno spazio dedicato, o l'inserimento delle tecnologie di stimolazione sensoriale e regolazione dell'ambiente all'interno dell'abitazione, in alcune stanze specifiche, come nella camera da letto o nel bagno;

- sono da favorire, quando utili e/o necessarie, sistemi di comunicazione facilitata e supporto alle abilità quotidiane attraverso immagini (step by step, regole visive, pecs, ecc.);

- è da favorire l'utilizzo di spazi esterni ed in particolare degli ambienti naturali, come giardini o orti, considerati anche terapeutici; sono di particolare interesse $\mathrm{i}$ giardini sensoriali (Figura 06).

\subsection{L'ambiente educativo}

Per quanto riguarda la scuola, condivido a pieno l'idea di inclusione, per cui ogni bambino debba avere gli stessi diritti all'istruzione in un ambiente comune e non separato, affinché l'alunno con DSA (o con altre disabilità) non sia visto come un ospite nella scuola e nella classe, ma come parte integrante della stessa (Cottini, 2009 ${ }^{590}$; Cottini, 2011 $1^{591}$ ). Allo stesso tempo risulta necessaria una sperimentazione sulla trasformazione e l'adeguamento dell'ambiente scolastico tradizionale in funzione delle necessità dei bambini con autismo, anche per comprendere se un contesto facilitato possa in realtà risultare utile al potenziamento dell'apprendimento di tutti gli studenti, attraverso l'applicazione dei modelli già sperimentati e convalidati a livello internazionale in altri ambiti di 'scuole o classi speciali' all'interno del contesto di scuola inclusiva. Come visto, sono state fatte, o sono in corso, ricerche e sperimentazioni sull'adeguamento dei programmi formativi, dell'organizzazione e della partecipazione della classe al fine di migliorare la qualità dell'inclusione stessa, ma vi è ancora poco per quanto riguarda l'adeguamento degli spazi e la valutazione, in questo senso, dei requisiti che potrebbero rilevarsi utili per tutta la popolazione studentesca.

\footnotetext{
${ }^{590}$ Cottini, L. (2009) Il Bambino con autismo in classe. Quattro parole chiave per l'integrazione, Giunti, Firenze.

${ }^{591}$ Cottini, L. (2011) L'autismo a scuola. Parole chiave per l'integrazione, Carocci, Roma
} 
Anche in questo caso sarebbe necessario un lavoro di ricerca totalmente concentrato su questo ambito e può rappresentare un interessante sviluppo della ricerca, anche attraverso l'utilizzo dei contatti sviluppati durante il percorso dottorale.

Si possono comunque riassumere alcuni requisiti specifici rilevati all'interno del percorso di ricerca, che comportano una particolare attenzione per le esigenze dei bambini/ragazzi con DSA e che in ogni caso sono convergenti con i regolamenti generali degli ambienti scolastici:

- il vecchio modello di classe basato sulla lezione didattica frontale è ormai superato per cui sarà sicuramente utile una flessibilità degli spazi ed una modularità degli arredi, al fine di adattare lo spazio in base alle diverse attività (individuali, piccoli o grandi gruppi);

- la strutturazione del layout degli spazi, sulla base del percorso educativo del singolo bambino e delle diverse attività, è da progettare per ogni singolo caso, attraverso una collaborazione multidisciplinare delle competenze psicologiche, del corpo docente, ma anche della famiglia, come mediatore rispetto alle esigenze specifiche del bambino e facilitatore nella comprensione delle reazioni e dei suoi comportamenti. L'esempio della classe speciale progettata da Magda Mostafa all'interno della sperimentazione presso l'Advance Society for Developing Skills of Special Needs Children in Cairo (Figura 07), riproduce un'organizzazione degli spazi sulla base della sequenza di attività, secondo una routine, attraverso la divisione in 'stazioni', anche con divisioni fisiche e visive; è compresa in questa strutturazione anche un escape space, per i momenti di riequilibrazione sensoriale;

- è maggiormente interessante integrare diversi spazi all'interno dello spazio classe, anche attraverso una forma articolata che preveda uno spazio più raccolto ed isolato, che consenta percorsi 'one to one' all'interno della classe stessa, piuttosto che l'utilizzo di stanze separate per il sostegno, che non è favorevole per l'inclusione del bambino. Un esempio interessante è la Northen School for Autism a Reservoir (Australia), dove l'aula è flessibile e prevede diversi spazi per lo studio e le altre attività, oltre ad un'area separata più raccolta per l'apprendimento individuale o un'area di quiete nei momenti necessari di isolamento e riequilibrazione sensoriale. Dalla sala lavoro si accede inoltre ad un cortile esterno privato e protetto, dove i bambini possono giocare in modo indipendente, ma sicuro;

- di conseguenza ai primi punti, dovrà essere valutata la dimensione, la forma $\mathrm{e}$ la distribuzione che si riveli maggiormente favorevole nello spazio classe, al fine di ottimizzare la superficie dell'aula disponibile. Una dimensione maggiore dell'aula risulta favorevole poiché dà la possibilità di strutturare l'ambiente in diverse zone di lavoro e aumentarne la flessibilità, oltre a non creare sovraccarico di stimoli e sovraffollamento. In questo senso, un numero inferiori di bambini può essere di supporto, per aumentare i livelli di concentrazione e favorire l'apprendimento;

- l'ambiente di ingresso nella scuola è il punto maggiormente critico, poiché è l'area dove c'è maggiore affluenza di bambini, che può generare confusione e disorientamento. Bisognerà valutare quindi non solo l'ampiezza, ma anche l'organizzazione e l'arredo di questo spazio, al fine di non sovraccaricare a livello sensoriale, prossemico e propriocettivo. Nella Forbush School (Baltimora) (Figura 08 - 09), ad esempio, lo spazio di ingresso dell'istituto scolastico è ampio, per favorire prossemica e propriocezione, ed è definito nelle volumetrie dai diversi colori; la main street 


\section{Elena Bellini}

conduce da quest'area ai diversi ambienti dell'edificio attraverso linee curve e colori vivaci ma non troppo forti, mentre l'illuminazione è zenitale, sia artificiale (luci testate non a neon, che non producano sfarfallio), che naturale (lucernari circolari a soffitto);

- allo stesso modo i collegamenti funzionali possono rivelarsi critici, quindi dovranno essere ampi e non troppo lunghi per evitare il sovraccarico sensoriale; la presenza di spazi come quiet room o escape space in quest'area potrebbe rilevarsi utile per superare questi momenti e facilitare le relazioni tra i diversi bambini. Talvolta può essere utile collocarsi in un'aula il più possibile vicino all'ingresso, o a piano terra, per facilitare il momento di arrivo a scuola e i collegamenti, anche in indipendenza. Può essere utile inoltre collocarsi in un'aula che abbia servizi igienici vicini, per facilitare l'accesso, anche rispetto ad alcune criticità legate ad eventuali comorbidità. La strutturazione planimetrica della Forbush School (Baltimora) (Figura 08), ad esempio, è interessante poiché si ha un sistema distributivo unico da cui entrare nelle aule e agli altri servizi della scuola, posti sul lato opposto; l'aula è conformata in modo da avere diversi spazi per le attività singole o di gruppo, spazi ripostiglio, una ressource room condivisa tra due diverse aule e un bagno a cui si accede direttamente. Risulta interessante anche l'esempio della New Struan School ad Alloa (UK) (Figura 10 - 11), dove l'atrio, dalla forma di una grande "strada", rappresenta il cuore sociale dell'edificio ed è importante per sviluppare l'orientamento ed il wayfinding, mantenendo una chiara visibilità dell'intera scuola e promuovendo il movimento indipendente tra le diverse aree. La street di collegamento è inondata dalla luce naturale attraverso un atrio vetrato e ben ventilato; la sua ampiezza favorisce inoltre la prossemica e la propriocezione, dando la possibilità a gruppi di bambini di fruirne contemporaneamente senza scontrarsi o avere la sensazione di caos e confusione; i muri curvi conducono da un'area all'altra, riducendo il numero di angoli acuti, angoli ciechi e porte nascoste, diminuendo quindi il livello di ansia e favorendo gli spostamenti. Lo spazio è arricchito dai disegni dei bambini fatti a scuola, per rendere lo spazio personalizzato e familiare, opposto all'idea di istituzionalizzazione;

- un ambiente di filtro tra corridoio e classe può essere inoltre utile per ricalibrarsi, concentrarsi e prepararsi all'attività di apprendimento; potrebbe essere uno spazio ricavato in corrispondenza dell'ingresso che prevede anche un piccolo guardaroba ed alcune sedute, diventando anche un momento di relazione e scambio, come nella New Struan School di Alloa (UK), dove si accede alle aule dall'atrio attraverso spazi di soglia, definiti "baie" (Figura 11), che funzionano appunto da filtro; i pannelli di vetro su ogni porta delle aule danno inoltre la possibilità al bambino di guardare all'interno dello spazio classe, prepararsi ed assimilare le informazioni prima di entrare;

- comfort visivo: al fine di limitare il sovraccarico visivo, è necessario un ambiente che sia il più possibile neutro, sia nei colori, che nei materiali, che negli elementi decorativi o comunque applicati a parete; spazi adeguati di ripostiglio consentono di limitare il sovraccarico visivo e quindi la distrazione del bambino; possono essere condivisi anche tra diverse aule, ponendosi al centro di più strutture, in modo flessibile ed adattabile;

- comfort luminoso: è preferibile una luce naturale zenitale o comunque indiretta, la possibilità di oscurare, regolare o filtrare la luce del sole, anche in base all'o- 
rientamento. Si dovrà prevedere inoltre l'eliminazione di luci fluorescenti o neon (spesso usate in ambienti scolastici), benché una luce fredda sia forse preferibile per la concentrazione, come negli ambienti di lavoro;

- comfort acustico: uno tra gli aspetti maggiormente importanti è l'acustica, sia come protezione dai rumori esterni che interni, ma anche e soprattutto dal riverbero, non solo nella classe, ma anche negli ambienti comuni (palestre, piscine, corridoi, sale mensa, ecc.), per cui si dovrà prevedere un'ottima prestazione di isolamento $\mathrm{e}$ qualità acustica del suono, soprattutto nelle aree di massima concentrazione;

- comfort termo-igrometrico: è preferibile la possibilità di controllare la temperatura nelle diverse aree, utilizzando sistemi radianti a pavimento, che sono anche maggiormente sicuri, non visibili e non rumorosi (ronzio o ventilazione possono creare disagio);

- la dotazione di spazi sensoriali come una quiet room o una sensory room è stata rilevata utile non solo per l'autismo, ma anche per i disturbi del neurosviluppo, disabilità intellettiva in generale, $\mathrm{ADHD}$, stress o depressione, e come supporto e potenziamento dello sviluppo e dell'apprendimento del bambino neurotipico. Questi spazi possono essere direttamente connessi alla classe o usati come spazi di servizio comune a tutta la scuola;

- l'integrazione di sistemi tecnologici di supporto alla didattica può essere di supporto all'apprendimento di tutti i bambini e favorire anche l'inclusione e l'integrazione;

- l'integrazione di sistemi di supporto visivi, sia per l'orientamento all'interno della struttura (colore, grafiche, immagini), che come supporto e potenziamento all'apprendimento durante la didattica. Questo sistema potrebbe anche in questo caso rivelarsi utile per tutti i bambini, soprattutto nei più piccoli, e potrebbe rappresentare un sistema intelligente di integrazione ed inclusione;

- dallo stato dell'arte non si rilevano particolari raccomandazioni in merito alla sicurezza in casi di emergenza, salvo affermare che si confermano le vigenti normative antincendio. In ogni caso, sarebbe utile poter utilizzare sistemi di allarme vocale, invece che sonoro, al fine di non turbare e agitare ancor più il bambino/ragazzo, ma fornendogli una spiegazione di ciò che sta succedendo. Il posizionamento della classe a piano terra, vicino all'ingresso o ad un'uscita di sicurezza, può favorire la riduzione dei tempi di emergenza e quindi dello stress correlato.

Una scuola ben progettata prevede molte delle indicazioni considerate come miglioramento della qualità dell'apprendimento di tutta la popolazione studentesca, salvo porre una particolare attenzione ad alcune esigenze specifiche legate ai bambini/ragazzi con autismo, che andranno valutate attraverso il Piano Educativo Individualizzato, redatto in collaborazione di insegnanti, equipe medica, esperti del settore e genitori, ma che possono essere comunque facilmente integrate in un contesto educativo che sia dinamico e flessibile.

\subsection{L'ambiente sanitario}

Nell'ambiente sanitario non è possibile arrivare ad un livello di personalizzazione basata sulla singola persona per ovvi motivi, inerenti le problematiche sanitarie emergenti sullo specifico carattere del paziente. Valgono comunque per l'autismo gli 


\section{Elena Bellini}

approcci al progetto dell'ospedale centrati sulle esigenze del paziente, sull'umanizzazione degli spazi, sulla riduzione dello stress, sul rispetto dei ritmi di privacy e cura. Si possono evidenziare all'interno di questi approcci quei requisiti volti a ridurre il disagio della persona e supportarne l'accessibilità, che nel caso dell'autismo hanno importanza a tutti i livelli di età e gravità.

All'interno dello studio dello stato dell'arte e dei casi studio si sono riscontrati alcuni obiettivi progettuali ricorrenti, che si possono riassumere in:

- umanizzazione degli ambienti ospedalieri, al fine di confrontarsi con un contesto il più possibile familiare ed assimilabile ad un ambiente domestico;

- abbattimento dello stress, soprattutto nelle situazioni di emergenza ospedaliera, ma anche in generale riguardo all'accesso ai processi sanitari, diagnostici, ambulatoriali, fino al ricovero, poiché l'ambiente sanitario rappresenta una vera e propria fonte di agitazione e ansia nella persona con autismo. Come si è visto infatti, anche dai risultati delle indagini quali-quantitative, il Pronto Soccorso, o in generale gli ambienti sanitari, sono considerati tra gli ambienti maggiormente critici in casi di autismo, per la loro imprevedibilità, difficoltà di comprensione, presenza di tante persone sconosciute in ambienti altrettanto sconosciuti, ma anche per l'elevata concentrazione di stimoli a livello sensoriale che vanno a bombardare e aumentare il livello di arousal della persona;

- favorire l'orientamento e la comprensione all'interno degli spazi ospedalieri, per aumentare la prevedibilità dell'ambiente, anche attraverso programmi di training e formazione, ma anche consentire il wayfinding e la fruibilità degli spazi;

- potenziare le soluzioni di comunicazione tra pazienti, famiglia e operatori/medici, anche attraverso immagini e sistemi tecnologici di interazione.

Attraverso l'analisi dello stato dell'arte e dei diversi casi studio si è cercato quindi di mettere in evidenza quelle soluzioni ad oggi utilizzate, specificatamente per l'autismo, anche se non molto frequenti ancora, oppure in generale soluzioni efficaci in ambito sanitario che potrebbero evolversi e rappresentare soluzioni di comfort anche in casi di Disturbo dello Spettro Autistico. Si è guardato soprattutto ad ospedali pediatrici poiché presentano una particolare attenzione al paziente e alle sue fragilità e vi sono esempi molto interessanti di umanizzazione degli ambienti ospedalieri e soluzioni di orientamento e wayfinding per colore e immagini.

Si specifica, inoltre, che tutte le soluzioni presentate sono state rilevate in ambito ospedaliero, ma che potrebbero essere utili anche rispetto ai servizi sanitari distribuiti sul territorio, come poliambulatori o case della salute. Anche attraverso le interviste sono state evidenziate molte criticità rispetto ai luoghi sanitari anche in ambito odontoiatrico, o ginecologico, o in merito ad altre visite specialistiche, che spesso si rivelano necessarie. Un ambiente adeguato in questo senso fornirebbe un supporto importante nel favorire l'accesso alla cura e, più in particolare, la prevenzione del paziente, riducendo anche le situazioni di emergenza e criticità ad esse relative.

Si può definire quindi un ambiente sanitario autism-friendly quella struttura che riesce a ridurre lo stress del paziente e prendersi carico dei suoi "bisogni speciali", che non prevedono servizi sanitari specifici, quanto piuttosto un adeguamento degli spazi e una formazione del personale.

In particolare, si possono considerare soluzioni efficaci di progettazione di ambienti sanitari per l'autismo: 
- uno spazio di attesa dedicato, particolarmente utile nelle situazioni di emergenza dove i livelli di stress e agitazione sono molto alti, ma anche in ambito poliambulatoriale o di diagnostica. Sono da considerarsi particolarmente favorevoli spazi neutri, che riducano la stimolazione sensoriale del paziente, o meglio che diano la possibilità al paziente stesso di autoregolarla, anche attraverso il supporto di assistive technologies o l'integrazione di sistemi ed ambienti sensoriali, come ad esempio: la sensory room Sea Life a Brighton (Figura 12); le strategie dell'Emma Children's Hospital, dove l'area di attesa prevede diverse soluzioni di gioco, interazione, sedute e stimolazione sensoriale (Figura 13); del Nemour Children's Hospital (Orlando, USA), dove è stata inserita un'area di interazione e gioco sensoriale all'ingresso (Figura 14); o dell'Evelina Children's Hospital (London, UK), dove è stato installato un sistema di proiezione interattivo che permette di giocare con il colore come se si dipingesse in modo virtuale (Figura 15). Questi ambienti, integrati alla tecnologia, permettono di personalizzare lo spazio sulla base delle esigenze specifiche della persona, in estrema flessibilità e adattabilità, anche in una situazione in cui il servizio viene utilizzato in modo differenziato da una grande varietà di utenza;

- un ambiente di visita dedicato, particolarmente utile nelle situazioni di diagnostica, come anche un semplice prelievo venoso, con le stesse caratteristiche dell'ambiente di attesa, pur integrate con le dotazioni mediche necessarie. L'integrazione degli apparati medicali è da considerarsi in questo caso molto utile, anche attraverso soluzioni di pareti attrezzate, in modo da non impattare e spaventare il paziente, come ad esempio all'interno del programma REACH del Nemours Children's Hospital. L'integrazione di sistemi sensoriali è inoltre utile sia per l'abbattimento dello stress, che per la distrazione del paziente, o per la collaborazione, offrendo la stimolazione sensoriale come "ricompensa" a seguito dell'intervento medico. Il metodo di interazione con il paziente è da condividere con il genitore o caregiver, che rappresenta in questo caso una figura fondamentale di facilitatore, conoscendo in modo approfondito il paziente. Questo tipo di spazi dedicati possono rivelarsi utili non solo in ambito ospedaliero, ma anche in situazioni ambulatoriali, come miglioramento della rete di servizi sul territorio, che possono rilevarsi particolarmente critici in questo senso, come ad esempio il dentista, il ginecologo, ecc.. ma anche lo stesso prelievo sanguigno;

- la conformazione degli ambienti interni al fine di favorire l'umanizzazione degli spazi ospedalieri, come si riporta nella letteratura generale legata al comfort sanitario. A questo proposito, come unica differenziazione, si pone l'attenzione sulla conformazione degli spazi, l'uso del colore o di effetti luminosi particolari che, se eccessivi, potrebbero causare un sovraccarico di stimoli, aumentando il livello di arousal del paziente. E' da favorire in questo senso un ambiente neutro, caratterizzato da interventi puntuali di colore o arredo, sulla base della funzionalità e dell'orientamento; l'utilizzo di luci calde, diffuse ed uniformi, possibilmente provenienti dall'alto o con raggio luminoso indiretto; evitare l'utilizzo di neon o luci fluorescenti; evitare l'uso di materiali riflettenti, sia a parete, che in particolare a pavimento, poiché potrebbe creare disagio nella fruibilità degli spazi; favorire la ventilazione e la possibilità di avere ambienti inodori; favorire l'ampiezza degli spazi, e dei collegamenti in particolare, per promuovere prossemica e propriocezione e ridurre il senso di 
stress dovuto al sovraffollamento di persone; favorire il comfort acustico ed in particolare l'eliminazione di eco e riverbero.

- sistemi di orientamento e wayfinding attraverso l'utilizzo di colore e grafiche illustrative, che differenzino le diverse aree e rendano comprensibile il funzionamento della struttura ed il rapporto della persona rispetto al contesto. L'uso di immagini e simboli grafici, piuttosto che di colori, permette di rendere la comprensione 'universale' e favorire anche l'inclusività della persona. Queste informazioni visive devono essere coordinate e diventare parte integrante dello spazio, senza sovraccaricare l'utente, ma anzi caratterizzandolo ed evidenziandolo dove serve. All'interno dei casi studio rappresentano esempi significativi e particolarmente riusciti come: l'Evelina Children's Hospital, dove i percorsi di distribuzione all'interno dei diversi reparti hanno colorazioni neutre, evidenziando l'ascensore centrale di colore rosso ed il sistema di grafiche, che è stato differenziato per piano attraverso l'utilizzo di una "gang" di personaggi e "paesaggi" (Figura 16 e 17), per favorire la riconoscibilità di ogni diverso reparto e quindi l'orientamento e la comprensione, oltre a promuovere l'umanizzazione dell'ambiente ed il gioco; e l'Emma Children's Hospital, dove è stato realizzato un sistema di grafica integrata che coordina un sistema di riconoscimento per immagine, simbolo, colore e grafica di riferimento (Figura 18), differenziato sulla base delle diverse funzioni e reparti e collocato a terra, in alto o a parete sullo sfondo neutro degli spazi di distribuzione (Figura 19); ogni camera ha inoltre a terra un riquadro di colore che esce dal perimetro della stanza, evidenziandosi nel corridoio dove è stato posto anche un grande numero di riconoscimento (Figura 18);

- sistemi di privacy e di relazione controllata con il contesto esterno e le altre persone, come l'esempio interessante del programma REACH al Nemours Children's Hospital;

- sistemi di organizzazione della stanza di degenza, preferibilmente ad uso singolo e con bagno privato nella stanza, che promuovano la strutturazione dello spazio in modo flessibile, che dia la possibilità di sviluppare diverse attività e non solo lo stare nel letto; che permetta di mantenere una presenza costante di un familiare; che preveda una parete attrezzata per l'integrazione dei sistemi medicali e magari anche un letto per il familiare stesso; che preveda uno spazio filtro adeguato a mantenere privacy e comfort visivo e acustico rispetto agli spazi comuni o di collegamento, o altre soluzioni, attraverso la direzione delle vedute, la distribuzione ed il layout delle stanze, anche in rapporto agli altri spazi ospedalieri, che offra magari anche l'integrazione di sistemi di stimolazione sensoriale. La stanza di degenza dell'Evelina Children's Hospital, ad esempio, è una stanza singola, dove gli impianti medicali sono stati completamente integrati e nascosti in una parete attrezzata dove è possibile anche aprire un letto per uno dei due genitori; la stanza ha colori neutri salvo la parete attrezzata, dove vengono usati colori vivaci ma non forti; un grande numero favorisce la riconoscibilità dello spazio; è importante inoltre il rapporto con il contesto, pur mantenendo la privacy e favorendo la tranquillità ed il comfort acustico, ponendo l'affaccio della stanza verso l'esterno invece che verso l'atrio e dando la possibilità di schermare l'apertura, in caso di necessità di privacy; l'illuminazione, sia naturale che artificiale, è diffusa. Un altro esempio interessante è la stanza di degenza del Nemours Children's Hospital, Orlando (USA), dove si ha una varietà degli spazi all'interno della camera, in modo da poter svolgere altre attività oltre a restare a let- 
to; un sistema di luci colorate permette di abbattere lo stress e favorire la stimolazione sensoriale controllata; il sistema di illuminazione crea una luce diffusa ed è possibile regolare l'illuminazione naturale attraverso schermature orizzontali esterne; le colorazioni neutre inoltre sono predisposte in modo da non sovraccaricare a livello visivo. L'esempio del Pronto Soccorso di Pordenone è interessante invece poiché è stata predisposta una stanza dedicata alle persone con autismo (Figura 18), per favorire una migliore assistenza specifica; lo spazio è fondato sull'assenza di stimoli: l'ambiente è completamente bianco, tutte le strumentazioni e gli impianti medicali sono nascosti all'interno della parete; la luce naturale è schermata dall'interno per un maggiore comfort visivo.

All'interno delle stanze di degenza sarebbe inoltre utile poter garantire una personalizzazione attraverso riferimenti familiari e a materiali, come ad esempio lenzuola che diano un comfort tattile, o arredi che promuovano il rilassamento. Sarebbe infine utile prevedere la maggior parte degli interventi medici all'interno della stanza, al fine di restare in un contesto 'protetto' e favorevole, anche attraverso sistemi organizzativi che possano prevedere la concentrazione di più professionisti in tempi brevi, attraverso protocolli specifici, come ad esempio il caso DAMA;

- sistemi di tecnologia comunicativa per immagini attraverso supporti tecnologici che facilitino l'interazione tra paziente e operatore, prevedano sistemi d'interazione del paziente con il luogo di cura, come la visione degli spazi e la comprensione dei dispositivi prima di effettuare gli interventi medici, ancor meglio se coadiuvati da un percorso di training e formazione precedente, come il programma utilizzato dalla Fondazione Bambini e Autismo di Pordenone (Fgura 21). Un esempio riuscitissimo di questo è il caso studio dell'Evelina Children's Hospital, dove c'è inoltre una corrispondenza e un'interazione tra tecnologia, grafica comunicativa e spazi sanitari (Figura 22 - 23 - 24).

Si è scelto quindi di focalizzare l'attenzione su questo ambito, all'interno del percorso di ricerca, poiché innovativo dal punto di vista non solo italiano, ma anche internazionale, presentando ancora pochi e recenti esempi di adeguamento degli spazi e programmi specifici di assistenza per l'autismo, ma anche poiché c'è stata la possibilità di progettare e realizzare una sperimentazione diretta all'interno del Pronto Soccorso dell'Azienda Ospedaliero-Universitaria di Careggi, a Firenze. Si è scelto, inoltre, di affrontare il tema delle nuove frontiere dei contesti ambientali, attraverso l'integrazione di uno spazio multisensoriale all'interno del Pronto Soccorso, utilizzato come spazio di attesa dedicato alle persone con autismo. Alla base del progetto vi è, da un lato la determinazione dell'ambiente e la progettazione degli spazi; dall'altro, lo sviluppo e l'integrazione delle tecnologie, attraverso un software in grado di consentire l'automazione e la regolazione dell'ambiente e favorire la comunicazione con un sistema visivo digitale di relazione tra paziente, familiare/caregiver e operatore/medico.

Questo prototipo di stanza di attesa multisensoriale autism-friendly rappresenta lo sviluppo delle esigenze riscontrate nell'analisi dello Stato dell'Arte dei Casi Studio, ovvero la trasformazione dell'idea di progettazione degli ambienti sanitari, non basandosi soltanto sullo spazio fisico come tale, ma anche sulla flessibilità dell'ambiente, visto come dinamico e trasformabile attraverso l'uso delle assistive technolo- 


\section{Elena Bellini}

gies, e quindi così adattabile e adeguabile alle esigenze specifiche di ogni persona che sopraggiunge al Pronto Soccorso.

Il caso sperimentale sarà rappresentato e descritto nel dettaglio del prossimo Capitolo 6 . 


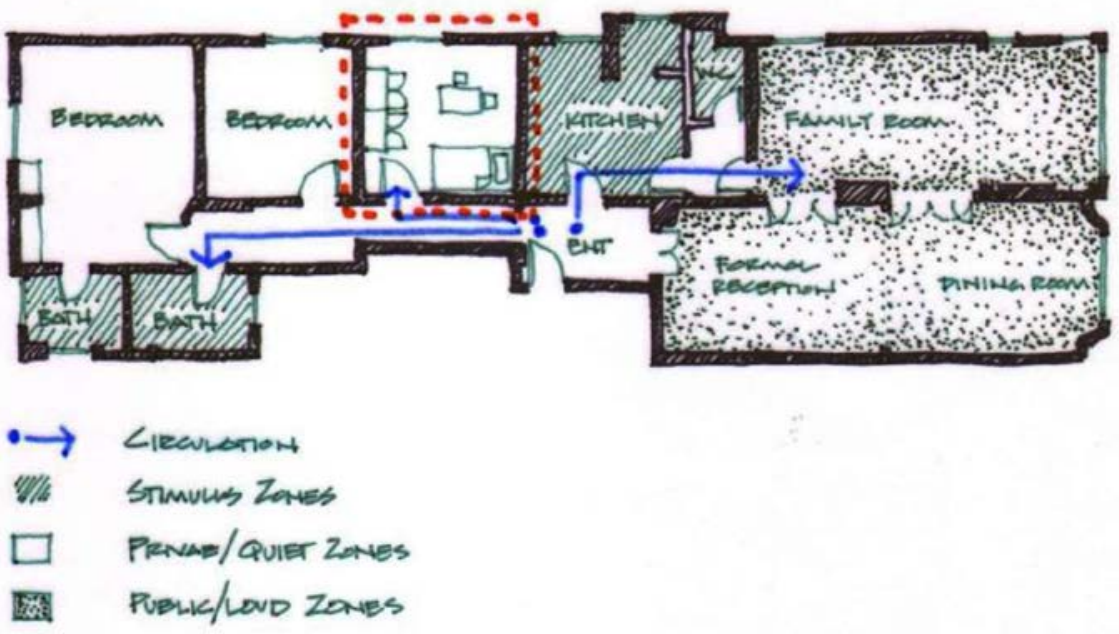

Figura 01. layout di abitazione privata tratta dall'analisi progettuale effettuata da Magda Mostafa nell'articolo "Mostafa, M. (2014) Architecture for Autism: Application of the Autism ASPECTSS ${ }^{\text {TM }}$ Design Index to Home Environments, in The International Journal of the Constructed Environment, Volume 4, Issue 2, April 2014, pp.25-38" (Copyright to Progressive Architects and Magda Mostafa)

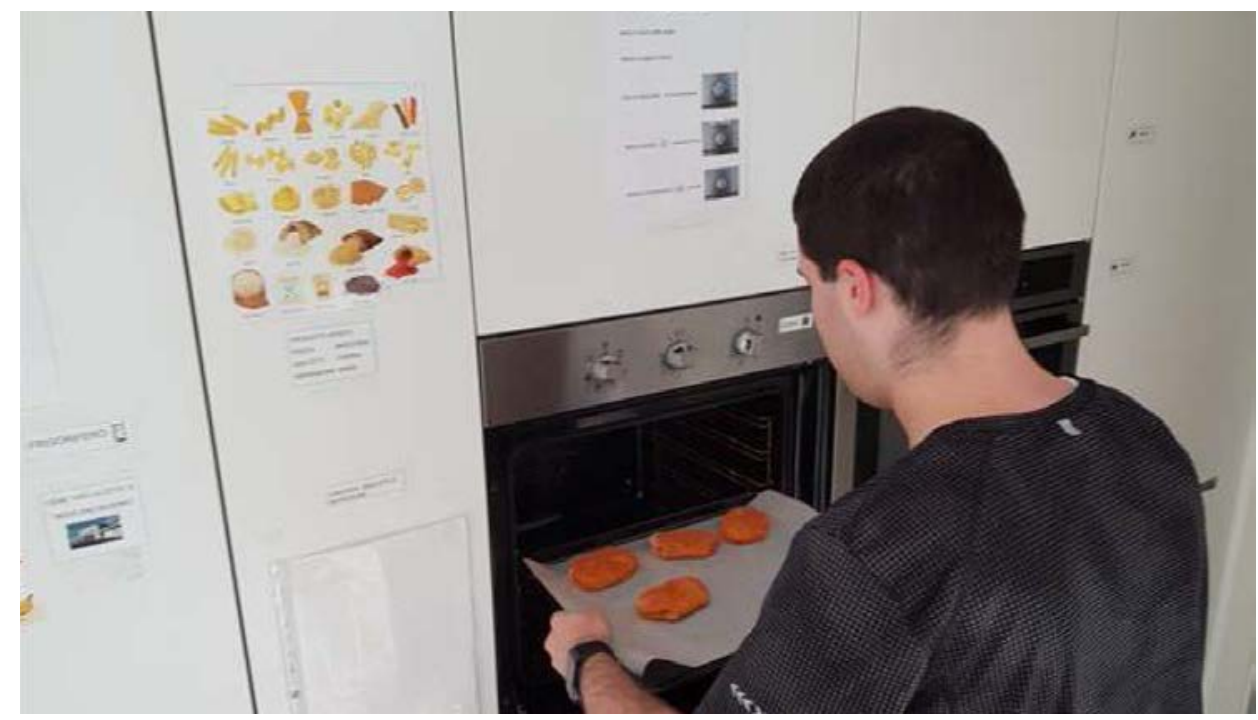

Figura 02. Villa Le Rogge Pordenone: utilizzo delle PECS e delle regole visive in cucina. (source: Trigari, M. (2017) Mamma, vado a vivere da solo (o con gli amici), SuperAbile Inail, agosto-settembre 2017, vol 8-9, p. 12-21) 


\section{Elena Bellini}

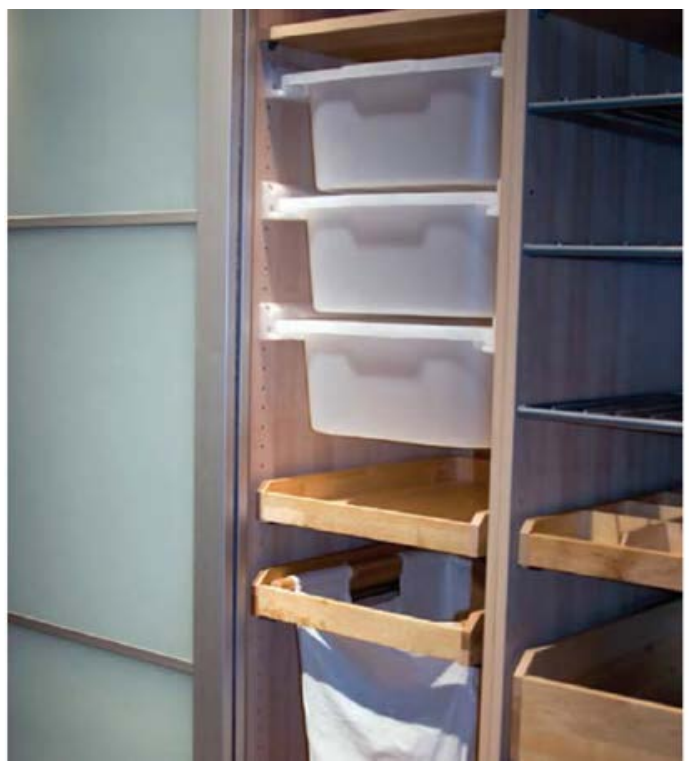

Figura 03. Esempio di guardaroba, per facilitarne un uso indipendente. (source: Ahrentzen, S. Steele, K. (2009) Advancing Full Spectrum Housing: Design for Adults with Autism Spectrum Disorders Technical report, Arizona Board of Regents, Phoenix)

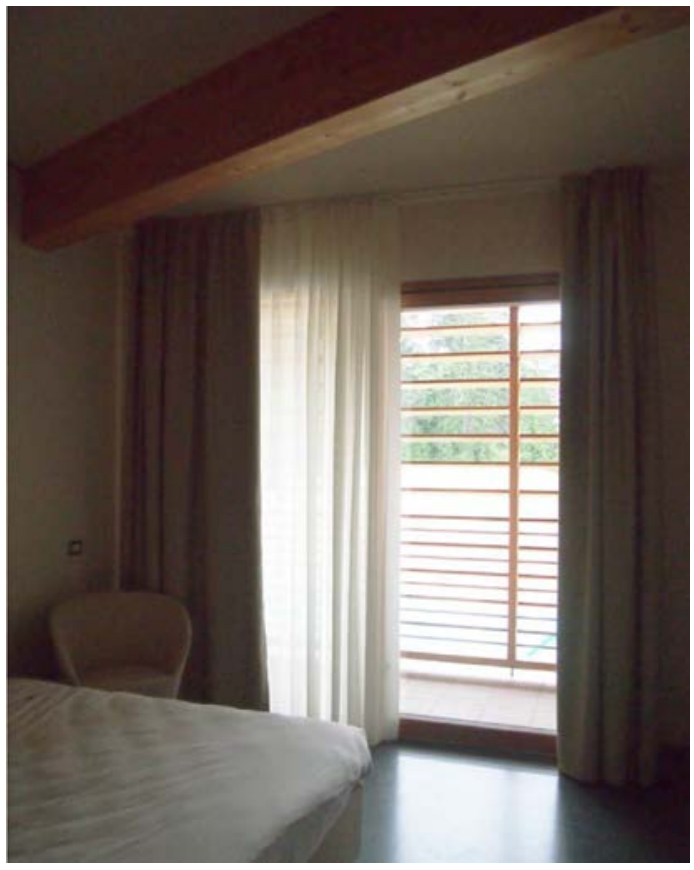

Figura 04. Camera da letto della residenza temporanea Casa Sebastiano a Coredo (TN) (foto dell'autore, aprile 2017) 


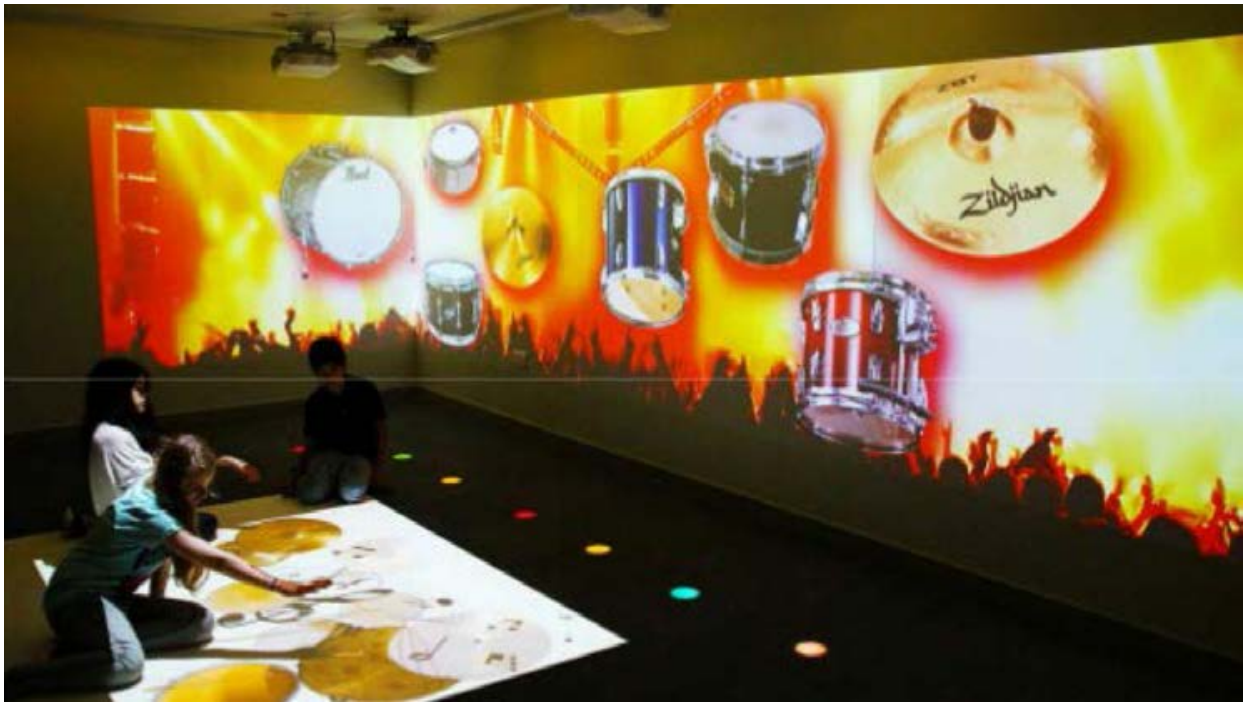

Figura 05. Casa Sebastiano, Coredo (TN): un sistema innovativo di proiettori, usica, luci colorate e aromi, creano un sistema multisnesoriale immersivo e interattivo. (source: http://www.ildolomiti.it/societa/coredo-il-nuovo-tecnologico-centro-bambini-e-ragazzi-conautismo-sara-il-primo-italia-ad)

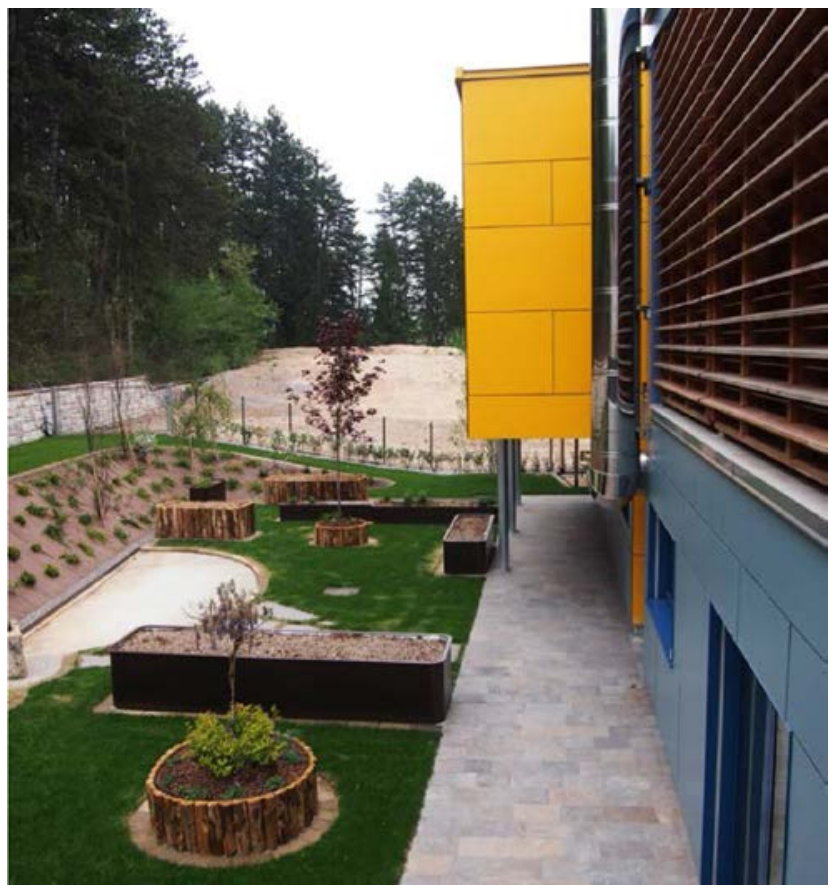

Figura 06. Casa Sebastiano, Coredo (TN): giardino sensoriale terapeutico con orto e piante aromatiche, per stimolare la sensorialità. (foto dell'autore, aprile 2017) 


\section{Elena Bellini}
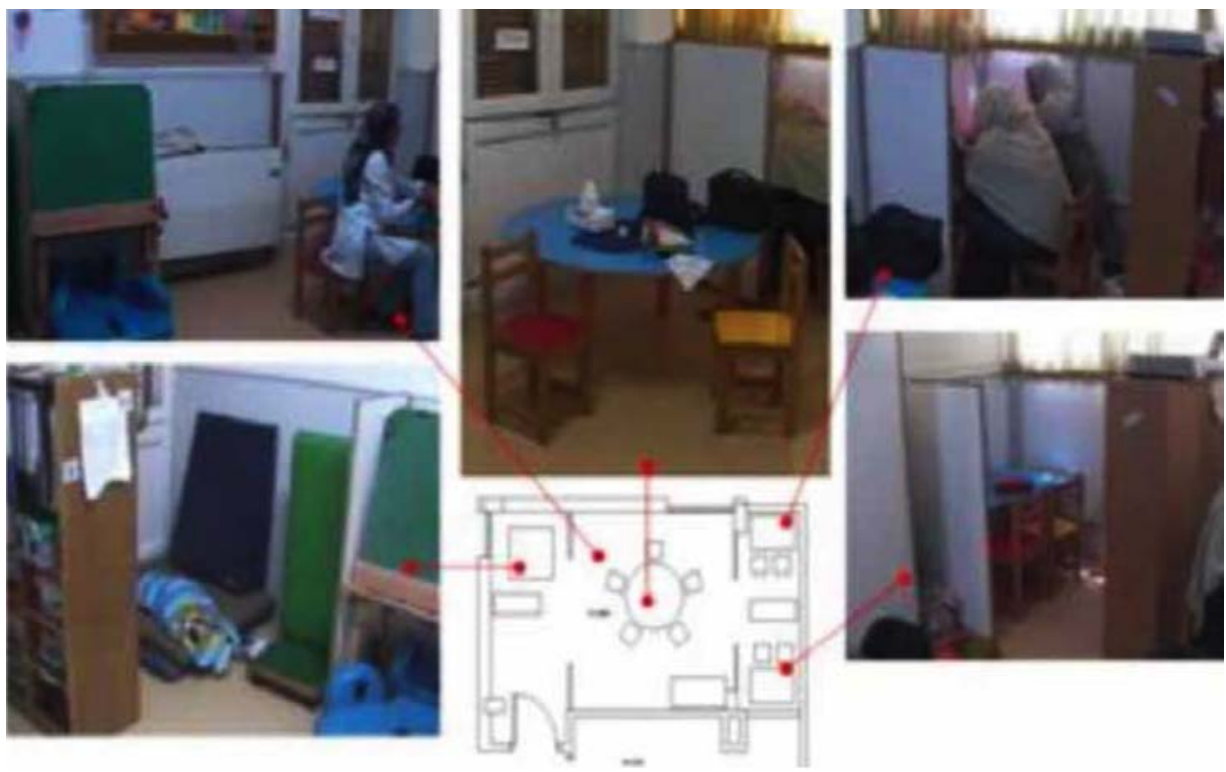

Figura 07. Planimetria della compartimentazione di una classe speciale progettata da Magda Mostafa nella sperimentazione presso l'Advance Society for Developing Skills of Special Needs Children in Cairo (source: Mostafa, M. (2008) An architecture for autism: Concepts of design intervention for the autistic user, in ArchNet-IJAR : International Journal of Architectural Research , vol.2(1), pp. 189-211 - Copyright to Progressive Architects, Magda Mostafa)

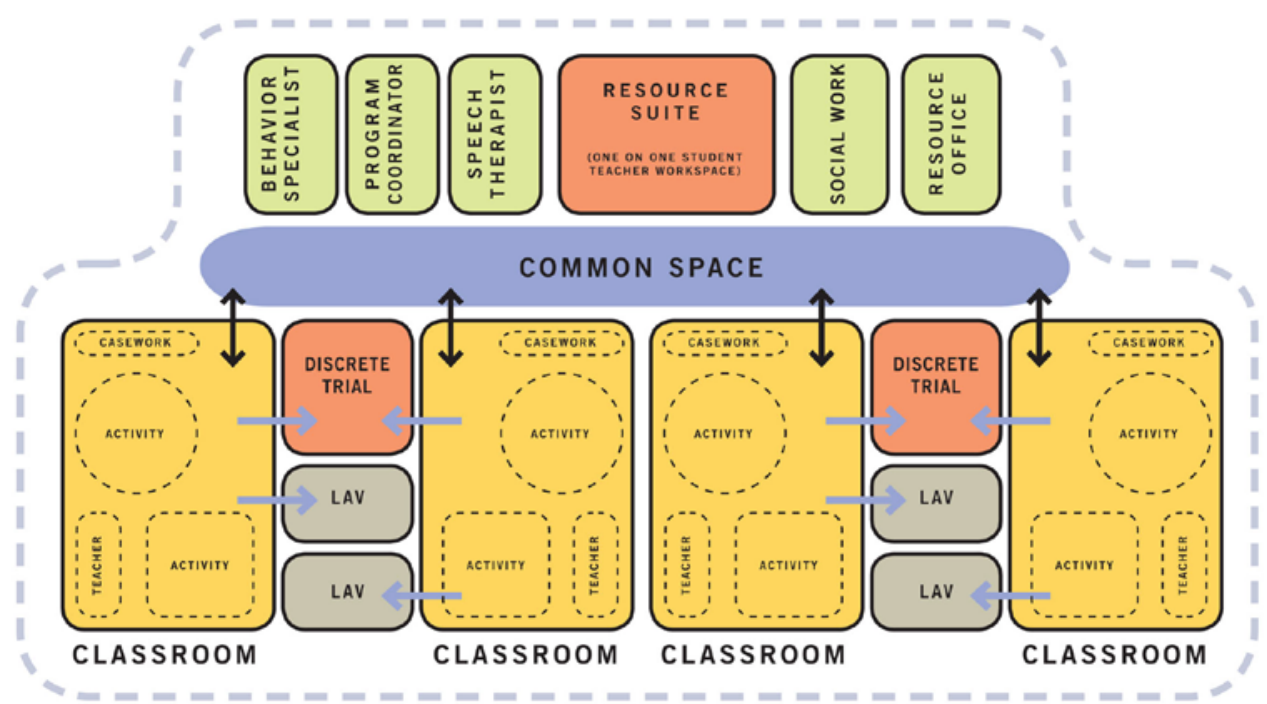

Figura 08. Forbush School for Autism: planimetria (Source: Universal Design Case Study Collection at: https://www.universaldesigncasestudies.org) 
Ambienti sensoriali "terapeutici" che rendano Abili

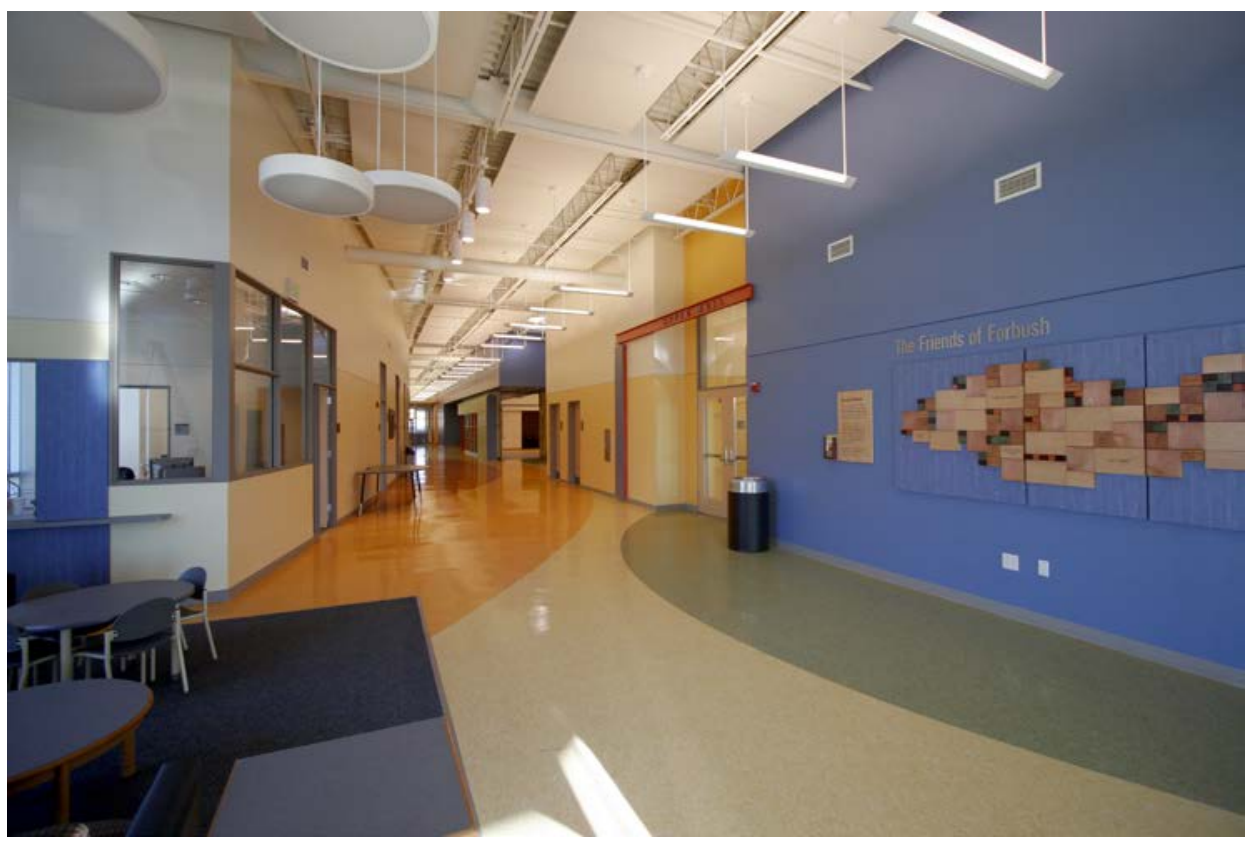

Figura 09. Forbush School for Autism: ingresso della scuola (Source: Universal Design Case Study Collection at: https://www.universaldesigncasestudies.org) 


\section{Elena Bellini}

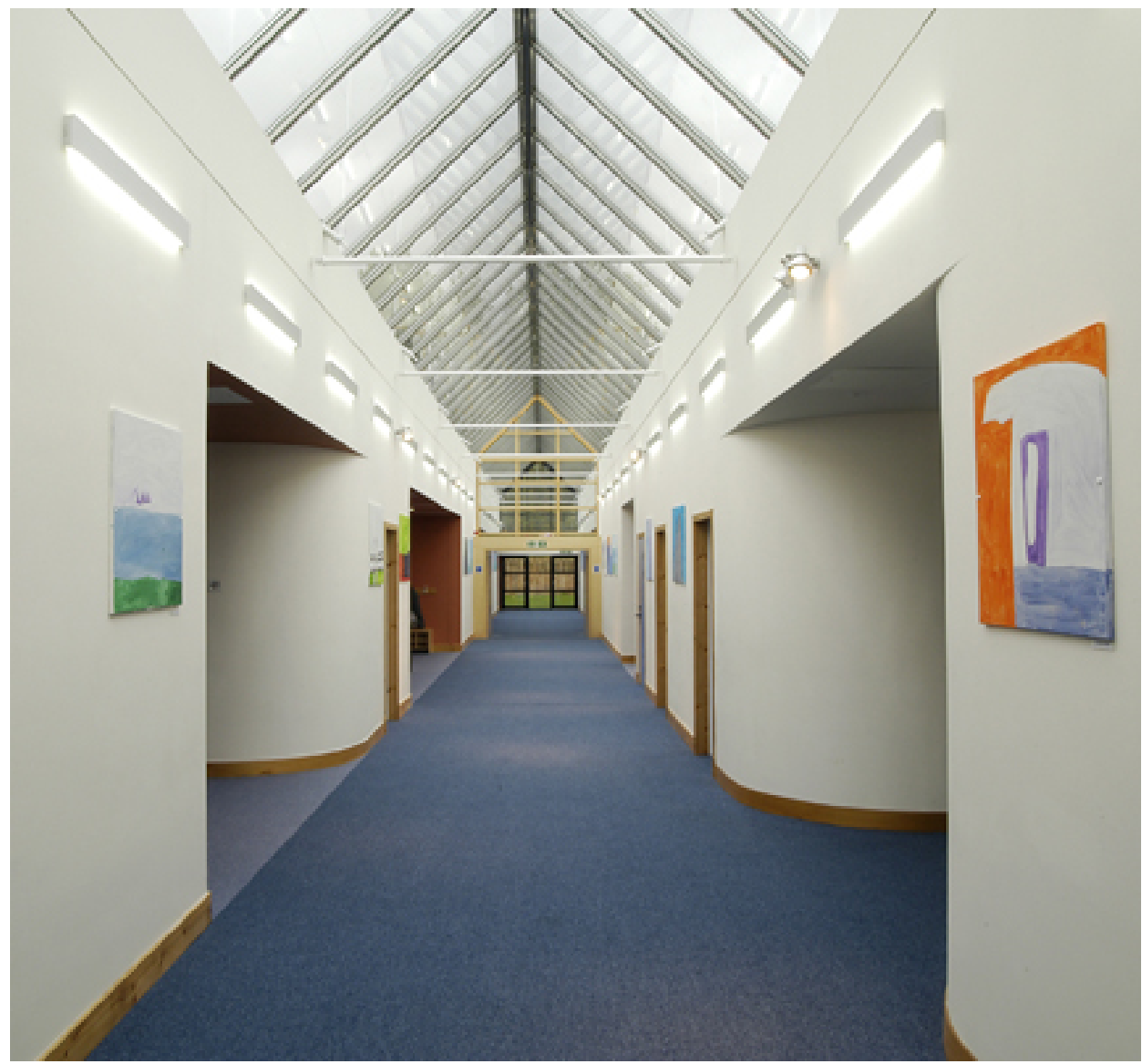

Figura 10. New Struan School (Source: Scottish Autism, photograpgher: Tina Norris) 
Ambienti sensoriali "terapeutici" che rendano Abili

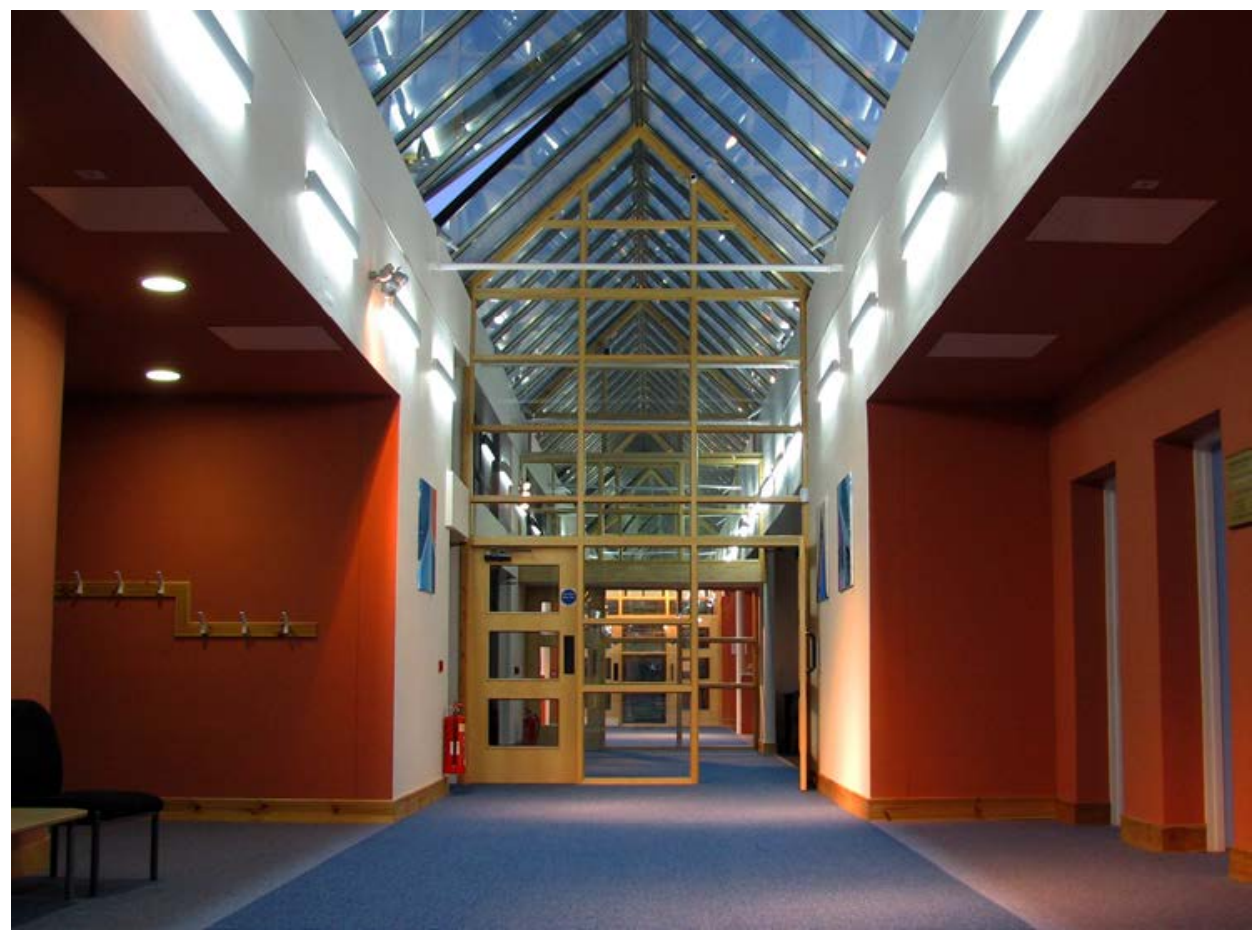

Figura 10. New Struan School (Source: Scottish Autism, photograpgher: Tina Norris)

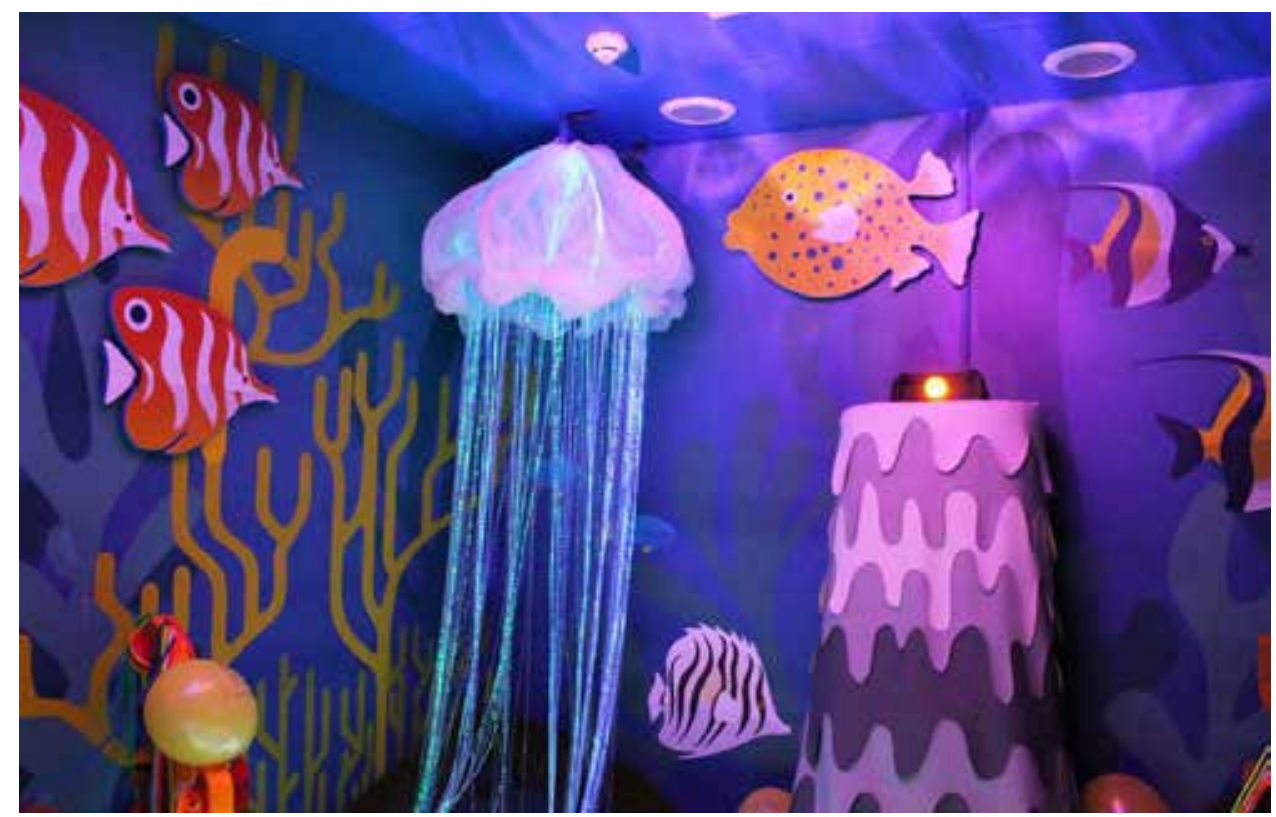

Figura 12. Royal Alexandra Children's Hospital: stanza multisensoriale (Source: Rockinghorse Children's Charity) 


\section{Elena Bellini}

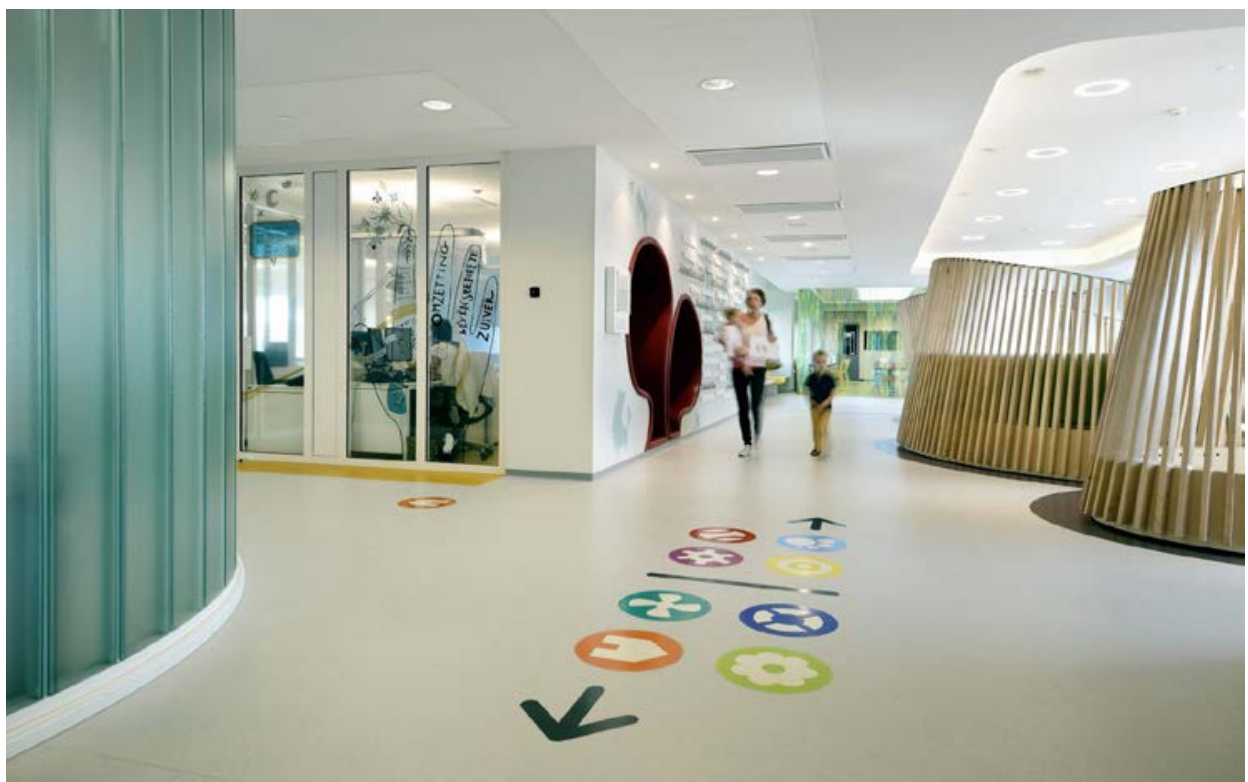

Figura 13. Emma Children's Hospital (Source: Defesche van den Putte, in short: DVDP photographer: Mike Bink)

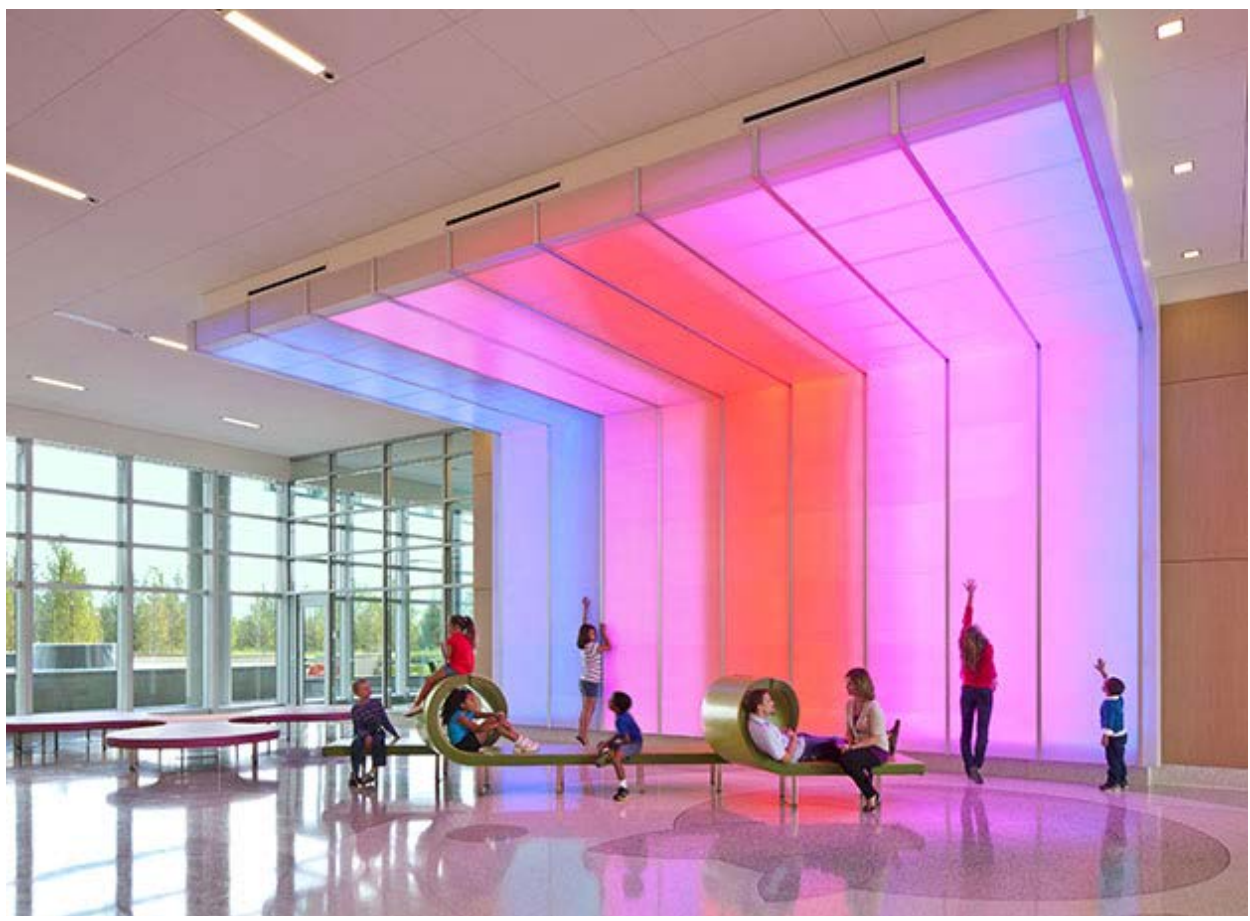

Figura 14. Nemours Children's Hospital: area di aspetto, installazione multisensoriale (Source: EYP Architecture \& Engineering) 
Ambienti sensoriali "terapeutici" che rendano Abili

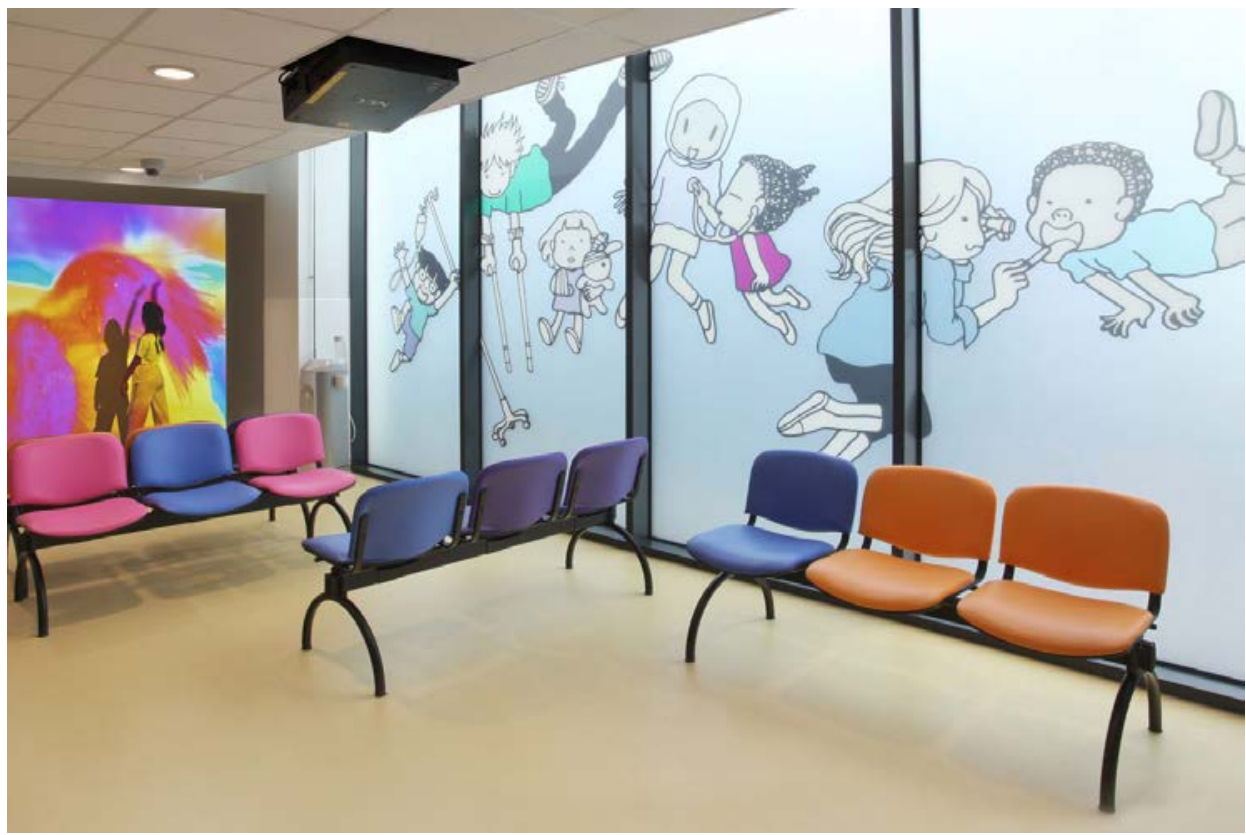

Figura 15. Evelina Londron Children's Hospital: sistema di grafica a parete, parete interattiva multisensoriale (Source: Art in Site)

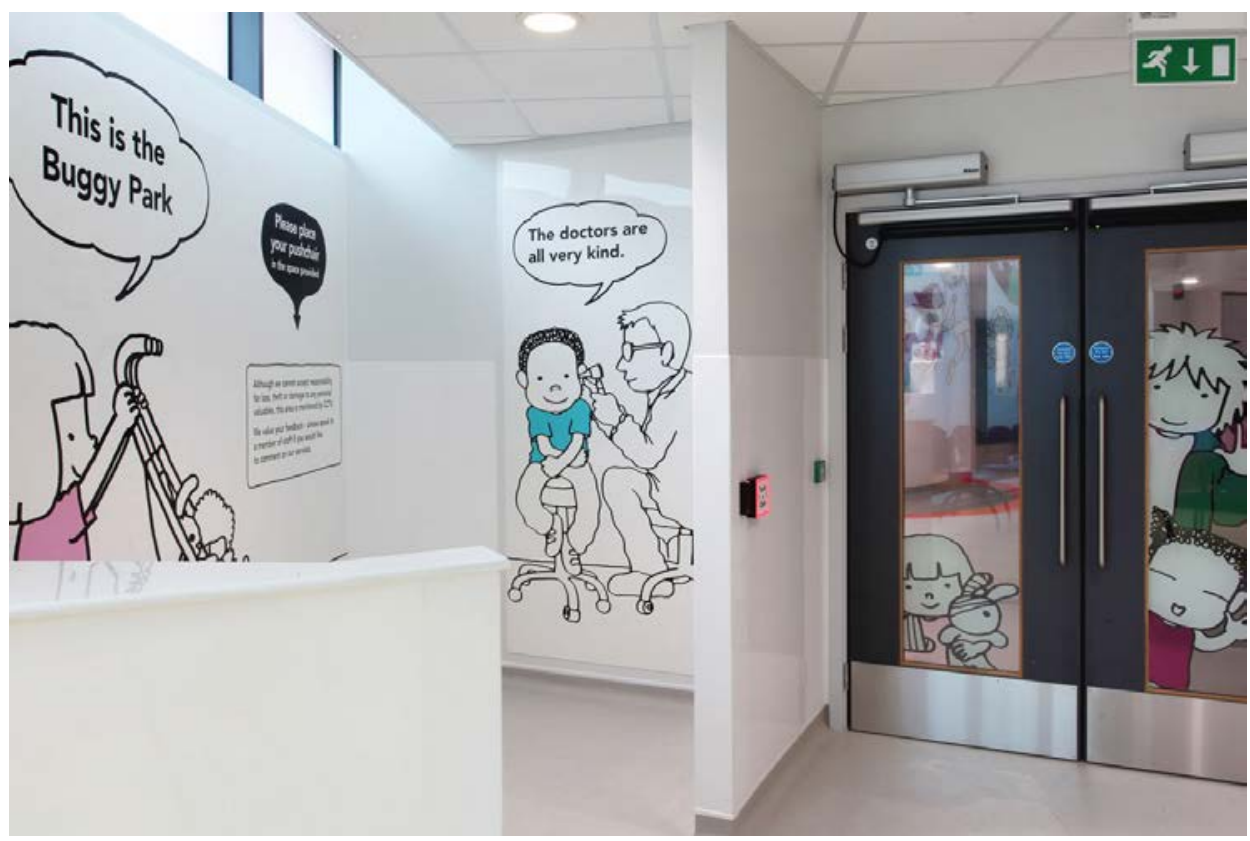

Figura 16. Evelina Londron Children's Hospital: sistema di grafica a parete (Source: Art in Site) 


\section{Elena Bellini}

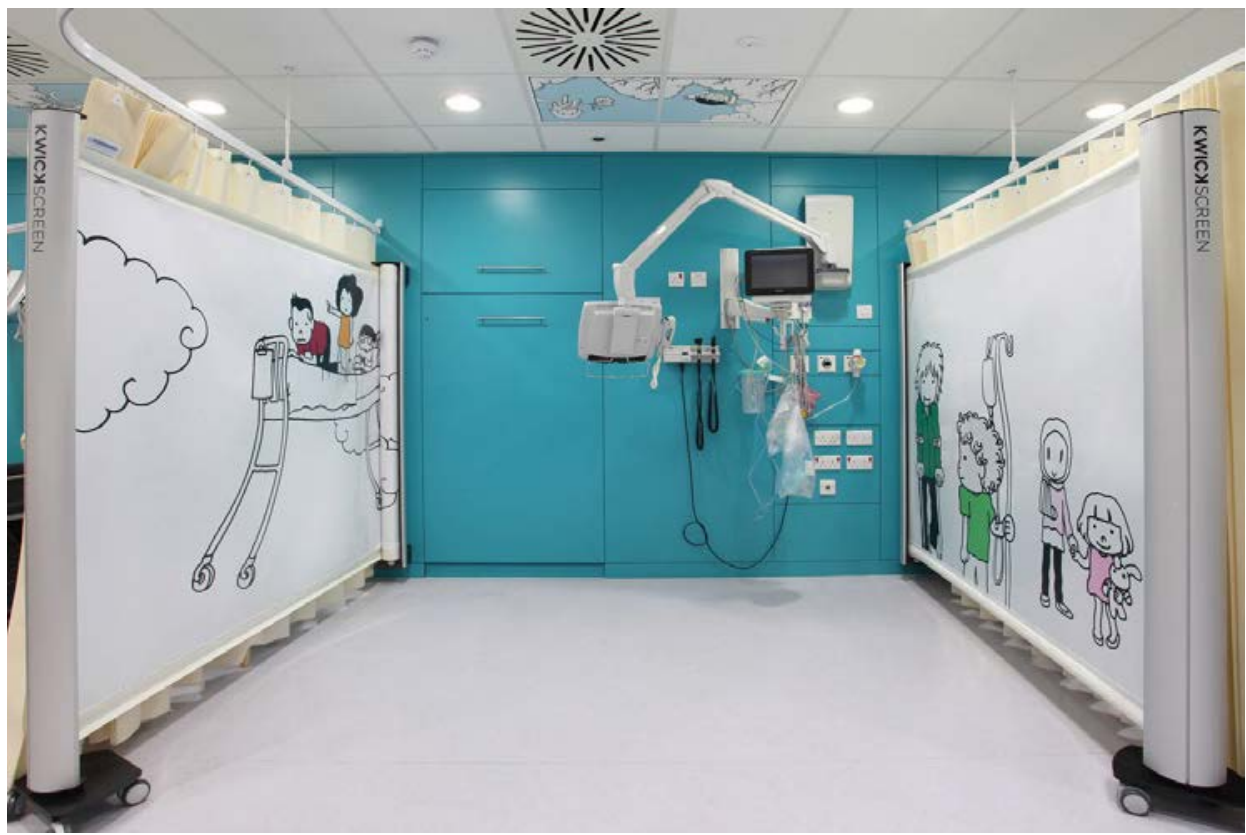

Figura 17. Evelina Londron Children's Hospital: sistema di grafica a parete (Source: Art in Site)

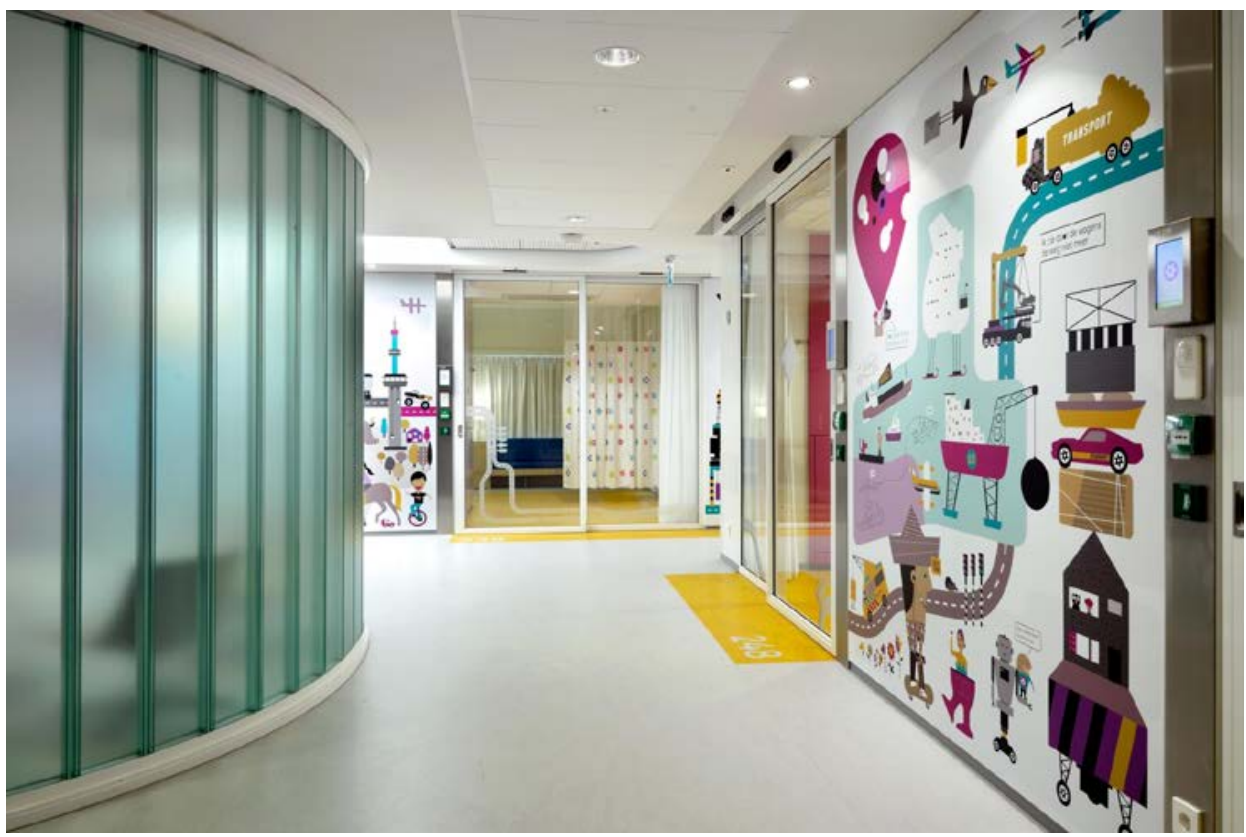

Figura 18. Emma Children's Hospital (Source: Defesche van den Putte, in short: DVDP, an office for urban design and architecture,located in Amsterdam, The Netherlands - photographer: Mike Bink) 


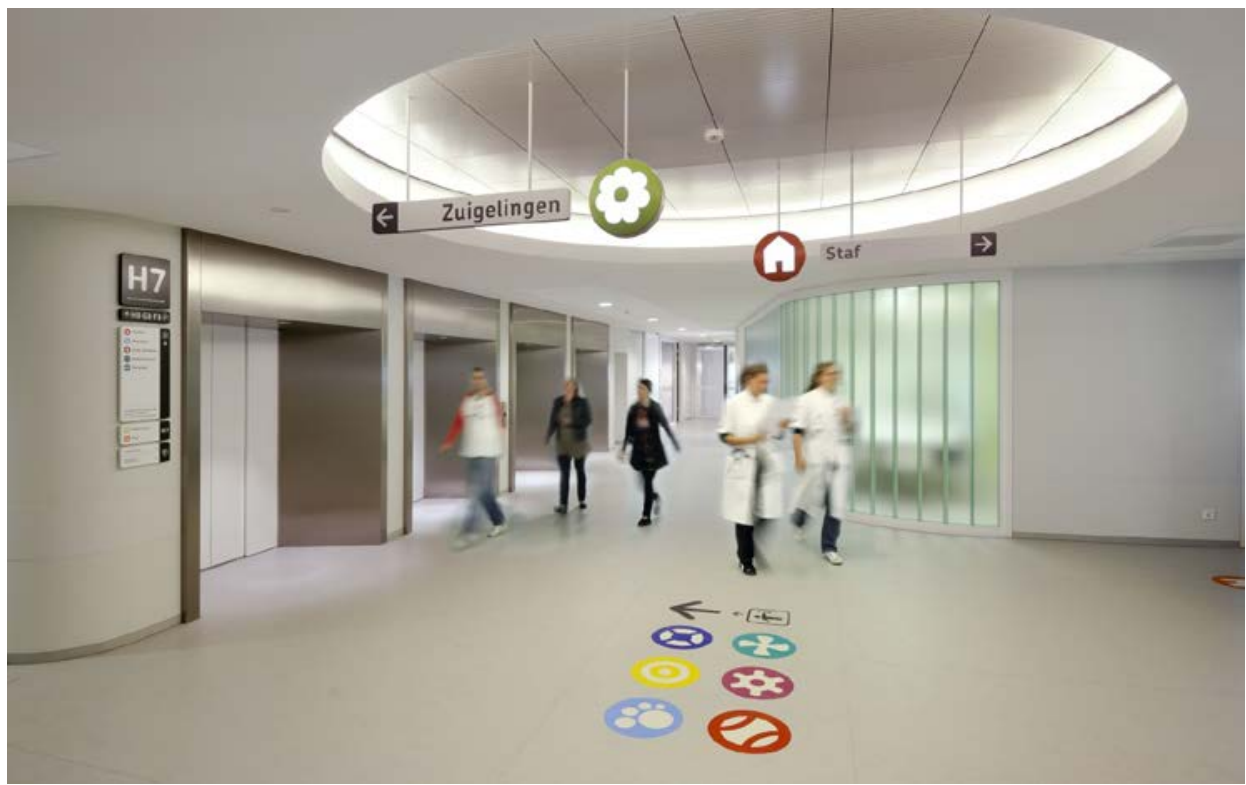

Figura 19. Emma Children's Hospital (Source: Defesche van den Putte, in short: DVDP, an office for urban design and architecture,located in Amsterdam, The Netherlands - photographer: Mike Bink)

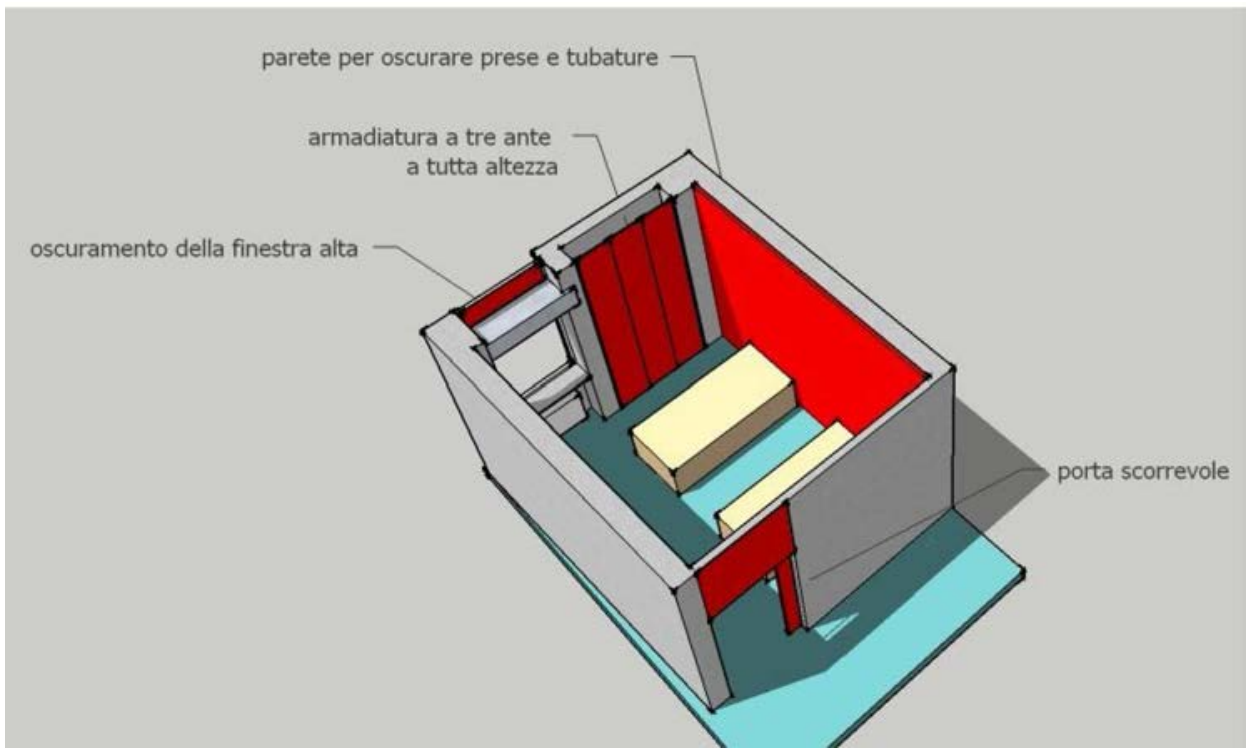

Figura 20. Pronto Soccorso, Pordenone: schema 3d della stanza dedicata alle persone con autismo. (source: Fondazione Bambini e Autismo Onlus Pordenone) 


\section{Elena Bellini}

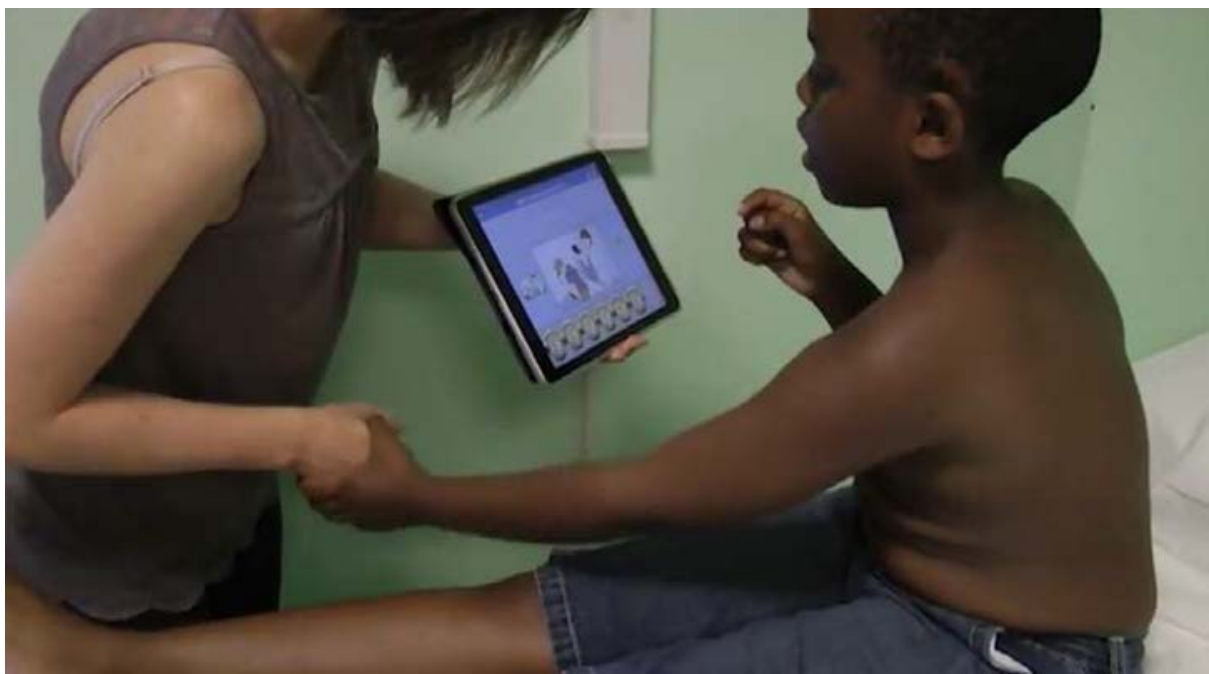

Figura 21. Vi.co Hospital, applicazione digitale progettata per la preparazione di bambini e adulti con autismo, prima di sottoporsi a qualsiasi esame diagnostico o visita medica. (source: Fondazione Bambini e Autismo Onlus Pordenone)

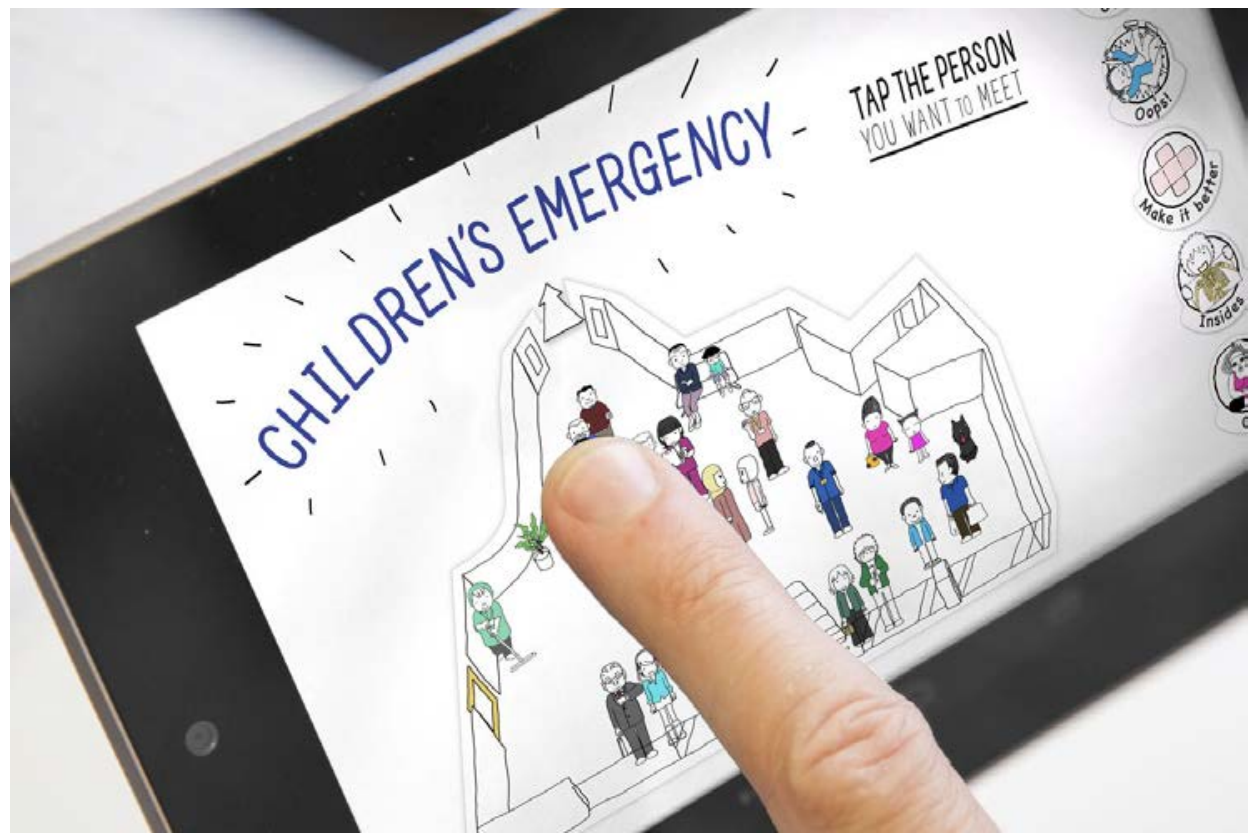

Figura 22. Evelina Londron Children's Hospital: sistema di grafica e applicazione digitale per la comunicazione (Source: Art in Site) 
Ambienti sensoriali "terapeutici" che rendano Abili

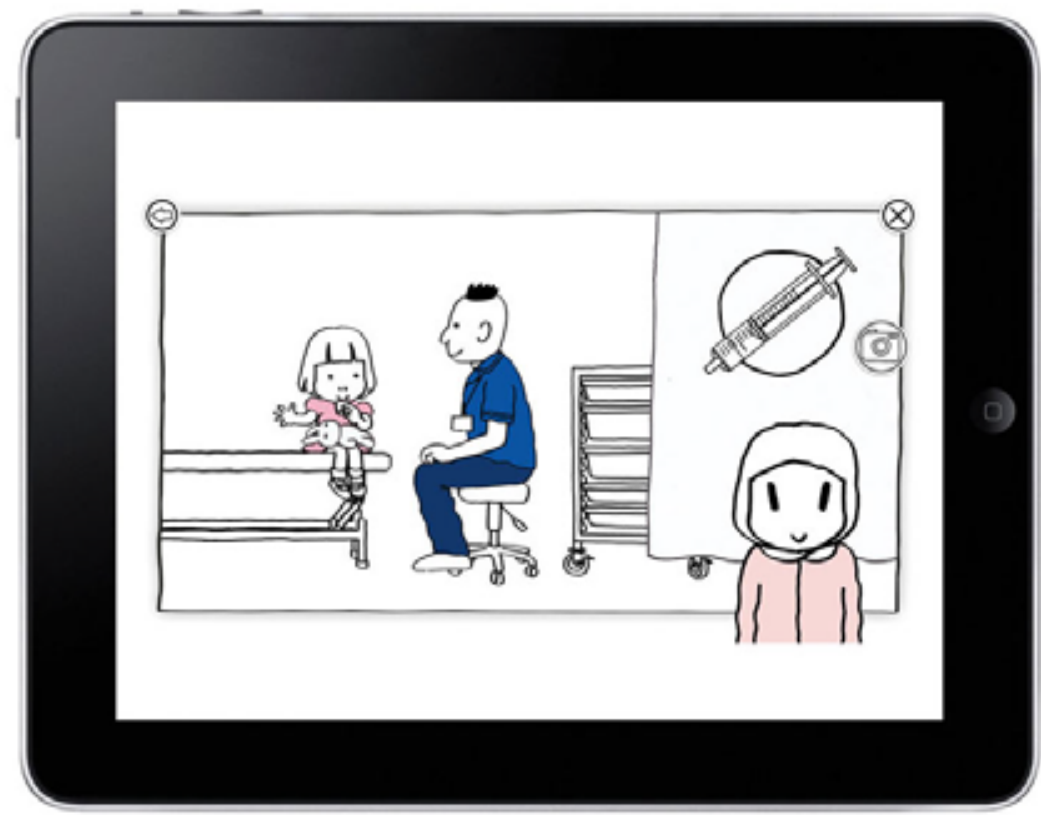

Figura 23. Evelina Londron Children's Hospital: sistema di grafica e applicazione digitale per la comunicazione (Source: Art in Site)

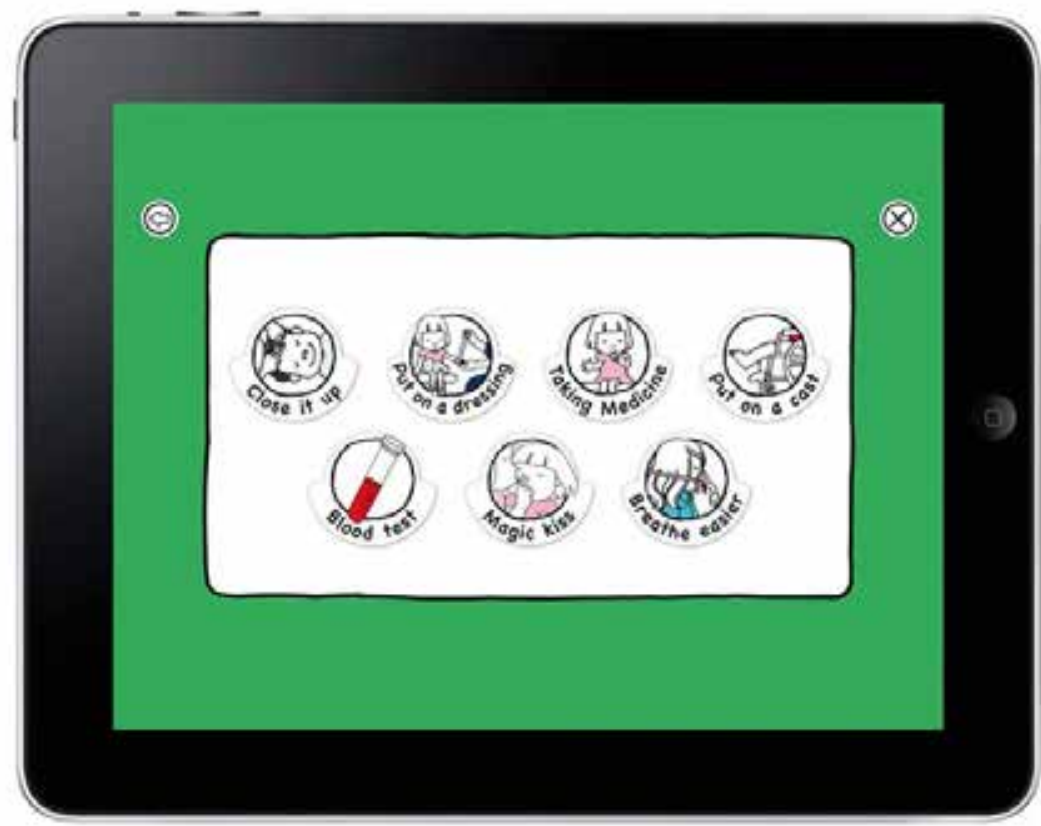

Figura 24. Evelina Londron Children's Hospital: sistema di grafica e applicazione digitale per la comunicazione (Source: Art in Site) 


\section{Capitolo 6}

\section{Proposta di un Ambiente Sperimentale: Lo Spazio Filtro All'emergenza Sanitaria}

\subsection{Una stanza multisensoriale di attesa autism-friendly presso il Pronto Soc- corso dell'Azienda Ospedaliero-Universitaria di Careggi a Firenze}

Nel luglio 2016 è stato attivato presso il Pronto Soccorso dell'Azienda Ospedaliero-Universitaria di Careggi di Firenze, un programma denominato "Codice H", ovvero un protocollo di intervento dedicato all'accessibilità dei servizi sanitari delle persone con disabilità, realizzato in collaborazione con la Direzione Sanitaria e lo staff di Careggi e la compartecipazione dei diversi attori interessati, tra cui la Commissione dei Servizi Sociali di Firenze e la Consulta dei Diritti degli Invalidi e degli Handicappati del Comune di Firenze. E' il primo progetto espressamente rivolto alle persone con grave disabilità intellettiva, nello specifico dei ricoveri di urgenza, nell'ottica di creare un percorso preferenziale che riduca i tempi di attesa e permetta al familiare di essere sempre presente. Con questo intervento si prevede inoltre una formazione mirata del personale sanitario e la strutturazione di ambienti adeguati all'accoglienza della persona ed in particolare all'attesa dell'intervento medico, momento molto critico per il paziente.

Con questa occasione ho avuto la possibilità, in collaborazione con l'Associazione e Centro per l'Autismo P.A.M.A.P.I., con cui ho instaurato un rapporto all'interno del percorso di tesi dottorale per l'esperienza e la competenza in ambito dei Disturbi dello Spettro Autistico ed in particolare in casi di adulti gravi e gravissimi, di progettare e realizzare uno spazio per l'attesa autism-friendly, all'interno del Pronto Soccorso dell'Ospedale di Careggi, sperimentando le competenze acquisite in ambito di progettazione per l'autismo negli specifici contesti dell'emergenza sanitaria. La progettazione è stata inoltre supportata da focus groups con i professionisti del Centro per l'Autismo P.A.M.A.P.I., i medici, gli operatori sanitari e lo staff tecnico dell'Ospedale di Careggi, che ha anche finanziato la realizzazione dello spazio di attesa come primo prototipo al Pronto Soccorso di Careggi di Firenze (Figura 1).

Anche sulla base della recente letteratura, dello studio dello Stato dell'Arte e dell'analisi delle interviste fatte, ho scelto di progettare lo spazio di attesa come un ambiente multisensoriale, che sia in grado di contenere le esperienze emozionali del paziente, abbattere lo stress e favorire uno stato di calma, attraverso la trasformazione degli aspetti sensoriali dell'ambiente fisico, grazie ad un sistema di assistive technology. Questo spazio per l'attesa autism-friendly viene concepito come un prototipo, primo esempio in Italia di ambiente multisensoriale in un Pronto Soccorso e 
uno tra $\mathrm{i}$ pochi in ambito europeo ed internazionale, come rilevato anche dallo stato dell'arte e dai casi studio presentati, un modulo ripetibile grazie alla sua ampia flessibilità data della struttura tecnologica, modulare, facile e veloce da installare, e dalla softwarizzazione del sistema tecnologico di interazione digitale. L'idea è quella di avere un ambiente neutro, inizialmente privo di stimoli, che per la sua conformazione sia accogliente e sicuro, ma che dia la possibilità, attraverso il sistema di assistive technology, di attivare o disattivare i diversi stimoli sensoriali e regolarli, trasformando il contesto ambientale sulla base delle singole esigenze della persona, consentendo quindi ad un ambiente "pubblico" di raggiungere un alto grado di personalizzazione secondo i bisogni individuali, utile in questi momenti di estremo stress ed ansia per il paziente. Il progetto prevede inoltre la progettazione di un sistema di comunicazione e interazione tra paziente, familiare e operatore/medico, facilitato anche attraverso immagini, di cui è stata studiata la grafica e la struttura digitale.

Il supporto tecnologico di realizzazione dell'installazione e programmazione del software di interazione è stato coadiuvato dalla startup innovativa DU IT s.r.l., di cui faccio parte, avendo la possibilità di costruire fisicamente il primo prototipo di stanza di attesa multisensoriale e sperimentarlo nel suo funzionamento all'interno del Pronto Soccorso.

Questi interventi, in ogni caso, non possono prescindere una formazione adeguata del personale sanitario e dalla strutturazione di un protocollo specifico di intervento in casi di accesso ospedaliero di persone con autismo, che rappresenterà una tappa fondamentale del percorso di supporto e assistenza sanitaria nello sviluppo del progetto.

\subsection{Pronto Soccorso dell'AOU Careggi}

L'ospedale di Careggi (Figura 2) è un centro di riferimento primario nel panorama sanitario regionale, presentando eccellenze anche a livello nazionale. Il progetto del nuovo Pronto Soccorso è stato inaugurato a settembre 2015, prevedendo un ambiente assolutamente all'avanguardia per quanto riguarda le funzionalità e l'efficacia a livello clinico e l'umanizzazione dell'ambiente ospedaliero. In particolare, la struttura prevede la suddivisione in diverse isole completamente indipendenti a livello clinico, differenziate per colore, attraverso il rivestimento a pavimento e a parete ed una segnaletica composta da numeri e lettere, suddivise a loro volta in diversi box per gli interventi medici, distribuiti attorno ad una postazione centrale di desk infermieristico (Figura 3). All'ingresso, entrando dal viale Pieraccini, si trova subito frontalmente il bancone della reception (Figura 4), che accoglie i pazienti e i familiari, ed una grande sala di attesa laterale (Figura 5), luminosa, arredata con sedute e con una vista verso la corte verde.

L'ambiente scelto per l'attesa delle persone con Disturbi dello Spettro Autistico è nell'isola 5 (Figura 6) - area gialla, in una zona "protetta", sia rispetto all'affollamento delle persone, che per i rumori provenienti dal contesto esterno, come vedremo meglio all'interno della descrizione di progetto. La posizione è stata espressamente scelta perché si trova in un angolo appartato dell'area di emergenza, a cui si accede attraverso un corridoio utilizzato in modo quasi esclusivo. Vicino a questo spazio è presente inoltre un accesso secondario dall'esterno (Figura 7), che potrebbe essere 


\section{Elena Bellini}

utile nel caso in cui non si voglia far entrare il paziente direttamente nelle aree di emergenza. In corrispondenza di quest'area di accesso è inoltre presente un servizio igienico, benché si sia valutato che sarebbe stato meglio averlo direttamente in connessione con lo spazio di attesa sensoriale, anche se purtroppo al momento non è stato possibile.

\subsection{Il progetto della stanza di attesa autism-friendly}

Come visto nell'analisi dello stato dell'arte, le persone con Disturbo dello Spettro Autistico presentano spesso alti livelli di disagio e stress durante l'attesa al Pronto Soccorso, a causa della loro elevata sensibilità sensoriale, che può essere disturbata soprattutto dai livelli di rumore, di illuminazione o di odore solitamente presenti nel Pronto Soccorso; dell'ansia che spesso provano rispetto alle novità e all'imprevedibilità, come visto anche nelle interviste, valutazione gli ambienti sconosciuti come luoghi di maggiore criticità; e soprattutto delle difficoltà di comunicazione, che rendono complessa l'interazione paziente operatore/medico.

Un ambiente di attesa dedicato è il primo passo verso un ambiente ospedaliero autism-friendly, che permetta che il paziente attenda in uno spazio dedicato, di privacy ed adeguato alle sue necessità, invece che ritrovarsi in una sala di attesa piena di persone, caos e rumore, luci forti ed abbaglianti ed altre situazioni tipiche di disagio sensoriale o percettivo. In questo ambiente la persona con autismo ha la possibilità di abbattere lo stress provocato dal contesto ambientale e dalla sofferenza della malattia, autoregolandosi sulla base del controllo delle stimolazioni sensoriali, ma anche distrarsi e quindi sopportare più facilmente l'attesa dell'intervento medico. Attraverso l'interazione digitale il paziente può inoltre avere accesso a sistemi di comunicazione facilitata che possono rendere più semplice l'interazione e la comunicazione tra paziente, familiare/caregiver e personale sanitario.

E' stato dimostrato anche dallo studio dello Stato dell'Arte che un ambiente in grado di ridurre il disagio in questi momenti di emergenza e sofferenza ha una notevole influenza sui pazienti in termini di riduzione dello stress, sopportazione dei tempi di attesa, ma anche efficacia e velocità dell'intervento terapeutico a cui sarà sottoposto. Conseguentemente questo impatto ricadrà direttamente sui familiari, sugli operatori sanitari, sullo stress correlato e quindi sulla qualità del loro lavoro e della cura.

Il progetto si è basato su alcuni principi fondamentali:

- la conformazione di un ambiente neutro, dalla forma accogliente e rassicurante, con un aspetto non sanitario ed istituzionale, che tranquillizzi il paziente appena entra nello spazio;

- la strutturazione di un sistema tecnologico e di controllo domotico che consenta di trasformare l'ambiente, rendendolo dinamico e adattabile, ovvero capace di rispondere alle diverse necessità del paziente;

- lo sviluppo di un sistema di grafica e comunicazione attraverso i-pad che permetta di facilitare l'interazione tra paziente, familiare/caregiver e personale sanitario.

Il primo aspetto importante è stata la scelta del luogo dove andare ad inserire la stanza multisensoriale, come visto precedentemente, ovvero in un'area appartata del Pronto Soccorso, rispetto alla confusione, all'affollamento e ai ritmi veloci e diso- 
rientanti di un tipico Dipartimento di Emergenza. Un angolo sicuro e protetto, sia acusticamente che visivamente.

La stanza del Pronto soccorso, scelta per essere riprogettata, non era molto grande (circa $14 \mathrm{mq}$ ) ed era conformata come una classica stanza per le visite/ambulatorio sanitario (Figura 8). Lo spazio era rivestito a pavimento con un linoleum grigio e alle pareti con una tinteggiatura di colore arancione, molto acceso, che avrebbe potuto creare stress e sovraccarico visivo. Il sistema di illuminazione a soffitto (Figura 9), molto forte, prevedeva inoltre corpi illuminanti a neon, da evitare in casi di autismo, come approfondito anche nello stato dell'arte. La stanza non aveva aperture verso l'esterno, ma questo non è stato considerato un problema, dato che l'ambiente multisensoriale ha necessità di essere oscurato per la riproduzione degli effetti luminosi e delle proiezioni video.

L'impianto planimetrico è stato quindi totalmente stravolto (Figura 10), conformando l'ambiente attraverso delle contropareti con angoli stondati (Figura 11), che riconformassero l'ambiente come curvilineo e avvolgente, favorendo la sensazione di sicurezza e contenimento. La conformazione curvilinea delle pareti inoltre, oltre a ingentilire lo spazio e renderlo maggiormente accogliente, ha anche percettivamente aumentato l'ampiezza della stanza, che era piuttosto contenuta nelle dimensioni.

Il gioco volumetrico delle contropareti, oltre ad ampliare ulteriormente la percezione della profondità della stanza, è stata utile per alloggiare, al di sotto della porzione superiore di controparete, una striscia LED RGBW per illuminare lo spazio e soprattutto effettuare giochi di luce in accordo o differenziandosi rispetto ai corpi illuminanti a soffitto.

La strutturazione di contropareti ha dato inoltre la possibilità di alloggiare uno strato di isolamento acustico ad alta prestazione (Figura 12), per proteggere l'ambiente dai rumori provenienti dall'esterno e non turbare i pazienti con i movimenti del Pronto Soccorso. Tutte le forassiti e le componenti elettriche e tecnologiche sono state alloggiate all'interno delle contropareti (Figura 13), sfruttando il controsoffitto esistente per il passaggio all'interno della stanza; in ogni caso sono state concentrate in un'unica colonna verticale montante, che con uno sfondo verso l'esterno ricongiunte le componenti al quadro elettrico e a tutti i dispositivi tecnologici posti nel rack nel corridoio adiacente. All'interno delle contropareti sono inoltre stati nascosti tutti i dispositivi medicali e i quadri con le prese elettriche (Figura 14), sia per non impattare alla vista degli utenti, che per favorire la sicurezza dell'ambiente; degli sportelli scorrevoli con chiusura a chiave chiudono a filo la superficie della parete.

La struttura delle contropareti è stata infine conformata con moduli preformati in legno, attraverso pannelli modulari componibili sul posto, per velocizzare il cantiere e non produrre polvere o sostanze che avrebbero potuto infastidire la porzione di Pronto Soccorso adiacente, ovviamente rimasta in funzione durante i lavori. Tutto l'allestimento infatti è stato concentrato in due settimane, ma si è valutato come sarebbe stato possibile ottimizzare ancora di più le fasi lavorative al fine di eseguire il tutto in una sola settimana.

La finitura delle pareti è stata effettuata con una vernice sanificabile di colore bianco, per avere un ambiente completamente neutro e non sovrastimolante, facile da personalizzare attraverso la luce; il colore bianco permette infatti la diffusione del 


\section{Elena Bellini}

colore delle luci, permettendo di trasformare tutto l'ambiente sulla base del colore applicato all'illuminazione.

E' stata modificata anche la finitura del pavimento (Figura 15), attraverso un pellicola vinilica adesiva stampata con una texture geometrica personalizzata sui toni del grigio, a finitura opaca, molto tenue, al fine di creare un contrasto con le pareti e migliorare la percezione dell'ambiente a livello propriocettivo, ma non impattare troppo sul sovraccarico visivo degli utenti. E' stata scelta una pellicola vinilica poiché ha uno spessore millimetrico e permette in questo modo di adattarsi totalmente, attraverso una modellazione a caldo, alle superfici sottostanti; dall'esterno è visibile inoltre una porzione di superficie al fine di identificare immediatamente lo spazio sensoriale.

Il soffitto è uniforme e vi sono state applicate due strutture in legno tonde che ricalano, da cui far uscire l'illuminazione, posizionate in corrispondenza delle due poltrone che compongono l'arredo della stanza, per offrire la possibilità di avere due bagni di luce colorata, per un maggiore rilassamento, se graditi dal paziente. L'illuminazione avviene infatti attraverso una composizione di diversi corpi illuminanti, una linea di luce uniforme e bassa a parete e due coni di luce a soffitto, e può essere totalmente regolata dall'utente, sia come colore che come intensità.

La particolarità dello spazio è infatti, come si diceva, la possibilità di trasformare i caratteri fisici dell'ambiente e quindi gli stimoli sensoriali, attraverso un sistema di controllo domotico che è stato reso facilitato attraverso un'applicazione software su i-pad, in modo che possa essere direttamente gestito dall'utente o dal familiare/caregiver (Figura 16). L'i-pad è posto all'interno dello spazio, a lato dell'ingresso, attraverso un dispositivo di dock station che ne permette la ricarica (Figura 17), oltre che sorreggerlo a parete. La persona quindi entra nello spazio, che di per sé ha una forma calda e accogliente, "abbracciandolo" con le linee curvilinee, ed una luce soffusa, per poi decidere, in modo assolutamente personale e indipendente, se e quali stimoli attivare e come regolarli sulla base delle proprie preferenze.

Il sistema domotico gestisce infatti luci, musica, video, e vibrazione tattile di una pedana posta a pavimento al lato sinistro della stanza. In quest'area è stato creato un angolo morbido (Figura 18) per rispondere alla necessità di flooring, dimostrata in casi di autismo. La pedana trasforma, attraverso particolari diffusori audio, la musica in vibrazione, trasmessa a sua volta alla superficie in legno della pedana. Sdraiandosi o sedendosi é quindi possibile godere di questa stimolazione tattile e aumentare il rilassamento. A perimetro di quest'area di pedana sono stati posti diversi cuscini a parete di forma quadrata, che garantiscono la sicurezza dello spazio, proteggendo da eventuali urti contro la parete. I cuscini sono staccabili e riattaccabili attraverso una superficie a velcro, per cui l'utente stesso può decidere come posizionarli a parete, o usarli come cuscini sulla pedana; questo dispositivo è anche un sistema di distrazione per il paziente.

Le due poltrone (Figura 19) sono invece state conformate in modo da poter essere ampie e comode e avvolgere la persona con una porzione di tessuto, attraverso un movimento di abbraccio. Questa conformazione, che si inspira al concetto dell'abbraccio di Temple Grandin, consente di rilassare l'utente attraverso una stimolazione tattile, inserendo le mani all'interno delle "tasche" di tessuto della poltrona, e contenitiva, facendosi comprimere e stringere dal tessuto con la pressione scelta e appli- 
cata dall'utente stesso. Una delle due poltrone ha inoltre la parte inferiore disegnata affinchè sia possibile dondolarsi, favorendo ancora una volta il rilassamento e la calma.

Nella stanza sono infine previsti anche un proiettore video (Figura 20), dei diffusori audio per la musica integrati a soffitto e un dispositivo per la diffusione di un aroma naturale (fuori dalla portata della persona), molto tenue, al fine di nascondere l'odore degli igienizzanti tipico degli ambienti ospedalieri; l'aroma è ovviamente cambiabile e personalizzabile. Un time-timer (Figura 21) permette inoltre, attraverso un sistema manuale, di visualizzare, attraverso la porzione colorata dell'orologio, la porzione di tempo che il paziente deve aspettare nella stanza, prima dell'intervento medico, al fine di facilitare la previsione e la comprensione da parte del paziente.

Delle videocamere contrapposte danno inoltre la possibilità di guardare il paziente all'interno della stanza dall'esterno, attraverso un monitor adiacente alla porta, o dal desk degli infermieri, consentendo di lasciare il paziente anche da solo all'interno dello spazio sensoriale, in completa sicurezza.

La collocazione delle due poltrone favorisce la presenza di un accompagnatore, familiare o caregiver, e stimola l'interazione, non solo con la famiglia, ma anche con gli operatori e medici, per il primo colloquio o visita preventiva, che può in questo modo avvenire in un'area maggiormente accogliente e rilassante.

In ogni caso la formazione del personale risulta fondamentale, per apprendere le strategie di relazione con le persone con Disturbi dello Spettro Autistico, anche attraverso indicazioni semplici quali abbassare il tono della voce, o fare movimenti cauti e pacati, che però possono rivelarsi di notevole importanza in una situazione di stress ed emergenza. Il familiare o caregiver è in ogni caso il punto di riferimento per la conoscenza del paziente e va seguito, come possibile, nelle indicazioni su come interagire con la persona.

\subsection{Il sistema di Assistive Technology}

Il lavoro di ricerca e progettazione ha previsto anche la strutturazione del sistema tecnologico di domotica e gestione dello spazio, anche grazie al supporto del Centro per l'Autismo P.A.M.A.P.I. per la scelta delle stimolazioni, delle grafiche e dei sistemi di comunicazione, sulla base della loro esperienza con persone adulte con Disturbi dello Spettro Autistico gravi e gravissimi; la startup innovativa DU IT ha progettato quindi il software che ha reso possibile la gestione coordinata del sistema tecnologico di interazione digitale.

Tutta la gestione della stanza avviene attraverso un i-pad, uno strumento conosciuto e spesso usato dalle persone con autismo, anche per i sistemi di comunicazione facilitata (Figura 22). La prima schermata è dedicata ai bisogni primari (Figura 23), ovvero dà la possibilità a coloro che sono a basso funzionamento o che hanno difficoltà comunicative, di poter informare il familiare/caregiver o il personale sanitario delle proprie esigenze, ed in particolare se ha bisogno dei servizi igienici, se ha sete, se sente dolore e, in questo caso, mostrare in quale porzione del corpo sente male, sempre attraverso le immagini dell'i-pad. La grafica scelta è molto semplice, con pochi colori molto tenui e tratti semplici in nero su sfondo bianco; le immagini 


\section{Elena Bellini}

sono grandi, suddividendo il quadrante in quattro, per chi ha anche problemi di ipovisione, e si associano scritte a disegni raffiguranti il bisogno.

Entrando nella sezione "gioco con la stanza" è possibile personalizzare lo spazio scegliendo come prima opzione tra alcuni scenari sensoriali integrati precostituiti (Figura 24), valutati insieme al Centro per l'Autismo P.A.M.A.P.I., oppure direttamente regolare in modo autonomo e differenziato ogni carattere della stanza dal colore e l'intensità delle luci, al video proiettato, alla musica preferita, entrando nella sezione "io voglio". Gli scenari integrati sono una composizione di tre temi naturalistici, efficaci in ambito sanitario in particolare per favorire lo stato di rilassamento, che attivano una proiezione video dal tema ripetitivo e ciclico, come lo scorrere di una cascata, lo scendere della neve nel cielo, o la luce che passa tra i rami di una foresta; musiche dolci o suoni della natura, abbinati al video proiettato, che attivano anche la stimolazione della pedana a ritmo ovviamente della musica scelta, trasformando il suono in vibrazione; luci tenui dai colori associati allo scenario di riferimento per non sovrastimolare. E' stato scelto di attivare alcuni scenari precostituiti poiché non è detto che tutti abbiano la capacità, in modo indipendente, di accedere a tutte le sezioni di personalizzazione, che avviene in modo graduale in base alle abilità del singolo, consentendo comunque ad ognuno di usufruire delle stesse possibilità di stimolazione sensoriale. In ogni caso, come precedentemente detto, è possibile agire indipendentemente su ogni stimolo ed eventualmente anche non usufruirne, attivando o disattivando ogni stimolazione.

Un'altra caratteristica importante è la possibilità di portare da casa o ricercare su internet i propri video o la propria musica preferita, cosa spesso molto utile nelle persone con autismo che potrebbero avere delle stereotipie o delle selettività verso alcune immagini, musiche o video specifici. All'esterno un computer permette l'inserimento dei propri files attraverso una porta USB, ritrovandoli dall'i-pad, mentre sul dispositivo è possibile ricercare le proprie preferenze direttamente sul web (Figura 25). Queste tecniche sono utili per l'abbattimento dello stress del paziente, ma anche per distrarre e supportare durante l'attesa.

Il processo dovrebbe inoltre prevedere in futuro l'inserimento di video che spiegano i trattamenti medici, attraverso tecniche di video modeling, al fine di aumentare la prevedibilità e quindi ridurre l'ansia del paziente.

\subsection{Feedback, limiti e sviluppi futuri del progetto}

I primi feedback sullo spazio sensoriale sono stati molto positivi, rilevati attraverso il colloquio con il personale sanitario del Pronto Soccorso, e partirà proprio adesso un percorso di valutazione dell'efficacia dell'ambiente rivolto agli utenti finali. Le prime rilevazioni hanno mostrato inoltre un dato molto interessante, ovvero che la flessibilità dell'ambiente, supportata dal sistema tecnologico integrato, ne ha consentito l'utilizzo a diversi tipi di utenza, oltre alle persone con Disturbi dello Spettro Autistico. Oltre ad essere efficace con altre disabilità di tipo intellettivo, come mostrato anche dalla letteratura e dallo Stato dell'Arte, lo spazio sensoriale è risultato utile in generale per l'abbattimento dello stress.

L'ambiente ha riscontrato infatti un utilizzo efficace nell'accesso persone del Codice Rosa, ovvero donne che sono state vittime di violenza o abusi e dei loro figli, 
proprio per la conformazione dello spazio, accogliente, rassicurante e protettivo; il posizionamento della stanza in un'area riservata e "protetta", anche lontana dall'accesso principale e con un ingresso secondario per uscire con discrezione, quando necessario; il layout dello spazio, la chiusura e l'isolamento, anche dal punto di vista acustico, e l'integrazione degli stimoli sensoriali, oltretutto personalizzabile, favoriscono il relax e lo stato di calma della persona, ma anche, come conseguenza, la comunicazione e l'interazione con il personale sanitario; i dispositivi di gioco e interazione sono utili infine per il comfort, il rilassamento e la distrazione anche dei bambini, soprattutto mentre la madre effettua i colloqui con il personale.

Un altro utilizzo correlato è stato l'abbattimento dello stress dello stesso personale sanitario, soggetto ad alti livelli di stress e burn out per la tipologia di lavoro e per i ritmi associati alla realtà del Dipartimento di Emergenza.

Questo ha dimostrato quindi una notevole flessibilità dell'ambiente, utile in diverse situazioni di fragilità nell'abbattimento di stress, paura e livelli di ansia, anche grazie alla possibilità di personalizzazione attraverso il sistema tecnologico. Questo riconduce inoltre alla definizione di "benessere" e "salute mentale", vissuta allo stesso modo per ogni essere umano, anche se differenziata nell'entità e nel livello rispetto alle determinate condizioni di fragilità.

Gli sviluppi futuri del progetto (Figura 26) prevedranno in ogni caso la valutazione dell'ambiente sensoriale, come anticipato all'inizio, al fine di comprenderne l'efficacia nello specifico delle persone con autismo. Questo rappresenta probabilmente il limite del percorso finale di ricerca all'interno del progetto, che non è stato possibile per necessità di tempi ed organizzazione con la struttura ospedaliera. L'Ospedale di Careggi sta in ogni caso avviando proprio adesso un processo di formazione specifica e di definizione dei criteri di valutazione dello spazio di attesa multisensoriale.

Potrebbero essere utilizzate strategie di raccolta dati di tipo fisiologico per misurare l'abbattimento dello stress attraverso la saturazione e la pressione sanguigna del paziente. Si dovranno utilizzare dispositivi che non siano d'impatto per la persona, come ad esempio nuove tecnologie a "braccialetto", ma necessitano ancora di una valutazione adeguata. Sarebbero utili inoltre questionari di gradimento rivolti ai genitori o agli accompagnatori del paziente, come mediatori, per comprendere anche quali aspetti dello spazio sono risultati nello specifico più efficaci. Infine, questionari o interviste agli operatori sanitari sarebbero in ogni caso utili per definire le criticità e le positività dell'intervento sul personale e sulla qualità del servizio sanitario.

I risultati delle valutazioni potranno inoltre innescare un sistema di modifica e ottimizzazione dello spazio, anche attraverso la revisione del sistema tecnologico di controllo domotico.

L'obiettivo finale infine dovrà essere la conformazione di un protocollo d'intervento validato e ripetibile in altre strutture ospedaliere.

Questo si trova in completo accordo con la Delibera n. 666 della Regione Toscana del 19/06/2017, che ha approvato le Linee di Indirizzo PASS - Percorsi Assi- 


\section{Elena Bellini}

stenziali per Soggetti con bisogni Speciali. ${ }^{592}$ Questo programma e modello organizzativo si propone di migliorare l'assistenza delle persone con disabilità, l'accesso alla cura e la qualità dei servizi sanitari a loro rivolti, sia come prevenzione che intervento. In particolare, prevede prima di tutto la tracciabilità delle persone con disabilità e delle informazioni sui loro "bisogni speciali" attraverso il portale Toscana Accessibile $^{593}$; la predisposizione di una scheda anamnestica condivisa e resa consultabile e implementabile a seguito di tutti gli accessi sanitari; la riduzione, per quanto possibile, dei casi di ricovero, privilegiando gli interventi ambulatoriali; la riduzione dei tempi di attesa attraverso percorsi preferenziali, soprattutto al momento dell'accesso in struttura sanitaria; la formazione obbligatoria del personale sanitario e amministrativo, a diversi livelli, in ambito della disabilità e delle metodologie di interazione e relazione con il paziente e i familiari; la presenza di un "facilitatore" come mediatore tra paziente e servizio sanitario; percorsi specifici di "accesso protetto" al Pronto Soccorso; adeguamento degli spazi, anche attraverso ausili, per facilitare i processi di cura e favorire il comfort del paziente e la qualità dei servizi sanitari offerti.

Il contatto stabilito con la dott.ssa Maria Teresa Mechi, dirigente responsabile della Regione Toscana per i "Diritti di Cittadinanza e Coesione Sociale", e del dott. Luca Puccetti, responsabili del programma PASS, anche a seguito del progetto della stanza di attesa del Pronto Soccorso di Careggi, apre le porte ad un nuovo sviluppo nell'ambito della progettazione di ambienti sanitari adeguati alle esigenze delle persone con bisogni speciali e, nello specifico, delle persone con Disturbi dello Spettro Autistico.

\footnotetext{
${ }^{592}$ DGR 666/2017 del 19/06/2017, Equità di accesso ai servizi sanitari delle persone con disabilità. Approvazione delle linee di indirizzo e del modello di intervento regionale PASS - Percorsi Assistenziali per Soggetti con bisogni Speciali, Regione Toscana

${ }_{593} \mathrm{http}: / /$ open.toscana.it/web/toscana-accessibile
} 
Ambienti sensoriali “terapeutici” che rendano Abili

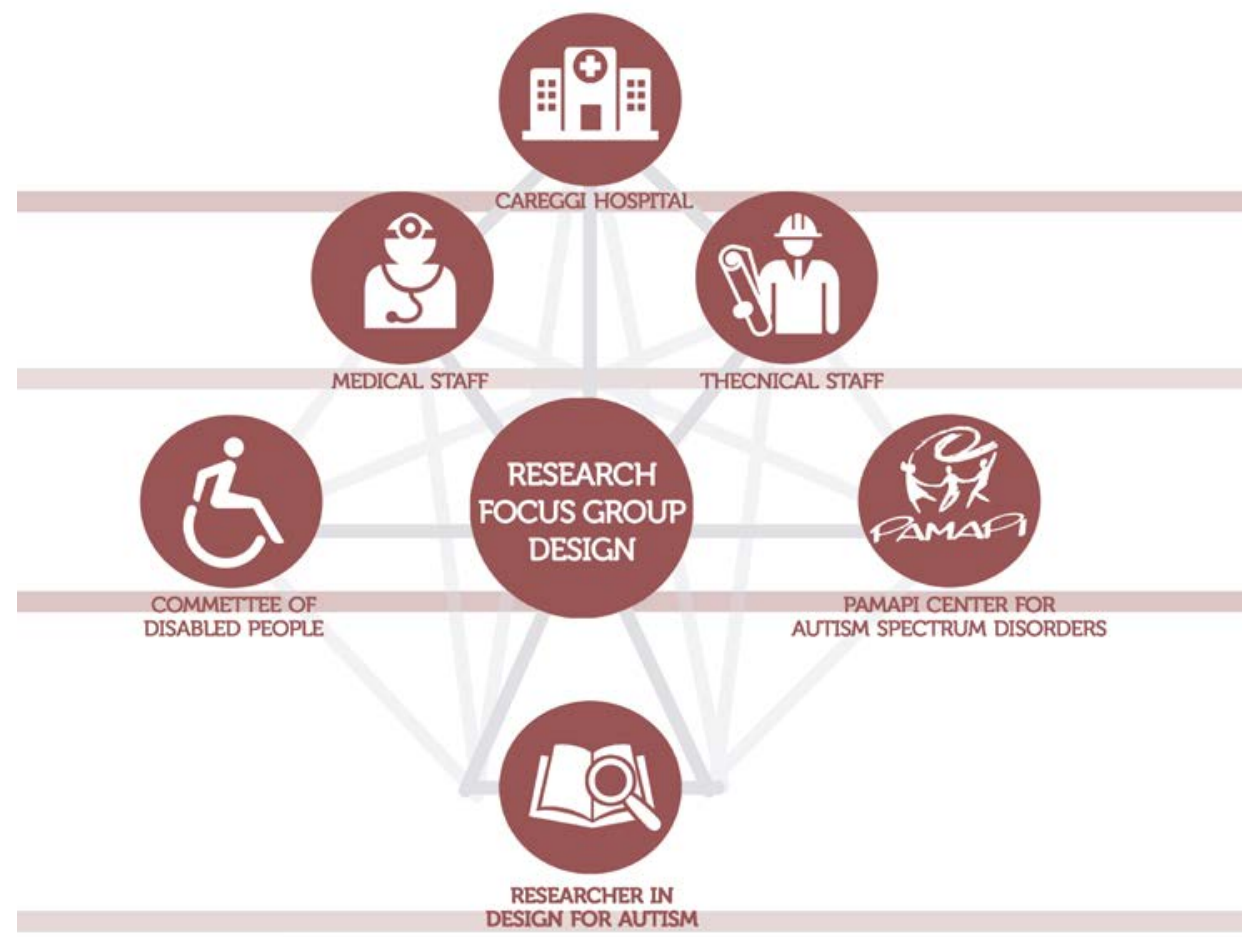

Figura 1. Schema di sviluppo del disegno di ricerca sull'ambiente multisensoriale di attesa autism-friendly presso il Pronto Soccorso di Careggi a Firenze.

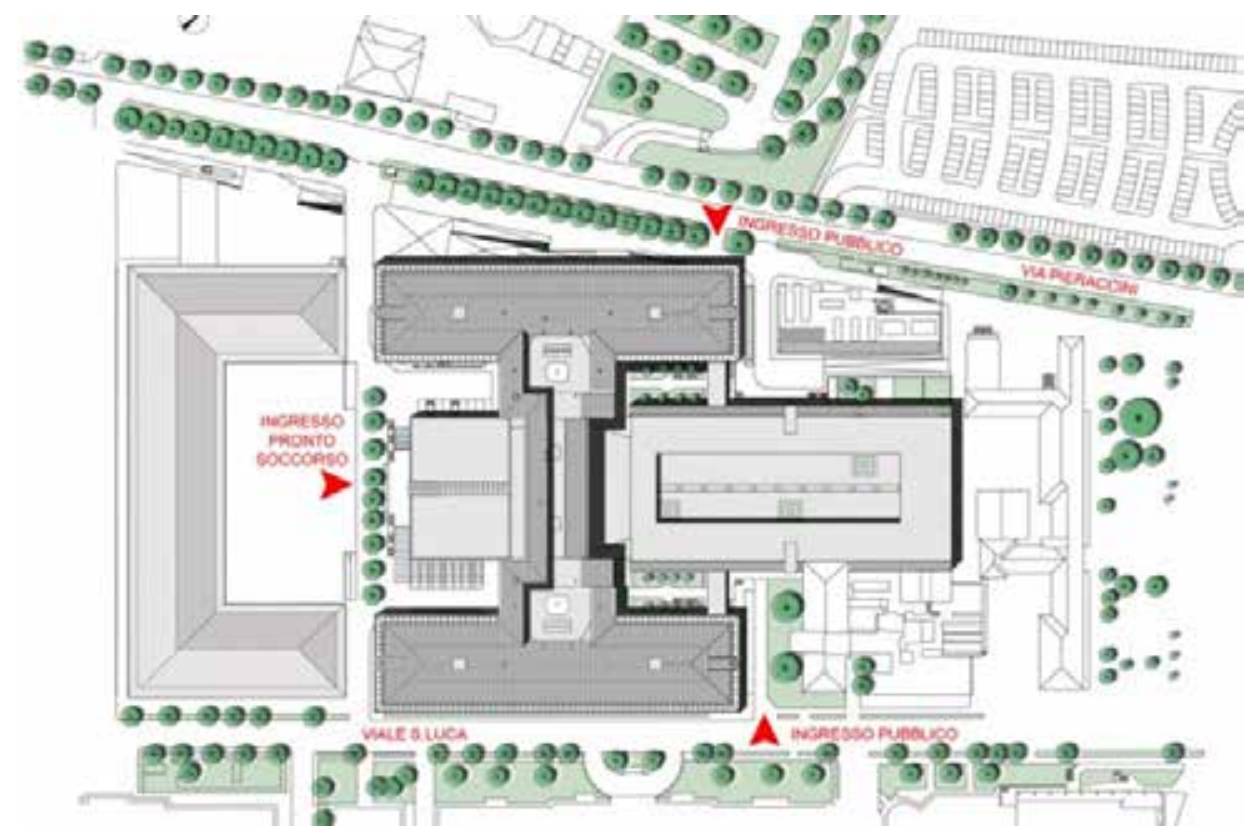

Figura 2. Una planimetria generale del Pronto Soccorso dell'Ospedale di Careggi. 


\section{Elena Bellini}

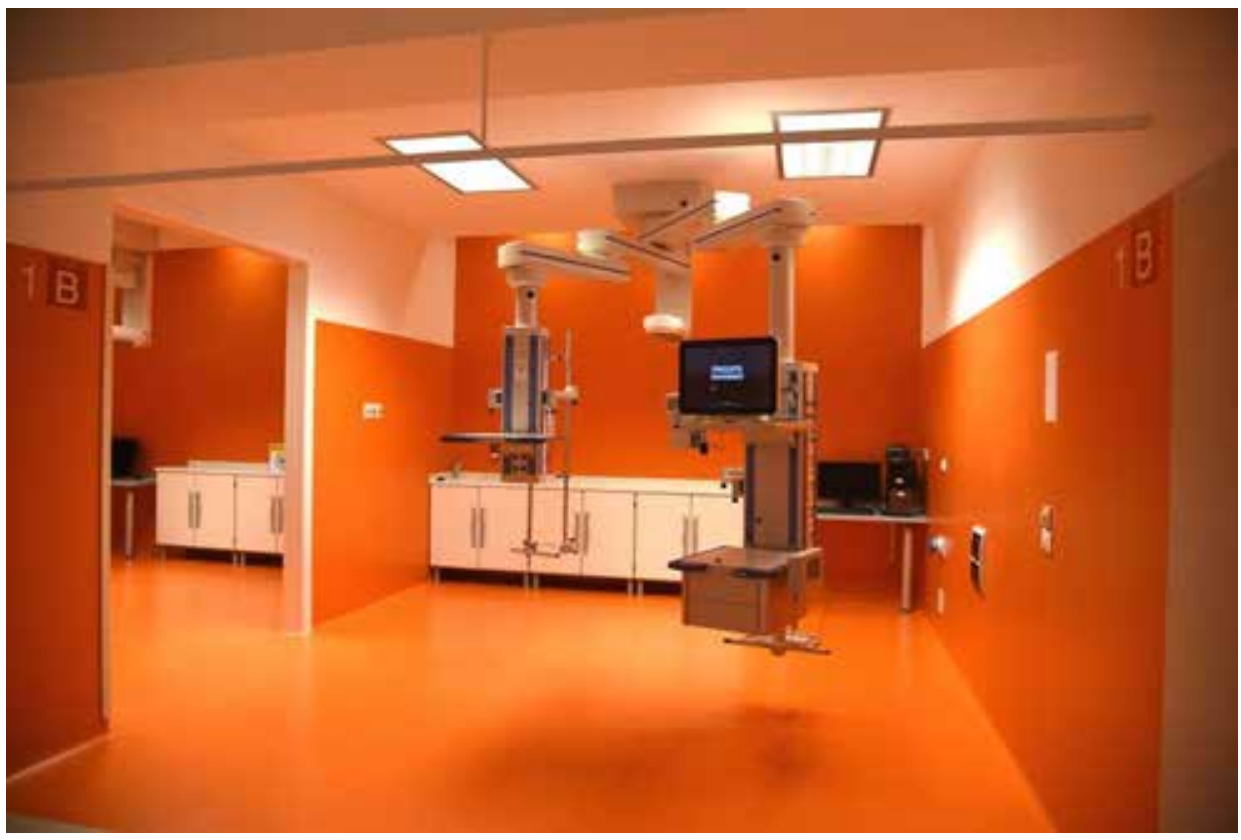

Figura 3. Una delle diverse isole del Pronto Soccorso.

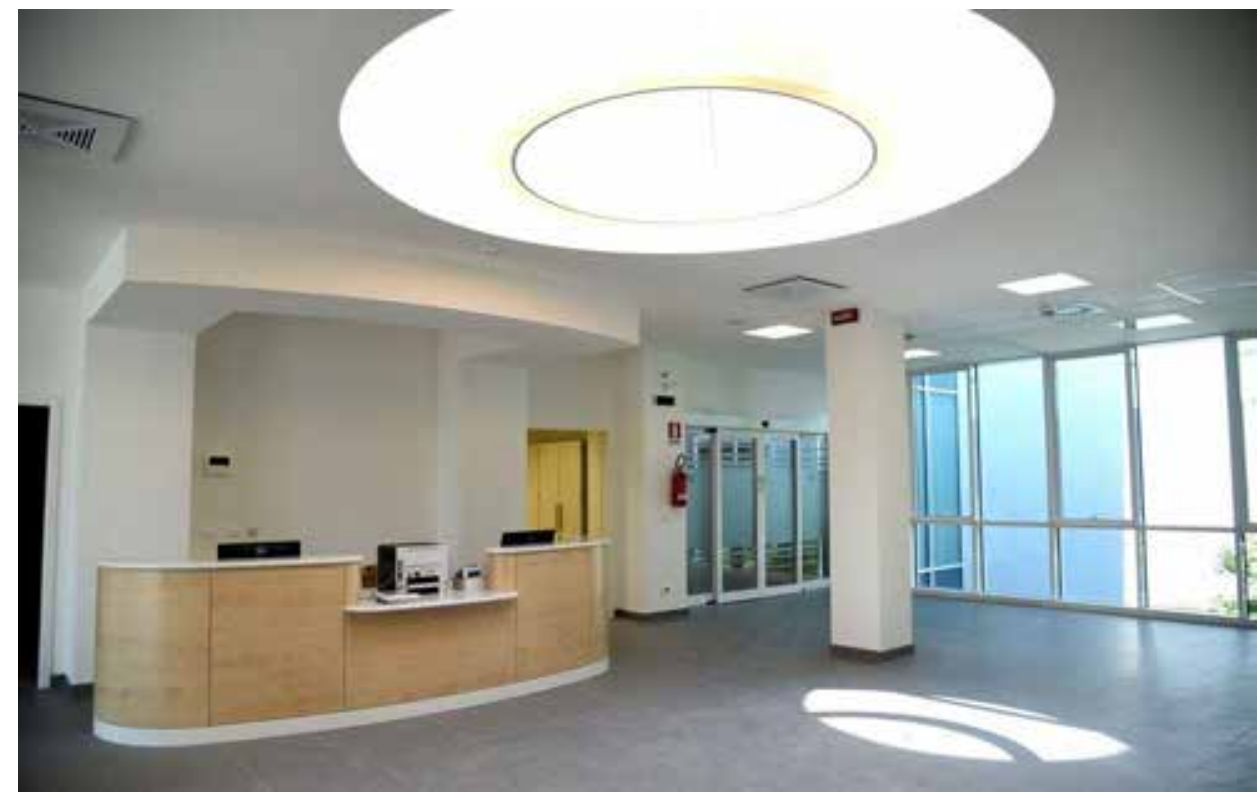

Figura 4. La reception. 
Ambienti sensoriali "terapeutici" che rendano Abili

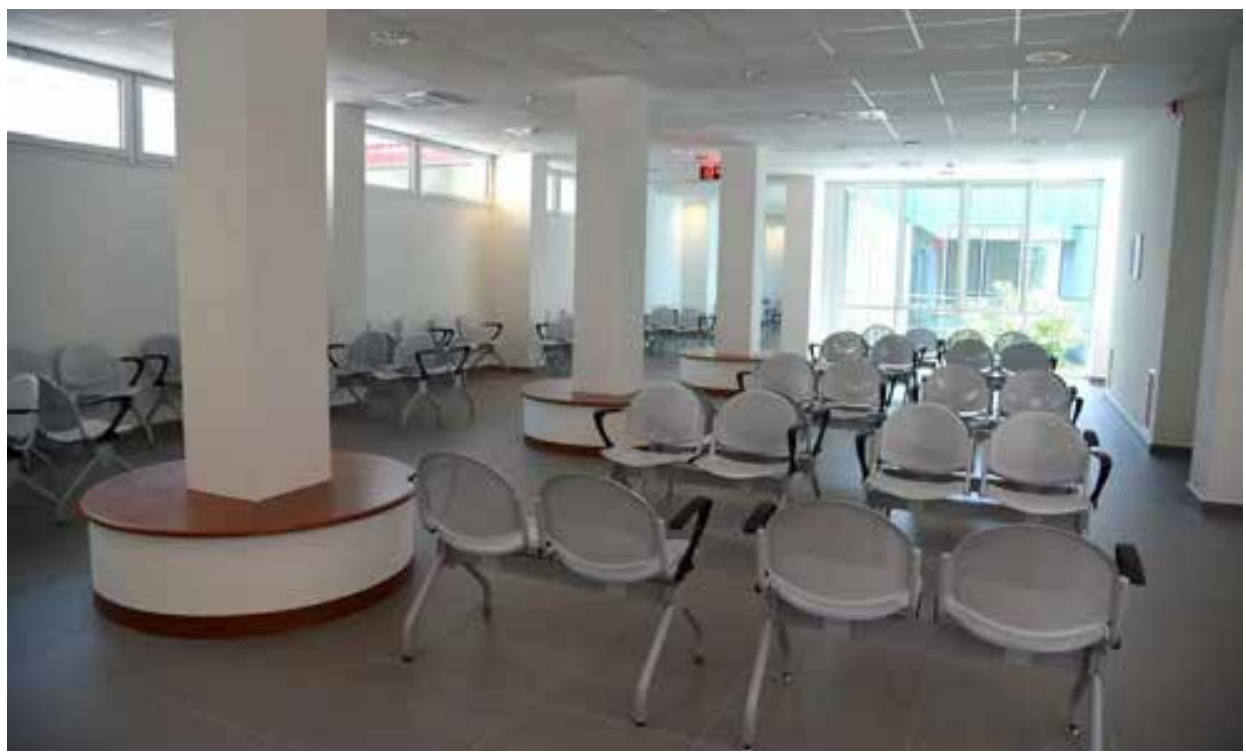

Figura 5. La sala di attesa.

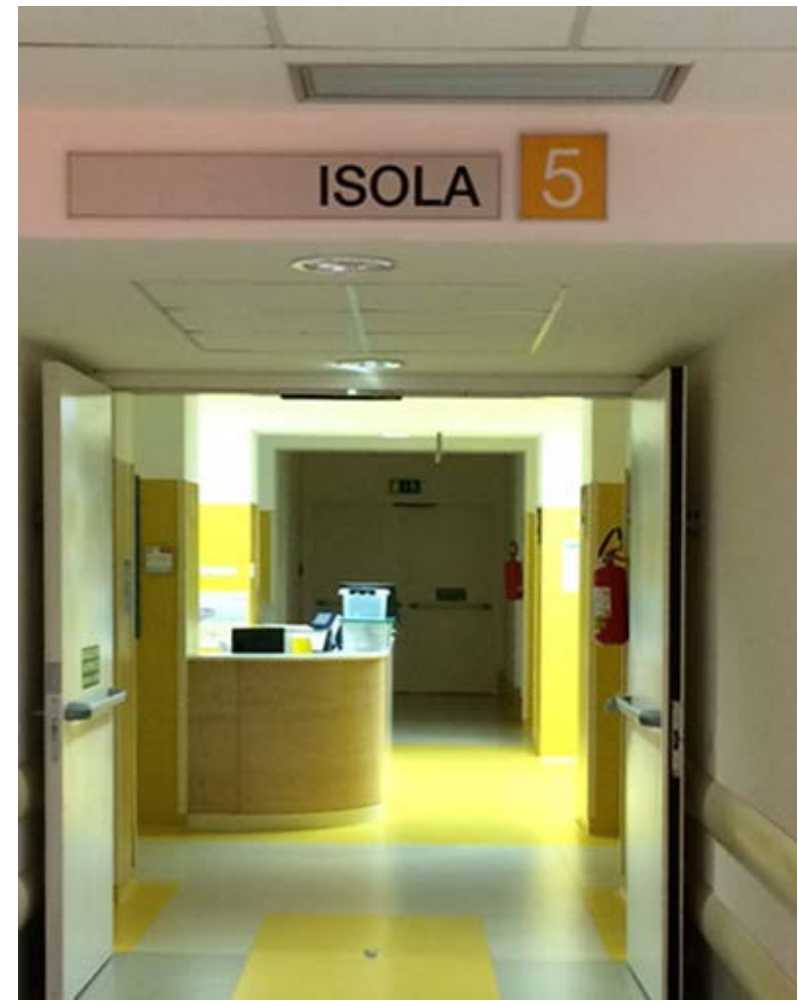

Figura 6. L'isola 5 del Pronto Soccorso. 


\section{Elena Bellini}

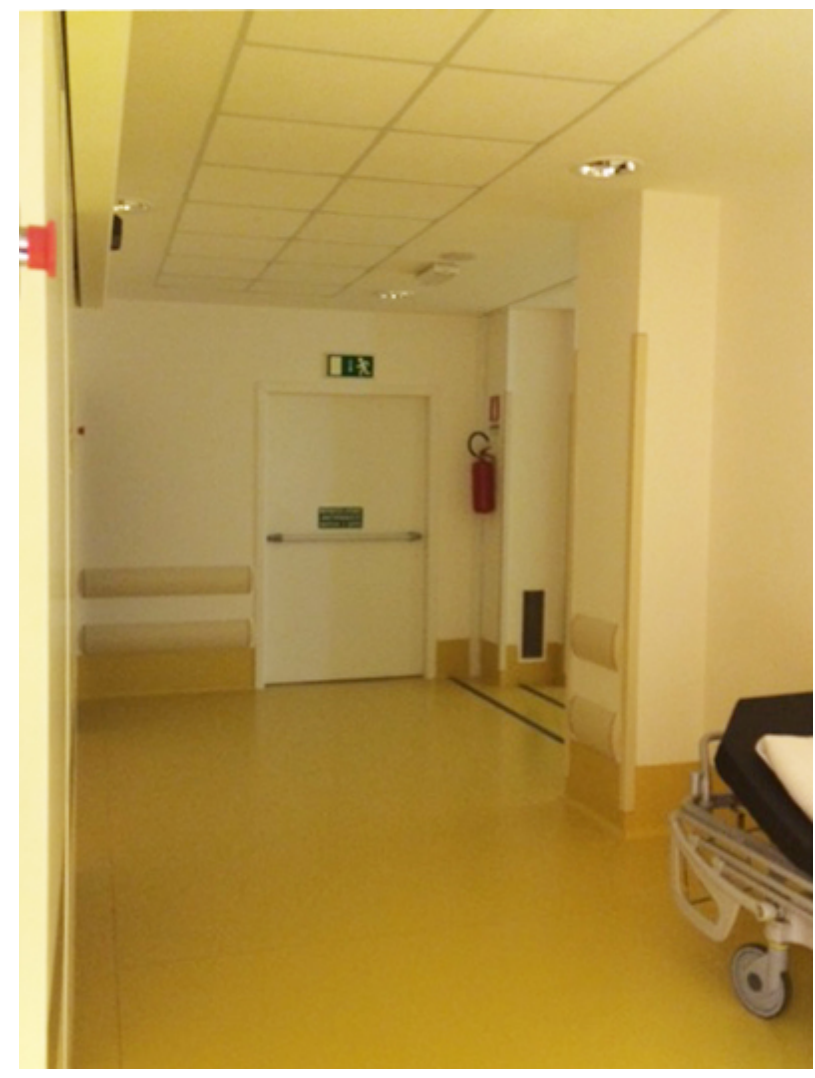

Figura 7. accesso secondario verso l'esterno; "corridoio protetto", filtro tra area di emergenza e stanza multisensoriale. 
Ambienti sensoriali "terapeutici" che rendano Abili

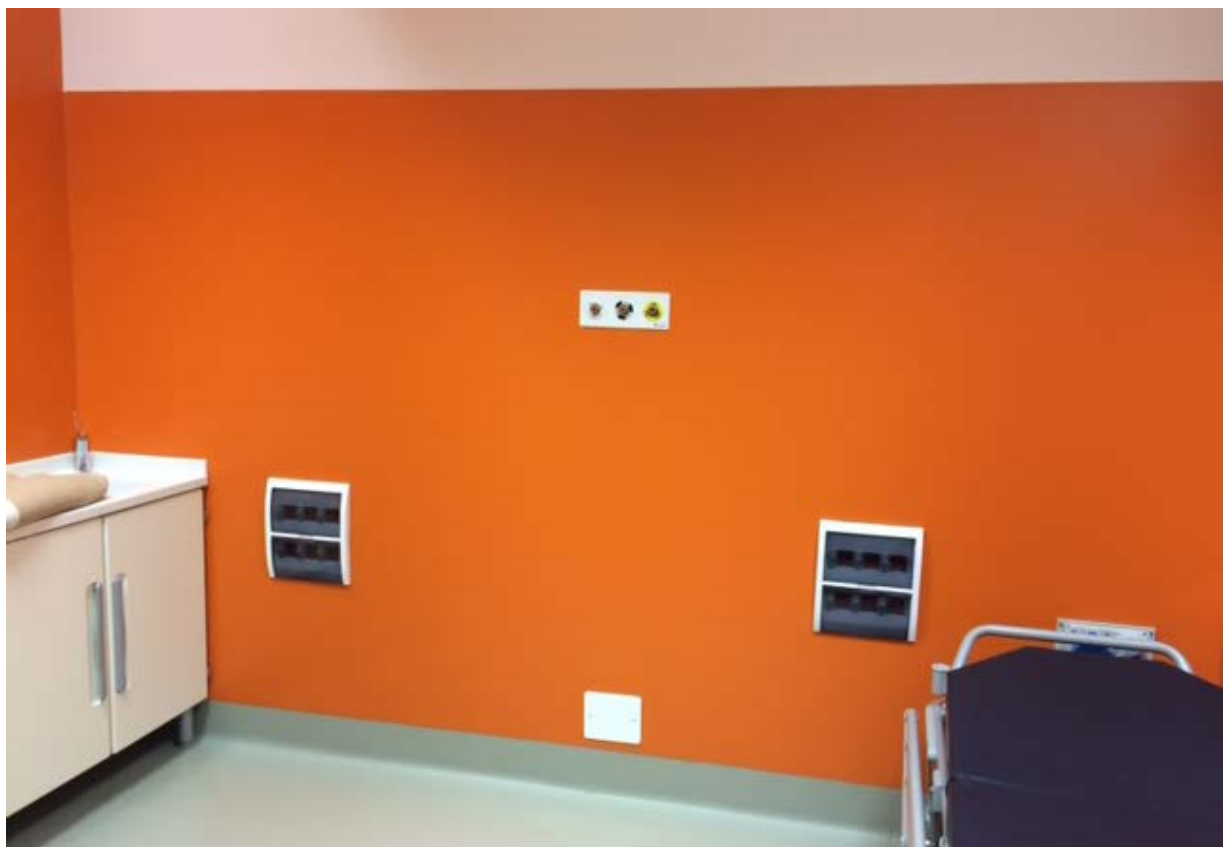

Figura 8. La stanza esistente.

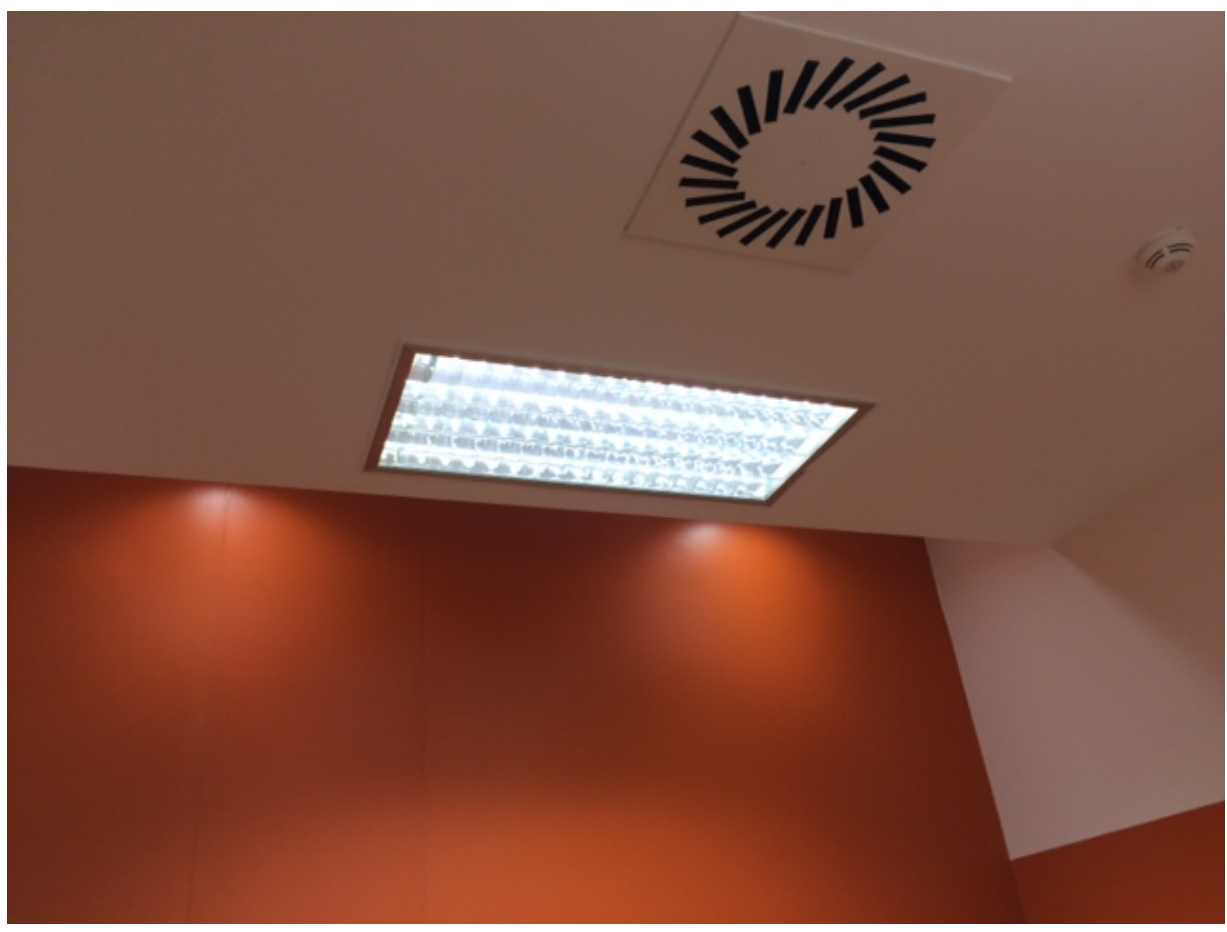

Figura 9. Il sistema di illuminaizone esistente. 
Elena Bellini

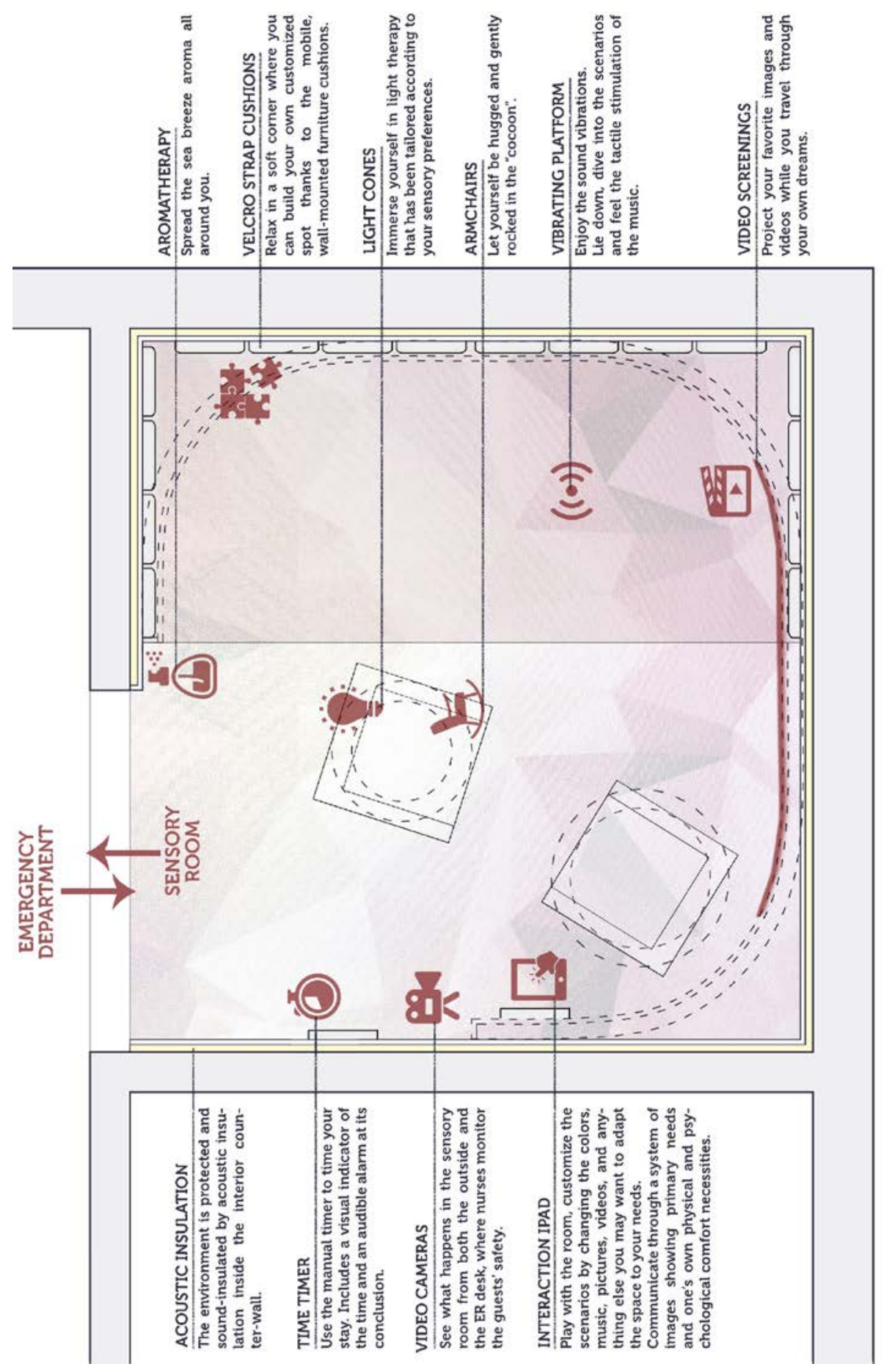

Figura 10. Plianimetria riassuntiva della sala di attesa multisensoriale. 
Ambienti sensoriali "terapeutici" che rendano Abili

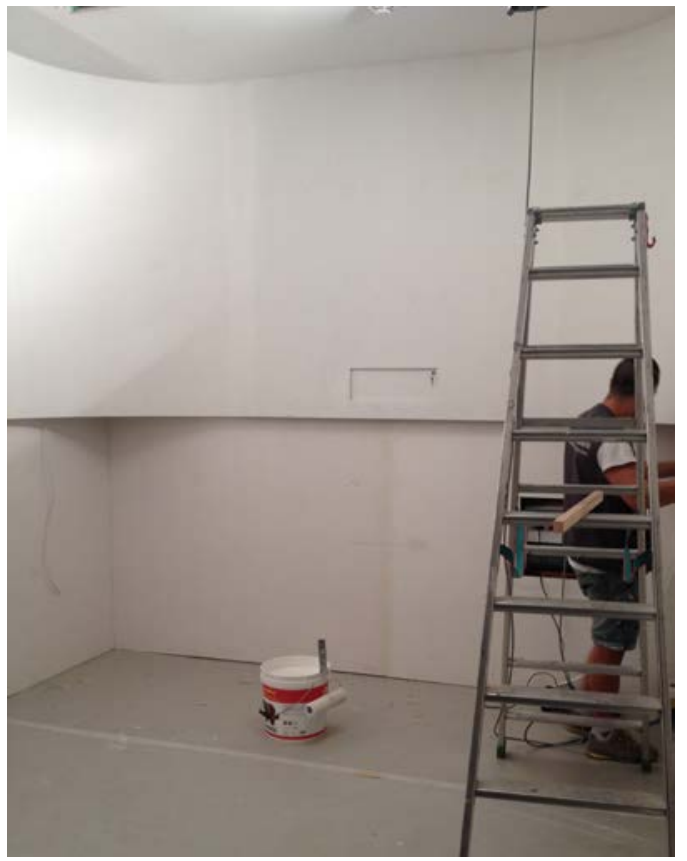

Figura 11. Il sistema di contropareti curvilinee in legno.

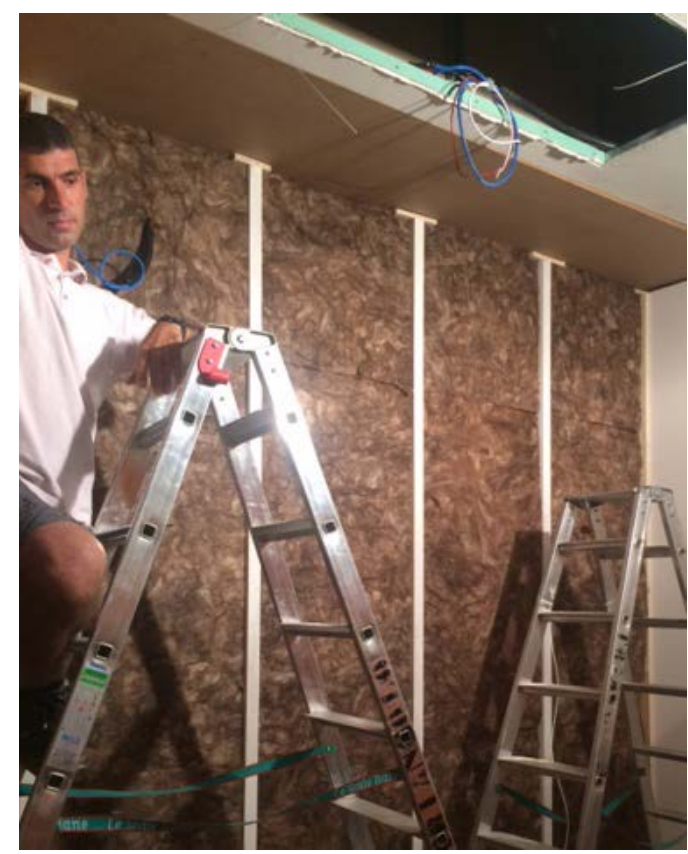

Figura 12. Il sistema di isolamento acustico in controparete. 


\section{Elena Bellini}

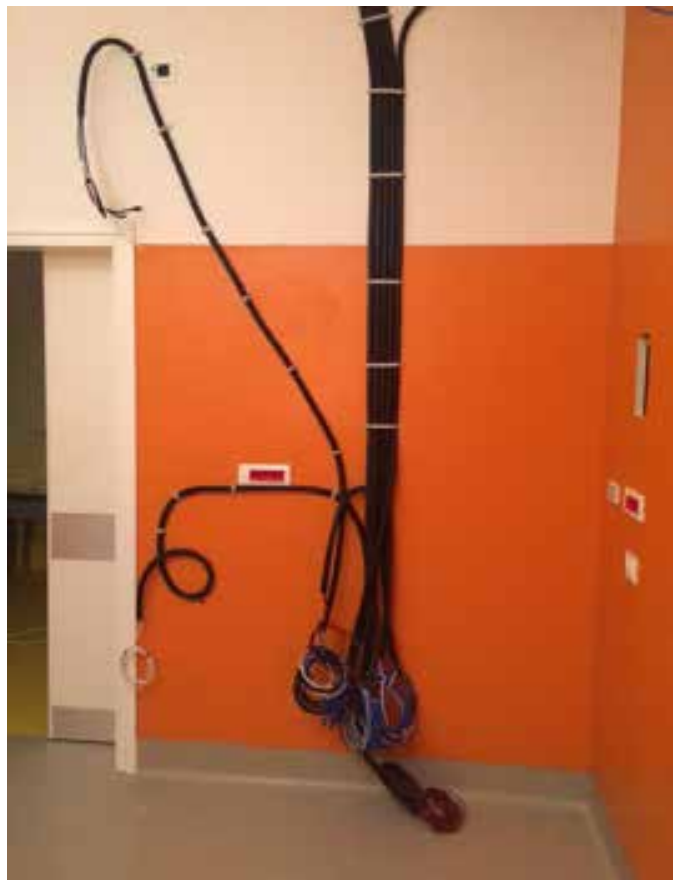

Figura 13. Colonna montante degli impianti elettrici.

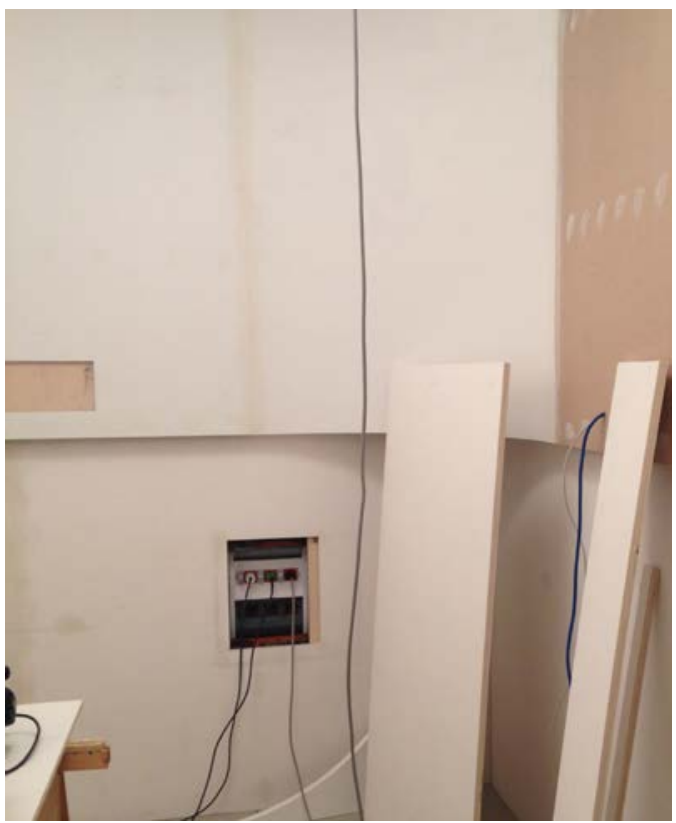

Figura 14. Sportello a scomparsa per nascondere le prese elettriche. 
Ambienti sensoriali "terapeutici” che rendano Abili

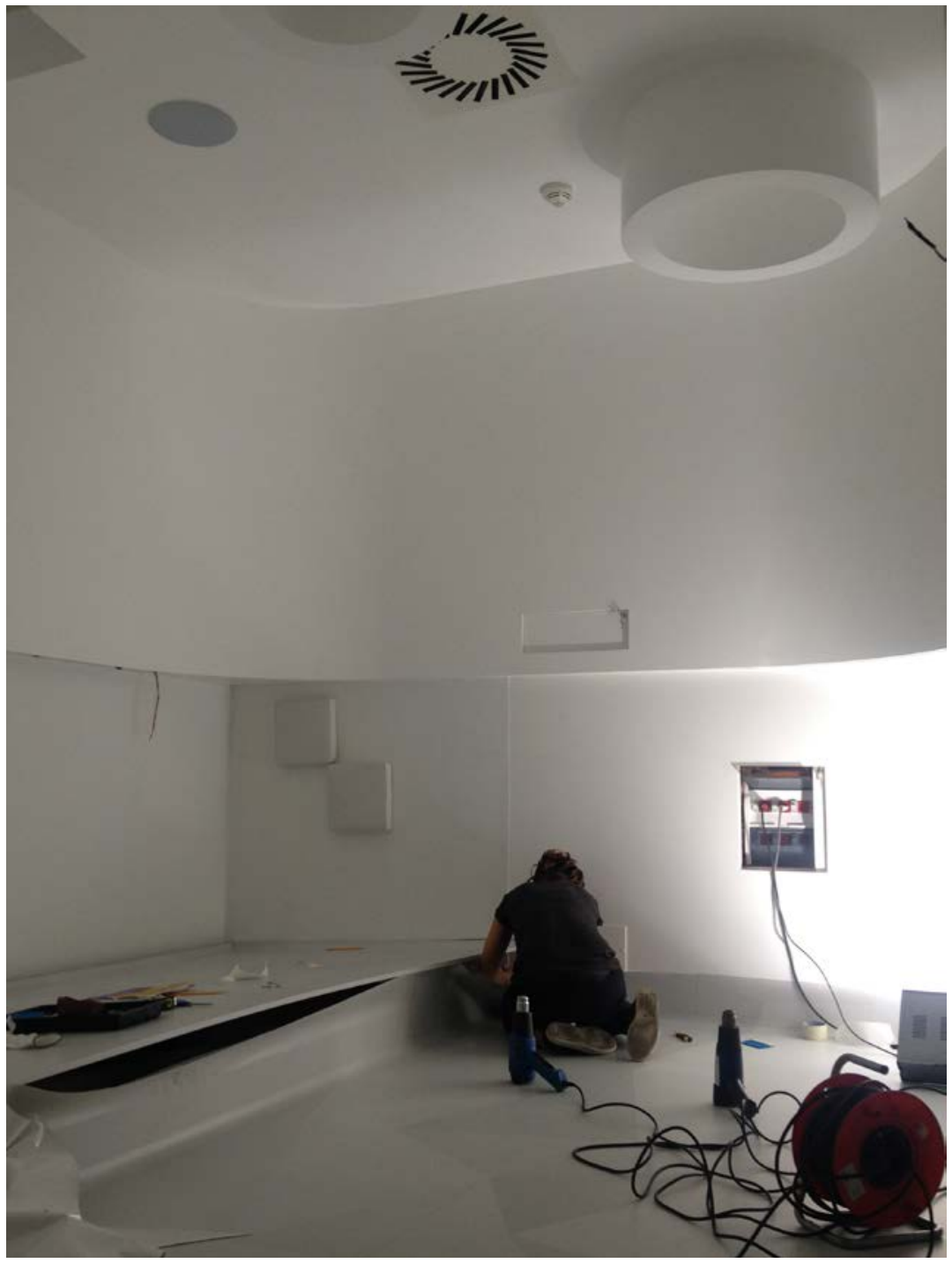

Figura 15.Il rivestimento a pavimento in pellicola vinilica. 


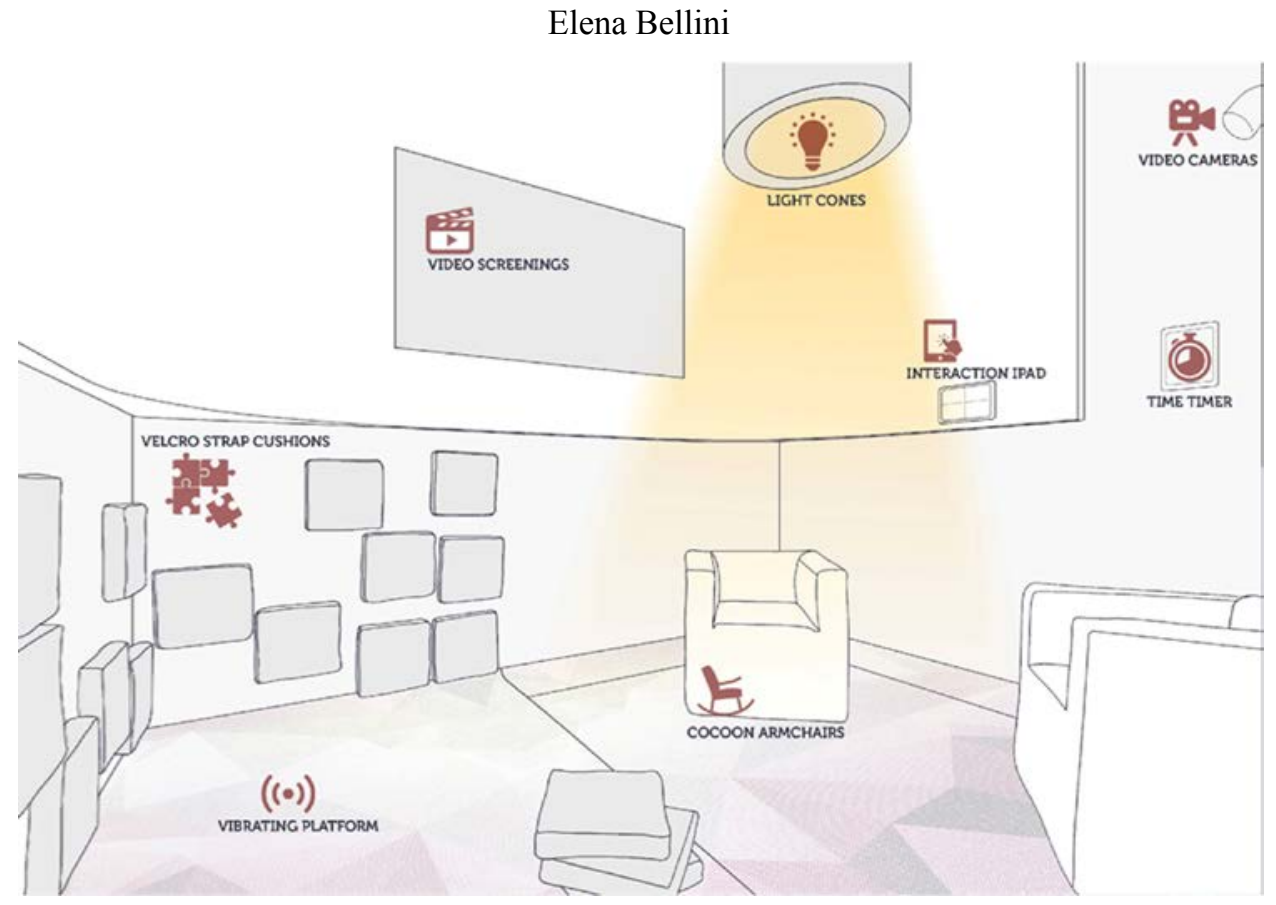

Figura 17. Schema $3 d$ dello spazio sensoriale.

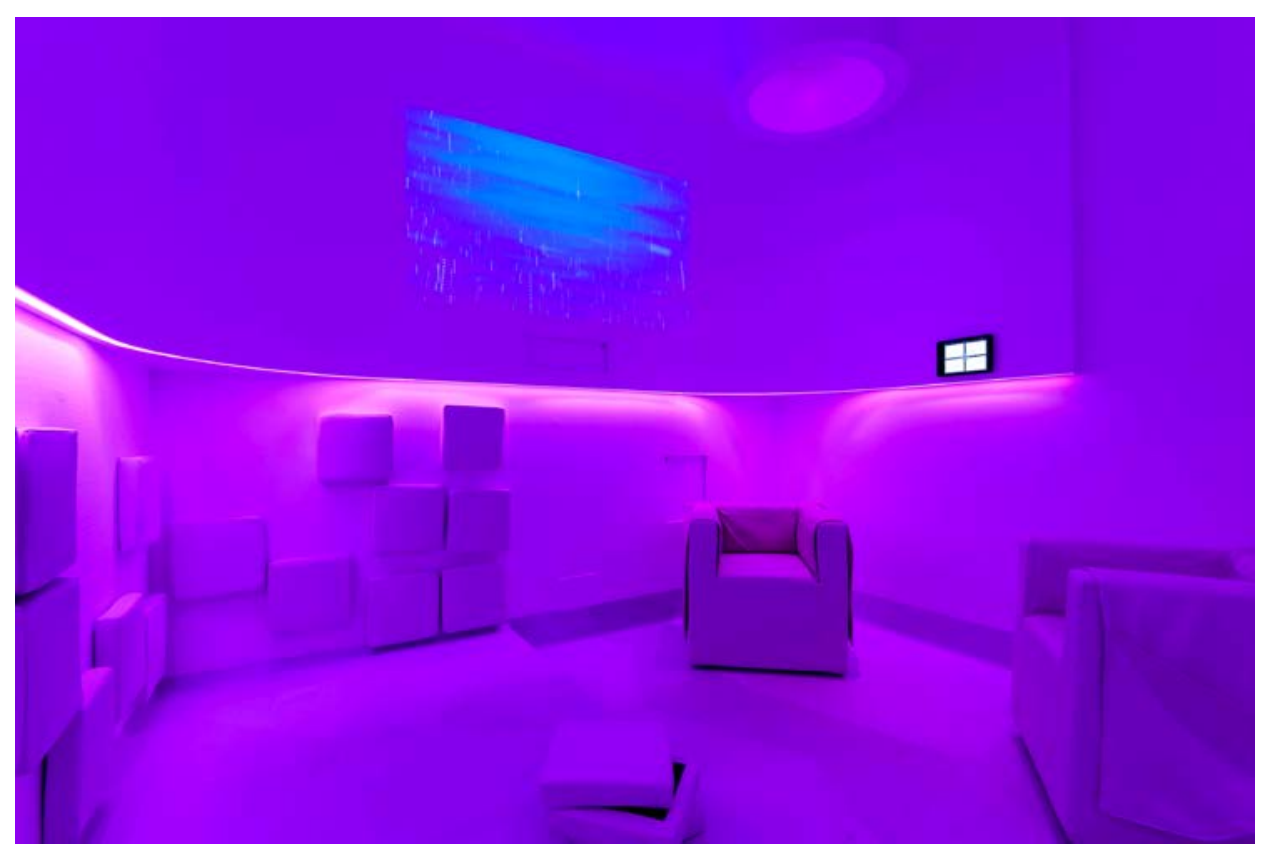

Figura 16. Supporto i-pad a lato dell'ingresso per gestire lo spaizo a livello domotico. 


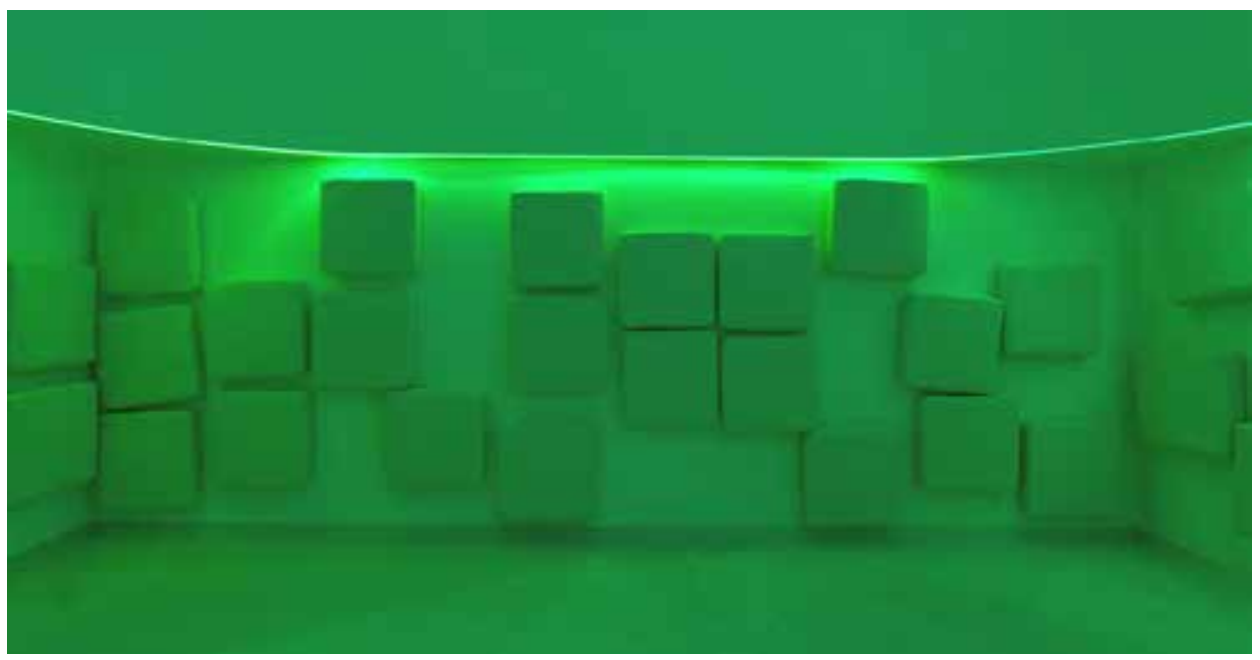

Figura 17. L'angolo morbido sulla pedana sonora vibrante.

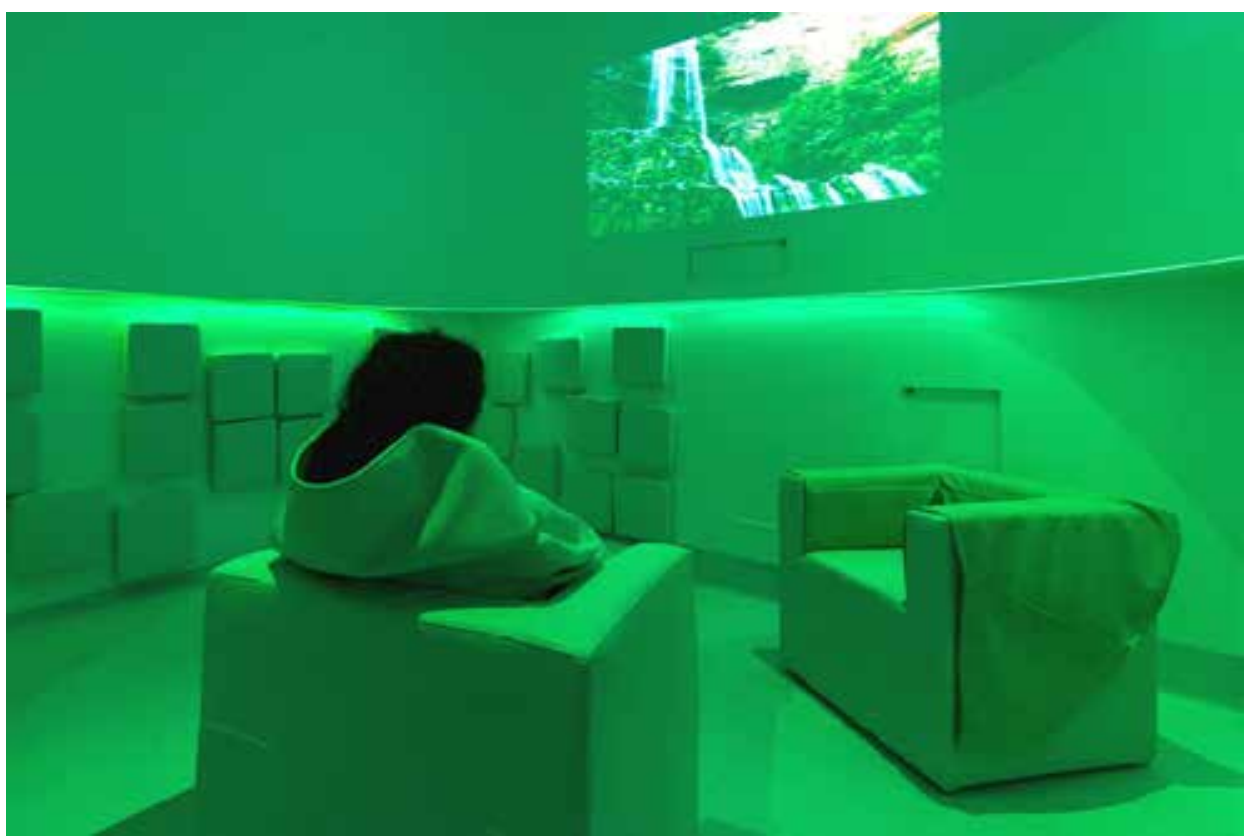

Figura 18.Le poltrone avvolgenti. 


\section{Elena Bellini}

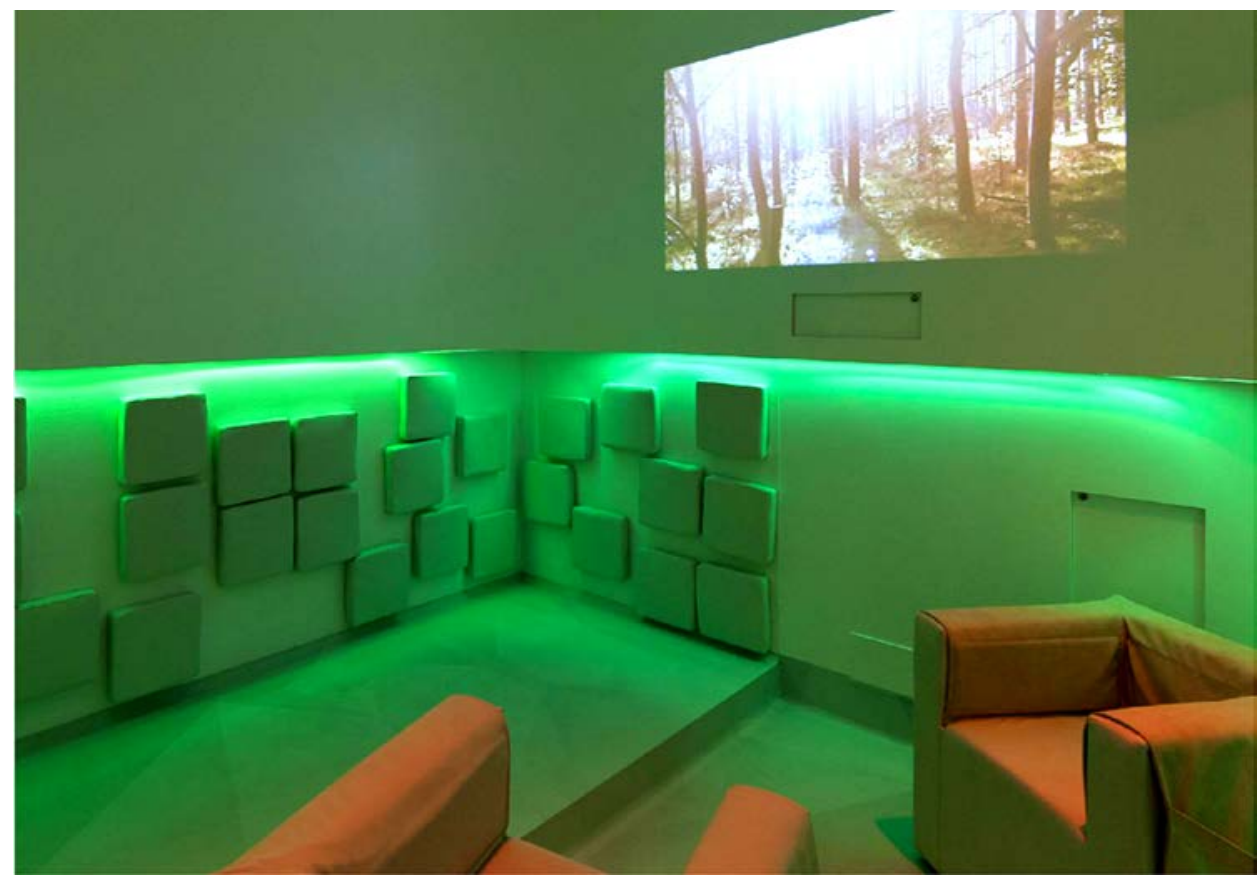

Figura 19. La proiezione video e gli scenari illuminotecnici.

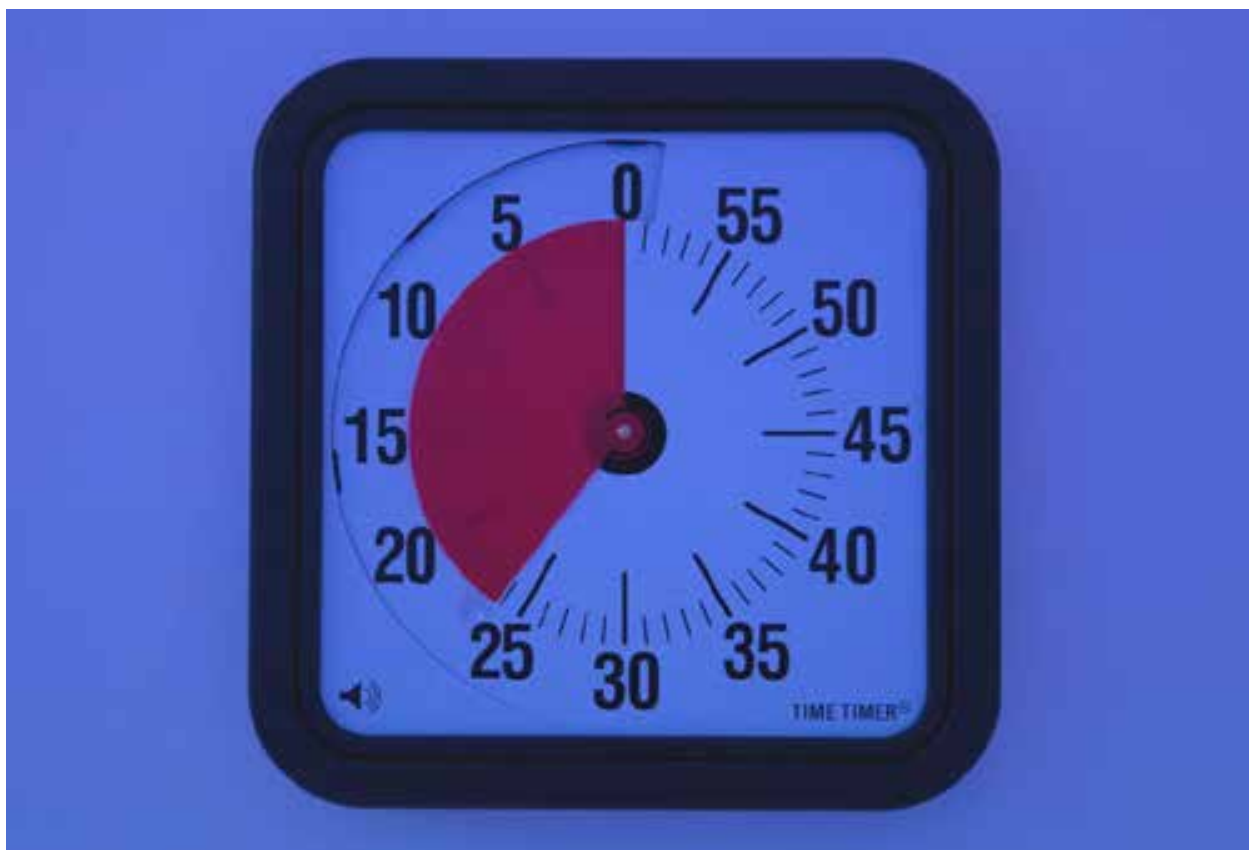

Figura 20. Il time-timer. 


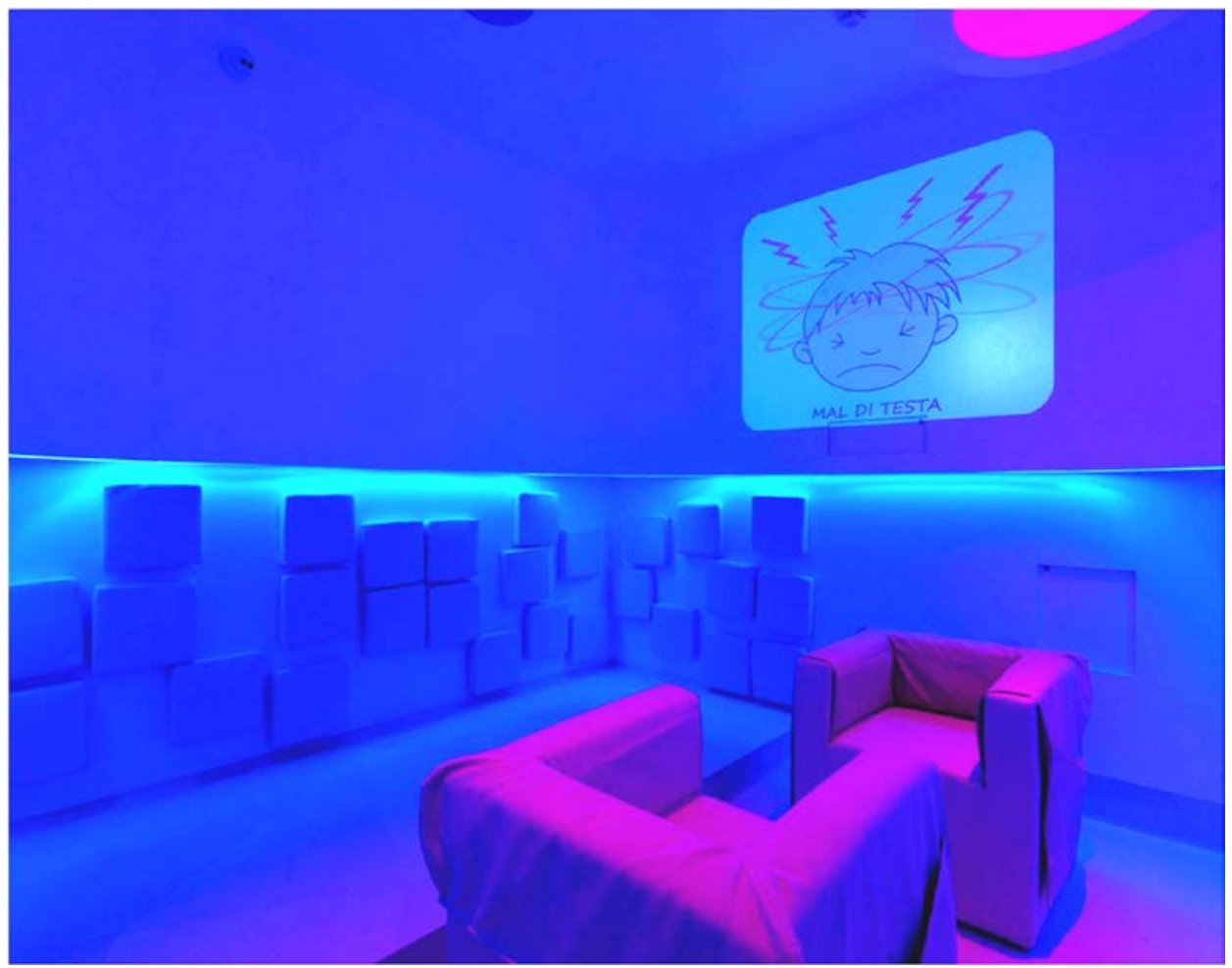

Figura 21. Il sistema di comunicazione per immagini proiettato a parete.

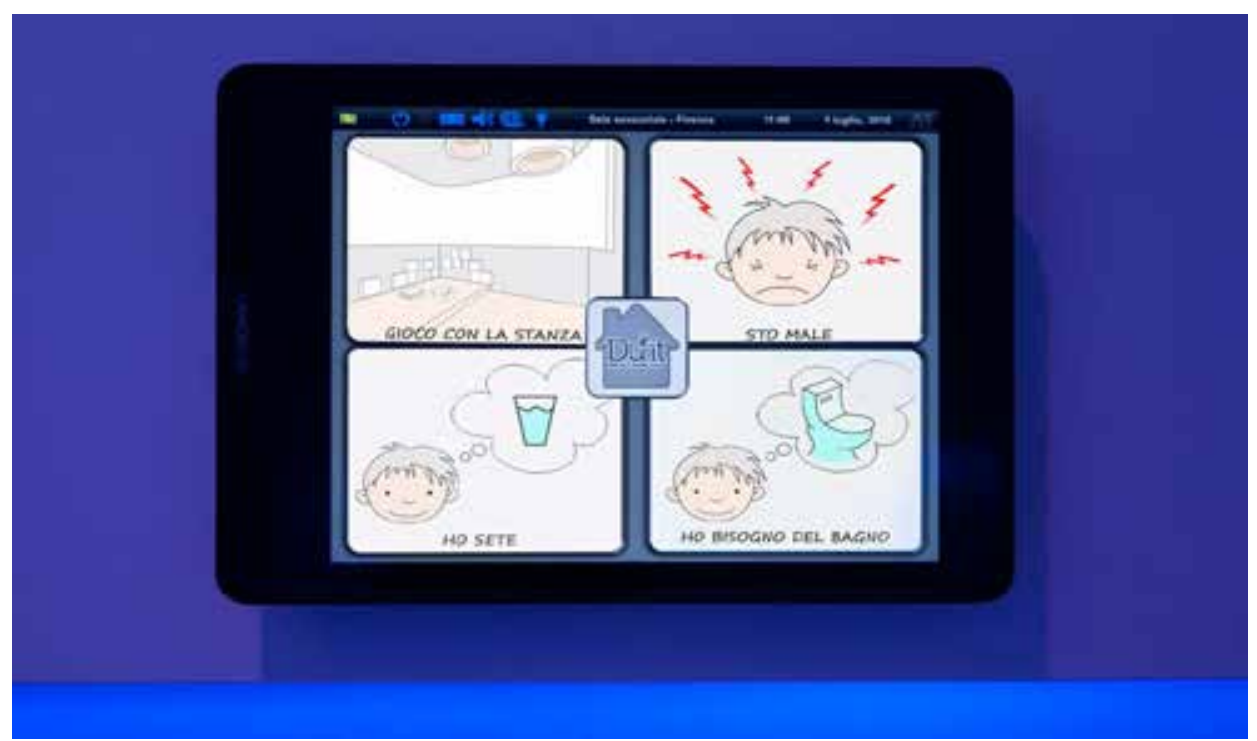

Figura 22. Home page dell'i-pad di gestione del controllo domotico dello spazio sensoriale; bisogni primari e area di personalizzazione dell'ambiente. 


\section{Elena Bellini}

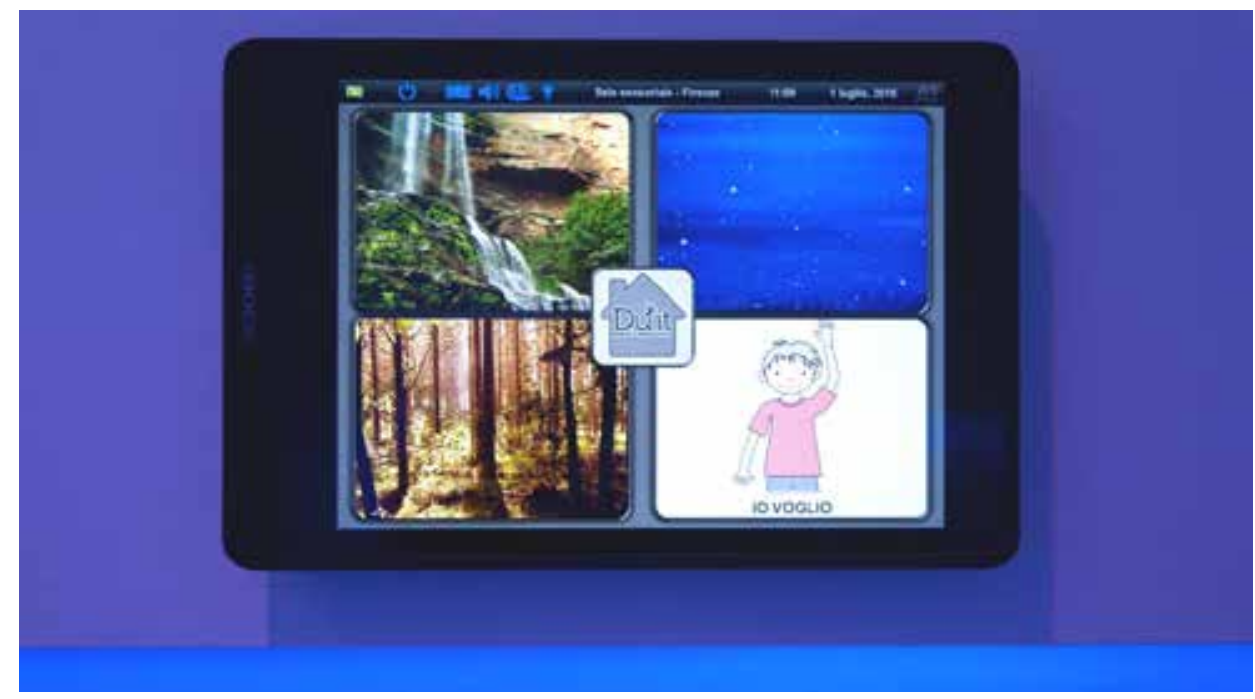

Figura 23. La schermata che permette di scegliere tra gli scenari naturali integrati (video, suono, vibrazione, luce, colore) precostituiti e entrare nella sezione "io voglio" per personalizzare ogni tipo di sitmolazione.

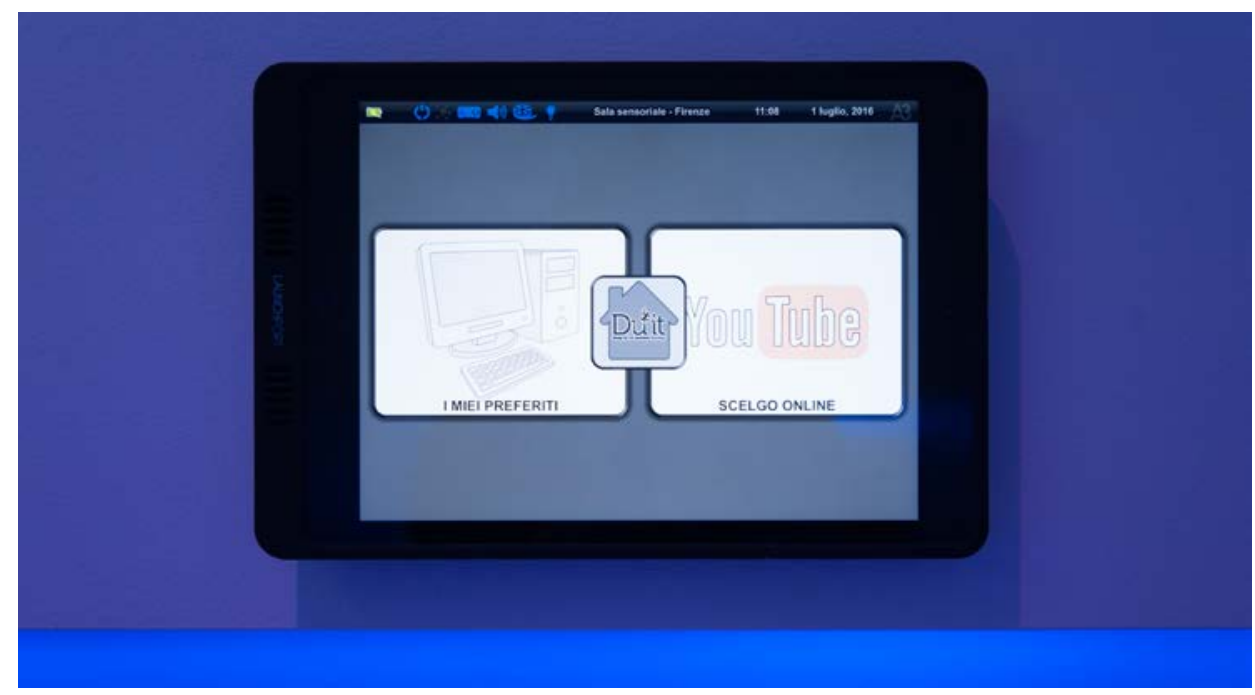

Figura 24. Scelta degli scenari "preferiti" dall'utente o portati direttamente da casa, al fine di ampliare la familiarità dell'ambiente e favorire la personalizzazione dei sistemi di relax e abbattimento dello stress. 
Ambienti sensoriali "terapeutici" che rendano Abili

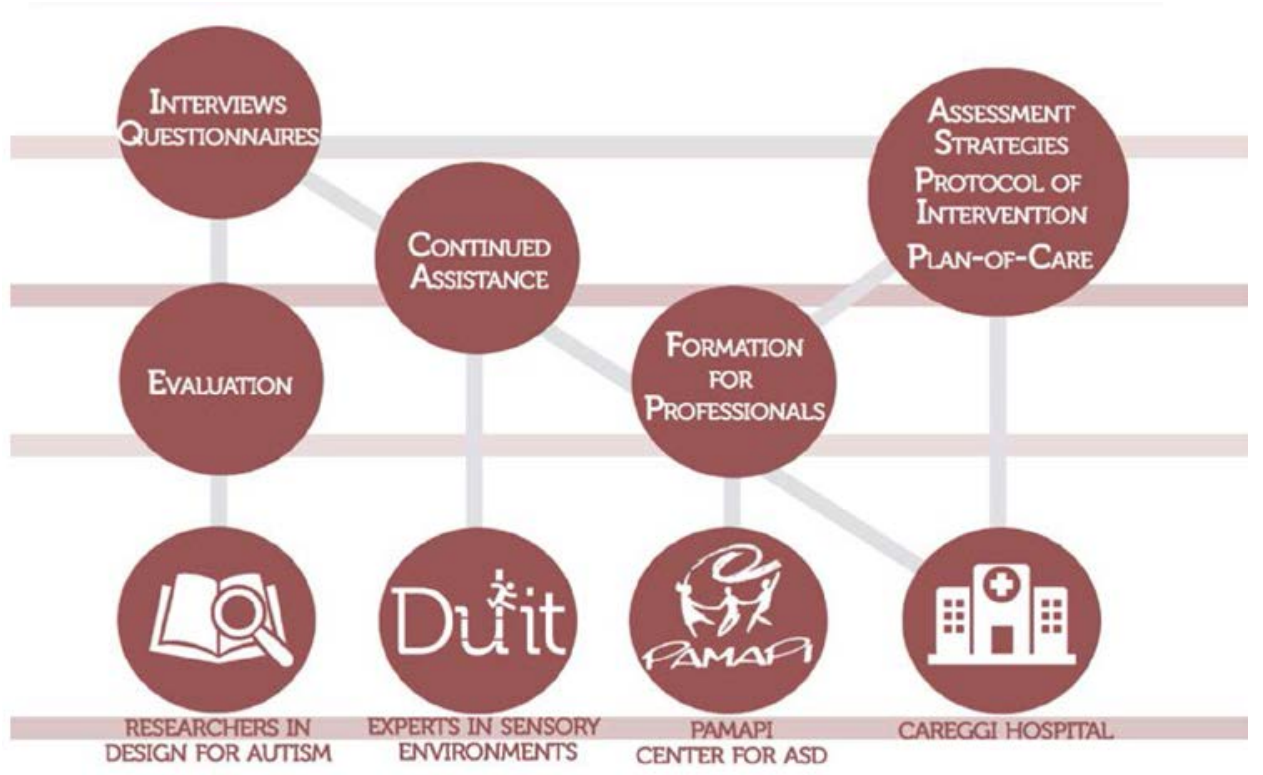

Figura 25. Schema di sviluppo del progetto di ricerca. 


\section{Capitolo 7 \\ Proposta di un aPiattaforma per il Co-design: \\ Condivisione di dati e conoscenze a supporto della progettazione partecipata}

\subsection{Introduzione}

L'aspetto più difficile nel momento in cui ci si trova a progettare per l'autismo è trovare le informazioni. Esistono ad oggi ancora un limitato numero di pubblicazioni sull'argomento, sparse e difficilmente recepibili. Spesso non seguono una metodologia specifica, talvolta sono tra di loro contrastanti, o riportano le opinioni di qualche professionista piuttosto che essere supportate da un background di ricerca. Spesso si riferiscono ad ambiti molto specifici, o al contrario talvolta molto vaghe, parlando in modo generico di come progettare per persone con autismo. Il lavoro più grande ed importante di questo progetto di ricerca è stato quindi rilevare la bibliografia ad oggi presente, filtrarla e riorganizzarla in modo critico secondo una metodologia rigorosa, basata sulle esigenze dalle persone, rilevate dalla studio dell'arte, ma anche e soprattutto dai questionari e dalle interviste dirette, che hanno fornito uno spaccato 'reale' del mondo dell'autismo e soprattutto dell'ambito italiano. Come premesso infatti, la maggior parte della letteratura è internazionale, in un contesto sociale, culturale e normativo, spesso molto diverso dal nostro; è pertanto necessario ed interessante anche riportare tutte le informazioni, applicandole al nostro contesto specifico.

Il fine ultimo di questa ricerca è quindi rendere accessibile tutto questo bagaglio di conoscenza, gli studi fatti, le esperienze rilevate interessanti, i casi studio sperimentati, e divulgarlo in modo semplice ed il più possibile diffuso. Il destinatario finale sono le famiglie di persone con autismo, o le Associazioni, le Fondazioni ed i Centri per l'autismo che sono formati dalle famiglie stesse ed il cui presidente è quasi sempre uno dei genitori. Durante questi anni di ricerca e scoperta, in cui ho visitato tante associazioni e parlato con molte persone, ho riscontrato in tutti un forte bisogno di conoscenza sull'argomento e la mancanza di punti di riferimento, o di professionisti preparati. Talvolta, alcuni presidenti sono anche psicologi e/o professionisti esperti in autismo, ed hanno avuto la possibilità di sviluppare delle ricerche o andare all'estero e visitare alcune strutture, ma talvolta invece non sono riusciti a trovare persone adeguatamente formate o esperti di questo ambito, soprattutto per quanto riguarda l'architettura e la progettazione. E' stato già dimostrato come l'ambiente sia di fondamentale importanza, in particolare parlando di Disturbi dello Spettro Autistico, dove la sensibilità sensoriale è molto forte, e si è ancora più influenzati nel benessere dalle condizioni del contesto ambientale. Non si può esimere quindi da 
una progettazione adeguata degli ambienti integrata con il progetto di vita della persona con autismo, rispondendo a tutti i suoi bisogni, coordinandosi con le altre discipline, compreso l'apporto terapeutico, per raggiungere un miglioramento della qualità di vita.

Come raggiungere allora il destinatario finale, le famiglie?

E' già stato evidenziato come non sia possibile definire delle Linee Guida progettuali che siano adeguate per i diversi ambiti e soprattutto per le diverse persone, così varie da aver bisogno di risposte notevolmente differenziate. C'è bisogno quindi di uno strumento dinamico che sia in grado di contenere tutte le informazioni di supporto alla progettazione e organizzarle in modo da poter trovare una risposta pronta e veloce rispetto alle definizione delle esigenze del destinatario finale, dove la tecnologia e l'ICT si presenta come una strategia utile, non solo in riferimento alla diffusione di informazioni e conoscenza, ma anche alla definizione di un processo di co-design nel quale informazioni e conoscenze siano condivise con il fine di una progettazione collaborativa in cui il destinatario finale sia coinvolto. DARE (sense) vuol essere una piattaforma web open source, che "dia senso" alle tante e sparse informazioni sul tema disponibili e sia di supporto alla progettazione sulla base delle proprie esigenze specifiche, come sviluppo dell'idea sostenuta con la ricerca di tesi.

Un altro aspetto fondamentale del processo è che la piattaforma sia dinamica non solo nell'interazione con l'utente finale, ma anche nell'essere implementabile nel tempo, aggiornabile nel corso del tempo, sulla base della letteratura o di un nuovo progetto o caso studio realizzato o sperimentato. Una sezione importante è anche dare spazio alle persone stesse, attraverso le loro esperienza, rendendo la piattaforma uno scambio continuo e reciproco, pur guidato da un comitato scientifico che ne valuti l'interesse e la valenza.

\subsection{Piattaforme web per il design. Cosa già esiste e cosa no per l'autismo}

E' stata fatta una ricerca sul web per capire se questa esigenza è stata già riscontrata e come si è provato a dare risposta a questo problema. Ci sono molti siti e piattaforme web sull'autismo, che si pongono come supporto alle famiglie. La maggior parte cerca di spiegare "cos'è l'autismo", come si diagnostica e come si può intervenire, diffondere novità in ambito della ricerca. Sono perlopiù realizzate e gestite da Associazioni, Fondazioni o Centri di Ricerca in ambito dei Disturbi dello Spettro Autistico, quindi hanno un profilo di tipo medico-psicologico o socio-assistenziale, fornendo spesso anche una panoramica dei servizi promossi nel territorio. Sono molto comuni anche piattaforme per il supporto dell'apprendimento e dello sviluppo delle abilità sociali e relazionali; spesso sono però gestite da privati e professionisti, che offrono le loro prestazioni di consulenza. Vi sono poi, anche molti blog creati direttamente da famiglie o da persone con autismo, per raccontare le proprie storie e le proprie esperienze, diffondere la consapevolezza sull'autismo, sensibilizzare la popolazione, promuovere la conoscenza e favorire lo sviluppo di rapporti e relazioni, supportando le famiglie e/o le persone con autismo, non facendole sentire sole e isolate nel rispondere alle difficoltà di tutti i giorni, dando anche spesso suggerimenti rispetto alle abilità quotidiane. Sono invece molto rari siti e piattaforme che parlino della progettazione per l'autismo e presentino un focus sull'ambiente e sull'architet- 


\section{Elena Bellini}

tura, benché in tanti riportino il grande impatto che i disturbi sensoriali generano sul benessere e sulle abilità della persona, l'importanza della strutturazione degli ambienti di vita, o le criticità di accessibilità di alcuni spazi quali la scuola o gli ambienti sanitari. Nei siti in cui queste informazioni sono presenti, inoltre, si riferiscono a linee guida o rimandano ad alcune pubblicazioni in questo ambito, ma non esaustive e non organizzate secondo i bisogni della persona, non prevedendo un'interazione con l'utente finale. $\mathrm{Ci}$ sono invece, piattaforme di co-design per interior design o web design, ma non rivolte a specifiche problematiche, quali l'autismo o altre.

Si riportano alcuni esempi di siti web e portali esistenti per comprendere i riferimenti e lo stato dell'arte in questo ambito e quindi definire alcuni spunti ed ispirazioni per costituire una piattaforma che risponda ai bisogni espressi dalle stesse famiglie.

La piattaforma più conosciuta e diffusa in ambito internazionale è Autism Speaks - https://www.autismspeaks.org/. Autism Speaks è stata fondata nel 2005 da Bob and Suzanne Wright, nonni di un bambino con autismo, attraverso la coesione di tre organizzazioni in ambito di autismo - Autism Coalition for Research and Education (ACRE), the National Alliance for Autism Research (NAAR) and Cure Autism Now (CAN) - per sviluppare e promuovere ricerca, conoscenza, assistenza e supporto alle persone con autismo e alle loro famiglie. Nello specifico la piattaforma si occupa di divulgare la conoscenza in ambito di autismo; promuovere la diagnostica precoce; offrire una panoramica completa dei servizi e degli interventi esistenti e più all'avanguardia in casi di autismo, continuamente aggiornati attraverso il blog, anche in merito allo specifico della ricerca; dare tutte le informazioni possibili in ambito dell'innovazione e del supporto di ogni genere per l'autismo, offrendo anche suggerimenti pratici alle famiglie, strumenti e tool boxes; trovare contributi e finanziamenti. Autism Speaks è un'organizzazione diffusa in tutti gli Stati Uniti, ma conosciuta e promossa in tutto il mondo.

Un'altra piattaforma interessante e molto diffusa in ambito americano è The Autism Society of America - http://www.autism-society.org/, un network nazionale di servizi e informazioni di supporto alle persone con autismo e alle loro famiglie, fondato nel 1965 da Dr. Bernard Rimland, Dr. Ruth Sullivane altri genitori di bambini con autismo. The Autism Society ospita anche la più grande conferenza nazionale sull'autismo, divulgando così informazioni e conoscenza, oltre che con il sito web ed il National Contact Center rivolto alle famiglie, e servizi diretti di supporto alle famiglie su tutti gli ambiti che migliorino la qualità di vita delle persone con autismo.

Il corrispettivo in ambito della Gran Bretagna è The NAS - National Autistic Society - http://www.autism.org.uk/, un'associazione no-profit esistente da più di 50 anni, che si occupa di divulgare la conoscenza e la sensibilizzazione in ambito di autismo, oltre a offrire scuole speciali e servizi di assistenza sul territorio e numerose conferenze ed eventi formativi, rivolti anche a professionisti del settore. Ha inoltre anche una Online Community, dove è possibile scrivere contributi e rispondere ad altri interventi, partecipando ai diversi forum, organizzati per età o argomento. Tra le conferenze organizzate negli anni, the NAS ha sponsorizzato anche conferenze in ambito specifico del design for autism ed è, infatti, una tra le poche piattaforme ad avere una sezione completa in ambito della progettazione per l'autismo, correlata da indicazioni e riferimenti, pubblicazioni scientifiche e professionisti esperti nel setto- 
re. Nello specifico, riporta indicazioni rispetto al rapporto tra ambiente e persona, anche riguardo alle sensibilità sensoriali, linee guida di riferimento sulla progettazione degli ambienti di vita (Figura 1), link e riferimenti a normative presenti in Gran Bretagna, ed in particolare riguardo al tema scuola - Building Bulletin 102: disabled children and children with SEN (bisogni educativi speciali), pubblicazioni scientifiche sull'argomento e risorse correlate. Il metodo di interazione con l'utente finale è comunque di tipo didascalico, offrendo linee guida o articoli, senza esplicazioni visive o applicazioni e casi studio di riferimento. Nella pagina dello shop offre comunque la possibilità di acquistare le pubblicazioni e gli atti di convegno passati.

Si riportano a titolo esemplificativo, inoltre, alcuni blog incentrati sulla divulgazione delle informazioni in abito di autismo di particolare interesse, tra cui: http://autismnow.org/, https://www.autism.com/, http://www.autismcommunity.com/, http://www.thinkingautismguide.com/, https://www.autismparentingmagazine.com/, http://blog.theautismsite.com/. http://nationalautismnetwork.com/, https://ollibean.com/,

Oltre a questi si segnalano come esempi particolari alcune piattaforme che si riferiscono nello specifico del supporto alla progettazione, tra cui: Autism Toolbox $\mathrm{http}: / / w w w . a u t i s m t o o l b o x . c o . u k /$ (Figura 2), ovvero uno strumento realizzato in compartecipazione tra il governo scozzese, Scottish Autism e Autism Network Scotland, per guidare e supportare l'inclusione di bambini e ragazzi con Disturbi dello Spettro Autistico nei servizi di istruzione tradizionale in Scozia. Anche se non rappresenta uno strumento tecnico e specifico dedicato alla progettazione, questo Toolbox si rivela interessante poiché offre delle indicazioni pratiche e dei suggerimenti per realizzare interventi nell'ambiente scolastico, anche attraverso raccomandazioni specifiche rivolte ad esempio all'ambiente dell'aula, corredate da alcuni strumenti operativi come check-list di valutazione degli spazi e delle risorse; è possibile dal sito scaricare anche un pdf completo dello strumento operativo.

Un altro esempio interessante, soprattutto poiché pensato per l'interazione dell'utente finale, almeno in parte, con la piattaforma web è la Car Autism Roadmap https://www.carautismroadmap.org/ (Figura 3). Questa mappa virtuale identifica i servizi presenti sul territorio, divulga le innovazioni in ambito di ricerca e diffonde le informazioni che possano essere di supporto per la persona con autismo e la sua famiglia, nell'ambito di scuola, residenza, sanità e intervento terapeutico, normative e legislatura, supporto alla comunità, sicurezza e inserimento lavorativo. La maggior parte degli articoli presenti sul blog sono stati scritti dai ricercatori e professionisti del Center For Autism Research (CAR) all'interno del Children's Hospital Of Philadelphia (CHOP) e revisionati da un team di professionisti, genitori e sostenitori, affinché se ne garantisse la validità e la facilità di lettura. Questa piattaforma è interessante proprio per la sua struttura e per il design, che sarà fonte di ispirazione per la realizzazione della piattaforma DARE (Sense). In particolare, stabilisce un'interazione con l'utente finale, attraverso delle domande e delle selezioni su icone che rappresentano le aree di riferimento, andando in modo semplice a selezionare gli articoli di specifico interesse della persona che naviga il sito, all'interno dell'enorme bacino di informazioni presente (Figura 4). Nella home, infatti, si fa subito una prima selezione sulla base della tipologia di utenza, se rappresenta una persona con autismo che ha appena avuto la diagnosi, o al contrario che è interessata ad avere in- 


\section{Elena Bellini}

formazioni poiché sono stati percepiti dei sintomi che potrebbero far pensare ad un caso di autismo, o che semplicemente cercano idee e risorse di supporto. Entrando nel sito quindi è possibile subito definire l'ambito specifico di riferimento, come anticipato, in modo da ridurre ulteriormente le risorse informative. Entrando in una specifica di queste sezioni è infine possibile affinare la ricerca in base ad un menu laterale (Figura 5), che fa scegliere tra indicazioni specifiche sull'età, il genere, il luogo di residenza, piuttosto che informazioni specifiche sulla singola area che potrebbero essere di maggiore interesse per la persona. Questo sistema è molto utile e funzionale poiché riesce a definire le informazioni sulla base delle singole esigenze. Tra le diverse sezioni ci sono anche alcune indicazioni specifiche sull'architettura e la progettazione di ambienti di vita per autismo, ma anche in questo caso, come negli altri, si rimanda a linee guida di riferimento, pubblicazioni o articoli d'interesse, ma senza indicazioni grafiche o metodi rappresentati che ne facilitino la comprensione o lo rendano uno strumento maggiormente operativo. $E^{\prime}$ possibile anche in questo caso scaricare le informazioni/linee guida in pdf (Figura 6).

Uno strumento creato invece, infine, appositamente per l'ambito della progettazione per l'autismo è Architetture for Autism - http://www.architectuur-voorautisme.org/, una piattaforma web che nasce dal lavoro e dall'esperienza della Dr. Leo Kannerhuis (inizialmente una "casa", nominata a partire da Leo Kanner, oggi centro di riferimento nazionale olandese nel campo della diagnosi, del trattamento e del supporto delle persone con autismo) e dalle ricerche più recenti di Flip Schrameijer (Figura 7). Inizialmente è stata la trasformazione e la rielaborazione dei volumi pubblicati sulla ricerca in ambito dell'architettura per l'autismo (Schrameijer, Flip, Met het oog op autisme. Bouwen \& inrichten voor mensen met autisme, Doorwerth, Dr. Leo Kannerhuis, november 2013) e, ad oggi, viene implementata dal continuo lavoro di ricerca e best practices sperimentate. Il sito prevede quindi una raccolta di "raccomandazioni" sul design degli spazi usati dalle persone con autismo e nello specifico residenze, scuole, centri per l'autismo. Queste raccomandazioni derivano in parte dallo studio dello stato dell'arte, in parte dall'esperienza pratica del centro. Ad oggi vi sono un totale di 194 raccomandazioni di cui 133 sono state sperimentate: 46 nel contesto "casa per bambini", 44 nella scuola, 43 nelle soluzioni di vita indipendente. Le raccomandazioni sono quindi organizzate rispetto al contesto, ovvero "casa per bambini", scuola, residenza indipendente (Figura 8), case di cura, residenze permanenti (anche se per adesso sono disponibili solo "casa per bambini", scuola e residenza indipendente), oppure per capitoli riferiti ad argomenti specifici, ovvero location (il luogo ed il contesto maggiormente adatto per l'edificio da progettare), spazi esterni e giardini, spazi architettonici, interior design, dispositivi ed impianti (Figura 9 e 10). Oltre a questi, vi sono poi "temi" specifici dei diversi capitoli o trasversali alle diverse raccomandazioni, che talvolta ricorrono anche più volte all'interno di differenti capitoli, come ad esempio luce e vista, rumore ed acustica, cucina, spazi comuni, colore, ecc.

Benché il sistema sia molto preciso ed interessante, è stato rilevato un sistema piuttosto complesso, non facilmente navigabile e leggibile. Sono testi lunghi, assimilabili a linee guida e non riportano esemplificazioni grafiche, immagini o riferimenti ad applicazioni reali che ne aiuterebbero la comprensione. Sono linee guida navigabili, interessanti, ma senza prevedere una particolare interazione da parte dell'utente 
finale e soprattutto un'organizzazione rispetto alle sue esigenze specifiche, come era presente ad esempio nella precedente piattaforma. Il metodo di classificazione per contesto e capitoli è molto vicino a quello di un volume scritto, molto ampio e generico nella definizione dei temi, e le raccomandazioni sono molto lunghe e discorsive. Il lavoro che è stato fatto è in ogni caso sicuramente di stimolo ed ispirazione, anche per quanto riguarda la possibilità dei visitatori di condividere contributi e opinioni rispetto alle raccomandazioni. $E^{\prime}$ presente infine un blog in cui viene costantemente aggiornato il sito con le ultime ricerche ed innovazioni in questo ambito.

Un altro riferimento interessante in questo senso è il sito web della ricercatrice

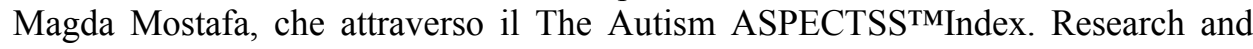
Design Services - https://www.autism.archi/ divulga la conoscenza della strutturazione della propria sensory matrix, anche se si pone solo come descrizione dei diversi criteri di design, come sintesi di linee guida progettuali, senza interazione da parte dell'utente finale o applicazione pratica su alcuni casi studio. Prevede anche una sezione sulle news, che riporta i progressi nella ricerca dell'Autism

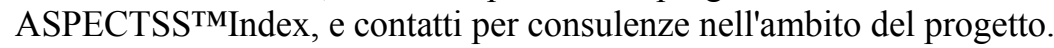

Un altro aspetto interessante riguardo alla diffusione di informazioni, che vorrebbero essere raccolti all'interno della piattaforma DARE (sense) sono le esperienze e le testimonianze dirette delle persone con autismo o dei loro familiari. Esistono ad oggi vari personal blog che utilizzano questo sistema di scambio e relazione, anche se poco organizzato per tematiche, cosa che invece sarebbe interessante nel caso della piattaforma, poiché darebbe un'interazione diretta tra raccomandazioni, esperienze, applicazioni e testimonianze.

Si riportano, in ogni caso, alcuni esempi da cui prendere spunto per la sezione del blog: http://autismjourney.org/blog/, interessante per la varietà di informazioni e tematiche, la freschezza della grafica e della rappresentazioni e la facilità di navigazione; http://atypicalfamilia.com/, interessante poiché riporta il punto di vista di tutta la famiglia, all'interno dei diversi ambiti della vita quotidiana della persona con autismo; http://www.justalilblog.com/, blog scritto e gestito da un padre di una bambina di 11 anni con autismo, interessante per il linguaggio, per il modo efficace di comunicare, spesso anche divertente, che arriva direttamente alle persone, tra cui ad esempio la sezione dedicata alle "memes": http://www.justalilblog.com/.

E per quanto riguarda l'Italia, invece, cosa c'é? Cosa succede?

Il portale sicuramente più completo ed efficace è Per noi autistici http://www.pernoiautistici.com/, una piattaforma di informazione edita da Insettopia Onlus e fondata dai giornalisti e genitori di un ragazzo con autismo, Gianluca Nicoletti e Natalia Poggi, dove le informazioni sono comunque filtrate da un comitato scientifico. Il portale prevede informazioni generali sull'autismo, la diagnosi ed il trattamento, sulla scuola, sulla ricerca e l'innovazione aggiornata ed implementata nel tempo, alcune esperienze dirette di persone con autismo o familiari, una web radio per esprimersi e raccontate, oltre ad una sezione speciale che mappa le strutture e i servizi autism-friendly sul territorio e la possibilità di scaricare una app che dà la possibilità di avere tutte queste informazioni a portata di mano in ogni momento (Figura 11).

In ambito dell'informazione e della ricerca scientifica invece Emergenza Autismo - http://www.emergenzautismo.org/ (Figura 12), è un portale valido e ricono- 


\section{Elena Bellini}

sciuto per la diffusione della conoscenza in ambito di autismo e neurosviluppo, soprattutto di profilo medico-psicologico e con sezioni dedicata all'ABA e all'apprendimento scolastico. Anche in questo caso vengono riportate esperienze e testimonianze, perlopiù di genitori, e una sezione dedicata alle news. Il portale è fondato sull'esperienza di familiari ed associazioni ed è infatti rappresentativo di un movimento per la diffusione della conoscenza di un gruppo di associazioni, ovvero: Associazione Genitori e Autismo, Associazione Con noi e Dopo di noi, Associazione Neomera Calabria, Comitato GAV - Genitori Autismo Veneto, Comitato Contro Autismo Lombardia, S.A.V - Servizi di Accoglienza alla Vita, Associazione ALBA Onlus, Associazione Cancellautismo Onlus, Comilva Puglia, Associazione La Forza del Silenzio, Associazione Daniele Onlus. Come contenuti è molto interessante, come grafica e navigabilità è invece meno aggiornato ed efficace rispetto al precedente. In ogni caso è sicuramente rappresentativo del bisogno espresso dalle famiglie e dalle associazioni di avere accesso all'informazione e all'aggiornamento sulla ricerca in ambito di autismo.

Sempre nello stesso ambito è interessante anche il portale della Fondazione ARES - http://www.fondazioneares.com/, centro di ricerca e sviluppo nazionale in ambito di autismo. In questo caso è un sito di informazione e centro di documentazione scientifica.

Un portale invece più completo ed interattivo è quello di Autismo Online http://autismo.inews.it/ (Figura 13), "nato dalla profonda esigenza di due genitori (Sonia e Roberto Rusticali) di dare un panorama il più completo possibile del mondo dell'Autismo" ad altri genitori che come loro condividono quest'esperienza. Gli argomenti trattati sono "cos'è l'autismo", la diagnosi, le patologie collegate, le terapie farmacologiche e riabilitative, articoli scientifici di riferimento e convegni sull'argomento, servizi ed indirizzi utili. Anche in questo caso interessante come esempio e come definizione del grande bisogno di conoscenza sentito dalle famiglie, ma non prevede le argomentazioni dell'architettura e del design, né si può considerare risolutore nella definizione della piattaforma DARE (sense).

Si cerca quindi di dare risposta a questo quesito: come progettare una piattaforma partecipativa in grado di coinvolgere l'utente e dare risposte/raccomandazioni efficaci all'interno del grande bagaglio conoscitivo, basate sulle esigenze e sull'interesse specifico della persona? Esistono esempi di piattaforme di progettazione, magari non per l'autismo, ma che in qualche modo possono essere fonte di ispirazione?

Non è stato facile trovare piattaforme di questo tipo, soprattutto fondate sullo scambio e l'interazione con il destinatario finale. Come anticipato, vi sono alcuni esempi nell'interior design, ma non mirati alla definizione di DARE (sense). Sono stati in ogni caso selezionati due esempi rappresentativi per la strutturazione della piattaforma e delle strategie di progettazione e design.

Il primo é WBDG - Whole Building Design Guide - https://www.wbdg.org/ (Figura 14), una piattaforma di linee guida navigabili online che fornisce informazioni aggiornate sulle tecniche e le tecnologie di progettazione integrate all'interno edificio. Lo sviluppo del WBDG è uno sforzo di collaborazione tra agenzie federali, società del settore privato, organizzazioni senza scopo di lucro e istituzioni educative, per dare supporto e assistenza alla comunità edilizia dall'Istituto Nazionale delle Scienze della Costruzione (NIBS). Un consiglio di amministrazione e un comitato 
consultivo, composto da rappresentanti di oltre 25 agenzie federali partecipanti, guidano lo sviluppo del portale, con sede a di Washington. Le pagine di risorse di linee guida per l'edilizia sono organizzate al momento in tre categorie principali: Orientamento alla progettazione, Gestione del progetto e Operazioni e manutenzione. Il portale definisce alcuni criteri principali di progettazione, ovvero accessibilità, estetica, conservazione storica, programmazione funzionale e operativa (prestazioni dell'edificio), costo e efficacia (life cycle design), comfort fisico e psicologico delle persone che vivono gli edifici, sicurezza e sostenibilità. Ogni criterio è inoltre analizzato in rapporto alla tipologia di edificio o alla tipologia di spazi all'interno di questo. Ogni "Ressource Page" prevede quindi una prima sezione introduttiva che sintetizza il criterio di riferimento, una parte che riporta gli standard presenti, l'applicazione in casi studio di riferimento, anche attraverso immagini e rappresentazioni grafiche, risorse aggiuntive per approfondire l'argomento (Figura 15). Questo esempio è molto interessante per la metodologia di integrazione dei diversi aspetti e la navigabilità dei contenuti; allo stesso tempo è rivolto però ad un'utenza specifica di professionisti tecnici quindi non è assimilabile per l'utilizzo del linguaggio e per l'interattività della piattaforma basata non tanto su requisiti e prestazioni dell'edificio, ma su esigenze specifiche della singola persona.

Un ultimo esempio interessante è il portale di The Center for Health Design https://www.healthdesign.org/, una piattaforma incentrata sull'ottimizzazione delle strutture sanitarie. Fondata nel 1993, è gestita da un'organizzazione no-profit che mira, attraverso la ricerca, a migliorare la qualità dei luoghi di cura, l'esperienza del paziente, la soddisfazione e le prestazioni del fornitore e/o del personale, attraverso un team di professionisti, con sede in California. Ci sono alcune parti del sito e raccomandazioni open access, altre invece solo rivolte agli affiliati, per cui è necessario registrarsi; non molto immediato quindi nella navigabilità, ma interessante soprattutto dal punto di vista grafico e della rappresentazione di raccomandazioni e progetti, all'interno delle strategie di design. I temi affrontati sono: design clinico, comunicazione, flusso del Pronto Soccorso, riforma della salute, impatto dell'invecchiamento, controllo delle infezioni, rumore, percezione della pulizia, salute della popolazione, sicurezza, sostenibilità, tecnologia. Sono pertanto organizzati rispetto a temi generali e non sempre specifici rispetto all'architettura. Ogni sezione prende poi risorse organizzate in "toolboxes" che raccolgono una breve introduzione del tema, interviste, casi studio, strategie di design, strumenti operativi utili, contributi attraverso il blog, eventuale formazione prevista sull'argomento e risorse correlate. Vi sono infine, all'interno degli strumenti, "toolboxes" rivolti sia alla progettazione, che alla valutazione degli ambienti. E' stata rilevata di particolare interesse la sezione sulle stanze di degenza (Figura 16), suddivise in reparto di chirurgia, terapie intensive, maternità e stanze per gli esami legati alla prima assistenza. In particolare, questo sistema è interessante poiché prevede una descrizione grafica che rappresenta lo spazio in $3 \mathrm{~d}$, consentendo al professionista di interagire direttamente l'ambiente e scegliere quale aspetto andare ad approfondire. Le raccomandazioni riguardano tutti gli aspetti dello spazio, dalla sua conformazione architettonica, ai materiali, agli arredi, fino ai dispositivi e agli impianti. E' stato considerato interessante soprattutto per la sua capacità di interazione, di sintesi nelle raccomandazioni, che non sono testi lunghi ma organizzate per punti e soprattutto sulla base di un outcome da raggiungere. 


\section{Elena Bellini}

Si riassumono quindi, a conclusione di questo paragrafo, i criteri e gli spunti acquisiti per la definizione della piattaforma DARE (sense):

- il bisogno di diffusione di conoscenza;

- la necessità di arrivare direttamente alle famiglie, alle associazioni di familiari, o alle stesse persone con autismo;

- l'utilizzo quindi di un linguaggio che non sia specificatamente tecnico, ma sia leggibile e comprensibile da ogni persona;

- la facilità di navigazione e la semplicità del percorso;

- l'utilizzo di una grafica semplice e pulita, con pochi stimoli visivi, chiara e comunicativa;

- la centralità delle esigenze delle persone, che siano alla base dell'organizzazione delle informazioni raccolte;

- la possibilità di effettuare delle domande dirette all'utente finale, all'inizio, in modo da affinare la ricerca delle "raccomandazioni" e, non solo, ma coinvolgerlo direttamente nel processo di progettazione partecipata;

- l'integrazione di raccomandazioni, casi studio applicativi, prodotti ed esperienze dirette degli utenti, come risposta completa al destinatario finale;

- l'utilizzo di testi brevi e molte immagini o schemi grafici, per facilitare la comprensione e l'accessibilità delle informazioni;

- dare la possibilità all'utente finale di scaricare in pdf i propri "risultati";

- dare la possibilità all'utente finale di approfondire la ricerca, attraverso fonti scientifiche e di riferimento, relative ad ogni "risultato";

- dare la possibilità all'utente finale di partecipare nel progetto, fin dall'accesso alla piattaforma, ma anche come contributo all'innovazione e scambio di conoscenza, attraverso la propria esperienza;

- avere uno strumento dinamico di progetto e implementabile nel tempo;

- dare uno strumento operativo al familiare e al professionista che lo affiancherà nel briefing di progetto.

\subsection{La proposta: DARE(sense)}

DARE (sense) vuol essere quindi una piattaforma open source che si basa sulle persone e parte da loro (Figura 17). E' strutturata per organizzare in modo dinamico le informazioni di supporto alla progettazione, sulla base della definizione stessa dell'utenza finale e del suo progetto di vita:

- ambito di riferimento, ovvero residenza, scuola, ambiente sanitario, lavoro, centri per l'autismo, e magari altri ambiti nel tempo, includendo il tempo libero;

- l'età della persona con autismo (bambini, ragazzi, adulti, anziani);

- la definizione del profilo senso-percettivo della persona con autismo, ovvero quali sensibilità sensoriali sono maggiormente percepite, o considerate prioritarie dall'utente nella condizione di disagio e/o benessere (visiva, acustica, propriocettiva, tattile, olfattiva, vestibolare, gustativa, o nessuna);

- la scelta delle proprie esigenze, definite e raggruppate sulla base delle risultanti dello studio dello stato dell'arte e le informazioni raccolte nei questionari e nelle interviste, ovvero "riduzione del disagio sensoriale ed ambientale", "salute e benesse- 
re", "ambienti sicuri e durevoli", "orientamento e comprensione", "autonomia e indipendenza. Abilità", "relazioni sociali e integrazione. Inclusività".

$E^{\prime}$ interessante come ci sia stato, anche in questo caso, uno scambio di conoscenza, ponendo le persone al centro del progetto, nel partecipare al processo di codesign (di ricerca). Se da un lato, infatti, lo studio dello stato dell'arte è stato la base per la definizione dei questionari e delle interviste alle persone con autismo e/o ai loro familiari, dall'altro, i dati raccolti con queste metodologie quali-quantitative, hanno guidato l'organizzazione dello stato dell'arte e di tutte le informazioni che saranno alla base della piattaforma DARE (sense).

Tutte le esigenze raccolte per la definizione del profilo della persona con autismo si trasformano nella piattaforma, quindi, in parole chiave che vanno, in modo dinamico, a richiamare tutte le informazioni di supporto alla progettazione. Queste informazioni sono raccolte a loro volta in "raccomandazioni", "progetti" (casi studio), "prodotti" presenti sul mercato ed "esperienze" di vita, raccolte attraverso il dialogo con le stesse persone con autismo o loro familiari. Queste informazioni saranno continuamente implementate attraverso un blog ed ognuno avrà la possibilità di dare un contributo sottoponendo al Comitato Scientifico nuove pubblicazioni o nuovi progetti d'interesse in questo ambito.

\subsection{Struttura e funzionamento della piattaforma}

La piattaforma prevede quindi una pagina iniziale - home (Figura 18), dove è possibile scegliere l'ambito di progetto (residenza, scuola, ambienti sanitari, centri per l'autismo, lavoro). Si è lasciato la possibilità comunque di guardare anche all'insieme delle raccomandazioni, dei progetti, dei prodotti e delle esperienze, che saranno ad ogni volta "taggati" attraverso le parole chiave, per ritornare al sistema di definizione del profilo sensoriale e di esigenze.

E' stata utilizzata una grafica semplice, con pochi colori e non troppo accesi, per non sovraccaricare l'utente finale, che potrebbe essere anche direttamente una persona con autismo ad alto funzionamento o Asperger. In questo senso sono state consultate alcune pubblicazioni di sviluppo di web design dedicate a persone con autismo, in modo da pensare ad un prodotto finale coerente. In ogni caso il web design non rappresenta l'ambito specifico di questa ricerca e non si esclude che possa anche in parte essere trasformato nel momento dell'effettiva realizzazione della piattaforma.

Una volta scelto l'ambito di riferimento attraverso la home, il sistema informatico tiene in memoria la sezione scelta e apre una nuova pagine che indaga l'età della persona con autismo (Figura 19).

Allo stesso modo, memorizza la scelta del gruppo di utenza e passa ad indagare nello specifico il profilo della persona con autismo. Nella schermata successiva si può scegliere quindi un'esigenza di riferimento tra quelle proposte (Figura 20). Si è preferito dare la possibilità di scegliere una sola esigenza alla volta per non andare a sovraccaricare immediatamente l'utente di informazioni e, allo stesso tempo, non ridurre troppo la rosa di informazioni che presentino l'integrazione di più esigenze contemporaneamente. Questo metodo permette anche all'utente di fare un processo critico di definizione delle priorità, in un percorso che altrimenti potrebbe portare alla scelta di tutte le esigenze possibili, senza ragionare a fondo sulla persona e su 


\section{Elena Bellini}

ciò di cui ha realmente bisogno. Il progetto finale sarà ovviamente l'integrazione di tutte queste esigenze, che in ogni caso non entrano in contrasto l'una con l'altra, ma sono obiettivi conseguenti l'uno all'altro, partendo dalla riduzione del disagio sensoriale ed ambientale, fino al supporto all'autonomia personale e all'integrazione e all'inclusione all'interno della società. La sensorialità è trasversale rispetto a tutte le esigenze di progetto, come si è visto anche dallo stato dell'arte e dall'analisi dei casi studio. Sarà ovviamente prioritario nella riduzione del disagio sensoriale ed ambientale, ma comunque importante anche nella scelta delle indicazioni di supporto alla progettazione secondo le altre esigenze. Come ultima schermata si dà quindi la possibilità di scegliere tra le sensibilità sensoriali (Figura 21) e, in questo caso, sceglierne anche più di una in modo integrato, poiché ovviamente concorrono tutte alla definizione del profilo senso-percettivo della persona e una non può prescindere dall'altra. Una volta definiti e selezionati tutti questi aspetti si dà automaticamente avvio al motore di ricerca che trova le indicazioni di supporto sulla base delle parole chiave definite in base alla scelta dell'utente.

Come si è già visto nelle schede dei casi studio, ogni informazione che fa parte del Data Base è corredata da alcune parole chiave, che corrispondono a quelle scelte dall'utente e costituiscono un rimando automatico che raccoglie solo le informazioni utili per l'utente finale. Nel caso dei progetti sono state selezionate parole chiave definite dall'analisi del caso studio, mentre nel caso dello stato dell'arte si attua un richiamo o un'indicizzazione alle parole chiave presenti direttamente all'interno del testo. Le esperienze delle persone, raccolte nel blog, e la pagina dei prodotti, vanno infine a organizzarsi attraverso $i$ tag attribuiti ai diversi post.

Nella pagina finale (Figura 22) quindi si presentano all'utente finale, a seguito di questo processo informatico di raccolta dati, tutte le raccomandazioni utili come supporto allo specifico progetto di quella persona con autismo, i progetti che rappresentano esempi di applicazione di questi principi, i prodotti presenti sul mercato interessanti e utili per lo specifico profilo della persona e, infine, le esperienze simili di altre persone con autismo o loro familiari, che possono essere un supporto ed una guida di best practices.

Le raccomandazioni (Figura 23) sono rappresentate da un "ponte", che simboleggia il percorso di connessione tra esigenze e indicazioni di supporto al progetto. A questa sezione della piattaforma non corrisponde un lungo testo scritto, che racconti "come progettare per l'autismo" ma piuttosto pillole che in modo breve e conciso danno delle raccomandazioni pratiche di supporto al progetto specifico dell'utente finale, selezionate appunto, come visto precedentemente, rispetto alle sue esigenze. Queste raccomandazioni, che derivano dallo studio dello stato dell'arte, dall'analisi dei risultati delle indagini quali-quantitative e delle scelte di progetto dei casi esistenti, vengono rappresentate da un'icona grafica e da un breve testo scritto con un linguaggio semplice e familiare, pensato per l'utente finale, che non è detto sia un professionista del settore. Come accennato, ogni indicazione corrisponde ad una fonte ed è implementabile nel tempo, ogni qual volta si presentino nuove ricerche e pubblicazioni di riferimenti, analizzate in modo critico dal Comitato Scientifico che dirige il funzionamento della piattaforma. E' possibile far parte del processo stesso, attraverso un contributo, ma anche commentare implementando il blog ed il patrimonio di esperienze e favorendo lo scambio di conoscenza e l'arricchimento 
scientifico. La pagina delle raccomandazioni infine presenta un'icona di connessione con gli esempi di progetto, da cui è possibile direttamente accedere ai progetti correlati.

La pagina dei progetti (Figura 24) riporta invece le schede di casi studio analizzate, considerate utili per lo specifico profilo senso-percettivo della persona e soprattutto per il suo profilo esigenziale. Le parole chiave che definiscono le esigenze della persona si riferiscono, infatti, direttamente alla sezione specifica della scheda di progetto, organizzata secondo gli stessi criteri, e correlata ad una o più immagini significative dell'ambito specifico (riduzione del disagio, inclusività, autonomia, ecc.), richiamate attraverso i tag. In ogni caso, si ha sempre un'indicazione del titolo di progetto, sede, anno di realizzazione, utenza e uso specifico, ed è possibile anche visitare o scaricare la scheda completa di progetto, qualora la persona sia interessata a vederlo nel suo complesso. Anche in questo caso l'utente finale può direttamente contribuire segnalando altri progetti interessanti visitati o conosciuti sul territorio, implementando il bagaglio di conoscenze, ma anche favorendo la diffusione delle più recenti innovazioni in ambito di progettazione per l'autismo.

Allo stesso modo avviene infine per i prodotti (Figura 25), pubblicati secondo tag di riferimento. Questa sezione può essere un altro aspetto interessante di sviluppo poiché le famiglie si trovano tutti i giorni a fare i conti con tantissime aziende che producono prodotti di interesse e tante altre che speculano invece sulla condizione di autismo. Partecipare a questa sezione potrebbe rappresentare una sorta di "lista etica" delle aziende, valutata sempre in modo critico nel suo contenuto scientifico dal Comitato, e potrebbe rivelarsi anche una risorsa per il mantenimento della piattaforma stessa open source (vedi paragrafo 7.5).

La sezione delle esperienze (Figura 26) infine dà accesso a storie di persone con autismo o loro familiari, attraverso i testi di biografie e autobiografie selezionate, la selezione dei racconti raccolti durante le interviste e tutte le altre informazioni che verranno raccolte attraverso il blog. Questa sezione sarà molto importante poiché si è visto come, da un lato, per comprendere le persone con autismo sia necessario leggere o parlare di loro con loro ed entrare in empatia con la diversità; dall'altro, l'utente finale, la persona con autismo o il familiare, può sentirsi meno solo e sfruttare la piattaforma stessa, oltre che come confronto e scambio di conoscenza, come sviluppo delle relazioni sociali, e non per ultimo come dialogo con professionisti ed esperti del settore.

Nella sezione info e contatti sarà infatti possibile anche vedere il pool di professionisti che stanno alla base del Comitato Scientifico, conoscerli e contattarli per avere un contributo specifico in ambito del progetto. Infine in questa sezione vi sarà indicata una bibliografia ed una sitografia di riferimento, come rimando alle fonti, ma anche come possibilità di approfondire e sviluppare la conoscenza.

\subsection{Fattibilità e funding. Conclusioni, limiti della ricerca e sviluppi futuri}

La fattibilità della piattaforma è stata verificata attraverso tecnici del settore dell'ICT nello specifico del web design. Se ne è studiato anche il funzionamento attraverso un mockup, che permette di navigare, in modo parziale ma esemplificativo, all'interno della piattaforma. 


\section{Elena Bellini}

Come sviluppo futuro della ricerca si prevede la sperimentazione attraverso alcuni utenti finali, opportunamente selezionati, che potrebbero rappresentare inoltre una base di dati necessari per affrontare una campagna di raccolta fondi e/o crowdfunding, per coprire i costi di sviluppo e programmazione della piattaforma. Questa metodologia di finanziamento è molto efficace, soprattutto su tematiche come quella dell'autismo, ad alto scopo sociale, e si potrebbe perseguire in collaborazione con alcune Associazioni del Terzo Settore, che rappresenterebbero l'espressione stessa del bisogno e la testimonianza della sperimentazione effettuata. Come anticipato, durante il percorso della ricerca sono stati sviluppati numerosi contatti e rapporti con le Associazioni di familiari, alla base dello sviluppo del progetto stesso, discutendo anche il risultato finale del percorso di ricerca, con feedback molto positivi.

Un limite della ricerca è stato forse la mancanza di una sperimentazione diretta sulla piattaforma completa, ma sarebbe stato al momento troppo oneroso a livello economico; questo si considera pertanto uno sviluppo importante da percorrere.

Un'altra metodologia di finanziamento potrebbe essere quella anticipata nel precedente paragrafo, ovvero delle sponsorizzazioni da parte di aziende o professionisti del settore, per la promozione dei loro prodotti. Ovviamente questo processo dovrebbe essere protetto a livello etico, attraverso una valutazione critica dei prodotti da parte del Comitato Scientifico, attraverso gli standard stabiliti per la Responsabilità sociale d'Impresa. In ogni caso, questo permetterebbe di avere una quota di finanziamento costante nel tempo per coprire i costi di gestione del sito.

Un altro sviluppo interessante potrebbe essere includere nel comitato anche persone con autismo ad alto funzionamento o Asperger, al fine di renderli parte integrante della costruzione della piattaforma, non solo come esperienze, ma anche come professionalità. Questo apre l'orizzonte a tante altre forme di evoluzione, che potrebbero ampliare la piattaforma in numerosi altri modi, lasciando finestre aperte sul campo del lavoro, dello sport, degli interessi e del tempo libero, e creando un network di persone e servizi.

Allo stesso modo potrebbe essere l'occasione di mettere in contatto i professionisti del settore e creare servizi di consulenza e progettazione integrati. 


\section{Autism and building design}

Many autistic people suffer from a high degree of stress. which in turn can have an effect on their behaviour. They may display obsessional behaviour, be hyperactive, or have severe tantrums, all of which can have implications for the environment that they live and work in.

A high proportion of autistic adults also have associated learning disabilities. This combination of factors can give rise to:

- Inappropriate use of domestic fittings

- poor co-ordination and balance

- a need for assistance with basic self-care functions

- a need to control access to areas of risk

- a need for large-capacity hot water storage and a limit to hot water temperature

- a need for an increased surface area and reduction in surface temperature for radiators.

A significant proportion of autistic adults suffer from epilepsy, resulting in a need for.

- outward opening doors on WC/bath/shower rooms

- avoiding sharp edges on domestic worktops and radiators

- beds positioned away from windows, radiators and room entrances

\section{Share page: $\mathbf{n}$ Y Thed 0}

Find local autism services

I'm looking for:

Enter service, or keyword(s)

In or around:

Place, postcode

\section{Also of interest}

On the NAS website:

Building design factors

Everyday life

The Autism Friendly Award

Figura 1. The NAS - National Autistic Society - http://www.autism.org.uk/ 


\section{Elena Bellini}

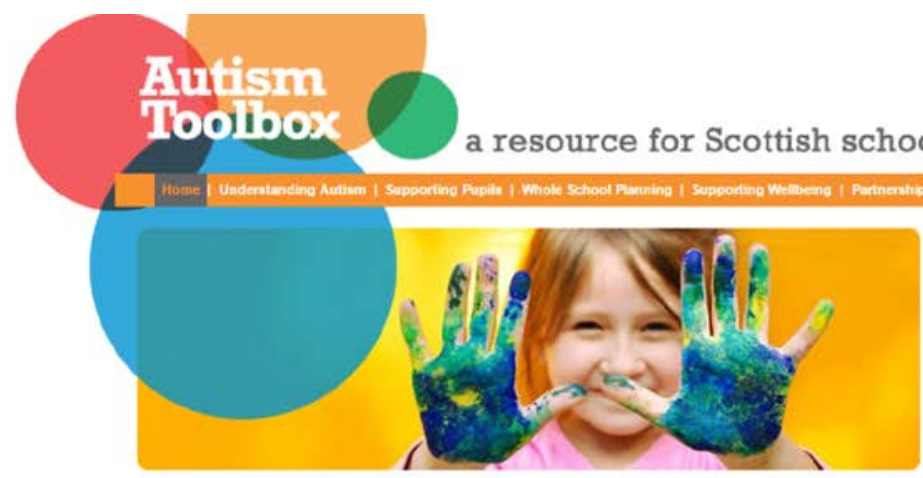

Introduction to Autism Toolbox

The Autism Toolbox website is brought to you by the Scottish Government in partnership with the national charify, Scottish Autism with support from Autism Network Scotland.

This online resource complements the original Autism Toolbox developed by the National Centre for Autism Studies and launched in 2009. We will continue to reference the original toobox throughout this site. For a full list of contributors, please see our Resources section.

The Aurism Toolbox is a resource to support the inclusion of children and young people with autism spectrum disorder in meinstream education services in Scotland. As well as introducing and describing some of the more cormmon challenges a pupil with autism might face, it provides real life case studies from Scottish schools and practical examples of supports that you can translate and use in your own school setting it also signposts you to other websites you may find useful
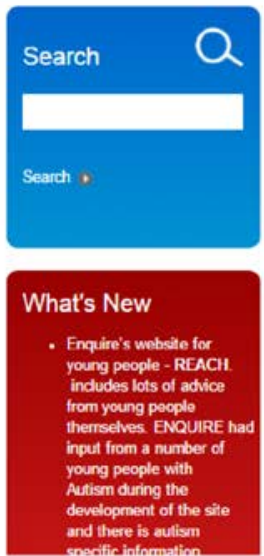

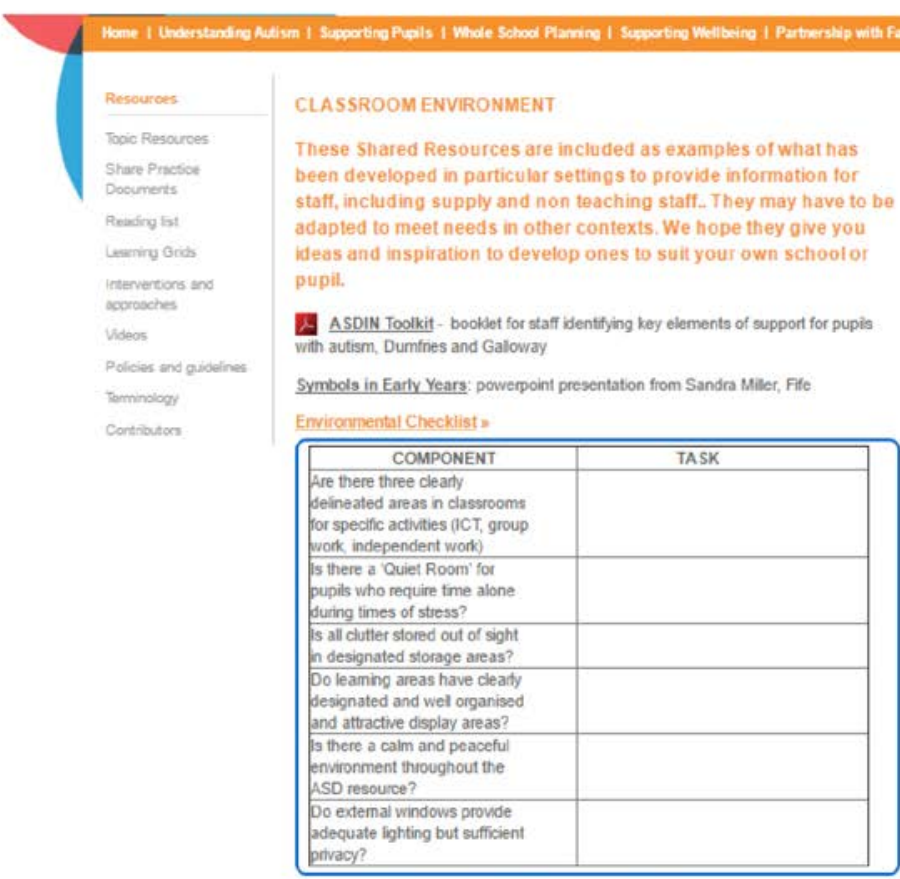

Creating a focused work atea within a msinstream class *

Figura 2. Autism Toolbox - http://www.autismtoolbox.co.uk/ 
Ambienti sensoriali “terapeutici” che rendano Abili

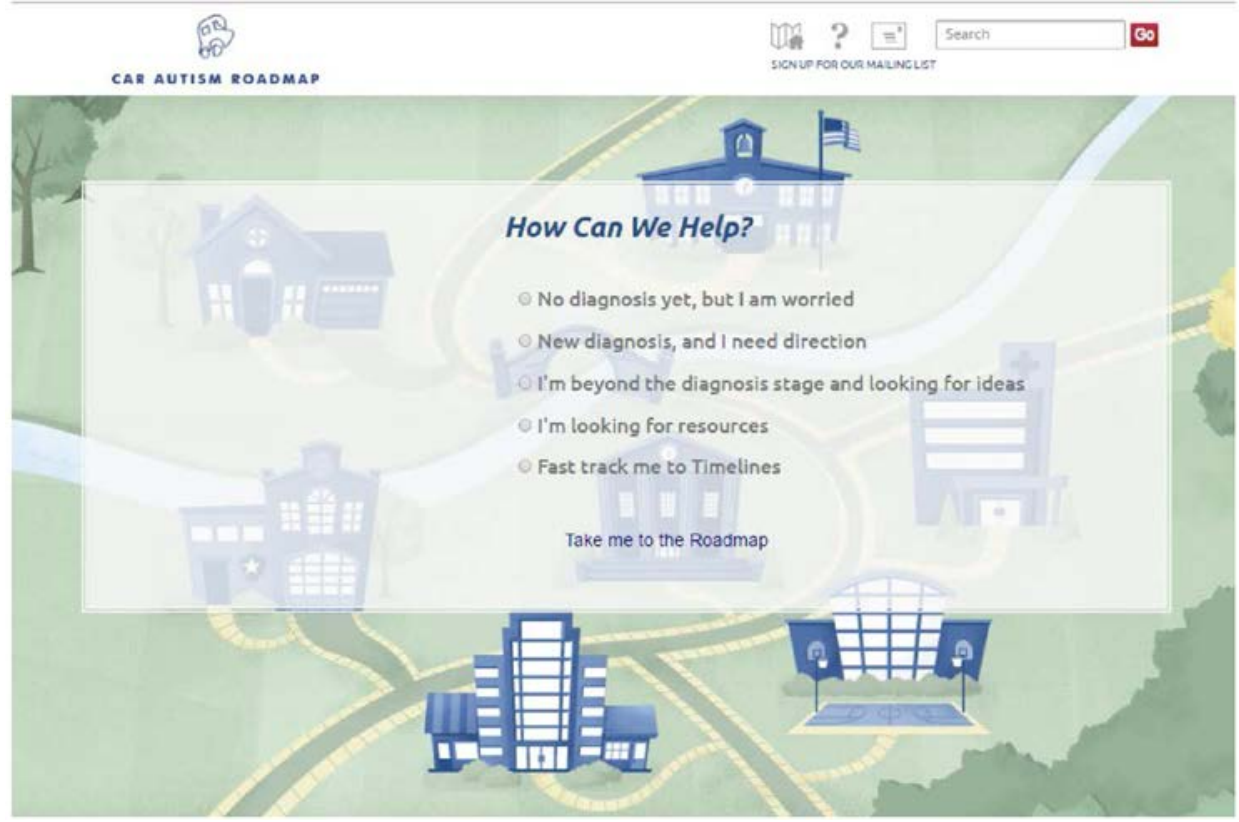

Figura 3. Car Autism Roadmap - https://www.carautismroadmap.org/
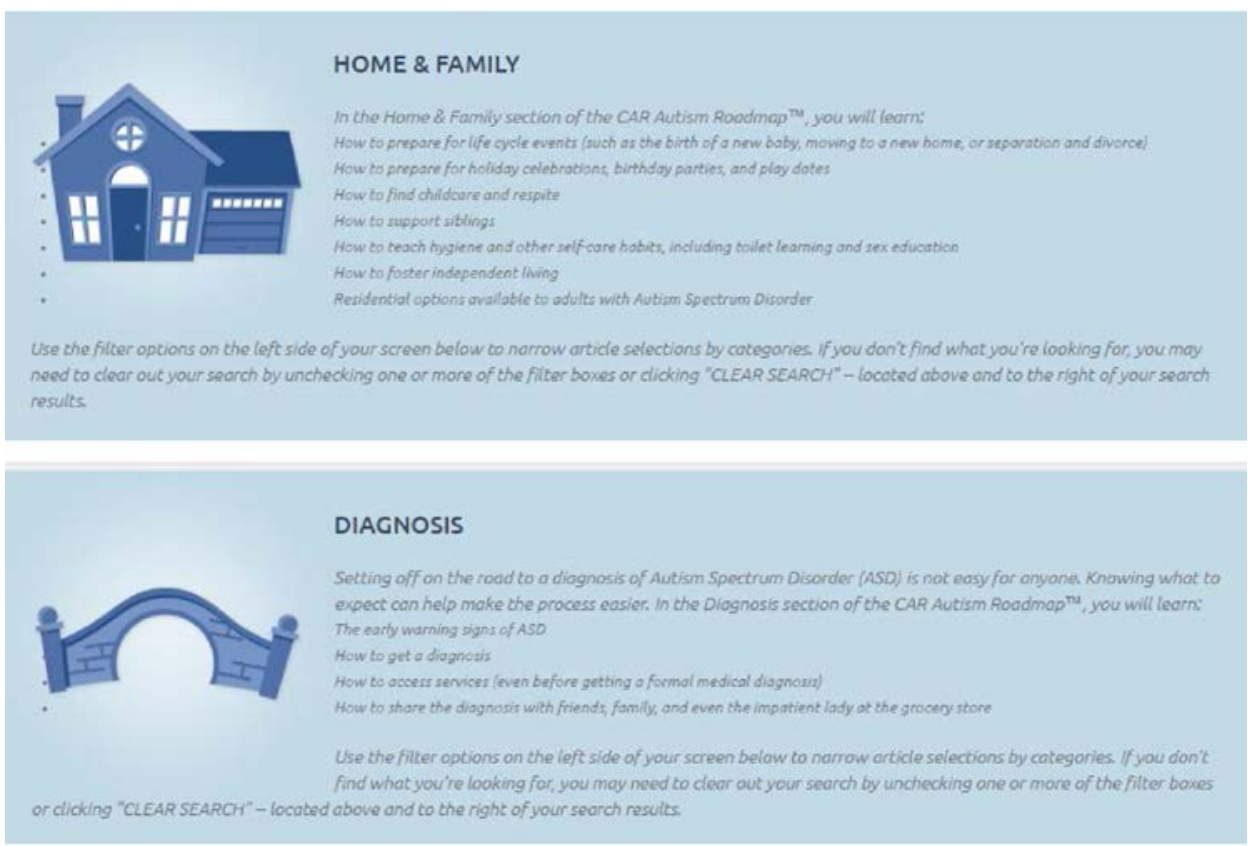

Figura 4. esempio della Car Autism Roadmap - https://www.carautismroadmap.org/ 


\section{Elena Bellini}

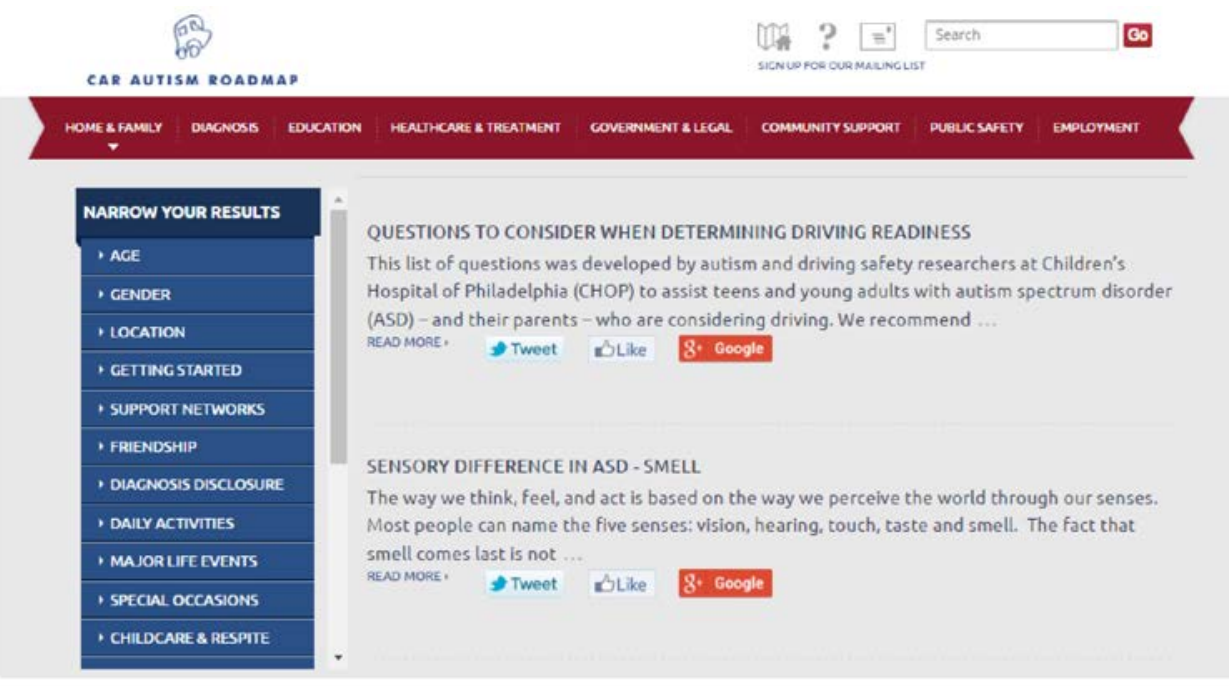

Figura 5. Car Autism Roadmap - https://www.carautismroadmap.org/

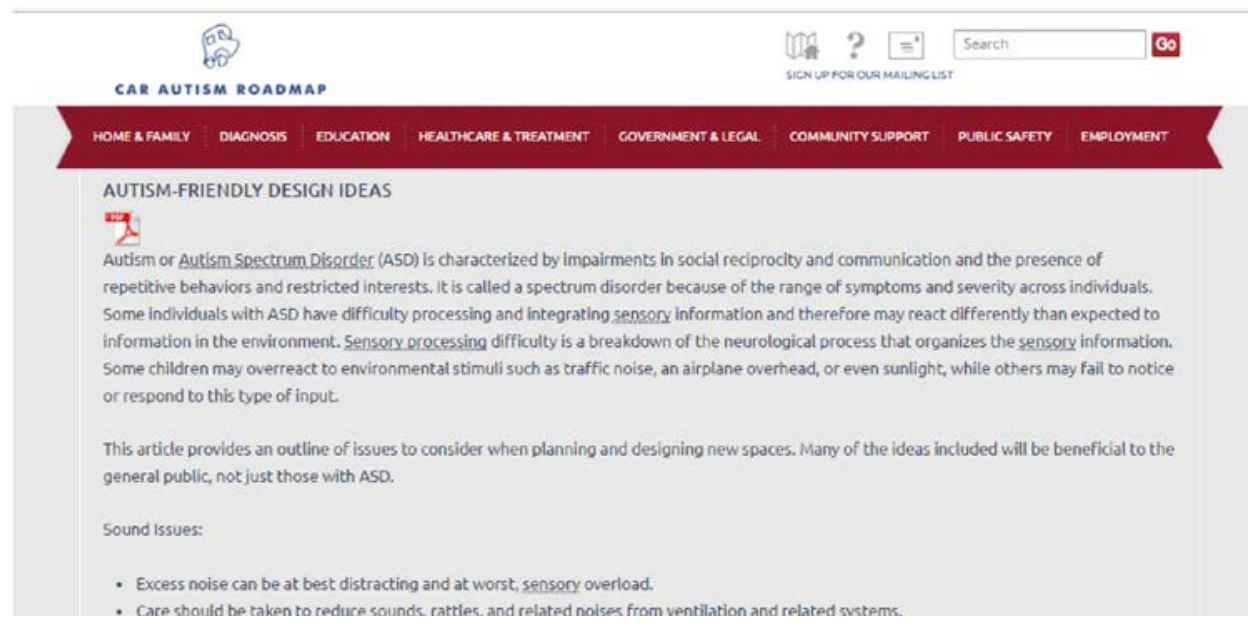

Figura 6. pdf scaricabile della Car Autism Roadmap - https://www.carautismroadmap.org/ 


\section{ARCHITECTURE FOR AUTISM}

mission recommendations acknowledgments contact blog-en

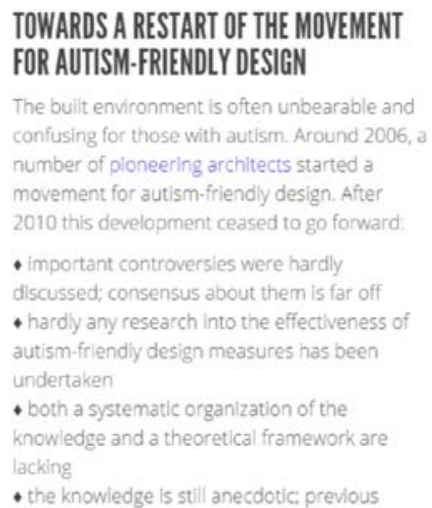

\author{
Presentation "About autism-friendly design" online \\ 7 myths about autism-friendly design \\ Introduction to autism-friendly design
} Autism-Friendly Design: A View on its Present and Future
State

Figura 7. Architetture for Autism - http://www.architectuur-voor-autisme.org/

\section{CONTEXT INDEPENDENT LIVING}

Almost everyone on the spectrum prefers their own place to live, where they feel safe, are not disturbed or distracted by others and where there is sufficient opportunity to pursue one's favorite pastimes. In short a place where they can be themselves.

Generally, people who live independently are more socially capable than others on the spectrum. However, being on the spectrum for them too means having difficulties which permeate all spheres of life and put them in a delicate balance. A wide range of supports should exist to heip the independent individual; among which special measures related to the overall design of their homes.

According to a rather recent US national survey $84 \%$ of caregivers reported that the person with autism currently lives at home. Asked about the ideal INing situations for the individuals in their care, slightly more than half (4696) answered living with his or her family. A group home would be better said $129 \%$ and 1096 preferred a home of their own with a roommate Because of the large number of newly diagnosed youths, the dernand for any sort of living arrangements - among which (seml-jindependent ones - will continue its unprecedented rise. An article in the New York Times estimated half a million people on the autism spectrum will become of age in the next decade.

A wider but somewhat older study (2008) found $96 \%$ of those between 17 en 30 years of age lived with their parents or guardian. (7696 if defined more strictly) Two thirds of their parents were concerned with their housing needs. Only $26 \%$ of these parents believe their child will always have a place to live (compared with 6096 of non special needs parents), while $15 \%$ said their child has the life skills to be able to live independently.

Briefly, the estimated percentage of youths living at home lies between 76 and $96 \%$, those who want to and probably are able to live independently in the future is around 10 to $15 \%$.

Figura 8. estratto di una scheda di Architetture for Autism - http://www.architectuur-voor-autisme.org/ 


\section{Elena Bellini}

\section{LOCATION}

\section{Location}

5 The best place to live is strongly individually determined

GARDEN / OUTSIDE AREA

\section{Demarcation}

6 Borders around the home

7 Entrance at home, flow / security/ wardrobe / hallway

10 Avoiding distraction and visual exposure

14 Garden or beneficial outside area

\section{ARCHITECTURAL SPACES}

\section{Zoning}

30 Zoning of the home

33 Situating the laundry area

34 Separating bathroom and toilet

Sightlines

36 Oversight at home

\section{Living rooms}

40 Spatial properties of tiving rooms

\section{Bathrooms and toilets}

47 Bathroom and toilet

\section{Kitchens}

50 Open-plan or closed kitchens?

\section{Stairs and corridors}

61 Generous stairs and corridors

63 Stairs and corridors: orlentation

64 Stairs and corridors: finishing touches

\section{Sound / Noise and acoustics}

69 Contact noise in new homes

70 Contact noise existing homes

71 Noise from outside

73 Stairwell noise

76 Muting reverberation

\section{Light and sight}

77 Incoming sunlight (large windows)

79 Day lighting

80 Avoiding daylight avoidance

Smell

82 Avoiding odorous/noxious building materials

Temperature

84 Cooling of new buildings

85 Cooling of existing building:

86 Heat-filtering glass

Flexibility

90 Anticipation of the "smart home"

\section{INTERIOR DESIGN}

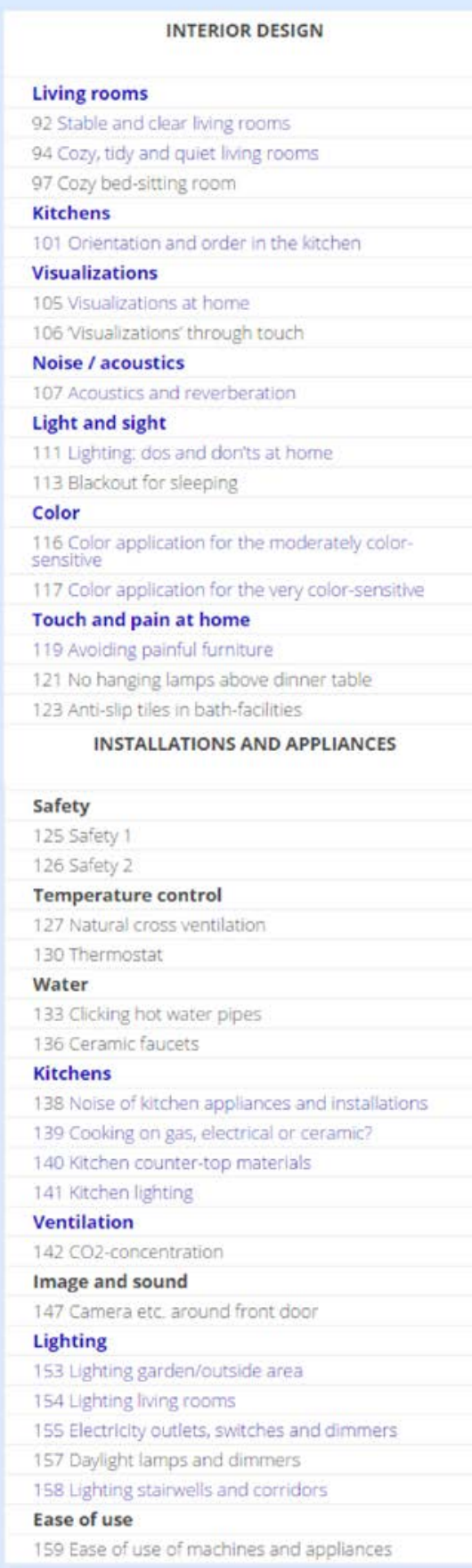

Figura 9. Architetture for Autism - http://www.architectuur-voor-autisme.org/ 


\section{Ambienti sensoriali "terapeutici" che rendano Abili}

\section{LIGHTING: DOS AND DONTS AT HOME}

\section{Recommendation 111}

Artificial lighting: provide generous, even and soft lighting

Dimmers for transitions from waking to sleeping

Avoid:

- abundant/harsh light

- fluorescent lighting and its attendant transformer humming

- high contrast lighting schemes or deep shadow

\section{Chapter (theme)}

Interior design (light and sight)

\section{Because of}

sensory sensitivity, disturbed perception, Central coherence and sleep disorders

\section{In order to}

- address sensory sensitivitys for light (and sound-yeffects

- to assist in falling asleep

- to prevent the familiar, daytime reality from turning into something strange and frightful

\section{Elaboration}

Artificial lighting generally promotes oversight and a sense of security when it is even and relatively soft and not harsh. Strong or harsh lighting, by contrast, produces annoying shadows. Therefore many prefer subdued light of compact fluorescent lamps (CFLs) and dimmers.

An important reason to strive for lighting the whole space is that change of a few elements of a situation may lead to the experience of a whole new situation, because of weak Central coherence. In such a case it's quite possible that the familiar room of the daytime isnt recognized at night. Of course the chances of such disorienting experiences rise as the number and size of unlit areas in the room go up.

Another reason for lighting the whole house is shadows can be wrongly perceived. A shadow behind a piece of furniture, for instance, can be seen as a hole in the floor and/or the wall

Strong fluorescent lighting is to be avoided. $T 1$ is in fact light which flashes rapidly, Because people with Asd often have more acute senses, they can experience a stroboscopic effect, such as in disco's. Th-light is usually accompanied by a high whistling-sound above the threshold of the normal ear, but within the reach of many people on the spectrum. Lindsey Nebeker has this to say about th-lighting: "White fluorescent tube lighting is (also) painful to my eves, and leaves me nauseated, irritable, and feeling like l'm visiting an interrogation room"

Dimmers which help create soft lighting, can also achieve a gradual weakening of the light which is a proven means to stimulate sleep. One of the operant mechanisms is most probably the influence of receding light on melatonin-production (the 'sleep hormone). Sleeping disorders are common among people with Asd.

\section{INDEPENDENT LIVING}

Back to previous page

- Acoustics and reverberation

- Avoiding daylight avoidance

- The best place to live is strongly individually determined

- Lighting: dos and donts at home

- Entrance at home flow/security/wardrobe/hallway

- Cozy, tidy and quiet living rooms

- Color application for the moderately color-sensitive

- Color application for the very colorsensitive

- Noise of kitchen appliances and installations

- Muting reverberation

- Situating the laundry area

- Stable and clear living rooms

- Avoiding odorous/noxious building materiais

, Stairwell noise

- Lighting garden / outside area

- Visualizations at home

- Zoning of the home

- Spatial properties of flving rooms

- Incoming sunlight (large windows)

- Electricity outlets, switches and

- Lighting living rooms

- Open-plan or closed kitchens?

- Contact noise in new homes

- Orientation and order in the kitchen

- Cooking on gas, electrical or ceramic?

- Kitchen counter-top materials

- Contact noise in existing homes

- Avoiding painful furniture

- Noise from outside

- Cooling of new buildings

- Cooling of existing buildings

- Borders around the home

- Separating bathroom and toilet

- Avoiding visual exposure

- Kitchen lighting

- A garden or benevolent outside area

- Oversight at home

- Bathroom and toilet

Figura 10. Architetture for Autism - http://www.architectuur-voor-autisme.org/ 


\title{
Elena Bellini
}
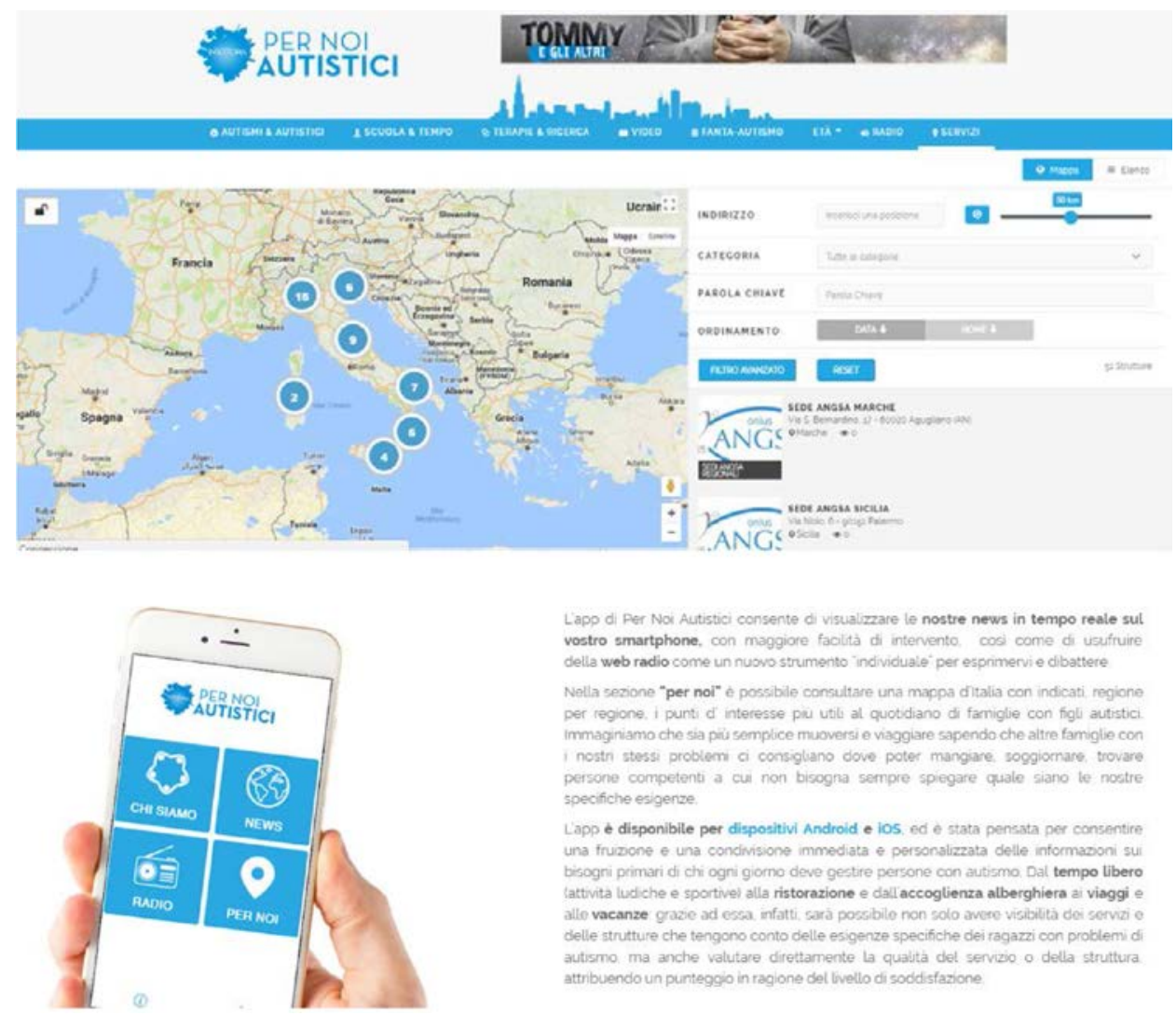

\begin{abstract}
Lapp di Per Noi Autistici consente di visualzzare le nostre news in tempo reale sul vostro smartphone, con maggiore facilta di intervento cos come di usufruire della web radio come un nusvo strumento individuale per esprimervi e dibattere

Nella sezione "per noi" é possibile consultare una mappa ditalia con indicati regione per regione. i punti of interesse pau utit al gughidiano di famiclie con foli autistic immagiviamo che sia piu semplice muoversi e vaggiare sapendo che altre famiglie con i nosth stessi problemi oi consiglano dove poter mangiare, soggiomare, trovare persone competenti a cul non bisogna sempre spiegare quale siano te nostre specifiche esigenze

Lapp e disponibile per dispositivi Android e ios ed e stata pensata per consentire una frumcoe e una concivisione immediata e personalizzata delle informazoni su bisogni primari di chi ogni giorno deve gestire persone con autismo. Dal tempo tibero (attivita ludiche e sportive) alla ristorazione e dall accoglienza alberghiera ai viaggi

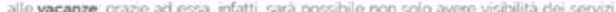
delle strutture che tenoono conto delle esioenze specifiche dei ragazzi con problemi di autismo ma anche valutare direttarnente la qualta del servizio o della struttura attribuendo un punteggio in ragione del livello di soddisfazione
\end{abstract}

Figura 11. Per noi autistici - http://www.pernoiautistici.com/

\section{WWW EMERGENZAUTISMOORG}

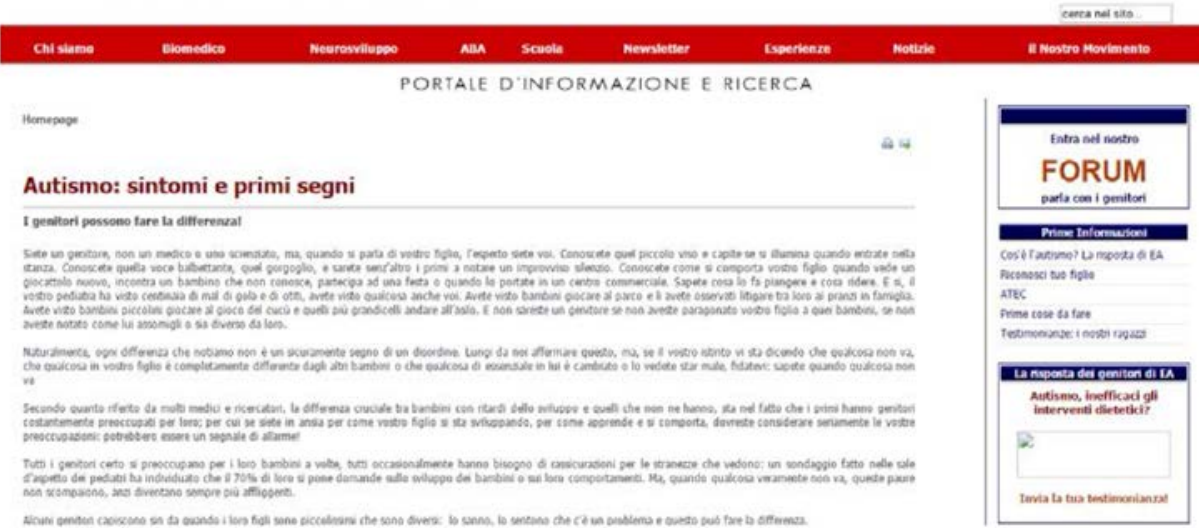

Figura 12. Emergenza Autismo - http://www.emergenzautismo.org/ 
Ambienti sensoriali "terapeutici" che rendano Abili

\section{AutismoOnline}

Visitate le sezioni Convegni, Leggi e Scuola che sor

" Chi siamo $n$ Contattaci $n$ Newsletter " Home

$\begin{array}{ll}\text { @를 MENU } & \\ \text { " Autismo } & \text { " Leggi } \\ \text { " Convegni } & \text { " Scuola } \\ \text { " Riflessioni } & \text { " Articoli } \\ \text { " Test } & \text { " Indirizzi utili } \\ \text { " Patologie } & \text { " Ospedali } \\ \text { " Progetti } & \text { " ANGSA } \\ \text { " Terapie } & \text { " Contatti } \\ \text { " T. Biochimica } & \text { " Newsletter } \\ \text { " Dieta } & \text { " Racconti }\end{array}$

IN PRIMO PIANO

Editoriale del 24-12-12

Raramente ho visto degli Auguri cosi belli e coinvolgenti........ non pensate che lo affermo perchè sono di parte,

(visto che è una produzione di Angsa Veneto, di cui mia Moglie è Presidente

Regionale), e credo che dopo esservi gustati il Video, anche Voi la penserete cosil....... e sono certo che lo inoltrerete agli amici e ai parenti...... (I disegni da Ragazzi con Autismo).

TANTI AUGURI....... " OGNI VOLTA, LA GIOIA DEL NATALE" continua

Invia il link di questo video a tutti i tuo amici. La conoscenza crea cultura e rispetto, la condivisione dà forza es motivazione.

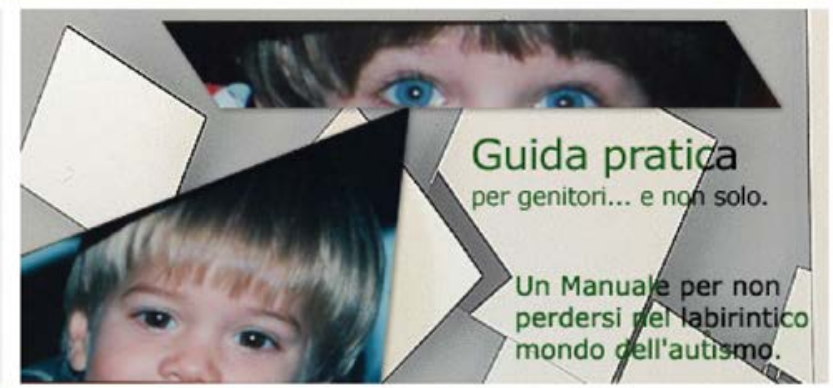

Vacanze a

EDITORIAE 30/01/2011

Il piacere di Collaborare con Validi Professionisti

Venerdi ho passato qualche ora in piacevole compagnia di una persona, anzi, di un Amico, col quale non ci si era mai visti di persona, ma sentiti solo via Telefono, Email, Skipe e Facebook (potenza dei mezzi di comunicazione moderni....). Ovviamente, avendo egli una bambina Autistica, si è anche parlato di Autismo, ma fortunatamente ne abbiamo parlato a 360 gradi perché è una persona aperta e soprattutto non e un fanatico...

continua a leggere

\section{Londra}

Le famiglie con portatori

di Handicap sono le benvenute: informazioni

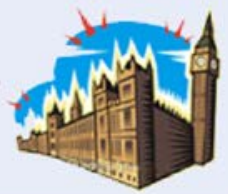

L' ESPERTO

Domande scientifiche sull'Autismo ? DOtt.GORAN DZINGALASEVIC

\section{RISPONDE}

Domande

sulle Leggi

sull'Handicap ?

Dott.SALVATORE. NOCERA

Figura 13. Autismo Online - http://autismo.inews.it/ 


\title{
Elena Bellini
}

\section{WBDG

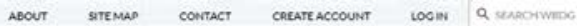

\begin{tabular}{|c|c|}
\hline & ESIGN OBJECTIVES \\
\hline & Accessible \\
\hline & Provide Equal Access and \\
\hline & Flexibility \\
\hline & History of Accessible Facility \\
\hline & Design \\
\hline & Beyond Accessabisty to \\
\hline & Universal Design \\
\hline & Universal Design and Heatth \\
\hline & Mesthetics \\
\hline & Cost-Elfective \\
\hline & Functional / Operational \\
\hline & Historic Preservation \\
\hline & Productive \\
\hline & Secure / Sate \\
\hline & Sustainable \\
\hline
\end{tabular}

\section{UNIVERSAL DESIGN AND HEALTH}

the WBDG Accessible Committee and Updated: 02-15-2017

\section{OVERVIEW}

As the eight Goals of Universal Design iSteinfeld and Maisel, 2012) suggest, an integral part of detigting high performance spoces for people with disabilities is Incorporating heaith and welloess into the by t tenvironment. In fact many of the pubichealth concerns targeted by health and wellness design stratesies oualify

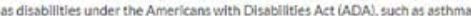

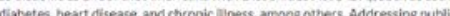
health isous through designcan contribute to overall heath promotion.

WITHIN THIS PAGE

- Overvien

- Introduction

- Application

- Major Resource

\section{INTRODUCTION}

\section{EMERGING STANDARDS}

There are several emerging design initiatives that promote the improvement of health ond overall human wellness, including, but not limited to:

\section{AIA DESIGNING FOR HEALTH}

AIA National launched its Designing for Health initiative in 2014 with a goal to enhance the physical and mental well being of buliding occupants. The initiative outlines six design strategies to achieve hesith in the built emvironment including: satety, socia connectedness, emvironmental quality, sensory environments, physical activity, and access to natural systems

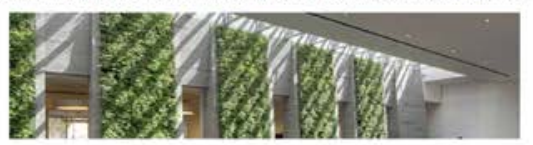

\section{WBDG}

\begin{abstract}
APPLICATION
The USO Warrior and Famlly Center at Walter Reed in Rectiesda. Maryland is a 16.000 seuare foot project designed as a facility for wounded soldiers and their families and caregivers: A focus group was led during the planging atage of the proiect to determine what types of features were important for likely building occupants, which included elements such as normalcy recreation, education, work, and respite. The Center is desioned to address inluries that impact both physical and cogniltive abilities: as well as mental welliness. The Center demonstrates both health and Universal Design strategies; and as such was the recipient of the ALA|DC Presidential Citation for Universal Design in 2014, and the AIA DC Presidential Citation for Design and Welibeing in 2015
\end{abstract}
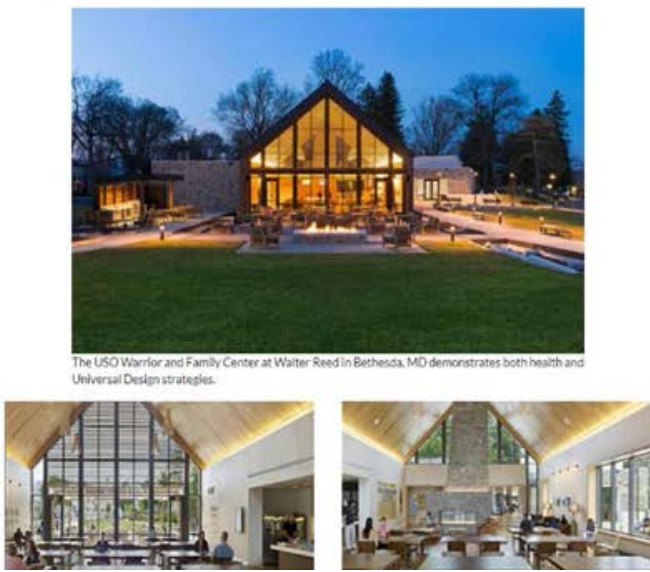

Figura 14. Autismo Online - http://autismo.inews.it/ 
Ambienti sensoriali "terapeutici" che rendano Abili

\begin{tabular}{|c|c|c|c|c|c|c|c|c|c|c|c|c|}
\hline & 3 The $\mathrm{C}$ & er for $\mathrm{He}$ & h Design & & & & JON & 6 & ACCOUNI & $8 \operatorname{tog} N$ & STOFE & ㅂ. \\
\hline * & About Us - & Topics & Insights \& Solutions - & Tools - & Research Services - & & Cert & & Soutreach- & Events & Search & 0 \\
\hline
\end{tabular}

n s Insights \& Soluntons : Design insights \& Strategies Tod

\section{Design Insights \& Strategies Tool}

November 2015 Tool

The patient room, the most repetitive space in acute care facilies, offers many opportunities to incorporate evidence-based design features. This set of interactive diagrams provides access to the healthcare design evidence base in an access ble and actionable format

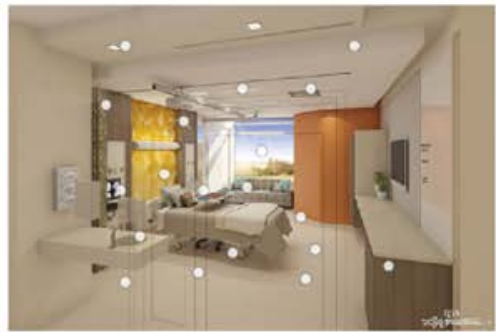

Medical-Surgical Patient Room

Medical-surgical patient rooms constitute a significant component of a hospital building project, with a large body of research evidence and best practice knowledge for designers to draw upon in order to create a best

design that successfully addresses unique issues in the design of each project.

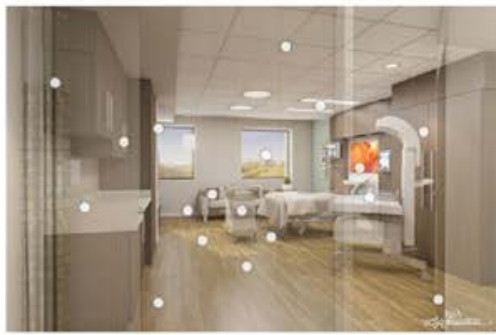

\section{Maternity Care Patient Room}

Maternity care patient rooms (typically LDR and LDRP rooms) represent a specialty design area that requires expertise, knowledge, and attention on the whole birthing process, which is often a time of great expectations. excitement, as well as pain and anxiety.

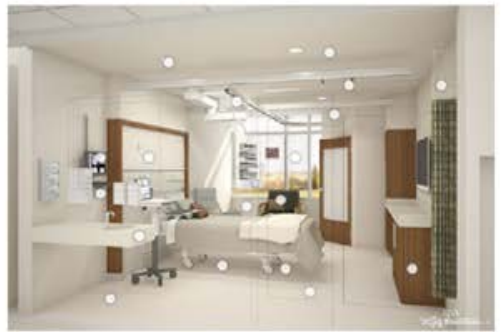

\section{Intensive Care Unit Patient Room}

Intensive care unit (ICU) patient rooms serve critically ill patients. With requirements for close monitoring and observation, as well as specialized equipment and technology, the physical environment can be frightening for families and requires special considerations to optimize staff efficiency. promote quality care and safety of patients, and support loved ones.

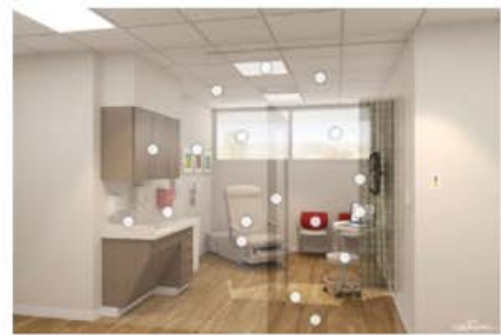

\section{Primary Care Exam Room}

Primary care exam rooms are becoming the center of the heslthcare experience. Technological advances, point-of-care testing and improved organizational flow models are streamlining the patient experience.by designing the exam room to be more patient-centric, providers and care teams can significantly enhance effectiveness, efficiency, comfort, safety. and quality of care

Figura 15. The Center for Health Design - https://www.healthdesign.org/ 


\section{Elena Bellini}

Overview

Medical-surgical patient rooms constitute a significant component of a hospital building project, with a large body of research evidence and best practice knowledge for designers to draw upon in order to create a best design that successfully addresses unique issues in the design of each project.

Click on one of the dots or the thumbnails below to learn more
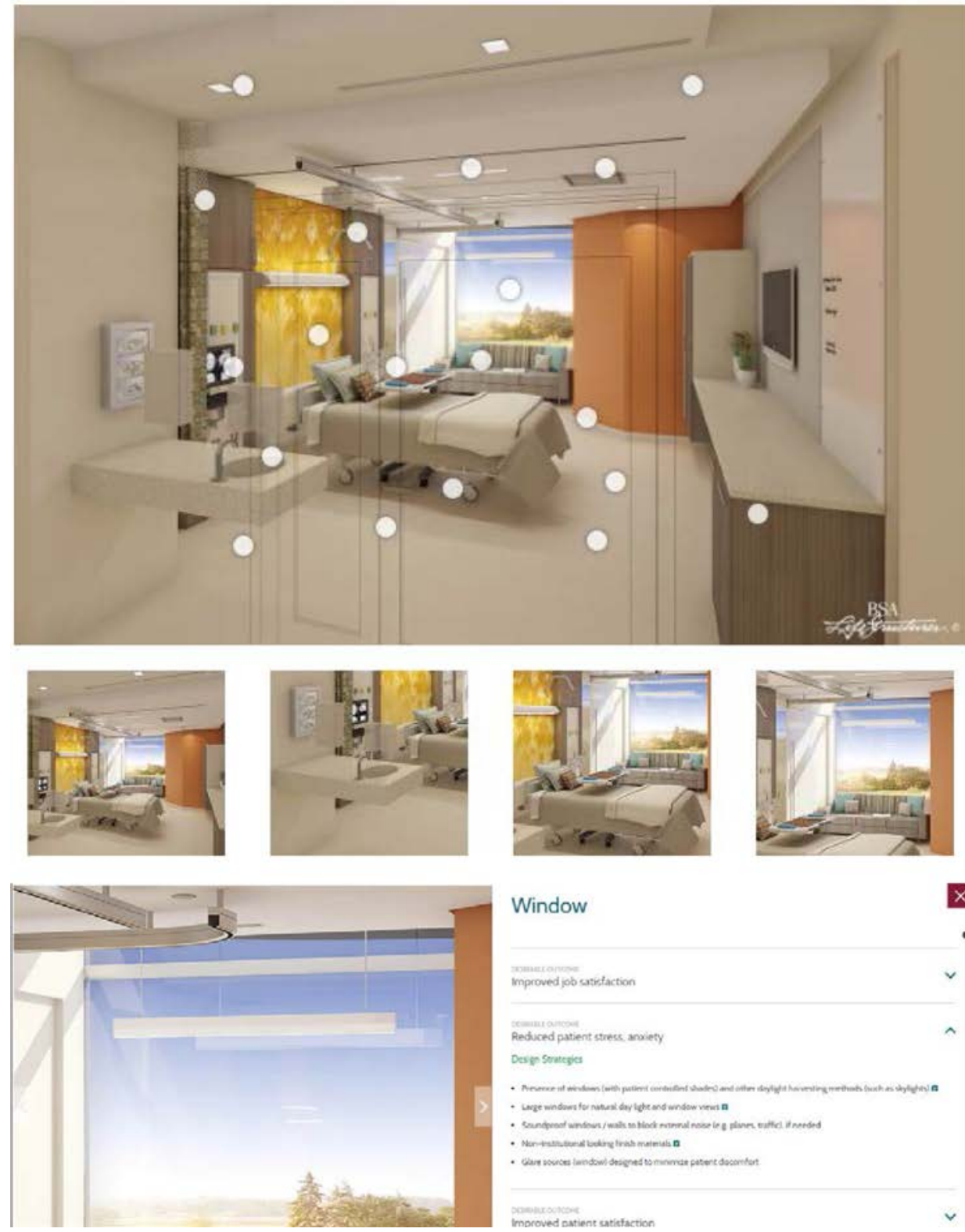

Window

improved iob custaction

Despe Srategie:

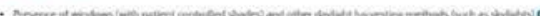

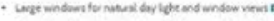

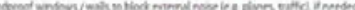

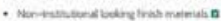

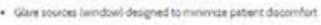

Figura 16. The Center for Health Design - https://www.healthdesign.org/ 


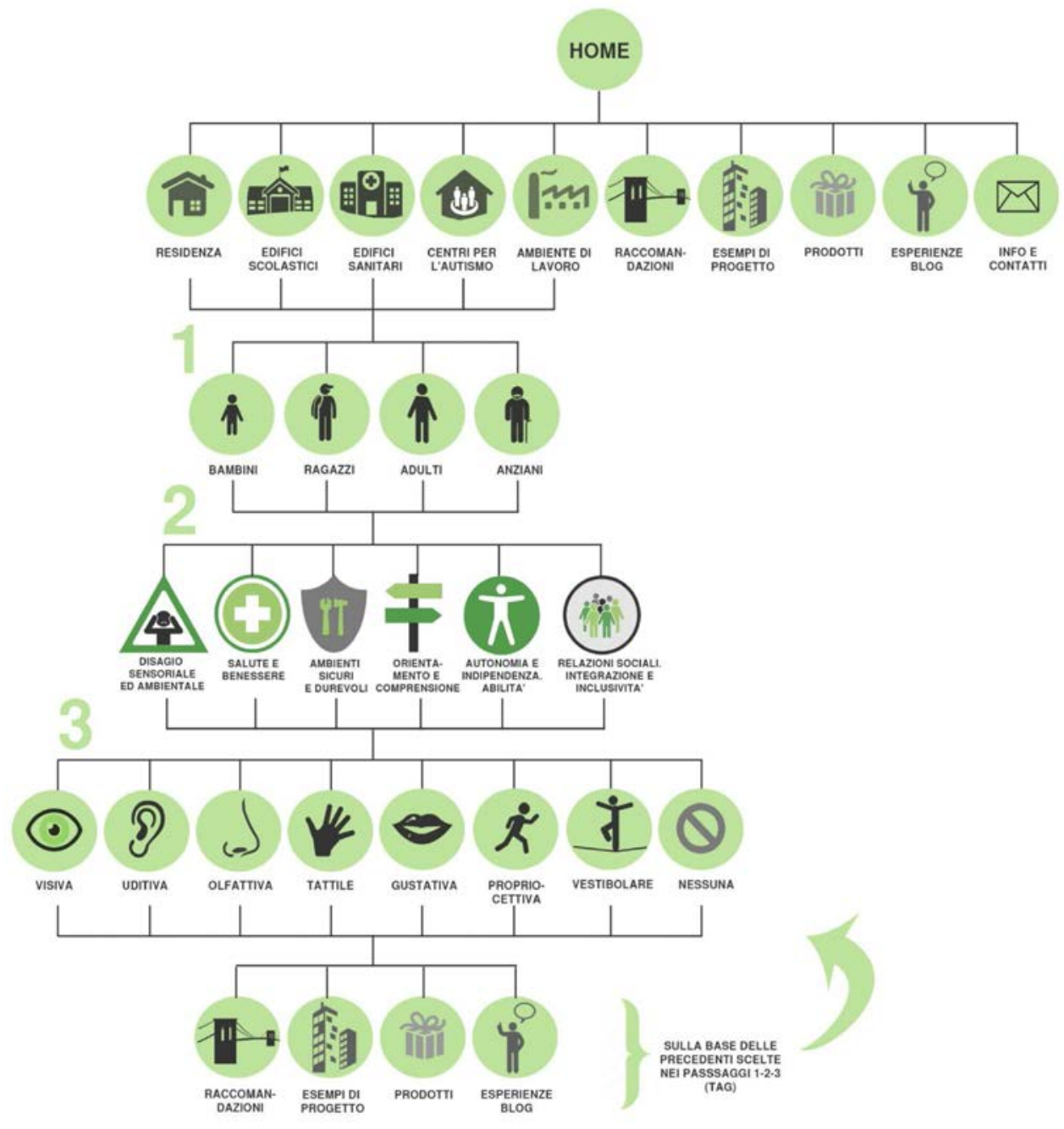

Figura 17. Site map di DARE (sense) 

DARE (sense)

HOME | RACCOMANDAZIONI | PROGETI | PRODOTI | ESPERIENZE | BLOG | INFO E CONTATT

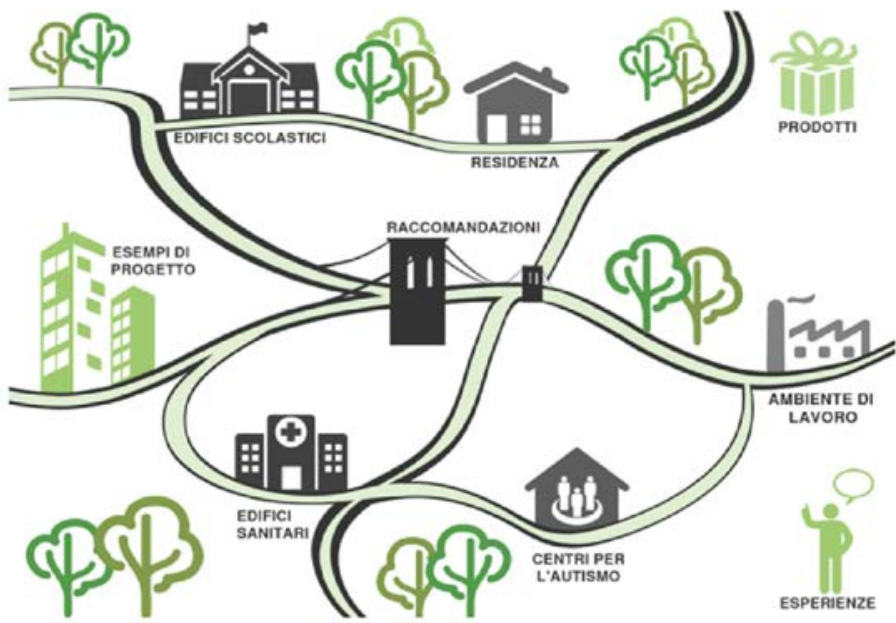

per maggiori info o contribuire scrivi sul blog

Figura 18. DARE (sense) Home page

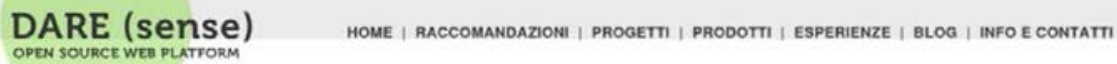

OPEN SOURCE WEB PLATFORM

per maggiori info o contribuire scrivi sul blog

Figura 19. DARE (sense) pagina di definizione dell'età dell'utenza 
Ambienti sensoriali “terapeutici” che rendano Abili

\section{DARE (sense) \\ OPEN SOURCE WEB PLATFORM}

HOME | RACCOMANDAZIONI | PROGETI | PRODOTI | ESPERIENZE | BLOG | INFO E CONTATT

\section{Quale esigenza senti come proritaria?}
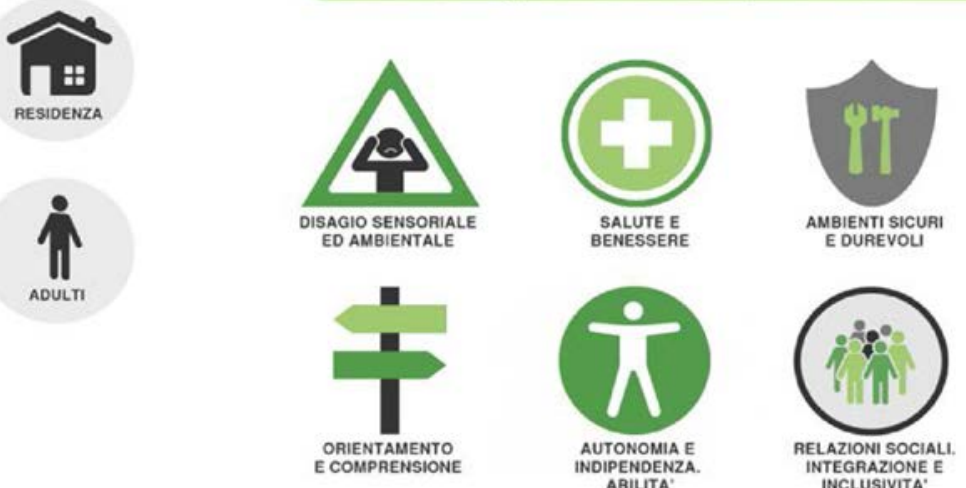

per maggiori info o contribuire scrivi sul blog

Figura 20. DARE (sense) pagina di deifnizione delle macro-esigenze

DARE (sense) OPEN SOURCE WEB PLATFORM
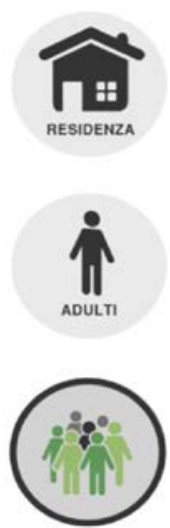

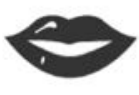

GUSTATIVA

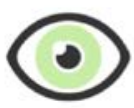

VISIVA

\section{Quale sensibilita' sensoriale percepisci maggiormente?}

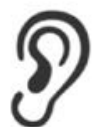

UDITIVA

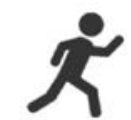

PROPRIOCETTIVA

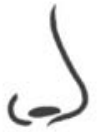

OLFATTIVA

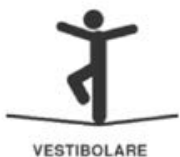

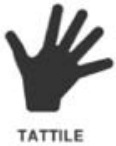

NESSUNA

per maggiori info o contribuire scrivi sul blog

Figura 21. DARE (sense) pagina di definizione delle sensibilità sensoriali 


\section{Elena Bellini}

DARE (sense)

HOME | RACCOMANDAZIONI | PROGETT | PRODOTI | ESPERIENZE | BLOQ | INFO E CONTATT

OPEN SOURCE WER DLATTORM

\#residenza \#adulti \#inclusivita \#visiva \#uditiva \#propriocettiva
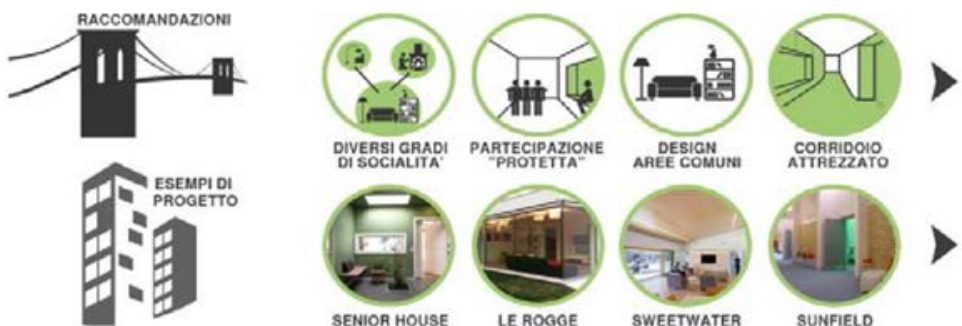
DIVERSI GRAD

PARTECIPAZIONE AREE COMUN

CORAIDOIO
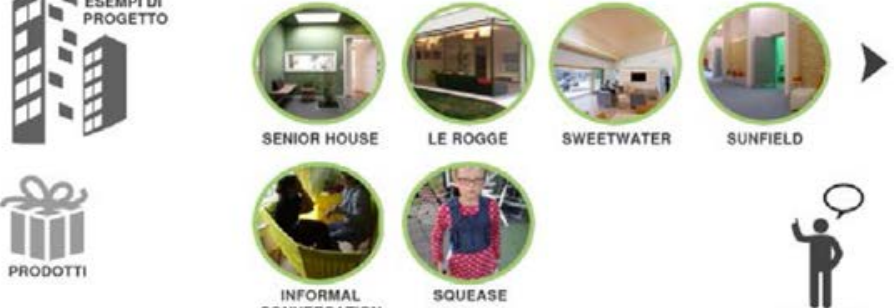

SUNFIELD

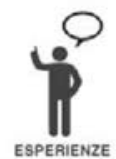

per maggiori info o contribuire scrivi sul blog

Figura 22. DARE (sense) pagina dei risultati - mockup

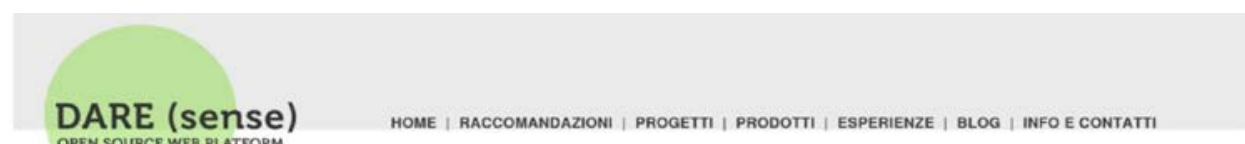

HOME | RACCOMANDAZIONI | PROGETT | PRODOTT| | ESPERIENZE | BLOG | INFO E CONTATT
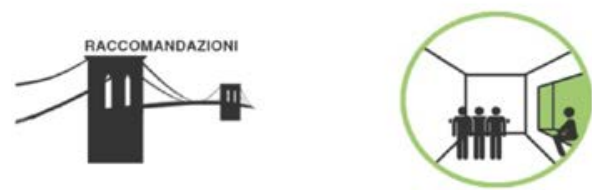

\section{PARTECIPAZIONE "PROTETTA"}

Una nicchia, un angolo o un'area protetta all'interno di spazi comuni più ampi, da cui guardare la vita sociale e farne parte. di scegliere quando e come relazionarsi con gli altri, attraverso diversi gradi di socialita.

Consente inoitre di isolarsi e appartarsi in un'area di quiete. dove poter autoregolare propri sensi, evitando it sovraccarico visivo ed uditivo, e riequilibrarsi neile situazioni di stress. Si tratta quindid di unarea neutra senza stimoli, se non gesti. attraverso la forma ele dimensioni contenute.

Figura 23. DARE (sense) pagina delle "raccomandazioni", scelta dell'icona tra i risultati (mockup) 
Ambienti sensoriali "terapeutici" che rendano Abili

\section{DARE (sense) \\ OPEN SOURCE WEB PLATFORM \\ SENIORS HOUSE}

Sede: Hinnerup (Danimarca) Data: 2014

Uso: residenza permanente per

adultianziani con autism

N. Utenti: 16 resident

Ogni residenza è separata ed autonoma, ma dirotta.

Flessibilita degli allogoi, attraverso parett divisotie arted mobill, per rispondere alit evoluzione delle abilis arred mobit, per rispondere airevoluzione deile abitits la vita indipendente e lautonomia della persona:

Nicchie/alcova con sedute in prossimita degli spazi di circolazione e delle diverse residenze per tavorire th telazione tra i residenti, ma anche far parte della vita comune pur isolandosi leggermente e riequilibrandosi a livello sensoriale:

Vista e rapporto continuo con gi spazi esterni ed il verde;

Finestra della cucina che affaccia sull'area centrale comune di collegamento tra le residenze, per mante. nere una relazione con il mondo esterno e ridurre if senso di solitudine.

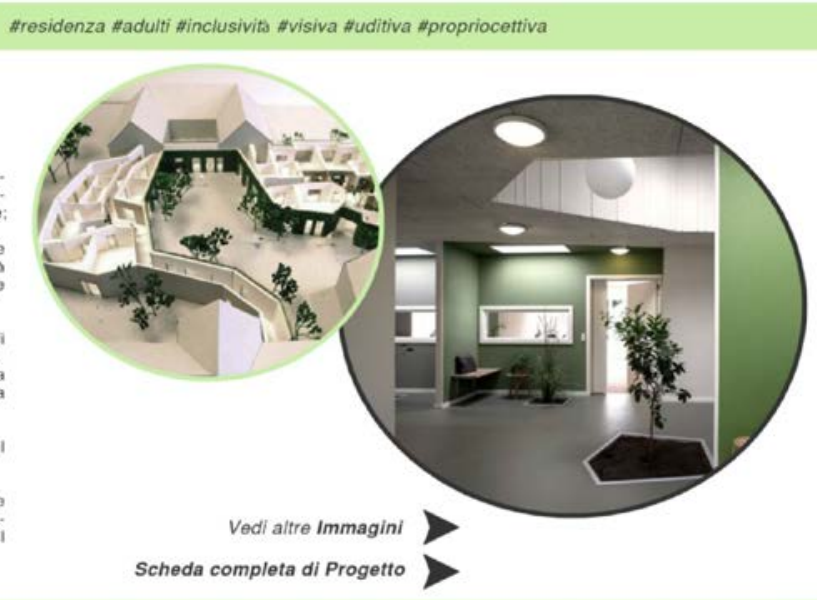

per maggiori info o contribuire scrivi sul blog

Figura 24. DARE (sense) pagina dei "progetti", scelta dell'icona tra i risultati (mockup)

DARE (sense) OPEN SOURCE WEB PLATFORM
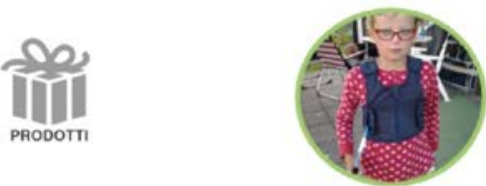

\section{SQUEASE}

Un gilet gonfiabile che consente alla persona di regolare in modo individuale la pressione prodotta sul proprio corpo attraverso il

Permette di rilassarsi nei momenti di maggiore stress favorendo tattilità, pressione e contenimento, come ad esempio la relazione con gli altri in luoghi pubblici o comuni.

Per visitare il sito di produzione del prodotto clicca qui

per maggiori info o contribuire scrivi sul blog

Figura 25. DARE (sense) pagina dei "prodotti", scelta dell'icona tra i risultati (mockup) 


\section{Elena Bellini}

DARE (sense)

HOME | RACCOMANDAZIONI | PROGETI | PRODOTI | ESPERIENZE | BLOG | INFO E CONTATI

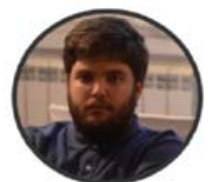

Federico De Rosa, 2016 \#residenza \#adulti \#inclusivita \#visiva \#uditiva \#propriocertiva

Ho bisogno di non stressare troppo le mie fin capacit percertive, quindi odlo gill ambient rumoros.

con molte luci e molta gente che paria

Per una passeggiata, quindi, meglio le atmoster
ovattate di un bosco che il caos di un centre.

commerciale. Datemi poi un input per volta, perche sono

molto meno elficace di voi nel decadificare la vostra

comunicazione. Posso capirvi ma comunicate piano

State tranquill poi, porche se siote in ansia.

State tranquilih, poi, porche se siote in ansia

La mia presenza, to che ho ransta di non capire bene

la sivazione ponseno che sieva in aglazione

Spiegatemi pacatamente e lentamente dove andiamo.

a tare cosa e dove. Per vol san ovie ma per me no.

E pol state in silenzio ogni tanto.

Essere diversi e bello e arricchisce.

Figura 26. DARE (sense) pagina delle "esperienze", scelta dell'icona tra i risultati (mockup) 



\section{Bibliografia}

La bibliografia è stata suddivisa per capitolo, in modo da facilitarne la consultazione, dato che per ogni sezione si trattano argomenti molto diversi tra loro. In particolare nel Capitolo dedicato ai "casi studio" è stata suddivisa la bibliografia e la sitografia relativa al singolo progetto. Nell'ultimo Capitolo infine è indicata la sitografia, poichè si riferisce allo studio della piattaforma web di progettazione e co-design.

\section{Capitolo 1}

American Psychiatric Association (APA) (2002) DSM IV, Diagnostic and Statistical Manual of Mental Disorders IV

American Psychiatric Association (APA) (2013) DSM V, Diagnostic and Statistical Manual of Mental Disorders V

Asperger, H. (1944) Die “Autistiche psychopathen” im Kindesalter, Archiv fur psychiatrie und Nervenkrankheiten, vol. 117, trad. in Frith, U. (1991) Autism and Asperger syndrome, New York: Cambridge University press, pp.37-92

Baker, J. P. (2013) Autism at 70 - Redrawing the Boundaries, in The New England Journal of Medicine, 369:1089-1091

Ballerini, L. (2013) I criteri diagnostici per l'autismo. 1943-2013: 70 anni di confusione, contributo al $1^{\circ}$ simposio Prima rappresentanza e psicopatologia. La psicopatologia precoce del 16 novembre 2013

Barale, F. et al. (2009) L'autismo a partire dall'età adulta: nuove conoscenze, criticità, implicazioni abilitative, NÓO $\varsigma, 15(3): 257-91$

Baron-Cohen, S. et al. (2000) Understanding other mind. Perspectives from developmental neuroscience, Oxford University Press, Oxford

Bartolomeo, S. Cerquiglini, A. (2010) Il quotidiano dei soggetti autistici: definizione dei programmi terapeutici e di socializzazione e fabbisogno di servizi, in Giofré, F. (a cura di) (2010) Autismo. Protezione sociale e architettura, Alinea, Firenze, pp. 17-27

Bertelli,M. et al. (2015) L'impatto della tipologia residenziale sul percorso riabilitativo della persona con Disturbi dello Spettro Autistico, Autismo e Disturbi dello Sviluppo, vol 13, n. 2, pp. 283.291

Bettheleim, B. (1967) The Empty Fortress: Infantile Autism and the Birth of the Self, The Free Press, New York Christensen, D.L. Baio, J. Van Naarden Braun, K. et al. (2016) Prevalence and characteristics of autism spectrum disorder among children aged 8 years-Autism and Developmental Disabilities Monitoring Network, 11 sites, United States, 2012, MMWR - Morbity and Mortality Weekly Report, Surveillance Summary 2016, 65(No. SS-3): 1 -23, CDC - Centers for Disease Control and Prevention, United States Department of Health and Human Services

Coccia, G. (2010) La dimensione del fenomeno nelle statistiche ufficiali e nelle indagini ad hoc, in Giofré, F. (a cura di) (2010) Autismo. Protezione sociale e architettura, Alinea, Firenze 
Ddl. n. 344, approvato il 18 marzo 2015, Disposizioni in materia di diagnosi, cura e abilitazione delle persone con disturbi dello spettro autistico e di assistenza alle famiglie

Elsabbagh, M. et al. (2012) Global prevalence of autism and other pervasive developmental disorders, Autism Res, 5:160179

Fombonne, E. (2009) Epidemiology of Pervasive Developmental Disorders, in Pediatric Research, vol. 65, n. 6, pp. 591-598, International Pediatric Research Foundation, Inc.

Fréjaville, E. Ciceri, F. (a cura di) (2007) Autismo che fare. Emilia Romagna a confronto con le altre regioni italiane sui modelli di intervento, Atti del VII Worhshop, Agenzia Regionale Sanitaria, Regione Emilia Romagna

Frith, U. (1989) Autism: Explaining the Enigma, Wiley-Blackwell, Hoboken, New Jersey, Stati Uniti

Giani, L. (2010) Norme e loro attuazione a favore delle persone con problemi cognitivocomportamentali, in Giofré, F. (a cura di) (2010) Autismo. Protezione sociale e architettura, Alinea, Firenze, pp. 29-39

Giofré, F. (a cura di) (2010) Autismo. Protezione sociale e architettura, Alinea, Firenze

Happé, F. Frith, U. (1996), The neuropsychology of autism, Brain 119:1377-1400

Hertz-Picciotto, I. (2011) Le cause ambientali dell'autismo: lo studio Charge, in Venerosi A, Chiarotti F (Ed.) (2011) Autismo: dalla ricerca al governo clinico, Roma: Istituto Superiore di Sanità (Rapporti ISTISAN 11/33)

Hobson, R. P. et al. (1989) Naming emotion in faces and voices: Abilities and disabilities in autism and mental retardation, British Journal of Developmental Psychology, vol. 7, n. 3, Blackwell Publishing Ltd, pp. 237-250

Hobson, R.P. (1993) Autism and the development of mind, Hove, Erlbaum, Sussex

Irish, J. E. N. (2013) What is Autism Spectrum Disorder (ASD)?, Design for All Institute of India, Special Issue, December 2015, Vol. 10, No. 13, pp. 22-24

ISS - Istituto Superiore della Salute (2012) Linee di indirizzo per la promozione ed il miglioramento della qualità della qualità e dell'appropriatezza degli interventi assistenziali nei Disturbi pervasivi dello sviluppo (DPS), con particolare riferimento ai disturbi dello spettro autistico, Ministero della Salute, Roma

ISS - SNLG (2011) Linee Guida 21 - Il trattamento dei disturbi dello spettro autistico nei bambini e negli adolescenti, Ministero della Salute, Roma (aggiornate nel 2015)

Kanner, L. (1943) Autisic disturbances and affective contact, The nervous Child, vol. 2, $\mathrm{n}^{\circ} 3$, pp. 217-250 - Traduzione Italiana: Disturbi autistici e contatto affettivo, Psicoterapia e scienze umane, $n^{\circ} 2$ e 3,1989

King, M. Bearman, P. (2009) Diagnostic change and the increased prevalence of autism, International Journal of Empidiemology, Oct 38(5):1224-34

L. 26 maggio 2016, n. 2232, in materia di Disposizioni in materia di assistenza in favore delle persone con disabilità grave prive del sostegno familiare

Levi, G. (a cura di) (2005) Linee Guida Per L'autismo Raccomandazioni Tecniche-Operative Per I Servizi Di Neuropsichiatria Dell'eta' Evolutiva, SINPIA - Societa' Italiana Di Neuropsichiatria Dell'infanzia E Dell'adolescenza, Roma

Maenner, M. J. et al. (2014) Potential Impact of DSM-5 Criteria on Autism Spectrum Disorder Prevalence Estimates, JAMA Psychiatry, 2014 March, 71(3): 292-300

Milton, D. (2012) So what exactly is autism?, AET Competence framework for the Department for Education, Birmingham

Nardocci, F. (2014) I Disturbi dello Spettro Autistico: evoluzioni per la classificazione, Relazione svolta al convegno Disabilità intellettive e dello sviluppo quadro diagnostico e interventi efficaci, Milano 10 Marzo 2014

Nevison, C. D. (2014) A comparison of temporal trends in United States autism prevalence to trends in suspected environmental factors, Environmental Health, 2014 Sep 5, 13:73 


\section{Elena Bellini}

NICE - National Institute for Health and Care Encellence (2012) Autism spectrum disorders in adults: diagnosis and management. Clinical guideline, NICE (aggiornato nel 2016)

Ozonoff, S. (1997) Components of executive function deficits in autism and other disorders, in Russel J. (Ed.) (1997) Autism as an executive disorder, Oxford University Press, Oxford, pp. 179-211

Pennington, B.F. Ozonoff, S. (1996) Executive functions and developmental psychopathology, Journal of Child Psychology and Psychiatry, 37: 51-87

Rimland, B. (1964) Infantile Autism: The Syndrome and Its Implications for a Neural Theory of Behavior, Englewood Cliffs, NJ: Prentice-Hall

Sacks, O. (1995) Un antropologo su Marte, Adelphi, Milano

Silberman, S. (2015) Neuro Tribes. The legacy of Autism and the Future of Neurodiversity, AVERY, New York (ed. Italiana 2016)

Trevarthen, C. Aitken, K.J. (2001) Infant intersubjectivity: research, theory, and clinical applications, Journal of Child Psychology and Psychiatry, 42: 3-48

United Nations (2006) Convention on the Rights of Persons with Disabilities and Optional Protocol

Vagni, D. (2015) Lo spettro Autistico, risposte semplici. Per una bonifica semantica dagli stereotipi dell'autismo, Asperger ONLUS per la condivisione della conoscenza

Venerosi A, Chiarotti F (Ed.) (2011) Autismo: dalla ricerca al governo clinico, Roma: Istituto Superiore di Sanità (Rapporti ISTISAN 11/33)

World Health Organization (WHO) (2007) ICD10, International Classification of diseases 10th revision

\section{Capitolo 2}

Ahrentzen, S. Steele, K. (2009) Advancing Full Spectrum Housing: Design for Adults with Autism Spectrum Disorders Technical report, Arizona Board of Regents, Phoenix

Amendola, G. Laurìa, A. Maldonado, T. Torricelli, M. C. (2004) Innovazione tecnologica per l'architettura: un diario a più voci, Edizioni ETS, Pisa

American Psychiatric Association (APA) (2002) DSM IV, Diagnostic and Statistical Manual of Mental Disorders IV

American Psychiatric Association (APA) (2013) DSM V, Diagnostic and Statistical Manual of Mental Disorders V

ARCA (2009) Providing Housing for People with Developmental Disabilities: An Analysis of Current Practices and Programs, Guiding Principles, Challenges and Recommendations, Association of Regional Center Agencies, Sacramento

Arenghi, A. et al. (2016) On the Relationship Between 'Universal' and 'Particular' in Architecture, in Petrie, H. et al. (2016) Universal Design 2016: Learning from the Past, Designing for the Future, IOS Press, pp. 31-39

Arneill, A. B. Devlin, A. S. (2002) Perceived Quality Of Care: The Influence Of The waiting Room Environment, in Journal of Environmental Psychology (2002) 22, 345-360, Elsevier Science Ltd

Aspy, R. \& Grossman, B. G. (2008) The Ziggurat Model: A Framework for Designing Comprehensive Interventions for Individuals with High-Functioning Autism and Asperger Syndrome, Shawnee Mission, KS: Autism Asperger Publishing Company

Assirelli, M. L. (2010) I servizi sociosanitari e relazionali per persone con disturbi dello spettro dell'autismo in Inghilterra: linee di ricerca, indicazioni di progetto e casi studio, in Giofré, F. (a cura di) (2010) Autismo. Protezione sociale e architettura, Alinea, Firenze, pp. $89-102$

Azéma, B. et al. (2011)L'habitat des personnes avec TED: du chez soi au vivre ensemble, DGCS - Direction Générale de la Cohésion Sociale, ANCREAI 
Ambienti sensoriali "terapeutici" che rendano Abili

Barale, F. et al. (2009) L'autismo a partire dall'età adulta: nuove conoscenze, criticità, implicazioni abilitative, $\mathrm{NÓ}_{\varsigma}, 15(3): 257-91$

Baumers, S. Heylighen, A. et al. (2010) Beyond the Designers' View: How People with Autism Experience Space, Proceedings of the Design Research Society Conference 2010" Design and Complexity", 7-9 July 2010, Montreal

Beaver, C. (2011) Designing environments for children and adults on the autism spectrum, in Good Autism Practice (GAP), 12(1), pp.7-11

Berliri, L. V. Pannocchia, N. (2014) Persone con disabilità e ospedale: principi, esperienze, buone prassi, Erickson, Trento

Bertelli, M. et al. (2015) L'impatto della tipologia residenziale sul percorso riabilitativo della persona con Disturbi dello Spettro Autistico, Autismo e Disturbi dello Sviluppo, vol 13, n. 2, pp. 283.291

Biggeri, M. (a cura di) (2010) Dalla relazione di cura alla relazione di prossimità. L'approccio della capability alle persone con disabilità, Liguori, Napoli

Biggeri, M. et al. (2011) Rethinking policies for persons with disabilities through the capability approach: the case of the Tuscany Region, ALTER, European Journal of Disability Research, 5(2011), 177-191

Bishop, K. (2012)The Role of Art in a Paediatric Healthcare Environment from Children's and Young People's Perspectives, in Procedia - Social and Behavioral Sciences ,38 ( 2012 ) $81-88$, Elsevier

Bogdashina O. (2003) Le percezioni sensoriali nell'autismo e nella sindrome di Asperger, Uovonero, Crema (edizione italiana del 2011)

Bonaiuto, M. Bilotta, E. Fornara, F. (2004) Che cos'è la psicologia architettonica, Carocci editore, Roma

Bowman, S. Jones, R. (2016) Sensory Interventions for Psychiatric Crisis in Emergency Departments-A New Paradigm, Journal of Psychiatry and Mental Health, 1(1)

Brand, A. (2010) Living in the community, London: Helen Hamlyn Centre, Royal College of Art

Brand, A., and Gaudion, K. (2012) Exploring sensory preferences; Living environments for adults with autism, The Helen Hamlyn Centre for Design, The Royal College of Art, London, UK

Brown,I. et al. (1997) Quality of Life Instrument package for adults with development disabilities, Toronto, Centre for Health Promotion, University of Toronto

Cama, R. (2009) Evidence-based healthcare design, J. Wiley, Hoboken

Campos Andrade, C. et al. (2012) Users' views of hospital environmental quality: Validation of the Perceived Hospital Environment Quality Indicators (PHEQIs), Journal of Environmental Psychology, 32 (2012) 97e111, Elsevier

Campos Andrade, C. et al. (2013) Inpatients' and outpatients' satisfaction: The mediating role of perceived quality of physical and social environment, Health \& Place, 21 (2013) 122-132, Elsevier

CARD - The Center for Autism \& Related Disabilities, Autism and the Hospital Emergency Room. A practical guide for health professionals to meet the needs of individuals with Autism Spectrum Disorders, CARD, TAMPA, Florida

Casey, L. B. et al. (2015) Emergency department visits by children with and without autism spectrum disorder: An initial comparison evaluating multiple outcome measures at one urban children's hospital, in Research in Autism Spectrum Disorders, 9 (2015) 144-150, Elsevier

Cattanei, M. (2009) Il Progetto DAMA un convegno per presentare e stimolare. Infermiere a Pavia, 21(1), 4-5

Cattanei, M. (2010) Progetto D.A.M.A. L'avvio a Pavia. Infermiere a Pavia, 22(2), 5-6 


\section{Elena Bellini}

Champagne, T. (2004) Sensory Approaches in Inpatient Psychiatric Settings. Innovative Alternatives to Seclusion \& Restraint, Journal of Psychological Nursing, VOL. 42, NO. 9

Coleman, R. Clarkson, J. Dong, H. Caasim, J. (2007) Design for inclusivity. A practical guide to accessible, innovative and user-centred design, Gower Publishing Limited, Aldershot

Corona, M. Ghelma, F. Maioli, M. Perazzoli, S. Mantovani, A. (2003) Il Progetto DAMA all'Ospedale San Paolo: una proposta di accoglienza medica rivolta ai disabili con ritardo mentale, AJMR (Ed. Italiana), 1(2), 100-106

Costa, M. (2013) Psicologia ambientale e architettonica. Come l'ambiente e l'architettura influenzano la mente e il comportamento, Franco Angeli, Milano

Cottini, L. (2009) Il Bambino con autismo in classe. Quattro parole chiave per l'integrazione, Giunti, Firenze

Cottini, L. (2011) L'autismo a scuola. Parole chiave per l'integrazione, Carocci, Roma

Cottini, L. Morganti, A. (2015) Evidence-Based Education e pedagogia speciale. Principi e modelli per l'inclusione. Carocci, Roma

Cottini, L. (2017) Le sfide dell'inclusione del bambino autistico a scuola, atti del XII Convegno Nazionale sulla Qualità della Vita. Progettare il Futuro, 11-12 settembre 2017,Università Cattolica del Sacro Cuore, Milano

Croen, L. A. et al. (2006) A Comparison of Health Care Utilization and Costs of Children With and Without Autism Spectrum Disorders in a Large Group-Model Health Plan, Pediatrics, Volume 118, Number 4, October 2006, pp. e1203-1211

Del Nord, R. (2006) Lo stress ambientale nel progetto dell'ospedale pediatrico. Indirizzi tecnici e suggestioni architettoniche, Motta Architettura, Milano

Del Nord, R. (2009) The culture for the future of healthcare architecture. Proceedings of 28tn International Public Health Seminar, Alinea, Firenze

Del Nord, R. (2014) Il ruolo strategico della ricerca intersettoriale nella progettazione delle strutture ospedaliere: il contributo del Centro Ricerche TESIS, TECHNE

Del Nord, R. Marino, D. Peretti, G. (2015) L'umanizzazione degli spazi di cura: una ricerca svolta per il Ministero della Salute italiano, in TECHNE, vol. 09, pp. 224-229, FUP

Department for Children, Schools and Families, UK (2012) Building Bulletin 102: Designing for Disabled Children and Children with Special Educational Needs, http://media.education.gov.uk/assets/files/pdf/b/building\%20bulletin\%20102.pdf

Devlieger, P. Rusch, F. Pfeiffer, D. (a cura di) (2003) Rethinking disability, Antwerp: Garant

D.M. Dicembre 1975, in materia di Norme tecniche aggiornate relative all'edilizia scolastica, ivi compresi gli indici minimi di funzionalità didattica, edilizia ed urbanistica da osservarsi nella esecuzione di opere di edilizia scolastica

Dijkstra, K. et al. (2006) Physical environmental stimuli that turn healthcare facilities into healing environments through psychologically mediated effects: systematic review, Integrative literature reviews and meta-analyses, Blackwell Publishing Ltd

Dunn, W. Little, L. (2013) Designing Sensory Friendly Environments for Children with Autism Spectrum Disorder (ASD), Design for All Institute of India, Special Issue, December 2015, Vol. 10, No. 13, pp. 26-32

Evans, G. W. (2003) The Built Environment and Mental Health, in Journal of Urban Health: Bulletin of the New York Academy of Medicine, vol. 80, n. 4, dicembre 2003

Fernandez-Andres, I. Pastor-Cerezuela , G. Sanz-Cervera, P. Tarraga-Minguez, R. (2015) A comparative study of sensory processing in children with and without Autism Spectrum Disorder in the home and classroom environments, in Research in Developmental Disabilities, 38 (2015) 202-212, Elsevier

Ferrante, T. (2013) Valutare la qualità percepita: uno studio pilota per gli Hospice, Franco Angeli, Milano

Ferrante, T. (2012) Architetture e design per l'hospice pediatrico, Alinea, Firenze 
Ambienti sensoriali "terapeutici” che rendano Abili

Fornara, F. (2005) Users' evaluative responses to spatio-physical humanization: the case of hospital environments, in Martens, B., Keul, A.G. (a cura di) Designing Social Innovation: Planning, Building, Evaluating, Hogrefe \& Huber, Gottingen (Germany), pp. 231240

Fornara, F., Bonaiuto, M., Bonnes, M. (2006) Perceived hospital environment quality indicators: a study of orthopaedic units, Journal of Environmental Psychology 26, 321-334

Frumkin, H. \& Fox, J. (2011) Healthy schools, in Dannenberg, A. L. Frumkin, H. Jackson, J. (a cura di) (2011) Making Healthy Places: Designing and Building for Health, Wellbeing, and Sustainability, pp. 217-28, Washington, DC: Island Press

Gaudion, K. (2013) Designing everyday activities: Living environments for adults with autism, The Helen Hamlyn Centre for Design, The Royal College of Art, London, UK.

Gaudion, K. (2014) Picture-it. A digital tool to support living with autism, The Helen Hamlyn Centre for Design Royal College of Art, London

Gaudion, K., Hall, A., Myerson, J., and Pellicano, L. (2015) Design and wellbeing: Bridging the empathy gap between neurotypical designers and autistic adults, in Mani, M. Kandachar, P. Design for Sustainable Well-being and Empowerment, IISc Press and TU Delft, $61-77$

Gibson, J. J. (1977) The theory of affordances, in Shaw, R. Bransford, j. (eds.) (1977) Perceiving, acting and knowing, Eribaum, Hillsdale, NJ, USA

Gibson, J. J. (1979) The ecological approach to visual perception, Houghton Mifflin, Boston, USA

Giddan, J. J. Ucelli Di Nemi, S. (2003) L'alternativa non urbana: le farm communities per adulti con autismo, in Barale F, Ucelli di Nemi S, Carrà G, Segagni G, eds. (2003) Il disturbo autistico in età adulta, $9: 4$, pp. 341-350

Ghelma, F. (2017) Mascheramento diagnostico e compliance al trattamento, atti del XII Convegno Nazionale sulla Qualità della Vita. Progettare il Futuro, 11-12 settembre 2017,Università Cattolica del Sacro Cuore, Milano

Giovenale, A. M. (a cura di) (2006) Qualità degli spazi e prevenzione dello stress. Indirizzi tecnici per la progettazione del Day Hospital, Alinea, Firenze

Grandin, T. (1995) Thinking in pictures and other reports from my life with autism, Vintage Books, New York

Gurney, J. G. et al. (2006) Parental Report of Health Conditions and Health Care Use Among Children With and Without Autism, National Survey of Children's Health, Arch Pediatr Adolesc Med, 160:825-830

HCUP (2010) Nationwide Emergency Department (NEDS), Healthcare Cost and Utilization Project (HCUP), Agency for Healthcare Research and Quality, Rockville, MD (USA)

Henriksen, K. Kaup, M-1. (2010) Supportive Learning Environments for Children with Autism Spectrum Disorders, in Undergraduate Research Journal for the Human Sciences, 9

Henry, C. (2013) A critical analysis of sensorysensitive and "neuro-typical" simulated architectural design in schools for autism, in Design for All Institute of India, Special Issue, December 2015, Vol. 10, No. 13, pp. 53-66

Henry, C. (2015) A critical analysis of sensorysensitive and "neuro-typical" simulated architectural design in schools for autism, Design for All Institute of India, Special Issue, December 2015, Vol. 10, No. 13, pp. 53-66

Heylighen, A. Neyt, E. Baumers, S. Herssens, J. Vermeersch, P. (2010) Conservation Meets Inclusion. Model Meets Reality, in Clarkson, P. J. Langdon, P. Robinson, P. (a cura di) (2010) Proceedings of the 5th Cambridge Workshop on Universal Access and Assistive Technology, Cambridge (UK), 22-25 March 2010

Hoffman, H. G. Et Al. (2001) The Effectiveness Of Virtual Reality For Dental Pain Control: A Case Study, Cyberpsychology \& Behavior, Volume 4, Number 4, 2001, Mary Ann Liebert, Inc. 


\section{Elena Bellini}

$\mathrm{Hu}$, J. et al. (2009) Effects of dental 3D multimedia system on the performance of junior dental students in preclinical practice: a report from China, Advanced in Health and Science Education, 14:123-133, Springer Science+Business Media

Hogg, J. et al. (2001) The use of 'Snoezelen' as multisensory stimulation with people with intellectual disabilities: a review of the research in Research in Developmental Disabilities, 22 (2001) 353-372, Pergamon

Humphreys, S. (2011) Architecture et Autisme, in Autisme Europe, vol. 55, pp.9-13, Bruxelles

Iannuzzi, D. et al. (2015) Brief Report: Emergency Department Utilization by Individuals with Autism, Journal of Autism and Development Disorders 45:1096-1102

Kaplan, H. et al. (2006) Snoezelen multi-sensory environments: Task engagement and generalization, in Research in Developmental Disabilities, 27 (2006) 443-455, Elsevier

Khare, R., Mullick, A. (2008) Educational Spaces for Children with Autism, Design Development Process, CIB W 084 Proceedings, Building Comfortable and Liveable Environment for All, Atlanta, USA, pp. 66-75

Khare, R., Mullick, A. (2009) Incorporating the Behavioral Dimension in Designing Inclusive Learning Environment for Autism, in ArchNet-IJAR: International Journal of Architectural Research, 3(3), pp.45-64

Khare, R. Mullick, A. (2010) Universally Beneficial Educational Space Design for Children with Autism, presented in 'Designing for Children' with focus on 'Play+Learn' Bombay, India

Kinnaer, M. Baumers, S. Heylighen, A. (2014) How do People with Autism (Like to) Live?, in Langdon, P. M . Lazar, J. Heylighen, A. Dong, H. (a cura di ) (2014) Inclusive Designing. Joining Usability, Acessibility and Inclusion, Springer Cham Heidelberg New York Dordrecht London, part IV, pp. 175-185

Kinnaer et al. (2016) Autism-friendly architecture from the outside in and the inside out: an explorative study based on autobiographies of autistic people, Journal of Housing and the Built Environment, 31:179-195, Springer Science+Business Media Dordecht

Kluth, P. (2013) Supporting Students with Autism: 10 Ideas for Inclusive Classrooms, in Design for All Institute of India, Special Issue, December 2015, Vol. 10, No. 13, pp. 36-42

Krauss, M. V. Seltzer, N. M. Jacobson, J. T. (2005) Adults with autism living at home or in non-family settings: positive and negative aspects of residential status, in Journal of Intellectual Disability Research, vol. 49, parte II, pp. 111-124, Blackwell Publishing Ltd

Langdon, P. Clarkson, J. Robinson, P. (2013) Designing inclusive interactions, in Universal Access in the Information Society, 12:233-235, Springer-Verlag Berlin Heidelberg

Langdon, P. M . Lazar, J. Heylighen, A. Dong, H. (a cura di ) (2014) Inclusive Designing. Joining Usability, Acessibility and Inclusion, Springer Cham Heidelberg New York Dordrecht London

Laurìa, A. (a cura di) (2003) Persone "reali" e progettazione dell' ambiente costruito. L'accessibilità come risorsa della qualità ambientale, Maggioli Editore, Santarcangelo di Romagna (RN)

Lotan, M. Gold, c. (2009) Meta-analysis of the effectiveness of individual intervention in the controlled multisensory environment (Snoezelen) for individuals with intelectual disability, Journal of mental and intellectual disabilities, 34(3):207-15

Lotan, M. Shapiro, M. (2005) Management of young children with Rett disorder in the controlled multi-sensory (Snoezelen) environment, in Brain \& Development 27 (2005) S88S94, Elsevier

Lowe, C. Gaudion, K. McGinley, C. Kew , A. (2014)Designing living environments with adults with autism, Tizard Learning Disability Review, Vol. 19 Iss 2 pp. 63-72

Lunsky, Y. et al. (2015) Predictors of emergency service use in adolescents and adults with autism spectrum disorder living with family, Emerg Med Journal, 32:787-792 
Mace, W. M. (1977) James J. Gibson's strategy for perceiving: Ask not what's inside your head, but what your head's inside of, in Shaw, R. Bransford, j. (eds.) (1977) Perceiving, acting, and knowing: Toward an ecological psychology, Erlbaum, Hillsdale, NJ, USA, 43-67

Maier, J. R. A. Fadel, G.M. (2009) An affordance-based approach to architectural theory, design, and practice, Design Studies, 30(4), 393-414, Elsevier

Maiocchi, M. (a cura di) (2007) La comunicazione emozionale negli ambienti ospedalieri, Maggioli Editore, Santarcangelo di Romagna (RN)

Marion, M. (2006) Bringing The World to the Classroom, EP Magazine, April: 32-35

Martin, C. S. (2014) Exploring the impact of the design of the physical classroom environment on young children with autism spectrum disorder (ASD), in JORSEN - Journal of Research in Special Educational Needs, pp. 1-19, NASEN. Helping Everyone Achieve

Mazzone, L. (2015) Un autistico in famiglia, Mondadori, Milano

McAllister, K. (2010) The ASD friendly classroom - Design complexity, challenge \& characteristics, Paper presented at Design Research Society International Conference (pp. 118). Montreal, Canada

McAllister, K. Maguire, B. (2012) A design model: The autism spectrum disorder classroom design kit, in British Journal of Special Education, 39(4), 201-208

McAllister, K. Maguire, B. (2012) Design considerations for the autism spectrum disorderfriendly Key Stage 1 classroom, in Support for Learning, vol 27, n. 3, pp. 103-112, NASEN

McAllister, K. and Hadjri, K. (2013) Inclusion and the special educational needs (SEN) resource base in mainstream schools: physical factors to maximise effectiveness, Support for Learning, 28: 57-65

Menzinger, B. Jackson, R. (2009) The effect of light intensity and noise on the classroom behaviour of pupils with Asperger syndome, Support for Learning, Volume 24, Number 4, NASEN

Milton, E. M. D. (2012) The ontological status of autism: The 'double empathy problem', Disability and Society, 1-5, 2

MIUR - Ministero dell'Istruzione, dell’Università e della Ricerca (2013) Linee guida per l'edilizia scolastica

Mostafa, M. (2006) Let Them be Heard: Appropriate Acoustics for Autism- Special Needs School Design, Cairo, Egypt: ArchCairo 2006 Appropriating Architecture and Taming Urbanism in the Decades of Transformation, Cairo University

Mostafa, M. (2006) Viewpoint: Enabling Architecture, in Communication Magazine, The National Autistic Society, London

Mostafa, M. (2008) An architecture for autism: Concepts of design intervention for the autistic user, in ArchNet-IJAR : International Journal of Architectural Research, vol.2(1), pp. $189-211$

Mostafa, M. (2010) Housing Adaptation for Adults with Autistic Spectrum Disorder, Open House International, Urban International Press, 35, 37-48

Mostafa, M. (2013) Editorial: Expanding Normal: Towards a More Inclusive Approach to Designing the Built Environment, in Open House International, Urban International Press, 38, 4-7

Mostafa, M. (2014) Architecture For Autism: Autism ASPECTSS ${ }^{\mathrm{TM}}$ in School Design, in Archnet-IJAR, Volume 8 - Issue 1 - March 2014 - (143-158)

Mostafa, M. (2015) Architecture for Autism: Built Environment Performance in Accordance to the Autism ASPECTSS ${ }^{\text {TM }}$ Design Index, in Design Principles and practices: an International Journal - Annual Review, vol. 8, pp.55-71,Common Ground Publishing, Champaign, Illinois, USA 


\section{Elena Bellini}

MIND (2015) Supporting people living with autism spectrum disorder and mental health problems. A guide for practitioners and providers, MIND, London

Nayfack, A. M. et al. (2014) Hospitalizations of Children with Autism Increased from 1999 to 2009, Journal of Autism and Development Disorders, 44:1087-1094

Nicholas, D.B. Zwaigenbaum, L. Muskat, B. et al. (2016) Toward Practice Advancement in Emergency Care for Children With Autism Spectrum Disorder, Pediatrics, 137(S2): S206-S211

Notbohm, E. (2015) 10 cose che ogni bambino con autismo vorrebbe che tu sapessi, Edizioni Centro Studi Erickson, Trento (ed. italiana di Notbohm, E. (2012) Ten Things Every Child With Autism Wishes You Knew, Future Horizons Inc., Arlington)

Novak, T. et al. (2012) Pilot study of a sensory room in an acute inpatient psychiatric unit, Australasian Psychiatry Vol 20, Issue 5, pp. 401-406

Nusssbaum, M. C. Sen A. K. (a cura di ) (1993) The quality of life, Claredon Press, Oxford

Nussbaum, M. C. (2000) Women and Human Development: The Capabilities Approach, The Press Syndicate of the University of Cambridge

Nussbaum, M. C. (2007) Le nuove frontiere della giustizia. Disabilità, nazionalità, appartenenza di specie, Il Mulino, Bologna

Pessina, A. (2010) Barriere della mente e barriere del corpo. Annotazioni per un' etica della soggettività empirica, in Paradoxa, Etica della condizione umana, Vita e Pensiero, Milano

Pratt, K. et al. (2011) Ensuring successful admission to hospital for young people with learning difficulties, autism and challenging behaviour: a continuous quality improvement and change management programme, Blackwell Publishing Ltd, Child: care, health and development, 38, 6, 789-797

Rebel, R. (2014) Specialized hospitals : design \& planning, North Point: Design media publishing limited

Resnik, D. D. et al. (2009) Opening Doors: A discussion of residential options for adults living with autism and related disorders, Collaborative report by the Urban Land Institute Arizona, Southwest Autism Research \& Resource Center and Arizona State University

Robison, J. E. (2007) Look Me in the Eye: My Life with Asperger's, New York : Crown Publishers

Royal Cornwall Hospital, NHS Trust (2014) Protocol for Children and Adults with Autism Spectrum Conditions

Russel, A. J. et al. (2016) The mental health of individuals referred for assessment of autism spectrum disorder in adulthood: A clinical report, Autism, July 2016, 20(5): 623-7

Scarpinato, N. et al. (2010) Caring for the Child With an Autism Spectrum Disorder in the Acute Care Setting, Journal for specialists in Pediatric Nursing, Wiley Periodicals, Inc.

Schrameijer, F. (2016) About autism-friendly design, Workshop Inservice Autism, November 9 through 11, 2016, Oost Malle, Belgium

Sanchez, P. A., Vazquez, F. S., \& Serrano, L. A. (2011) Autism and the built environment, in Williams, T. (a cura di) (2011) "Autism spectrum disorders", Croatia: InTech, pp. 363380

Schrameijer, F. (2016) About autism-friendly design, Workshop Inservice Autism, November 9 through 11, 2016, Oost Malle, Belgium

Scott, I. (2009) Designing learning spaces for children on the autism spectrum, in Good Autism Practice (GAP), 10(1), 36-51

Scott, I.(2011) Analysis of a project to design the ideal classroom undertaken by a group of children on the autism spectrum and students of architecture, Good Autism Practice (GAP), 12(1), pp.13-25

Segado Vàzquez, F. Segado Torres, A. (2013) Autism and Architecture, Recent Advances in Autism Spectrum Disorders - Volume II (9), pp. 178-186, InTech

Sen, A. K. (1999) Development as freedom, Oxford University Press, Oxford 
Sen, A. K. (2004) Disability and Justice, keynote speech, second international disability conference, World Bank

Shabha, G. Gaines, K. (2011) Therapeutically enhanced School Design for Pupils with Autism Spectrum Disorders (ASD): a Comparative Study of the United States and United Kingdom, Conference proceedings: Make no little plans - Environmental Design Research Association, Chicago, 25-28/05/2011

Silberman, S. (2015) Neuro Tribes. The legacy of Autism and the Future of Neurodiversity, AVERY, New York (ed. italiana 2016)

Sing, N. N. et al. (2004) Effects of Snoezelen room, Activities of Daily Living skills training, and Vocational skills training on aggression and self-injury by adults with mental retardation and mental illness, in Research in Developmental Disabilities, 25 (2004) 285-293, Elsevier

Spes contra Spem (2013) Carta Dei Diritti Delle Persone Con Disabilità In Ospedale, Roma

Spinelli, F. Bellini, E. Bocci, P. Fossati, R. (1994) Lo spazio terapeutico. Un metodo per il progetto di umanizzazione degli spazi ospedalieri, Alinea, Firenze

Standen, P. J. Brown, D. J. (2005) Virtual Reality in the Rehabilitation of People with Intellectual Disabilities: Review, in Cyberpsychology \& Behavior, Volume 8, Number 3, 2005, Mary Ann Liebert, Inc

Steele, K. Ahrentzen, S. (2016) At home with autism. Designing housing for the spectrum, Policy Press, Bristol

Sutton, D. et al. (2013) Optimizing arousal to manage aggression: A pilot study of sensory modulation, International Journal of Mental Health Nursing (2013) 22, 500-511

Tommaso d'Aquino, Quaestiones disputatae de veritate, vol 1: De veritate, q. 2 a. 3 a. 19

Tufvesson, C. Tufvesson, J. (2009) The building process as a tool towards an all-inclusive school. A Swedish example focusing on children with defined concentration difficulties such as ADHD, autism and Down's syndrome, Journal of Housing and the Built Environment, 24(1), 47-66

Ulrich, R. Zimring, C. Xiaobo, Q. Anjali, J. Choudhary, R (2004) The role of the physical environment in the hospital of the 21th century: a once-in-a-lifetime opportunity, Report to The Center for Health Design for the Designing the 21st Century Hospital Project, Concord (USA)

Vogel, C. (2008) Classroom design for living and learning with autism, Autism Aspergers Digest, May-June

United Nations (2006) Convention on the Rights of Persons with Disabilities and Optional Protocol

Verderber, S. (2010) Innovations in Hospital Architecture, Routledge, London

Vohra, R. (2016) Emergency Department Use Among Adults with Autism Spectrum Disorders (ASD), Journal of Autism and Development Disorders, 46:1441-1454

Weingerg, A. Creed, F. (2000) Stress and psychiatric disorder in health care professionals and hospital staff, in "The Lacet", 355, February 12, pp.533-537

Whitehurst, T. (2006) The impact of building design on children with autistic spectrum disorders, in Good Autism Practice (GAP), 7(1), 31-38

Whitehurst, T. (2006) Evaluation of features specific to an ASD designed living accommodation, in Designing living \& learning environments for children with autism, Sunfield Research Institute

Wiederhold, M. D. Et Al. (2014) Clinical Use Of Virtual Reality Distraction System To Reduce Anxiety And Pain In Dental Procedures, Cyberpsychology, Behavior, And Social Networking Volume 17, Number 6, 2014, Mary Ann Liebert, Inc

Wing, L. and Gould, J. (1979) Severe impairments of social interaction and associated abnormalities in children: Epidemiology and classification, Journal of Autism and Developmental Disorders, 9, 11-29 


\section{Elena Bellini}

Woo, C. C. Donnelly, J. H. Steinberg-Epstein, R. Leon, M. (2015) Environmental Enrichment as a Therapy for Autism: A Clinical Trial Replication and Extension, in Behavioral Neuroscience, Vol. 129, No. 4, 412-422, American Psychological Association

Zwaigenbaum, L. et al. (2016) Perspectives of Health Care Providers Regarding Emergency Department Care of Children and Youth with Autism Spectrum Disorder, Journal of Autism and Development Disorders, 46:1725-1736

\section{Capitolo 3}

Antonello, F. Antonello, A. (2015) Sono graditi visi sorridenti, Feltrinelli, Milano

Arduino, M. (2014) Il bambino che parlava con la luce. Quattro storie di autismo, Einaudi, Torino

Arenghi, A. et al. (2016) On the Relationship Between 'Universal' and 'Particular' in Architecture, in Petrie, H. et al. (2016) Universal Design 2016: Learning from the Past, Designing for the Future, IOS Press, pp. 31-39

Cottini, L. (2009) Il Bambino con autismo in classe. Quattro parole chiave per l'integrazione, Giunti, Firenze

Cottini, L. (2011) L'autismo a scuola. Parole chiave per l'integrazione, Carocci, Roma

De Rosa, F. (2014) Quello che non ho mai detto. Io, il mio autismo e ciò in cui credo, San Paolo Edizioni, Alba (CN)

Francis, P. Balbo, S. Firth, L. (2009) Towards co-design with users who have autism spectrum disorders, Universal Access in the Information Society, 8:123-135, Springer-Verlag

Gaudion, K., Hall, A., Myerson, J., and Pellicano, L. (2015) Design and wellbeing: Bridging the empathy gap between neurotypical designers and autistic adults, in Mani, M. and Kandachar, P. (2015) Design for Sustainable Well-being and Empowerment, IISc Press and TU Delft, 61-77

Gerland, G. (2003) A real person: Life on the outside, London, England: Souvenir Press

Grandin, T. (2001) Pensare in immagini e altre testimonianze della mia vita di autistica, Edizioni Centro Studi Erickson, Trento; ed. italiana di Grandin, T. (1995) Thinking in pictures and other reports from my life with autism, Vintage Books, New York

Mazzone, L. (2015) Un autistico in famiglia, Mondadori, Milano

Milton, E. M. D. (2012) The ontological status of autism: The 'double empathy problem', Disability and Society, 1-5, 2

Milton, D. (2012) So what exactly is autism?, AET Competence framework for the Department for Education, Birmingham

Morreau, L. E. Bruininks, R. H. (1991) Checklist of Adaptive Living Skills (CALS), Riverside Publishing

Nicoletti, G. (2013) Una notte ho sognato che parlavi. Così ho imparato a fare il padre di mio figlio autistico, Mondadori, Milano

Nicoletti, G. (2014) Alla fine qualcosa ci inventeremo. Che ne sarà di mio figlio autistico quando non sarò più al suo fianco, Mondadori, Milano

Notbohm, E. (2015) 10 cose che ogni bambino con autismo vorrebbe che tu sapessi, Edizioni Centro Studi Erickson, Trento; ed. italiana di Notbohm, E. (2012) Ten Things Every Child With Autism Wishes You Knew, Future Horizons Inc., Arlington

Peete, H. R. Peete, R. E. (2010) My Brother Charlie

Petrie, H. et al. (2016) Universal Design 2016: Learning from the Past, Designing for the Future, IOS Press, pp. 31-39

Rizzo, F. (2009) Strategie di co-design. Teorie, metodi e strumenti per progettare con gli utenti, Franco Angeli, Milano

Sachs, O. (1995) Un antropologo su Marte, Adelphi Edizioni, Milano 
Ambienti sensoriali "terapeutici” che rendano Abili

Silberman, S. (2015) Neuro Tribes. The legacy of Autism and the Future of Neurodiversity, AVERY, New York (ed. Italiana 2016)

Sparrow, S. Cicchetti, D. V. Balla, D. A. (1984) Vineland Adaptive Behavior Scales, Circle Pines, MN: American Guidance Service

Üstün, T.B. Kostanjsek, N. Chatterji, S. Rehm, J. (a cura di) (2010) Measuring Health and Disability: Manual for WHO Disability Assessment Schedule (WHODAS 2.0), World Health Organization

\section{Capitolo 4}

New Struan School - Alloa (UK) - 2005:

Henry, C. (2011) Designing for Autism: Lighting, ArchDaily, https://www.archdaily.com/177293/designing-for-autism-lighting

Scott, I. (2009) Designing learning spaces for children on the autism spectrum, in Good Autism Practice (GAP), 10(1), 36-51

http://www.scottishautism.org/new-struan-school/

http://www.scottishautism.org/about-autism/research-and-training/design-autism

DLC- Developmental Learning Center - Warren (New Jersey, USA) - 2007

Henry, C. (2015) A critical analysis of sensorysensitive and "neuro-typical" simulated architectural design in schools for autism, Design for All Institute of India, Special Issue, December 2015, Vol. 10, No. 13, pp. 53-66

Marion, M. (2006) Bringing The World to the Classroom, in EP Magazine, April: 32-35

Henry, C. (2011) Designing for Autism: The 'Neuro-Typical' Approach, ArchDaily http://www.archdaily.com/181402/designing-for-autism-the-neuro-typical-approach https://www.mujc.org/domain/72

USA Architects, http://www.usaarchitects.com/project/developmental-learning-center

Forbush School for Autism - Hunt Valley (USA) - 2008:

Universal Case Studies, Institute for Human Centered Design http://universaldesigncasestudies.org/education/specialized-purpose/forbush-school

Cho Benn Holback + Associates - http://www.cbhassociates.com/projects/details/forbushschool-for-autism/

Advance School for Developing Skills of Special Needs Children - Qattameya (Cairo) 2008:

Mostafa, M. (2015), Architecture for Autism: Built Environment Performance in Accordance to the Autism ASPECTSS ${ }^{\text {TM }}$ Design Index, in Design Principles and Practices: An International Journal - Annual Review, vol 8, pp. 55-71

Mostafa, M. (2014) Architecture For Autism: Autism ASPECTSS ${ }^{\mathrm{TM}}$ in School Design, in Archnet-IJAR, Volume 8 - Issue 1 - March 2014 - (143-158)

Mostafa, M. (2008) An architecture for autism: Concepts of design intervention for the autistic user, in ArchNet-IJAR : International Journal of Architectural Research, vol.2(1), pp. $189-211$

Quirk, V. (2013) An Interview with Magda Mostafa: Pioneer in Autism Design, ArchDaily, https://www.archdaily.com/435982/an-interview-with-magda-mostafa-pioneer-in-autismdesign

Northen School for Autism - Reservoir (Australia) - 2013: 


\section{Elena Bellini}

Mostafa, M. (2015), Architecture for Autism: Built Environment Performance in Accordance to the Autism ASPECTSS ${ }^{\text {TM }}$ Design Index, in Design Principles and Practices: An International Journal - Annual Review, vol 8, pp. 55-71

Association for Learning Environments, http://a4le.org.au/awards/awards-2013/2013regional-award-winners-and-commendations/northern-school-for-autism

Bozikovic, A. (2015) 'Design empathy' builds inclusive spaces for people with autism, https://www.theglobeandmail.com/life/home-and-garden/design/design-empathy-buildsinclusive-spaces-for-people-with-autism/article23966012/

http://www.northernautism.vic.edu.au/?page_id=84

Hede Arhcitects, http://www.hedearchitects.com.au/Northern-School-for-Autism/

Villa delle Rogge - Pordenone - 2012:

Copat, O. (2013) L'inclusione lavorativa delle persone con autismo: l'esperienza della Fondazione Bambini e Autismo ONLUS, relazione a 'Autismo: i punti fermi per la promozione dell'integrazione educativa e sociale', Milano, 31 Maggio 2013

Del Duca, D. Raffin, C. Sedran, E. (2003) Un weekend di respiro - Un modello nuovo di programma respiro per persone con autismo, Milano: Franco Angeli.

Design for all Udine https://designforalludine.wordpress.com/2013/02/27/limportanza-dellaprogettazione-

condivisa-villa-delle-rogge/

Canevaro, A. Ianes, D. (a cura di) (2003) Diversabilità. Storie e dialoghi nell'anno europeo delle persone disabili, Ed. Erickson, Trento

Raffin, C. (2001a) La violence qui se cache derrière le problème de l'autisme, Thérapie Familiale, 22 (1): 21-38

Raffin C. (2001b) Multidisciplinary work for children with autism, Educational and Child Psychology, 18 (2):15-27

Raffin, C. (2001c) Educazione strutturata: quando e con chi, Percorsi di integrazione, 26/27:65-72

Superando (2012) A Pordenone si inaugura una "palestra per l'autonomia",

http://www.superando.it/2012/11/29/a-pordenone-si-inaugura-una-palestra-per-lautonomia/

Trigari, M. (2017) Mamma, vado a vivere da solo (o con gli amici), SuperAbile Inail, agostosettembre 2017, vol 8-9, p. 12-21

http://www.bambinieautismo.org/

http://www.bambinieautismo.org/contenuti-video

http://www.bambinieautismo.org/index.php?option=com_content\&view=article\&id=61

intervista alla Presidente dott.ssa Cinzia Raffin

intervista alla psicologa dott.ssa M. Filippini

intervista a utenti e genitori della Fondazione Bambini e Autismo Onlus

sopralluogo diretto

Sweetwater Spectrum Community - Sonoma (USA) - 2013:

Ahrentzen, S. Steele, K. (2009) Advancing Full Spectrum Housing: Design for Adults with Autism Spectrum Disorders Technical report, Arizona Board of Regents, Phoenix

Resnik , D. D. et al. (2009) Opening Doors: A discussion of residential options for adults living with autism and related disorders, Collaborative report by the Urban Land Institute Arizona, Southwest

Autism Research \& Resource Center and Arizona State University 
Ambienti sensoriali "terapeutici" che rendano Abili

King, J. (2016) Welcome Home. A community for adults with autism shows the power of an understated landscape, in Landscape Architecture Magazine, vol. 10 Land Matters, February 2016 , pp. $68-80$

Maytum, M. Fuertes, G. (2016) Finding the balance point, High Performing Buildings, Winter 2016, pp. 14-22

Henry, C. (2011) Designing for Autism: More Able Not Less Disabled, ArchDaily http://www.archdaily.com/190322/designing-for-autism-more-able-not-less-disabled/

LMS Architects (2013), Sweetwater Spectrum Community / LMS Architects, ArchDaily http:/www.archdaily.com/446972/sweetwater-spectrum-community-lms-architects

The American Institute of Architects, Sweetwater Spectrum Community http://www.aiatopten.org/node/369

http://www.lmsarch.com/projects/sweetwater-spectrum-community?f1=all

Seniors House - Hinnerup (Danimarca) - 2014:

Frier Architecture, http://www.frierarchitecture.dk/?p=599

Seniors House. The building and the architectural ideas behind Denmark's first homes for the elderly designed for people with autism, Specialområde Autisme, Hinnerup

http://www.sau.rm.dk/siteassets/om-os/about-us/seniors-house-specialist-area-autism.pdf http://www.sau.rm.dk/om-os/about-us/physical-framework/seniors-house/

Salman, S. (2013) We need to know and do more about ageing with autism, The Guardian, social care network: adult social care, https://www.theguardian.com/social-carenetwork/2013/aug/15/ageingwith-autism-know-more

Casa Sebastiano - Coredo (Trento) - 2017:

https://www.fondazionetrentinaautismo.it/it/

https://www.fondazionetrentinaautismo.it/it/progetti/

InfoBuild (2017) Casa Sebastiano, una vera casa accogliente, innovativa e tecnologica,

http://www.infobuild.it/2017/08/casa-sebastiano/

Pernoiautistici (2017) Benvenuti a Casa Sebastiano, il Paradiso sulle Dolomiti degli Autistici, http:/www.pernoiautistici.com/2017/09/benvenuti-a-casa-sebastiano-il-paradiso-sulle-

dolomiti-degliautistici/

Superando (2017) Casa Sebastiano: la realizzaizone di un sogno,

http://www.superando.it/2017/03/27/casa-sebastiano-la-realizzazione-di-un-sogno/

Visetti, G. (2017) La casa sulle Dolomiti per i ragazzi autistici: "Qui imparano a crescere da soli", La

Repubblica

http://www.repubblica.it/cronaca/2017/03/31/news/la_casa_sulle_dolomiti_per_i_ragazzi_aut istici q

ui_imparano_a_crescere_da_soli_-161837544/

Film «Tommy e gli altri», regia Massimiliano Sbrolla, scritto da Gianluca Nicoletti intervista all'arch. Giovanni Berti

sopralluogo diretto

Royal Alexandra Children's Hospital - Brighton (UK) - 2007/2016:

Architecture \& Design Scotland, Royal Alexandra Children's Hospital, http://www.ads.org.uk/royalalexandria-childrens-hospital/

BDP, Humanising Healthcare, http:/www.bdp.com/globalassets/about/publications/healthcare-bybdp.pdf 


\section{Elena Bellini}

http://www.theroyalalex.co.uk/

BDP, Lighting, http://www.bdp.com/globalassets/about/publications/lighting-by-bdp.pdf

BDP, Design, http://www.bdp.com/globalassets/about/publications/interior-design.pdf

BDP (2016) Vibrancy, http://www.bdp.com/globalassets/about/publications/vibrancy.pdf

http://www.bdp.com/en/projects/p-z/Royal-Alexandra-Childrens-Hospital/

Sea Life sensory room, http://www.rockinghorse.org.uk/news/sea-life-sensory-room/

https://www.youtube.com/watch?v=U3qjPviIR4Q

Emma Children's Hospital - Amsterdam (Paesi Bassi) - 2014:

Pinhão, C. S. F. (2016)Children's Hospitals. The role of architecture in children's recovery and development, tesi di Architettura al Técnico Lisboa

Wagenaar, C. (ed.) (2006) The architecture of hospitals, Rotterdam : NAI Publishers

Mens, N. Wagenaar, C. (2010) Health care architecture in the Netherlands, Rotterdam : NAI Publishers

http://www.opera-amsterdam.nl/projects.php?c=3

http://www.dvdp.nl/en/work/emma-childrens-hospital-amsterdam

Nemours Children's Hospital - Orlando (USA) - 2014/2016:

Wilson, J. (2016) Redesigned ER Model limits stressors for Children with Autism Spectrum Disorders.

Pilot Program Improves Care by Reducing Sensory Overload, Orlando

https://www.nemours.org/about/mediaroom/press/florida/redesigned-er-model-limitsstressors-forchildren-with-autism-spectrum-disorder.html

https://www.nemours.org/welcome.html

http://stanleybeamansears.com/project/nemours_childrens_hospital/

Nemours Children's Hospital / Stanley Beaman \& Sears (2013) ArchDaily, https://www.archdaily.com/439396/nemours-children-s-hospital-stanley-beaman-andsears

IDEO, A Hospital Centered on the Patient Experience, https://www.ideo.com/case-study/ahospitalcentered-on-the-patient-experience

Programma REACH, http://www.orlandosentinel.com/health/os-nemours-autism-friendly-er-

20160221-story.html

iDesign production, http://idesignproductions.com/projects/nemours/

Luthra, S. (2016) Helping patients with autism navigate the stressful ER, Kaiser Health News, http://edition.cnn.com/2016/02/29/health/autism-patient-care-er/index.html

Miller, N. S. (2016) Emergency Rooms Go Sensory-Friendly, Disability Scoop, https://www.disabilityscoop.com/2016/02/23/emergency-rooms-sensory/21946/Evelina

London Children's Hospital - Londra (UK) - 2016:

Clift, S. Camic, P. C. (a cura di) (2016) Oxford Textbook of Creative Arts, Health, and Wellbeing:International Perspectives on Practice, Policy and Research, Oxford University Press

Criddle, J. Jones, M. (2017) Taking the "ouch!" out of emergency: using illustrative and digital arts to guide, reassure and role-model behaviours at the children's emergency department, Evelina London Children's Hospital, atti di convegno dell'European Healthcare Design 2017, 12-13 giugno 2017

Del Nord, R. (a cura di) (2006) Lo stress ambientale nel progetto dell'ospedale pediatrico: indirizzi tecnici e suggestioni architettoniche, Motta Architettura, Milano 
Ambienti sensoriali "terapeutici” che rendano Abili

Marcus, C. C. (2017) Healing gardens in healthcare - the necessity of nature, atti di convegno dell'European Healthcare Design 2017, 12-13 giugno 2017

Verderber, S. (2010) Innovations in Hospital Architecture, Routledge, London

Buxton, B. (2015) Designing out stress on paediatric wards, Riba J, Architecture Information\&Inspiration, https://www.ribaj.com/products/designing-out-stress-on-paediatricwards

http://evelinalondon.nhs.uk/Home.aspx

http://www.hopkins.co.uk/projects/6/9/

https://www.burohappold.com/projects/evelina-childrens-hospital/

virtual tours - http://www.evelinalondon.nhs.uk/parents-and-visitors/getting-here/virtualtours/ outside-evelina-london.aspx

neuroscience department - http://www.evelinalondon.nhs.uk/ourservices/

hospital/neurosciences/overview.aspx

Art in Site - http://www.artinsite.co.uk/evelina-childrens-hospital-and-paediatric-oral-health

Demo of the App - https://vimeo.com/205356030/4ad8b62c4a

\section{Capitolo 5}

Arneill, A. B. Devlin, A. S. (2002) Perceived Quality Of Care: The Influence Of The waiting Room Environment, in Journal of Environmental Psychology (2002) 22, 345-360, Elsevier Science Ltd.

Berliri, L. V. Pannocchia, N. (2014) Persone con disabilità e ospedale: principi, esperienze, buone prassi, Erickson, Trento

Bishop, K. (2012)The Role of Art in a Paediatric Healthcare Environment from Children's and Young People's Perspectives, in Procedia - Social and Behavioral Sciences ,38 ( 2012 ) 81 - 88, Elsevier

Bonaiuto, M. Bilotta, E. Fornara, F. (2004) Che cos'è la psicologia architettonica, Carocci editore, Roma

Bowman, S. Jones, R. (2016) Sensory Interventions for Psychiatric Crisis in Emergency Departments-A New Paradigm, Journal of Psychiatry and Mental Health, 1(1)

Cama, R. (2009) Evidence-based healthcare design, Hoboken : J. Wiley

Campos Andrade, C. et al. (2012) Users' views of hospital environmental quality: Validation of the Perceived Hospital Environment Quality Indicators (PHEQIs), Journal of Environmental Psychology, 32 (2012) 97e111, Elsevier

Campos Andrade, C. et al. (2013) Inpatients' and outpatients' satisfaction: The mediating role of perceived quality of physical and social environment, Health \& Place, 21 (2013) 122-132, Elsevier

CARD - The Center for Autism \& Related Disabilities, Autism and the Hospital Emergency Room. A practical guide for health professionals to meet the needs of individuals with Autism Spectrum Disorders, CARD, TAMPA, Florida

Casey, L. B. et al. (2015) Emergency department visits by children with and without autism spectrum disorder: An initial comparison evaluating multiple outcome measures at one urban children's hospital, in Research in Autism Spectrum Disorders, 9 (2015) 144-150, Elsevier

Cattanei, M. (2009) Il Progetto DAMA un convegno per presentare e stimolare. Infermiere a Pavia, 21(1), 4-5

Cattanei, M. (2010) Progetto D.A.M.A. L'avvio a Pavia. Infermiere a Pavia, 22(2), 5-6

Champagne, T. (2004) Sensory Approaches in Inpatient Psychiatric Settings. Innovative Alternatives to Seclusion \& Restraint, Journal of Psychological Nursing, VOL. 42, NO. 9 


\section{Elena Bellini}

Corona, M. Ghelma, F. Maioli, M. Perazzoli, S. Mantovani, A. (2003) Il Progetto DAMA all'Ospedale San Paolo: una proposta di accoglienza medica rivolta ai disabili con ritardo mentale, AJMR (Ed. Italiana), 1(2), 100-106

Croen, L. A. et al. (2006) A Comparison of Health Care Utilization and Costs of Children With and Without Autism Spectrum Disorders in a Large Group-Model Health Plan, Pediatrics, Volume 118, Number 4, October 2006, pp. e1203-1211

Del Nord, R. (2006) Lo stress ambientale nel progetto dell'ospedale pediatrico. Indirizzi tecnici e suggestioni architettoniche, Motta Architettura, Milano

Del Nord, R. (2009) The culture for the future of healthcare architecture. Proceedings of 28tn International Public Health Seminar, Alinea, Firenze

Del Nord, R. (2014) Il ruolo strategico della ricerca intersettoriale nella progettazione delle strutture ospedaliere: il contributo del Centro Ricerche TESIS, TECHNE

Del Nord, R. Marino, D. Peretti, G. (2015) L'umanizzazione degli spazi di cura: una ricerca svolta per il Ministero della Salute italiano, in TECHNE, vol. 09, pp. 224-229, FUP

DGR 666/2017 del 19/06/2017, Equità di accesso ai servizi sanitari delle persone con disabilità. Approvazione delle linee di indirizzo e del modello di intervento regionale PASS Percorsi Assistenziali per Soggetti con bisogni Speciali, Regione Toscana (http://open.toscana.it/web/toscana-accessibile)

Dijkstra, K. et al. (2006) Physical environmental stimuli that turn healthcare facilities into healing environments through psychologically mediated effects: systematic review, Integrative literature reviews and meta-analyses, Blackwell Publishing Ltd

Dunn, W. Little, L. (2013) Designing Sensory Friendly Environments for Children with Autism Spectrum Disorder (ASD), Design for All Institute of India, Special Issue, December 2015, Vol. 10, No. 13, pp. 26-32

Ferrante, T. (2012) Architetture e design per l'hospice pediatrico, Alinea, Firenze

Ferrante, T. (2013) Valutare la qualità percepita: uno studio pilota per gli Hospice, Franco Angeli, Milano

Fornara, F. (2005) Users' evaluative responses to spatio-physical humanization: the case of hospital environments, in Martens, B., Keul, A.G. (a cura di) Designing Social Innovation: Planning, Building, Evaluating, Hogrefe \& Huber, Gottingen (Germany), pp. 231240

Fornara, F., Bonaiuto, M., Bonnes, M. (2006) Perceived hospital environment quality indicators: a study of orthopaedic units, Journal of Environmental Psychology 26, 321-334

Ghelma, F. (2017) Mascheramento diagnostico e compliance al trattamento, atti del XII Convegno Nazionale sulla Qualità della Vita. Progettare il Futuro, 11-12 settembre 2017,Università Cattolica del Sacro Cuore, Milano

Giovenale, A. M. (a cura di) (2006) Qualità degli spazi e prevenzione dello stress. Indirizzi tecnici per la progettazione del Day Hospital, Alinea, Firenze

Gurney, J. G. et al. (2006) Parental Report of Health Conditions and Health Care Use Among Children With and Without Autism, National Survey of Children's Health, Arch Pediatr Adolesc Med, 160:825-830

Hoffman, H. G. Et Al. (2001) The Effectiveness Of Virtual Reality For Dental Pain Control: A Case Study, Cyberpsychology \& Behavior, Volume 4, Number 4, 2001, Mary Ann Liebert, Inc.

Hogg, J. et al. (2001) The use of 'Snoezelen' as multisensory stimulation with people with intellectual disabilities: a review of the research in Research in Developmental Disabilities, 22 (2001) 353-372, Pergamon

HCUP (2010) Nationwide Emergency Department (NEDS), Healthcare Cost and Utilization Project (HCUP), Agency for Healthcare Research and Quality, Rockville, MD (USA) 
$\mathrm{Hu}$, J. et al. (2009) Effects of dental 3D multimedia system on the performance of junior dental students in preclinical practice: a report from China, Advanced in Health and Science Education, 14:123-133, Springer Science+Business Media

Iannuzzi, D. et al. (2015) Brief Report: Emergency Department Utilization by Individuals with Autism, Journal of Autism and Development Disorders 45:1096-1102

Kaplan, H. et al. (2006) Snoezelen multi-sensory environments: Task engagement and generalization, in Research in Developmental Disabilities, 27 (2006) 443-455, Elsevier

Lotan, M. Gold, c. (2009) Meta-analysis of the effectiveness of individual intervention in the controlled multisensory environment (Snoezelen) for individuals with intelectual disability, Journal of mental and intellectual disabilities, 34(3):207-15

Lotan, M. Shapiro, M. (2005) Management of young children with Rett disorder in the controlled multisensory (Snoezelen) environment, in Brain \& Development 27 (2005) S88S94, Elsevier

Lunsky, Y. et al. (2015) Predictors of emergency service use in adolescents and adults with autism spectrum disorder living with family, Emerg Med Journal, 32:787-792

Maiocchi, M. (a cura di) (2007) La comunicazione emozionale negli ambienti ospedalieri, Maggioli Editore, Santarcangelo di Romagna (RN)

Nayfack, A. M. et al. (2014) Hospitalizations of Children with Autism Increased from 1999 to 2009, Journal of Autism and Development Disorders, 44:1087-1094

Nicholas, D.B. Zwaigenbaum, L. Muskat, B. et al. (2016) Toward Practice Advancement in Emergency Care for Children With Autism Spectrum Disorder, Pediatrics, 137(S2): S206-S211

Novak, T. et al. (2012) Pilot study of a sensory room in an acute inpatient psychiatric unit, Australasian Psychiatry Vol 20, Issue 5, pp. 401-406

Pessina, A. (2010) Barriere della mente e barriere del corpo. Annotazioni per un' etica della soggettività empirica, in Paradoxa, Etica della condizione umana, Vita e Pensiero, Milano

Pratt, K. et al. (2011) Ensuring successful admission to hospital for young people with learning difficulties, autism and challenging behaviour: a continuous quality improvement and change management programme, Blackwell Publishing Ltd, Child: care, health and development, 38, 6, 789-797

Rebel, R. (2014) Specialized hospitals : design \& planning, North Point : Design media publishing limited Royal Cornwall Hospital, NHS Trust (2014) Protocol for Children and Adults with Autism Spectrum Conditions

Scarpinato, N. et al. (2010) Caring for the Child With an Autism Spectrum Disorder in the Acute Care Setting, Journal for specialists in Pediatric Nursing, Wiley Periodicals, Inc.

Sing, N. N. et al. (2004) Effects of Snoezelen room, Activities of Daily Living skills training, and Vocational skills training on aggression and self-injury by adults with mental retardation and mental illness, in Research in Developmental Disabilities, 25 (2004) 285-293, Elsevier

Spes contra Spem (2013) Carta Dei Diritti Delle Persone Con Disabilità In Ospedale, Roma

Spinelli, F. Bellini, E. Bocci, P. Fossati, R. (1994) Lo spazio terapeutico. Un metodo per il progetto di umanizzazione degli spazi ospedalieri, Alinea, Firenze

Standen, P. J. Brown, D. J. (2005) Virtual Reality in the Rehabilitation of People with Intellectual Disabilities: Review, in Cyberpsychology \& Behavior, Volume 8, Number 3, 2005, Mary Ann Liebert, Inc

Sutton, D. et al. (2013) Optimizing arousal to manage aggression: A pilot study of sensory modulation, International Journal of Mental Health Nursing (2013) 22, 500-511

Ulrich, R. Zimring, C. Xiaobo, Q. Anjali, J. Choudhary, R (2004) The role of the physical environment in the hospital of the 21th century: a once-in-a-lifetime opportunity, Report to The Center for Health Design for the Designing the 21st Century Hospital Project, Concord (USA) 


\section{Elena Bellini}

Verderber, S. (2010) Innovations in Hospital Architecture, Routledge, London

Vohra, R. (2016) Emergency Department Use Among Adults with Autism Spectrum Disorders (ASD), Journal of Autism and Development Disorders, 46:1441-1454

Weingerg, A. Creed, F. (2000) Stress and psychiatric disorder in health care professionals and hospital staff, in "The Lacet", 355, February 12, pp.533-537

Wiederhold, M. D. Et Al. (2014) Clinical Use Of Virtual Reality Distraction System To Reduce Anxiety And Pain In Dental Procedures, Cyberpsychology, Behavior, And Social Networking Volume 17, Number 6, 2014, Mary Ann Liebert, Inc

Woo, C. C. Donnelly, J. H. Steinberg-Epstein, R. Leon, M. (2015) Environmental Enrichment as a Therapy for Autism: A Clinical Trial Replication and Extension, in Behavioral Neuroscience, Vol. 129, No. 4, 412-422, American Psychological Association

Zwaigenbaum, L. et al. (2016) Perspectives of Health Care Providers Regarding Emergency Department Care of Children and Youth with Autism Spectrum Disorder, Journal of Autism and Development Disorders, 46:1725-1736

\section{Capitolo 7}

Atipical Familia - http://atypicalfamilia.com/ -

Architetture fo Autism - http://www.architectuur-voor-autisme.org/ -

Schrameijer, F. (2013) Met het oog op autisme. Bouwen \& inrichten voor mensen met autisme, Doorwerth, Dr. Leo Kannerhuis, november 2013

Schrameijer, F. (2016) About autism-friendly design, Workshop Inservice Autism, November 9 through 11, 2016, Oost Malle, Belgium

Autism - https://www.autism.com/-

Autism Community - http://www.autism-community.com/-

Autism Journey - http://autismjourney.org/blog/ -

Autism Now - http://autismnow.org/-

Autismo Online - http://autismo.inews.it/ -

Autism Parenting Magazine -https://www.autismparentingmagazine.com/-

Autism Toolbox - http://www.autismtoolbox.co.uk/-

Car Autism Roadmap - https://www.carautismroadmap.org/-

Department for Children, Schools and Families, UK (2012) Building Bulletin 102: Designing for Disabled

Children and Children with Special Educational Needs,

http://media.education.gov.uk/assets/files/pdf/b/building\%20bulletin\%20102.pdf

Emergenza Autismo - http://www.emergenzautismo.org/ -

Francis, P. Balbo, S. Firth, L. (2009) Towards co-design with users who have autism spectrum disorders, Universal Access in the Information Society, 8:123-135, Springer-Verlag

Fondazione ARES - http://www.fondazioneares.com/ -

Gaudion, K., Hall, A., Myerson, J., and Pellicano, L. (2015) Design and wellbeing: Bridging the empathy gap between neurotypical designers and autistic adults, in Mani, M. and Kandachar, P. (2015) Design for Sustainable Well-being and Empowerment, IISc Press and TU Delft, 61-77

Just a Lil blog - http://www.justalilblog.com/ -

National autism network - http://nationalautismnetwork.com/-

Ollibean - https://ollibean.com/-

Per noi autistici - http://www.pernoiautistici.com/ -

The Autism Society of America - http://www.autism-society.org/ -

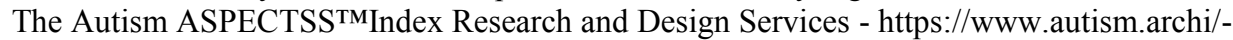
Mostafa, M. (2014) Architecture For Autism: Autism ASPECTSS ${ }^{\text {TM }}$ in School Design, in Archnet-IJAR, Volume 8 - Issue 1 - March 2014 - (143-158) 
Ambienti sensoriali "terapeutici" che rendano Abili

Mostafa, M. (2014) Architecture for Autism: Application of the Autism ASPECTSSTM Design Index to Home Environments, in The International Journal of the Constructed Environment, Volume 4, Issue 2, April 2014, pp.25-38

Mostafa, M. (2015) Architecture for Autism: Built Environment Performance in Accordance to the Autism ASPECTSS ${ }^{\text {TM }}$ Design Index, in Design Principles and practices: an International Journal - Annual Review, vol. 8, pp.55-71,Common Ground Publishing, Champaign, Illinois, USA

The Autism Site - http://blog.theautismsite.com/-

The Center for Health Design - https://www.healthdesign.org/-

The NAS - National Autistic Society - http://www.autism.org.uk/ -

Thinking Autism Guide -http://www.thinkingautismguide.com/-

WBDG - Whole Building Design Guide - https://www.wbdg.org/- 


\section{Ringraziamenti}

Ringrazio prima di tutto le persone che hanno avuto voglia di raccontarmi le loro storie, che mi hanno regalato il loro tempo e che pazientemente hanno risposto a tutte le mie domande. Ringrazio quindi le famiglie che hanno aderito alla diffusione dei questionari e tutte le persone, le Associazioni e i Centri per l'Autismo che si sono resi disponibili a questo, oltre a farmi visitare le loro strutture. In particolare, tra questi, ringrazio:

La Fondazione Bambini e Autismo Onlus di Pordenone, che mi ha aiutato nella composizione dei questionari, mi ha fatto visitare i loro centri e ospitato per più volte nella loro struttura facendomi conoscere i loro bambini e ragazzi, le famiglie e gli operatori che vi lavorano ogni giorno. La dott.ssa Filippin e la dott.ssa Raffin, che fin dall'inizio hanno accolto con entusiasmo e disponibilità questa collaborazione di ricerca.

ANGSA Novara-Vercelli, che mi ha fatto conoscere tante belle persone, disponibili a raccontarmi di sé, di cui conservo sempre una vivida fotografia stampata nella mente. Prima tra tutti Fiorella, che da subito mi ha colpito con le sue parole soavi, la precisione nei racconti, la pazienza e i dolci sorrisi. Ringrazio quindi Lorenza e tutte le altre psicologhe e operatrici del centro, per tutto quello che mi hanno dato, la gentilezza e l'accoglienza, oltre ovviamente alla professionalità.

PAMAPI di Firenze ed in particolare Luciano, Daniele e Michele, che mi hanno coinvolto nella ricerca sugli spazi di attesa autism-friendly al Pronto Soccorso, dandomi la possibilità di sperimentare su Careggi il prototipo di stanza sensoriale. Le famiglie, a partire da Luciano, babbo e presidente, per il calore e l'accoglienza con cui mi hanno aperto la porta.

Ringrazio tutti i professionisti e gli esperti che mi ha regalato tempo e conoscenza, permettendomi di andare avanti in questo percorso complesso e ricco di tante discipline. Tra questi, il CIRPA, il prof. Bonaiuto e Gloria, che mi hanno accolto durante lo stage di ricerca.

Non per ultimo, ringrazio poi il mio tutor, la prof.ssa Torricelli, Nicoletta e tutti coloro con cui ho collaborato durante questi anni all'interno del Dipartimento, o che mi hanno in qualche modo guidato e consigliato lungo la strada.

Nominare tutti coloro che mi sono stati vicini in questi anni sarebbe impossibile, avrei bisogno di dedicare almeno una pagina a ciascuno di loro e non sarebbe sufficiente, mentre preferisco farlo in modo diverso ogni giorno quando siamo vicini. In fondo, li ringrazio non tanto per questo, ma per continuare a starmi accanto, fisicamente o meno. Chi lavora al mio fianco e condivide con me fatiche e soddisfazioni, chi è riuscito a regalarmi una risata, chi ci ha creduto più di me, chi mi ha supportato con le parole, una telefonata, un abbraccio o un semplice sorriso... e ovviamente chi mi aspetta a casa, con pazienza.

Solo un ringraziamento particolare va a Maria, che in fondo ha un po' di co-responsabilità in tutto questo, anche solo per avermi fatto conoscere la possibilità ed il significato di un dottorato di ricerca. 

ANNO 2007

Bracardi M., La Materia e lo Spirito. Mario Ridolfi nel paesaggio umbro

Coppi E., Purines as Transmitter Molecules. Electrophysiological Studies on Purinergic Signalling in Different Cell Systems

Mannini M., Molecular Magnetic Materials on Solid Surfaces

Natali I., The Ur-Portrait. Stephen Hero ed il processo di creazione artistica in A Portrait of the Artist as a Young Man

Petretto L., Imprenditore ed Università nello start-up di impresa. Ruoli e relazioni critiche

ANNO 2008

Bemporad F., Folding and Aggregation Studies in the Acylphosphatase-Like Family

Buono A., Esercito, istituzioni, territorio. Alloggiamenti militari e «case Herme» nello Stato di Milano (secoli XVI e XVII)

Castenasi S., La finanza di progetto tra interesse pubblico e interessi privati

Colica G., Use of Microorganisms in the Removal of Pollutants from the Wastewater

Gabbiani C., Proteins as Possible Targets for Antitumor Metal Complexes: Biophysical Studies of their Interactions

ANNO 2009

Decorosi F., Studio di ceppi batterici per il biorisanamento di suoli contaminati da Cr(VI)

Di Carlo P., I Kalasha del Hindu Kush: ricerche linguistiche e antropologiche

Di Patti F., Finite-Size Effects in Stochastic Models of Population Dynamics: Applications to Biomedicine and Biology

Inzitari M., Determinants of Mobility Disability in Older Adults: Evidence from Population-Based Epidemiologic Studies

Macrì F., Verso un nuovo diritto penale sessuale. Diritto vivente, diritto comparato e prospettive di riforma della disciplina dei reati sessuali in Italia

Pace R., Identità e diritti delle donne. Per una cittadinanza di genere nella formazione

Vignolini S., Sub-Wavelength Probing and Modification of Complex Photonic Structures

ANNO 2010

Fedi M., "Tuo lumine». L'accademia dei Risvegliati e lo spettacolo a Pistoia tra Sei e Settecento

Fondi M., Bioinformatics of genome evolution: from ancestral to modern metabolism. Phylogenomics and comparative genomics to understand microbial evolution

Marino E., An Integrated Nonlinear Wind-Waves Model for Offshore Wind Turbines

Orsi V., Crisi e Rigenerazione nella valle dell'Alto Khabur (Siria). La produzione ceramica nel passaggio dal Bronzo Antico al Bronzo Medio

Polito C., Molecular imaging in Parkinson's disease

Romano R., Smart Skin Envelope. Integrazione architettonica di tecnologie dinamiche e innovative per il risparmio energetico

ANNO 2011

Acciaioli S., Il trompe-l' $e i l$ letterario, ovvero il sorriso ironico nell'opera di Wilhelm Hauff

Bernacchioni C., Sfingolipidi bioattivi e loro ruolo nell'azione biologica di fattori di crescita e citochine

Fabbri N., Bragg spectroscopy of quantum gases: Exploring physics in one dimension

Gordillo Hervás R., La construcción religiosa de la Hélade imperial: El Panhelenion

Mugelli C., Indipendenza e professionalità del giudice in Cina

Pollastri S., Il ruolo di TAF12B e UVR3 nel ciclo circadiano dei vegetali

Salizzoni E., Paesaggi Protetti. Laboratori di sperimentazione per il paesaggio costiero euro-mediterraneo 
ANNO 2012

Evangelisti E., Structural and functional aspects of membranes: the involvement of lipid rafts in Alzheimer's disease pathogenesis. The interplay between protein oligomers and plasma membrane physicochemical features in determining cytotoxicity

Bondì D., Filosofia e storiografia nel dibattito anglo-americano sulla svolta linguistica

Petrucci F., Petri Candidi Decembrii Epistolarum iuvenilium libri octo. A cura di Federico Petrucci

Alberti M., La 'scoperta' dei disoccupati. Alle origini dell'indagine statistica sulla disoccupazione nell'Italia liberale (1893-1915)

Gualdani R., Using the Patch-Clamp technique to shed light on ion channels structure, function and pharmacology

Adessi A., Hydrogen production using Purple Non-Sulfur Bacteria (PNSB) cultivated under natural or artificial light conditions with synthetic or fermentation derived substrates

Ramalli A., Development of novel ultrasound techniques for imaging and elastography. From simulation to real-time implementation

ANNO 2013

Lunghi C., Early cross-modal interactions and adult human visual cortical plasticity revealed by binocular rivalry

Brancasi I., Architettura e illuminismo: filosofia e progetti di città nel tardo Settecento francese

Cucinotta E., Produzione poetica e storia nella prassi e nella teoria greca di età classica

Pellegrini L., Circostanze del reato: trasformazioni in atto e prospettive di riforma

Locatelli M., Mid infrared digital holography and terahertz imaging

Muniz Miranda F., Modelling of spectroscipic and structural properties using molecular dynamics

Bacci M., Dinamica molecolare e modelli al continuo per il trasporto di molecole proteiche - Coarsegrained molecular dynamics and continuum models for the transport of protein molecole

Martelli R., Characteristics of raw and cooked fillets in species of actual and potential interest for italian aquaculture: rainbow trout (oncorhynchus mykiss) and meagre (argyrosomus regius)

ANNO 2014

Lana D., A study on cholinergic signal transduction pathways involved in short term and long term memory formation in the rat hippocampus. Molecular and cellular alterations underlying memory impairments in animal models of neurodegeneration

Lopez Garcia A., Los Auditoria de Roma y el Athenaeum de Adriano

Pastorelli G., L'immagine del cane in Franz Kafka

Bussoletti A., L'età berlusconiana. Il centro-destra dai poli alla Casa della Libertà 1994-2001

Malavolti L., Single molecule magnets sublimated on conducting and magnetic substrates

Belingardi C., Comunanze urbane. Autogestione e cura dei luoghi

Guzzo E., Il tempio nel tempio. Il tombeau di Rousseau al Panthéon di Parigi

ANNO 2015

Lombardi N., MEREAFaPS: uno Studio di Farmacovigilanza Attiva e Farmacoepidemiologia in Pronto Soccorso

Baratta L., "A Marvellous and Strange Event». Racconti di nascite mostruose nell'Inghilterra della prima età moderna

Richichi I.A., La teocrazia: crisi e trasformazione di un modello politico nell'Europa del XVIII secolo

Palandri L., I giudici e l'arte. Stati Uniti ed Europa a confronto

Caselli N., Imaging and engineering optical localized modes at the nano scale

Calabrese G., Study and design of topologies and components for high power density dc-dc converters

Porzilli S., Rilevare l'architettura in legno. Protocolli metodologici per la documentazione delle architetture tradizionali lignee: $i$ casi studio dei villaggi careliani in Russia 
ANNO 2016

Martinelli S., Study of intracellular signaling pathways in Chronic Myeloproliferative Neoplasms

Abbado E., "La celeste guida”. L'oratorio musicale a Firenze: 1632-1799

Focarile P., I Mannelli di Firenze. Storia mecenatismo e identità di una famiglia fra cultura mercantile e cultura cortigiana

Nucciotti A., La dimensione normativa dell'imprenditorialità accademica. Tre casi di studio sugli investigatori principali, i loro gruppi di ricerca e i fattori di innesco dell'imprenditorialità accademica

Peruzzi P., La inutilizzabilità della prestazione

Lottini E., Magnetic Nanostructures: a promising approach towards RE-free permanent magnets

Uricchio T., Image Understanding by Socializing the Semantic Gap

ANNO 2017

Valenti R., Cerebral Small Vessel Disease and Cerebral Amyloid Angiopathy: neuroimaging markers, cognitive features and rehabilitative issues

Starnini M., L'uomo tutto intero. Biografia di Carlo Livi, psichiatra dell'Ottocento

Verardi D., La scienza e i segreti della natura a Napoli nel Rinascimento: la magia naturale di Giovan Battista Della Porta

Minicucci G., Il dolo nella bancarotta. Alla ricerca della tipicità soggettiva della fattispecie patrimoniale

Pattelli L., Imaging light transport at the femtosecond scale: a walk on the wild side of diffusion

Egea Molines M.T., Etnobotánica en el Alto Valle del Reno (Toscana y Emilia-Romaña, Italia). Etnobotanica nell'Alta Valle del Reno (Toscana ed Emilia-Romagna, Italia)

Romano I.M., Pressione turistica sul Centro Storico di Firenze - sito UNESCO. Un modello per la valutazione dell'impatto percettivo

ANNO 2018

Costa A., Histaminergic neurotransmission as a gateway for the effects of the fat sensing molecule Oleoylethanolamide. Focus on cognition and stress-reactivity

Solera D., "Sotto l'ombra della patente del Santo Officio». I familiares dell'Inquisizione romana tra XVI e XVII secolo

Landi G., Secession and Referendum. A new Dimension of International Law on Territorial Changes?

Sacchetti A., La costituente libertaria di Camillo Berneri. Un disegno politico tra federalismo e anarchismo

Livi L.F., New quantum simulations with ultracold Ytterbium gases

Bellini E., Ambienti sensoriali "terapeutici” che rendano Abili. Un progetto integrato di vita per persone con Disturbi dello Spettro Autistico

Piscitelli L.R., Serviceability and post-failure behaviour of laminated glass structural elements 
